\title{
Untersuchungen zur Schutzwirkung des Fahrradhelms
}

Klaus Bauer

Sylvia Schick

Anja Wagner

Ketuo Zhou

Steffen Peldschus

Unfallforschung

Axel Malczyk der Versicherer 


\section{Untersuchungen zur Schutzwirkung des Fahrradhelms}

Dipl.-Ing. (FH) Klaus Bauer Dr. med. Sylvia Schick, MPH

Dipl.-Biol. Anja Wagner

Ketuo Zhou, M.Sc. (Comp. Mechanics)

Prof. Dr. rer. biol. hum. Dipl.-Ing. Steffen Peldschus Dr. Axel Malczyk

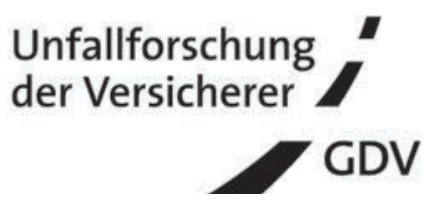




\section{Impressum}

Gesamtverband der Deutschen Versicherungswirtschaft e. V.

Unfallforschung der Versicherer

Wilhelmstraße 43/43G, 10117 Berlin

Postfach 0802 64, 10002 Berlin

E-Mail: unfallforschung@gdv.de

Internet: www.udv.de

Facebook: www.facebook.com/unfallforschung

Twitter: @unfallforschung

YouTube: www.youtube.com/unfallforschung

ISBN-Nr.: 978-3-939163-60-2

Redaktion: Dr. Axel Malczyk

Bildnachweis: UDV und siehe Quellenangaben

Erschienen: 04/2015 


\section{Untersuchungen zur Schutzwirkung des Fahrradhelms}

bearbeitet durch:

Institut für Rechtmedizin München Unfallforschung/Biomechanik

Dipl.-Ing. (FH) Klaus Bauer Dr. med. Sylvia Schick, MPH

Dipl.-Biol. Anja Wagner

Ketuo Zhou, M.Sc. (Comp. Mechanics)

Prof. Dr. rer. biol. hum. Dipl.-Ing. Steffen Peldschus

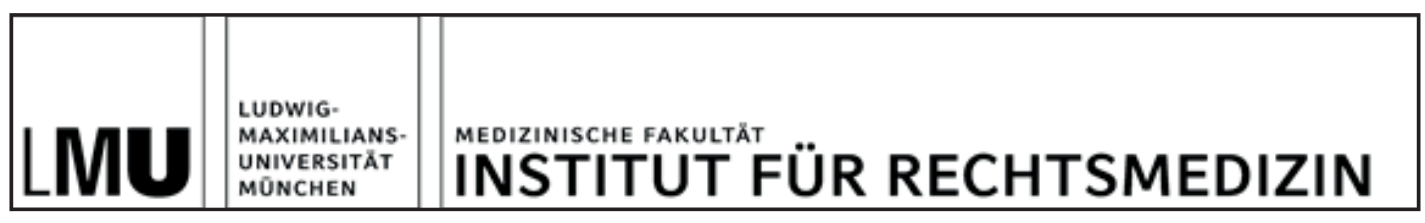

Bei der UDV betreut von:

Dr. Axel Malczyk

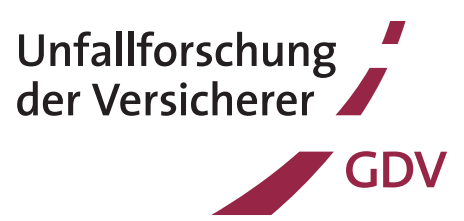





\section{Inhaltsverzeichnis}

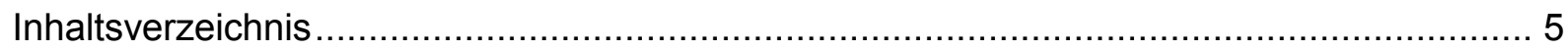

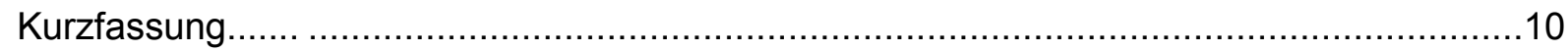

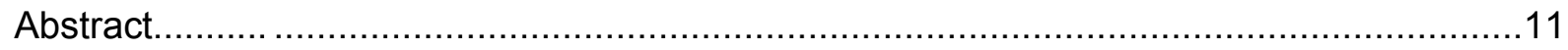

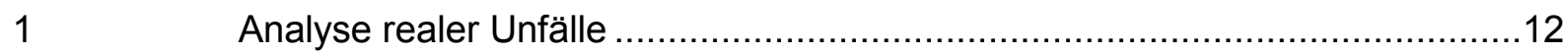

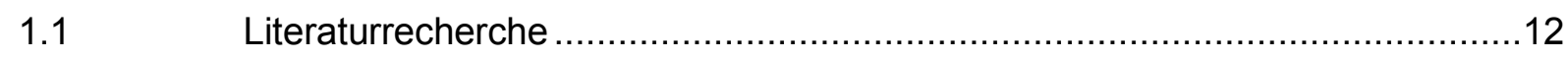

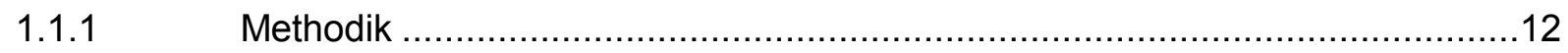

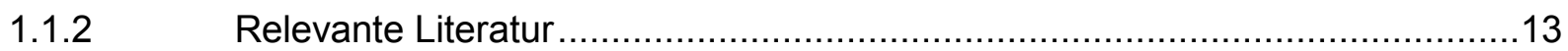

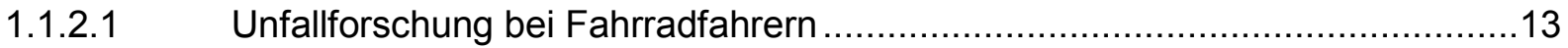

1.1.2.2 Risikoreduktion für Kopfverletzungen durch Helmtragen ..............................17

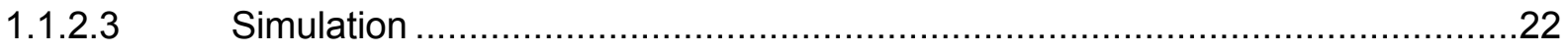

1.1.2.4 Helmtests und Helmeigenschaften .................................................25

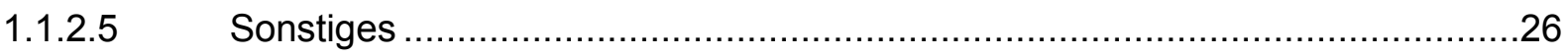

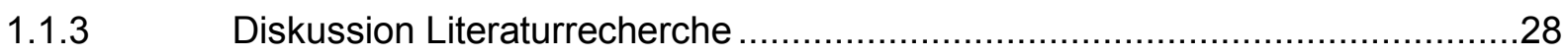

$1.2 \quad$ Definition helmrelevanter Parameter ..........................................................

1.3 Auswahl der im Detail zu rekonstruierenden Unfälle .................................32

1.3.1 Vorliegendes Material und Datenbankaufbau SUD …................................33

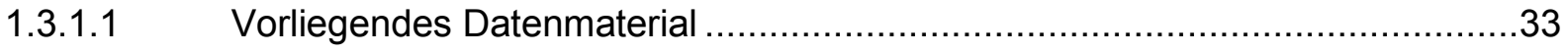

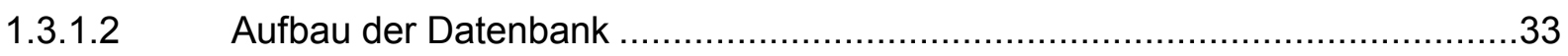

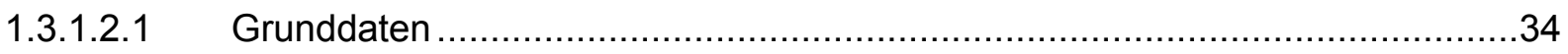

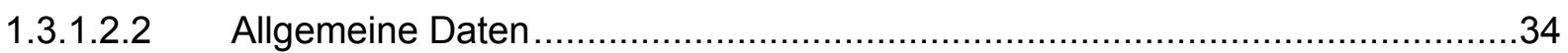

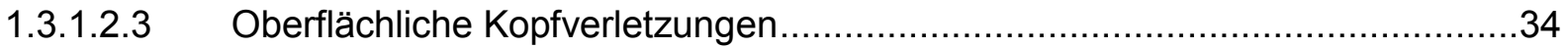

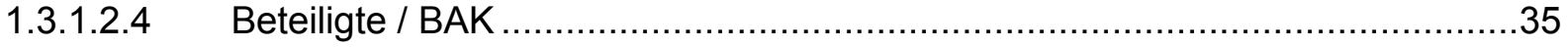

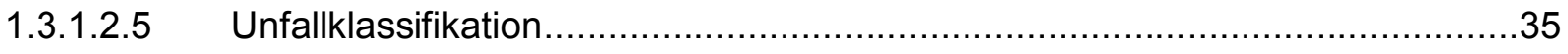

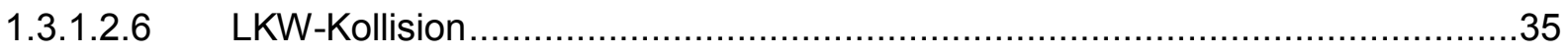

1.3.1.2.7 Geschwindigkeiten / Schleuderweiten / Anprall Körper...................................35

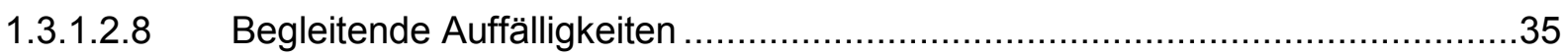

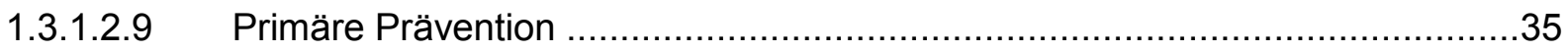




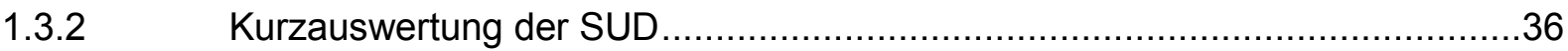

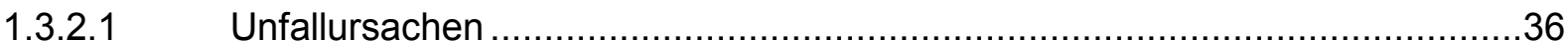

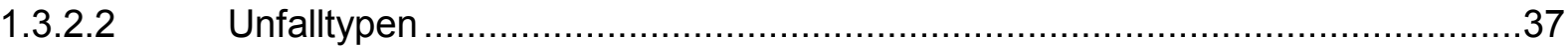

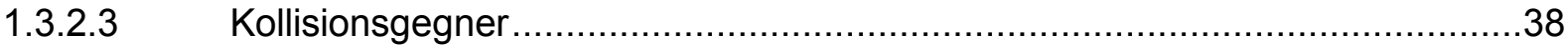

1.3.2.4 Charakteristika bei Unfällen mit Pkw-Beteiligung .....................................38

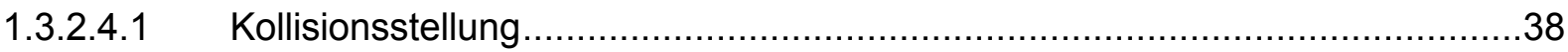

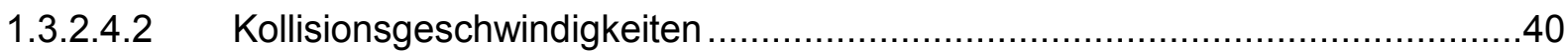

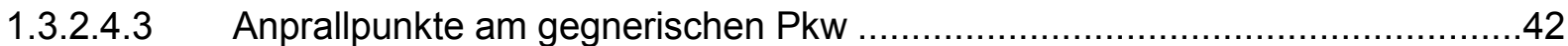

1.3.2.5 Charakteristika der Alleinunfälle .............................................................42

1.3.3 Diskussion der im Detail zu rekonstruierenden Unfälle ................................43

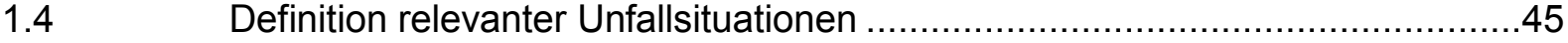

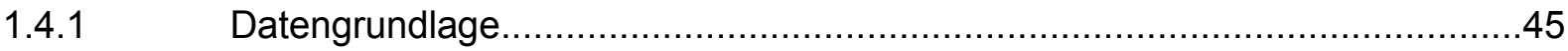

1.4.1.1 Daten des Instituts für Rechtsmedizin München ........................................45

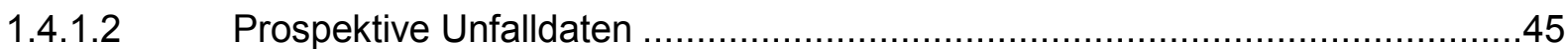

1.4.1.2.1 Daten des Universitätsklinikums München ............................................46

1.4.1.2.2 Daten des Universitätsklinikums Münster ..............................................

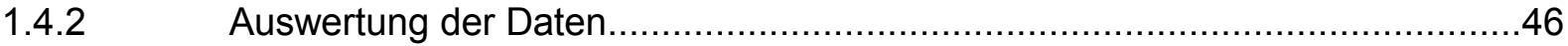

1.4.2.1 Alter der verunfallten Fahrradfahrer.......................................................46

1.4.2.2 Geschlecht der verunfallten Fahrradfahrer ..............................................48

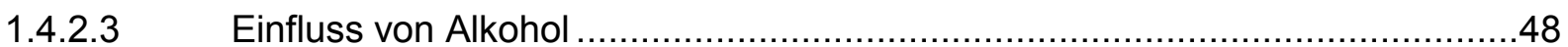

1.4.2.4 Kollisionsart und Unfallursachen ..............................................................

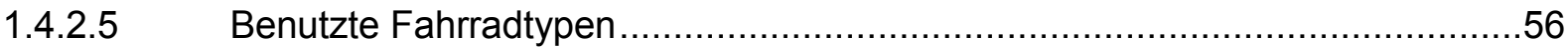

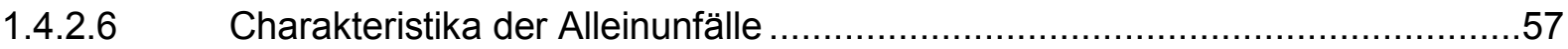

1.4.2.7 Kollisionsstellungen/-geschwindigkeiten bei Unfällen mit Pkw-Beteiligung ......61

1.4.2.8 Anprallpunkte am gegnerischen Pkw, Form der Fahrzeugfront .....................65

1.4.2.9 Helmtragequote, Beschädigungen am Helm ............................................68

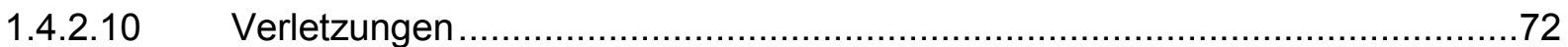

1.4.2.10.1 MAIS, Körperregion des MAIS, ISS .......................................................

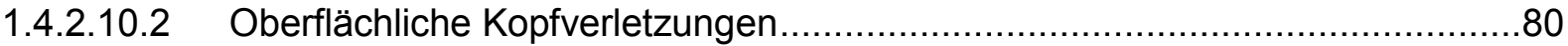


1.4.3 Diskussion relevanter Unfallsituationen .82 .86

2.1 Übersicht und Einschätzung existierender Helmnormen / Testverfahren. .86

2.1.1 Detaillierter Vergleich der Fahrradhelmnormen / Testverfahren ...................86

2.1.1.1 Übersicht der betrachteten Fahrradhelmnormen / Testverfahren ...................86

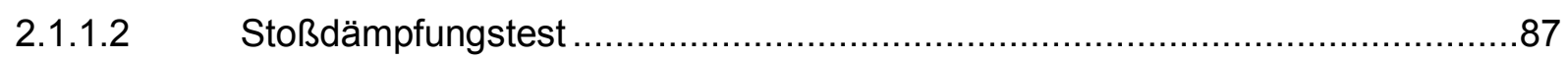

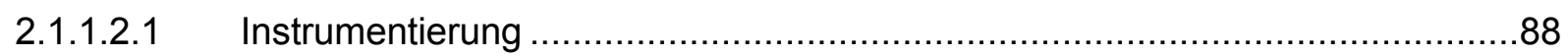

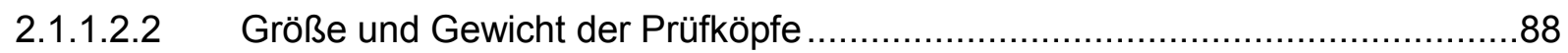

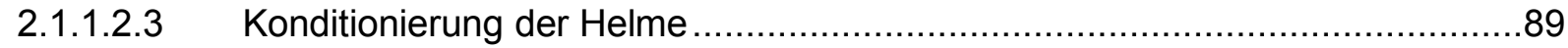

2.1.1.2.4 Prüfgeschwindigkeiten und Testsockel......................................................90

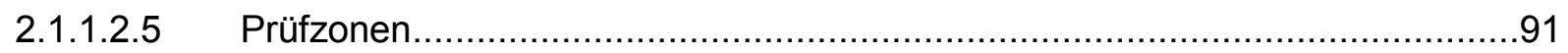

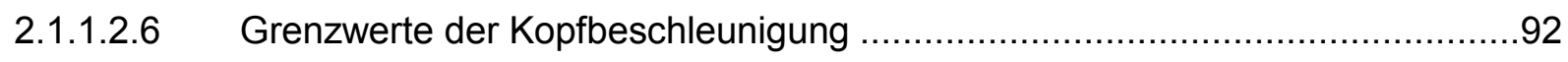

2.1.1.3 Festigkeit und Wirksamkeit der Trageeinrichtung ..................................92

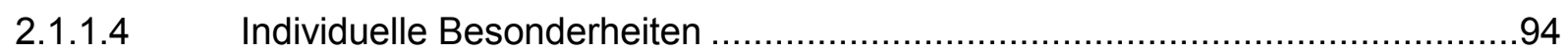

2.1.2 Übersicht und Vergleich weiterer Helmnormen / Testverfahren ......................95

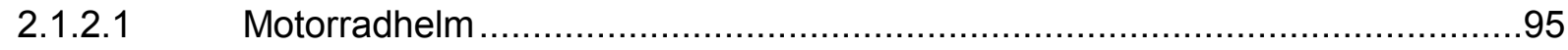

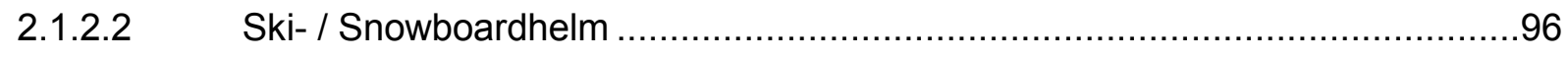

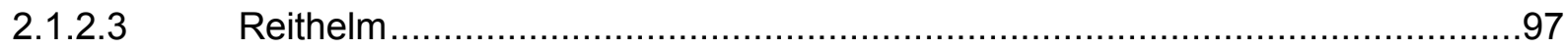

2.1.3 Einschätzung der verschiedenen Helmnormen / Testverfahren.....................98

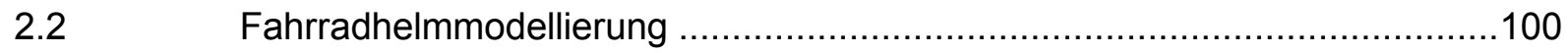

2.2.1 Auswahl eines geeigneten Helms und geometrische Beschreibung ..............100

2.2.2 Segmentierung Helmgeometrien, Export / Netzerstellung FEM ...................101

2.2.3 Entwicklung und Validierung der Materialbeschreibung des Helmmodells.....105

2.2.3.1 Entwicklung einer Materialbeschreibung für quasi-statische Belastung .........105

2.2.3.2 Dynamische Materialprüfung und -modellierung .....................................107

2.2.3.3 Bewertung und Kopplung mit dem Kopfmodell.....................................119

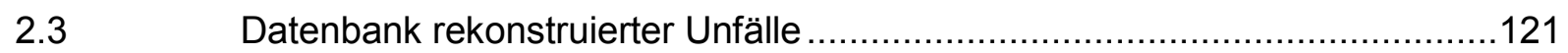

2.4 Vergleichende Einschätzung der Testnormen in Relation zu 1.4 und 2.3 ......121

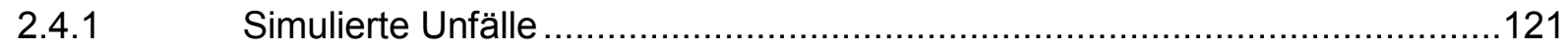




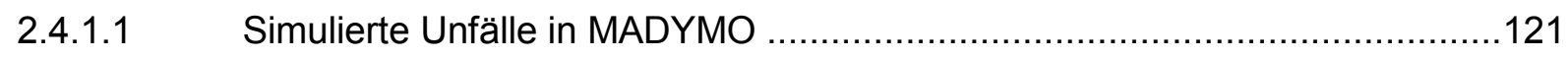

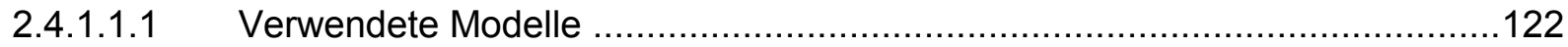

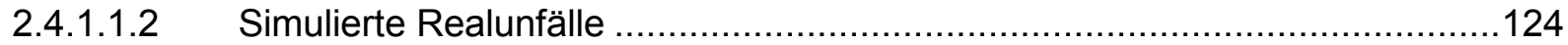

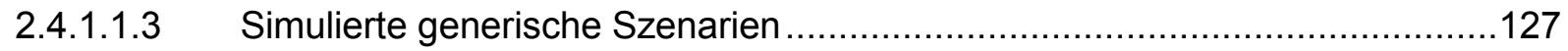

2.4.1.2 Anprallsimulation in FE $\quad$.

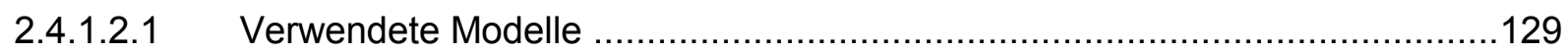

2.4.1.2.2 Simulierte Kopfanprallszenarien und Kopfverletzungsrisiken.......................131

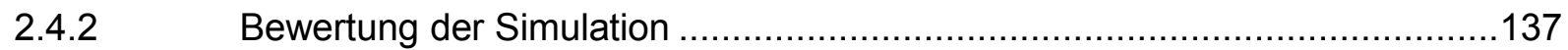

2.4.3 Vergleich der Kontaktparameter Unfallgeschehen/Norm ..........................138

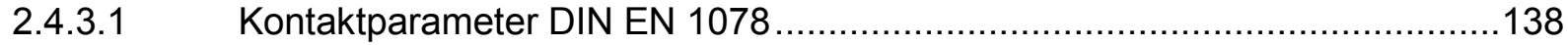

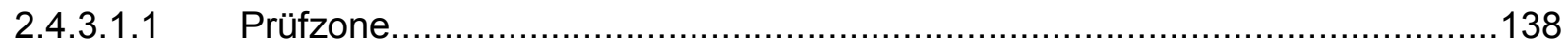

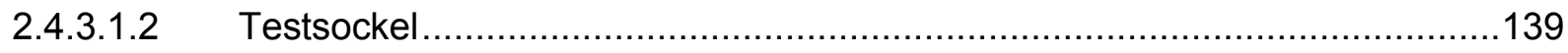

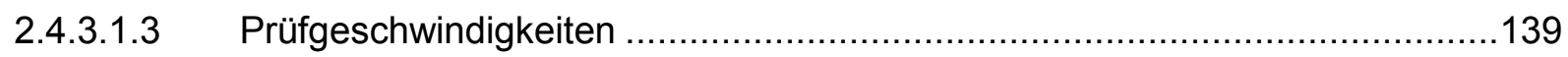

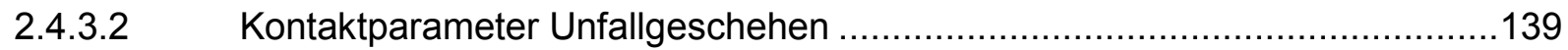

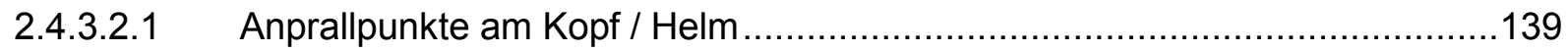

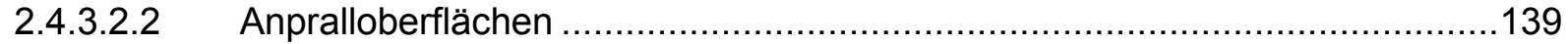

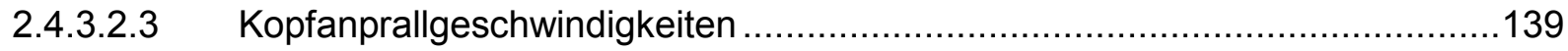

2.4.4 Diskussion der Anforderungen durch Testnorm........................................140

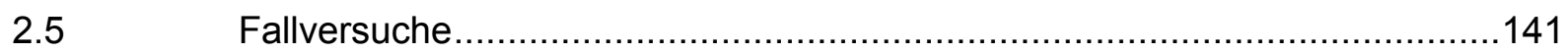

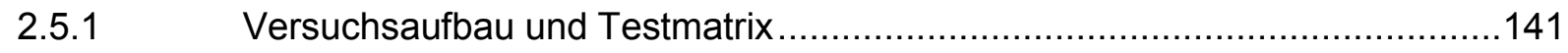

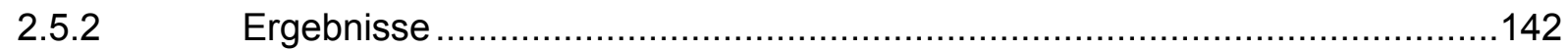

$3 \quad$ Empfehlung für verbesserte Fahrradhelmtests .....................................149

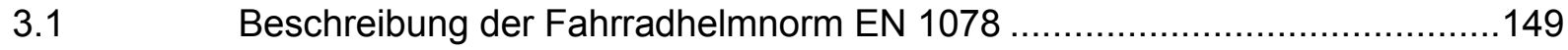

Empfehlung für verbesserte Fahrradhelmtests .....................................149

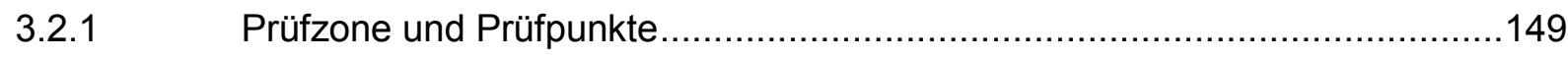

3.2.2 Prüfgeschwindigkeiten und Testsockel.............................................150

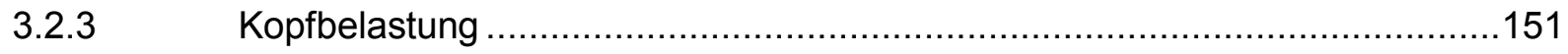

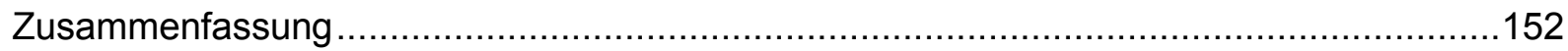

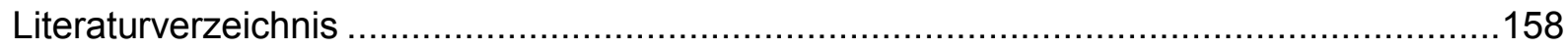


Abbildungsverzeichnis .

163

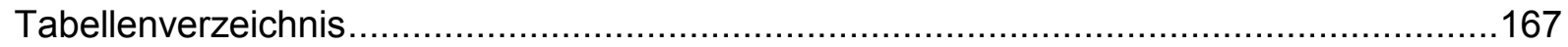

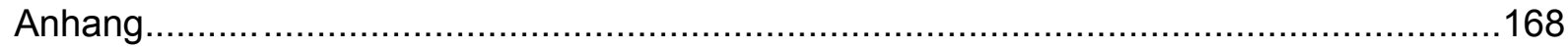




\section{Kurzfassung}

Im dreijährigen Forschungsprojekt „Fahrradhelm“ der UDV wurden durch detaillierte Realunfallanalyse und darauf aufbauende biomechanische Betrachtungen Empfehlungen für verbesserte Testverfahren für Fahrradhelme erarbeitet.

Als Datengrundlage dienten 117 retrospektiv erfasste gut dokumentierte tödliche Fahrradunfälle aus der Sicherheits-Unfall-Datenbank (SUD) des Instituts für Rechtsmedizin München, 500 prospektiv erfasste Unfälle mit leicht- bis schwerverletzten Fahrradfahrern aus dem Universitätsklinikum Münster und 71 prospektiv erfasste Unfälle mit leicht- bis schwerverletzten Fahrradfahrern aus dem Universitätsklinikum München.

Durch Auswertung dieser Fallkollektive konnten der Alleinunfall und die Kollision des Fahrradfahrers mit der Front eines Pkw als typische Unfallszenarien erarbeitet werden. Häufigste Variante des Alleinunfalls ist der Sturz seitlich bei eher geringer Geschwindigkeit, gefolgt vom Sturz nach vorne über den Lenker. Bei Unfällen mit Pkw-Beteiligung trifft typischerweise die Front des Pkw den Fahrradfahrer seitlich in annähernd rechtem Winkel. Die typischen Kollisionsgeschwindigkeiten bewegen sich dabei um $15 \mathrm{~km} / \mathrm{h}$ und um $40 \mathrm{~km} / \mathrm{h}$.

Anschließend wurden ausgewählte typische Realunfälle rekonstruiert, mit generischen Szenarien ergänzt und in zwei Schritten simuliert. Dabei wurde die Kinematik des Fahrradfahrers im Unfallablauf erarbeitet, insbesondere die Parameter des Kopfanpralls auf die Straße oder den beteiligten Pkw. Mit Finite-Elemente-Simulationen unter Verwendung des Kopfmodells der Universität Straßburg (SUFEHM) wurden die Risiken ausgewählter Kopfverletzungsarten für die häufigsten Kopfanprallszenarien bestimmt. Zusätzlich wurde mit einem selbst erstellten detaillierten FE-Modell eines aktuellen Fahrradhelms der mögliche Nutzen eines Fahrradhelms in diesen Kopfanprallszenarien aufgezeigt.

Durch die Analyse der Realunfälle wurde deutlich, dass Fahrradhelme, die nur den Minimalanforderungen der Europäischen Norm EN 1078 entsprechen, noch Optimierungspotential bieten. So fanden sich Kopfanprallpunkte bei Unfällen unbehelmter Fahrradfahrer, die schwerste Verletzungen davontrugen, häufig im Bereich der Schläfen und der unteren Stirn. Zum Teil liegen diese Bereiche außerhalb des unmittelbaren Prüfbereichs des aktuellen Testverfahrens. Daher sollte über eine Ausweitung des Prüfbereichs nachgedacht werden.

Die Prüfgeschwindigkeit in aktuellen Testverfahren erscheint im Kontext von Alleinunfällen ausreichend. Bei schweren Kollisionen mit einem Pkw werden jedoch oft weit höhere Kopfanprallgeschwindigkeiten erreicht. Für zukünftige Testverfahren erscheint es deshalb sinnvoll, auch höhere Prüfgeschwindigkeiten für den Stoßdämpfungstest in Betracht zu ziehen, um mit einem Fahrradhelm auch in solchen Unfallsituationen noch besser geschützt zu sein. Verschärfungen der Normanforderungen müssen allerdings auch gegen Faktoren wie Helmgewicht und Helmbelüftung abgewogen werden, die Einfluss auf die Akzeptanz von Fahrradhelmen haben können. 


\section{Abstract}

The research project „bicycle helmet“, which was funded by the German Insurers Accident Research (UDV) and lasted three years had the aim to develop recommendations for improved test methods for bicycle helmets, based on detailed analysis of real world accidents and combined biomechanical considerations.

The data base consisted of 117 well documented and retrospectively recorded fatal bicycle accidents from the safety accident database (SUD) of the Institute of Legal Medicine, Munich and 500 prospectively recorded accidents with minorly to severely injured cyclists from the University Hospital of Münster and 71 prospectively recorded accidents with minorly to severely injured cyclists from the University Hospital of Munich.

By analyzing this data two typical accident scenarios were identified: the single-vehicle accident of the bicycle rider and the collision with the front part of a car. The most common situation of the single-vehicle accident is falling sideways at rather low speed and by falling off the bicycle forward over the handlebar. Regarding the accidents with a car, the bicycle rider typically collides with the front part of the car approximately under a right angle. The typical collision speeds are around $15 \mathrm{kph}$ and $40 \mathrm{kph}$.

Then, selected typical accident scenarios were reconstructed, supplemented with generic scenarios and simulated in two steps. In the first step, the kinematics of the bicycle rider, especially the parameters of the head contact on the road surface or the car were developed. In the second step (finite element simulation), the risks for selected head injuries were determined using the head model of the University of Strasbourg (SUFEHM). In addition, the benefit of a bicycle helmet has been shown in these head impact scenarios using a detailed finite element model of a bicycle helmet which was developed within this project.

By analyzing the real-world accidents it became clear that bicycle helmets, which only meet the minimum requirements of the European standard EN 1078, still offer potential for optimization. For severely injured bicycle riders head impact points were derived from the location of soft tissue injury on the skull and face which are often found in the temporal region and the lower part of the forehead. Some of these areas are not covered by existing testing procedures. Therefore, extending the test area should be considered.

The test speed in current test methods appears to be sufficient regarding single-vehicle accidents. In a severe collision with a car, far higher head impact speeds can occur. Therefore, it seems to be sensible to include higher testing speeds for the shock absorption test in future testing methods to be even better protected in such accident scenarios. However, tightening the requirements of the test standard must be weighed against such factors like helmet weight and helmet ventilation, which may have an impact on the acceptance of bicycle helmets. 


\section{$1 \quad$ Analyse realer Unfälle}

\section{$1.1 \quad$ Literaturrecherche}

Um zu Beginn des Fahrradhelmprojekts (im Jahr 2011/2012) einen Überblick über bisherige Untersuchgen und Erkenntnisse im Bereich der Fahrradunfälle und Fahrradhelme zu erlangen, wurden insgesamt 42 Studien näher betrachtet.

Sechzehn Studien sind retro- oder prospektive Fallstudien, die Daten für diese Studien stammen aus Deutschland (Chen et al. 2008, (Fredriksson et al. 2012), Richter et al. 2007, Tschernitschek et al. 2010), Australien (Heesch et al. 2011, Mclntosh et al. 1998, Pang et al. 2009, Williams 1991), den USA (Kim et al. 2007), Schweden ((Eilert-Petersson et al. 1997), Oström et al. 1993, Scheiman et al. 2010), Belgien (Depreitere et al. 2004), Israel (SimanTov et al. 2012), Singapur (Heng, K W J et al. 2006) oder sind länderübergreifend (COST 327).

Acht Studien sind Fall-Kontroll-Studien, die Datengrundlage stammt aus Frankreich (Amoros et al. 2012), Schweden (Berg et al. 2007), Kanada (Linn et al. 1998), Deutschland (Otte et al. 2008) Norwegen (Hansen et al. 2003) und den USA (Rivara et al. 1997, Thompson et al. 1989, Thompson et al. 1996).

Zwölf Studien sind Experimentalstudien oder Finite-Elemente-Analysen, darunter die Studien von Bourdet et al. 2012, Deck et al. 2012, Depreitere et al. 2004, Fahlstedt et al. 2012, Ghajari et al. 2011, (Mills et al. 2006), (Mills et al. 2008a), (Mills et al. 2008b), Milne et al. 2012, Pang et al. 2008, van Schijndel et al. 2012, Watson et al. 2009.

Unter den verbleibenden sechs Studien ist eine Meta-Analyse (Attewell et al. 2001), Überprüfungen, Bewertungen und Reviews (Curnow 2003, Curnow 2005, Elvik 2011, Thompson et al. 2009) und eine Logit-Analyse (Moore et al. 2011).

\subsubsection{Methodik}

Nach relevanter Literatur wurde in den Bänden 39 - 48 (Jahre 2007-2012) der Zeitschrift Accident Analysis and Prevention gesucht. Zusätzlich wurden die Tagungsbände der IRCOBI und Stapp-Konferenzen von 2005 bis 2011 betrachtet. Des Weiteren wurde im Internet mit Hilfe der Portale PubMed, Google scholar und Scirus gesucht. Als Schlagworte wurden "bicycle, bicylce helmet, effectiveness, head injury" sowie die deutschen Synonyme verwendet. Zudem wurden Studien betrachtet, die häufig durch andere Fachautoren zitiert wurden.

Prinzipiell vernachlässigt wurden Studien, die sich mit Auswirkungen einer Helmtragepflicht beschäftigen. Diese Problematik wird aktuell äußerst kontrovers diskutiert, trägt aber für dieses Projekt nicht zur zielführenden Diskussion bei.

Ebenso wurden Studien vor 1990 nicht primär betrachtet, es sei denn, sie besitzen heute noch hohe Relevanz und werden häufig zitiert.

Es wurden Studien mit unterschiedlichen Fragestellungen aufgenommen. Zum einen wurden Fallstudien und Fall-Kontroll-Studien betrachtet, die möglichst detaillierte Informationen über Unfälle mit Fahrradfahrerbeteiligung enthalten. Dazu zählen typische Unfallsituationen von 
Fahrradfahrern, typische Verletzungen und Verletzungsmechanismen und eine mögliche Wirksamkeit von Fahrradhelmen. Auch aktuelle Überprüfungen und Bewertungen teilweise veralteter, aber häufig zitierter Studien waren Bestandteil der Recherche.

Zum anderen wurden Studien aufgenommen, die als Zielstellung eine möglichst detaillierte Darstellung von Kollisionsabläufen (mit und ohne Helm) haben. Dabei liegt der Fokus auf biomechanischen Prozessen und der Vorhersage von möglichen Verletzungen.

\subsubsection{Relevante Literatur}

\subsubsection{Unfallforschung bei Fahrradfahrern}

Depreitere, B., Van Lierde, C., Maene, S., Plets, C., Vander Sloten, J., Van Audekercke, R., Van der Perre, G., Goffin, J. (2004). Bicycle-related head injury: a study of 86 cases. Accident Analysis \& Prevention, Vol. 36, No. 4, S. 561-567 (Depreitere et al. 2004)

Datengrundlage dieser Studie sind 86 verunfallte Fahrradfahrer der Jahre 1990 bis 2000, die sich im Universitätsklinikum in Leuven (Belgien) einem neurochirurgischen Eingriff unterziehen mussten. Daten wurden aus Polizeiberichten, medizinischen Berichten, CTBildern des Kopfs und einem Patientenfragebogen erhoben.

In drei Fällen trug der Fahrradfahrer einen Helm. 44 Unfälle waren Kollisionen mit einem motorisierten Fahrzeug, 42 Unfälle waren Stürze. Die häufigsten Aufprallpunkte waren seitlich (57\%) und frontal (27\%) am Kopf lokalisiert. Die häufigsten Verletzungen waren Schädelbrüche (86\%) und Hirnprellungen (73\%). Die Verletzungen mit dem ungünstigsten Outcome sind Subarachnoidalblutungen, multiple und schwere Hirnprellungen, Subduralhämatome und Hirnschwellungen. Die Hirnprellungen sind dabei vermutlich die primäre Verletzung in einem komplexen Mechanismus und sollten als hauptursächlich für das Outcome angesehen werden.

Eilert-Petersson, E., Schelp, L. (1997). An epidemiological study of bicycle-related injuries. Accident Analysis and Prevention, Vol. 29, No. 3, S. 363-72 (Eilert-Petersson et al. 1997)

Datengrundlage dieser Studie sind 1022 Fahrradfahrer, die im Zeitraum von November 1989 bis Oktober 1990 in der Schwedischen Provinz Västmanland verunfallten.

Häufigster Unfalltyp ist der Alleinunfall (80\%). Bei einer Kollision mit einem anderen Verkehrsteilnehmer war der Gegner in über der Hälfte der Unfälle ebenfalls ein Fahrradfahrer. Der Alleinunfall wurde überwiegend ausgelöst durch Fahrfehler, glatte Straßen oder einer Kollision mit Bordstein. Knapp 10 \% der Fahrradfahrer waren alkoholisiert, wobei die Autoren hier nicht konkreter werden.

Kopfverletzungen (inklusive Gesichtsverletzungen) waren die häufigsten (21\%) und schwersten Verletzungen, speziell bei Kindern und Senioren.

Die Autoren fordern zwar eine verstärkte Werbung für Helmnutzung, treffen in ihrer Studie aber keine Aussagen zur Helmtragequote oder zum Einfluss des Helms auf Kopfverletzungen. 
Fredriksson, R., Rosén, E. (2012). Priorities for Bicyclist Protection in Car Impacts - a Real life Study of Severe Injuries and Car Sources. Proc. IRCOBI 2012, Nr. 83 (Fredriksson et al. 2012)

In dieser Fallstudie wurden 2327 Fahrradfahrer und 1195 Fußgänger mit einer Verletzung AIS 1+ betrachtet, die mit der Front eines Pkw kollidierten. Die Daten stammen aus der GIDAS-Datenbank (1999-2010). Die Autoren verglichen die typischen Verletzungen und Kollisionsstellungen zwischen Pkw-Front und Fahrradfahrer / Fußgänger.

Es wurden 121 Fahrradfahrer und 144 Fußgänger schwer (AIS 3+) verletzt; 18 Fahrradfahrer und 47 Fußgänger wurden getötet. Das Hauptunfallszenario war die Kollision Pkw-Front gegen Seite des Fahrradfahrers/Fußgängers.

Fahrradfahrer wurden am häufigsten (27\%) in der Kollisionsstellung Kopf-Frontscheibe schwer (AIS 3+) verletzt. Die Aufprallpunkte der Fahrradfahrer auf der Frontscheibe liegen höher als die der Fußgänger. Schutzsysteme, die Schutzpotential für Fußgänger bei Kollision mit der Pkw-Front bieten, haben auch einen protektiven Effekt für Fahrradfahrer, insbesondere wenn auch höhere Teile des Fahrzeugs abgedeckt werden.

Kim, J.K., Kim, S., Ulfarsson, G.F., Porrello, L.A. (2007). Bicyclist injury severities in bicycle-motor vehicle accidents. Accident Analysis and Prevention, Vol. 39, No. 2, S. 238-51 (Kim et al. 2007)

In dieser Fallstudie werden Risikofaktoren für die Verletzungsschwere von Fahrradfahrern bestimmt. Datengrundlage sind 2394 verunfallte Fahrradfahrer, die in den Jahren 1997-2002 in North Carolina, USA von der Polizei erfasst wurden. Zwingendes Aufnahmekriterium in die Studie war eine Kollision des Fahrradfahrers mit einem KfZ.

Das Risiko für tödliche Verletzungen steigt mit größerer Ausgangsgeschwindigkeit des Fahrzeugs oder einem Lkw als Kollisionsgegner. Ebenfalls ist die Verletzungsschwere bei Unfällen mit Beteiligung eines berauschtem Fahrrad- oder KfZ.-Fahrer und bei Kollisionen mit dem Kopf voran höher. Fahrradfahrer mit einem Alter über 55 sind generell schwerer verletzt als jüngere. Der größte Risikofaktor für schwerste und tödliche Verletzungen ist eine Fahrzeuggeschwindigkeit (des beteiligten $\mathrm{Kfz}$ ) über $80 \mathrm{~km} / \mathrm{h}(50 \mathrm{mph})$.

Linn, S., Smith, D., Sheps, S. (1998). Epidemiology of bicycle injury, head injury, and helmet use among children in British Columbia: a five year descriptive study. Injury Prevention, 1998, Vol. 4, S. 122-125 (Linn et al. 1998)

Datengrundlage dieser Studie sind 1462 verletzte Fahrradfahrer (Alter 1-19 Jahre), vom British Columbia Children's Hospital der Jahre 1991-1995. Die Daten wurden anhand eines Fragebogens erhoben, den die Eltern oder Begleiter der Kinder im Krankenhaus ausfüllen mussten.

Ziel der Studie war die Beschreibung der Epidemiologie von Verletzungen, Helmnutzung und dem Auftreten von Kopfverletzungen. Mehr als $70 \%$ der verletzten Fahrradfahrer trugen keinen Helm. Kopf- und Gesichtsverletzungen traten häufiger auf, wenn kein Helm getragen wurde. Es wurde keine Häufung von leichten Kopfverletzungen bei Fahrradfahrern ohne Helm betrachtet.

Mclntosh, A., Dowdell, B., Svensson, N. (1998). Pedal cycle helmet effectiveness: a field study of pedal cycle accidents. Accident Analysis and Prevention, Vol. 30, No. 2, S. 161-168 (Mclntosh et al. 1998)

Es wurden 42 Unfälle betrachtet, in denen der Fahrradfahrer einen Helm getragen hat, der einem Aufprall ausgesetzt war. Vier davon waren tödlich, nur ein Fahrradfahrer starb an einer isolierten Kopfverletzung. Das Datenmaterial stammt aus dem Großraum Sydney und New South Wales und wurde im Jahr 1991 aufgenommen. 
Es wurden nur Unfälle betrachtet, in denen die Datengrundlage sehr gut war, d.h.

Verletzungen wurden von Fachpersonal exakt dokumentiert und die Unfälle von der Polizei aufgenommen. Am häufigsten waren Alleinunfälle.

Neun (23\%) der verletzten Fahrradfahrer erlitten eine Kopfverletzung AIS $\geq 2$

(Kopfverletzungen AIS = 1 wurden ausgeschlossen, da hier von keinem neurologischen

Schaden ausgegangen wird). 17 (45\%) verletzte Fahrradfahrer hatten mehrere Verletzungen

AIS $\geq 2$.

Diese Studie zeigt auf, dass ein Aufprallpunkt in der temporoparietalen Region des Kopfes (seitliche Schläfenregion / über den Ohren) ein erhöhtes Verletzungsrisiko nach sich zieht.

$25 \%$ der direkten Aufschläge fanden in dieser Region statt, diese verursachten $75 \%$ aller AIS 2+ Verletzungen. Dabei ist es nicht bekannt, ob dies am Aufbau des menschlichen Schädels oder an den Eigenschaften des Helms liegt. Es wird die Empfehlung ausgesprochen, die Testlinie der Fahrradhelmnormen zu überdenken.

Die Objekte, auf denen der Helm aufprallte, waren meist flach und starr.

Zusätzlich wurde aufgezeigt, dass in Unfällen, an denen ein zusätzliches Fahrzeug beteiligt war, ein höheres Risiko besteht, verletzt oder getötet zu werden. Dieser Trend verstärkte sich bei Pkw, die schneller als $30 \mathrm{~km} / \mathrm{h}$ fuhren.

Moore, D.N., Schneider, W.H. 4th, Savolainen, P.T., Farzaneh, M. (2011). Mixed logit analysis of bicyclist injury severity resulting from motor vehicle crashes at intersection and non-intersection locations. Accident Analysis and Prevention, Vol. 43, No. 3, S. 621 - 30 (Moore et al. 2011)

In dieser Logit-Analyse werden 10029 Unfälle mit Fahrradfahrerbeteiligung der Jahre 2002 bis 2008 aus Ohio, USA betrachtet. Es werden Faktoren dargestellt, die die Verletzungsschwere des Fahrradfahrers beeinflussen (Vergleich zwischen Kollisionen an und außerhalb einer Kreuzung/Einmündung)

Bei Kollisionen an einer Kreuzung steigt das Gesamtverletzungsrisiko des Fahrradfahrers für schwere Verletzungen um 14,8\%, wenn kein Helm getragen wird, um 82,2\% falls der Kfz.Fahrer unter Alkoholeinfluss steht, um 141,3\% falls ein Van beteiligt ist, um 40,6\% falls der Pkw die Seite des Fahrrads trifft.

Bei Kollisionen, die nicht an einer Kreuzung stattfinden, steigt das Risiko für schwere Verletzungen um 53,5\%, falls der Pkw die Seite des Fahrrads trifft, um 150\% bei einem alkoholisierten Kfz.-Fahrer und um 100\% bei Beteiligung eines Lkw.

Oström, M., Bjornstig, U., Naslund, K., Eriksson, A. (1993). Pedal cycling fatalities in Northern Sweden. International Journal of Epidemiology 22, S. 483- 488 (Oström et al. 1993)

In dieser retrospektiven Fallstudie wurden über 10 Jahre (1975 - 1985) 146 tödliche Fahrradunfälle aus der nördlichen Hälfte von Schweden gesammelt und analysiert. Dabei wurden die Unfall- und Verletzungsmechanismen bei tödlichen Fahrradunfällen verdeutlicht. Das Durchschnittsalter der Verunglückten liegt bei 60 Jahren, 66\% der Opfer waren männlich. 88\% der Fahrradfahrer starben bei einer Kollision mit einem motorisierten Fahrzeug, davon $21 \%$ bei einer Kollision mit einem Lkw. Keiner der Verunglückten trug einen Fahrradhelm. Bei 69\% war eine Kopfverletzung Todesursache. In 91\% aller Fälle gab es eine Kopfverletzung AIS $\geq 3$.

Aufgrund dieser Zahlen gehen die Autoren von einem protektiven Effekt des Fahrradhelms aus 
Richter, M., Otte, D., Haasper, C., Knobloch, K., Probst, C., Westhoff, J., Sommer, K., Krettek, C. (2007). The Current Injury Situation of Bicyclists - A Medical and Technical Crash Analysis. The Journal of Trauma Injury, Infection and Critical Care 62, S. 11181122 (Richter et al. 2007)

Es wurden 4264 verletzte Fahrradfahrer aus den Jahren 1985 bis 2003 betrachtet. Diese Unfälle sind Bestandteil der GIDAS-Datenbank. Helmtragequote war 1,7\%.

$55 \%$ der Fahrradfahrer benutzten separate Fahrradwege vor dem Unfall, 16,8\% der Unfälle ereigneten sich direkt auf Fahrradwegen. Kollisionsgegner waren in 65,8\% der Fälle Pkw, gefolgt von Lkw $(7,2 \%)$ und anderen Fahrradfahrern (7,4\%). In 77,9\% lag die Kollisionsgeschwindigkeit unter $30 \mathrm{~km} / \mathrm{h}$. Hauptzonen der ersten Kollision lokalisiert am Fahrrad waren frontal (12 Uhr), links (9 Uhr) und rechts (3 Uhr).

$79 \%$ aller Fahrradfahrer waren leicht verletzt (MAIS = 1). 4,2\% hatten mindestens eine schwerere Verletzung (MAIS $\geq 3$ ). 64 Personen starben $(1,5 \%)$, davon 58 bevor sie in eine medizinische Einrichtung gebracht werden konnten.

Die Kopfverletzungen der unbehelmten Fahrradfahrer waren in $68 \%$ der Fälle im typischen Schutzbereich eines Fahrradhelms lokalisiert und wären somit potentiell mit einem Helm vermeidbar gewesen.

Rivara, F. P., Thompson, D. C., Thompson, R. S. (1997). Epidemiology of bicycle injuries and risk factors for serious injury. Injury Prevention 1997; Vol. 3, S.110-114 (Rivara et al. 1997)

Diese prospektive Fall-Kontroll-Studie hat die gleiche Datengrundlage wie die Studie von Thompson et al. (1996), es wurden 3390 verletzte Fahrradfahrer von 1992 bis 1994 in Seattle betrachtet.

Abweichend von der oben genannten Studie (Thompson et al. 1996) werden in der aktuellen Studie die Risikofaktoren für schwere Verletzungen von Fahrradfahrern bestimmt, die nicht im Zusammenhang mit einer Helmnutzung stehen.

$52 \%$ der verunfallten Fahrradfahrer hatten zwei oder weniger Verletzungen, $37 \%$ drei bis fünf Verletzungen, 11\% mehr als fünf Verletzungen. 22\% der Fahrradfahrer hatten Kopfverletzungen, 35\% Gesichtsverletzungen. 6\% hatten Gehirnverletzungen. $7 \%$ hatten einen ISS $>9$. Das Risiko für schwere Verletzungen stieg bei einer Kollision mit einem motorisierten Fahrzeug, bei einer selbst gefahrenen Geschwindigkeit $>15 \mathrm{mph}$ und bei einem Alter von jünger als sechs Jahren oder älter als 39 Jahren.

$51 \%$ der Fahrradfahrer trugen einen Helm; dieser hatte jedoch keinen statistisch signifikanten Einfluss auf die Reduktion von schweren Verletzungen (ISS > 8).

Scheiman, S., Moghaddas, H. S., Björnstig, U., Bylund, P.-O., Saveman, B.-I. (2010). Bicycle injury events among older adults in Northern Sweden: A 10-year population based study. Accident Analysis and Prevention, Vol. 42, No. 2, S. 758-763 (Scheiman et al. 2010)

Diese Studie vergleicht Verletzungsmechanismen und Verletzungen von Senioren (Alter 65+ Jahre), die sich beim Fahrradfahren verletzt haben, mit denen von jüngeren Fahrradfahrern und Senioren, die als Pkw-Insassen verletzt wurden. Datengrundlage sind 465 Fahrradfahrer über 65, die in den Jahren 1997 bis 2006 im Universitätsklinikum Umeå in Schweden behandelt wurden.

Am häufigsten (20\%) traten Verletzungen bei Stürzen beim Auf-/Absteigen von Fahrrad auf. Nur 6\% der Fahrrad fahrenden Senioren zogen sich ihre Verletzungen bei einer Kollision mit einem anderen Verkehrsteilnehmer zu. 10\% der Senioren hatten eine Hirnverletzung (75\% 
Gehirnerschütterung, 25\% intrakranielle Blutungen), davon trugen 20\% einen Helm. Drei Senioren starben (einer nach fünf Tagen mit MAIS 5, zwei am Unfallort mit MAIS 6), zwei davon nach Kollision mit einem Pkw. Die Verletzungen von Senioren als Fahrradfahrer (52\% MAIS 2+) waren schwerer als die der jüngeren Fahrradfahrer (34\% MAIS 2+) und PkwInsassen (32\% MAIS 2+).

Siman-Tov, M., Jaffe, D.H., Israel Trauma Group, Peleg, K. (2012). Bicycle injuries: a matter of mechanism and age. Accident Analysis and Prevention, Vol. 44, No. 1, S. 135-9 (Siman-Tov et al. 2012)

Datengrundlage dieser retrospektiven Fallstudie sind 5529 verletzte Fahrradfahrer aus elf Traumazentren in Israel der Jahre 2001 bis 2007.

Ziel der Studie ist der Vergleich der Verletzungen von erwachsenen Fahrradfahrern mit denen von Kindern.

Fahrradfahrer, die in Kollisionen mit weiteren Fahrzeugen verwickelt wurden, trugen schwerere Verletzungen davon und hatten ein schlechteres Outcome als allein Verunfallte. $37 \%$ der Erwachsenen wurden stationär aufgenommen, im Gegensatz dazu nur $27 \%$ bei den Kindern. Kinder hatten mehr Kopf- und Gehirnverletzungen als Erwachsene.

\subsubsection{Risikoreduktion für Kopfverletzungen durch Helmtragen}

\section{Retrospektive Studien}

Amoros, E., Chiron, M., Martin, J.-L., Laumon, B. (2011). Bicycle helmet wearing and the risk of head, face, and neck injury: a French case-control study based on a road trauma registry. Inj Prev. 2012 Feb;18(1), S. 27-32 (Amoros et al. 2012)

Datengrundlage dieser Fall-Kontroll-Studie sind 13797 Fahrradunfälle im Département Rhône in Frankreich aus den Jahren 1998 bis 2008. Alle Verletzungen wurden nach AIS kodiert.

Bei 8373 Fahrradfahrern war der Helmtragestatus dokumentiert, davon trugen 1720 (26\%) während des Unfalls einen Helm.

Als Fälle wurden Fahrradfahrer mit Kopfverletzungen (bzw. Gesichts- oder Nackenverletzungen) definiert; die Kontrollgruppe waren verletzte Fahrradfahrer, die keine Kopfverletzungen aufwiesen ( $n=5153$ ).

Es konnte für den Helm eine statistisch signifikante Reduzierung des Risikos um $31 \%$ für Kopfverletzungen aller Schweregrade (AIS 1+) nachgewiesen werden. Die Reduktion des Risikos für schwere Kopfverletzungen (AIS 3+) liegt sogar bei $66 \%$ innerorts und $93 \%$ außerorts. Das Risiko für Gesichtsverletzungen ist mit Helm um $28 \%$ geringer als ohne Helm. Für Nackenverletzungen zeigt sich kein signifikanter Anstieg des Risikos mit Helm.

Attewell, R., Glase, K., McFadden, M., (2001). Bicycle helmet efficacy: a meta-analysis. Accident Analysis and Prevention, Vol. 33, No. 3, S. 345-352 (Attewell et al. 2001)

In dieser Meta-Analyse wurden nur Studien aufgenommen, in denen Daten über individuelle Verletzungen sowie die Benutzung eines Helmes vorlagen. Es sind insgesamt 16 Studien aus verschiedenen Ländern der Jahre 1987-1998 betrachtet worden. Darunter befinden sich auch die separat betrachteten Studien von Thompson et al. (1989) und Thompson et al. (1996). 
Es wurde ein statistisch signifikanter protektiver Effekt von Fahrradhelmen nachgewiesen. Diese können schwere und tödliche Verletzungen verhindern. In drei Studien wurden aufgezeigt, dass das Tragen eines Fahrradhelms ein höheres Risiko für Nackenverletzungen birgt, was aber nach Meinung der Autoren mit den heutigen leichteren Helmen nicht mehr zutreffend sein kann, wobei der Beweis basierend auf den betrachteten Studien nicht vorgebracht wird. Nach konservativen Annahmen gibt es mit Helm eine Risikoreduktion von mindestens 45\% für Kopfverletzungen, 33\% für Gehirnverletzungen, 27\% für Gesichtsverletzungen und 29\% für tödliche Verletzungen.

\section{Berg, P., Westerling, R. (2007). A decrease in both mild and severe bicycle-related head injuries in helmet wearing ages - trend analyses} in Sweden. Health Promotion International, Vol. 22 No. 3, S. 191-197 (Berg et al. 2007)

Diese Studie betrachtet die komplette schwedische Bevölkerung von 1987 bis 1996. Es wurden 49758 verletzte Fahrradfahrer aus einem zentralen Register aufgenommen. Hauptargumentationslinie dieser Studie ist, dass bei Kindern die Häufigkeit an Kopfverletzungen abnahm, während die Helmtragequote zunahm. Kombiniert mit der Tatsache, dass die Häufigkeit aller anderen Verletzungen (und Unfälle) annähernd stagnierte, muss der Helm somit einen schützenden Effekt haben. Bei Erwachsenen stieg sowohl die Verletzungshäufigkeit für Kopfverletzungen wie auch für andere Verletzungen (bei gleichbleibender Helmtragequote).

Es wird zudem davon ausgegangen, dass Fahrradhelme auch vor schweren Verletzungen schützen können.

\section{Elvik, R. (2011). Publication bias and time-trend bias in meta-analysis of bicycle helmet efficacy: A re-analysis of Attewell, Glase and McFadden, 2001. Accident Analysis and Prevention Vol. 43, No. 3, S. 1245-1251 (Elvik 2011)}

Die Meta-Analyse von Attewell et al. (2001) wurde kritisch auf mögliche Fehler untersucht, da Attewell die Möglichkeit verzerrter Ergebnisse in seiner Diskussion erwähnt. Anschließend wurde das Datenmaterial mit aktuellen Studien von Hausotter (2000), Hansen et al. (2003), Heng et al. (2006) und Amoros et al. (2009) ergänzt und neu ausgewertet. Untersucht wurden die Einflüsse möglicher publication bias, time trend bias und zero count bias. Diese Fehlerquellen traten im Fallmaterial auf und wurden mit statistischen Methoden korrigiert, die zum Zeitpunkt des Reviews von Attewell noch nicht entwickelt waren. Es zeigt sich, dass die Korrekturen für zero count bias und publication bias die von Attewell berichteten Effekte nur unwesentlich verändern. Wesentliche Effektänderungen zeigen sich allerdings durch den vorhandenen time trend bias. Ältere Studien berichten von größeren Risikoreduktionen durch Helme als neuere Studien. Elvik diskutiert dafür die vermehrte Verwendung von Softshell-Helmen in den Datenkollektiven der neueren Studien, deren geringeres protektives Potential gegenüber den in den häufiger in älteren Studien vorkommenden Hardshell-Helmen bekannt sei.

Im Einzelnen ist nach Korrektur davon auszugehen, dass durch das Tragen von Helmen das Risiko für Kopfverletzungen um 42\% reduziert wird (OR 0,58 95\% KI [0,45; 0,75]), das Risiko für Gesichtsverletzungen um 17\% (nicht signifikant) reduziert wird, und das Risiko für Nackenverletzungen möglicherweise um 32\% erhöht wird (hierbei kann jedoch nicht für die publication bias adjustiert werden). Ohne time trend Betrachtung, aber unter Miteinbeziehung der publication bias berichtet Elvik eine Risikoreduktion für tödliche Verletzungen bei Tragen eines Helmes um $77 \%$.

Wesentliche Erkenntnis liegt in der Betrachtung des Risikos für Kopf- oder Gesichts- oder Nackenverletzung: dieses Risiko wird durch das Helmtragen um 15\% reduziert. 
Hansen, K.S., Engesæter, L.B., Viste,A. (2003). Protective effect of different types of bicycle helmets. Traffic Injury and Prevention, Vol. 4, S. 285-290 (Hansen et al. 2003)

Die Fall-Kontroll Studie untersucht das protektive Potential des Tragens von HardshellHelmen und Softshell-Helmen im Vergleich zu unbehelmten Fahrradfahrern bezüglich Kopfund Gesichtsverletzungen. Fälle waren kopf- oder gesichtsverletzte Fahrradfahrer, Kontrollgruppe 1 waren verletzte Fahrradfahrer, die aber keine Kopf oder Gesichtsverletzung aufwiesen und Kontrollgruppe 2 waren alle verunfallten Fahrradfahrer unabhängig davon, ob sie eine Verletzung erlitten hatten oder nicht. Die Helmtragequoten in allen Gruppen wurden verglichen und das Schutzpotential mittels logistischer Regression ermittelt.

Ergebnisse: Hardshell-Helme reduzieren das Kopfverletzungsrisiko um 64\% und SoftshellHelme zeigen keinen signifikanten Effekt. Ebenso konnten weder Hardshell- noch SoftshellHelme signifikant das Risiko von Gesichtsverletzungen reduzieren.

Heng, K.W.J., Lee, A.H., Zhu, S., Tham, K.Y., Seow,E. (2006). Helmet use and bicycle-related trauma in patients presenting to an acute hospital in Singapore. Singapore Medical Journal, Vol. 47, S. 367-372 (Heng, K W J et al. 2006)

In diese Fallstudie wurden alle 160 verunfallten Fahrradfahrer, die sich in einer Notaufnahme in Singapur zwischen September 2004 und Mai 2005 vorstellten, aufgenommen. die Helmtragequote lag bei 10,6\%. Einflussfaktoren auf die Verletzungsschwere (ISS) waren das Tragen eines Helmes, die Kollision mit einem motorisierten Fahrzeug und Alter. Die Häufigkeiten von Kopf- und Gesichtsverletzungen unterschieden sich signifikant zwischen Helmträgern und Nicht-Helmträgern (5,9\% zu $40 \%$ und 5,9\% zu $37,1 \%)$.

Otte, D., Haasper, C., Wiese, B. (2008). Wirksamkeit von Fahrradhelmen bei Verkehrsunfällen von Radfahrern auf Kopfverletzungshäufigkeit und Verletzungsschwere. Verkehrsunfall und Fahrzeugtechnik, Heft Nr. 11, S. 2-12 (Otte et al. 2008)

Datengrundlage dieser Studie sind 3525 an Unfällen beteiligte Fahrradfahrer aus der GIDASDatenbank der Jahre 1999 bis 2006. Diese Unfälle stammen aus den Großräumen Hannover und Dresden. 269 Fahrradfahrer (7,6\%) benutzten einen Fahrradhelm.

Die Studie vergleicht die Kopfverletzungen der Fallkollektive "mit Helm" und "ohne Helm". Es blieben 72,7\% der Radfahrer mit Helm am Kopf unverletzt, 27,3\% wiesen Kopfverletzungen auf. Ohne Helm waren lediglich 61,3\% am Kopf unverletzt und 38,7 \% kopfverletzt. Zudem ist der Anteil schwerster Kopfverletzungen AIS 3+ für Fahrradfahrer mit Helm $(1,2 \%)$ geringer als für Personen ohne Helm $(1,8 \%)$.

Die häufigsten Anprallbereiche sind zum einen Frontscheiben von Pkw sowie in über $50 \%$ aller Fälle die Straßenoberfläche. Die häufigsten Anprallbereiche am Kopf sind an der Stirn, Schädelseite und Hinterhaupt lokalisiert.

Pang, T.Y., Thai, K.T., Rankin, T., Curtis, K., Schilter, E., McIntosh, A.S. (2009). Risk of Head, Facial and Neck Injury in Bicycle and Motorcycle Crashes in relation to Helmet Use. 2009 Australasian Road Safety Research, Policing and Education Conference (Pang et al. 2009)

Pang et al. (2009) betrachteten in ihrer Studie 84 Fahrrad- und 130 Motorradfahrer (insgesamt $n=214$ ), die im Zeitraum von Juni 2008 bis Juni 2009 im St. George Public Hospital, New South Wales, behandelt wurden. 
Die Helmtragequote unter den Fahrradfahrern lag bei 64,3\%. 28\% der Fahrer hatten eine Kopf-, Gesichts- oder Nackenverletzung, 27,6\% eine Gehirnerschütterung.

In der Studie wird abgeschätzt, dass das Risiko für behelmte Fahrer von Zweirädern, eine Kopf-, Nacken- oder Gesichtsverletzung zu erleiden, gegenüber unbehelmten Fahrern um $80 \%$ abnimmt. Der Helm vermindert nur das Risiko eines leichten oder moderaten Kopftraumas.

Thompson, D.C., Rivara, F., Thompson, R. (2009). Helmets for preventing head and facial injuries in bicyclists (Review). The Cochrane Library 2009, Issue 1 (Thompson et al. 2009)

In diesen systematischen Review wurden fünf Studien aufgenommen (Thompson (1996), Maimaris (1994), Thomas (1994), McDermott (1993), Thompson (1989)).

Die zugrunde liegenden Daten wurden von zwei Personen unabhängig betrachtet. Die Ergebnisse von Kopf- und Gehirnverletzungen wurden unter Zuhilfenahme von Techniken einer Meta-Analyse zusammengefasst.

Das Risiko für Kopf-, Gehirn- und schwere Gehirnverletzungen in allen Altersgruppen sinkt mit einem Fahrradhelm um 63 bis $88 \%$. Helme bieten bei Unfällen mit Beteiligung eines motorisierten Fahrzeugs und allen anderen Unfällen das gleiche Schutzpotential.

Verletzungen im oberen und mittleren Gesichtsbereich reduzieren sich mit Helm um 65\%.

Thompson, D.C., Rivara, F.P., Thompson, R.S. (1996). Effectiveness of Bicycle Safety Helmets in Preventing Head Injuries. The Journal of the American Medical Association 276, S. 1968-1973 (Thompson et al. 1996)

Diese Studie wird an dieser Stelle separat betrachtet, obwohl sie in der Meta-Analyse von Attewell et al. (2001) aufgeführt ist.

Thompson et al. errechneten bereits 1989 ein um 85\% reduziertes Risiko für Kopfverletzungen für Fahrradfahrer mit Helm. Diese Studie stammt von den gleichen Autoren, es wurden 3390 verletzte Fahrradfahrer von 1992 bis 1994 in Seattle betrachtet. $22,3 \%$ der Fahrradfahrer hatten eine Kopfverletzung, 1,8\% eine schwere Kopfverletzung. Die Helmtragequote der Fallgruppe (verunfallte Fahrradfahrer mit Kopfverletzungen) war 29\%; die der Kontrollgruppe (verunfallte Fahrradfahrer mit Verletzungen ausschließlich unterhalb des Kopfes) $56 \%$.

Fahrradhelme waren sowohl in Unfällen mit motorisierten Fahrzeugen als auch ohne deren Beteiligung gleich effektiv. Außerdem konnten keine signifikanten Unterschiede zwischen den verschiedenen Helmtypen (Soft-, Hard- und Microshell) sowie deren Zertifizierungen (Snell, ANSI) aufgedeckt werden.

Behelmte Fahrradfahrer tragen ein um 69\% reduziertes Risiko für Kopfverletzungen sowie ein um $65 \%$ reduziertes Risiko für Hirnverletzungen, verglichen mit Fahrern ohne Helm. Dieses Ergebnis gilt für alle Altersgruppen.

Die etwas niedrigeren Zahlen verglichen mit der ersten Studie 1989 erklären die Autoren damit, dass in der aktuellen Studie keine populationsbasierte Kontrollgruppe betrachtet wurde. Somit kann der mögliche Effekt von Helmen möglicherweise unterschätzt worden sein.

Thompson, R.S. Frederick, P.R. Thompson, D.C. (1989). A Case-Control Study of the Effectiveness of Bicycle Safety Helmets. New England Journal of Medicine 320, No. 21, S. 1361-1367 (Thompson et al. 1989) 
Diese Studie ist bereits in der ebenfalls aufgeführten Meta-Analyse von Attewell et al. (2001) enthalten, wird aber an dieser Stelle separat behandelt, da sie seit Jahrzehnten überdurchschnittlich oft zitiert wird.

Es wurde eine Gruppe von 235 verunfallten Fahrradfahrern mit Kopfverletzungen mit zwei Kontrollgruppen verglichen. Eine Kontrollgruppe bestand aus 433 Fahrradfahrern, die in denselben Krankenhäusern in Seattle aufgrund eines Unfalls behandelt wurden, aber keine Kopfverletzungen erlitten hatten. Die zweite Kontrollgruppe bestand aus 558 Fahrradfahrern, die im vergangenen Jahr einen Fahrradunfall hatten. Die Autoren errechneten, dass Fahrradfahrer mit Helm ein um $85 \%$ reduziertes Risiko für Kopfverletzungen und ein um $88 \%$ reduziertes Risiko für Hirnverletzungen hatten.

Tschernitschek, J., Hannawald, L., Koch, R., Zwipp, H. (2010). Evaluation of the protective effect of bicycle helmets (A GIDAS-analysis 2000-2007). 4th International Conference on ESAR "Expert Symposium on Accident Research", S.1-8 (Tschernitschek et al. 2010)

Datengrundlage dieser Studie sind 2691 verunfallte Fahrradfahrer aus der GIDASDatenbank. Die Fälle wurden in den Großräumen Hannover und Dresden in den Jahren 2000 - 2007 erhoben. Ziel der Studie ist die Bewertung des protektiven Effekts von Fahrradhelmen in Hinblick auf Kopf- und Gesichtsverletzungen. Im betrachteten Unfallkollektiv kamen 26 Fahrradfahrer ums Leben, zwei davon trugen einen Helm. Nur ein Fahrer starb an einer isolierten Kopfverletzung, die restlichen 25 an Polytraumata.

Kopf- und Gesichtsverletzungen waren schwerer, wenn der Fahrradfahrer keinen Helm trug. Verunfallte Fahrradfahrer ohne Helm hatten häufiger Verletzungen am Kopf (13\%) verglichen mit anderen Körperregionen. Fahrradfahrer mit Helm erlitten seltener Kopfverletzungen (6\%). Es wurde bei allen verunfallten Fahrradfahrern mit Helm in keiner Körperregion eine Verletzung AIS 4+ gefunden.

\section{Experimentalstudien}

Depreitere, B., Van Lierde, C., Vander Sloten, J., Van der Perre, G., Van Audekercke, R., Plets, C., Goffin, J. (2007). Lateral Head Impacts and Protection of the Temporal Area by Bicycle Safety Helmets. The Journal of Trauma Injury, Infection, and Critical Care 62, S. 1440-1445 (Depreitere et al. 2007)

Depreitere et al. (2007) betrachten die Wirksamkeit von Fahrradhelmen in der Schläfenregion bei einem seitlichen Anprall. Dazu führten sie elf Leichenversuche durch. Durch ein Stahlpendel mit flacher Auftrefffläche wurde eine Kraft von $15 \mathrm{kN}$ auf den Kopf aufgebracht. In acht Versuchen wurde ein durchschnittlicher Fahrradhelm benutzt, drei Versuche wurden mit einem Helm durchgeführt, der einen größeren Bereich in der temporalen Region abdeckte (Helmkante $<10 \mathrm{~mm}$ über der Frankfurter Horizontalebene). Die Frankfurter Horizontalebene ist dabei wie folgt definiert: "Die gedachte horizontale Linie, die durch den tiefstgelegenen Punkt des Unterrandes der Augenhöhle und durch den höchsten Punkt des äußeren knöchernen Gehörgangs (Porus acusticus externus, Porion) im menschlichen Schädel verläuft" (Lehmann, 2005).

Von den acht Tests mit durchschnittlichen Helmen kam in sieben Fällen ein direkter Kontakt zwischen Kopf und Pendel zustande, in einem Fall wurde ein Schädelbruch dokumentiert. Bei den drei Helmen mit größerem Schutzbereich wurde ein solcher Kontakt verhindert. Das führt die Autoren zu der Aussage, dass sie die Schutzwirkung von aktuellen Helmen in der Schläfenregion anzweifeln. 


\subsubsection{Simulation}

\section{Kinematik des Fahrradsturzes}

Bourdet, N., Deck, C., Carreira, R. P., Willinger, R. (2012). Head impact conditions in the case of cyclist falls. Proceedings of the Institution of Mechanical Engineers, Part P: Journal of Sports Engineering and Technology 2012 (Bourdet et al. 2012)

Diese Parameterstudie betrachtet die Anprallbedingungen für einen Kopfanprall bei stürzenden Fahrradfahrern, insbesondere die Aufprallpunkte und die Aufprallgeschwindigkeit des Kopfs.

Es wurden 1024 Simulationen mit dem TNO 50 Perzentil-Fußgängermodell und einem eigenem Fahrradmodell unter Madymo® durchgeführt.

Es wurden zwei unterschiedliche Situationen untersucht. Zum einen der Sturz eines Fahrradfahrers bedingt durch Rutschen und zum anderen nach Kollision mit einem Bordstein (jeweils mit einer Ausgangsgeschwindigkeit von $5,5 \mathrm{~m} / \mathrm{s}$ und $11,1 \mathrm{~m} / \mathrm{s}$ ).

Die bei den Simulationen auftretenden Aufprallpunkte sind oft an oder unter der Helmkante lokalisiert. Die Geschwindigkeit des Kopfs enthält eine wesentliche tangentiale Komponente, die Normalgeschwindigkeit beträgt knapp $5,5 \mathrm{~m} / \mathrm{s}$.

Chen, Y., Yang, J., Otte, D. (2008). Load and Impact Conditions for Head Injuries in Car-to-Pedestrian and Car-to-Cyclist Accidents - A Comparison of Real Accidents and Simulations. 4th International Conference on ESAR "Expert Symposium on Accident Research" (Chen et al. 2008)

In dieser Studie werden zum einen die Aufprallkonstellationen und daraus resultierenden Verletzungen von Fahrradfahrern und Fußgängern bei Kollisionen mit einem Pkw verglichen. Zum anderen werden verletzungsrelevante Parameter wie HIC bestimmt und aufgetretene Kopfverletzungen mit Hilfe von Rekonstruktion und Simulation erklärt.

Datengrundlage für epidemiologische Betrachtungen sind 402 Fußgänger und 940 Fahrradfahrer aus der GIDAS-Datenbank der Jahre 1999 bis 2007. Es wurden nur Fußgänger und Fahrradfahrer ausgewählt, die in annähernd rechtem Winkel mit einem Pkw kollidiert sind (links oder rechts). Es wurden 40 Unfälle mit Hilfe von PC-Crash und Madymo rekonstruiert und simuliert.

Kollisionen des Kopfes mit dem Rahmen der Frontscheibe und rahmennahen Teilen ziehen eher schwere Verletzungen nach sich. Fahrzeuggeschwindigkeiten von $44,8 \mathrm{~km} / \mathrm{h}$ und $57,9 \mathrm{~km} / \mathrm{h}$ ziehen mit 50\% Wahrscheinlichkeit Kopfverletzungen von AIS 2+ und AIS 3+ nach sich. Fahrradfahrer haben ein generell geringeres Risiko als Fußgänger sich zu verletzen (bei identischer Unfallschwere). Die kopfverletzungsrelevanten Parameter und Aufprallkonstellationen sind ebenfalls unterschiedlich.

Fahlstedt, M., Baeck, K., Halldin, P., Vander Sloten, J., Goffin, J., Depreitere, B., Kleiven, S. (2012). Influence of Impact Velocity and Angle in a Detailed Reconstruction of a Bicycle Accident. Proc. IRCOBI 2012, Nr. 84 (Fahlstedt et al. 2012)

Es wurde der Unfall eines 68-jährigen Fahrradfahrers betrachtet, der schwere Kopfverletzungen erlitten hat. Mit Hilfe eines numerischen Kopfmodells wurde der Einfluss der Variablen „Ausgangsgeschwindigkeit“ und „Orientierung des Kopfs“ auf die zu 
erwartenden Belastungen des Gehirns überprüft. Es wurde verdeutlicht, dass die tangentiale Geschwindigkeitskomponente darauf einen großen Effekt hat.

Pang, T. Y., Sakeran, H., Short, A., McIntosh, A. S., Rechnitzer, G., Thai, K. (2008). Numerical analysis of real-world cyclist crashes: impact speed, collision mechanism and movement trajectories. 2008 Australasian Road Safety Research, Policing and Education Conference 7, 10-12 November 2008, Adelaide, South Australia (Pang et al. 2008)

In dieser FE-Analyse wurde ein Mehrkörpersystem eines Fahrrads erstellt. Dieses wurde mit einem Hybrid III Dummy Modell (TNO) kombiniert. Damit wurden drei reale Unfälle rekonstruiert (Fahrrad-Fahrrad, Fahrrad-Pkw, Fahrrad-Leitplanke). Die Simulationen konnten das reale Unfallgeschehen plausibel darstellen. Das Modell wird im Verlauf weiterer Projekte zur Bestimmung der Geschwindigkeit des Fahrradfahrers vor dem Unfall und zur Verdeutlichung von kinematischen Abläufen verwendet.

van Schijndel, M., de Hair, S., Rodarius, C., Fredriksson, R. (2012). Cyclist kinematics in car impacts reconstructed in simulations and full scale testing with Polar dummy. Proc. IRCOBI 2012, Nr. 85 (van Schijndel et al. 2012)

In dieser Studie wurden drei Ansätze zur Bestimmung der typischen Kinematik von Fahrradfahrern und Fußgängern bei Kollisionen mit einem Pkw betrachtet: Unfallforschung, Simulation und Crashversuche. Bei der Simulation wurden Modelle von Fahrrad und Fahrer in MADYMO verwendet. Die realen Crashtests wurden mit einem Polar II Dummy, Fahrrad, und einem Volvo V70 durchgeführt.

In über $60 \%$ der Unfälle ist die Seite des Fahrrads (rechts oder links) der Anprallpunkt an der Pkw-Front. Sowohl die Simulation als auch die realen Crashtests zeigen, dass die Frontscheibe häufig von Kopf und Torso getroffen wird. Die Crashversuche zeigen außerdem, dass zum effektiven Schutz von Fahrradfahrern die Schutzmaßnahmen in Pkw auch höhere Bereiche (als die Fußgängerschutzsysteme) der Frontscheibe adressieren sollten. Die Kopfaufprallgeschwindigkeiten der Fahrradfahrer waren generell höher als die der Fußgänger.

Watson, J., Hardy, R., Kayvantash, K. (2009). Understanding the Nature of Cyclists Head Impacts. Proc. IRCOBI 2009, S. 301-313 (Watson et al. 2009)

In dieser FE-Analyse wurden Kopfanprallszenarien von Fußgängern und Fahrradfahrern auf ein Fahrzeug untersucht (insbesondere Aufprallgeschwindigkeit, Aufprallort und Auftreffwinkel). Es wurden ein numerisches Mensch-Modell sowie vier verschiedene Fahrzeugfronten verwendet. Es wurden 24 Simulationsszenarien für Fahrradfahrer und 22 Szenarien für Fußgänger durchgeführt.

Die Aufprallgeschwindigkeit für Fahrradfahrer und Fußgänger ist vergleichbar, jedoch trifft der Kopf eines Fahrradfahrers typischerweise in einem anderen Winkel und an einem anderen Punkt (weiter hinten an der Frontscheibe in Richtung Dach) auf. Deshalb sind Fußgänger- und Fahrradfahrer-Anprallszenarien nicht direkt miteinander vergleichbar.

\section{Kopfbelastungen/ Verletzungskriterien}

Ghajari, M., Galvanetto, U., lannucci, L., Willinger, R. (2011). Intracranial Response in Helmet Oblique Impacts. Proc. IRCOBI 2011, S. 90-93 (Ghajari et al. 2011)

In dieser FE-Analyse wurde das numerische menschliche Kopfmodell der Universität Straßburg (SUFEHM) und das Modell eines menschlichen Körpers (THUMS) verwendet. Es wurde der Einfluss eines Körpers bei seitlichen Helmtests betrachtet, insbesondere in 
Hinblick auf Effekte im Gewebe des menschlichen Kopfs (Diffuser axonaler Schaden DAI, Subduralhämatom SDH). Ergebnis dieser Analyse ist, dass der Körper einen nicht zu vernachlässigen Einfluss auf die intrakranielle Antwort ausübt (Anstieg der intrakraniellen Belastungswerte um bis zu 25\%).

Bei Tests mit einem isolierten Kopf wurden realistischere Ergebnisse erreicht, wenn die Masse des Kopfs und die Trägheitsmatrix angepasst wurden. Sowohl die lineare Beschleunigung als auch die Rotationsbeschleunigung üben einen Einfluss auf die Verletzungswahrscheinlichkeit des Gehirns aus.

Mills, N.J., Gilchrist, A. (2008a). Finite-element analysis of bicycle helmet oblique impacts. International Journal of Impact Engineering, Vol. 35, No. 9, S. 1087-1101 (Mills et al. 2008a)

Anhand der Ergebnisse der vorher durchgeführten Experimentalstudie (Mills, Gilchrist 2008) wurden FE-Analysen durchgeführt, um die lineare Beschleunigung und Rotationsbeschleunigung der Kopfform zu überprüfen. Dabei wurden sowohl die Helmrotation auf der Kopfform als auch die Interaktionen der Befestigungsbänder des Helms mit der Kopfform betrachtet. Es wurden exakte Materialeigenschaften des Helms (Schaumstoff, Schale und Befestigungsbänder) definiert.

Es wurden Simulationen durchgeführt, die das seitliche Auftreffen des Helms auf eine Straße nachbilden.

Die auftretenden Kräfte sind stark abhängig von Reibungskoeffizienten, die von der Zerstörung der Innenschale abhängig sind. Die Rotation des Helms ist begrenzt durch die Interaktion zwischen der harten Innenschale und dem menschlichen Schädel, solange die Gurte den Helm auf dem Kopf halten. Vorwärtsrotation ist möglicherweise vom Kontakt des Helms mit der Nase begrenzt, weitere Rotation wird von der ovalen Form des Kopfs begrenzt. Da die innere Form der Schale aber etwas größer ist als die des Kopfs, ist eine gewisse Rotation unvermeidlich.

Die maximale Rotationsbeschleunigung wird hauptsächlich von der Tangentialkraft auf die Helmoberfläche verursacht. Rotationsbeschleunigung tritt ebenfalls in direkten vertikalen Aufprallszenarien auf, wenn die Richtung der auftretenden Kraft nicht durch den Schwerpunkt des Kopfs zeigt und hängt wahrscheinlich ebenfalls eng mit der menschlichen Kopfform zusammen.

Die Ergebnisse wurden im Vergleich mit den realen Experimenten validiert und die Methode zur Ermittlung der maximalen linearen Beschleunigung bestätigt. Die Reibungskoeffizienten konnten ermittelt werden.

Die vorhergesagten maximalen Rotationsbeschleunigungen sind abhängig von Aufprallstelle und -richtung. Die Werte werden stark vom niedrigen Reibungskoeffizienten zwischen Helm und Kopf beeinflusst.

Milne, G., Deck, C., Bourdet, N., Carreira, R. P., Allinne, Q., Willinger, R. (2012). Development and validation of a bicycle helmet: Assessment of head injury risk under standard impact conditions. Proc. IRCOBI 2012, Nr. 86 (Milne et al. 2012)

In dieser Studie wurde ausgehend von Fallversuchen mit einem realen Fahrradhelm ein FEModell dieses Helms erstellt. Dabei wurden 90 reale Fallversuche in Anlehnung an die Testbedingungen der EN 1078 durchgeführt (5,42 m/s auf flachen Amboss, 4,57 m/s auf Bordsteinamboss), die dann zur Validation des Helmmodells ebenfalls simuliert wurden. Dazu wurden die auftretenden linearen Beschleunigungen abgeglichen. Dieses Helmmodell wurde anschließend mit einem Kopfmodell verknüpft, um das Kopfverletzungsrisiko bei diesen Aufprallszenarien darzustellen. Für die überprüften Aufprallpunkte (bis auf einige in der seitlichen Region des Helms) ist das Kopfverletzungsrisiko als akzeptabel 
einzuschätzen. Diese Studie betrachtet nur direkte Aufprallszenarien ohne Rotationsanteil, es wird jedoch darauf hingewiesen, dass in weiteren Studien auch seitliche Anprallszenarien betrachtet und überprüft werden, das diese häufig im realen Unfallgeschehen vorkommen.

\subsubsection{Helmtests und Helmeigenschaften}

Deck, C., Bourdet, N., Calleguo, A., Carreira, P. R., Willinger, R. (2012). Proposal of an improved bicycle helmet standards. Proc. ICRASH 2012 (Deck et al. 2012)

Diese Studie betrachtet die momentanen Testkriterien für Fahrradhelme kritisch. Basierend auf Betrachtungen des realen Unfallgeschehens sowie Experimenten werden Verbesserungsvorschläge für aktuelle Testverfahren, insbesondere die EN 1078, entwickelt. Es werden nur noch zwei Testtemperaturen vorgeschlagen $\left(0^{\circ}\right.$ und $\left.+50^{\circ} \mathrm{C}\right)$, der Test bei niedriger Temperatur $\left(-20^{\circ} \mathrm{C}\right)$ wird für überflüssig erachtet. Es wird nur noch ein Test mit flachem Sockel gefordert, die Testgeschwindigkeit soll mit 5,42 m/s nicht verändert werden. Zusätzlich sollen noch zwei seitliche Aufprallszenarien mit aufgenommen werden, beide mit einer Normalgeschwindigkeit von $5,45 \mathrm{~m} / \mathrm{s}$ und einer Tangentialgeschwindigkeit von $3,5 \mathrm{~m} / \mathrm{s}$ (resultierende Geschwindigkeit ist $6,5 \mathrm{~m} / \mathrm{s}$ ). Es soll damit eine Rotation um die seitliche Achse und die Längsachse des Kopfs initiiert werden.

Es wird vorgeschlagen, einen Hybrid III-Kopf für die Tests zu verwenden, da dieser den menschlichen Kopf realistischer abbildet als die für Helmtests gebräuchlichen Kopfformen. Idealerweise soll dieser auch mit einem Hybrid III-Halssystem verbunden werden.

Die zu testenden Punkte auf dem Helm werden im Gegensatz zur aktuellen Testnorm EN 1078 exakt definiert (vorne, hinten, seitlich und oben). Zudem werden alternative Testpunkte vorgeschlagen, falls es nicht möglich ist, diese in eine Linie mit dem Schwerpunkt des Kopfs zu bringen.

Zudem soll anstatt gebräuchlicher Kriterien zur Vorhersage von Kopfverletzungen (insbesondere HIC) ein FE-Kopfmodell verwendet werden, mit dem detaillierte Aussagen zu verschiedenen Verletzungsarten möglich ist.

Mills, N. J., Gilchrist, A. (2006). Bicycle helmet design. Proceedings of the Institution of Mechanical Engineers, Part L: Journal of Materials: Design and Applications, Vol. 220 No. 4, S. 167-180 (Mills et al. 2006)

Unter Verwendung eines validierten Kopf-/Helmmodells werden Aussagen zur möglichen Verbesserung von Fahrradhelmen getroffen. Insbesondere wird dabei auf die Dicke, Dichte und Art des Absorptionsmaterials eingegangen. Zudem werden auch die Geometrie der Fahrradhelme und die Oberfläche der äußeren Helmschale kritisch betrachtet. Die Ergebnisse sind: Das Schutzpotential eines Fahrradhelms kann durch dickeres Absorptionsmaterial (mit geringerer Dichte) verbessert werden. Es gibt kein aktuelles Material, das Vorteile gegenüber dem gebräuchlichen EPS hat. Zweischichtiges Absorptionsmaterial (mit unterschiedlichen Materialeigenschaften) bietet keine Vorteile. Die maximale Rotationsbeschleunigung bei seitlichen Aufprallszenarien auf eine Straße verursacht normalerweise keine rotationsbedingten Gehirnschäden. Die Stirn sowie die Schläfenregion sollten besser geschützt werden.

Mills, N.J., Gilchrist, A. (2008b). Oblique impact testing of bicycle helmets. International Journal of Impact Engineering, Vol. 35, No. 9, S. 1075-1086 (Mills et al. 2008b) 
In dieser Experimentalstudie wurde ein Versuchsaufbau realisiert, der es ermöglicht, mit einer horizontal verschiebbaren Platte am Aufprallpunkt den seitlichen Aufprall eines frei fallenden Fahrradhelms samt Prüfkopf zu ermöglichen. Der Prüfkopf wurde mit einer künstlichen Haut sowie einer Perücke ausgestattet, um möglichst realistische

Reibungsverhältnisse zwischen Kopf und Helm abzubilden. Die Helme wurden dem Prüfkopf nach einer exakt dokumentierten Prozedur aufgesetzt und dort fixiert. Gemessen wurden die linearen Beschleunigung sowie die Rotationsbeschleunigung in zwei Richtungen. Die Ergebnisse wurden ebenfalls für die oben aufgeführte FE-Analyse von Mills und Gilchrist (2008) verwendet.

Bei den Versuchen wurde keine Abweichung der relativen Position zwischen Helm und Kopf beobachtet. Die bei seitlichen Aufprallszenarien beobachtete Rotationsbeschleunigung ist nur geringfügig größer als bei einem direkten Aufprall ( $5 \mathrm{krad} / \mathrm{s}^{2}$ bei $4 \mathrm{~m} / \mathrm{s}$ ). Die gebräuchlichen Fahrradhelme bieten einen ausreichenden Schutz für seitliche Aufprallszenarien auf eine Straße, was die maximale lineare und Rotationsbeschleunigung des Kopfs betrifft.

Es wird vorgeschlagen, einen seitlichen Aufpralltest (Kopfform mit Haut und Perücke) in die EN 1078 aufzunehmen.

Williams, M. (1991). The protective performance of bicyclists' helmets in accidents. Accident Analysis and Prevention, Vol. 23, No. 2, S. 119-31 (Williams 1991)

Es wurden 64 Helme aus einem Fallkollektiv von 1892 verletzten Fahrradfahrern betrachtet. Die Fahrradfahrer wurden in den Jahren 1987 und 1989-1990 in den 11 öffentlichen Krankenhäusern in Victoria, Australien, behandelt.

Mit neuen Helmen wurden Versuche unter definierten Testbedingungen unternommen, um die im realen Unfall aufgetretenen Beschädigungen möglichst exakt nachzubilden, um somit Aussagen zur Unfallschwere treffen zu können (Aufprallgeschwindigkeit sowie aufgetretene Kopfbeschleunigung).

$39 \%$ der Unfälle $(n=25)$ waren Alleinunfälle. Die restlichen Unfälle waren Kollisionen mit anderen Verkehrsteilnehmern, davon überwiegend (85\%) Kollisionen mit einem motorisierten Fahrzeug. Bei diesen Kollisionen wurden alle aufgetretenen schweren Kopfverletzungen betrachtet.

$30 \%$ der Helme hatten mehr als einen Aufprall. Die Aufprallgeometrie war in allen Fällen flach, in 62\% war es die Oberfläche einer Straße. 63\% aller Aufpralle waren unterhalb des Testbereichs lokalisiert, den die australische Helmnorm AS 2063.1-1986 vorschreibt. Typischer Aufprallbereich war entweder an der Stirn oder der temporalen Region des Kopfs. Wenn der Helm auf dem Kopf fixiert blieb, trat keine schwerwiegende Hirnverletzung auf.

\subsubsection{Sonstiges}

COST 327 Final Report, online verfügbar unter: http://ec.europa.eu/transport/roadsafety_library/publications/cost327_final_report.pdf (COST 327)

Datengrundlage dieser Fallstudie sind 253 behelmte Motorradfahrer entweder mit Kopf- oder Halsverletzungen oder dokumentiertem Helmkontakt. Das Datenmaterial wurde in den Jahren 1996-1998 in Finnland, Deutschland und Großbritannien erhoben. Es wurden typische Unfallsituationen und Verletzungsmechanismen (Kopf und Hals) von behelmten Motorradfahrern untersucht.

$76 \%$ der Motorradfahrer hatten eine Kopfverletzung, 27\% eine Halsverletzung. Die mittlere Aufprallgeschwindigkeit beim Auftreten von Gehirnerschütterungen war $43 \mathrm{~km} / \mathrm{h}$, für 
Hirnverletzungen $60 \mathrm{~km} / \mathrm{h} .31 \%$ der Gehirnverletzungen wurden durch direkte Krafteinwirkung verursacht, 58\% durch indirekte Krafteinwirkung und $11 \%$ durch Krafteinwirkung auf der gegenüberliegenden Seite ("contre coup).

Bei Kopfverletzungen AIS $\geq 2$ wurde in über $60 \%$ eine Rotationsbewegung und in $30 \%$ eine lineare Bewegung beobachtet.

Zusätzlich wurde ein FE-Modell von Schädel, Gehirn, Hals und Helm erstellt. Damit wurden 13 Fälle aus der Datenbank simuliert. Damit konnten die Helmbeschädigungen von realen Unfällen nachgestellt werden. Ergebnis dieser Simulationen ist, dass AIS, HIC und GAMBIT nicht gut miteinander korrelieren.

\section{Curnow, W. J. (2003). The efficacy of bicycle helmets against brain injury. Accident} Analysis and Prevention, Vol. 35, No. 2, S. 287-292 (Curnow 2003)

Die Ergebnisse der Meta-Analyse von Attewell et al. (2001) werden in Hinblick auf die Berücksichtigung von Mechanismen der Entstehung von Gehirnverletzungen überprüft. Der Autor weist darauf hin, dass in dieser Studie nicht die bei Kollisionen auftretenden Rotationsbeschleunigungen berücksichtigt werden, obwohl diese für schwerste Gehirnverletzungen (diffuse axonal injury, DAI) verantwortlich scheinen. Somit sei die Annahme, dass Fahrradhelme das Risiko für Kopf- und Gehirnverletzungen deutlich reduzieren, aus wissenschaftlicher Sicht nicht haltbar. Es könne nicht nachgewiesen werden, dass Helme Verletzungen reduzieren, auf deren Effektivität sie nicht getestet werden.

\section{Curnow, W. J. (2005). The Cochrane Collaboration and bicycle helmets. Accident Analysis and Prevention, Vol. 37, No. 3, S. 569-573 (Curnow 2005)}

Die Ergebnisse des Cochrane Reviews "Helmets for preventing head and facial injuries in bicyclists" von Thompson et al. (2004) werden vom Autor aufgrund der verwendeten Daten und Methoden angezweifelt.

Nach Curnow gehen die Autoren nicht auf die Typen und Mechanismen von Gehirnverletzungen ein. Somit sei die Aussage, dass alle Arten von Fahrradhelmen das Risiko von Gehirnverletzungen reduzieren, nicht korrekt. Lediglich die Behauptung, dass die fast nicht mehr gebräuchlichen Hartschalenhelme vor Gehirnverletzungen schützen, die wiederum durch Beschädigungen des Schädels verursacht wurden, sei aus seiner Sicht haltbar.

Heesch, K. C., Garrard, J., Sahlqvist, S. (2011). Incidence, severity and correlates of bicycling injuries in a sample of cyclists in Queensland, Australia. Accident Analysis \& Prevention, Vol. 43, No. 6, S. 2085-2092 (Heesch et al. 2011)

Datengrundlage dieser Querschnittsstudien sind 2056 Fahrradfahrer, die im Jahr 2009 einen Fragebogen ausgefüllt haben. Die Fahrradfahrer stammen aus Queensland, Australien, und waren Mitglieder bei „Bicycle Queensland“, einer Vereinigung die sich für bessere Radfahrbedingungen einsetzt.

Es wurden die Häufigkeit und die Schwere von Verletzungen bei Fahrradfahrern in Abhängigkeit von den Unfallumständen betrachtet. 27\% der befragten Fahrradfahrer erlitten bereits Verletzungen beim Radfahren, $6 \%$ hatten bereits schwere Verletzungen. Schwere Verletzungen wurden häufiger bei Unfällen mit Fremdbeteiligung berichtet. 


\subsubsection{Diskussion Literaturrecherche}

Laut Gesundheitsberichtserstattung des Bundes (2014) starben im Jahr 2013 ca. 58\% aller getöteten Fahrradfahrer in Deutschland an Verletzungen des Kopfes. Bei weiteren $18 \%$ ist die Todesursache ein Polytrauma, was weitere Kopfverletzungen beinhalten kann.

Studien von Fife et al. (1983), Oström et al. (1993), (Wood et al. 1988) führen auf, dass in $69 \%$ bis $86 \%$ aller tödlichen Fahrradunfälle eine Kopfverletzung die direkte Todesursache war. Liers (2011) beschreibt, dass der Kopf der am häufigsten verletzte Bereich des Körpers bei MAIS 2+ verletzten Fahrradfahrern ist.

Somit sind von einer reduzierten Zahl von schwersten Kopfverletzungen bei Fahrradunfällen direkte positive Auswirkungen auf den Rückgang von getöteten Fahrradfahrern zu erwarten.

Thompson et al. (1989) beschreiben in ihrer sehr häufig zitierten Fall-Kontroll-Studie, dass sich bei Fahrradfahrern mit Helm das Risiko für Kopfverletzungen um 85\% und das Risiko für Hirnverletzungen um 88\% reduziert. In einer von der Methodik ähnlichen Studie der gleichen Autoren (Thompson et al. 1996) sind diese Zahlen geringer. Das Risiko für Kopfverletzungen sinkt demzufolge mit Helm um 69\%, das Risiko für Hirnverletzungen um 65\%. Jedoch merken die Autoren an, dass diese aktuelleren Zahlen möglicherweise zu niedrig sind, da die Kontrollgruppe nicht populationsbasiert ist, was eine mögliche Abweichung nach unten erklärt. In einem aktuellen Review der Cochrane Collaboration (Thompson et al. 2009), der ebenfalls von den oben genannten Autoren verfasst wurde, wird an diesen Zahlen festgehalten. Nach diversen Kritikern ist dieser Review jedoch nicht unabhängig, da das überwiegende Datenmaterial von Studien der gleichen Autoren stammt, deren Arbeiten selbst für ihre methodischen Fehler kritisiert wurden. Es wurden zudem nur Fall-KontrollStudien betrachtet. Zusätzlich wurde die Problematik des Einflusses von Rotationsbeschleunigungen nicht betrachtet.

Weitere kritische Stimmen bemängeln die Ergebnisse der Studie von 1989, da die Auswahl der Kontrollgruppen nicht zufällig war. Zudem wurde die generelle Helmtragequote zu diesem Zeitpunkt falsch eingeschätzt und auch das Risikoverhalten der Fahrradfahrer wurde nicht betrachtet. Die Studie von 1996 bietet ähnliche Einschränkungen.

Curnow (2005) kritisiert die Ergebnisse der aktuellen Review prinzipiell, da die Autoren seiner Meinung nach nicht auf die genauen Verletzungsmechanismen des Gehirns eingehen.

Eine ähnliche Kritik übt Curnow (2003) an der Meta-Analyse von Attewell et al. (2001), jedoch ist auch die Kritik von Curnow nicht unumstritten und detailliert zu diskutieren.

In der genannten Studie von Attewell et al. (2001) wird aufgezeigt, dass Fahrradfahrer mit Helm ein geringeres Risiko tragen, eine Kopf- oder Hirnverletzung zu erleiden.

Datengrundlage bilden 16 Studien aus den Jahren 1980 bis 1995. Die Werte für eine mögliche Reduktion der Kopf- und Gehirnverletzungen liegen dabei zwischen 33\% und 88\%. Laut Elvik (2011) sind diese Zahlen immer noch zu hoch angesetzt.

Elvik (2011) liefert die aktuellste Zusammenschau und den kritischsten Umgang mit dem vorhandenen Datenmaterial aus bisherigen Studien und Reviews. Daher ist der von inm präsentierte Wert (42\% für die Risikoreduktion von Kopfverletzungen) aufgrund der konservativen Schätzung am verlässlichsten.

Die Studien von Amoros et al. (2011), Otte et al. (2008), Pang et al. (2009) Richter et al. (2007), sprechen von einer deutlichen Reduktion des Risikos für alle Kopfverletzungen. Nach Otte et al. (2008), Amoros et al. (2011), Berg et al. (2007) kann ein aktueller Fahrradhelm 
auch schwerste (AIS 3+) Kopfverletzungen verhindern. Nach Amoros et al. (2011) ist die Risikoreduzierung für schwere Kopfverletzungen (-69\%) größer als für beliebige Kopfverletzungen (-31\%). Liers (2011) zeigt auf, dass die Helmbenutzung sowohl zu einem Rückgang von schweren Verletzungen als auch einem Anstieg der Zahl im Kopfbereich unverletzter Fahrradfahrer führen kann. Berg et al. (2007) stellen aufgrund ihrer Fall-KontrollStudie fest, dass der Helm einen protektiven Effekt hat.

Mclntosh et al. (1998) stellen in ihrer Fallstudie dar, dass in der temporoparietalen Region des Kopfs ein hoher Anteil an Aufprallpunkten lokalisiert ist. Aus diesem Grund denken die Autoren über eine Neuanordnung der Testlinie in aktuellen Testverfahren nach.

Depreitere et al (2007) kommen in ihrer Experimentalstudie ebenfalls zu dem Ergebnis, dass aktuelle Fahrradhelme die temporoparietale Region des Kopfs nur unzureichend schützen.

Die Experimentalstudien von Mills et al. 2008 und Mills et al. 2006 betrachten das Schutzpotential von Fahrradhelmen in realen Experimenten und FE-Analysen. Es wird dabei ein Kopfmodell verwendet, das mit Haut und Perücke ausgestattet ist, um den Einfluss von Reibungsparametern zwischen Helm und Kopf möglichst realistisch abbilden zu können. Dabei wird ebenfalls der aktuelle Schutz der Schläfenregion bemängelt. Prinzipiell wird das Schutzpotential aktueller Fahrradhelme (insbes. Stoßdämpfung) als ausreichend eingeschätzt.

Depreitere et al. (2004), Eilert-Petersson et al. (1997) und Fredriksson et al. (2012) beschreiben typische Unfallszenarien für Fahrradfahrer. Während Eilert-Petersson et al. (1997) den Alleinunfall mit über $80 \%$ als typischen Fahrradunfall einschätzen, ist es bei Depreitere et al. (2004) und Fredriksson et al. (2012) die Kollision mit einem anderen Fahrzeug. Diese Aussagen werden dadurch relativiert, dass bei der Studie von Depreitere et al. ein bias hin zu Kfz-RF-Kollisionen durch das Fallmaterial von Patienten, die eines neurochirurgischen Eingriffs bedurften (also besonders schwere Unfallfolgen) gegeben ist.

Fredriksson et al. haben sich in ihrer Studie Fallmaterial betrachtet, das von vorneherein auf Kollisionen zwischen Kfz. und RF beschränkt ist.

Oben genannte Autoren und Siman-Tov et al. (2012) sind sich einig, dass eine Kollision mit einem Fahrzeug schwerere Verletzungen nach sich zieht. Nach Kim et al. (2007) steigt die Verletzungsschwere dabei mit der Fahrzeuggeschwindigkeit. Nach Moore et al. (2011) steigt das Risiko des Fahrradfahrers für schwere Verletzungen bei Kollisionen an einer Kreuzung/Einmündung und wenn der Pkw die Seite des Fahrradfahrers trifft.

Oström et al. (1993) betrachteten tödliche Fahrradunfälle und stellten fest, dass $88 \%$ der Fahrradfahrer bei einer Kollision mit einem andern Fahrzeug zu Tode kamen. In 69\% war dabei eine Kopfverletzung die Todesursache. Tschernitschek et al. (2010) stellten fest, dass nur ein Fahrradfahrer von 26 an einer isolierten Kopfverletzung starb. Bei verunfallten Fahrradfahrern mit Helm wurde in keiner Körperregion eine Verletzung AIS $\geq 4$ gefunden.

Chen et al. (2008), Pang et al. (2008), van Schijndel et al. (2012) und Watson et al. (2009) haben die Kinematik von Fahrradunfällen simuliert. Dabei wurden ein Fahrradmodell und das Modell eines Dummys / Menschen gekoppelt und versucht, reale Unfallszenarien in ihrer Gesamtheit abzubilden. Bei Kollisionen mit der Pkw-Front wurde beobachtet, dass die Fahrradfahrer häufig auf der Frontscheibe aufprallen. Verglichen mit Fußgängeranprallen sind die Aufprallorte der Fahrradfahrer typischerweise weiter oben und hinten in Richtung Dachkante orientiert. Partnerschutzsysteme von Pkw sollten dementsprechend optimiert werden. 
Eine Forschungsgruppe der Universität Straßburg beschäftigt sich ebenfalls mit Fahrradhelmen und liefert interessante Ergebnisse. Deck et al. (2012) liefern konkrete Vorschläge, wie man aktuelle Testverfahren optimieren kann. Milne et al. (2012) haben ein numerisches Fahrradhelmmodell entwickelt und damit die aktuellen Testbedingungen in Simulationen überprüft. Nach ihren Ergebnissen bieten aktuelle Fahrradhelme zumindest für diese Testbedingungen ausreichendes Schutzpotential, was plausibel klingt, da die Hersteller ihre Produkte genau auf diese Anforderungen optimieren. Die Autoren stellten jedoch keine Verknüpfung zu realen Unfallszenarien her. Bourdet et al. (2012) haben in ihrer Parameterstudie die Anprallbedingungen eines Kopfanpralls simuliert. Dabei stellten sie fest, dass die Aufprallpunkte oft an oder unter der Helmkante lokalisiert sind. Ebenfalls deutlich wird der Einfluss der tangentialen Geschwindigkeitskomponente, die nach Ansicht der Autoren auch kritische Rotationsbeschleunigungen zur Folge haben kann.

Betrachtet man alle untersuchten Studien in ihrer Gesamtheit und versucht ein Fazit zu ziehen, bleibt festzuhalten, dass ein beträchtlicher Anteil an Fahrradfahrern schwere oder tödliche Verletzungen im Bereich des Kopfs erleidet. Dabei ist die Verletzungsentstehung und -mechanik noch nicht im Detail geklärt. Ein Fahrradhelm bietet Potential, diese Verletzungen zu verhindern oder abzuschwächen. Die aktuellen Anforderungen und Testbedingungen bieten eine gute Basis, um dieses Schutzpotential zu gewährleisten. Es sind jedoch weitere Optimierungsmaßnahmen nötig, um dieses Schutzpotential zu verbessern und an reale Unfallbedingungen anzupassen.

Einzelne Studien versuchen bisher nur, Lösungsvorschläge für einzelne Teile dieser komplexen Problematik anzubieten.

Der im vorliegenden Forschungsprojekt „Fahrradhelm“ realisierte Ansatz mit der Verknüpfung von Unfallforschung und Biomechanik schafft jedoch eine Möglichkeit für komplexere und weiterführende Betrachtungen. Diese können in Zukunft nicht nur als Grundlage für verbesserte Testverfahren für Fahrradhelme dienen, sondern auch als Diskussionsgrundlage für darauf aufbauende Problemstellungen zur Verfügung stehen.

\section{$1.2 \quad$ Definition helmrelevanter Parameter}

Folgende Parameter wurden als helmrelevant definiert:

- Geometrie des Helmes

- Materialeigenschaften des Absorptionsschaums

- Materialeigenschaften der Helmschale

- Passgenauigkeit / Befestigung des Helms

- Umweltfaktoren (Temperatur, Feuchtigkeit)

- Anprallpunkt / -richtung am Kopf / Helm

- Geometrie und Steifigkeit des Aufprallobjekts

- Geschwindigkeit des Fahrradfahrers

- Geschwindigkeit des Kollisionsgegners (falls kein Alleinunfall)

Die Geometrie des Fahrradhelms ist essentiell für seine mögliche Schutzfunktion. Nach McIntosh et al. (1998) ist die Geometrie ein möglicher Kritikpunkt an aktuellen Helmen. Insbesondere die Gestaltung im temporoparietalen Bereich bedarf weiterer Untersuchungen, was Depreitere et al. (2007) auch in Versuchen darstellten. Zusätzlich ist auch über eine Anpassung des Prüfbereichs im Stoßdämpfungstest der Fahrradhelmnormen nachzudenken. 
Deck et al. (2012) fordern vorgeschriebene Prüfpunkte, um auch das Schutzpotential der Seiten des Fahrradhelms ausreichend zu überprüfen.

Die Materialeigenschaften des Absorptionsschaums beeinflussen direkt die Werte der Kopfbeschleunigung. Je weicher der Schaum (geringere Druckfestigkeit), umso geringer die zu erwartende resultierende Kopfbeschleunigung, solange es zu keinem Durchschlag (d.h. Blockbildung des komprimierten Schaumes) kommt. Jedoch ist bei gleicher Dicke der maximale Absorptionsweg bei geringerer Krafteinwirkung erreicht, der Helm wirkt nur bei niedrigen Aufprallgeschwindigkeiten optimal. Für diese gegensätzlichen physikalischen Wirkprinzipien gilt es einen Kompromiss zu finden, der an das reale Unfallgeschehen angepasst ist. Mills et al. $(2006,2008)$ haben die Problematik des Absorptionsmaterials auf Basis von FE-Analysen betrachtet. Nach Aussage dieser Autoren kann das Schutzpotential von Helmen prinzipiell durch dickeres Material mit geringerer Dichte erhöht werden. Es ist aktuell kein Material verfügbar, das erkennbare Vorteile gegenüber aktuell gebräuchlichen EPS (Expanded Polystyrol)-Schäumen bietet.

Die Helmschale dient einerseits der optimalen Kraftverteilung eines Aufpralls, zum anderen sorgt die Schale für die Integrität des Helms bei schweren Kollisionen. Es soll ein Abbrechen von Absorptionsschaum oder sogar ein Zersplittern in mehrere Teile verhindert werden, was vor allem einen großen Effekt für Folgekollisionen hat. Außerdem soll die Schale ein problemloses Abgleiten des behelmten Kopfs von den Anprallobjekten, z.B. der Straßenoberfläche gewährleisten. Dies soll schwere Halsverletzungen verhindern. Die gängigste Schalen-Ausführung ist heutzutage die Microshell, d.h. über dem Absorptionsmaterials ist eine dünne Schale aus Polycarbonat (z.B. Makrolon) angebracht.

Des Weiteren gilt es, die Passform und Befestigung aktueller Fahrradhelme zu überprüfen und gegebenenfalls zu optimieren. Mills et al. (2008) führten ihre FE-Analysen mit einem exakt positionierten und einer an die Realität angepassten Befestigung durch. In verschiedenen Simulationsszenarien wurde die relative Bewegung zwischen Helm und Kopf beobachtet. Dabei wurde die aufgetretene Bewegung als vergleichsweise gering eingeschätzt.

Thai et al. $(2009 a+b)$ betrachteten, wie der Fahrradhelm in der Realität getragen wird. Dabei wurde deutlich, dass die meisten Fahrradfahrer die Helmgröße korrekt auswählen, jedoch bei über $60 \%$ der Helm nicht optimal auf dem Kopf sitzt.

Temperatur und Feuchtigkeit haben nach Liu et al. (2003) einen direkten Einfluss auf die Dämpfungseigenschaften des Absorptionsschaums. In der EN 1078 sind Tests in einem Temperaturbereich von $-20^{\circ}$ bis $+50^{\circ}$ Celsius sowie einem definierten Feuchtigkeitszustand vorgeschrieben. Deck et al. (2012) schlagen vor, Helmtests nur noch bei $0^{\circ}$ und $50^{\circ}$ Celsius durchzuführen, da niedrigere Temperaturen im realen Unfallgeschehen kaum vorkämen. Zudem könne das Absorptionsmaterial weiter optimiert werden, wenn der Test bei $-20^{\circ}$ entfiele.

Die Lokalisation des Anprallpunkts am Kopf hat direkte Auswirkungen auf die geometrische Gestaltung des Helms. Es sollte eine möglichst große Anzahl von typischen Anprallpunkten durch die Helmschale abgedeckt werden.

Dabei gilt es, die Schutzeigenschaften des Helms auf die Verletzungsanfälligkeit der verschiedenen Bereiche des menschlichen Schädels anzupassen. So ist unter anderem nach Mclntosh et al. (1998) und Depreitere et al. (2007) die temporoparietale Region des Schädels besonders anfällig für schwere Verletzungen. Es wäre somit über eine optimale 
Anpassung der Eigenschaften des Helms in dieser Region nachzudenken, z.B. durch darauf abgestimmte Dämpfungseigenschaften.

Insbesondere die Richtung des Anpralls (gepaart mit dem Anprallpunkt) ist eine wichtige Variable. Einige Studien (Deck et al. 2012, Bourdet et al. 2012, Mills et al. 2008, Milne et al. 2012) zeigen deutlich, dass eine tangentiale Geschwindigkeitskomponente

Rotationsbeschleunigungen verursachen kann. In aktuellen Testverfahren wird nur die lineare Beschleunigung des Helms beziehungsweise Prüfkörpers beurteilt, d.h. der resultierende Kraftvektor zeigt in Richtung des Schwerpunkts der Kopfform.

Rotationsbeschleunigungen werden dabei nicht erfasst. Nach Deck et al. (2012) besteht aber ein erhöhtes Risiko für Schädigungen am Gehirn, falls Rotationsbeschleunigungen wirken. Daher schlagen diese Autoren vor, einen zusätzlichen Test einzuführen, bei dem der Helm seitlich auf einem abgeschrägten Amboss abgleitet. Damit soll das Schutzpotential des Helms in Hinsicht auf Rotationskräfte untersucht werden.

Um weitere Aussagen über die typischen Krafteinwirkungen zu treffen und Anforderungen an einen optimalen Fahrradhelm zu stellen, sind zusätzlich Geometrie und Festigkeit des Aufprallobjekts, Geschwindigkeit des Fahrradfahrers und die Geschwindigkeit des Kollisionsgegners zu betrachten.

Basierend auf den realen Belastungen sollen optimierte Testanforderungen erarbeitet werden.

Folgende Parameter sollten dazu in den zu betrachtenden Unfällen möglichst exakt dokumentiert sein:

- Helmtragestatus

- Anprallpunkt am Kopf / Helm

- Geometrie und Steifigkeit des Anprallpunkts am Boden / am Kollisionsgegner

- Geschwindigkeit und Richtung des Fahrradfahrers

- Geschwindigkeit und Richtung des Kollisionsgegners.

\subsection{Auswahl der im Detail zu rekonstruierenden Unfälle}

Im vorliegenden Forschungsprojekt wurden auf Basis mehrerer Datenkollektive aus der Rechtsmedizin München, dem Universitätsklinikum München und dem Universitätsklinikum Münster typische Unfallsituationen herausgearbeitet, die anschließend anhand von gut dokumentierten Realunfällen rekonstruiert und simuliert wurden.

Zu Projektbeginn lag nur das retrospektiv erfasste Fallkollektiv des Instituts für Rechtsmedizin München vor. Die weiteren Fallkollektive aus den Universitätskliniken München und Münster wurden prospektiv über den Zeitraum von einem Jahr im Verlauf des Projekts erfasst und anschließend ausgewertet.

Um parallel mit Rekonstruktion und Simulation typischer Unfallszenarien beginnen zu können, wurde in der frühen Phase des Projekts eine Kurzauswertung des Fallmaterials des Instituts für Rechtsmedizin München durchgeführt.

Ziel war die Vorauswahl der typischen Unfallszenarien sowie die Selektion geeigneter, sehr gut dokumentierter Realunfälle zur weiteren Rekonstruktion und detaillierten Simulation. Zum Teil wird dies auch durch Ergebnisse der prospektiv erfassten Unfälle mit verletzten Radfahrern unterstützt. 
Auf den folgenden Seiten ist diese Kurzauswertung dargestellt. Die Auswertung und Analyse aller Fallkollektive und die endgültige Bestimmung der typischen Unfallsituationen folgt in Kapitel 1.4.

\subsubsection{Vorliegendes Material und Datenbankaufbau SUD}

\subsubsection{Vorliegendes Datenmaterial}

In der Datenbank des Instituts für Rechtsmedizin München (Sicherheits-Unfall-Datenbank, SUD) sind 117 Unfälle der Jahre 2003 bis 2009 enthalten, bei denen der beteiligte Fahrradfahrer an den Unfallfolgen verstarb. Um die Definition eines Verkehrstoten zu erfüllen, wurden ausschließlich die Fälle betrachtet, in denen der Tod nach maximal 30 Tagen eintrat. Die Unfälle ereigneten sich in Oberbayern, Niederbayern und Schwaben. Die Obduktion wurde dabei jeweils am Institut für Rechtsmedizin der Ludwig-MaximiliansUniversität München durchgeführt.

Im Einzugsbereich der Staatsanwaltschaft München 1 werden annähernd 100\% der Verkehrsunfalltoten obduziert, während in den übrigen Einzugsbereichen (München 2, Augsburg, Traunstein, Landshut) die Obduktionsquote deutlich geringer ist.

Dabei stand je nach Fall folgender Akteninhalt zur Verfügung:

- Polizeiliche Verkehrsunfallanzeige

- Unfallskizze

- Polizei- und Sachverständigenbilder

- Beteiligtenaussagen

- Zeugenaussagen

- Obduktionsgutachten

- Unfallanalytische Sachverständigengutachten

- Blutalkoholuntersuchung

- Toxikologisches Gutachten zur Feststellung des Drogenkonsums

- Rechtsanwaltskorrespondenz

Betrachtet wurden ausschließlich Fälle mit vollständigen Akten, bei denen mindestens ein Fahrradfahrer beteiligt war, der aufgrund der Unfallfolgen innerhalb von 30 Tagen verstarb.

\subsubsection{Aufbau der Datenbank}

Die Datenbank wurde unter Microsoft Access erstellt, es wurden für jeden Fall insgesamt neun verschiedene Unterrubriken angelegt:

1. "Grunddaten des verstorbenen Radfahrers"

2. "Allgemeine Daten (inkl. Verletzungskodierung nach AIS)"

3. "Oberflächliche Kopfverletzungen"

4. "Beteiligte / BAK"

5. „Unfallklassifikation“

6. "Lkw Kollision"

7. "Geschwindigkeiten / Schleuderweiten / Anprall Körper"

8. „Begleitende Auffälligkeiten“

9. „Primäre Prävention“ 


\subsection{Grunddaten}

Dieses Fenster beinhaltet die wichtigsten Daten auf den ersten Blick, die dem schnellen Vorselektieren der Fälle dienen sollen.

Die Informationen über das „Alter“, das „Geschlecht“, „Gegner“ (Beteiligter 02) und "Getöteter gleich Verursacher" wurden der polizeilichen Verkehrsunfallanzeige entnommen und gegebenenfalls mit dem Sachverständigengutachten abgeglichen. In dieser Datenbank ist der Getötete Radfahrer immer der Beteiligte 01, demgegenüber kann in der Verkehrsunfallanzeige auch der Unfallgegner der Beteiligte 01 und der Radfahrer Beteiligter 02 sein.

Dem Obduktionsgutachten entstammen die Informationen über die „Todesursache“, „Todesursächliche Körperregion“ und den „Todeszeitpunkt“.

\subsection{Allgemeine Daten}

Die Informationen stammen aus der jeweiligen polizeilichen Verkehrsunfallanzeige und den technischen Gutachten. Aufgeführt sind:

Datum, Wochentag, Uhrzeit, Ortslage (innerorts oder außerorts), Wetter (sonnig, bewölkt, regnerisch), Lichtverhältnisse (hell, dunkel, Dämmerung), Straßenverhältnisse (trocken, feucht, nass, unbefestigt). Straßenverlauf, Kurzbeschreibung des Unfalls.

Zusätzlich sind an dieser Stelle alle im Obduktionsgutachten aufgeführten Verletzungen nach AIS kodiert und tabellarisch aufgeführt.

\subsection{Oberflächliche Kopfverletzungen}

Hier sind alle im Obduktionsgutachten aufgeführten oberflächlichen Kopfverletzungen nach Art, Lokalisation und Ausdehnung der Verletzung dokumentiert. Dafür wird die Oberfläche des menschlichen Kopfes nach einem Schema unterteil, welches später (s. Kapitel 1.4) auch beim Fragebogen zur prospektiven Erhebung in München und Münster verwendet wird (Abbildung 1-1).
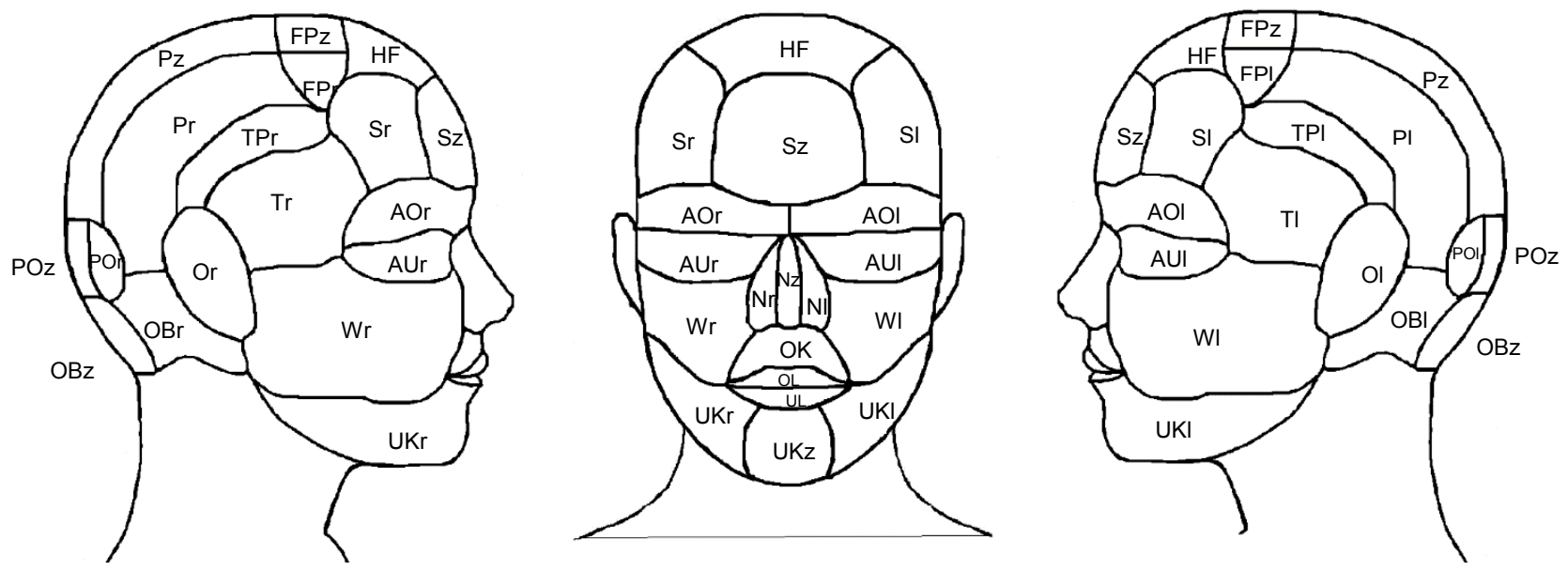

Abbildung 1-1: Einteilung der Kopfoberfläche 


\subsection{Beteiligte / BAK}

In dieser Rubrik sind Gewicht und Körpergröße der unfallbeteiligten Personen aufgeführt. Ebenfalls ist dokumentiert, ob die Beteiligten unter Alkohol-, Drogen- oder Medikamenteneinfluss standen.

\subsection{Unfallklassifikation}

Jeder Unfall wird nach den offiziellen Unfallursachen der Polizei kodiert. Zusätzlich erfolgt eine Einteilung in Unfallart und Unfalltyp analog des amtlichen Unfalltypen-Katalogs (GDV, 2003), wie er in Polizeiberichten Verwendung findet. Es erfolgt auch eine Einteilung des Anprallbereichs am Kfz, im Speziellen wird nach Frontscheibe, Motorhaube und Fahrzeugfront differenziert (falls möglich).

\subsection{LKW-Kollision}

Die Angaben in dieser Rubrik sind für das Fahrradhelmprojekt primär nicht relevant, sofern nicht Kopfverletzungen des Radfahrers dominierend sind. Aufgeführt sind das Verhalten des Lkw-Fahrers vor Kollision, Typ des Lkw und genauer Kollisionsbereich.

\subsection{Geschwindigkeiten / Schleuderweiten / Anprall Körper}

In diesem Bereich der Datenbank werden überwiegend Informationen zusammengetragen, die Aufschluss über Größe und Richtung der Krafteinleitung auf den Fahrradfahrer geben. Hierzu zählen die Fahr- und Kollisionsgeschwindigkeiten der Beteiligten und die Anprallzonen am Körper und Kfz/Hindernis.

Die Fahr- und Kollisionsgeschwindigkeiten der Beteiligten sind den unfallanalytischen Sachverständigengutachten entnommen oder wurden rekonstruiert.

Zur ersten Feststellung der Relevanz eines Fahrradhelmes sind die Anprallstellen am Kopf unter Zuhilfenahme der Obduktionsgutachten grob dargestellt (analog Abbildung 1-1).

\subsection{Begleitende Auffälligkeiten}

Die „Begleitenden Auffälligkeiten“ bilden weitere Schwerpunkte, mit denen die Entstehung von Verkehrsunfällen analysiert werden kann. Dabei erfolgt eine generelle Einteilung in drei Kategorien. Diese sind Mensch, Fahrzeug und Umfeld.

\subsection{Primäre Prävention}

Unter primärer Prävention sind generelle erste Einschätzungen zur Unfallvermeidung aufgeführt. Hier wird unterteilt zwischen möglichen Maßnahmen in den Bereichen Infrastruktur, Technik, Verkehrsteilnehmer und Gesellschaft. Diese sind jedoch nicht Gegenstand des vorliegenden Projekts und werden daher nicht näher betrachtet. 


\subsubsection{Kurzauswertung der SUD}

\subsubsection{Unfallursachen}

Für beide unfallbeteiligten Parteien wurden Unfallursachen nach dem amtlichen Katalog von den Polizeibeamten festgestellt und in den Verkehrsunfallanzeigen festgehalten.

In Abbildung 1-2 und Abbildung 1-3 sind die polizeilichen Unfallursachen getrennt nach der Unfallbeteiligung aufgezeigt.

Abbildung 1-2 zeigt ausschließlich die Unfallursachen bei den 117 getöteten Fahrradfahrern. Dominierend mit knapp 20\% ist die Ursache "Andere Fehler beim Fahrzeugführer" (z.B. ungeklärtes Abkommen von der Fahrbahn oder nicht erklärbare verzögerte Reaktion).

Darauf folgen mit kurzem Abstand die Unfallursachen "Vorfahrt, Vorrang",

"Straßenbenutzung", und "Verkehrstüchtigkeit". In 15\% der Fälle ist die erhebliche Alkoholisierung der Fahrradfahrer unfallursächlich.

Die weiteren Unfallursachen spielen bei den Fahrradfahrern eine untergeordnete Rolle.

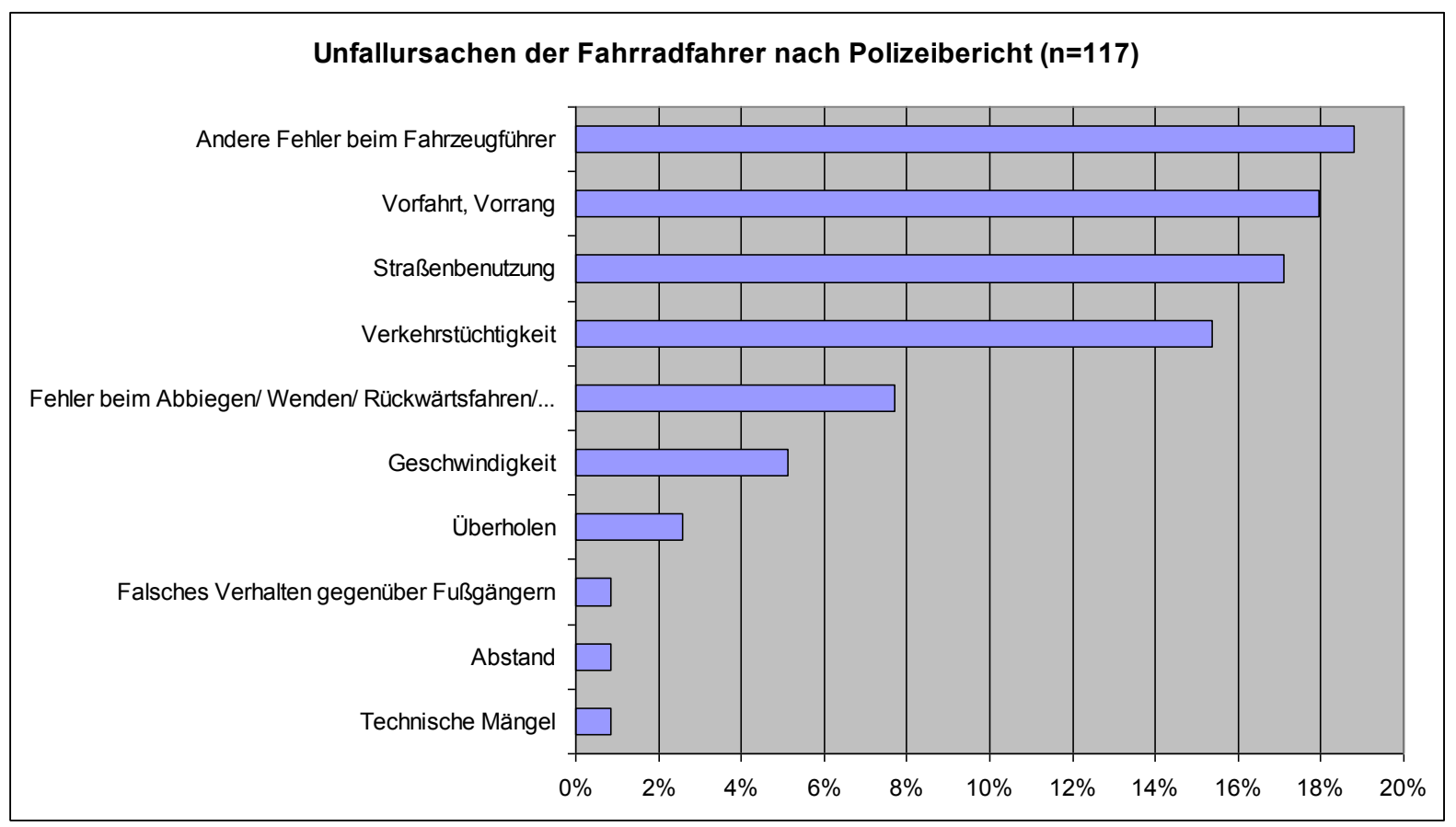

Abbildung 1-2: Unfallursachen der Fahrradfahrer nach Polizeibericht

Auf Abbildung 1-3 sind die Unfallursachen der Unfallgegner (Pkw, Lkw, ...) aufgetragen. Die 16 Alleinunfälle sind in dieser Betrachtung nicht enthalten, somit beträgt die Fallzahl 101.

An erster Stelle stehen "Fehler beim Abbiegen/Wenden/Rückwärtsfahren" mit über 20\%. Dahinter folgen mit etwas Abstand "Andere Fehler beim Fahrzeugführer" mit 12\% und "Geschwindigkeit" mit 11\%. "Verkehrstüchtigkeit" ist bei $8 \%$ der Unfallgegner eine Unfallursache, d.h. nur halb so oft wie bei den Fahrradfahrern. 


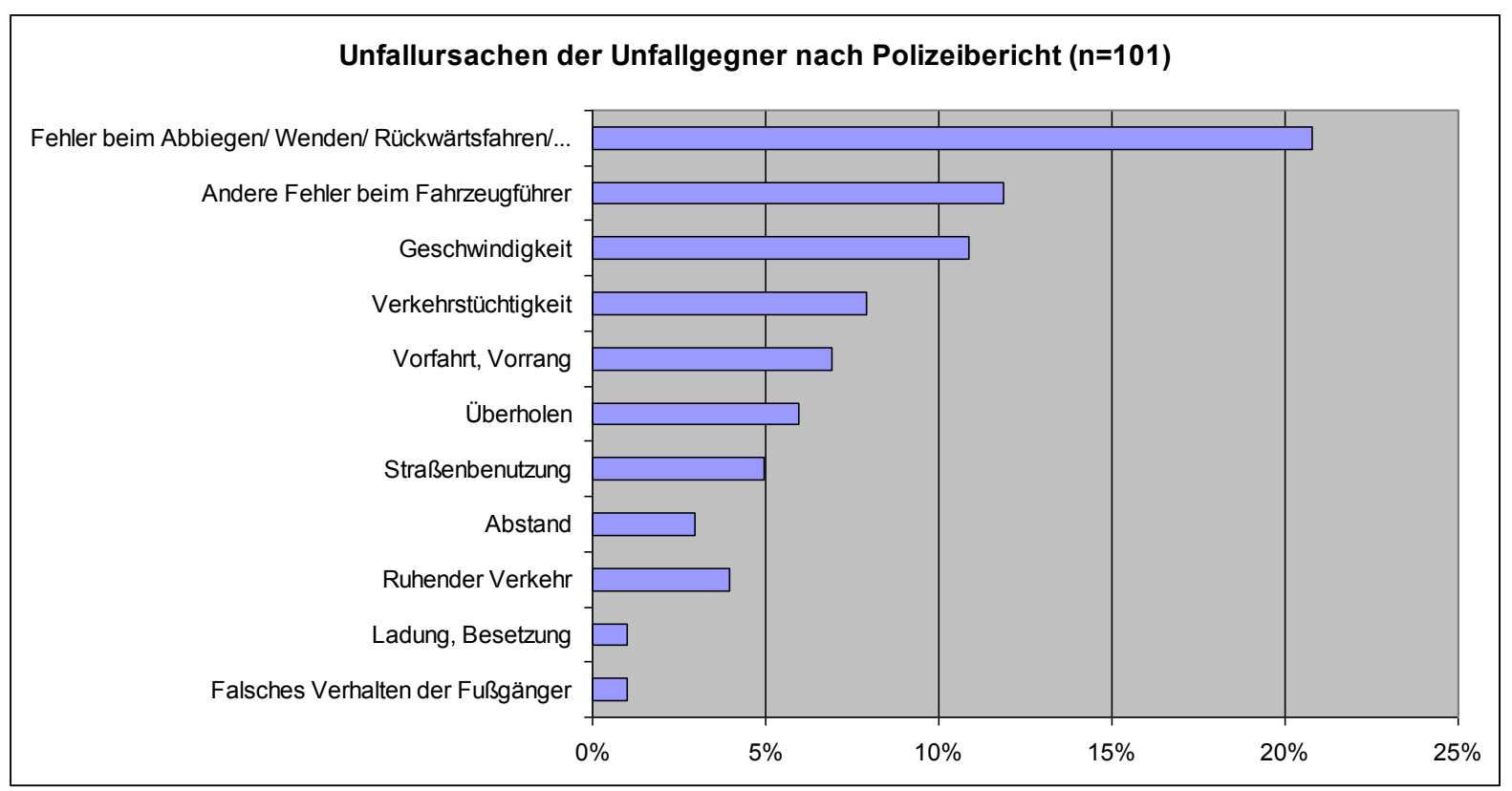

Abbildung 1-3: Unfallursachen der Unfallgegner nach Polizeibericht

\subsubsection{Unfalltypen}

Fast zwei Drittel der tödlichen Unfälle trugen sich innerhalb geschlossener Ortschaften zu. In knapp 60\% der Unfälle kamen die Fahrradfahrer bei Straßenquerungs- und Abbiegevorgängen (bezogen auf alle Beteiligten) ums Leben.

Abbildung 1-4 zeigt die Unfalltypen analog des amtlichen Unfalltypen-Katalogs (GDV 2003), wie er in Polizeiberichten Verwendung findet. Die Unfalltypen beschreiben die dem Unfall vorangegangene Konfliktsituation und lassen sich keinem Beteiligten zuordnen. Pro Unfall kann nur ein Unfalltyp vergeben werden.

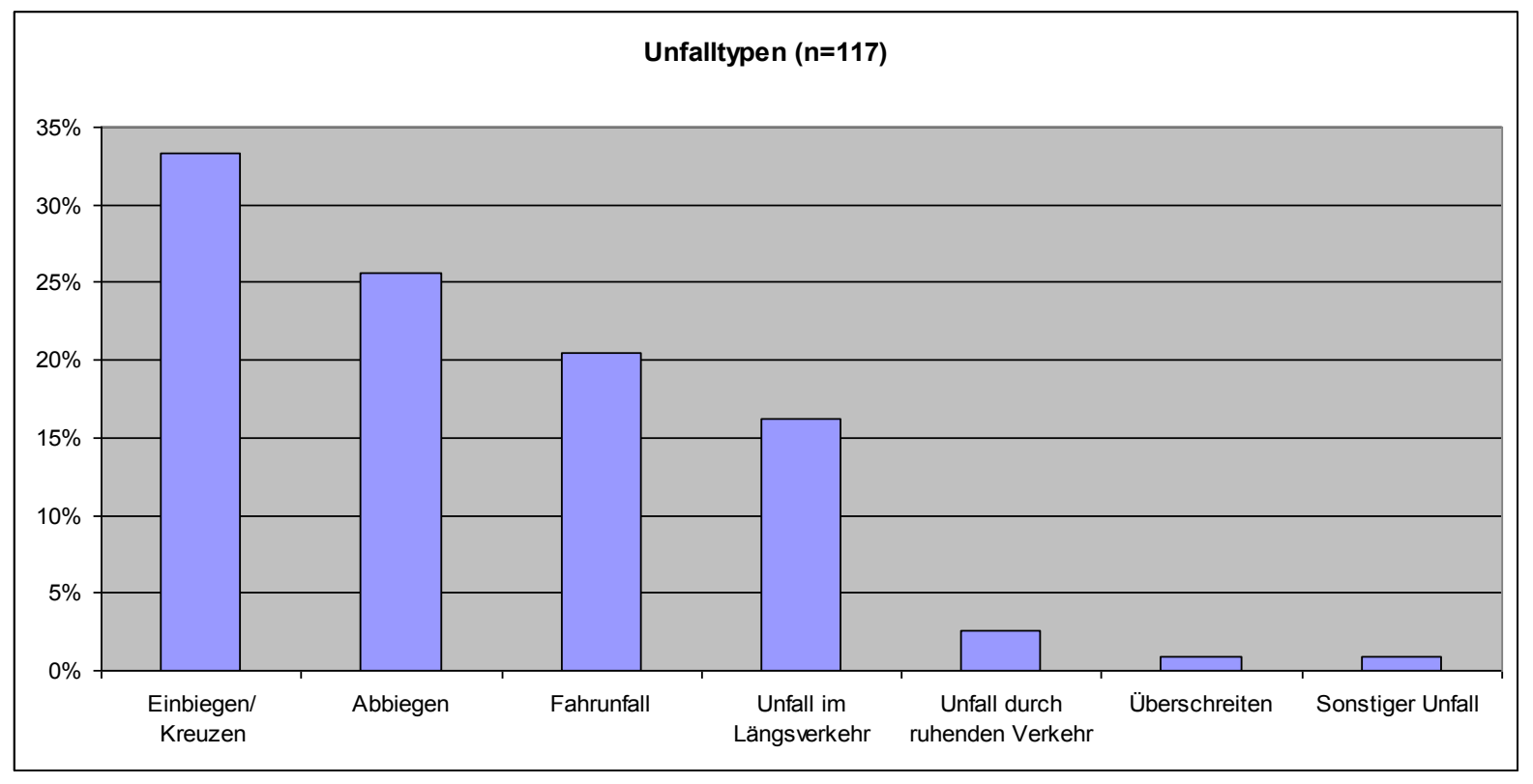

\section{Abbildung 1-4: Unfalltypen}




\subsubsection{Kollisionsgegner}

Wie aus Abbildung 1-5 zu ersehen ist, zog sich jeder zweite Fahrradfahrer aus dem untersuchten Kollektiv die tödliche Verletzung bei einer Kollision mit einem Pkw zu. Als zweithäufigster Kollisionsgegner ist der Lkw zu nennen. In 18 der 28 Unfälle mit LkwBeteiligung wurde der Fahrradfahrer dabei überrollt; bei den fünf Unfällen mit Kraftomnibus(KOM)-Beteiligung wurde kein Fahrradfahrer überrollt.

Knapp 14\% der tödlich verunglückten Fahrradfahrer kamen bei einem Alleinunfall ums Leben.

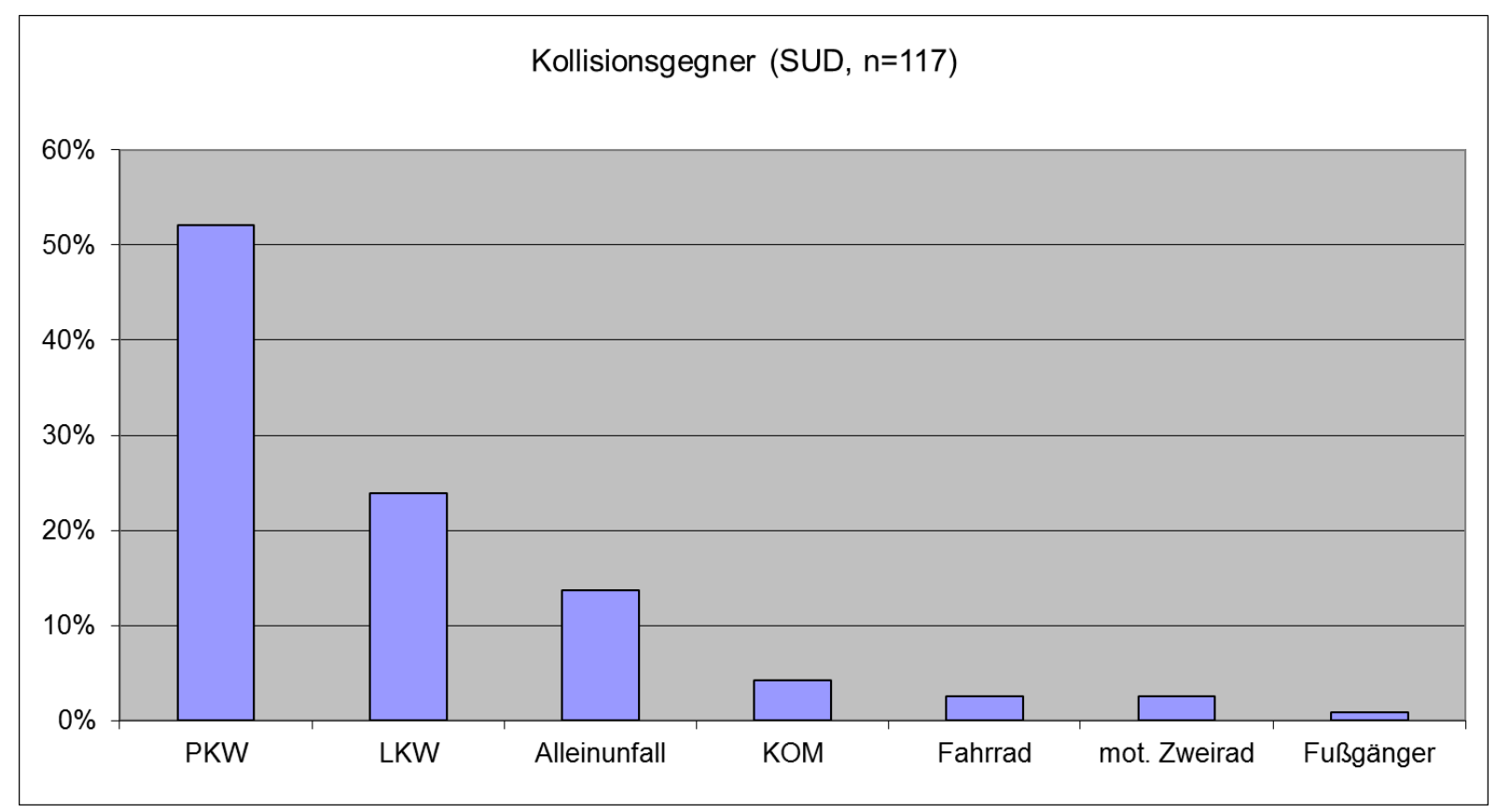

Abbildung 1-5: Tödlich verunglückte Fahrradfahrer nach Kollisionsgegner

\subsubsection{Charakteristika bei Unfällen mit Pkw-Beteiligung}

\subsection{Kollisionsstellung}

Wie auf Abbildung 1-5 zu sehen, ist bei über 50\% der tödlichen Unfälle ein Pkw der Kollisionsgegner. Deshalb wurden diese Unfälle einer genaueren Betrachtung unterzogen, insbesondere wurde die relative Kollisionsstellung des Fahrradfahrers und des Pkw zueinander bestimmt. Die Kollisionsstellungen wurden anschließend den von Bachmann (2011) entwickelten Gruppen zugeordnet, wie auf Abbildung 1-6 dargestellt.

Die häufigste Variante mit 19,7\% ist dabei Typ 1, bei der der Fahrradfahrer von hinten von der Pkw-Front erfasst wird. Diese Unfälle geschehen typischerweise außerorts auf Landstraßen und unter schwierigen Sichtbedingungen bei hohen Geschwindigkeiten des Pkws. Im Fallmaterial der prospektiv erhobenen Unfälle mit weniger schweren Unfallfolgen waren solche Unfallumstände hingegen äußerst selten.

Die dominierenden Kollisionsstellungen sind Typ 4 bis Typ 6, in denen der Fahrradfahrer von links von einer Pkw-Front erfasst wird. Die einzelnen Typen unterscheiden sich dabei im 
Winkel der Kollision, dieser reicht vom rechtwinkligen Anprall $\left(90^{\circ}\right.$, Typ, 4) bis zu Kollisionswinkeln von $45^{\circ}$ (Typ 6) und $135^{\circ}$ (Typ 5).

Fasst man diese drei Kollisionsstellungen zusammen, kommt man auf eine Fallzahl von 21, das entspricht 34\% aller Unfälle mit Pkw-Beteiligung.

Analog dazu sind auch die Kollisionsstellungen zwischen Pkw und Fahrrad dargestellt, bei denen das Fahrrad von rechts getroffen wird. Der Unterschied besteht darin, dass hier nur Winkel von $45^{\circ}$ (Typ 8) bis zu $90^{\circ}$ (Typ 7) abgedeckt werden, der Aufprall mit einem Winkel von über $90^{\circ}$ ist im dargestellten Fallkollektiv nicht zu verzeichnen.

Zusammengefasst ergeben Typ 7 und Typ 8 elf Fälle (18\%).

Insgesamt 32 von 61 Unfällen (53\%) mit Pkw-Beteiligung werden mit den Kollisionsstellungen Typ 4 bis Typ 8 abgebildet. Diese Unfälle werden zum relevanten Szenario 1 „Pkw-Front erfasst Fahrradfahrer seitlich" zusammengefasst.
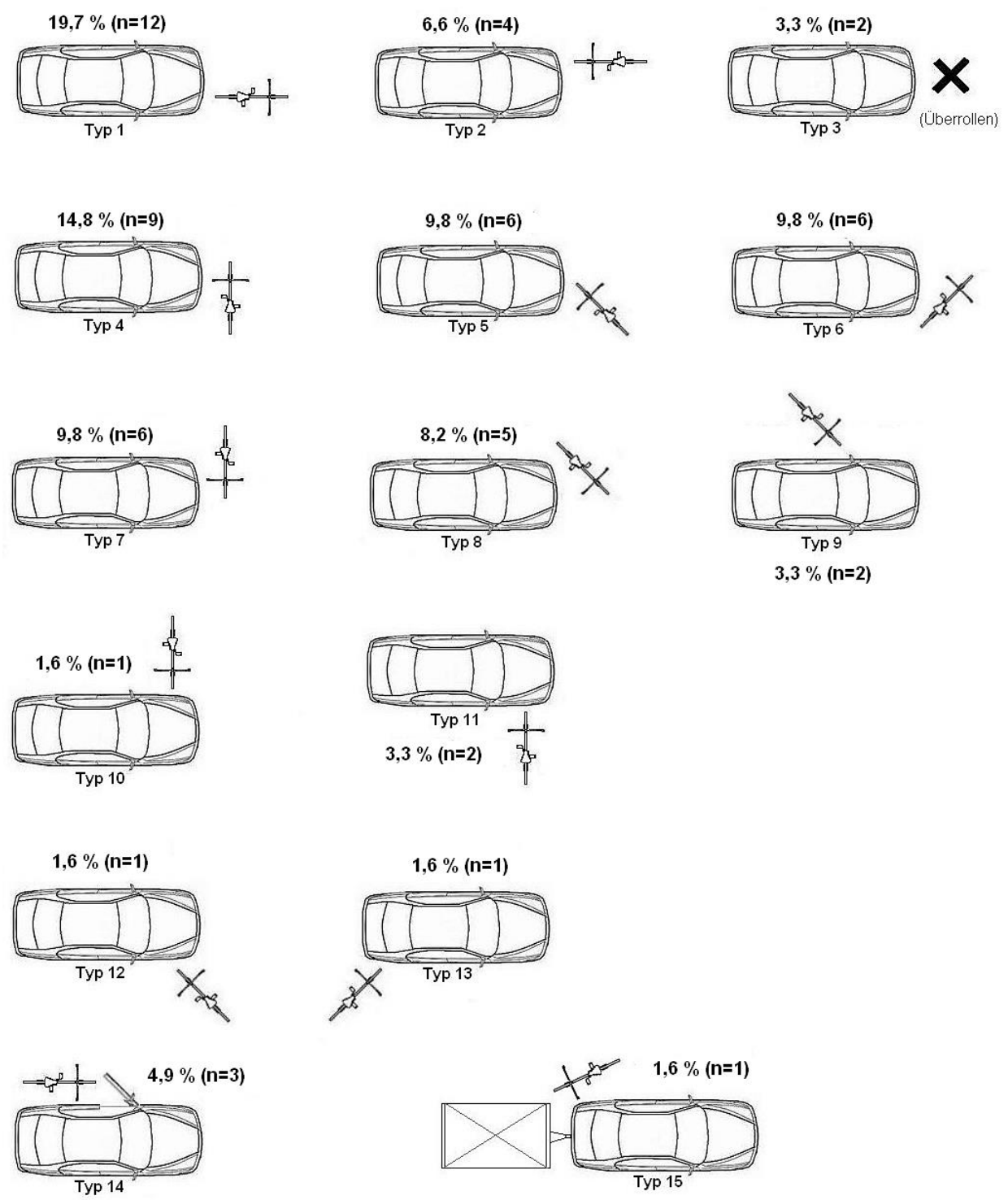

Abbildung 1-6: Kollisionsstellungen Unfallbeteiligte Fahrzeuge (Fahrrad - PKW), n=61 


\subsection{Kollisionsgeschwindigkeiten}

Betrachtet man die Kollisionsgeschwindigkeiten aller Unfallgegner (Pkw, Lkw, ...) auf Abbildung 1-7, erkennt man zwei Bereiche, in denen sich die Kollisionsgeschwindigkeit vermehrt befindet.

Die erste Häufung liegt in einem Geschwindigkeitsbereich von $10-20 \mathrm{~km} / \mathrm{h}$, die zweite in einem Bereich von $50-70 \mathrm{~km} / \mathrm{h}$.

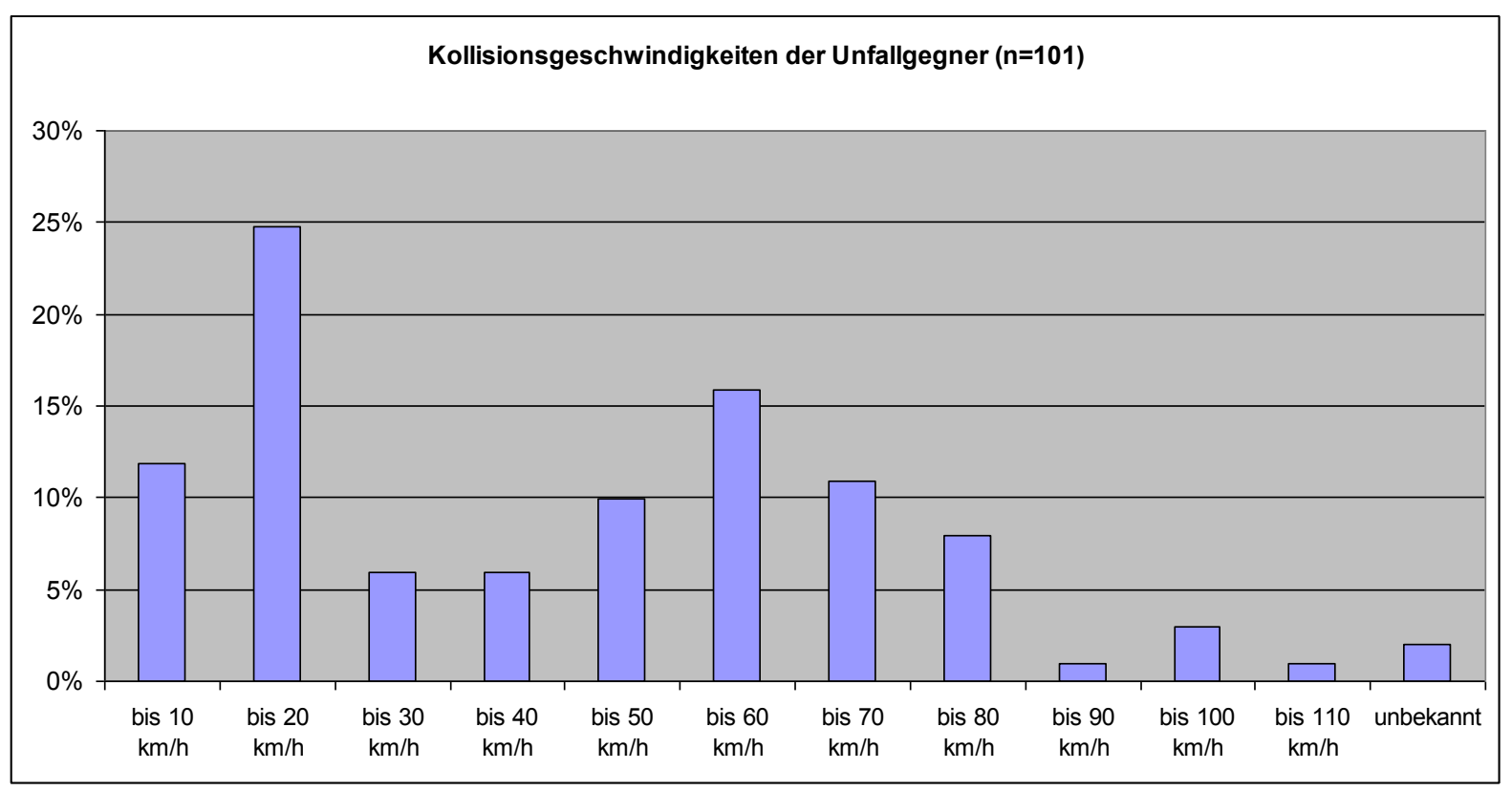

Abbildung 1-7: Kollisionsgeschwindigkeiten aller Kollisionsgegner

Auf Abbildung 1-8 sind nur die Kollisionsgeschwindigkeiten der Pkw bei den 61 Unfällen mit Pkw-Beteiligung dargestellt. Alle anderen Unfallgegner wurden bei dieser Betrachtung im Vergleich zu Abbildung 1-7 nicht dargestellt.

Es ist immer noch eine deutliche Häufung im Geschwindigkeitsbereich um $60 \mathrm{~km} / \mathrm{h}$ zu erkennen.

Die Häufung bei niedrigen Geschwindigkeiten ist nicht mehr ausgeprägt, weil die reinen Abbiegeunfälle innerorts mit Lkw-Beteiligung in dieser Darstellung ausgeklammert sind.

In 1.3.2.4.1 wurden bereits die typischen Kollisionsstellungen herausgearbeitet. Diese sind Typ 4 bis 8 nach der Einteilung von Bachmann (2011).

Auf Abbildung 1-9 sind die Kollisionsgeschwindigkeiten der beteiligten Pkw nur für diese Kollisionsstellungen dargestellt.

Es ist weiterhin eine Häufung im Bereich um $60 \mathrm{~km} / \mathrm{h}$ vorhanden.

Die Häufigkeitsverteilung der Kollisionsgeschwindigkeiten bis zu $30 \mathrm{~km} / \mathrm{h}$ ist abgeflacht, dennoch sind Unfälle mit geringer Geschwindigkeit in den typischen Kollisionsstellungen vorhanden.

Alleinunfälle nehmen im Kollektiv der prospektiv erhobenen Fahrradunfälle eine weitaus größere Rolle ein (s. 1.4). 


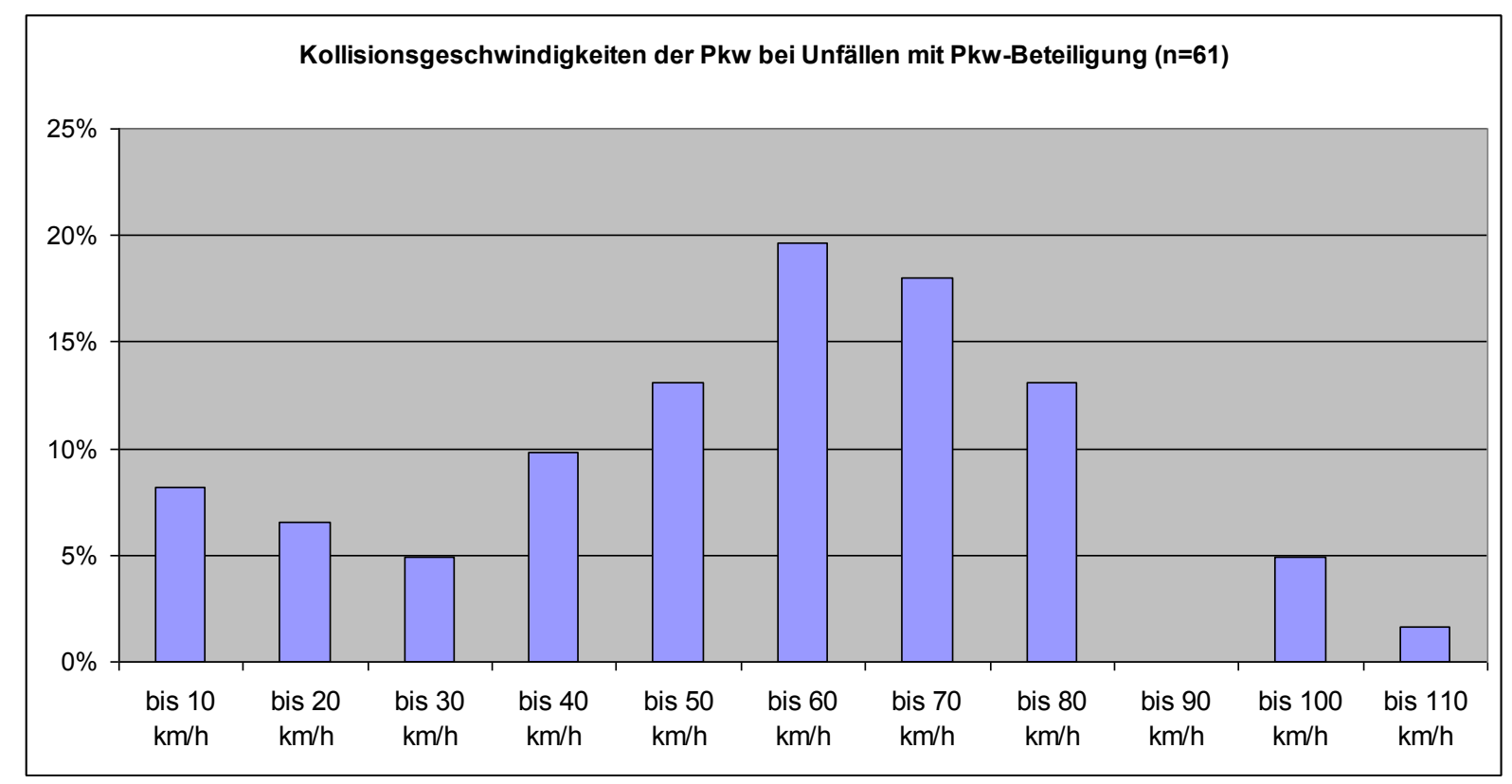

Abbildung 1-8: Kollisionsgeschwindigkeiten Pkw bei Unfällen mit Pkw-Beteiligung

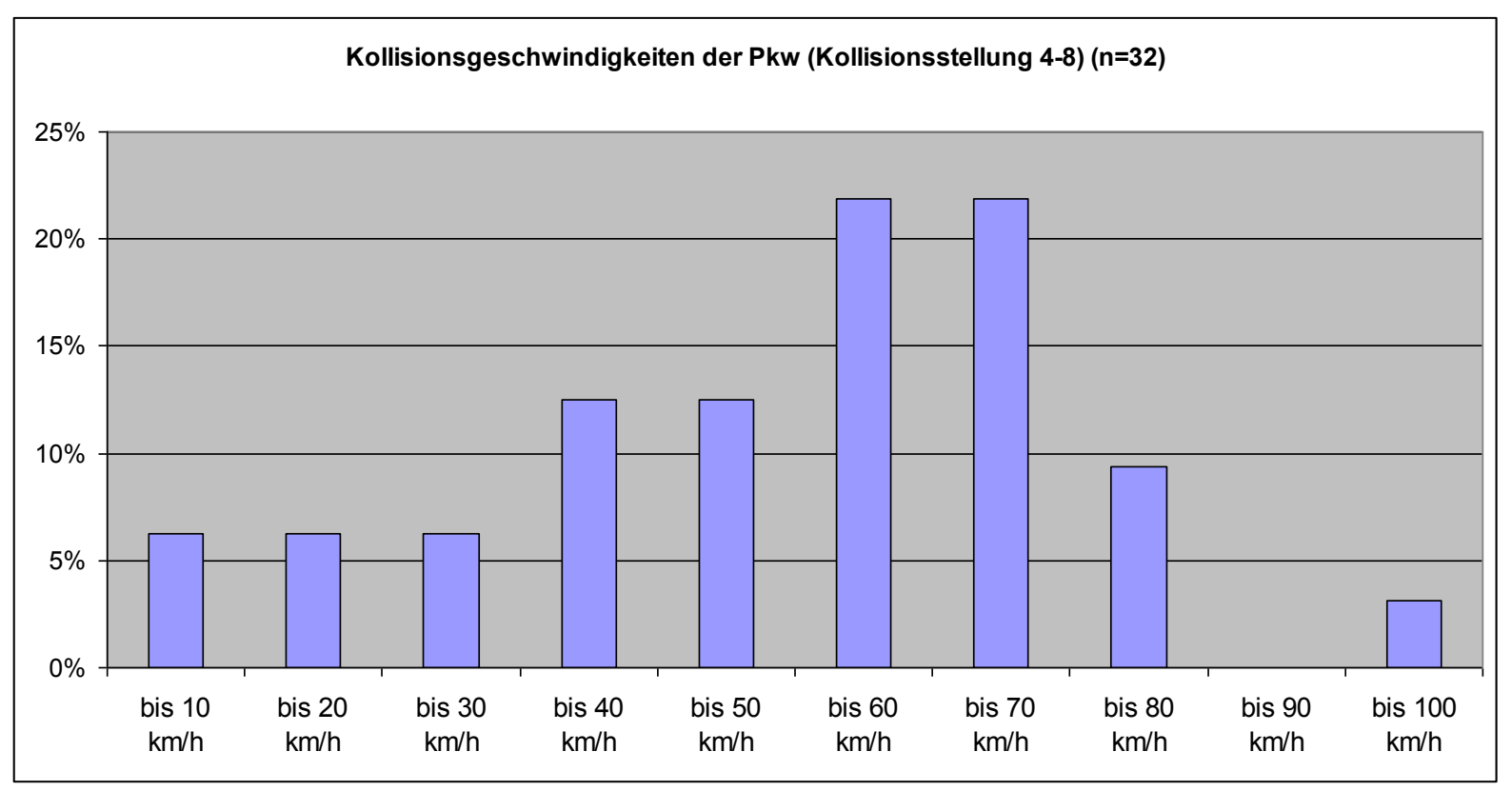

Abbildung 1-9: Kollisionsgeschwindigkeiten der Pkw bei den Kollisionsstellungen 4-8 


\subsection{Anprallpunkte am gegnerischen Pkw}

Für die Unfälle mit Pkw-Beteiligung $(n=61)$ sind die Anprallstellen des Fahrradfahrers am Pkw auf Abbildung 1-10 dargestellt. Dabei werden neben Kontakten des Kopfes auch die Kontakte anderer Körperteile erfasst.

Der Pkw wurde dabei in drei Bereiche unterteilt: Fahrzeugfront mit Stoßfänger, Motorhaube und Frontscheibe. Die Front wurde wiederum in drei Teile unterteilt, während bei Motorhaube und Frontscheibe jeweils neun weitere Unterteilungen getroffen wurden. Pro Unfall können (müssen aber nicht zwingend) maximal drei Anprallstellen vergeben werden, jeweils eine pro Bereich (Stoßfänger, Motorhaube, Frontscheibe).
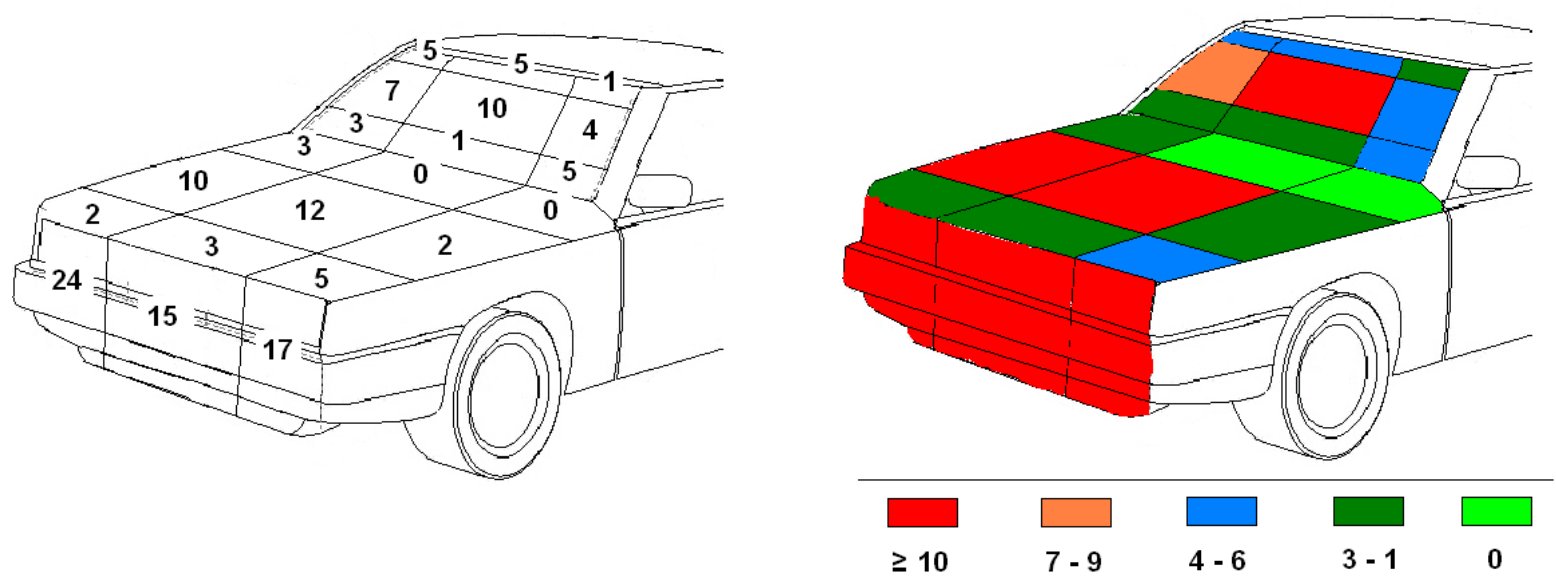

Abbildung 1-10: Anprallpunkte am gegnerischen

Pkw

Abbildung 1-10Fehler! Verweisquelle konnte nicht gefunden werden. zeigt die Verteilung der Anprallstellen. Die linke Abbildung zeigt dabei die absolute Anzahl von Anprallen, die rechte Abbildung stellt diese Verteilung farblich dar.

Es wird deutlich, dass bei der Motorhaube sowie der Frontscheibe der zentrale Anprallpunkt dominiert. Zudem ist eine Rechtstendenz zu erkennen. An der Front findet in 56 der 61 Unfälle mit Pkw-Beteiligung ein Kontakt statt.

\subsubsection{Charakteristika der Alleinunfälle}

Es wurde bereits dargestellt, dass 14\% $(n=16)$ der verunglückten Fahrradfahrer bei einem Alleinunfall ums Leben kamen.

In diesem Teilkollektiv fällt insbesondere der hohe Anteil an alkoholisierten Fahrradfahrern auf (ca. $60 \%$ ).

In neun der 16 Alleinunfälle stellte die Kopfverletzung die schwerste Verletzung dar, d.h. der Maximum AIS (MAIS) ist am Kopf lokalisiert.

Die gefahrene Geschwindigkeit der allein verunglückten Fahrradfahrer zum Unfallzeitpunkt ist auf Abbildung 1-11 dargestellt. Sie bewegt sich zwischen 0 und $30 \mathrm{~km} / \mathrm{h}$, lediglich in einem Fall ist eine weit höhere Geschwindigkeit dokumentiert. 


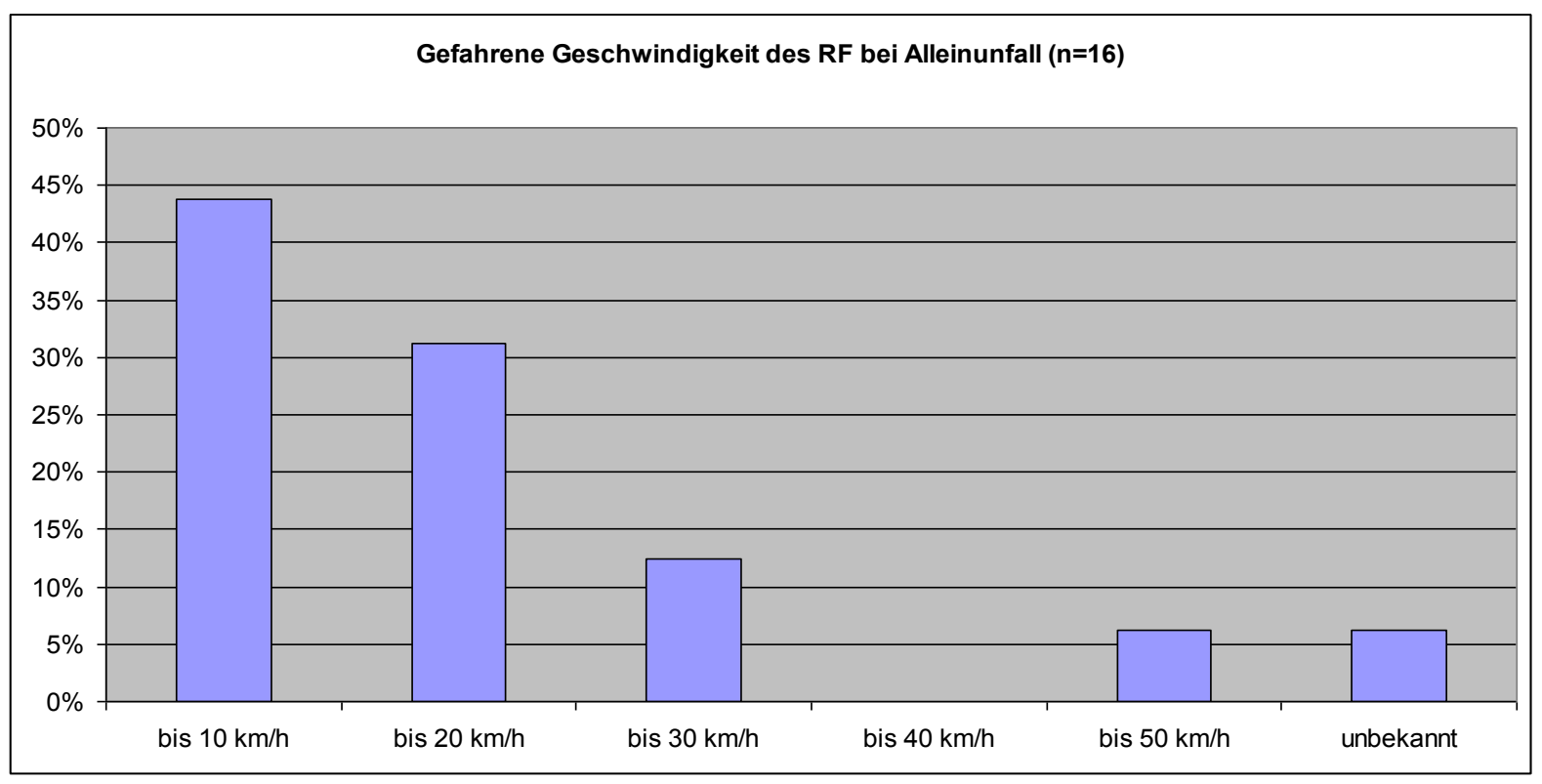

Abbildung 1-11: Gefahrene Geschwindigkeiten der Fahrradfahrer bei Alleinunfällen

\subsubsection{Diskussion der im Detail zu rekonstruierenden Unfälle}

Wie oben bereits dargelegt, handelt es sich bei dem in 1.3.1 betrachteten Fallkollektiv um Unfälle mit schwersten Folgen, die sich in ihrer Charakteristik von der Allgemeinheit der Fahrradunfälle mit Verletzungsfolge teilweise erheblich unterscheiden. Dies gilt nicht nur für spezielle Kollisionsstellungen bei Unfällen mit Pkw-Beteiligung, sondern auch für die Relevanz des Alleinunfalls.

So ist in 19\% $(n=3)$ der Fälle ein Abkommen von der Fahrbahn mit anschließendem Sturz über eine steile Böschung hinunter dokumentiert. 31\% $(n=5)$ der Fahrradfahrer stürzten seitlich bei geringer Geschwindigkeit, 19\% $(n=3)$ durch ein überbremstes Vorderrad nach vorne über den Lenker. Bei vier Unfällen ist die kein klarer Unfallablauf dokumentiert, anhand der zur Verfügung stehenden Daten ist jedoch in diesen Fällen ebenfalls von einem Sturz seitlich bei geringer Geschwindigkeit auszugehen.

Nach Diskussion aller vorliegenden Ergebnisse wurde der Alleinunfall als erstes typisches Unfallszenario bei Fahrradunfällen bestimmt.

Es lassen sich, auch unter Nutzung der Erkenntnisse aus den prospektiv erhobenen Fällen, zwei verschiedene Sturzmechanismen unterscheiden:

\section{1) Alleinunfall des Fahrradfahrers}

1a) Sturz seitlich (ca. $60 \%$ der Alleinunfälle getöteter Radfahrer) bei geringer Geschwindigkeit $(<10 \mathrm{~km} / \mathrm{h}$ ) durch Verlust des Gleichgewichts

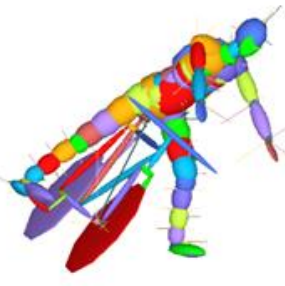


1b) Sturz über Lenker bei blockierendem Vorderrad (ca. $20 \%$ der Alleinunfälle getöteter Radfahrer) bei mäßiger Geschwindigkeit (15 - 20 km/h) durch Überbremsen oder sonstige Blockade des Vorderrades

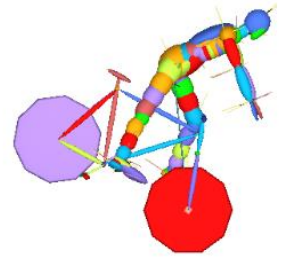

Diese Auswertung der Unfallabläufe findet bei der Bestimmung der typischen Unfallszenarien unter 1.4 Berücksichtigung.

Wie unter 1.3.2.3 dargestellt, sind die Kollisionen eines Fahrradfahrers mit einem Pkw in der SUD mit über 50\% dominierend. Somit wurde als zweites typisches Unfallszenario die Kollision des Fahrradfahrers mit einem Pkw bestimmt.

Es dominiert mit 53\% die Kollisionsstellung „Pkw-Front gegen Fahrrad seitlich“.

Bei Schwerstunfällen treten höhere Kollisionsgeschwindigkeit vermehrt auf, während bei Unfällen mit verletzten Fahrradfahrern geringe Kollisionsgeschwindigkeiten vorherrschend sind.

Dies führte zur Festlegung folgender typischer Unfallszenarien:

\section{2) Kollision "Pkw-Front gegen Fahrrad seitlich"}

2a) mit geringer Geschwindigkeit (ca. $15 \mathrm{~km} / \mathrm{h}$ )

2b) mit hoher Geschwindigkeit (ca. $60 \mathrm{~km} / \mathrm{h}$ )
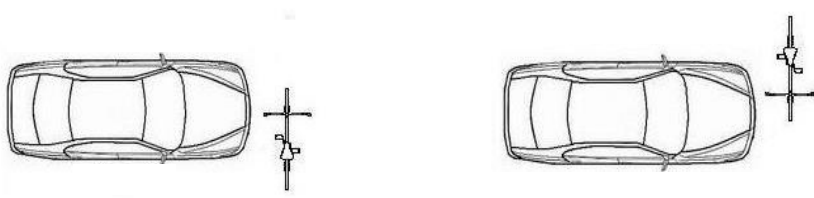

Abbildung 1-12: Kollisionsszenario 2

Der Kollisionswinkel ist dabei nicht fest auf $90^{\circ}$ festgelegt. Wie unter 1.3.2.4 schon verdeutlicht, sind hier Abweichungen von bis zu $45^{\circ}$ in beide Richtungen inbegriffen (Typ 4 bis Typ 8).

Die gefahrene Geschwindigkeit des Fahrradfahrers bewegt sich meist im Bereich von 15 $20 \mathrm{~km} / \mathrm{h}$ und hat wegen des Winkels zum Pkw (oft annähernd rechtwinklig) einen zu vernachlässigenden Einfluss auf die relative Geschwindigkeit beider Unfallbeteiligter. Somit lassen sich die dargestellten Geschwindigkeiten des Pkw auch vereinfachend als relative Kollisionsgeschwindigkeit zwischen Fahrrad und Pkw heranziehen.

Der Fahrradfahrer prallt mit seinem Kopf abhängig von der Kollisionsgeschwindigkeit meist gegen die Motorhaube oder die Frontscheibe. 
Da die unter 1.3.2.4 beschriebene Kollisionsstellung "Pkw-Front erfasst Fahrradfahrer von hinten" nur bei getöteten Radfahrern von Bedeutung ist, wurde dieses Szenario für weitere Rekonstruktionen und eine Simulation außer Betracht gelassen.

Aus der SUD wurden anhand der oben aufgeführten Parameter fünf Alleinunfälle und neun Unfälle mit Pkw-Beteiligung für die weitere Rekonstruktion/Simulation selektiert.

Diese Unfälle stimmen mit den ermittelten typischen Szenarien gut überein und eignen sich aufgrund einer sehr guten Dokumentationsgrundlage für detaillierte Betrachtungen.

Eine Übersicht dieser Unfälle ist Anhang 1 zu entnehmen.

\subsection{Definition relevanter Unfallsituationen}

Aufbauend auf die oben dargestellte Kurzauswertung der SUD wurden nach Vorliegen aller Daten aus den unterschiedlichen Kollektiven eine detaillierte Auswertung durchgeführt und die finalen relevanten Unfallsituationen bestimmt.

\subsubsection{Datengrundlage}

\subsubsection{Daten des Instituts für Rechtsmedizin München}

Der Aufbau der Datenbank des Instituts für Rechtsmedizin München und die erfassten Parameter wurden bereits unter 1.3.1 detailliert beschrieben, deshalb wird an dieser Stelle darauf verzichtet.

\subsubsection{Prospektive Unfalldaten}

Zusätzlich zu den vorliegenden retrospektiv erhobenen Unfalldaten der Rechtsmedizin München wurden Fahrradunfälle in München und Münster prospektiv über den Zeitraum von einem Jahr erfasst (vom 01.05.2012 bis zum 30.04.2013). Dabei handelt es sich um Unfälle, bei denen verletzte Fahrradfahrer im Universitätsklinikum München (UKLMU) respektive dem Universitätsklinikum Münster (UKM) eingeliefert wurden oder sich dort selbst vorstellten. Die verunfallten Fahrradfahrer, die im UKLMU behandelt wurden, kamen fast ausschließlich aus dem näheren Stadtgebiet. Im UKM wurden aufgrund des Status der Klinik als Maximalversorger auch Patienten behandelt, die nicht direkt im Stadtgebiet verunfallten, sondern auch aus dem umliegenden ländlichen Bereich. Der Anteil dieser tendenziell eher schwer verletzten Fahrradfahrer liegt bei knapp über $5 \%$.

Die gesamte Studie wurde der Ethikkommission der Ludwig-Maximilians-Universität mit der Projektnummer 108-12 vorgelegt und von dieser genehmigt. Die Daten wurden streng anonymisiert erfasst und ausgewertet.

Demografische Daten des beteiligten Fahrradfahrers und zum Unfallhergang wurden anhand eines Fragebogens (s. Anhang 2) erfasst. Dieser Bogen wurde in Zusammenarbeit mit dem Universitätsklinikum Münster entwickelt und basiert auf dem Erfassungsbogen, der bereits in der 2010 veröffentlichten „Fahrradunfallstudie Münster“ verwendet wurde. Dieser Bogen wurde vom Patienten auf freiwilliger Basis zusammen mit der verantwortlichen Krankenschwester in der Ambulanz / Notaufnahme der Klinik ausgefüllt. Die Bögen wurden in Papierform gesammelt und deren Inhalte von einem Studienmitarbeiter in eine Datenbank (Filemaker) eingetragen. Die Verletzungsdiagnosen wurden ebenfalls von geschultem Personal nach AIS kodiert und in die Datenbank überführt. Ergänzend zu den Informationen aus den Fragebögen konnten zu 37 polizeilich erfassten Unfällen in München die 
Verkehrsunfallanzeigen samt Fotodokumentation, Unfallskizzen und Zeugenaussagen eingeholt werden. Diese Unterlagen wurden primär dazu verwendet, die Angaben zum Unfallhergang zu bestätigen und fehlende Angaben, vor allem in Hinblick auf die Kollisionsbereiche am Fahrrad und beteiligtem Pkw, zu ergänzen. Die FileMaker-Datenbank wurde anschließend in eine MS Excel-Tabelle konvertiert und ausgewertet.

\subsection{Daten des Universitätsklinikums München}

Im Universitätsklinikum München (UKLMU) wurden 71 Fahrradunfälle erfasst. Die beteiligten Fahrradfahrer erlitten dabei leichte bis schwere Verletzungen. Aus diesem Datenkollektiv wurden für die weiteren Betrachtungen fünf Fahrradfahrer ausgeschlossen, die entweder unverletzt waren oder als Fußgänger ihr Fahrrad schoben. Somit standen für die Auswertungen 66 Fahrradfahrer aus dem UKLMU zur Verfügung.

\subsection{Daten des Universitätsklinikums Münster}

Im Universitätsklinikum Münster (UKM) wurden 500 Fahrradfahrer mit leichten bis schweren Verletzungen erfasst. Dort wurden für die weiteren Betrachtungen 23 Fahrradfahrer ausgeschlossen, die entweder unverletzt waren oder als Fußgänger ihr Fahrrad schoben, es wurden somit 477 Fälle ausgewertet.

\subsubsection{Auswertung der Daten}

\subsubsection{Alter der verunfallten Fahrradfahrer}

Auf Abbildung 1-13 sind die Altersverteilungen der erfassten verunfallten Fahrradfahrer des UKLMU und des UKM dargestellt.

Bei den erfassten Fällen des UKLMU ist der jüngste erfasste Fahrradfahrer 19 Jahre alt, der älteste 81, der Altersdurchschnitt liegt bei 42 Jahren. Das Alter der verunfallten Fahrradfahrer liegt überwiegend im Bereich von 25 bis 54 Jahren. Es sind verhältnismäßig wenige Fahrradfahrer im Alter 55+ Jahre vertreten.

Im Fallmaterial des UKM ist der jüngste erfasste Fahrradfahrer 4 Jahre alt, der älteste 91, der Durchschnitt liegt bei 37 Jahren.

Es ist eine sehr deutliche Häufung im Bereich von 15 bis 34 Jahren zu sehen. Da in Münster sehr viele Studenten wohnen, ist diese Auffälligkeit durchaus plausibel und findet sich in ähnlicher Weise auch in der früheren Fahrradunfallstudie aus Münster.

Im Gegensatz dazu liegen im Fallmaterial der Rechtsmedizin (Abbildung 1-14) bis zu einem Alter von 34 Jahren nur 14 verunglückte Fahrradfahrer vor. Diese Zahl steigt mit zunehmendem Alter kontinuierlich an, in der Altersgruppe "75+ Jahre" sind 33 tödlich verletzte Fahrradfahrer erfasst. Der Altersdurchschnitt liegt bei 59,8 Jahren. 


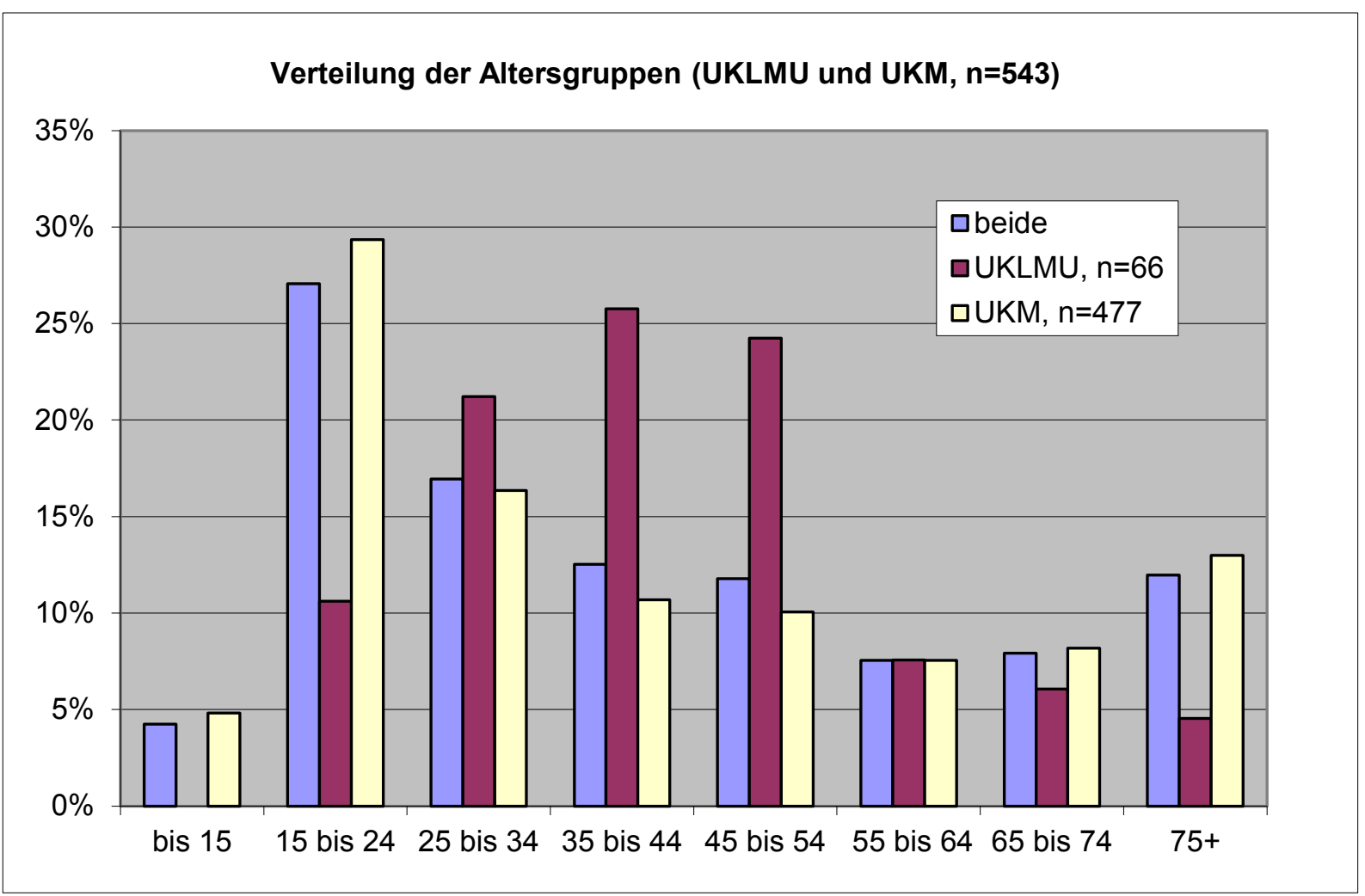

Abbildung 1-13: Verunfallte Fahrradfahrer nach Altersgruppen (UKLMU und UKM, n=543)

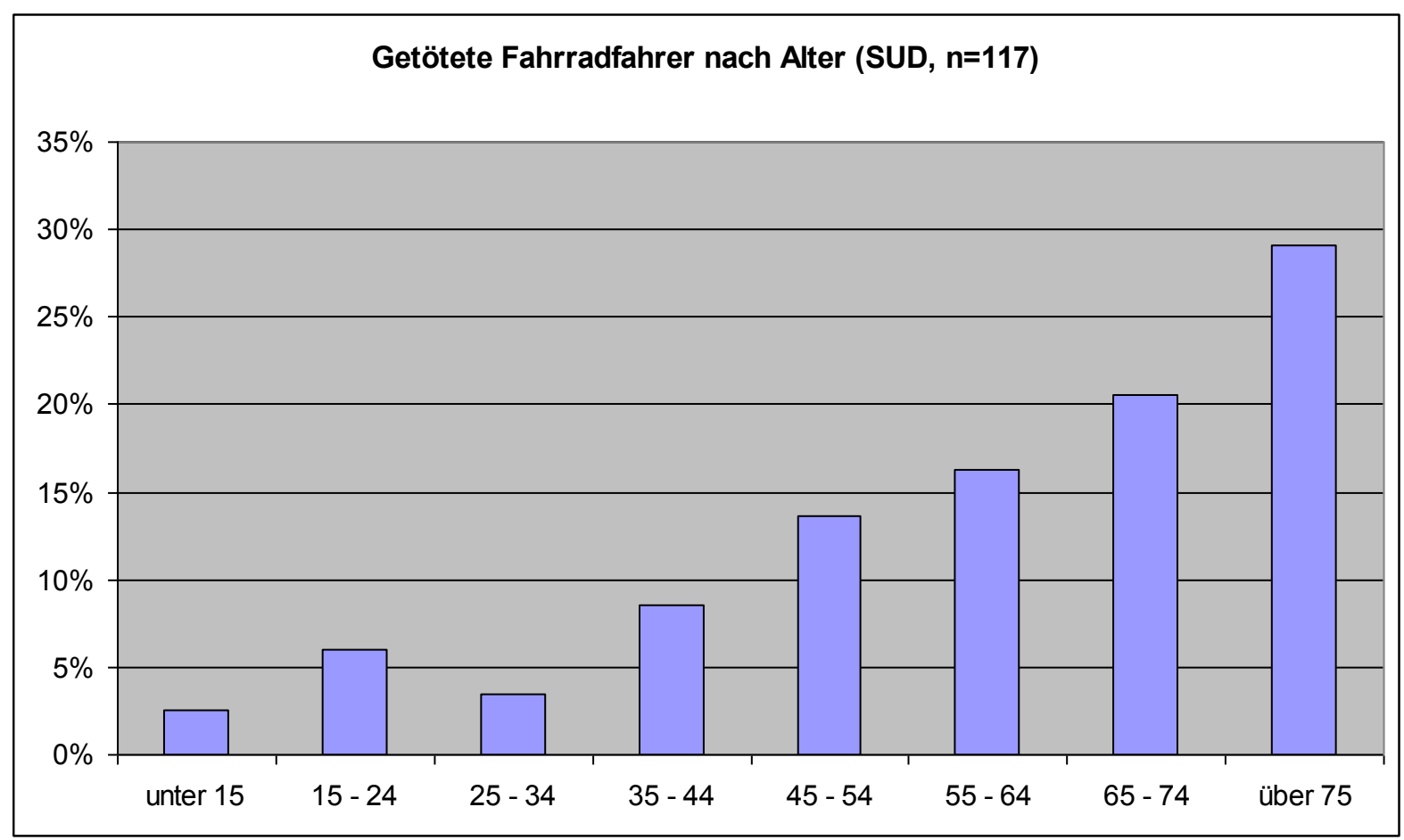

Abbildung 1-14: Tödlich verunglückte Fahrradfahrer nach Altersgruppen (SUD, n=117) 


\subsubsection{Geschlecht der verunfallten Fahrradfahrer}

Im Fallmaterial des UKLMU sind 35 verunfallte Fahrradfahrer männlich und 31 weiblich. Das entspricht einer Verteilung von $53 \%$ zu $47 \%$.

Im UKM sind 247 männliche und 229 weibliche Fahrradfahrer erfasst, in einem Fall wurde keine Angabe gemacht. Die prozentuale Aufteilung entspricht mit $52 \%$ zu $48 \%$ annähernd der des UKLMU.

Die Daten der SUD unterscheiden sich in diesem Punkt deutlich. Von den 117 getöteten Fahrradfahrern sind 80 männlich und 37 weiblich, was einer Verteilung von $68 \%$ zu $32 \%$ gleichkommt.

\subsubsection{Einfluss von Alkohol}

Im Patientenfragebogen konnten freiwillig Angaben zu Alkoholkonsum gemacht werden. Die Angaben aus dem UKLMU und dem UKM sind auf Abbildung 1-15 zu erkennen.

Im UKLMU gaben $80 \%$ an, keinen Alkohol vor dem Unfall getrunken zu haben. Weniger als $10 \%$ berichteten, Alkohol konsumiert zu haben.

Im UKM gaben $15 \%$ der verunfallten Fahrradfahrer an, Alkohol zu sich genommen zu haben, während knapp über $60 \%$ einen Alkoholkonsum verneinten.

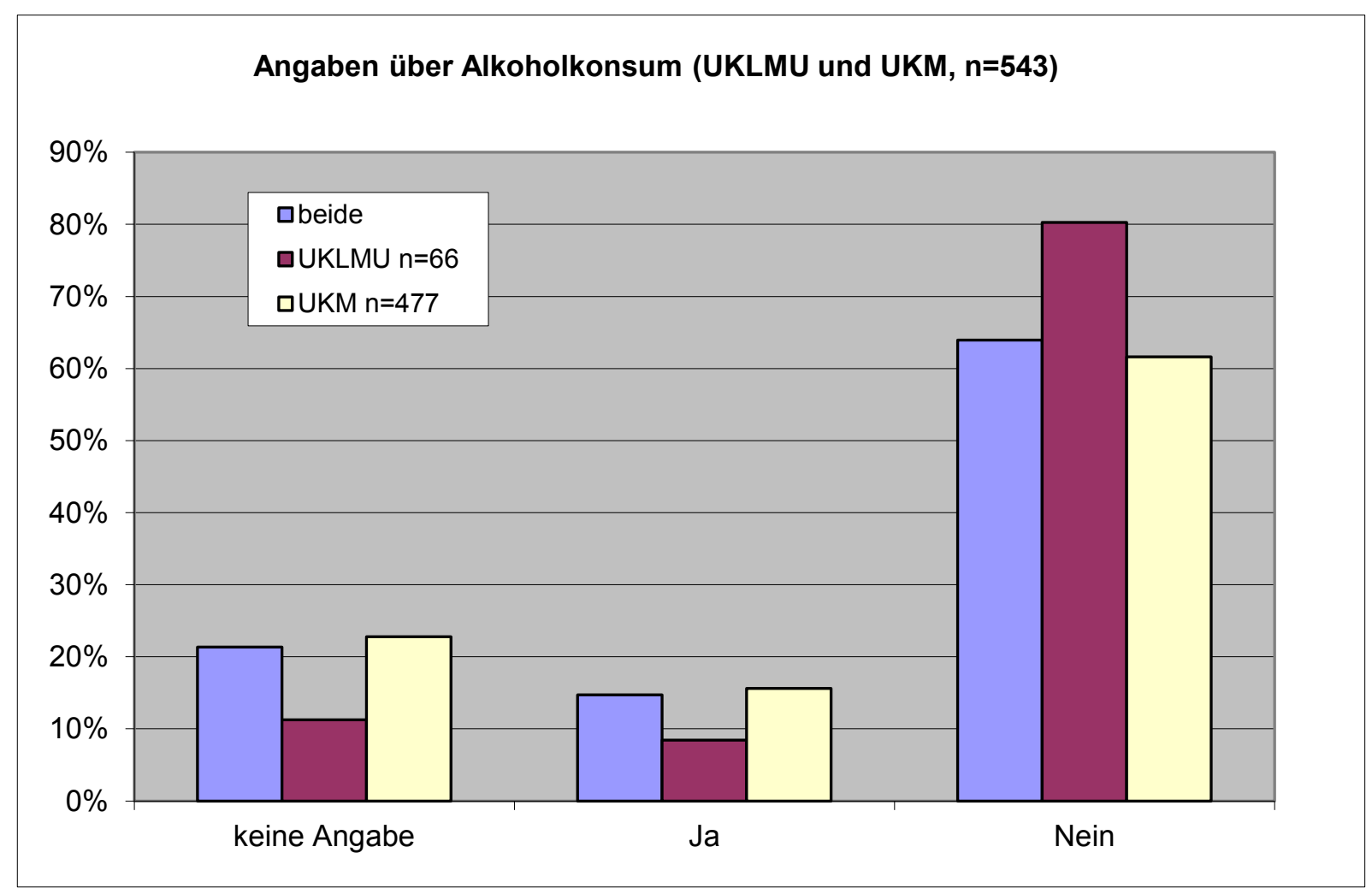

Abbildung 1-15: Angaben über Alkoholkonsum (UKLMU und UKM, n=543)

Ergänzend zu diesen Angaben wurde im UKM von 24 Patienten mit einem Schädelhirntrauma der Blutalkoholwert bestimmt, die Ergebnisse zeigt Abbildung 1-16.

Bei drei Patienten (13\%) konnte kein Alkohol im Blut nachgewiesen werden.

Sieben Patienten (29\%) lagen mit ihrem gemessenen Blutalkoholwert zwischen 1,1 und 1,59 Promille, 12 Patienten (50\%) teils deutlich über dem Grenzwert von 1,6 Promille für die „absolute Fahruntüchtigkeit“ bei Fahrradfahrern. 


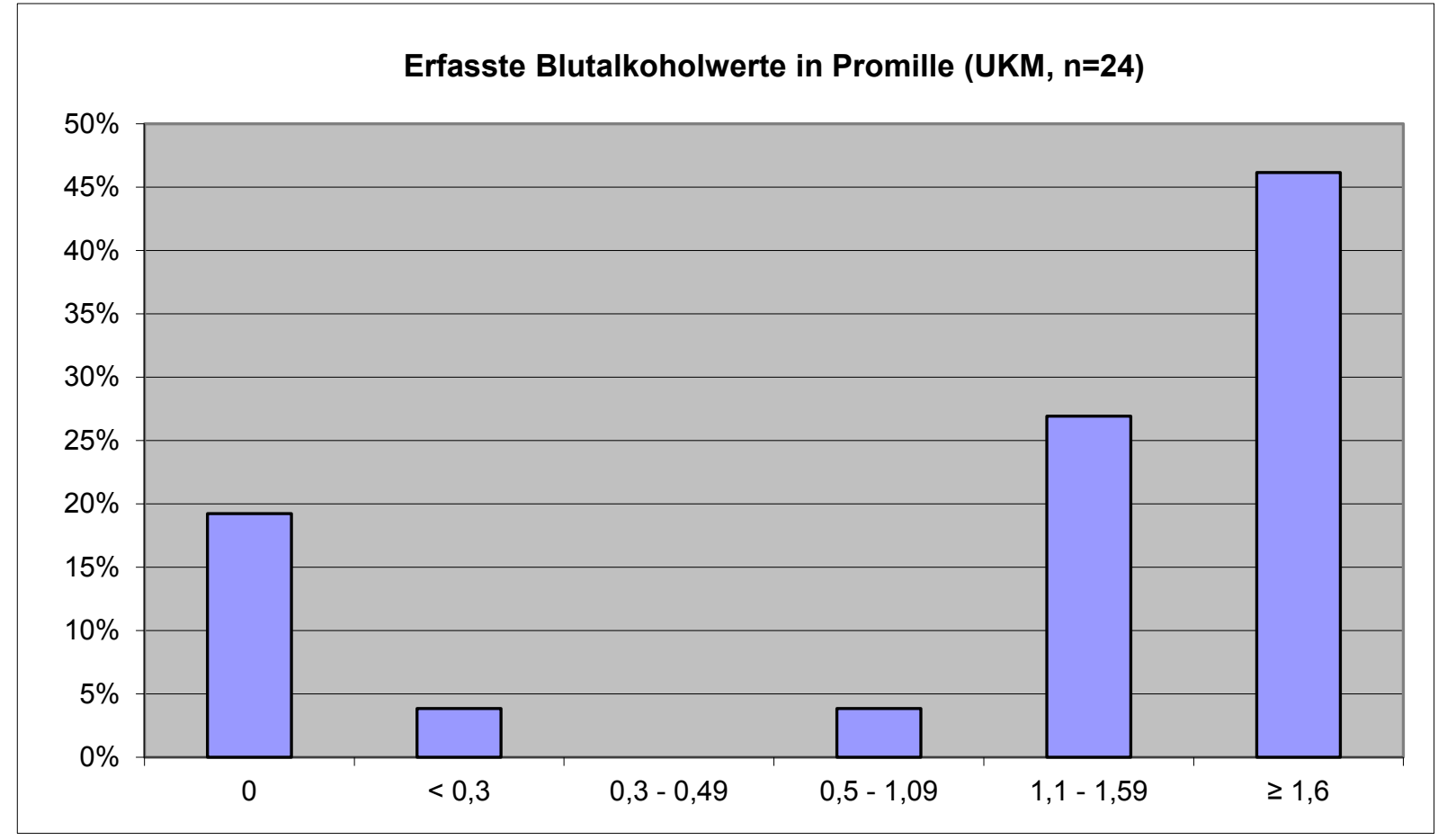

Abbildung 1-16: Erfasste BAK-Werte in Promille (UKM, n=24)

Im Fallmaterial der Rechtsmedizin lag für 65 getötete Fahrradfahrer eine Bestimmung des Blutalkoholwerts vor.

Von diesen 65 getöteten Fahrradfahrern wurde bei 38 Fahrern (58\%) ein von Null abweichender Blutalkoholpegel festgestellt (Abbildung 1-17).

Bei 6 Fahrradfahrern (9\%) wurde ein BAK-Wert zwischen 1,1 und 1,6 Promille nachgewiesen, weitere $10(15 \%)$ hatten einen Wert über 1,6 Promille. Zudem wurde bei zwei männlichen Verunglückten bei der Obduktion der psychoaktive Wirkstoff Tetrahydrocannabinol (THC) nachgewiesen.

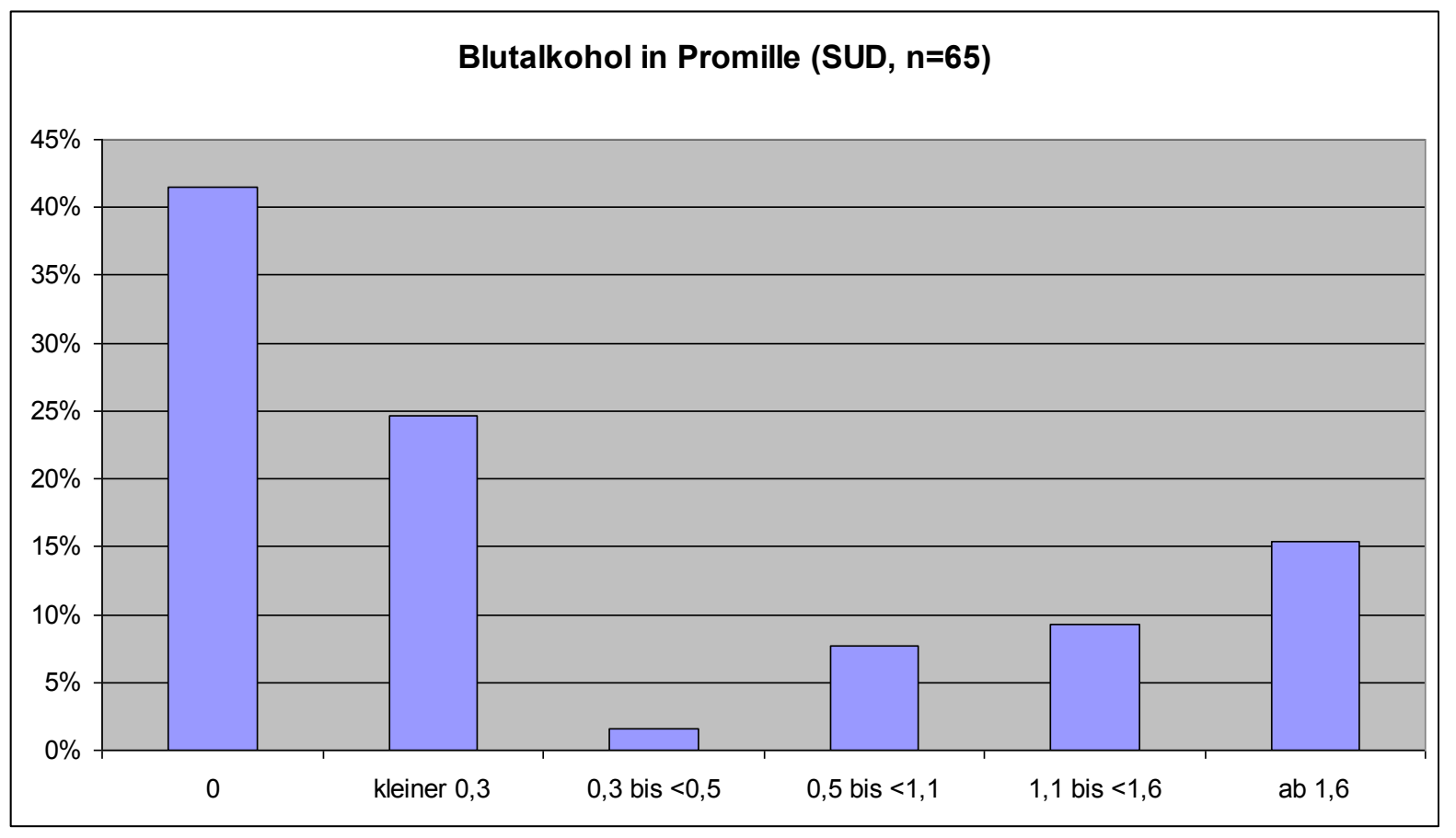

Abbildung 1-17: Erfasste BAK-Werte in Promille (SUD, $n=65$ ) 


\subsubsection{Kollisionsart und Unfallursachen}

Die Auswertung der Angaben zur Kollisionsart respektive dem Kollisionsgegner ist auf Abbildung 1-18 dargestellt.

Knapp die Hälfte aller Unfälle im Fallmaterial des UKLMU und des UKM sind Alleinunfälle.

Dieser Wert ist in der Münchener Klinik und der Klinik in Münster annähernd identisch.

Zweithäufigste Kollisionsart ist die Kollision mit einem fahrenden Pkw. Die Prozentzahl ist im UKLMU mit über 20\% größer als im UKM mit knapp 15\%. An dritter Stelle folgt die Kollision mit einem anderen Fahrradfahrer.

Die übrigen Kollisionsarten nehmen eine untergeordnete Rolle mit 5\% oder weniger ein.

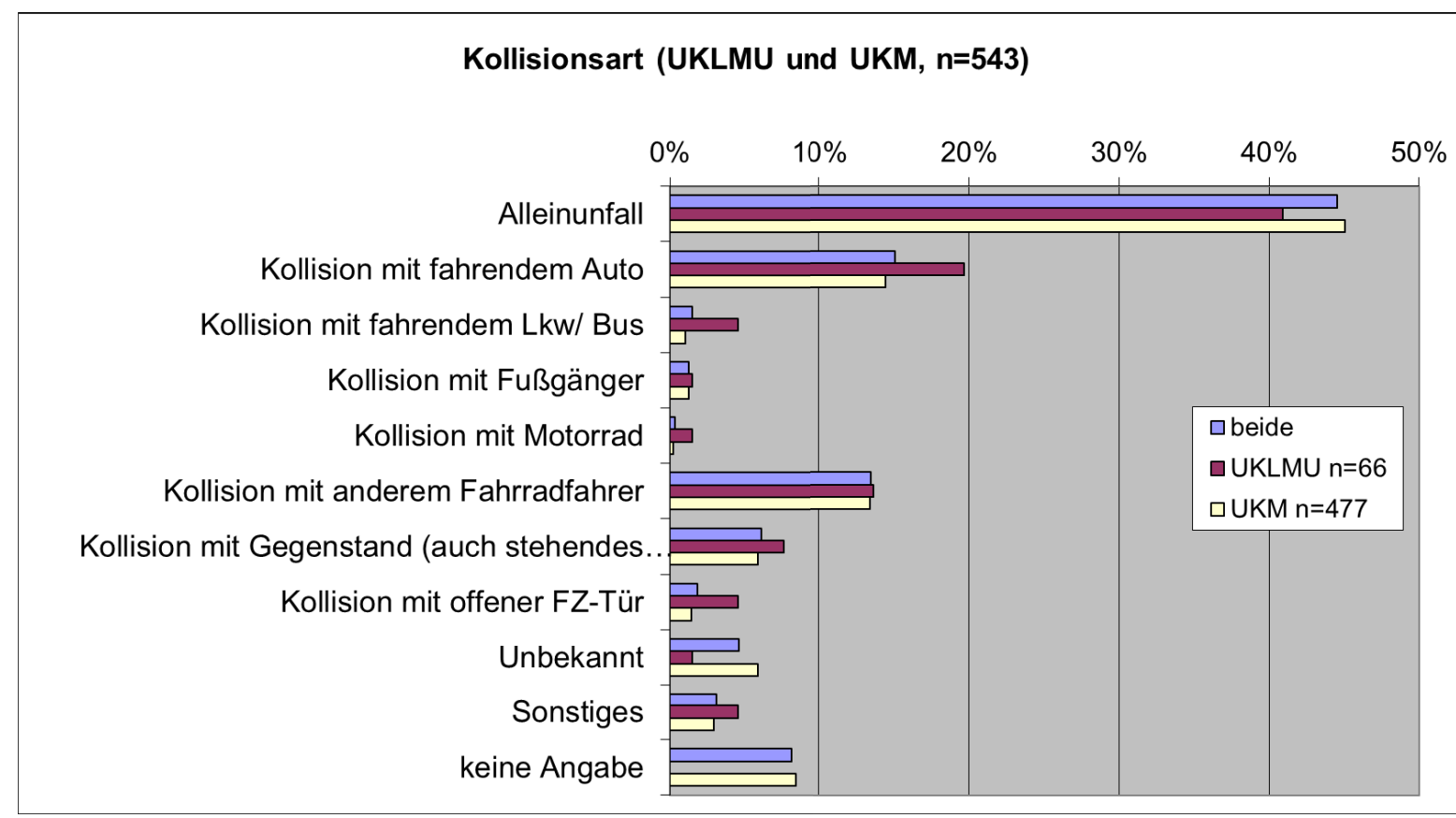

Abbildung 1-18: Kollisionsart (UKLMU und UKM, $n=543$ )

Ergänzend zur Kollisionsart bzw. dem Kollisionsgegner konnten die Patienten Angaben zur Unfallursache machen.

Die Auswertung auf Abbildung 1-19 zeigt, dass als häufigste Unfallursache ein schlechter Untergrund angegeben wurde. Dies beinhaltet zum Beispiel Straßenbahnschienen, Glatteis, Kopfsteinpflaster etc. Diese Unfallursache ist typischerweise bei Alleinunfällen zu finden. An zweiter Stelle ist die Unfallursache dem Unfallgegner zugeordnet, im Fallmaterial des UKLMU häufiger als im Fallmaterial des UKM. Naturgemäß steht diese Ursache nur bei Unfällen mit Fremdbeteiligung zur Auswahl. Überwiegend wurde diese Angabe bei Unfällen mit einem Pkw als Kollisionsgegner gemacht.

Der Verlust des Gleichgewichts wurde als primäre Unfallursache in knapp $10 \%$ aller Alleinunfälle angegeben. 
Unfallursache (UKLMU und UKM, $n=543$ )

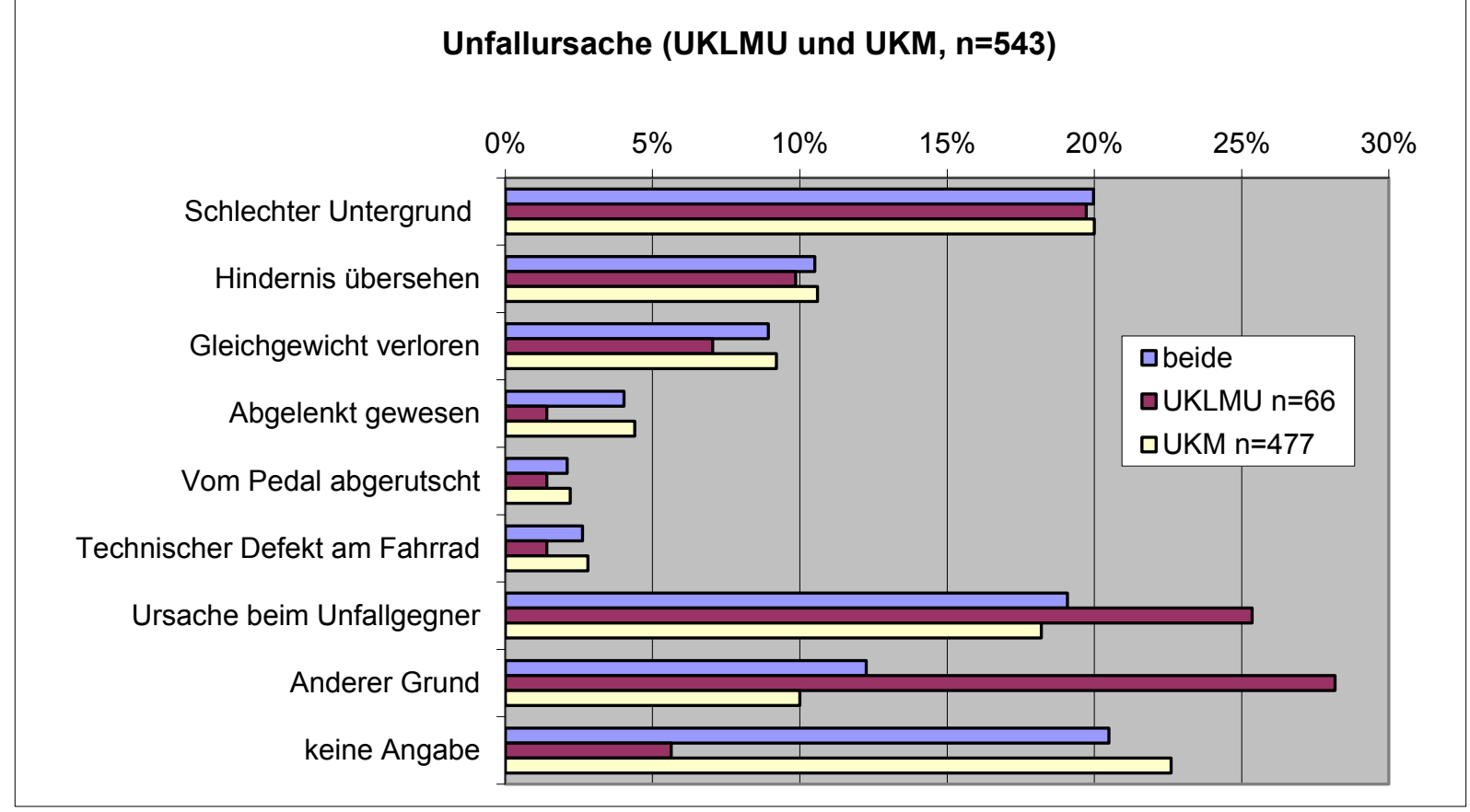

Abbildung 1-19: Unfallursache (UKLMU und UKM, n=543)

Die Verteilung der Kollisionsgegner stellt sich im Fallmaterial der Rechtsmedizin im Vergleich zu den Daten aus dem UKLMU und dem UKM deutlich abweichend dar.

Wie bereits in Abbildung 1-5 gezeigt, zogen sich über 50\% der Getöteten (SUD) ihre Verletzungen bei einer Kollision mit einem Pkw zu. Als zweithäufigster Kollisionsgegner ist der Lkw zu nennen. In 18 der 28 Unfälle mit Lkw-Beteiligung wurde der Fahrradfahrer dabei überrollt.

Deutlichste Abweichung zu den nicht getöteten Fahrradfahrern (UKLMU und UKM) ist die Anzahl der Alleinunfälle. Knapp 14\% der tödlich verunglückten Fahrradfahrer aus der SUD kamen bei einem Alleinunfall ums Leben.

Um mögliche Auffälligkeiten in Hinblick auf typische Unfallsituationen anhängig vom Alter des verunfallten Fahrradfahrers zu treffen, wurde die Kollisionsart für das Fallmaterial von UKLMU und UKM und der SUD in Abhängigkeit von ausgewählten Altersgruppen ausgewertet (Abbildung 1-20 bis Abbildung 1-23).

Auf Abbildung 1-20 ist die Auswertung für die Daten des UKLMU dargestellt. Die Altersgruppen wurden eingeteilt in Jugendliche/Junge Erwachsene (15-24), Erwachsene (2564) und Senioren (65+). Kinder unter 15 Jahren sind im Fallmaterial des UKLMU nicht vorhanden.

Es sind in allen 66 vorhandenen Fällen Angaben zur Kollisionsart gemacht worden.

Der Alleinunfall ist in der Altersgruppe von 15 bis 24 Jahren am häufigsten vertreten. In der Altersgruppe von 15 bis 24 Jahren ist die Kollision mit einem fahrenden Lkw/Bus überproportional oft vertreten. Die Erwachsenen von 25 bis 64 Jahren kollidieren häufiger als die anderen Altersgruppen mit einem fahrenden Pkw.

Bei den Senioren kommen Kollisionen mit fahrenden motorisierten Verkehrsteilnehmern überhaupt nicht vor, dort ist der Sturz nach Kollision mit einem festen Gegenstand (z.B. auch mit einem stehenden Auto) nach einem Sturz ohne Fremdeinwirkung die zweithäufigste Unfallart. Allerdings sind sowohl die Altersgruppen von 15 bis 24 Jahren wie auch über 64 Jahren mit acht bzw. sieben Fällen in diesem Kollektiv recht schwach besetzt. 


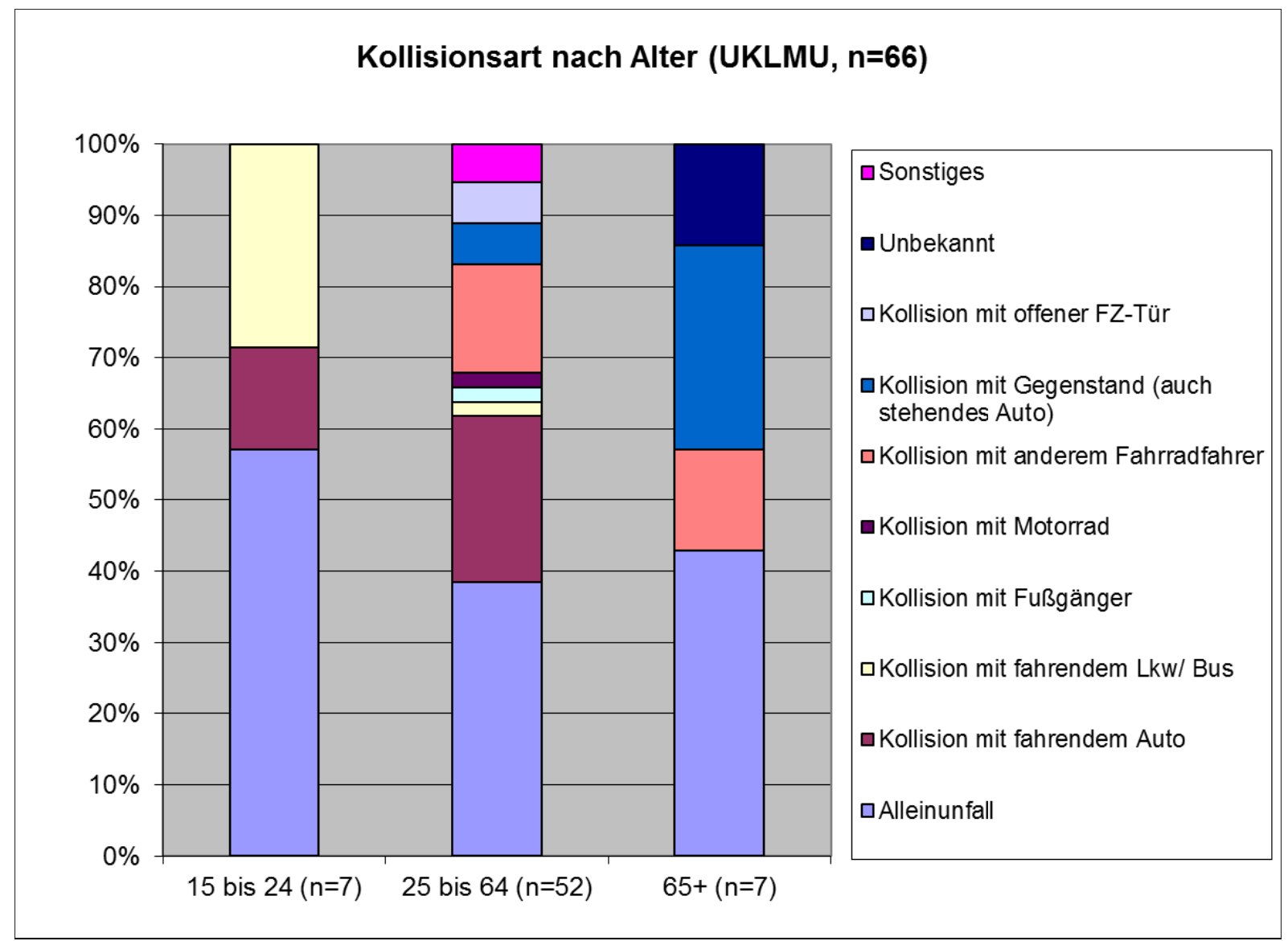

Abbildung 1-20: Kollisionsart nach Alter (UKLMU, $n=66$ )

Im Fallmaterial des UKM sind in 437 Fällen Angaben zur Kollisionsart vorhanden, in 40 Fällen wurde keine Angabe gemacht.

Die Altersgruppen wurden eingeteilt in Kinder (unter 15 Jahren), Jugendliche/Junge Erwachsene (15-24), Erwachsene (25-64) und Senioren (65+).

Die Auswertung auf Abbildung 1-21 zeigt, dass die Alleinunfälle mit höherem Alter seltener vorkommen. Die Häufigkeit von Alleinunfällen differiert aber über die gesamte Alterspanne hinweg nur zwischen 54\% und 45\%.

Die Kollision mit einem fahrenden Pkw ist in allen Altersgruppen annähernd gleich häufig vertreten, lediglich bei den Kindern und Senioren ist ein leicht erhöhter Prozentsatz zu verzeichnen.

Kollisionen mit einem fahrenden Lkw/Bus oder einem Fußgänger treten nur in den Altersgruppen von 15 bis 64 Jahren auf. Senioren kollidieren im Vergleich mit den anderen Altersgruppen seltener mit einem anderen Fahrradfahrer. 
Kollisionsart nach Alter (UKM, $n=437$ )

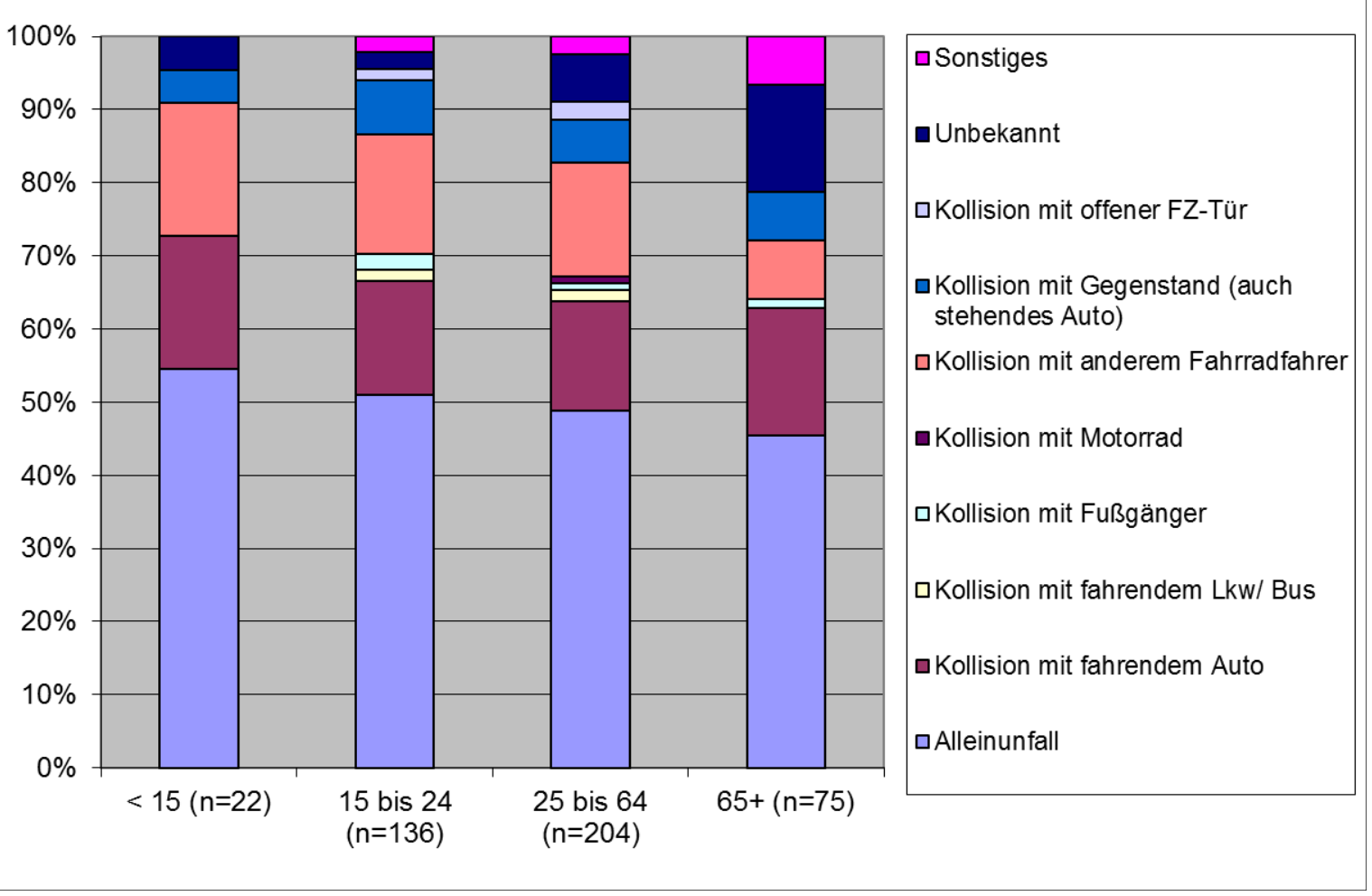

Abbildung 1-21: Kollisionsart nach Alter (UKM, n=437)

Betrachtet man die Unfalldaten des UKLMU und des UKM zusammen (Abbildung 1-22), ergibt sich ein ähnliches Bild wie für das UKM allein (Abbildung 1-21).

Dies ist insbesondere dem hohen Anteil von 437 Fällen geschuldet, die das UKM im Vergleich zu den 66 Fällen des UKMU im Fallmaterial einnimmt.

Zwischen den einzelnen Altersgruppen sind nur leicht differierende Häufigkeiten der Kollisionsarten zu erkennen. Der Alleinunfall tritt in höherem Alter seltener auf, während die Senioren weniger häufig in Unfälle mit anderen Fahrradfahrern verwickelt wurden.

In Hinblick auf Kollisionen mit anderen motorisierten Verkehrsteilnehmern sind keine deutlichen Unterschiede zwischen den einzelnen Altersgruppen zu erkennen. Einzige Ausnahme ist, dass Kollisionen mit einem fahrenden Lkw/Bus nur in den Altersgruppen von 15 bis 64 Jahren auftreten. 


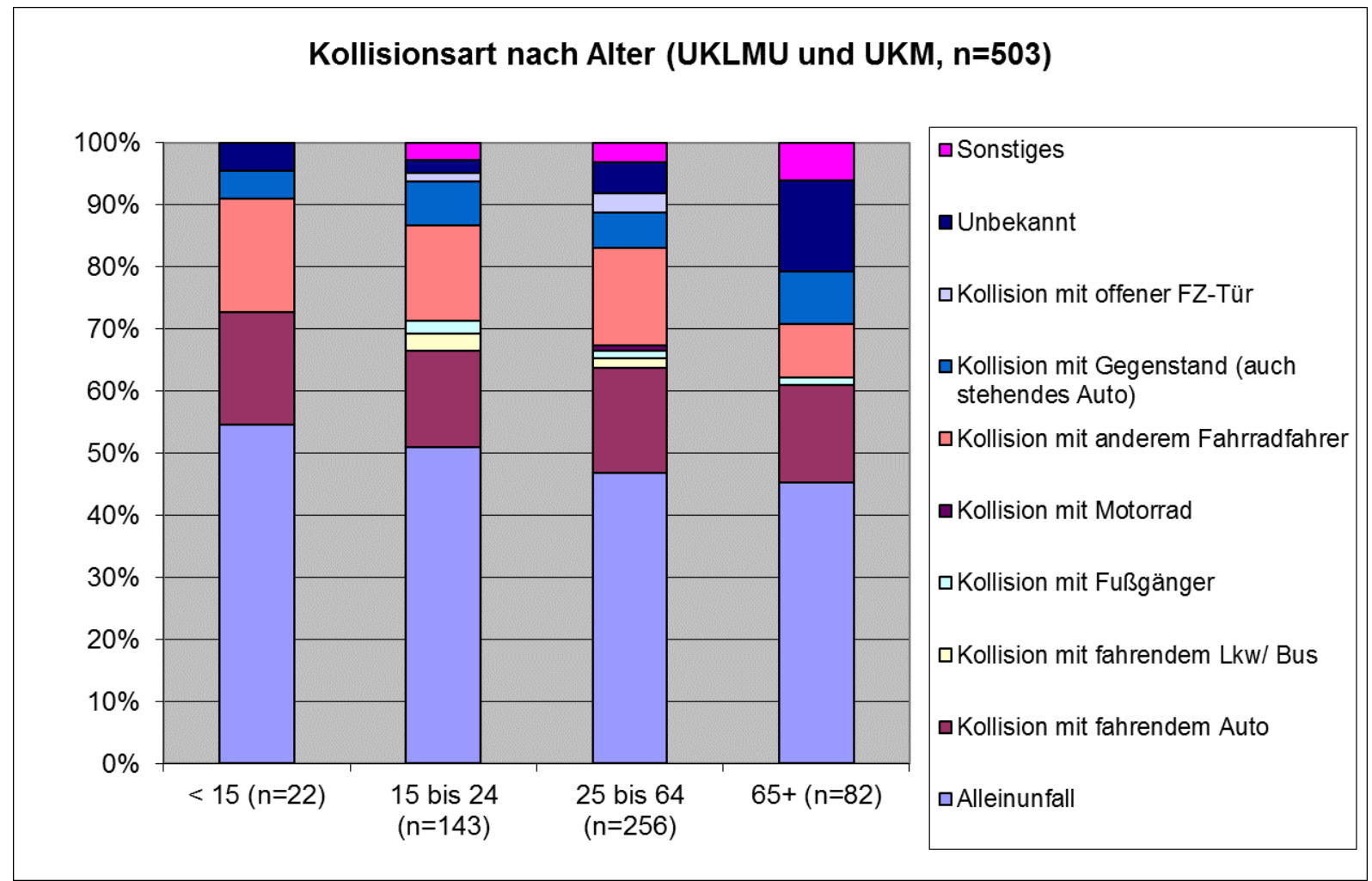

Abbildung 1-22: Kollisionsart nach Alter (UKLMU und UKM, n=503)

Im Fallmaterial der SUD (Abbildung 1-23) verunglückte kein Fahrradfahrer jünger als 24 Jahre bei einem Alleinunfall, wobei die ohnehin geringe Zahl von Getöteten in dieser Altersgruppe zu berücksichtigen ist.

Senioren über 65 verunglückten in Vergleich zu den Fahrern in der Altersgruppe von 25 bis 64 Jahren seltener bei einem Alleinunfall, dafür kollidierten die Senioren häufiger mit einem Pkw.

Ergänzend zu den oben gezeigten Abbildung 1-20 bis Abbildung 1-23, in denen die Kollisionsart abhängig vom Alter dargestellt wurde, ist auf Abbildung 1-24 die Verteilung der Kollisionsarten allgemein für die drei unterschiedlichen Datenkollektive (UKLMU, UKM und SUD) abgebildet.

Man erkennt deutlich, dass in den Fallkollektiven des UKLMU und des UKM der Alleinunfall dominiert, während im Schwerstunfallkollektiv der SUD die Kollision mit einem Pkw mit ca. $50 \%$ führend ist.

In der SUD sind ebenfalls verhältnismäßig viele Kollisionen mit einem Lkw/Bus vorhanden; im Fallmaterial des UKLMU und des UKM ist diese Kollisionsart von geringer Bedeutung. Kollisionen mit einem Lkw oder Omnibus sind häufig mit schwerwiegenden Folgen für den Fahrradfahrer (bis hin zum Tod) verbunden, somit überrascht diese Verteilung nicht. Auffällig ist in den Daten des UKLMU und des UKM der Anteil von Kollisionen mit einem anderen Fahrradfahrer; diese Kollisionsart wiederum ist in der SUD fast nicht vorhanden. 


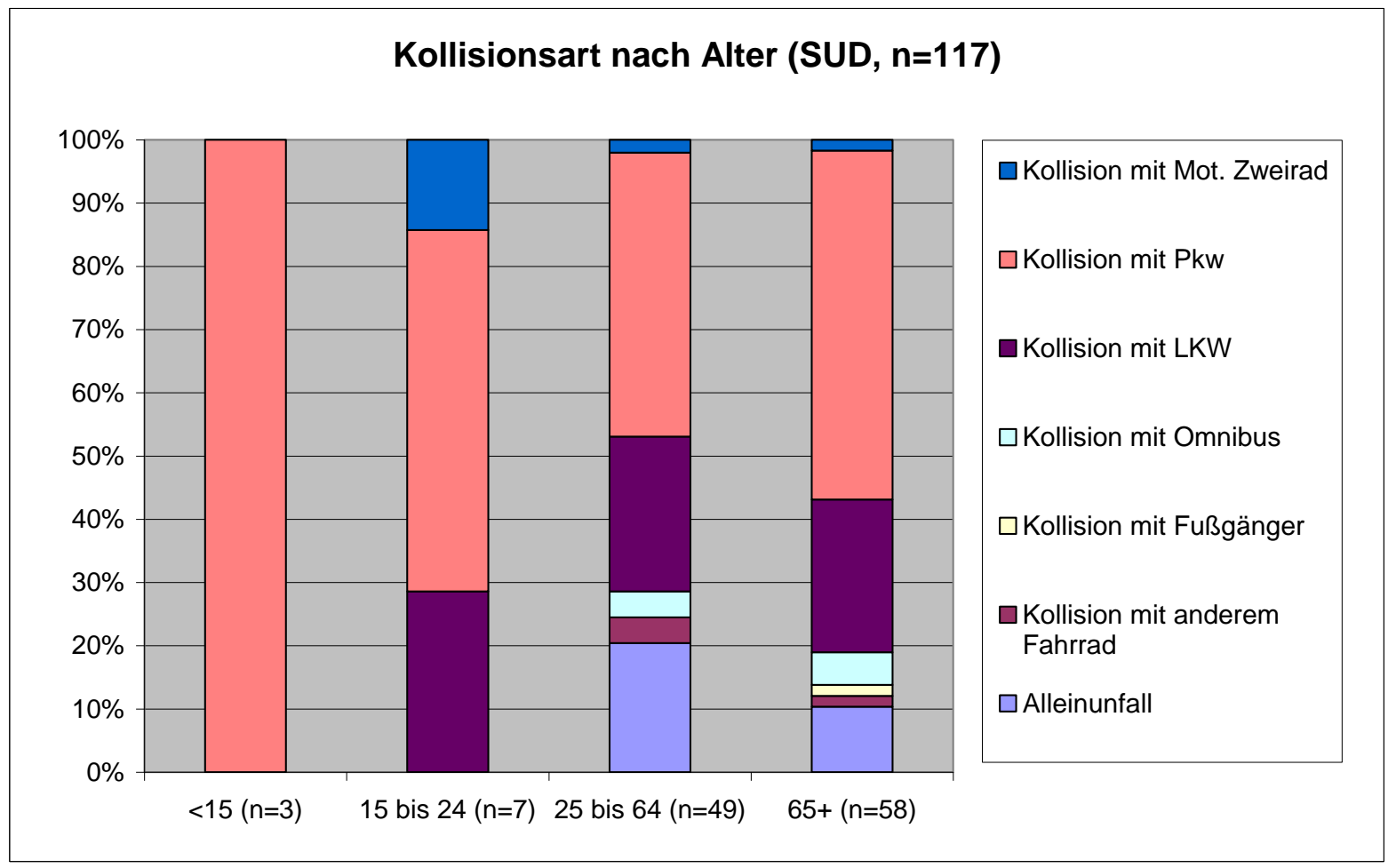

Abbildung 1-23: Kollisionsart nach Alter (SUD, n=117)

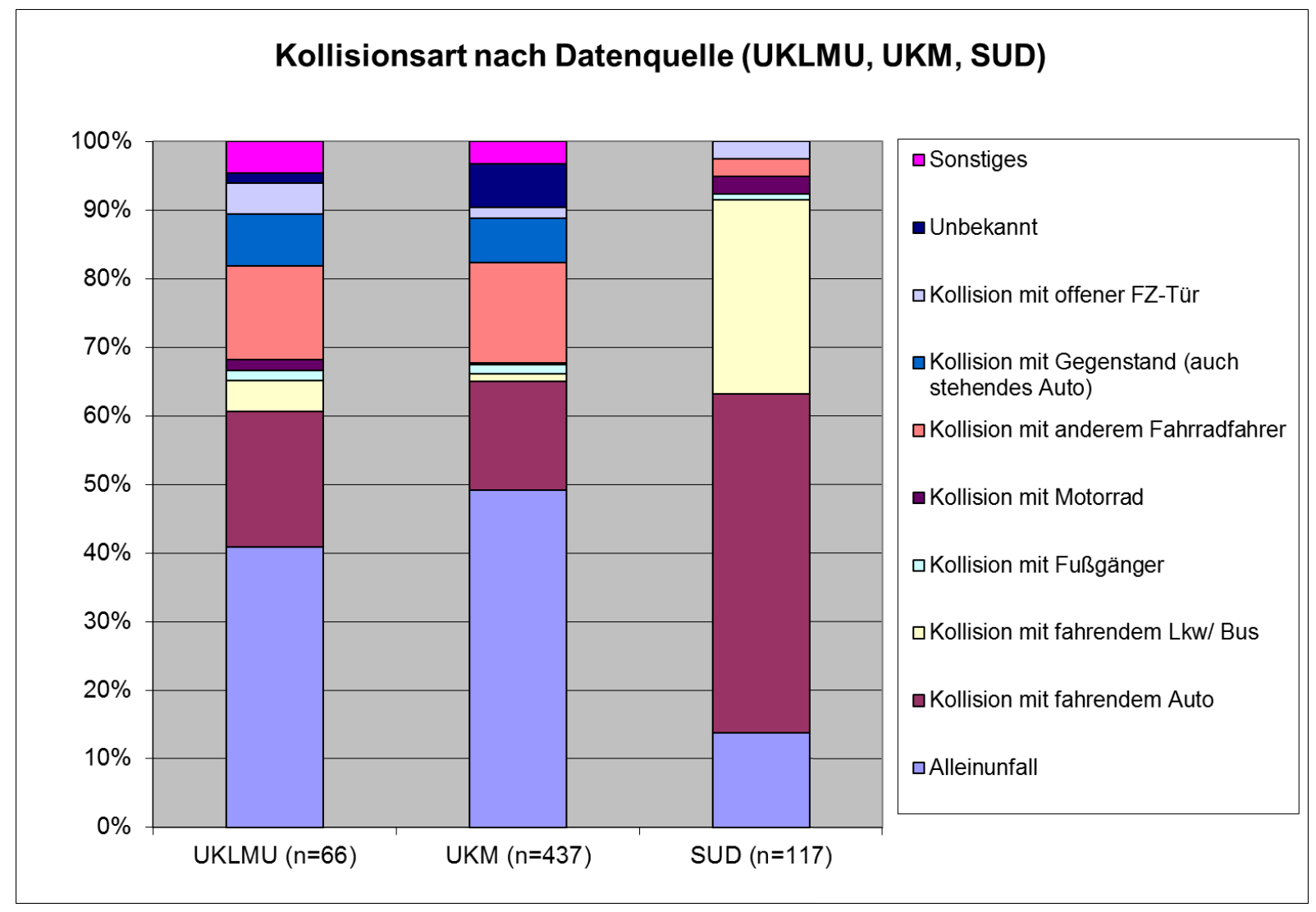

Abbildung 1-24: Kollisionsart nach Datenquelle (UKLMU, UKM, SUD) 


\subsubsection{Benutzte Fahrradtypen}

Auf Abbildung 1-25 ist die Auswertung der benutzten Fahrradtypen dargestellt. Im UKLMU fuhren knapp 60\% der verunfallten Radfahrer mit einem Sportrad, Rennrad, Mountain- oder Trekkingbike. Knapp über 30\% benutzten ein Holland- oder Citybike; E-Bikes oder Pedelecs wurden in München nicht festgestellt.

Im UKM stellt die Gruppe der Holland-/Citybike-Fahrer die Mehrheit mit über 40\%. Auf einem Sportrad, Rennrad, Mountain- oder Trekkingbike waren unter 30\% der verunfallten Radfahrer unterwegs. Im UKM wurden 15 Patienten (3\%) erfasst, die mit einem Pedelec oder E-Bike fuhren.

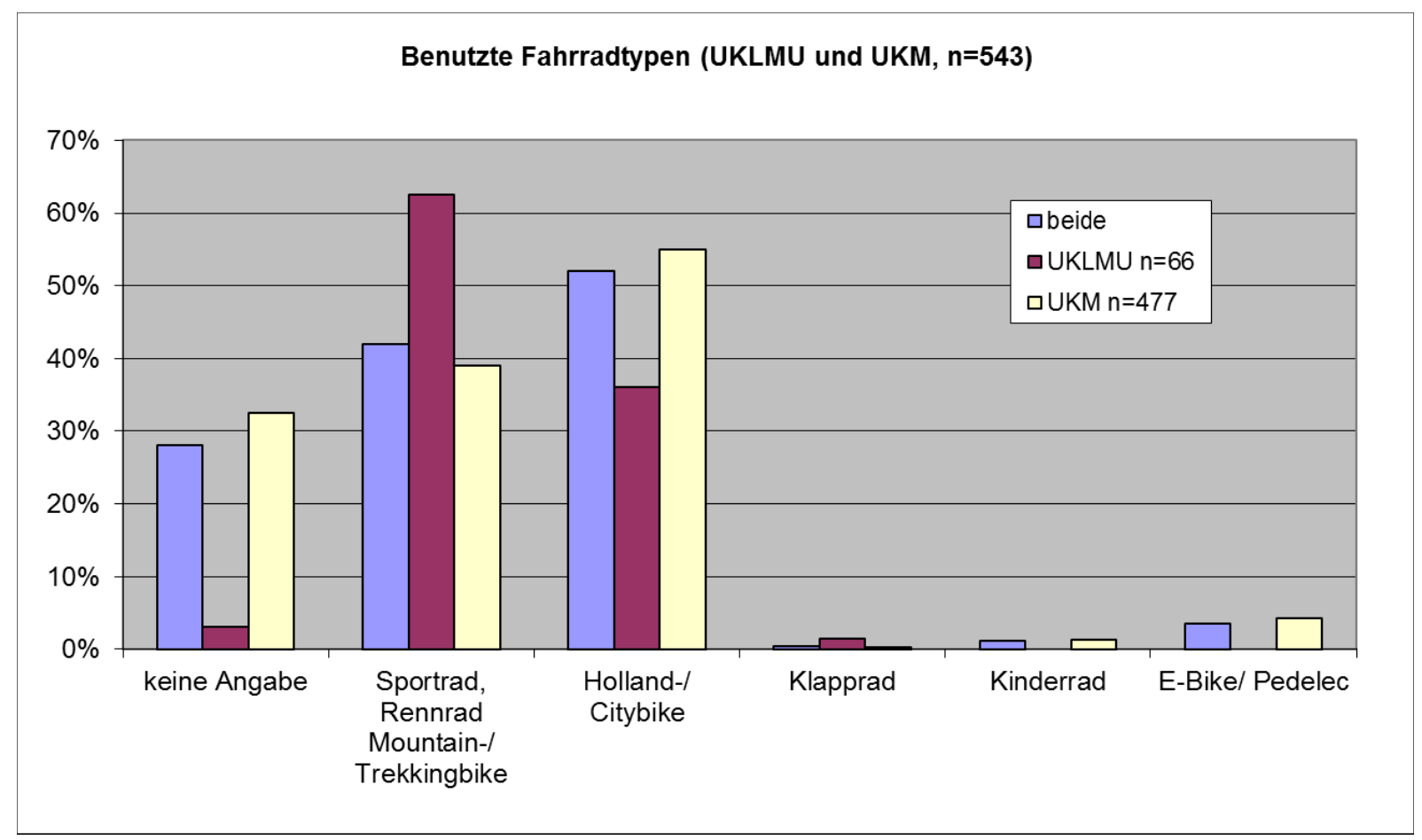

Abbildung 1-25: Benutzte Fahrradtypen (UKLMU und UKM, n=543)

In den Daten der Rechtsmedizin wurde eine andere Unterteilung der benutzten Fahrradtypen getroffen. Im Unterschied zum Patientenfragebogen der prospektiv erfassten Fälle wurden Rennräder, Mountainbikes und Trekkingräder getrennt betrachtet.

Wie in Abbildung 1-26 zu erkennen ist, fuhren jeweils knapp über 30\% der getöteten Fahrradfahrer (SUD) mit einem Citybike oder einem Trekkingrad. Mit einem Mountainbike waren ca. 20\% der Fahrradfahrer unterwegs, mit einem Rennrad 13\%. 


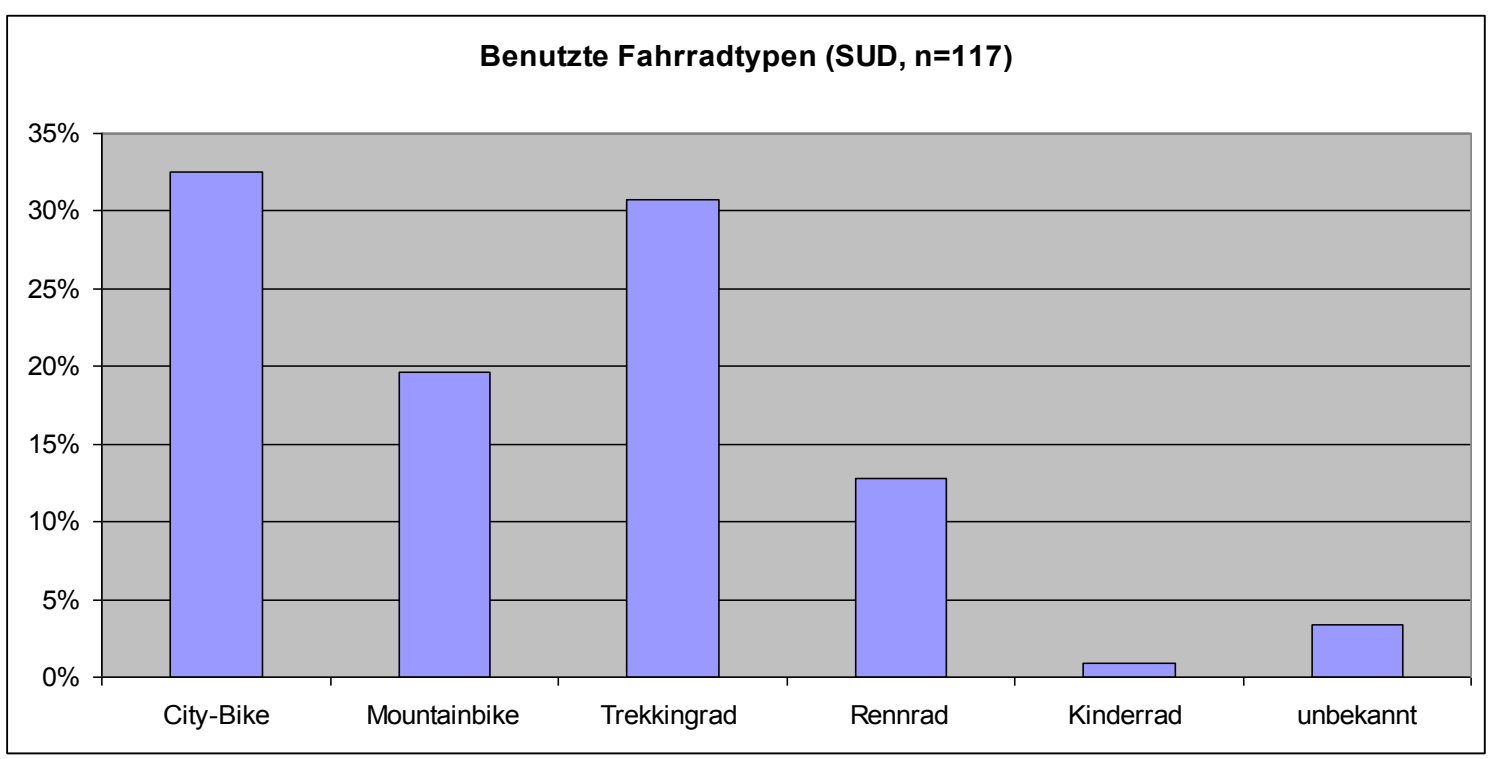

Abbildung 1-26: Benutzte Fahrradtypen (SUD, $n=117$ )

\subsubsection{Charakteristika der Alleinunfälle}

Wie unter 1.4.2.4 bereits dargestellt wurde, ist der Alleinunfall der häufigste Kollisionstyp der verletzten Fahrradfahrer, die im UKLMU oder UKM erfasst wurden. 45\% (242) aller Fahrradfahrer verunfallten ohne Fremdeinwirkung.

Im Fallmaterial der Rechtsmedizin ist dieser Kollisionstyp weniger stark repräsentiert.

Auf Abbildung 1-27 ist die Geschwindigkeit der bei einem Alleinunfall verletzten

Fahrradfahrer aus dem UKLMU und dem UKM abgebildet. Die Einschätzung wurde von den Radfahrern selbst vorgenommen, wofür der Patientenfragebogen vier verschiedene Kategorien vorsieht.

Die Patienten aus dem UKLMU gaben vermehrt an (60\%), zügig (> $20 \mathrm{~km} / \mathrm{h}$ ) unterwegs gewesen zu sein. Langsam gefahren (> $20 \mathrm{~km} / \mathrm{h}$ ) sind nach eigenen Angaben unter $30 \%$. Angefahren oder gestanden sind summiert nur ca. 10\% der erfassten Fahrradfahrer aus dem UKLMU.

Die Daten aus dem UKM unterscheiden sich hier deutlich. Über $50 \%$ der Fahrradfahrer aus dem UKM gaben an, langsam gefahren zu sein, nur knapp über $20 \%$ gaben an, zügig unterwegs gewesen zu sein. Die Zahl der stehenden oder anfahrenden Fahrradfahrer ist auch hier gering. 


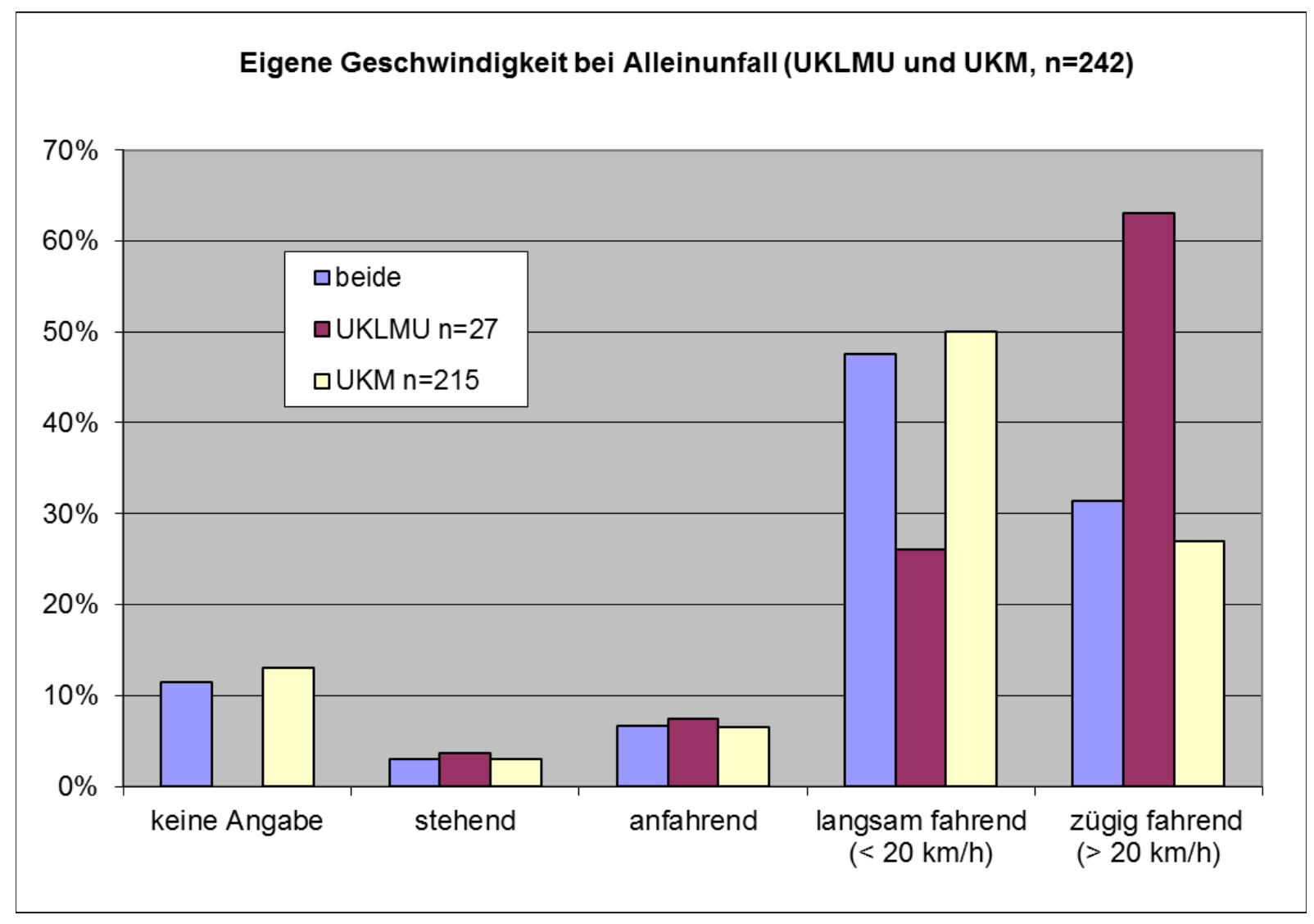

Abbildung 1-27: Eigene Geschwindigkeit bei Alleinunfall (UKLMU und UKM, n=242)

Wie auf Abbildung 1-28 zu sehen ist, wurde in der Mehrheit der Alleinunfälle (42\%) als Unfallursache ein schlechter Untergrund angegeben (z.B. Straßenbahnschienen, Kopfsteinpflaster etc.). Ca. 15\% gaben an, das Gleichgewicht verloren zu haben, gefolgt von der Angabe „Hindernis übersehen“ mit knapp 10\%. Mit um die 5\% sind technische Defekte am Fahrrad, eine Ablenkung oder das Abrutschen vom Pedal selten Unfallursache für den Alleinunfall.

Die Angabe „Anderer Grund“ wurde in 12\% aller Fälle als Ursache angegeben. Hier sind z.B. ein querender Hund oder eine sich verhakende Einkaufstasche die häufigsten Beschreibungen. 


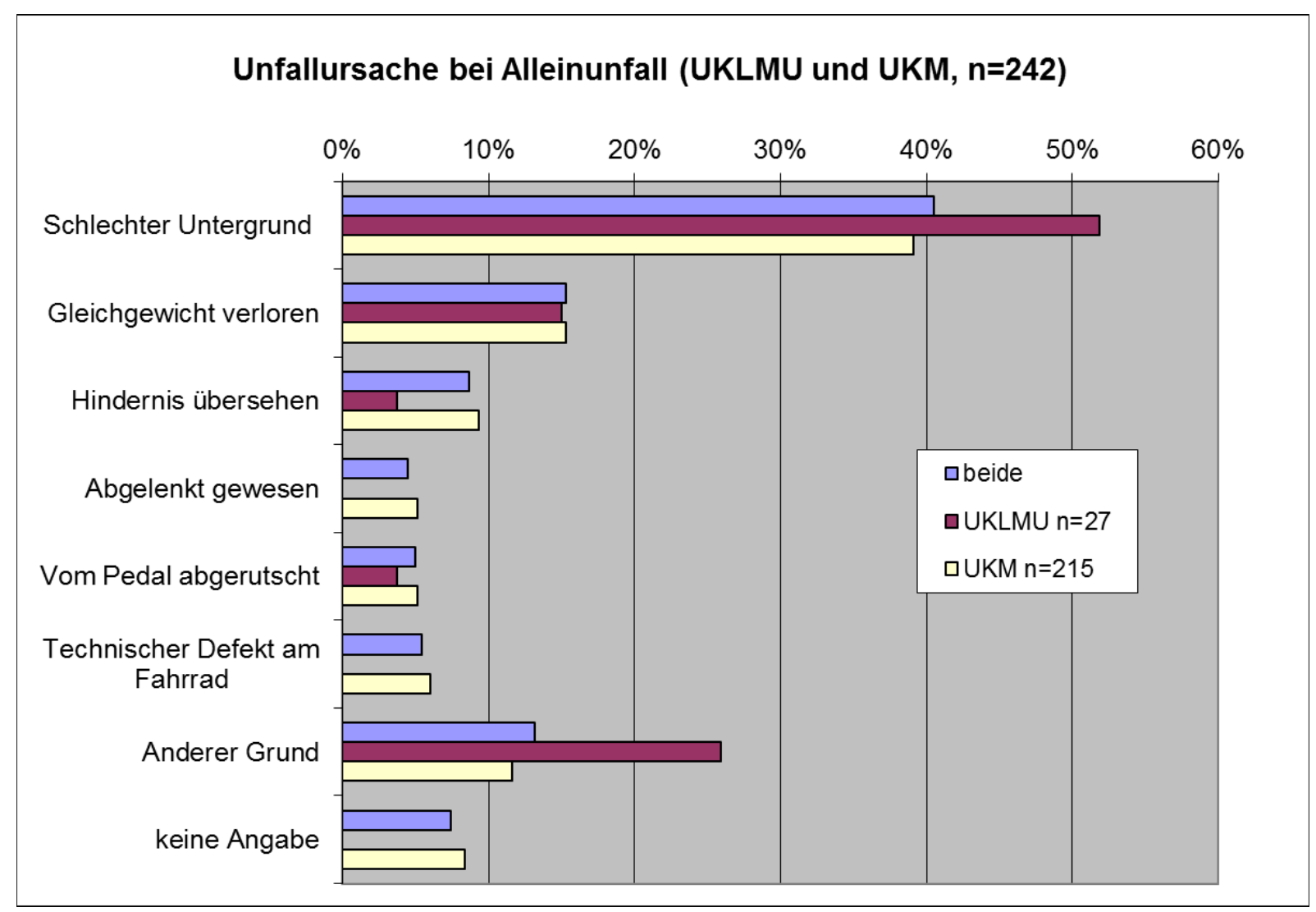

Abbildung 1-28: Unfallursache bei Alleinunfall (UKLMU und UKM, n=242)

In der SUD wurde bei den Alleinunfällen kein expliziter Unfallgrund dokumentiert. Die Analyse möglicher Unfallursachen erfolgte anhand von Einzelfallanalysen. Dabei konnten zwei typische Unfallabläufe festgestellt werden. Zum einen erfolgt der Sturz bei meist geringer Geschwindigkeit aufgrund von Gleichgewichtsverlust in einer seitlichen Bewegung. Zum anderen kommt es entweder aufgrund einer Überreaktion des Fahrradfahrers oder eines Hindernisses oder Gegenstands zum Sturz über das blockierende Vorderrad.

Die gefahrene Geschwindigkeit der allein tödlich verunglückten Fahrradfahrer zum Unfallzeitpunkt bewegt sich zwischen 0 und $30 \mathrm{~km} / \mathrm{h}$, lediglich in einem Fall ist eine weit höhere Geschwindigkeit dokumentiert.

Nach Betrachtung aller Fallkollektive (UKLMU, UKM, SUD) konnten zwei verschiedene typische Unfallmechanismen herausgearbeitet werden.

Der erste typische Alleinunfall geschieht durch schlechten Untergrund und/oder Verlust des Gleichgewichts. Der Fahrradfahrer fährt über schlechten Untergrund (z.B. einer Straßenbahnschiene oder einem Schlagloch), kommt aufgrund dieser unerwarteten Situation aus dem Gleichgewicht und stürzt in einer seitlichen Bewegung auf die Fahrbahn. Bei einem reinen Verlust des Gleichgewichts ist ebenfalls ein Sturz in seitlicher Richtung zu beobachten. Unterschied zwischen diesen zwei Mechanismen ist die Geschwindigkeit. Der Verlust des Gleichgewichts geschieht bei sehr geringen Geschwindigkeiten und annäherndem Stillstand, während beim Sturz aufgrund von schlechtem Untergrund die Geschwindigkeit etwas höher sein kann. Die Geschwindigkeit überschreitet dabei selten „langsam fahrend“ (UKLMU, UKM), bzw. 15 km/h (SUD). 
Der zweite typische Alleinunfall ist mit einem Sturz über den Lenker zu beschreiben. Aus den Angaben zum Unfalltyp im Patientenfragebogen des UKLMU und UKM (siehe Abbildung 1-26) ist dieser Unfallmechanismus nicht explizit zu erkennen. Dies wurde in Einzelfallanalysen und der Recherche in den Kurzbeschreibungen zum Unfall herausgearbeitet.

Der Sturz nach vorne geschieht entweder durch eine Fehlreaktion des Fahrradfahrers, der das Vorderrad überbremst, oder durch sonstige Blockade des Vorderrads. Diese Bewegung geschieht typischerweise längs in Fahrtrichtung mit geringer seitlicher Abweichung. Die gefahrene Geschwindigkeit ist tendenziell mit "zügig fahrend" zu beschreiben.

Bei einem Alleinunfall ist jedoch oft der exakte Unfallhergang nicht zu beschreiben, da in einigen Fällen weder Zeugen noch detaillierte Unfallspuren vorhanden sind.

Der mögliche Unfallmechanismus muss demnach interdisziplinär aus den Verletzungen des Fahrradfahrers und den vorhandenen Randparametern herausgearbeitet werden.

Dies geschah für die vorliegenden fraglichen Fälle aller Datenkollektive in der BiomechanikGruppe des Instituts für Rechtsmedizin in München.

Wie unter 1.3 bereits dargestellt, ist bei den Alleinunfällen der Sturz seitlich mit 31 bis $56 \%$ der häufigste Unfallmechanismus, gefolgt vom Sturz über den Lenker.

Im prospektiv erfassten Fallmaterial aus dem UKLMU und UKM erfolgte basierend auf den Kurzbeschreibungen eine vorsichtige Abschätzung der Verteilung der Unfallmechanismen. Am häufigsten (ca. 50\%) verunglückten die Fahrradfahrer durch einen Sturz seitlich; zweithäufigster Unfallmechanismus ist der Sturz über den Lenker mit ca. $14 \%$.

Für die weitere Rekonstruktion und Simulation sind an dieser Stelle die folgenden zwei typischen Unfallmechanismen des Alleinunfalls festzuhalten:

\section{1) Alleinunfall des Fahrradfahrers}

1a) Sturz seitlich bei geringer Geschwindigkeit

1b) Sturz über Lenker bei blockierendem Vorderrad durch Überbremsen oder sonstige Blockade des Vorderrads bei mäßiger bis hoher Geschwindigkeit

1a)

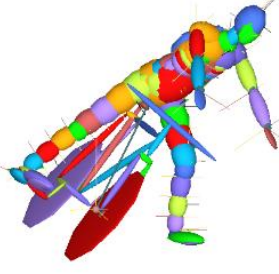

1b)

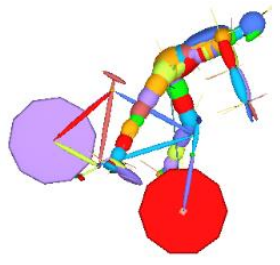

Abbildung 1-29: Kollisionsszenarien 1a) und 1b) 


\subsubsection{Kollisionsstellungen/-geschwindigkeiten bei Unfällen mit Pkw-Beteiligung}

Unter 1.4.2.4 wurde dargelegt, dass die Kollision des Fahrradfahrers mit einem fahrenden Pkw nach dem Alleinunfall die zweithäufigste Unfallsituation ist.

In $15 \%(n=82)$ der prospektiv erfassten Fälle aus dem UKLMU und dem UKM kollidierte der Radfahrer mit einem Pkw. Im Fallmaterial der Rechtsmedizin ist dieser Unfalltyp mit über $50 \%(n=61)$ dominierend.

Deshalb werden im Folgenden Charakteristika dieser Unfallsituation herausgearbeitet.

Auf Abbildung 1-30 ist der Anprallbereich am Fahrrad bei Unfällen mit Pkw-Beteiligung der Fälle aus dem UKLMU und dem UKM dargestellt.

Von den 82 Unfällen (UKLMU n=13, UKM n=69) mit Pkw-Beteiligung wurden von den Radfahrern in 24 Fällen Angaben zum Anprallbereich am eigenen Fahrrad gemacht.

Die Daten des UKLMU wurden durch die angeforderten Polizeiberichte ergänzt, so dass in 12 der 13 Fälle Angaben vorliegen. Im Fallmaterial des UKM liegen in 12 der 69 Unfälle mit Pkw-Beteiligung Angaben zum Anprallbereich vor.

Aus den Daten des UKLMU ist zu erkennen, dass der Anprall an die linke Seite des Fahrrads mit $42 \%$ führend ist, es folgen der Anprall an die Front des Fahrrads mit 33\% und der Anprall an die rechte Seite mit $25 \%$. Es wurde kein Fahrrad von einem Pkw von hinten erfasst.

Die Daten des UKM geben hier ein leicht abweichendes Bild. Hier ist Front des Fahrrads mit $42 \%$ der häufigste Anprallbereich, gefolgt von der rechten Seite und hinten mit jeweils $25 \%$. An letzter Stelle ist der Anprall an die linke Seite mit 9\% (entspricht einem Fall).

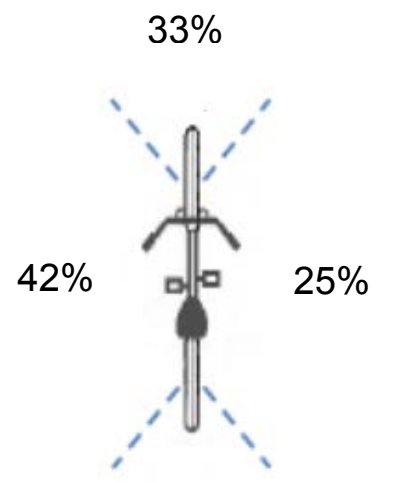

UKLMU, $n=12$

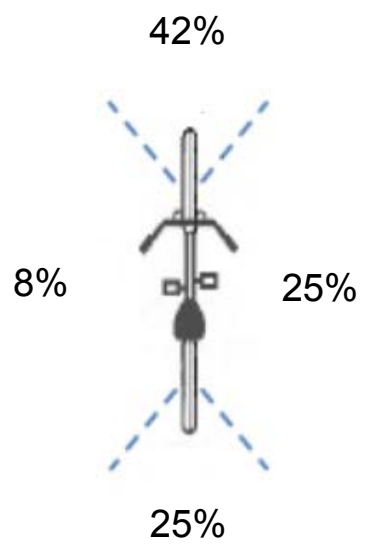

UKM, $n=12$

Abbildung 1-30: Anprallbereich am eigenen Fahrrad bei Unfällen mit Pkw-Beteiligung (UKLMU und UKM, $n=24)$

Um Angaben über mögliche Kollisionsstellungen treffen zu können, wurde auch der Anprallbereich am Pkw näher betrachtet (Abbildung 1-31). Hier sind in 15 der 16 Fälle aus dem UKLMU Angaben vorhanden, in den Daten aus dem UKM ist in 12 der 69 Fälle der Anprallbereich am Pkw bekannt. Dabei liegen nicht notwendiger Weise dieselben Unfallereignisse wie für die Bestimmung der Anprallstellen am Fahrrad zugrunde. 
Es dominiert in beiden Datensätzen der Anprall an die Front des Pkw. In den Daten des UKLMU ist die Mitte der Pkw-Front der häufigste Anprallbereich. Zusätzlich wurden auch beide Seiten des Pkw getroffen.

Im Fallmaterial des UKM sind Anpralle an die Seite des Pkw nicht vorhanden, dafür ist in $11 \%$ der Fälle das Heck des Pkw als Anprallort angegeben. Im Frontbereich des Pkw ist die linke Ecke mit 56\% dominierend, gefolgt von der Mitte.

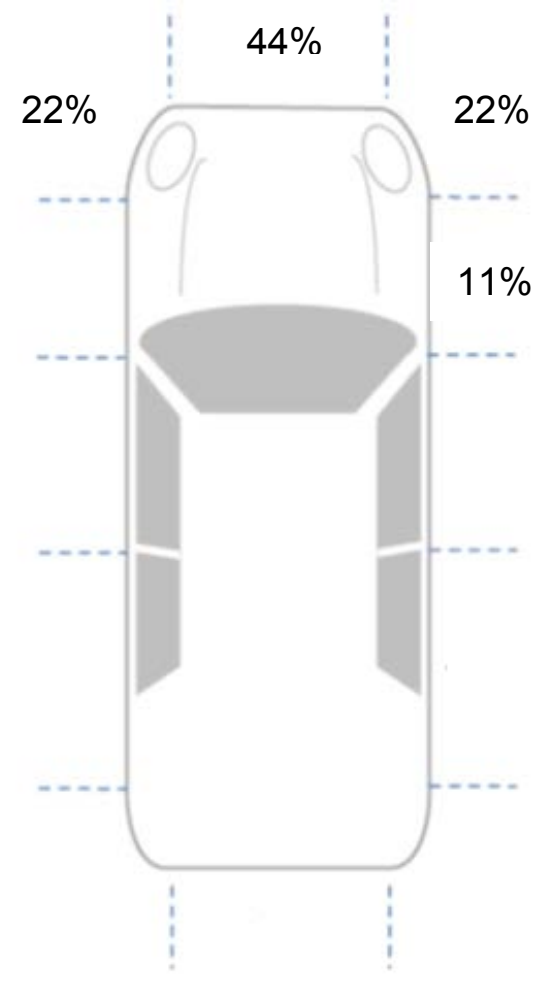

UKLMU, $\mathrm{n}=9$

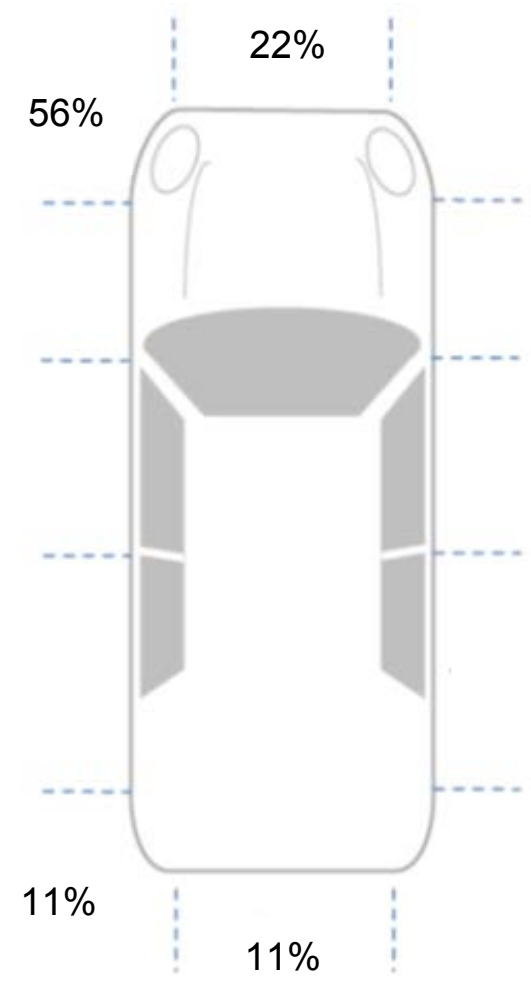

UKM, $n=9$

\section{Abbildung 1-31: Anprallbereich am gegnerischen Pkw (UKLMU und UKM, n=18)}

Aus der Kombination der auf Abbildung 1-30 und Abbildung 1-31 dargestellten Ergebnisse und einer Recherche der Kurzbeschreibungen (und falls vorhanden der Polizeiberichte) der jeweiligen Unfälle lässt sich auf zwei typische Kollisionsstellungen schließen.

Zum einen treffen Fahrradfahrer und Pkw bei einem Abbiegevorgang des Pkw aufeinander. Dabei trifft entweder der Pkw mit einer Ecke seiner Front die Seite des Fahrradfahrers oder der Fahrradfahrer fährt frontal in die vordere Ecke des Pkw. Dabei bewegt sich der Winkel des Fahrradfahrers zum abbiegenden Pkw im Bereich von $90^{\circ}$ (jeweils $45^{\circ}$ nach rechts und links).

Zum anderen erfasst der Pkw den kreuzenden Fahrradfahrer in einem annähernd rechten Winkel mit seiner Front oder einer der beiden vorderen Fahrzeugecken und trifft dabei die Seite des Fahrrads.

Die Kollisionsstellungen, in denen der Fahrradfahrer auf das Heck des Pkws auffährt oder der Pkw den Fahrradfahrer von hinten erfasst, werden aufgrund der geringen Fallzahlen an dieser Stelle nicht weiter betrachtet. 
Auf Abbildung 1-32 sind die Kollisionsgeschwindigkeiten der beteiligten Pkw gemäß Angabe durch den Radfahrer dargestellt. In 63 der 82 Unfälle mit Pkw-Beteiligung sind Angaben vorhanden.

Sowohl im Fallmaterial des UKLMU als auch des UKM ist die Geschwindigkeitsangabe von $10-30 \mathrm{~km} / \mathrm{h}$ am häufigsten (UKLMU 54\%, UKM 29\%). An zweiter Stelle liegt mit 31\% (UKLMU) und $28 \%$ (UKM) die Angabe von < $10 \mathrm{~km} / \mathrm{h}$. Höhere Geschwindigkeiten über 30 $\mathrm{km} / \mathrm{h}$ sind bei UKLMU und UKM mit jeweils $15 \%$ vertreten.

Diese von Radfahrern selbstberichteten Geschwindigkeitsangaben sind als grob qualitativ anzusehen und möglicherweise durch die eigene Wahrnehmung verzerrt.

Die vorhandenen Kurzbeschreibungen der Unfälle und Polizeiberichte lassen vermuten, dass Geschwindigkeiten unter $30 \mathrm{~km} / \mathrm{h}$ typisch für Abbiegevorgänge des Pkw sind und Geschwindigkeiten über $30 \mathrm{~km} / \mathrm{h}$ auf rechtwinklige Kollisionen mit dem Radfahrer oder ein Auffahren von hinten hindeuten.

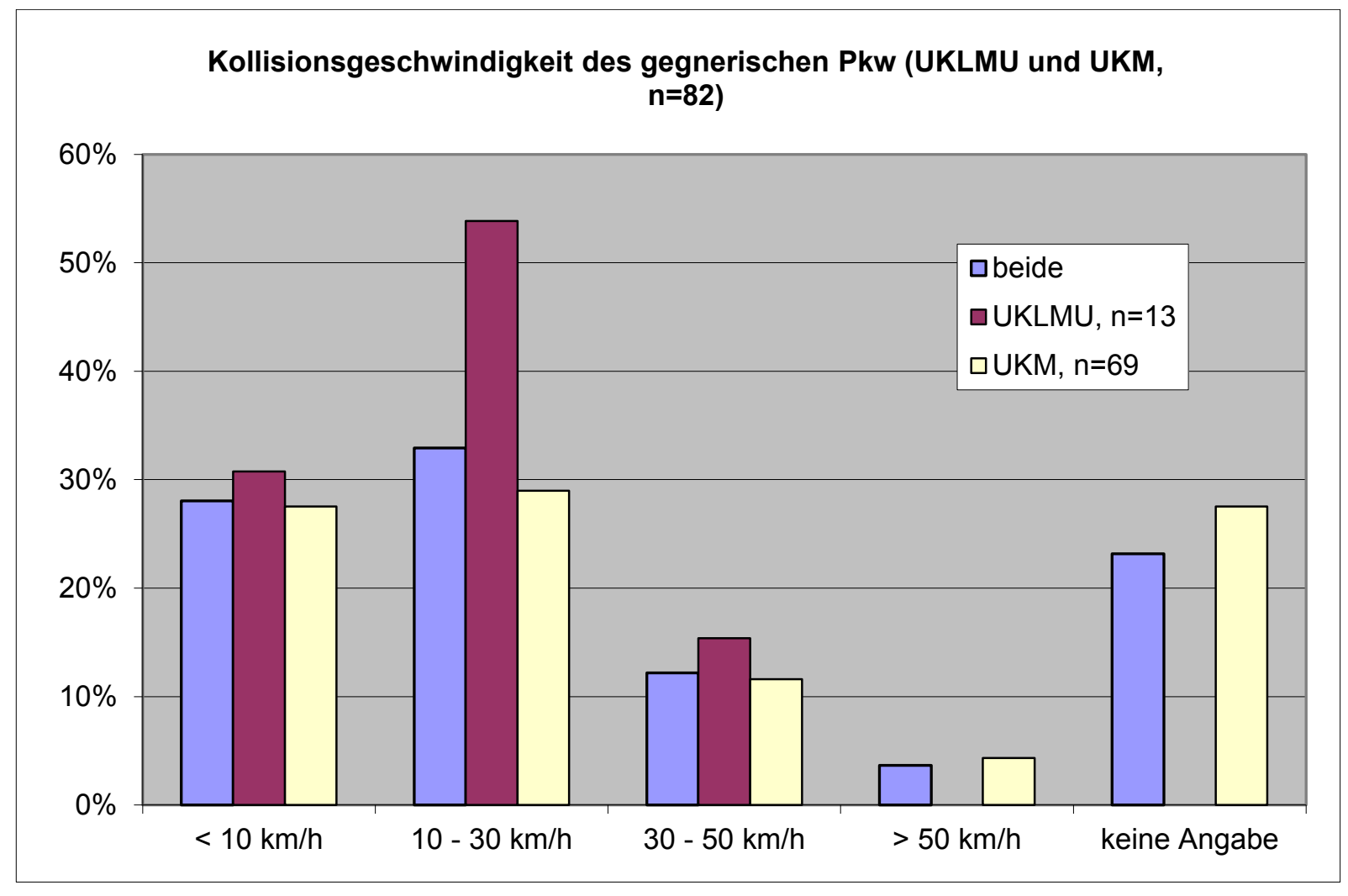

Abbildung 1-32: Kollisionsgeschwindigkeiten des gegnerischen Pkw (UKLMU und UKM, n=82)

Die Geschwindigkeit des Fahrradfahrers bei Unfällen mit Pkw-Beteiligung ist auf Abbildung 1-33 dargestellt. Hier sind in 69 der 82 Fälle Angaben vorhanden.

Deutlich an erster Stelle mit insgesamt über $50 \%$ ist die Angabe „langsam fahrend $(<20$ $\mathrm{km} / \mathrm{h}$ )“. Lediglich die verunfallten Fahrradfahrer des UKLMU waren mit über $50 \%$,zügig fahrend (> $20 \mathrm{~km} / \mathrm{h}$ )“ unterwegs, als sie mit einem Pkw kollidierten. Hier gilt ebenfalls die oben genannte Einschränkung, dass diese Angaben als grob qualitativ zu verstehen sind. 


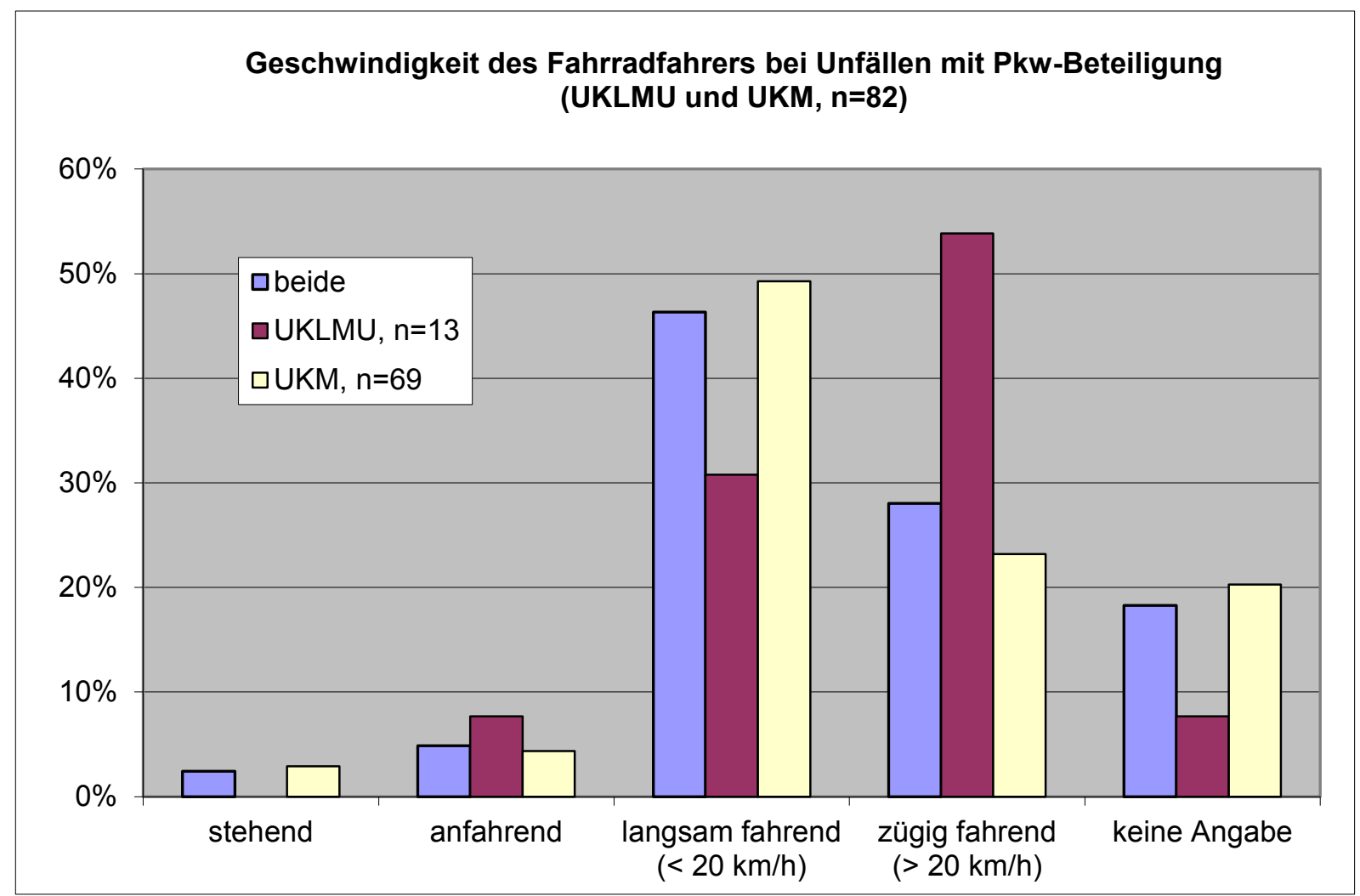

Abbildung 1-33: Fahrgeschwindigkeit des Fahrradfahrers bei Unfällen mit Pkw-Beteiligung (UKLMU und UKM, $n=82$ )

Analog zum prospektiv erfassten Fallmaterial aus dem UKLMU und dem UKM wurden auch die Daten der Rechtsmedizin ausgewertet.

Wie bereits unter 1.3.2.4 dargestellt, ist dabei die Kollisionsstellung „Pkw-Front erfasst Fahrradfahrer seitlich“ in annähernd rechtem Winkel mit 53\% dominierend.

Die Kollisionsgeschwindigkeit des Pkw bewegt sich bei diesen Schwerstunfällen häufig im Bereich um $60 \mathrm{~km} / \mathrm{h}$.

Fasst man die oben dargestellten Ergebnisse der Daten des UKLMU, des UKM und der SUD zusammen, lassen sich zwei typische Unfallkonstellationen für Unfälle mit Pkw-Beteiligung erkennen.

Sowohl bei den prospektiv erfassten Fällen mit verletzten Fahrradfahrern (UKLMU und UKM), wie auch bei den retrospektiven Fällen mit getöteten Fahrradfahrern der Rechtsmedizin (SUD) ist die Kollision des Fahrradfahrers mit der Front des beteiligten Pkw typisch, wobei der Anprall unter einem Winkel von $45^{\circ}$ bis $90^{\circ}$ erfolgt.

Dabei lassen sich für die Kollisionsgeschwindigkeit des Pkws zwei typische Bereiche eingrenzen.

In den Daten des UKLMU und des UKM mit verletzten Fahrradfahrern ist überwiegend eine geringe Kollisionsgeschwindigkeit von $<10 \mathrm{~km} / \mathrm{h}$ bis zu $30 \mathrm{~km} / \mathrm{h}$ dokumentiert. Im Fallmaterial der Rechtsmedizin mit tödlich verletzten Radfahrern gibt es ebenfalls eine erkennbare Häufung in diesem Geschwindigkeitsbereich, jedoch ist die weit größere Zahl bei Geschwindigkeiten um $60 \mathrm{~km} / \mathrm{h}$ zu finden. 
Die Resultate aus den drei Teilkollektiven können den Ergebnissen anderer Studien auf Grundlage von Fahrrad-Pkw-Kollisionen in Deutschland gegenübergestellt werden.

Die durchschnittliche Kollisionsgeschwindigkeit des Pkw bei Unfällen mit Fahrradfahrern beträgt nach Fredriksson et al. (2012) 36 km/h. In dieser Studie wurden Unfälle mit schwerverletzten, von Pkw erfassten Radfahrern betrachtet.

Eine Studie von Otte et al. (2013) zeigt, dass in 10\% der Kollisionen zwischen Fahrradfahrern und den beteiligten Fahrzeugen die Kollisionsgeschwindigkeit des anprallenden Kraftfahrzeugs über $35 \mathrm{~km} / \mathrm{h}$ liegt; in weiteren $10 \%$ zwischen 35 und $25 \mathrm{~km} / \mathrm{h}$. Die Autoren berichten, dass in Kollisionen mit einem Pkw bei Anprallgeschwindigkeiten bis 40 km/h 35,2\% der Radfahrer Kopfverletzungen erlitten, bei Geschwindigkeiten von über $40 \mathrm{~km} / \mathrm{h} 72,2 \%$.

Durch Analyse von Fällen aus der Unfalldatenbank der Versicherer (UDB) konnten ebenfalls typischen Geschwindigkeiten für Pkw ermittelt werden, die annähernd rechtwinklig mit Radfahrern kollidierten.

In 210 Fällen kollidierte ein Pkw mit einem querenden Fahrradfahrer und der Fahrradfahrer prallte entweder an der Front oder dem seitlichen Vorderwagen des Pkw an und wurde verletzt.

In $90 \%$ dieser Fälle betrug die Fahrgeschwindigkeit des Pkw 45 km/h oder weniger. $29 \%$ der Fahrradfahrer bei Kollisionsgeschwindigkeiten des Pkw bis zu 45 km/h wurden unter diesen Bedingungen MAIS 3+ verletzt (unter Betrachtung aller Körperregionen).

Bei weiterer Einschränkung der Kollisionsbedingungen (Pkw kollidiert annähernd rechtwinklig mit einem querenden Fahrradfahrer und der Fahrradfahrer wird dabei seitlich von der Front des Pkw erfasst und verletzt) zeigen sich vergleichbare Anteilswerte bezüglich der Fahrgeschwindigkeit des Pkw und der größten Verletzungsschwere (MAIS).

Für das unter 1.3 bestimmte typische Unfallszenario „Pkw gegen Fahrrad seitlich“ wurde die typische Kollisionsgeschwindigkeit des Pkws nach Betrachtung des Gesamtunfallkollektivs und unter Einbeziehung oben dargestellter Literatur in Szenario $2 \mathrm{~b}$ auf $40 \mathrm{~km} / \mathrm{h}$ festgelegt.

\subsubsection{Anprallpunkte am gegnerischen Pkw, Form der Fahrzeugfront}

Auf Abbildung 1-34 und Abbildung 1-35 sind die Anprallpunkte durch den Radfahrer gegen die Front des beteiligten Pkw dargestellt. Im UKLMU wurden bei neun Unfällen Angaben gemacht, pro Unfall sind dabei mehrere Nennungen möglich. Die Anprallpunkte des Fahrradfahrers werden getrennt von den Anprallpunkten des Fahrrads betrachtet. Es wird deutlich, dass der Fahrradfahrer in der überwiegenden Mehrheit der Fälle mit der Front des Pkw kollidiert. Die Frontscheibe und der obere Teil der Motorhaube wurden im Fallmaterial des UKLMU nicht getroffen. Durch die relativ geringen Geschwindigkeiten wird der Fahrradfahrer nicht auf den Pkw aufgeworfen, sondern von diesem oft nur umgestoßen oder abgewiesen. 

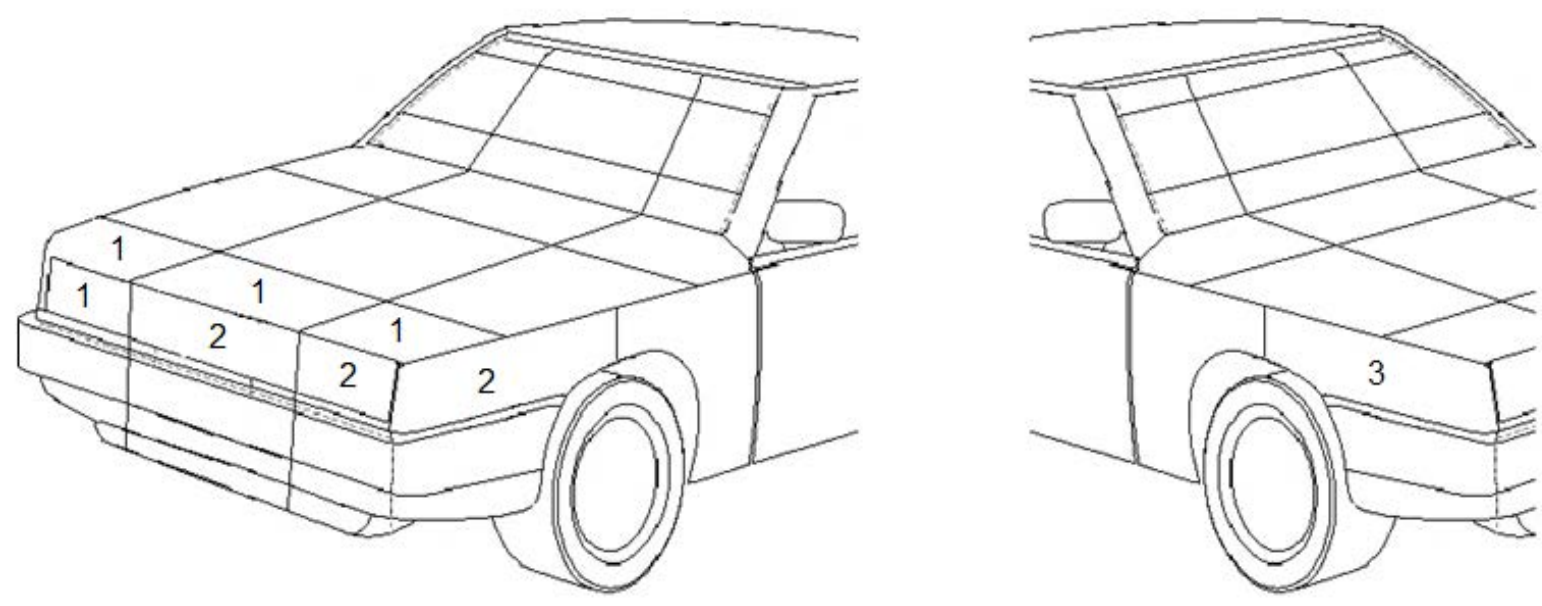

Abbildung 1-34: Anprallpunkte am gegnerischen Pkw (UKLMU, n=9)

Im Fallmaterial des UKM sind in neun Unfällen Angaben zum Anprallort vorhanden. Die Verteilung der Anprallpunkte des UKM auf Abbildung 1-35 unterscheidet sich leicht im Vergleich zum UKLMU. Es sind zwei Fälle dokumentiert, in denen der Fahrradfahrer an die Dachkante des Pkw geprallt ist. Zudem sind die Anprallpunkte linksseitig verteilt. Der obere Bereich der Motorhaube und Frontscheibe wurden nicht getroffen.

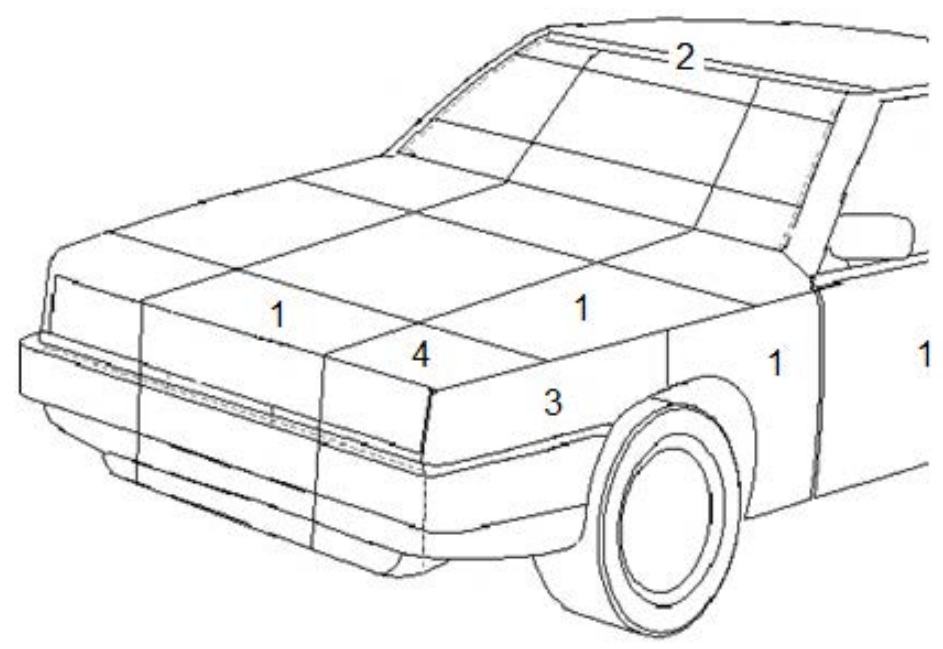

Abbildung 1-35: Anprallpunkte am gegnerischen Pkw (UKM, n=9)

Die Anprallstellen am Pkw wurden ebenfalls für die tödlichen Unfälle mit Pkw-Beteiligung $(n=61)$ aus der Rechtsmedizin ausgewertet (1.3.2.4.3).

Abbildung 1-10 zeigt die Verteilung der Anprallstellen des Körpers des Fahrradfahrers (nicht des Fahrrads). Die linke Abbildung zeigt dabei die absolute Anzahl von Anprallen, die rechte Abbildung stellt diese Verteilung farblich dar.

Dabei wird deutlich, dass der jeweilige zentrale Bereich der Motorhaube und der Frontscheibe zwar am häufigsten getroffen werden, den Randbereichen der Haube, der Frontscheibe und dem vorderen Dachrahmen aber auch erhebliche Bedeutung zukommt. 
Zudem ist eine Rechtstendenz zu erkennen. Der Stoßfänger wird in 56 der 61 tödlich verlaufenden Unfälle mit Pkw-Beteiligung getroffen.

Ergänzend zu den Anprallstellen am Pkw wurde die Frontform des Pkws betrachtet. Diese beruhen auf den Angaben zur Fahrzeugart, die die Patienten im Fragebogen machten.

Auf Abbildung 1-36 ist die entsprechende Verteilung aus dem Kollektiv des UKLMU und des UKM dargestellt. Im Fallmaterial des UKLMU sind bei 12 der 13 Unfälle mit Pkw-Beteiligung Angaben vorhanden, bei den Daten des UKM in 10 der 69 Pkw-Unfälle.

Am häufigsten mit knapp 60\% weist der am Unfall beteiligte Pkw die Form einer Limousine oder eines Kombis auf, welche in der Regel ähnlich geformte Fronten aufweisen, wie sie schematisch in Abbildung 1-34 und Abbildung 1-35 wiedergegeben ist. Auf eine langgezogene und relativ niedrige Motorhaube folgt eine schräg ansteigende Frontscheibe. Andere Fahrzeugformen spielten demgegenüber eine vergleichsweise geringe Rolle.

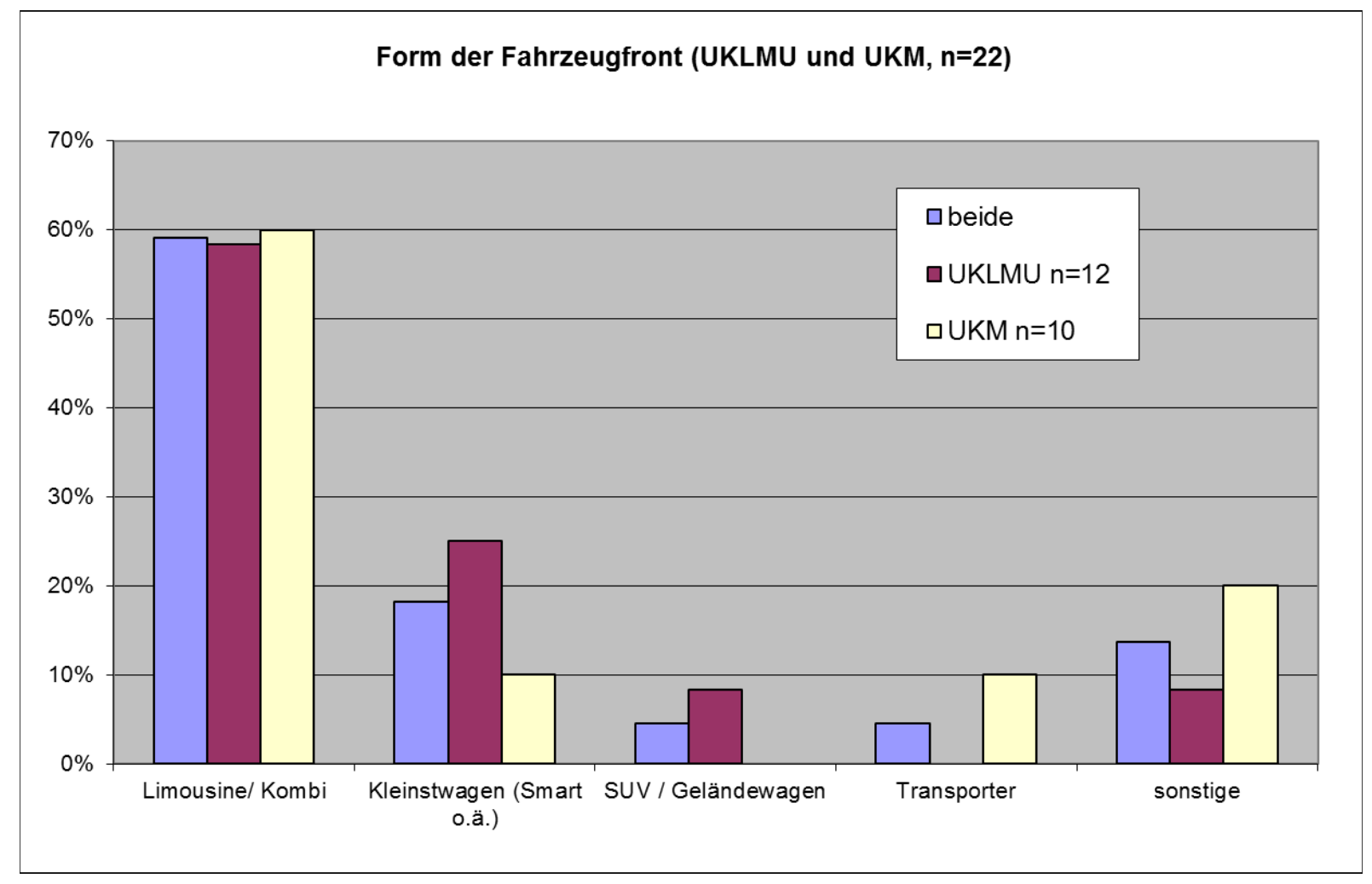

Abbildung 1-36: Form der Fahrzeugfront (UKLMU und UKM, n=22)

Die Auswertung des Fallmaterials der Rechtmedizin bezüglich der Fahrzeugform ist auf Abbildung 1-37 abgebildet. Hier dominiert ebenfalls mit knapp 60\% die typische Limousinenform, gefolgt vom Kleinstwagen mit knapp über 20\%. Mit einem SUV kollidierten $10 \%$ der bei einem Pkw-Unfall getöteten Fahrradfahrer. Diese Eingruppierung erfolgte durch Personal der LMU. 


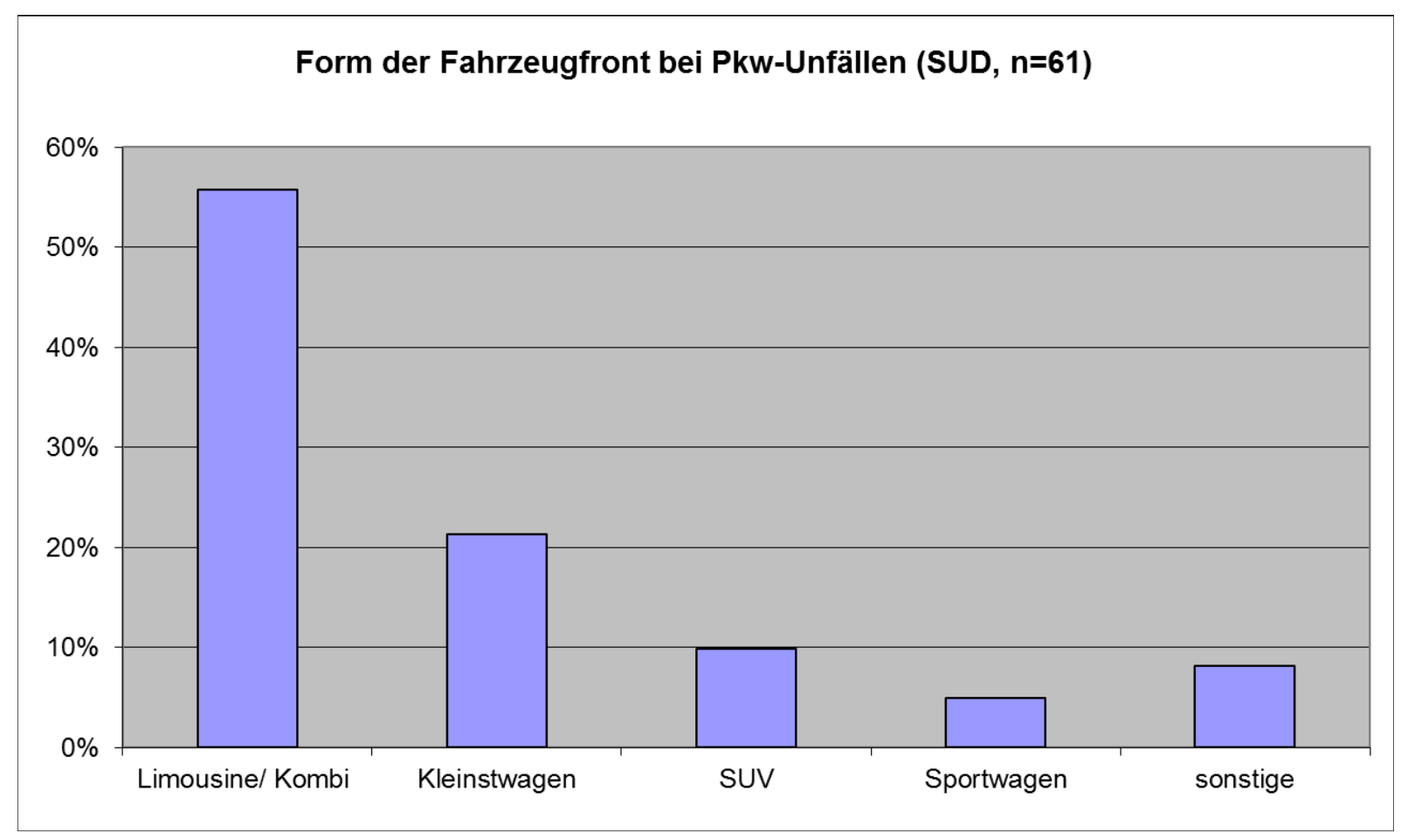

Abbildung 1-37: Form der Fahrzeugfront bei Pkw-Unfällen (SUD, n=61)

\subsubsection{Helmtragequote, Beschädigungen am Helm}

Gemäß der regelmäßig im Auftrag der Bundesanstalt für Straßenwesen innerorts erhobenen Radhelmquoten trugen 2012 über alle Altersgruppen hinweg 13 Prozent der Fahrradfahrer einen Schutzhelm (BASt 2013).

Auf Abbildung 1-38 ist die Häufigkeit der Fahrradhelmnutzung für die Unfälle aus dem UKLMU und dem UKM dargestellt. Diese wurde zum großen Teil von den in den Kliniken behandelten Radfahrern selbst berichtet, konnte aber oft auch anhand der bei Einlieferung mitgeführten Helme bestätigt werden. Demnach trugen 13\% der verunfallten Fahrradfahrer im Raum Münster (UKM) einen Fahrradhelm. In München (UKLMU) lag die Quote mit 21\% erheblich höher, die Quote für beide Fallkollektive kombiniert lag bei 17,2\%.

Im Material der SUD trugen nur sechs der 117 getöteten Fahrradfahrer (5\%) einen Helm.

Am häufigsten trugen Fahrer eines Sportrads, Rennrads, Mountain-/Trekkingbikes einen Helm. Wie auf Abbildung 1-39 abgebildet trugen in München (UKLMU) 30\% der 40 Benutzer eines solchen Fahrrads einen Helm. Von den 23 Benutzern eines Holland-/Cityrads nur knapp unter 5\%. Der einzige Fahrer eines Klapprads trug ebenfalls einen Helm. 


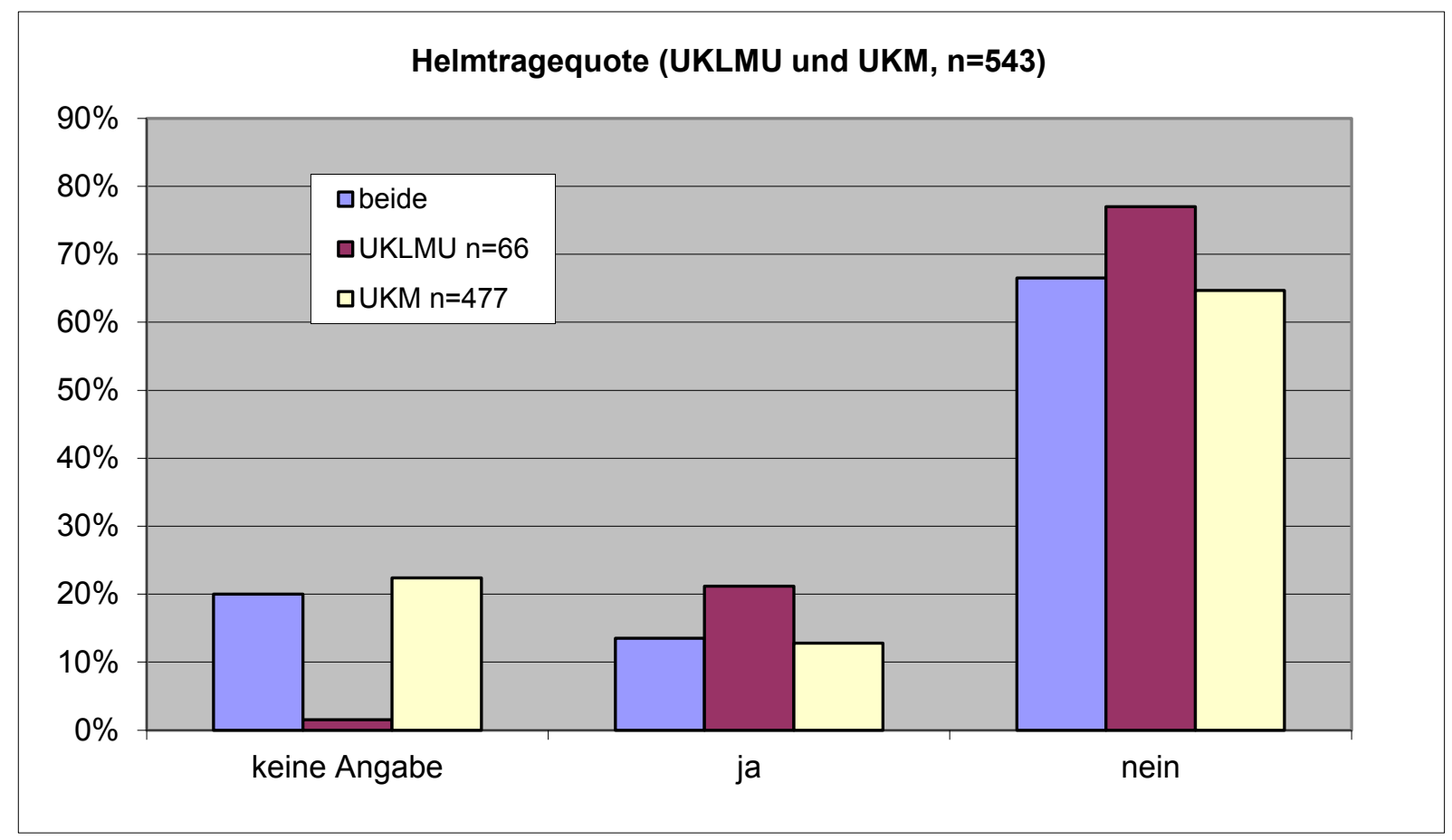

Abbildung 1-38: Helmtragequote (UKLMU und UKM, n=543)

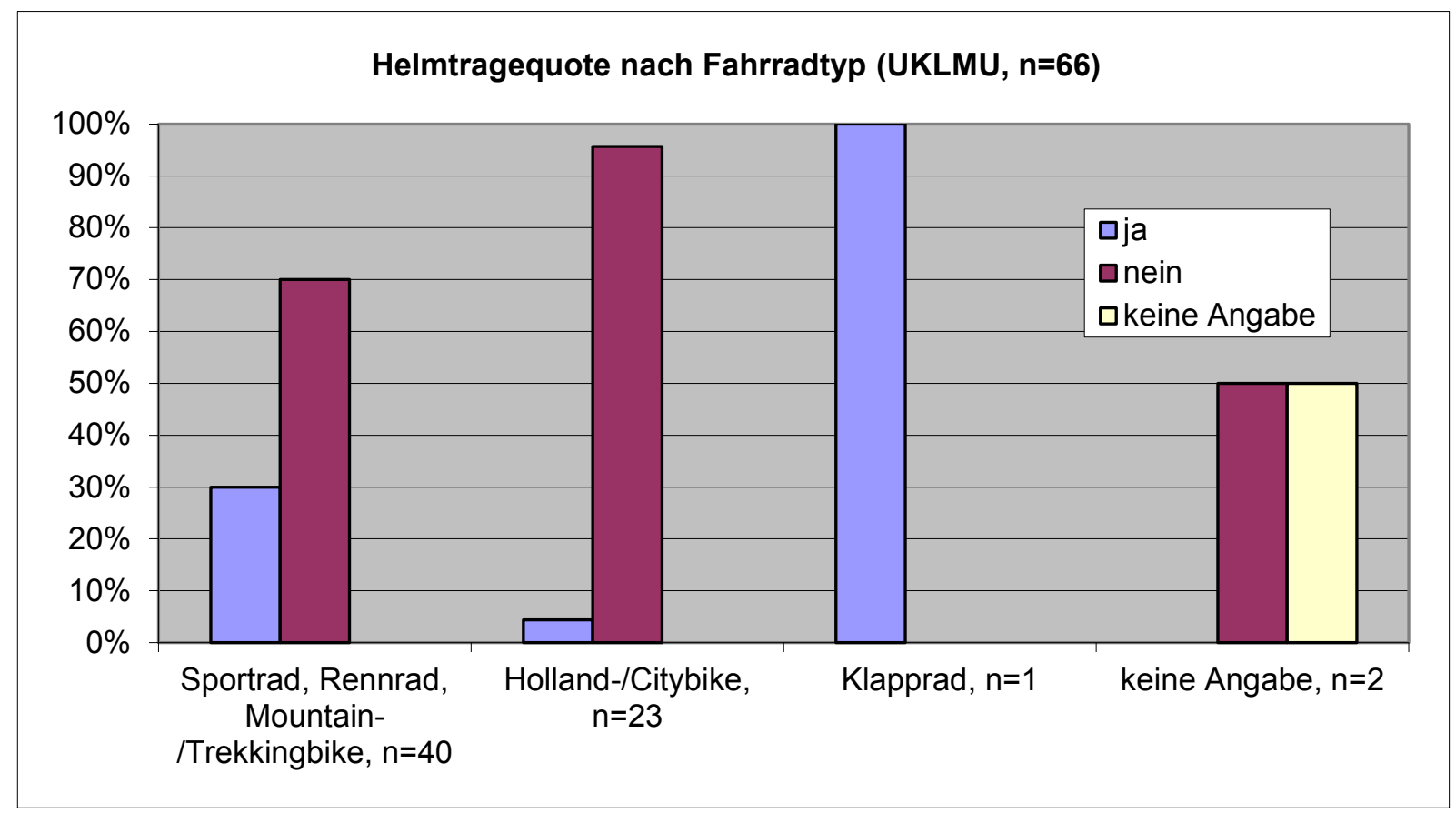

Abbildung 1-39: Helmtragequote nach Fahrradtyp (UKLMU, n=66)

In Münster (UKM) trugen über 20\% der 140 Sportrad-, Rennrad-, Mountain-/TrekkingbikeFahrer einen Helm (Abbildung 1-40). Von den 199 Fahrern eines Holland-/Citybikes trugen nur $5 \%$ einen Helm.

Von den fünf Nutzern eines Kinderrads wurde in drei Fällen eine Helmnutzung berichtet. Die Fahrer eines E-Bikes oder Pedelecs trugen in über 30\% der Fälle einen Helm. 


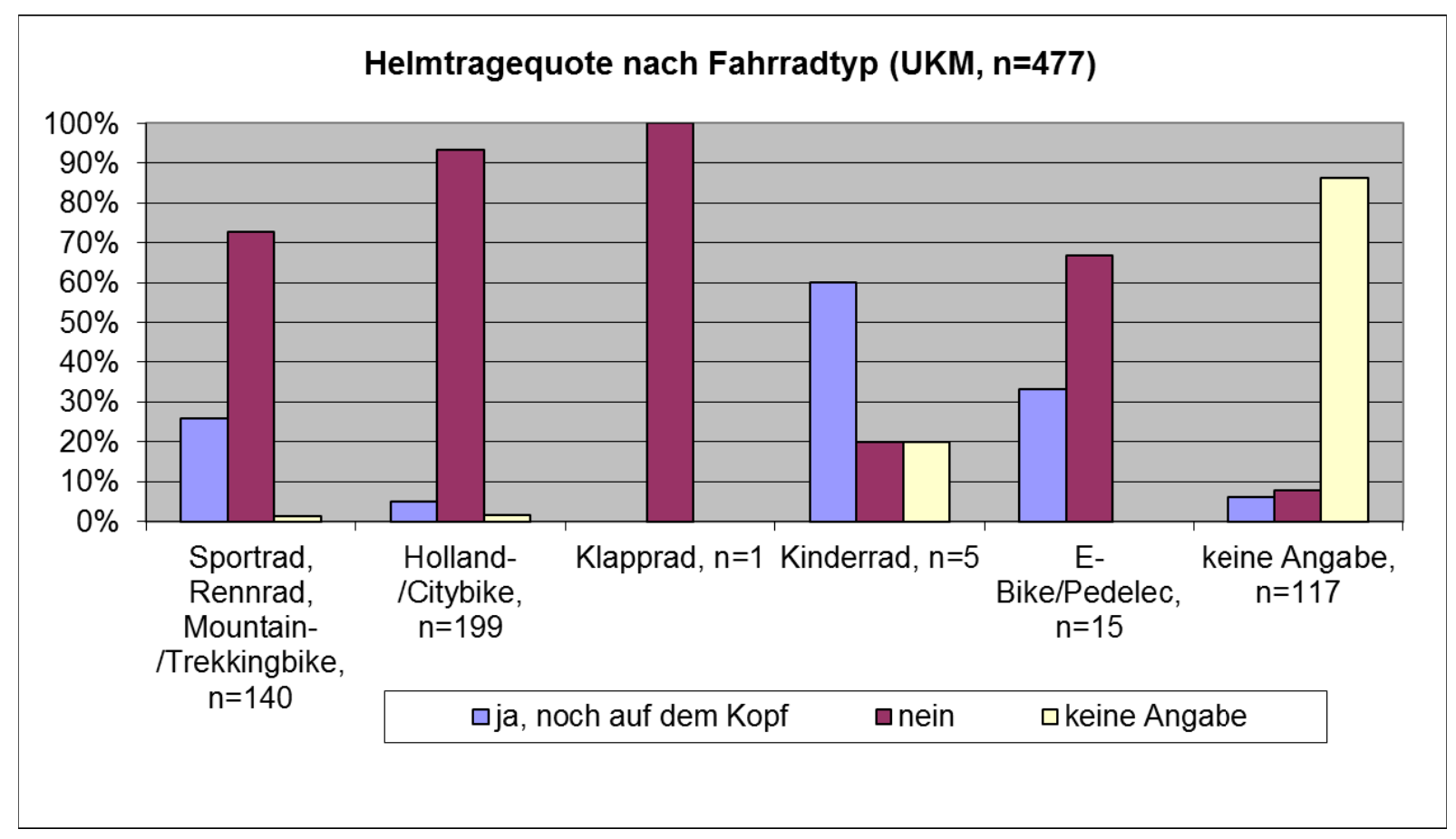

Abbildung 1-40: Helmtragequote nach Fahrradtyp (UKM, n=477)

Um Anhaltspunkte für die Lokalisation eines Anpralls des behelmten Kopfes gegen die Straße oder ein Objekt zu gewinnen, wurden die Beschädigungen am Helm zusammen mit dem Klinikpersonal nach Art und Lage der Beschädigung dokumentiert, wo dies möglich war. Für diese spezielle Auswertung werden ebenfalls vier Fahrradfahrer aus dem ursprünglichen Datenkollektiv mit 571 Fällen miteinbezogen, die unverletzt blieben, aber einen dokumentierten Helmkontakt aufwiesen.

Im Fallmaterial des UKLMU hatten 17 der 18 getragenen Fahrradhelme Kontakt zur Straße oder einem Objekt. Ein Helm hatte keinen Kontakt und auch keine Beschädigungen. Die Lokalisation der Beschädigungen dieser 17 Helme ist auf Abbildung 1-41 zu sehen. Pro Helm sind dabei mehrere Nennungen möglich, bei den 17 Helmen wurden insgesamt 35 Beschädigungen dokumentiert.

Am häufigsten ist der Anprall an die vordere untere Kante des Helms im Stirnbereich. Sieben der 17 Helme (41\%) hatten dort eine Beschädigung.

Generell ist die Tendenz zu erkennen, dass die Helme eher selten im oberen Bereich beschädigt wurden, sondern vielmehr an den Seiten im Bereich der unteren Helmkante.

In den Daten des UKLMU sind 50\% der Beschädigungen Kratzer, 28\% Dellen und 22\% Risse und Brüche. 

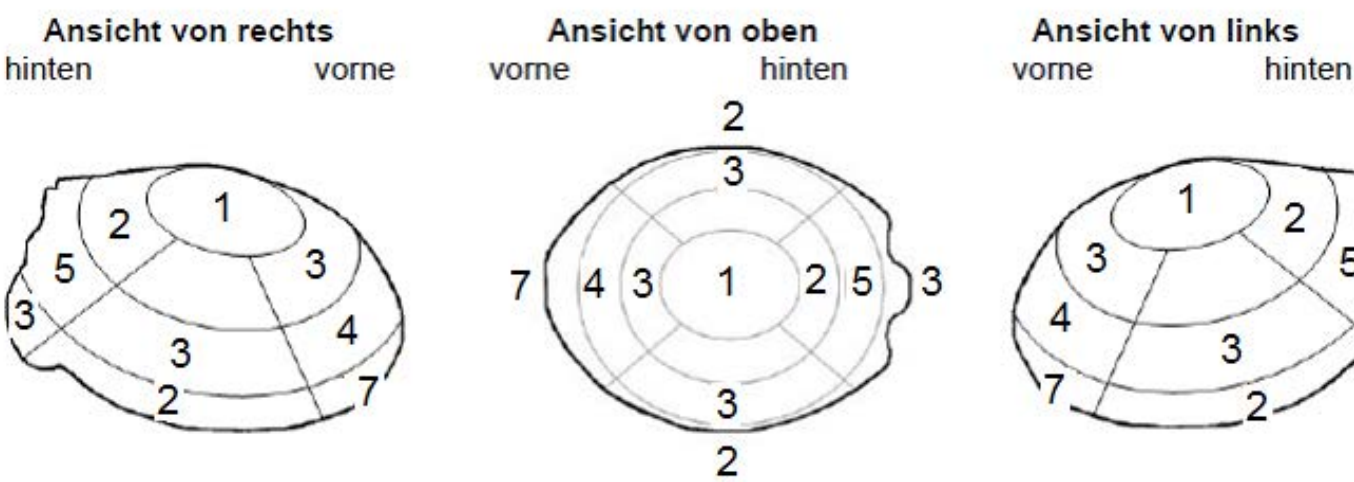

vorne hinten

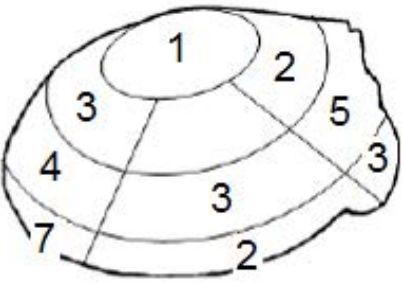

Abbildung 1-41: Lokalisation von Beschädigungen am Helm (UKLMU, n=17)

63 Fahrradfahrer, die im UKM behandelt wurden, trugen einen Helm. Bei 14 dieser Helme wurde angegeben, dass dieser Kontakt zur Straße oder einem Objekt hatte, bei den übrigen fehlen Angaben dazu.

Ein Helm mit Kontakt zu einem Objekt hatte keine Beschädigungen, einer war maximal zerstört. Von den übrigen 12 Helmen sind bei neun Helmen Angaben über insgesamt 23 Beschädigungen vorhanden. Der Ort der Beschädigungen dieser neun Helme ist auf Abbildung 1-42 dargestellt.

Sieben der neun dokumentierten Helme (78\%) hatten eine Beschädigung im Bereich der Unterkante der linken Seite. Hier ist ebenfalls die Tendenz zu erkennen, dass Schäden im oberen Bereich verhältnismäßig selten auftreten.

Im Fallmaterial des UKM sind 22\% der Beschädigungen Kratzer, 13\% Dellen und 65\% Risse und Brüche.
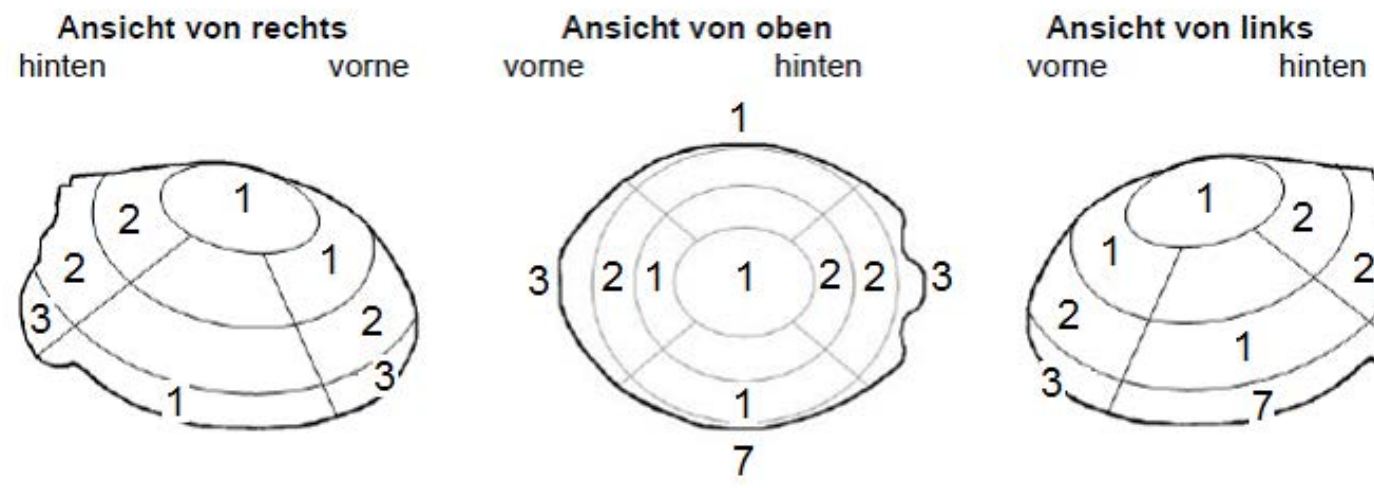

Abbildung 1-42: Lokalisation von Beschädigungen am Helm (UKM, n=9) 


\subsubsection{Verletzungen}

\subsection{MAIS, Körperregion des MAIS, ISS}

Bei den verunfallten Fahrradfahrern aus dem UKLMU und dem UKM sowie den getöteten Fahrradfahrern der SUD wurden die Verletzungen nach AIS 2005, Update 2008 (Abbreviated Injury Scale), (AAAM 2008) kodiert. Bei den 117 getöteten Fahrradfahrern der SUD wurde zusätzlich die Todesursache laut Obduktionsbericht bestimmt.

Auf Abbildung 1-43 ist die Verteilung des größten aufgetretenen AIS, des sogenannten MAIS (Maximum AIS) des UKLMU und des UKM dargestellt. Die Mehrheit der Fahrradfahrer (ca. $60 \%$ ) wies einen MAIS 1 auf. Schwer verletzt (MAIS 3+) im Sinne der AIS-Skala waren knapp unter 10\% aller Fahrradfahrer. Verletzungen mit einem AIS-Wert von 5 oder 6 kamen im UKLMU und UKM nicht vor.

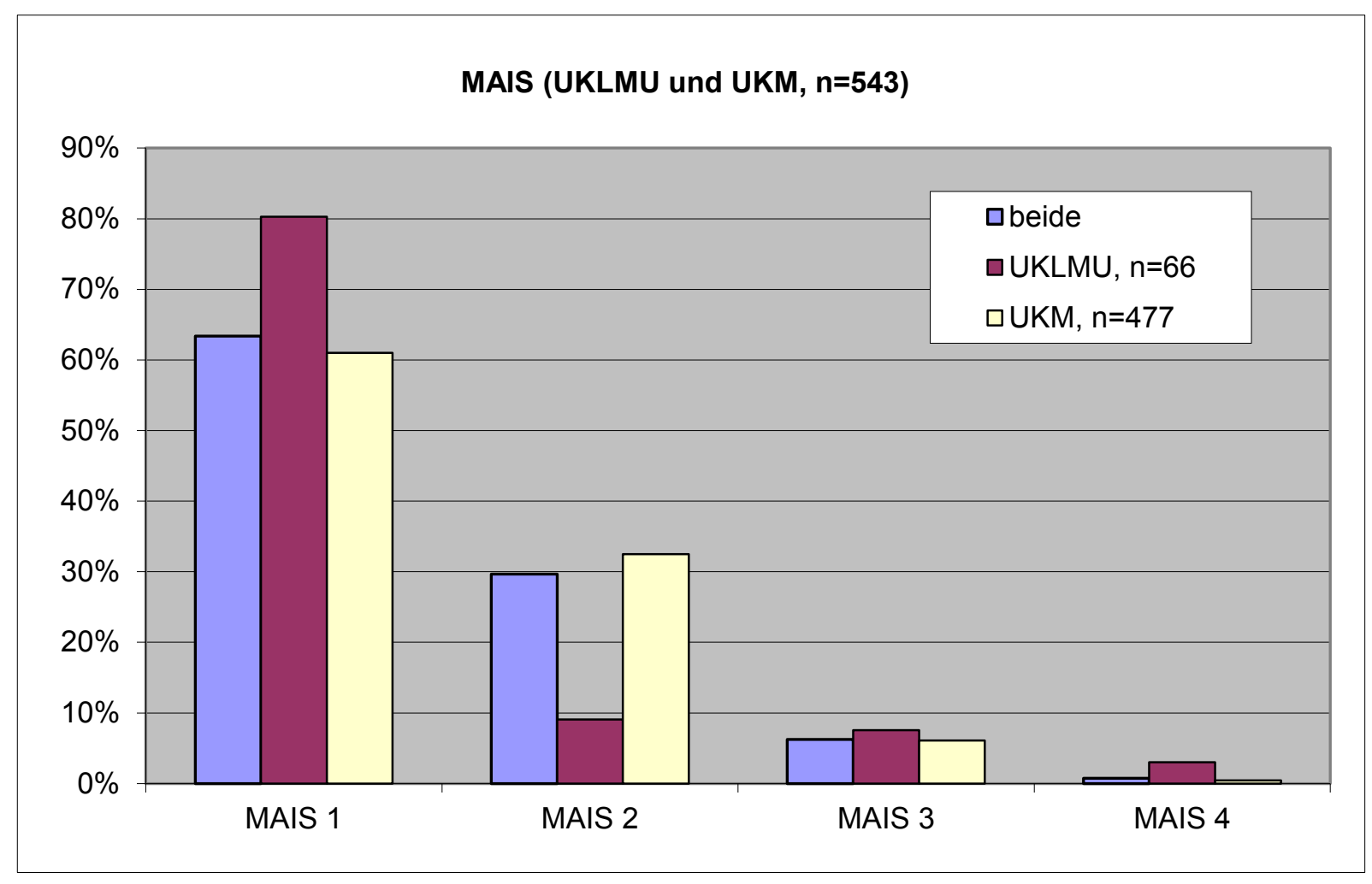

Abbildung 1-43: MAIS der verletzten Fahrradfahrer (UKLMU und UKM, $n=543$ )

Auf Abbildung 1-44 (UKLMU) und Abbildung 1-45 (UKM) sind die Körperregionen der schwersten Verletzungen dargestellt. Da bei einem verunglückten Radfahrer mehrere Verletzungen gleichzeitig den höchsten AIS-Wert aufweisen können, kann der MAIS auch in mehreren Körperregionen gleichzeitig vorliegen. Liegt z.B. für einen Patienten sowohl in der Kopf- als auch der Thoraxregion jeweils AIS 2 als höchste Verletzungsschwere vor (ohne dass in den übrigen Körperregionen ein AIS 2 erreicht wird), so wird die Kombination der Körperregionen Kopf/Thorax als am schwersten verletzte Körperregion angegeben. Hingegen sind diese Körperregionen in den AIS-Kodierungsrichtlinien gegeneinander abgegrenzt.

In den Daten des UKLMU sind die Körperregionen „Kopf" und „Gesicht“ mit jeweils 27\% isoliert die schwerstverletzte Körperregion. In 28 Fällen (42\%) ist der Kopf als schwerstverletzte Körperregion beteiligt. Das Gesicht zählt nach den Kodierrichtlinien des 
AIS als eigene Körperregion und schließt neben dem Gesichtsskelett auch die Weichteile der Stirn ein.

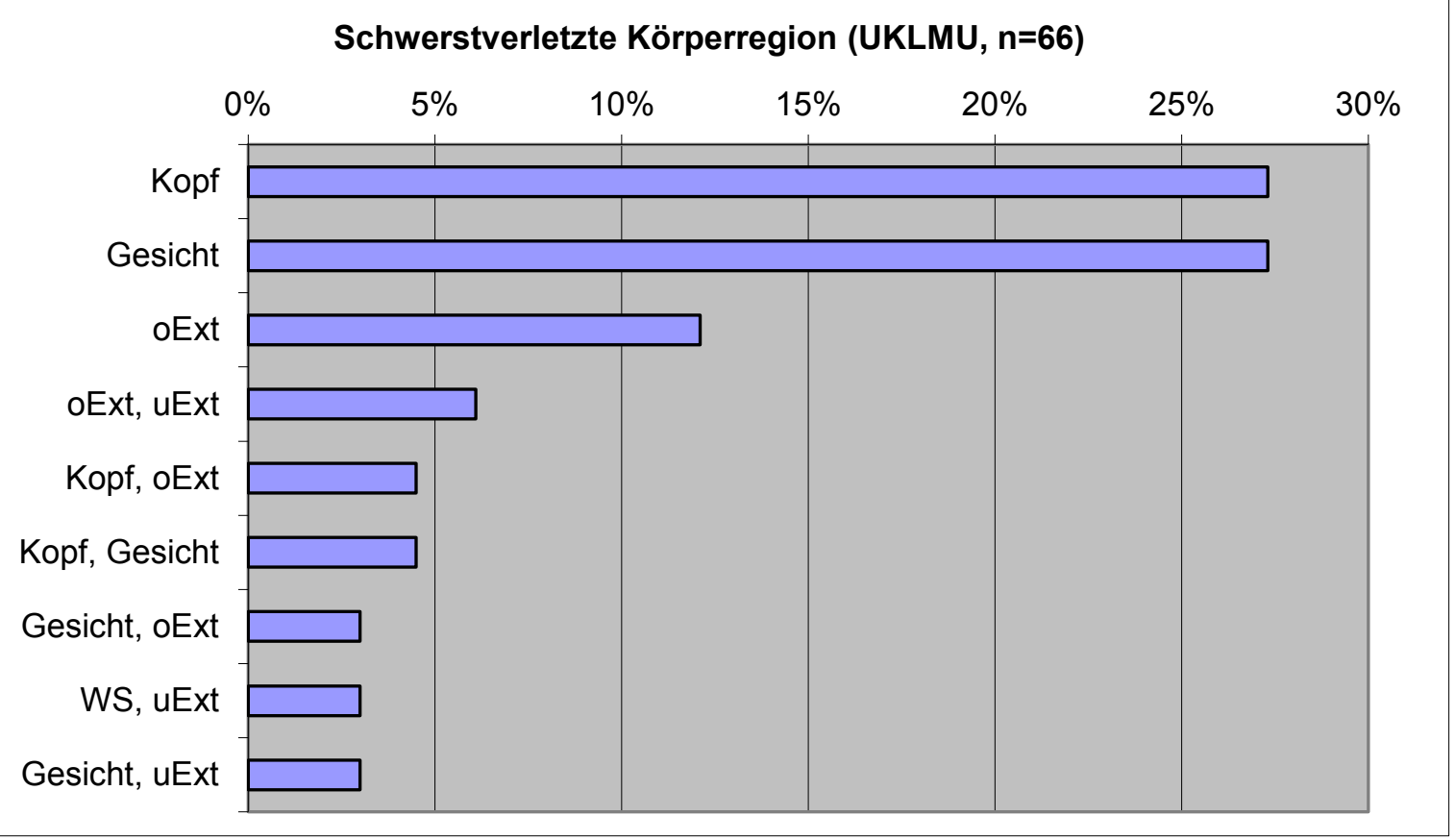

Abbildung 1-44: Körperregion des MAIS der verletzten Fahrradfahrer (UKLMU, n=66)

Im Fallmaterial des UKM sind die oberen Extremitäten mit 134 von 477 Fällen (28\%) und die unteren Extremitäten mit 102 Fällen (201) als alleinige Körperregion, in der der MAIS lokalisiert ist, führend. An dritter Stelle folgt das Gesicht mit 38 Fällen (8\%).

Der Kopf ist in 89 Fällen (19\%) bei der schwerstverletzten Körperregion beteiligt. Körperregionen oder Kombinationen von diesen, die bei weniger als vier Patienten den Ort der schwersten Verletzung darstellen, sind auf Abbildung 1-45 nicht mehr dargestellt, bei der Auswertung aber prinzipiell mit berücksichtigt. 


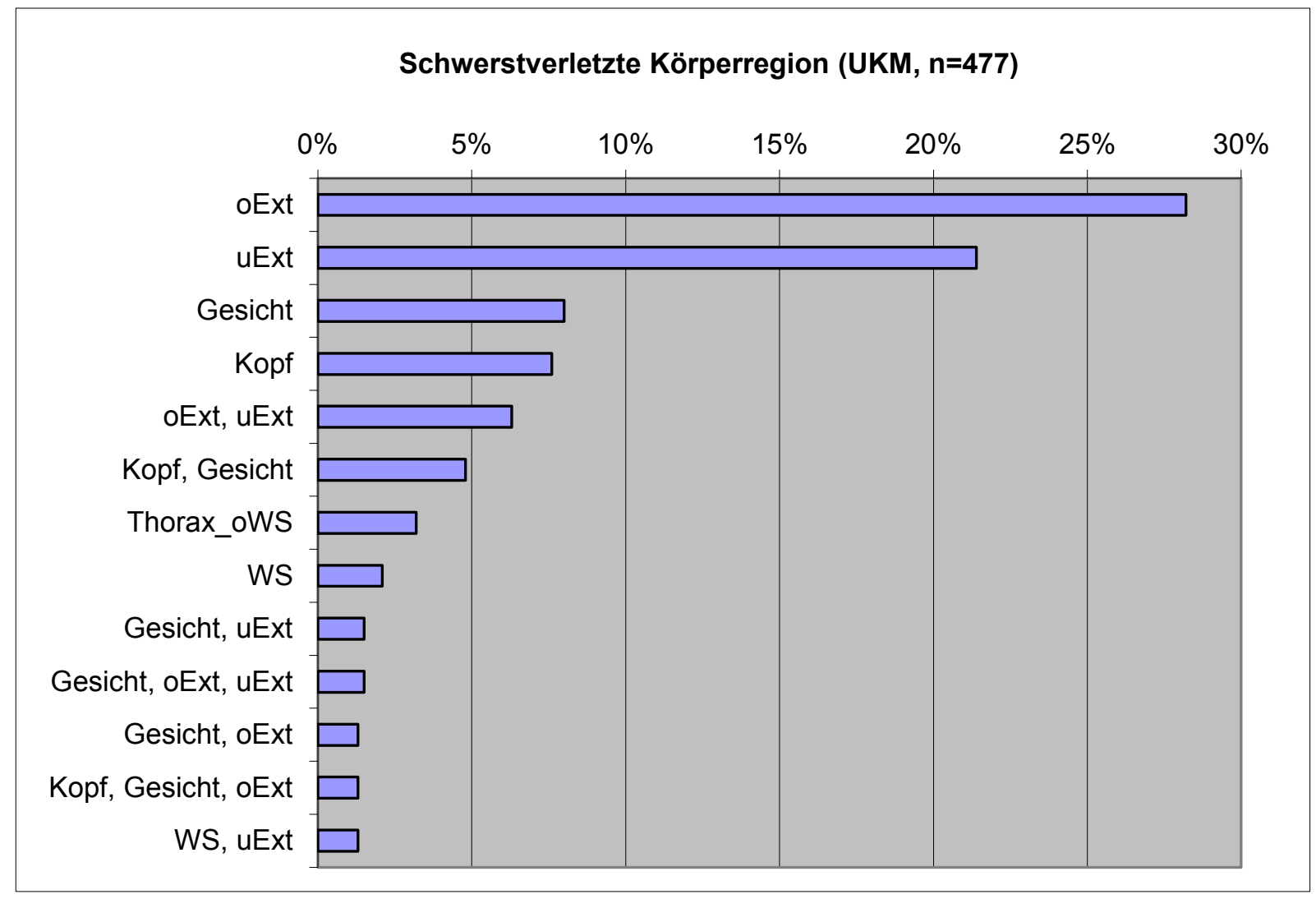

Abbildung 1-45: Körperregion des MAIS der verletzten Fahrradfahrer (UKM, n=477)

Aus den AIS-Kodierungen wurde die Gesamtverletzungsschwere, ausgedrückt durch den ISS (Injury Severity Score), berechnet. Auf Abbildung 1-46 ist die Häufigkeitsverteilung des ISS der verunfallten Fahrradfahrer des UKLMU und des UKM zu erkennen.

Mit über $60 \%$ hat die Mehrheit der verunfallten Fahrradfahrer einen ISS von 1 bis 3 . Einen ISS $\geq 16$, was als lebensgefährliche Verletzungsschwere gilt, hatten nur $2 \%$ aller Fahrradfahrer. Der höchste erreichte ISS-Wert lag bei 22. 


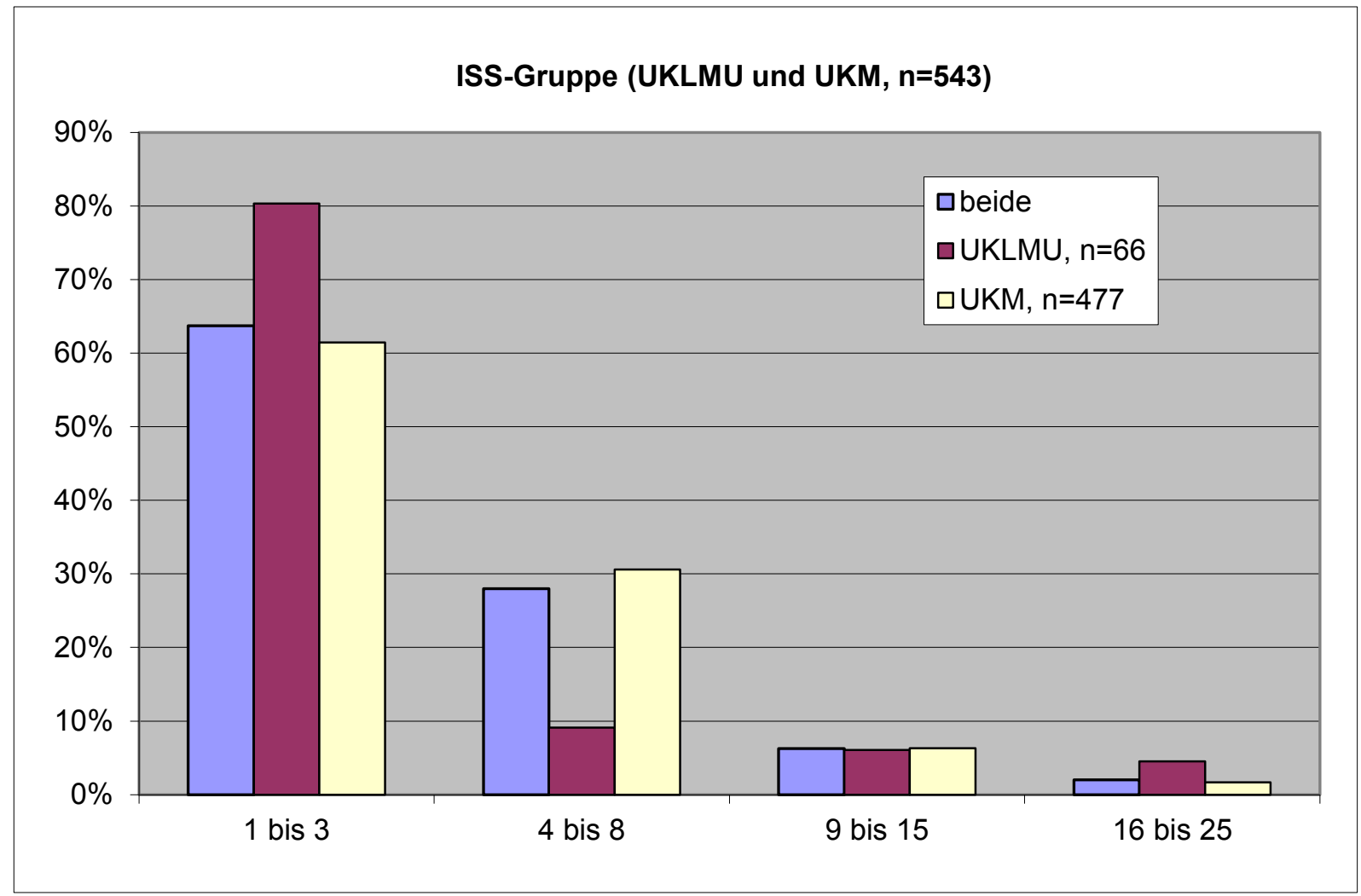

Abbildung 1-46: ISS der verletzten Fahrradfahrer (UKLMU und UKM, n=543)

Betrachtet man die MAIS-Werte der getöteten Fahrradfahrer auf Abbildung 1-47, so wird deutlich, dass im Fallkollektiv der SUD erwartungsgemäß schwerste Verletzungen dominieren. Knapp 90\% der Unfallopfer hatten Verletzungen MAIS 4+. Unfallopfer mit MAIS 1 lagen nicht vor. Es gab zwei Todesfälle mit MAIS 2; hierbei handelte es sich um Todesfälle durch Aspiration und Multiorganversagen.

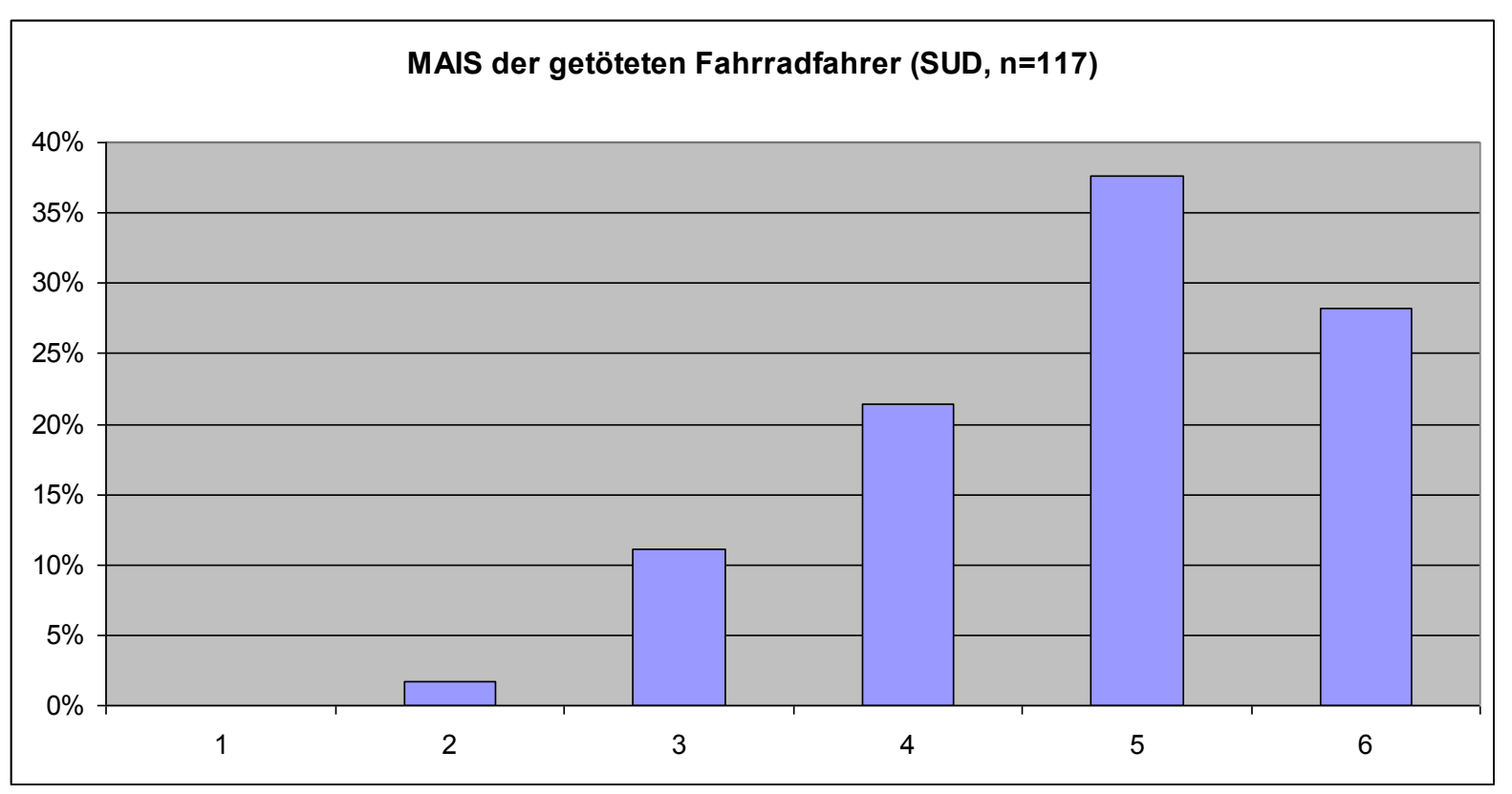

Abbildung 1-47: MAIS der getöteten Fahrradfahrer (SUD, n=117) 
Auf Abbildung 1-48 ist die Körperregion des MAIS der Fälle aus der SUD dargestellt.

Dominierend mit über $50 \%$ ist der Kopf, d.h. in über der Hälfte aller tödlichen Fahrradunfälle hatte der getötete Fahrradfahrer die schwerste Verletzung isoliert im Bereich des Kopfs. An zweiter Stelle ist die schwerste Verletzung isoliert im Bereich des Thorax, gefolgt von der Kombination Kopf und Thorax. Insgesamt ist der Kopf bei $69 \%$ der getöteten Fahrradfahrer als schwerstverletzte Körperregion beteiligt, der Thorax bei $39 \%$.

Alle anderen Verletzungsmuster sind nur mit sehr geringen Fallzahlen vertreten.

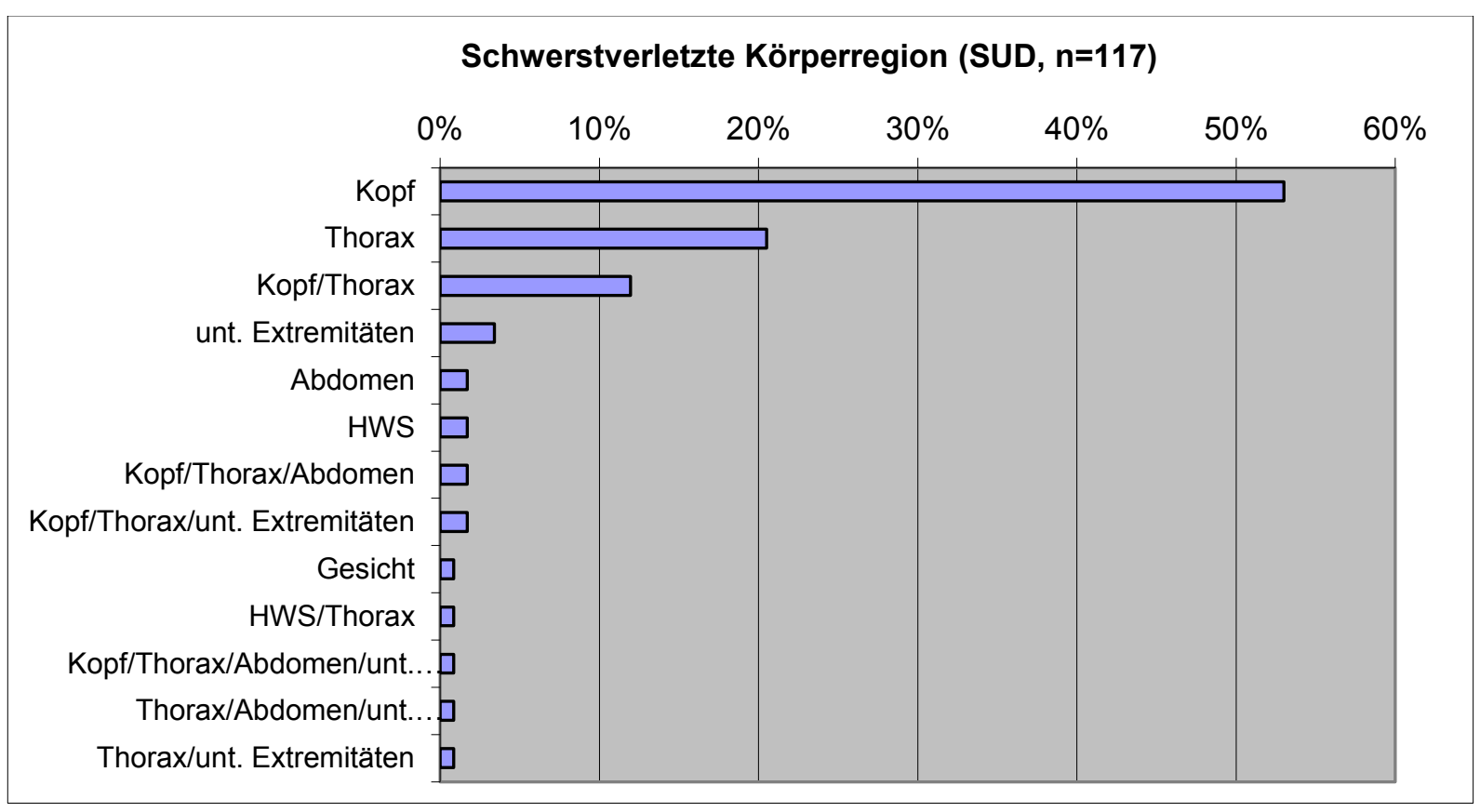

Abbildung 1-48: Körperregionen des MAIS der getöteten Fahrradfahrer (SUD, n=117)

Auf Abbildung 1-49 ist der ISS der getöteten Fahrradfahrer dargestellt. In 28\% der Fälle wurde ein ISS von 75 (maximale Verletzungsschwere) erreicht. Nur $9 \%$ hatten einen ISS von maximal 15.

Ergänzend zu der streng formalen Skalierung der Verletzungschwere nach MAIS und ISS sind die Todesursachen laut Obduktionsbericht auf Abbildung 1-50 dargestellt.

Deutlich führend ist mit über $50 \%$ das Schädel-Hirn-Trauma (SHT). Das SHT ist eine Sammelbezeichnung für gedeckte oder offene Schädelverletzungen (mit oder ohne Schädelfraktur) mit Gehirnbeteiligung (Pschyrembel 2012).

Mit jeweils knapp über $10 \%$ folgen das Thoraxtrauma und das Polytrauma. Das Polytrauma bezeichnet nach der Definition von Tscherne gleichzeitig vorliegende Verletzungen in mehreren Körperregionen, von denen eine oder die Kombination der Verletzungen lebensbedrohlich ist. 


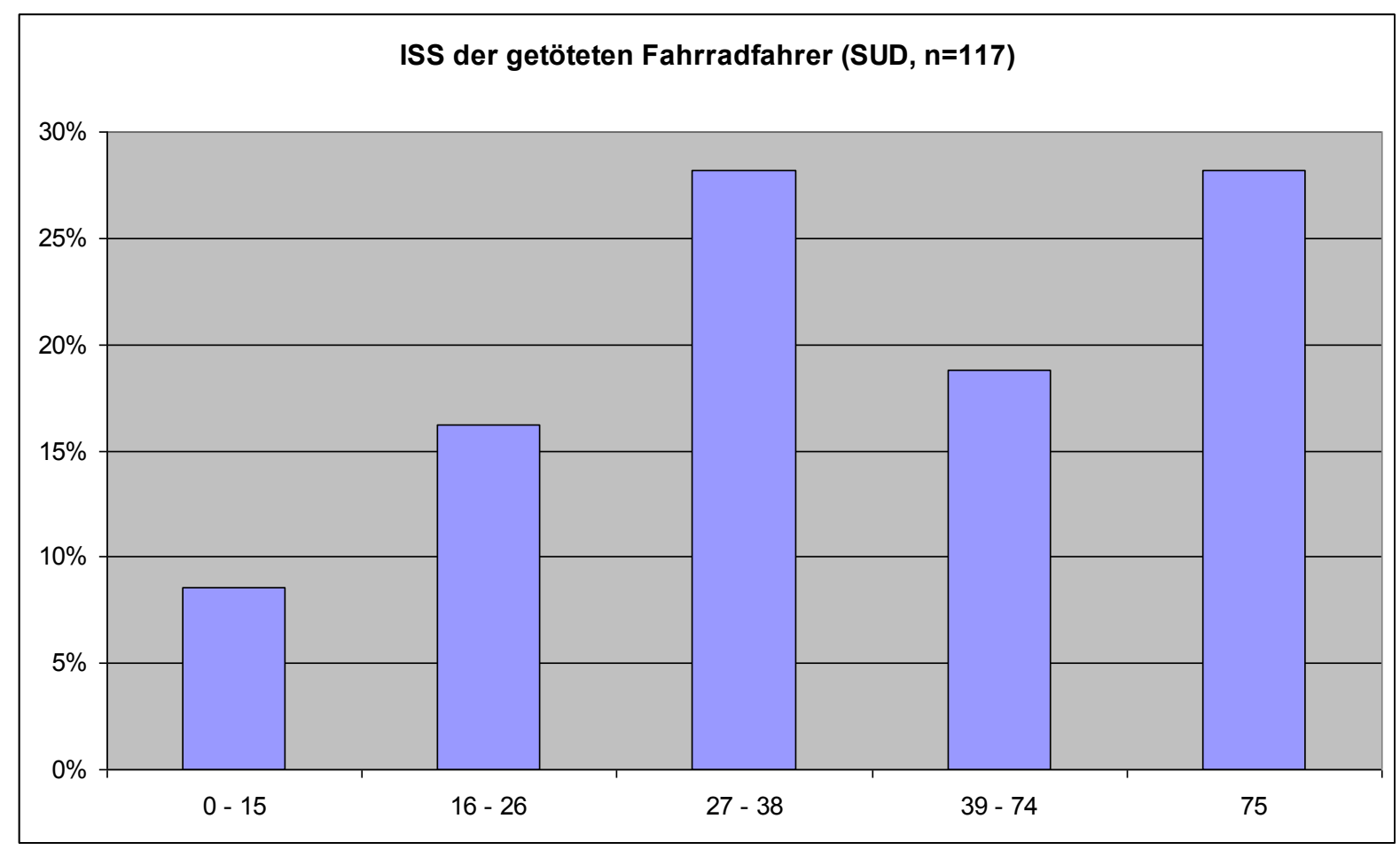

Abbildung 1-49: ISS der getöteten Fahrradfahrer (SUD, n=117)

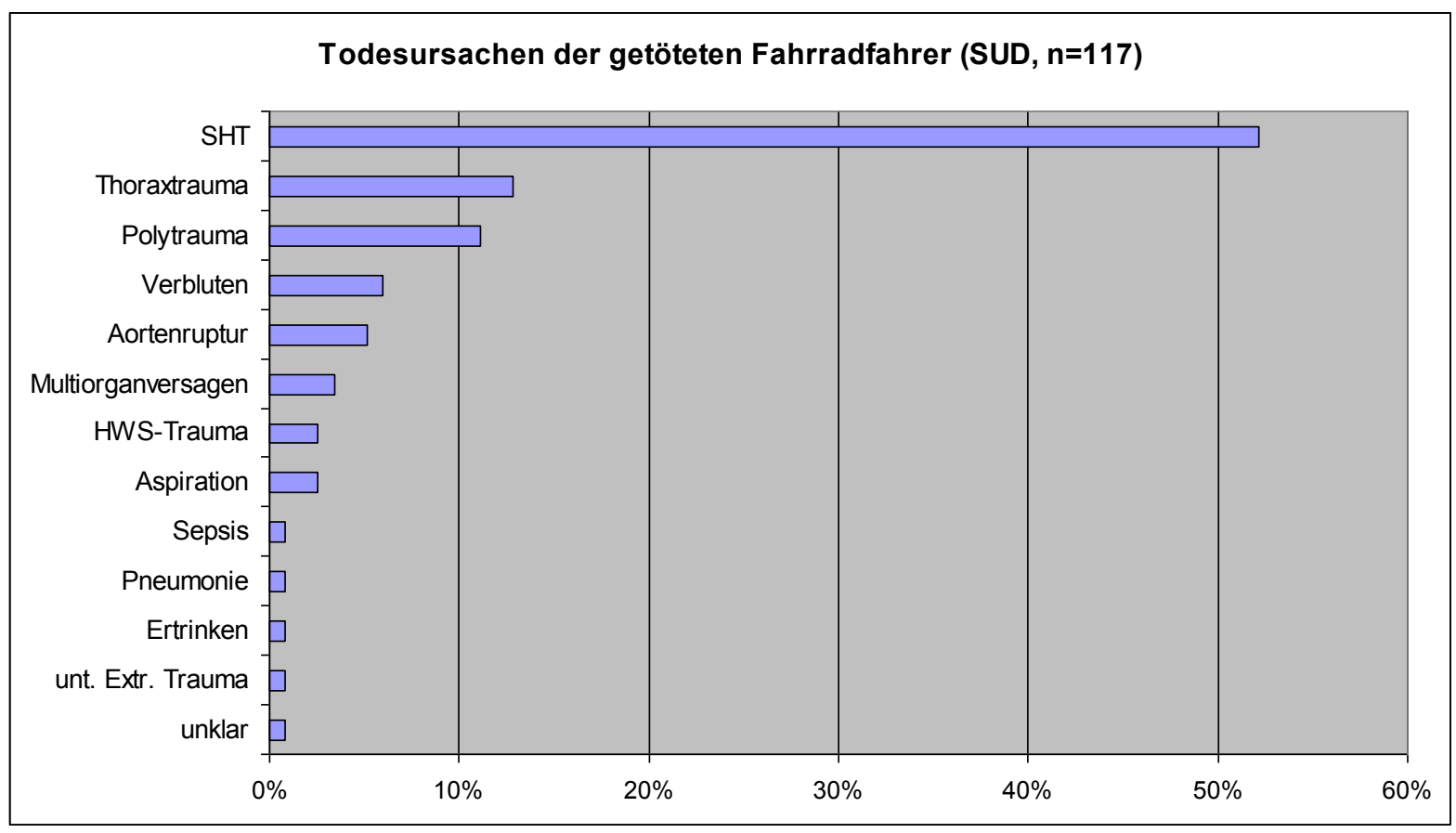

Abbildung 1-50: Todesursachen laut Obduktionsbericht (SUD, n=117)

Da die Kopfverletzungen in allen drei Fallkollektiven eine wichtige oder sogar dominante Rolle einnehmen, sind auf Abbildung 1-51 und Abbildung 1-52 die größten AIS-Werte der Region "Kopf" dargestellt.

Die prospektiv erfassten Fälle mit verletzten Fahrradfahrern aus dem UKLMU und dem UKM sind auf Abbildung 1-51 abgebildet. Über 70\% aller Fahrradfahrer sind am Kopf unverletzt. Das UKLMU unterscheidet sich hier deutlich vom UKM, was unter anderem darin begründet ist, dass im UKM weitgehend alle verletzten Radfahrer erfasst wurden, im UKLMU in erster 
Linie diejenigen mit Kopfverletzungen. In München (UKLMU) sind ca. 55\% der Fahrradfahrer nicht am Kopf verletzt, in Münster (UKM) knapp unter 80\%. Im Fallmaterial des UKLMU sind auch mehr Fahrradfahrer mit schweren Kopfverletzungen (AIS 3+) vorhanden. Deren Häufigkeit liegt jedoch unter 10\% (AIS 3), bzw. unter 5\% (AIS 4). Die schwerste Kopfverletzung in den Daten des UKLMU beträgt AIS 4, in den Daten des UKM AIS 3.

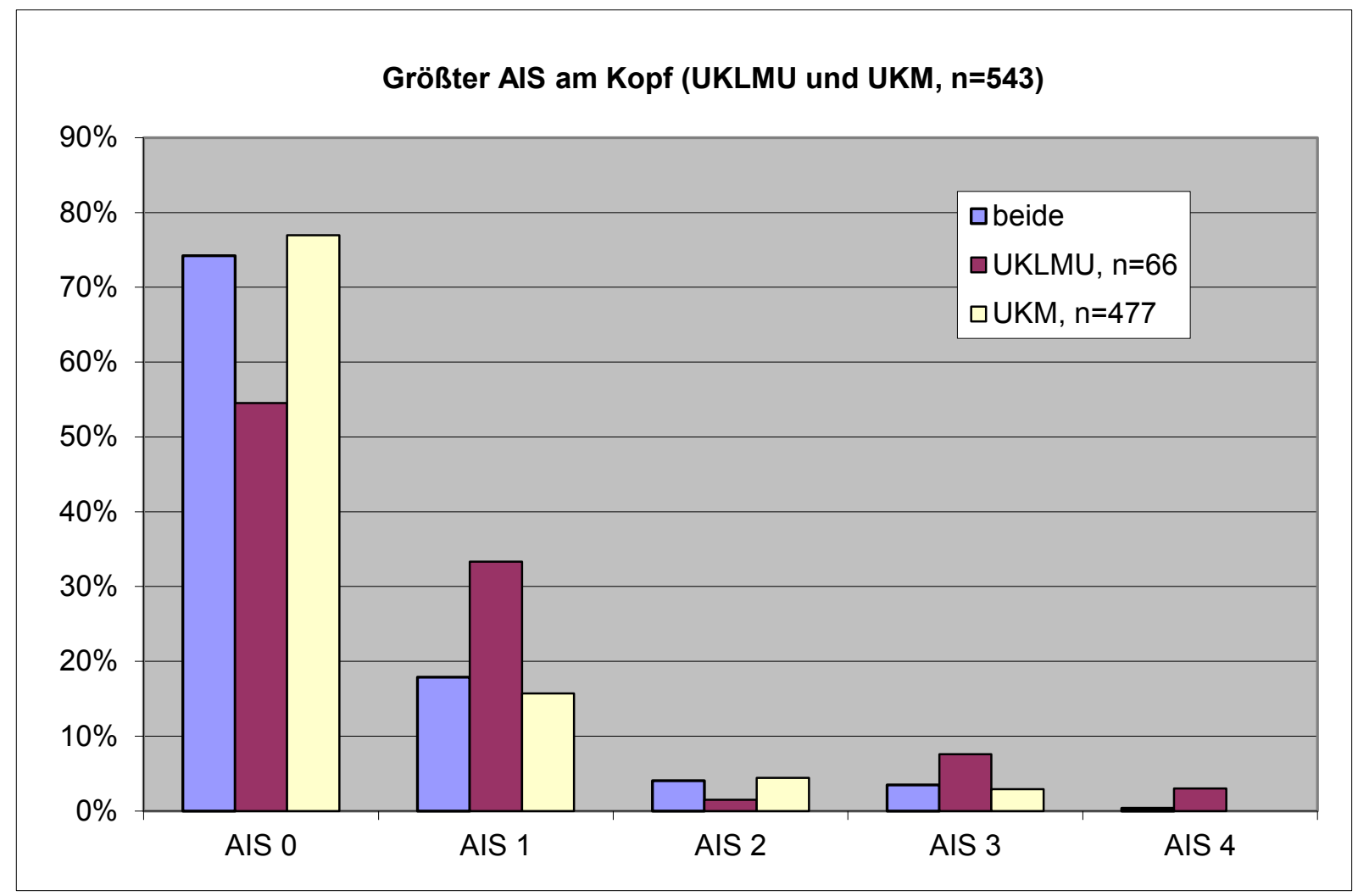

Abbildung 1-51: Größter AIS am Kopf der verletzten Fahrradfahrer (UKLMU und UKM, n=543)

Abbildung 1-52 zeigt den größten AIS-Wert im Bereich des Kopfs der 117 Fälle der SUD auf. Lediglich 4\% der getöteten Fahrradfahrer hatten keine Verletzung im Bereich des Kopfs. Weitere $14 \%$ hatten eine Verletzung AIS 1 oder 2.

Über $80 \%$ der getöteten Fahrradfahrer hatten schwerste Verletzungen AIS 3+ im Bereich des Kopfs. 


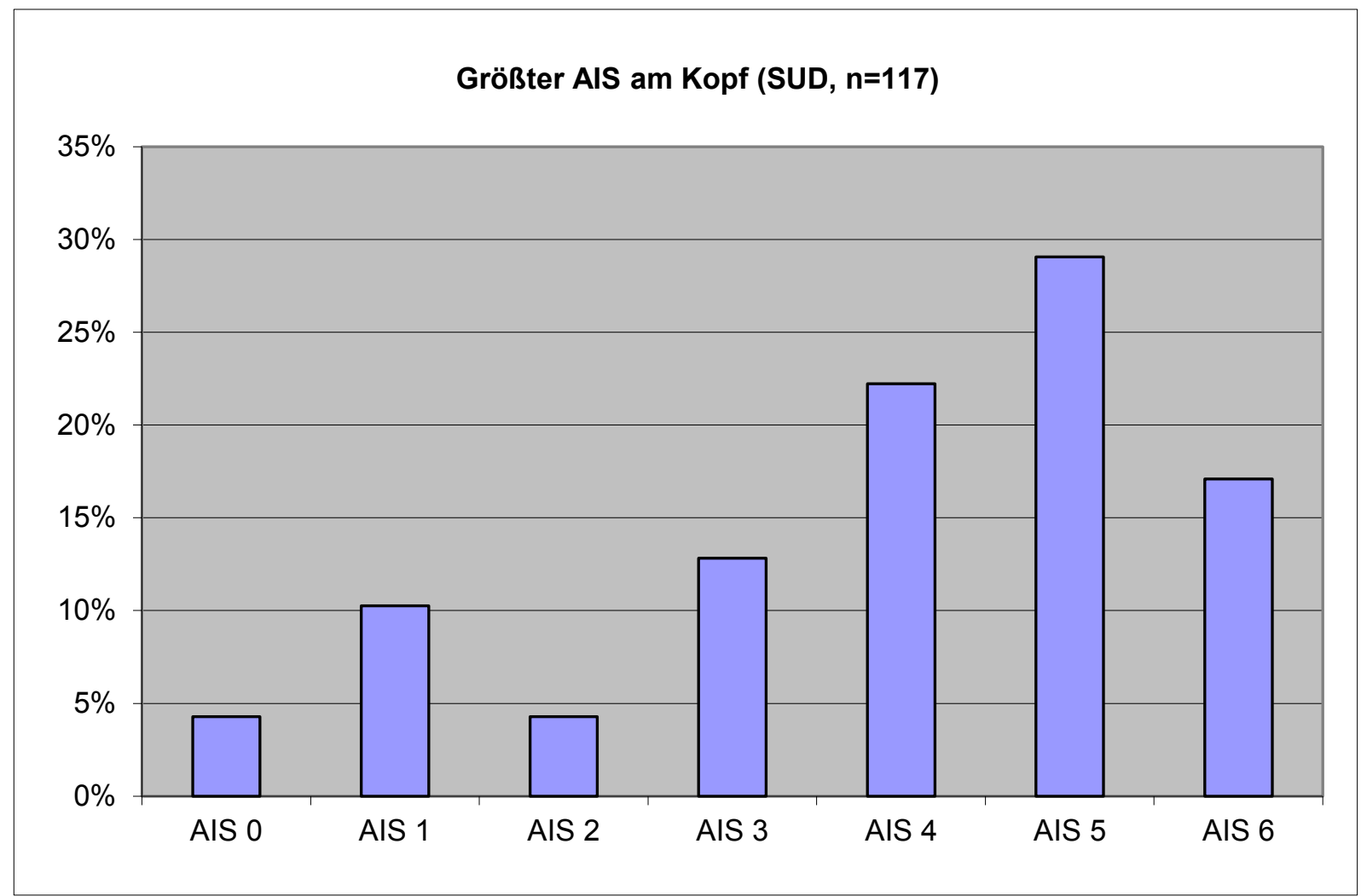

Abbildung 1-52: Größter AIS am Kopf der getöteten Fahrradfahrer (SUD, n=117)

Zusätzlich zum AIS-Wert im Kopfbereich wurde die Verletzungsschwere des Kopfs in Abhängigkeit von einem getragenen Helm ausgearbeitet.

Auf Abbildung 1-53 sind die Ergebnisse für die Daten des UKLMU und UKM kombiniert dargestellt. Angesichts der geringen Zahlen für Helmträger werden die beiden Fallkollektive nicht separat betrachtet.

Fahrradfahrer, die einen Helm trugen, erlitten maximal eine Kopfverletzung AIS 1 oder AIS 2. Kopfverletzungen AIS 3+ traten bei Helmträgern nicht auf.

Über $70 \%$ aller Fahrradfahrer mit Helm hatten keine Kopfverletzungen.

Von den Fahrradfahrern ohne Helm blieben ebenfalls über $70 \%$ ohne Kopfverletzungen.

Die Anzahl der leichten Kopfverletzungen AIS 1 beträgt bei den Helmträgern ca. 25\%, bei den Radfahrern ohne Helm knapp unter $20 \%$.

Schwere Kopfverletzungen AIS 3 und AIS 4 sind nur bei den Fahrradfahrern ohne Helm zu beobachten.

Für das Fallmaterial der SUD wurden aufgrund der sehr geringen Zahl an Helmträgern $(n=6)$ diese Betrachtungen nicht durchgeführt. 


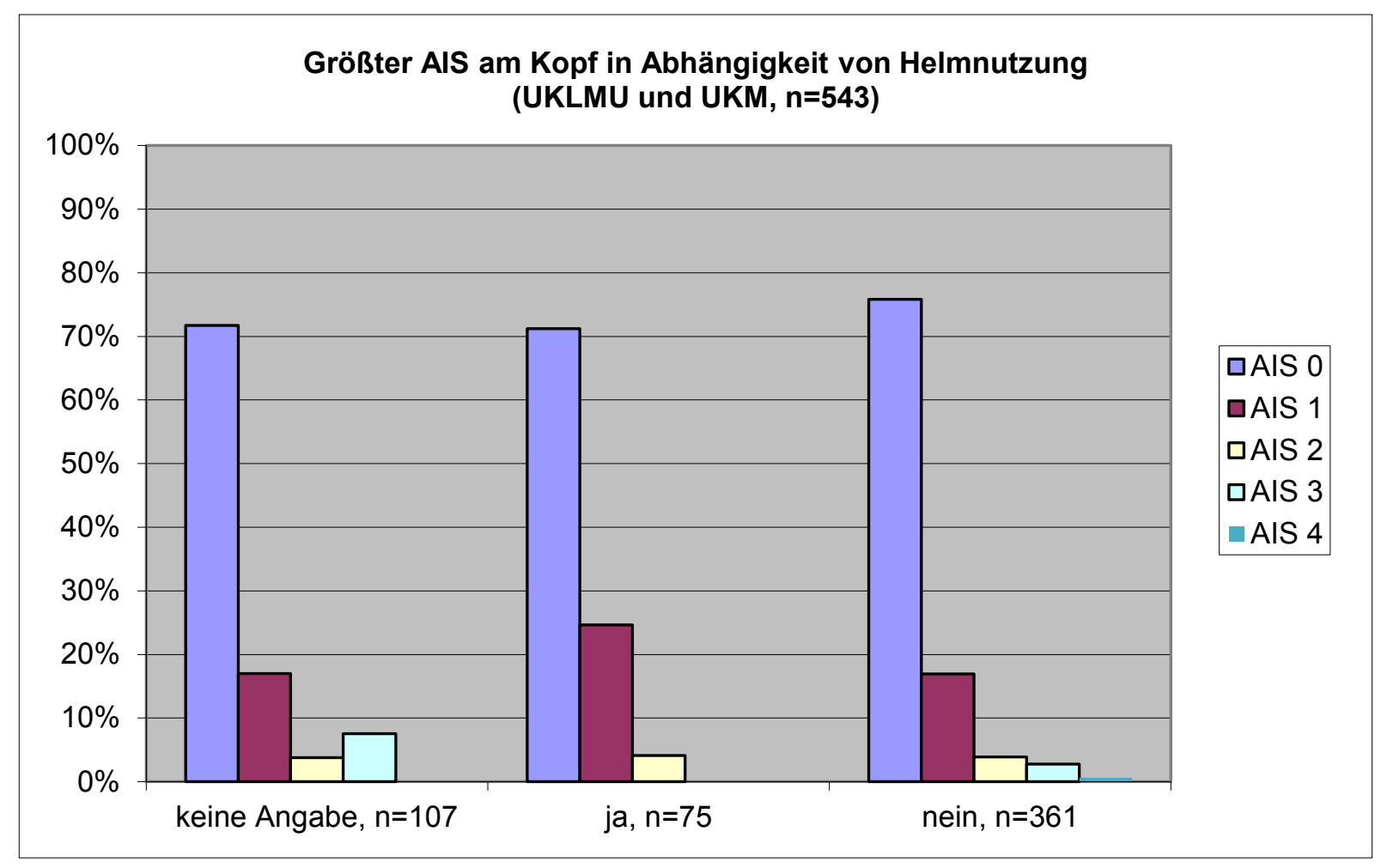

Abbildung 1-53: Größter AIS am Kopf in Abhängigkeit von Helmnutzung (UKLMU und UKM, n=543)

\subsection{Oberflächliche Kopfverletzungen}

Um Anhaltspunkte für die Lage der Anprallstellen am Kopf zu erhalten, wurden die oberflächlichen Kopfverletzungen der Fahrradfahrer dokumentiert.

Dabei wurde zwischen Schürfungen, Hämatomen, Schnittwunden und Riss-QuetschWunden differenziert.

183 der 543 Personen aus dem UKLMU und dem UKM haben äußerliche Kopfverletzungen. Bei 32 dieser Personen ist keine Angabe zum Helm vorhanden. 25 Personen trugen einen Helm, 126 Personen mit oberflächlichen Kopfverletzungen trugen keinen Helm.

Jeder Patient kann oberflächliche Kopfverletzungen in mehreren Regionen aufweisen.

Auf Abbildung 1-54 ist die Verteilung der oberflächlichen Kopfverletzungen der Fahrradfahrer ohne Helm aus den Datenkollektiven des UKLMU und des UKM dargestellt.

Am häufigsten sind Verletzungen im Bereich um die Augen, gefolgt vom Kinn und weiteren Bereichen im Gesicht.

Verletzungen im Bereich des Scheitels (Stirn bis Hinterkopf) sind selten. Lediglich im Bereich des Ober-/Hinterkopfs, des temporalen Bereichs und der Stirn sind oberflächliche Kopfverletzungen dokumentiert, die im möglichen Schutzbereich eines Fahrradhelms liegen. 


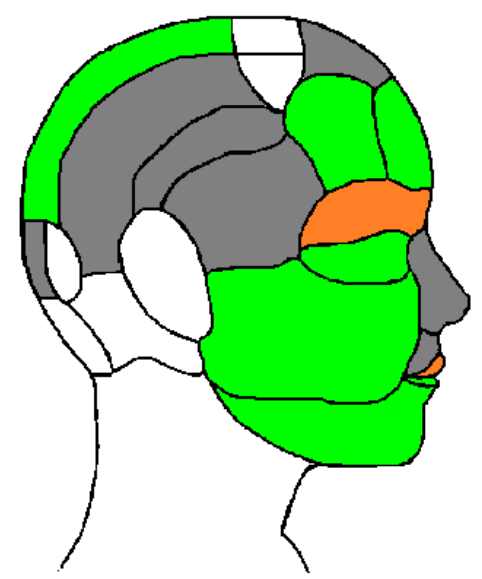

$20 \%$ und größer

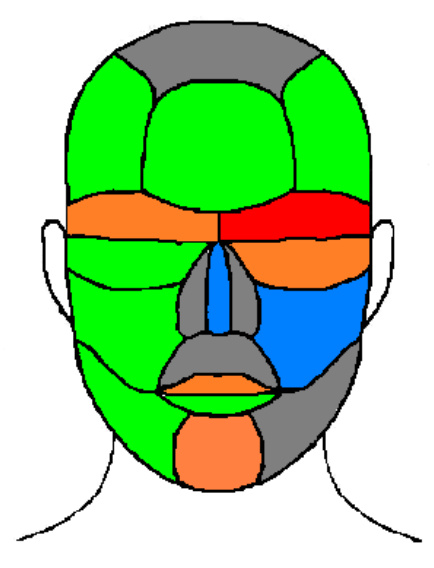

$10 \%$ bis $<15 \% \quad 5 \%$ bis $<10 \% \quad>0 \%$ bis $<5 \%$

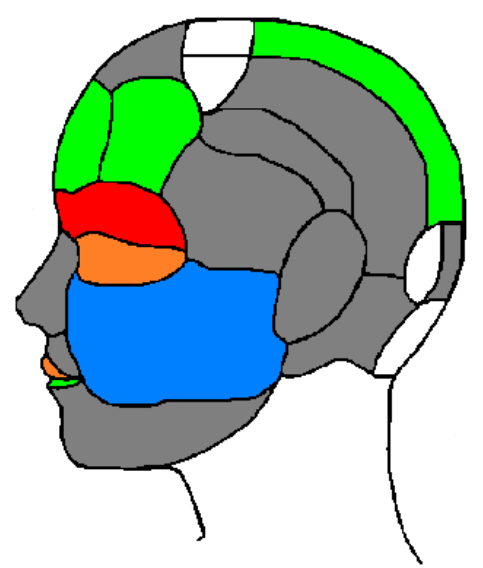

$15 \%$ bis $<20 \%$

(1)

Abbildung 1-54: Häufigkeit der oberflächlichen Kopfverletzungen der Fahrradfahrer ohne Helm (UKLMU und UKM, $n=126$ )

Auf Abbildung 1-55 ist die Verteilung der oberflächlichen Kopfverletzungen der Fahrradfahrer mit Helm abgebildet. Hier dominiert ebenfalls der vordere Gesichtsbereich, jedoch auch die Stirn. Der Oberkopf ist erwartungsgemäß nicht verletzt. Die Häufigkeit von Gesichtsverletzungen (z.B. Orbit, Kinn) ist bei den Helmträgern reduziert, obwohl diese nicht im unmittelbaren Schutzbereich des Fahrradhelms liegen.

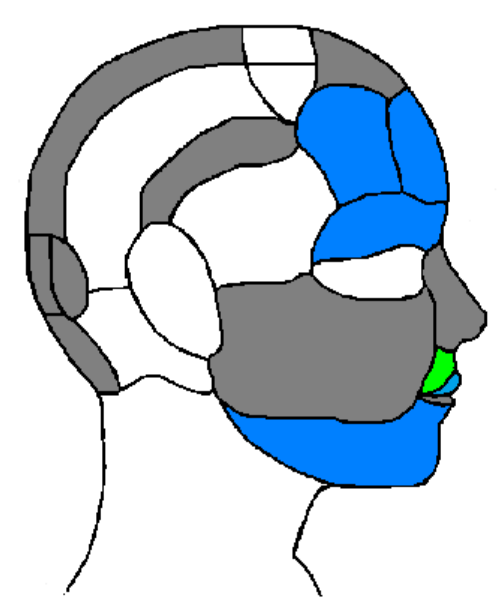

$15 \%$ bis $20 \%$

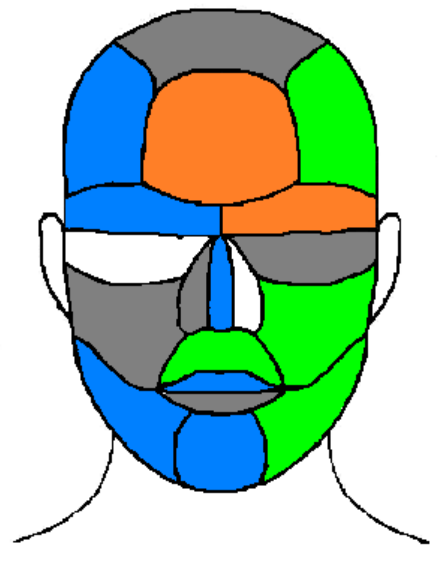

$10 \%$ bis $<15 \%$

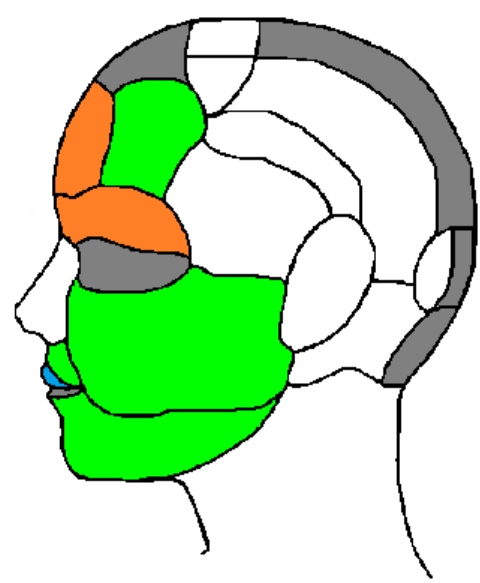

$>0 \%$ bis $<5 \%$

Abbildung 1-55: Häufigkeit der Oberflächlichen Kopfverletzungen der verletzten Fahrradfahrer mit Helm (UKLMU und UKM, $\mathrm{n}=25$ )

Auf Abbildung 1-56 ist die Verteilung der oberflächlichen Kopfverletzungen der getöteten Fahrradfahrer ohne Helm der SUD abgebildet. Von den 117 Fahrradfahrern erlitt jeder mindestens eine oberflächliche Kopfverletzung, meist sogar mehrere. 21 Fahrradfahrer erlitten großflächige Kopfschwarteneinblutungen oder einen sog. "Crush", eine erhebliche Deformation des gesamten Schädels. Diese am Kopf schwerstverletzten Fahrradfahrer wurden für die Abbildung nicht betrachtet. Von den übrigen 96 Fahrradfahrern hatten sechs einen Helm auf; diese sind ebenfalls nicht abgebildet. Grundlage für Abbildung 1-56 sind somit 90 Fahrradfahrer ohne Helm und mit abgrenzbaren einzelnen Kopfverletzungen. 
Die Bereiche, die im Fallmaterial der SUD am häufigsten verletzt wurden, unterscheiden sich sehr deutlich von den Fahrradfahrern, die nur verletzt wurden (UKLMU und UKM). In der SUD ist die temporale Region rechts und links sowie Stirn auf der linken Seite mit teils deutlich über $30 \%$ am häufigsten verletzt. Es folgen die Stirn rechts und Bereiche des unteren Hinterkopfs mit 25-30\%.

Die obere Augenpartie, die Nasenmitte, die parietale Region rechts und links sowie die parieto-okzipitale Region rechts und links sind in $20-25 \%$ aller Fälle verletzt.

Die Oberseite des Kopfs ist ähnlich wie im Material aus dem UKLMU und dem UKM verhältnismäßig selten verletzt.
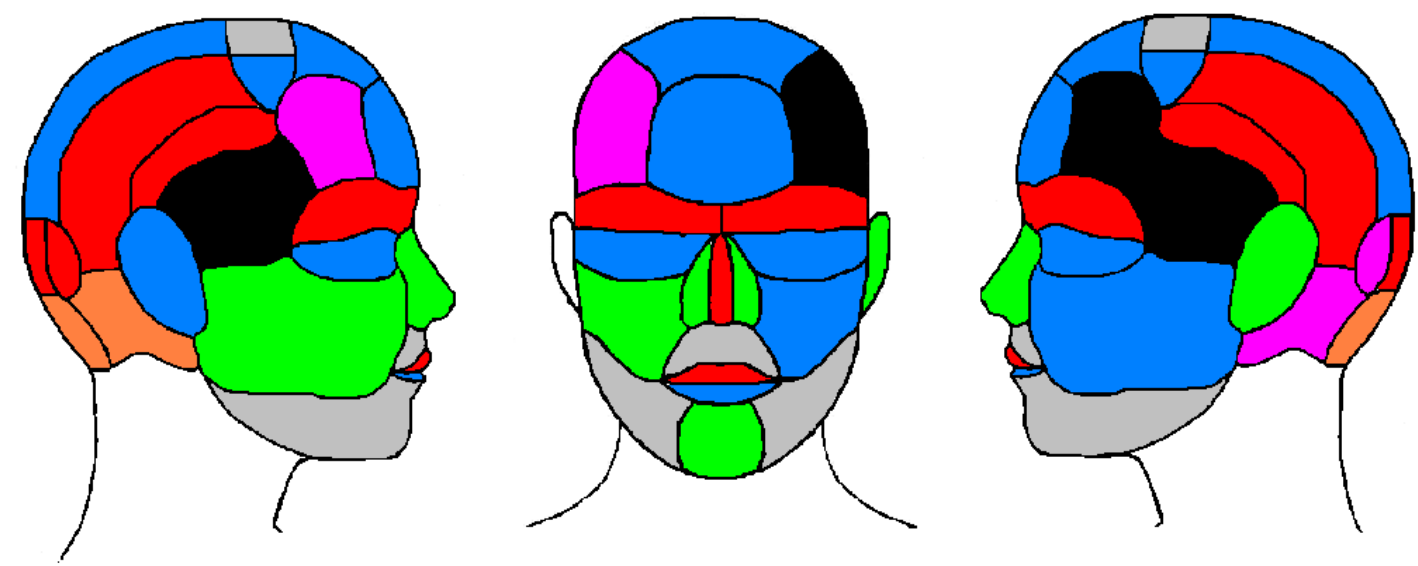

$30 \%$ und größer $25 \%$ bis $<30 \% \quad 20 \%$ bis $<25 \% \quad 15 \%$ bis $<20 \% \quad 10 \%$ bis $<15 \% \quad 5 \%$ bis $<10 \% \quad 0 \%$ bis $<5 \%$

Abbildung 1-56: Häufigkeit der oberflächlichen Kopfverletzungen der getöteten Fahrradfahrer ohne Helm (SUD, $\mathrm{n}=90$ )

\subsubsection{Diskussion relevanter Unfallsituationen}

Zur Auswertung typischer Unfallsituationen und Verletzungen standen drei Fallkollektive zur Verfügung.

Es wurden 71 Unfälle mit Fahrradfahrern im Universitätsklinikum München (UKLMU) und 500 Unfälle mit verletzten Fahrradfahrern im Universitätsklinikum Münster (UKM) prospektiv erfasst und in einer Datenbank zusammengetragen. Angaben zum Unfallhergang, benutztem Fahrradtyp und Fahrradhelm stammten großenteils aus Fragebogen, die von den Patienten auf freiwilliger Basis gemeinsam mit Klinikpersonal ausgefüllt und vereinzelt durch Informationen aus Polizeiberichten oder Bildmaterial ergänzt wurden. Nach erster Analyse wurden für die Auswertungen 34 Radfahrer ausgeschlossen, die entweder unverletzt blieben oder ihr Fahrrad als Fußgänger schoben. Somit standen insgesamt 543 Unfälle mit leicht- bis schwerverletzten Radfahrern zu Verfügung.

Zusätzlich konnte auf die Sicherheitsunfalldatenbank (SUD) des Instituts für Rechtsmedizin München zurückgegriffen werden. Hier wurden 117 tödliche Fahrradunfälle der Jahre 2003 bis 2009 retrospektiv erfasst.

Bei den Fahrradunfällen des UKLMU und des UKM ist die Mehrheit der Fahrradfahrer zwischen 15 und 64 Jahren alt. Es ist eine leichte Häufung von Senioren über 65 Jahren zu erkennen. In Münster verunfallten auffällig viele Fahrradfahrer in der Altersgruppe von 20 bis 
30 Jahren. Dies ist damit zu erklären, dass in Münster viele Studenten leben, die wiederum überdurchschnittlich viel Fahrrad fahren.

Bei den getöteten Fahrradfahrern in der SUD sind am häufigsten Fälle im Alter von 75+ Jahren erfasst. Die Geschlechterverteilung ist bei den Fällen des UKLMU und UKM annähernd gleich bei 50:50, bei den tödlichen Unfällen der SUD sind die männlichen Fahrradfahrer häufiger vertreten.

In der „Fahrradstadt“ Münster fuhr die Mehrheit der verunfallten Fahrradfahrer mit einem Holland-/Citybike, während in München klar die Fahrer eines Sportrads, Rennrads, Mountain- oder Trekkingbikes dominierten. Die Fahrer eines Sportrads, Rennrads, Mountainoder Trekkingbikes trugen sowohl in München als auch in Münster überdurchschnittlich häufig einen Fahrradhelm.

Insgesamt trugen 13\% der am UKM behandelten Fahrradfahrer einen Helm, was sich mit der von der BASt berichteten durchschnittlichen Tragequote für 2012 deckt (BASt 2013). Bei den im UKLMU behandelten Radfahrern ist diese Zahl erheblich höher. Hier trugen $21 \%$ der verunglückten Fahrradfahrer einen Helm.

In der SUD trugen nur sechs der 117 getöteten Fahrradfahrer (5\%) einen Helm.

Wie unter 1.4.2.4 dargestellt, ist der Alleinunfall des Fahrradfahrers häufigstes Unfallszenario bei den verletzten Fahrradfahrern des UKLMU und des UKM. In der SUD ist dieser Kollisionstyp mit $14 \%$ dagegen deutlich seltener.

Im prospektiv erfassten Fallmaterial aus dem UKLMU und UKM erfolgte basierend auf den Kurzbeschreibungen eine vorsichtige Abschätzung der Verteilung der Unfallmechanismen. Am häufigsten (ca. 50\%) verunglückten die Fahrradfahrer durch einen Sturz seitlich; zweithäufigster Unfallmechanismus ist der Sturz über den Lenker mit ca. 14\%. Im Fallmaterial der SUD ist bei den Alleinunfällen der Sturz seitlich mit 31 bis $56 \%$ der häufigste Unfallmechanismus, gefolgt vom Sturz über den Lenker mit 19\%.

Die Geschwindigkeit überschreitet dabei für den seitlichen Sturz selten „langsam fahrend“ (UKLMU, UKM), bzw. 15 km/h (SUD). Die Geschwindigkeit bei Sturz über den Lenker ist in den Datenkollektiven des UKLMU und UKM meist „zügig fahrend“ (> $20 \mathrm{~km} / \mathrm{h}$ ), in Fallkollektiv der SUD $\geq 15 \mathrm{~km} / \mathrm{h}$.

Es lassen sich für den Alleinunfall somit zwei typische Szenarien unterscheiden:

\section{1) Alleinunfall des Fahrradfahrers}

1a) Sturz seitlich

1b) Sturz über Lenker bei blockierendem Vorderrad

1a)

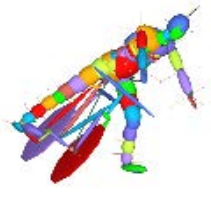

1b)

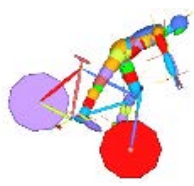

Zweithäufigstes Szenario ist die Kollision des Fahrradfahrers mit einem Pkw. Im Fallmaterial des UKLMU und des UKM werden $15 \%$ aller Fahrradfahrer bei diesem Kollisionstyp verletzt. In der SUD sind über 50\% der getöteten Fahrradfahrer bei einer Kollision mit einem Pkw zu verzeichnen. 
Die geschätzte Kollisionsgeschwindigkeit des Pkw liegt in den Fällen des UKLMU und des UKM typischerweise im Bereich zwischen $10 \mathrm{~km} / \mathrm{h}$ und $30 \mathrm{~km} / \mathrm{h}$. In der SUD beträgt die Kollisionsgeschwindigkeit des Pkw in der Mehrzahl der Fälle um 60 km/h. In der Studie Fredriksson et al. (2012) wurde eine durchschnittliche Kollisionsgeschwindigkeit des Pkw von $36 \mathrm{~km} / \mathrm{h}$ ermittelt. Otte et al. (2013) stellten in ihrer Studie dar, dass „bei Anprallgeschwindigkeiten bis $40 \mathrm{~km} / \mathrm{h}$ [...] 35,2\% der Radfahrer Kopfverletzungen [erlitten], während dies bei Geschwindigkeiten von über 40 km/h 72,2\% waren“. Durch Analyse von Fällen aus der Unfalldatenbank der Versicherer (UDB) konnte ermittelt werden, dass $29 \%$ der Fahrradfahrer bei Kollisionsgeschwindigkeiten des Pkw bis zu 45 km/h MAIS 3+ verletzt wurden.

Aufgrund der oben dargestellten Ergebnisse und Einteilungen der Kollisionsgeschwindigkeit der Pkw wurden für die folgenden Betrachtungen und Simulationen für die Kollision zwischen Fahrradfahrer und Pkw Geschwindigkeiten von $15 \mathrm{~km} / \mathrm{h}$ und $40 \mathrm{~km} / \mathrm{h}$ ausgewählt.

Die Karosserieform der beteiligten Pkw ist meist die einer Limousine.

Der Pkw kollidiert dabei typischerweise frontal gegen die Seite des Fahrradfahrers.

Daher wurden für diesen Kollisionstyp folgende typische Szenarien ausgewählt:

\section{2) Kollision "Pkw-Front gegen Fahrrad seitlich"}

2a) mit geringer Geschwindigkeit (ca. $15 \mathrm{~km} / \mathrm{h}$ )

2b) mit mittlerer Geschwindigkeit (ca. $40 \mathrm{~km} / \mathrm{h}$ )

Die Mehrheit der verletzten Fahrradfahrer (ca. 60\%) aus dem UKLMU und dem UKM zeigte eine maximale Verletzungsschwere MAIS 1. Schwer verletzt (MAIS 3+) waren knapp unter $10 \%$ aller Fahrradfahrer aus diesen Kliniken. Verletzungen mit AIS 5 oder AIS 6 kamen im UKLMU und UKM nicht vor. In der SUD hatten knapp 90\% der Unfallopfer Verletzungen MAIS 4+.

Knapp 70\% aller verletzten Fahrradfahrer des UKLMU und des UKM zusammengenommen blieben am Kopf unverletzt. Im Fallmaterial des UKLMU sind mehr Fahrradfahrer mit schweren Kopfverletzungen (AIS 3+) vorhanden. Schwerste Kopfverletzung ist in den Daten des UKLMU AIS 4, in den Daten des UKM AIS 3.

Im Gegensatz dazu hatten nur 4\% der getöteten Fahrradfahrer keine Verletzung im Bereich des Kopfs. Über $80 \%$ der getöteten Fahrradfahrer hatten schwerste Verletzungen AIS 3+ im Bereich des Kopfs. Bei den getöteten Fahrradfahrern der SUD ist der Kopf bei $69 \%$ der Unfälle als schwerstverletzte Körperregion beteiligt.

Fahrradfahrer, die einen Helm trugen, erlitten maximal eine Kopfverletzung AIS 1 oder AIS 2. Kopfverletzungen AIS 3+ traten bei Helmträgern nicht auf. Über $70 \%$ aller Fahrradfahrer mit Helm hatten keine Kopfverletzungen. Von den Fahrradfahrern ohne Helm blieben ebenfalls über $70 \%$ ohne Kopfverletzungen.

Die Anzahl der leichten Kopfverletzungen AIS 1 beträgt bei den Helmträgern ca. 25\%, bei den Radfahrern ohne Helm knapp unter 20\%. Schwere Kopfverletzungen AIS 3 und AIS 4 sind nur bei den Fahrradfahrern ohne Helm zu beobachten.

Ein Fahrradhelm kann schwere Kopfverletzungen nicht vollständig verhindern, aber das Risiko dafür deutlich reduzieren (Thompson et al. 2009). Dies kann eine mögliche Erklärung dafür sein, dass die Radfahrer mit Helm einen höheren Anteil an AIS 1 Kopfverletzungen aufweisen. In der tiefergehenden Analyse des Fallmaterials des UKLMU und UKM durch 
Malczyk et al. (2014) ist die Häufigkeit von schwereren Schädel-Hirn-Verletzungen sowie Schädel- und Gesichtsfrakturen bei den Helmträgern deutlich reduziert.

Zusätzlich zu den nach AIS kodierten Verletzungen wurden oberflächliche Kopfverletzungen in allen drei Fallkollektiven dokumentiert. Da eine solche Verletzung einen Kontakt zwischen Kopf und einem gegnerischen Fahrzeug, Objekt oder der Fahrbahn voraussetzt, kann diese als Indikator für einen Anprallpunkt am Kopf verwendet werden. Damit lässt sich die Relevanz des Schutzbereichs von Helmen im Unfallgeschehen einschätzen. 183 der 543 verunfallten Fahrradfahrer aus dem UKLMU und dem UKM zeigten äußerliche Kopfverletzungen. 25 Fahrradfahrer mit oberflächlichen Kopfverletzungen trugen einen Helm; 126 Personen mit Kopfverletzungen trugen keinen Helm.

Verletzungen im Bereich des Scheitels (Bereich von Stirn bis Hinterkopf) waren bei den unbehelmten Fahrradfahrern aus dem UKLMU und dem UKM sehr selten. Die untersuchten Fahrradhelme hatten in diesem Bereich ebenfalls selten Beschädigungen.

Lediglich im Bereich des Ober-/Hinterkopfs, des temporalen Bereichs und der Stirn sind oberflächliche Kopfverletzungen dokumentiert, die im unmittelbaren Schutzbereich eines Fahrradhelms liegen. Die Häufigkeit von Gesichtsverletzungen (z.B. Orbit, Kinn) ist bei den Helmträgern reduziert, obwohl diese nicht im unmittelbaren Schutzbereich des Fahrradhelms liegen. Eine mögliche Begründung dafür ist, dass der Helm beim Anprall gegen ebene Flächen durch die überkragende Helmkante auch Bereiche des Kopfes und Gesichtes schützen kann, die nicht im unmittelbaren Abdeckbereich des Helms liegen.

Es dominieren bei den Unfällen mit verletzten Fahrradfahrern der vordere Gesichtsbereich, insbesondere der Bereich um die Augen, die Nase und das Kinn.

Von den 117 getöteten Fahrradfahrern der SUD erlitt jeder mindestens eine oberflächliche Kopfverletzung, meist sogar mehrere oberflächliche Kopfverletzungen.

Die Bereiche, die am häufigsten oberflächliche Verletzungen zeigten, unterscheiden sich sehr deutlich zwischen den Getöteten und den Fahrradfahrern, die nur verletzt wurden. In der SUD ist die temporale Region rechts und links sowie Stirn auf der linken Seite mit teils deutlich über $30 \%$ am häufigsten verletzt. Es folgen die Stirn rechts und Bereiche des unteren Hinterkopfs mit 25-30\%.

Die Oberseite des Kopfs ist analog zu den Fällen aus dem UKLMU und dem UKM nur verhältnismäßig selten verletzt.

Die bei unbehelmten und behelmten Radfahrern festgestellte Lage von mutmaßlichen Anprallstellen an Schädel und Gesicht lässt vermuten, dass Radhelme nicht immer alle Bereiche wirksam abdecken, die im Unfallgeschehen beaufschlagt werden. Insbesondere gilt dies für die seitlichen Schädelregionen (Schläfen, untere Stirn) die bei getöteten Radfahrern häufig in Erscheinung treten, so dass über eine Anpassung oder Ausweitung der von Helmen geschützten Bereiche nachgedacht werden muss. 


\section{Biomechanische Rekonstruktion und Bewertung der Testverfahren}

\section{1 Übersicht und Einschätzung existierender Helmnormen I Testverfahren}

Aufbauend auf den Ergebnissen der Unfallanalyse anhand der als typisch ermittelten Unfallszenarien wurden die Belastungen des Fahrradfahrers im Unfallablauf ermittelt. Anschließend wurden die Anforderungen aus aktuellen Testverfahren für Fahrradhelme mit den realen Belastungen verglichen und auf Verbesserungspotential überprüft. Zunächst wird ein Überblick über etablierte Helmtestverfahren gegeben.

\subsubsection{Detaillierter Vergleich der Fahrradhelmnormen / Testverfahren}

Um einen möglichst umfassenden Eindruck von aktuellen Testnormen, den angewendeten Verfahren und nationalen Besonderheiten zu erlangen, wurden die am häufigsten angewandten Vorschriften im Bereich Fahrradhelm einem detaillierten Vergleich unterzogen. Namentlich sind das die Normen EN 1078 (Europa), CPSC 16 CFR Part 1203, ASTM F 1447-06 (USA), AS/NZS 2063:2008(Australien/ Neuseeland), JIS T 8134:2007(Japan) sowie die Richtlinie SNELL B95 (USA) und das Prüfverfahren der deutschen Stiftung Warentest und des ADAC.

Es lässt sich dabei eine Einteilung in Normen und Richtlinien vornehmen. So sind die Testverfahren der Snell Memorial Foundation ${ }^{\circledR}$, der Stiftung Warentest und des ADAC als reine Empfehlungen ohne gesetzliche Notwendigkeit zu verstehen, während die übrigen Verfahren Normen sind.

Die Einhaltung der Normen DIN EN 1078, CPSC 16 CFR Part 1203 und AS/NZS 2063:2008 ist in den jeweiligen Gültigkeitsbereichen darüber hinaus gesetzlich vorgeschrieben.

\subsubsection{1 Übersicht der betrachteten Fahrradhelmnormen / Testverfahren}

Helme, die in Deutschland kommerziell in Umlauf gebracht werden sollen, müssen die Norm DIN EN 1078 erfüllen. Diese Norm ist in Deutschland 1997 in Kraft getreten und seitdem inhaltlich annähernd unverändert. 2005 gab es eine Überarbeitung, die sich aber vor allem auf die Kennzeichnung der getesteten Helme bezog. Im April 2014 wurde eine neue Fassung veröffentlicht (DIN EN 1078:2014-04). Die Änderungen sind formaler Natur, die Prüfbedingungen und -verfahren bleiben davon unberührt.

Zudem werden in Deutschland von der Stiftung Warentest regelmäßig Fahrradhelme getestet, die Prüfanforderungen im Bereich Unfallschutz sind dabei an die DIN EN 1078 angelehnt. Die Kriterien, nach denen der ADAC testet, entsprechen der DIN EN 1078, weshalb auf eine genauere Betrachtung verzichtet wird.

In den USA gibt es zwei namentlich verschiedene Normen, zum einen die CPSC (Consumer Product Safety Commision, 16 CFR Part 1203), deren Einhaltung gesetzlich vorgeschrieben ist, zum anderen die ASTM F1447-06. Inhaltlich unterscheiden sich diese Normen nur in minimalen Details.

Eine besondere Stellung nimmt die in den USA ansässige Snell Memorial Foundation ${ }^{\circledR}$ ein. Diese Organisation wurde 1957 gegründet und hat sich zum Ziel gesetzt, besonders kritische Anforderungen an Helme zu stellen, die jedoch nur als Empfehlungen gewertet werden. 
In Australien wird seit über 20 Jahren intensiv über Fahrradhelme diskutiert, dort wurde in einigen Staaten 1991, im Rest des Landes 1996 auch eine Fahrradhelmpflicht eingeführt. Es gilt die gesetzlich verpflichtende Norm AS/NZS 2063:2008.

Zuletzt wird die japanische Fahrradhelmnorm JIS T 8134:2007 betrachtet.

Es werden in allen Prüfverfahren folgende Punkte getestet:

- Stoßdämpfung (der Helmschale)

- Festigkeit der Trageeinrichtung

- Wirksamkeit der Trageeinrichtung

Zusätzlich ist in einigen Verfahren ein Durchdringungstest der Helmschale und ein "load distribution test" (Lastverteilungstest) vorgeschrieben.

\subsubsection{Stoßdämpfungstest}

Der Prüfaufbau für den Test des Stoßdämpfungsvermögens ist bei allen Vorschriften vergleichbar. Wie auf Abbildung 2-1 zu erkennen, wird ein Prüfkopf mit dem darauf befestigten, zu prüfenden Helmmuster aus einer definierten Höhe mit einer definierten Geschwindigkeit auf einen starr befestigten Stahlsockel fallengelassen. Dabei ist der Kopf samt Helm entweder in alle drei Dimensionen frei beweglich (DIN EN 1078, JIS T 8143:2007 optional), oder er wird an einer Schiene geführt und ist nur in einer Dimension frei beweglich (CPSC, ASTM F1447-06, Snell B95, AS/NZS 2063:2008, JIS T 8134:2007 optional).

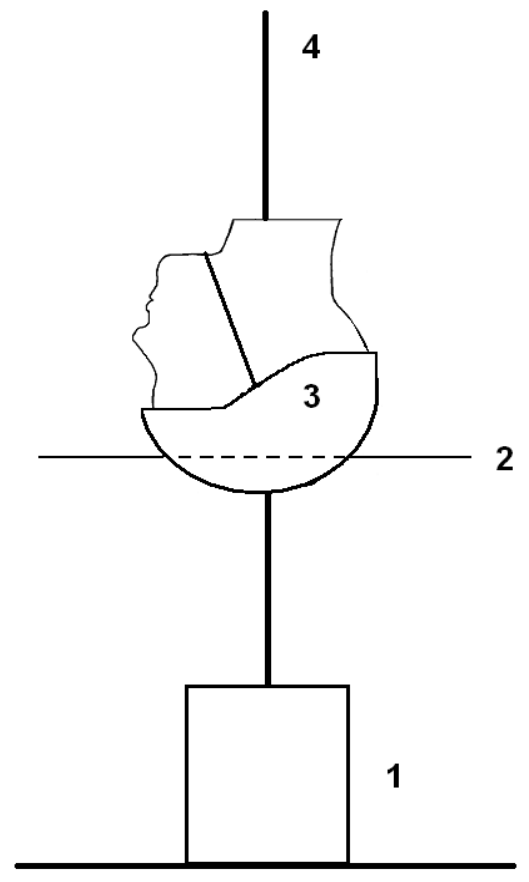

\footnotetext{
1 Sockel

2 Bewegliche Halterung

3 Prüfkopf mit Helm

4 Führungsschiene
}

Abbildung 2-1: Prüfaufbau Stoßdämpfungstest 


\subsection{Instrumentierung}

Abhängig von der gewählten Zahl der freien Dimensionen fällt auch die Instrumentierung der Prüfkopfe aus. Es ist dabei ein Beschleunigungsaufnehmer mit einer vorgeschriebenen maximalen Abweichung zum Schwerpunkt des Prüfkopfs zu montieren. Ist der Kopf in drei Dimensionen beweglich, muss ein triaxialer Beschleunigungsaufnehmer mit einer Masse von maximal 50 Gramm montiert werden, der Beschleunigungen bis $2000 \mathrm{~g}$ aufzeichnen kann, zudem müssen alle Prüfaufbauten der CFC (Channel Frequency Class;

"Kanalfrequenzklasse") 1000 entsprechen.

Ist der Kopf nur in einer Dimension beweglich, genügt ein uniaxialer

Beschleunigungsaufnehmer, der Beschleunigungen bis zu 1000g aufzeichnen kann. Hier muss ebenfalls durchgehend die CFC 1000 eingehalten werden.

\subsection{Größe und Gewicht der Prüfköpfe}

Der Stoßdämpfungstest wird in allen Vorschriften mit fünf verschiedenen Prüfkopfgrößen durchgeführt. Diese sind in der folgenden Tabelle 2-1 aufgeführt. Die Größenbezeichnung bezieht sich auf den Kopfumfang.

Tabelle 2-1: Größe der Prüfköpfe

\begin{tabular}{|c|c|}
\hline Kopfform & Innenumfang des Helms [mm] \\
\hline A & $500 \pm 5$ \\
\hline E & $540 \pm 5$ \\
\hline J & $570 \pm 5$ \\
\hline M & $600 \pm 5$ \\
\hline O & $620 \pm 5$ \\
\hline
\end{tabular}

Dabei sind die verwendeten Kopfformen in allen Vorschriften (DIN EN 960; AS/NZS 2512.3.1:2007; ASTM F 1446-11a) mit der Abstufung der gängigen Größenangaben XS bis XL vergleichbar, wobei die Form J der Größe M (medium) entspricht.

Die Prüfkopfgewichte sind in einzelnen Normen/Richtlinien unterschiedlich geregelt; wie in Tabelle 2-2 ersichtlich sind Kopfgewichte von 3100 Gramm bis hin zu 6500 Gramm möglich. Diese sind meist mit bestimmten Kopfgrößen verknüpft.

Tabelle 2-2: Kopfgewichte in Gramm

\begin{tabular}{|c|c|c|c|c|c|c|}
\hline Kopfform & EN 1078 & $\begin{array}{c}\text { CPSC 16 CFR } \\
\text { Part 1203 }\end{array}$ & $\begin{array}{c}\text { ASTM F1447- } \\
\text { 06 }\end{array}$ & Snell B95 & $\begin{array}{c}\text { AS/NZS } \\
2063: 2008\end{array}$ & $\begin{array}{c}\text { JIS T } \\
8134: 2007\end{array}$ \\
\hline $\mathrm{A}$ & $3100 \pm 100 \mathrm{~g}$ & $5000 \pm 100 \mathrm{~g}$ & $3100 \pm 100 \mathrm{~g}$ & $5000-6500 \mathrm{~g}$ & $3100 \pm 50 \mathrm{~g}$ & $3100 \pm 100 \mathrm{~g}$ \\
\hline $\mathrm{E}$ & $4100 \pm 120 \mathrm{~g}$ & $5000 \pm 100 \mathrm{~g}$ & $4100 \pm 120 \mathrm{~g}$ & $5000-6500 \mathrm{~g}$ & $4100 \pm 50 \mathrm{~g}$ & $4100 \pm 120 \mathrm{~g}$ \\
\hline $\mathrm{J}$ & $4700 \pm 140 \mathrm{~g}$ & $5000 \pm 100 \mathrm{~g}$ & $4700 \pm 140 \mathrm{~g}$ & $5000-6500 \mathrm{~g}$ & $4700 \pm 50 \mathrm{~g}$ & $4700 \pm 140 \mathrm{~g}$ \\
\hline $\mathrm{M}$ & $5600 \pm 160 \mathrm{~g}$ & $5000 \pm 100 \mathrm{~g}$ & $5600 \pm 160 \mathrm{~g}$ & $5000-6500 \mathrm{~g}$ & $5600 \pm 50 \mathrm{~g}$ & $5600 \pm 160 \mathrm{~g}$ \\
\hline $\mathrm{O}$ & $6100 \pm 180 \mathrm{~g}$ & $5000 \pm 100 \mathrm{~g}$ & $6100 \pm 180 \mathrm{~g}$ & $5000-6500 \mathrm{~g}$ & $6100 \pm 50 \mathrm{~g}$ & $6100 \pm 180 \mathrm{~g}$ \\
\hline
\end{tabular}


Lediglich die amerikanische CPSC schreibt ein Kopfgewicht von $5000 \pm 100$ Gramm vor, ungeachtet der Größe ("regardless of size") (CPSC 16 CFR Part 1203, 1998, S. 11718). In der Richtlinie Snell B95 ist die Prozedur zu den übrigen Normen leicht abweichend, dort wird für die Masse des Prüfkopfs lediglich ein Bereich zwischen 5000 und 6500 Gramm angegeben. Der Test muss aber mit einer bestimmten Aufprallenergie durchgeführt werden.

\subsection{Konditionierung der Helme}

Alle Testverfahren fordern eine Konditionierung der Helme vor dem Stoßdämpfungstest wie in Tabelle 2-3 dargestellt. Dazu werden sie über eine festgelegte Dauer Wärme, Kälte oder Nässe ausgesetzt, um verschiedene Witterungseinflüsse zu simulieren. In der EN 1078 wir zusätzlich ein Schnellalterungsverfahren gefordert. Im direkten Anschluss muss dann der Stoßdämpfungstest durchgeführt werden.

Tabelle 2-3: Konditionierung der Helme

\begin{tabular}{|c|c|c|c|c|c|}
\hline & Raumtemperatur & Wärme & Kälte & Schnellalterung & Nässe \\
\hline EN 1078 & - & $\checkmark$ & $\checkmark$ & $\checkmark$ & - \\
\hline Stiftung Warentest & $\checkmark$ & $\checkmark$ & $\checkmark$ & $\checkmark$ & $\checkmark$ \\
\hline $\begin{array}{c}\text { CPSC 16 CFR Part } \\
\text { 1203 }\end{array}$ & $\checkmark$ & $\checkmark$ & $\checkmark$ & - & $\checkmark$ \\
\hline ASTM F1447-06 & $\checkmark$ & $\checkmark$ & $\checkmark$ & - & $\checkmark$ \\
\hline Snell B95 & $\checkmark$ & $\checkmark$ & $\checkmark$ & - & $\checkmark$ \\
\hline AS/NZS 2063:2008 & $\checkmark$ & $\checkmark$ & $\checkmark$ & - & $\checkmark$ \\
\hline JIS T 8134:2007 & $\checkmark$ & $\checkmark$ & $\checkmark$ & - & $\checkmark$ \\
\hline
\end{tabular}

Raumtemperatur bewegt sich im Rahmen von 17 bis $27^{\circ} \mathrm{C}$, Wärme im Bereich von $+50^{\circ} \mathrm{C}$, Kälte im Bereich von $-20^{\circ} \mathrm{C}$. Typischerweise erfolgt die Temperierung über eine Dauer von 4 bis 24 Stunden. Konditionierung unter Nässe bedeutet, dass der Helm über eine bestimmte Dauer bei Raumtemperatur komplett in Wasser getaucht oder mit Wasser berieselt wird (wenige Minuten bis hin zu 24 Stunden). Unter Schnellalterung versteht man eine zuerst eine Bestrahlung des Helms mit einer UV-Lampe für 48 Stunden, im Anschluss hat eine Berieselung mit Wasser bei Raumtemperatur für 4 bis 6 Stunden zu erfolgen. Anschließend wird dann abhängig von der jeweiligen Vorschrift nach einem exakten Prüfschema der Stoßdämpfungstest durchgeführt. Die Prüfparameter der EN 1078 sind in der nachfolgenden Tabelle 2-4 dargestellt.

Die Einflussfaktoren werden in allen Prüfverfahren separat abgeprüft, es gibt keine Kombinationen der einzelnen Parameter. Der Test bei Nässe wird immer bei Raumtemperatur durchgeführt. 
Tabelle 2-4: Prüfparameter EN 1078

\begin{tabular}{|c|c|c|}
\hline Prüfmuster Nr. & Vorbehandlung & Sockel \\
\hline \multirow{2}{*}{1} & Wärme & Bordstein \\
\cline { 2 - 3 } & keine erneute Wärmelagerung & flach \\
\hline \multirow{2}{*}{2} & Kälte & flach \\
\cline { 2 - 3 } & keine erneute Kältelagerung & Bordstein \\
\hline \multirow{2}{*}{3} & Schnellalterung & Bordstein \\
\cline { 2 - 3 } & keine erneute Schnellalterung & flach \\
\hline
\end{tabular}

\subsection{Prüfgeschwindigkeiten und Testsockel}

Der Helm wird zuerst dem passenden Prüfkopf nach einer definierten Prozedur aufgesetzt und anschließend mit dem Kinnriemen fixiert. Der nächste Schritt ist dann der eigentliche Stoßdämpfungstest. Dazu trifft der Helm samt Prüfkopf mit vorgegebenen Prüfgeschwindigkeiten durch eine Vorrichtung geführt auf verschiedene Prüfsockel, wie in Tabelle 2-5 dargestellt. Gemäß EN 1078 wird der Helm mit Prüfkopf senkrecht mit 5,42 m/s auf einen flachen Sockel und mit $4,57 \mathrm{~m} / \mathrm{s}$ auf einen Bordsteinsockel fallen gelassen.

Tabelle 2-5: Prüfgeschwindigkeiten der Fahrradhelmnormen/ -richtlinien in Abhängigkeit der verwendeten Sockel

\begin{tabular}{|c|c|c|c|}
\hline & Flacher Sockel & Bordstein & Halbkugel \\
\hline & \multicolumn{2}{|c|}{ Prüfgeschwindigkeit [m/s] (Fallhöhe [m]) } \\
\hline EN 1078 & $5,42(1,5)$ & $4,57(1,1)$ & - \\
\hline Stiftung Warentest & $5,42(1,5)$ & $5,42(1,5)$ & - \\
\hline CPSC 16 CFR Part 1203 & $6,2(2)$ & $4,8(1,2)$ & $4,8(1,2)$ \\
\hline ASTM F1447-06 & $6,2(2)$ & $4,8(1,2)$ & $4,8(1,2)$ \\
\hline Snell B95 & $6,6^{*}(2,2)$ & $5,4^{*}(1,5)$ & $5,4^{*}(1,5)$ \\
\hline AS/NZS 2063:2008 & $5,4(1,5)$ & - & $4,57(1,1)$ \\
\hline JIS T 8134:2007 & $5,42(1,5)$ & - & - \\
\hline
\end{tabular}

*: Berechnet mit einem Prüfkopfgewicht von $5 \mathrm{~kg}$

Der flache Sockel ist eine runde, ebene Stahlplatte und soll die Straßenoberfläche abbilden. Der Bordstein-Sockel bildet die Kante eines Bordsteins ab und ist so gedreht, dass der Helm direkt auf die nach oben gerichtete Kante prallt. Der Halbkugel-Sockel ist eine nach oben gedrehte Halbkugel mit definiertem Radius $(48 \pm 1 \mathrm{~mm})$ und stellt an die lastverteilende Funktion des Helmes ebenso wie der Bordstein eine erhöhte Anforderung.

Die Prüfgeschwindigkeiten für diese beiden Sockel sind im Vergleich zum flachen Sockel reduziert. Abweichend davon testet die Stiftung Warentest sowohl auf dem flachen Sockel wie auch dem Bordsteinsockel aus einer Höhe von 1,5 Metern. Lediglich bei Nichtbestehen auf dem Bordsteinsockel wird der Helm bei einer Fallhöhe von 1,1 Metern geprüft. 
Generell fällt auf, dass die Snell B95 die härtesten Anforderungen stellt, gefolgt von den beiden amerikanischen Normen CPSC und ASTM F1447-06. Diese Vorschriften fordern auch den Test auf allen drei unterschiedlichen Sockeln. Die europäische, japanische und auch australische Norm schreiben im Vergleich geringere Prüfgeschwindigkeiten vor und prüfen nicht auf allen Sockeln; in Australien wird nur auf dem flachen Sockel getestet.

\subsection{Prüfzonen}

Die Zone des Prüfkopfs, die der Helm schützen muss (im Weiteren als Prüfzone bezeichnet), ist bei den unterschiedlichen Vorschriften sehr ähnlich. Abbildung 2-2 zeigt beispielhaft die Prüfzone, wie sie in der EN 1078 gefordert ist. Die Prüfzone deckt den Kopf oberhalb einer Umfangslinie um Stirn und Hinterkopf ab. Bei Durchführung des Stoßdämpfungstests auf den flachen Sockel gilt die Zone über der Line von 1 nach 3 als Prüfzone. Wird mit dem Bordsteinsockel getestet, gilt die Zone über der Linie zwischen den Punkten 1, 2 und 3' als Prüfzone. Im Vergleich zur Prüfzone unter Verwendung des flachen Sockels ist von der Mitte des Kopfs nach hinten ein Teil des unteren Hinterkopfs ausgespart.

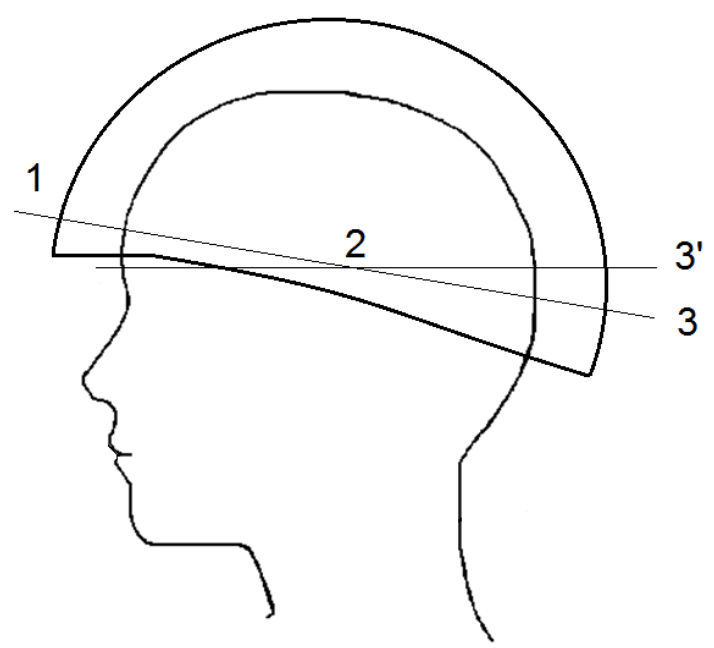

Abbildung 2-2: Prüfzone beim Stoßdämpfungstest in Anlehnung an die EN 1078

Bei einem Test nach der EN 1078 sind die Aufschlagpunkte in der Prüfzone vom Prüfer so auszuwählen, dass diese für den "schlimmsten Fall" stehen. Aufschläge auf jede als schwach anerkannte Zone innerhalb der Prüfzone werden durchgeführt. Die Aufschlagpunkte müssen mindestens $150 \mathrm{~mm}$ entlang der Sehne voneinander entfernt sein. 


\subsection{Grenzwerte der Kopfbeschleunigung}

Die verschiedenen Vorschriften fordern auch relativ ähnliche Grenzwerte für die Kopfbeschleunigung (angegeben in Vielfachem der Erdbeschleunigung g), wie in Tabelle 2-6 dargestellt.

Tabelle 2-6: Zulässige Grenzwerte der Kopfbeschleunigung der Fahrradhelmnormen/ -richtlinien

\begin{tabular}{|c|c|c|}
\hline & \multicolumn{2}{|c|}{ Zulässige Grenzwerte Kopfbeschleunigung } \\
\hline EN 1078 & $250 \mathrm{~g}$ & \\
\hline Stiftung Warentest & $250 \mathrm{~g}$ & \\
\hline CPSC 16 CFR Part 1203 & $300 \mathrm{~g}$ & \\
\hline ASTM F1447-06 & $300 \mathrm{~g}$ & \\
\hline Snell B95 & $300 \mathrm{~g}$ & \\
\hline AS/NZS 2063:2008 & $250 \mathrm{~g}$ & max. 3ms falls $>200 \mathrm{~g} ;$ max. $6 \mathrm{~ms}$, falls $>150 \mathrm{~g}$ \\
\hline JIS T 8134:2007 & $300 \mathrm{~g}$ & $<4 \mathrm{~ms}$, falls $>150 \mathrm{~g}$ \\
\hline
\end{tabular}

Alle amerikanischen Normen/ Richtlinien legen einheitlich den Maximalwert von $300 \mathrm{~g}$ fest, ohne Angabe über die maximale Einwirkdauer.

Die japanische Norm JIS T 8134 legt ebenfalls $300 \mathrm{~g}$ als Höchstwert fest, jedoch wird hier die zulässige Einwirkzeit von Beschleunigungen über $150 \mathrm{~g}$ auf 4 Millisekunden beschränkt. Die europäische Norm EN 1078 schreibt als Maximalwert $250 \mathrm{~g}$ vor, ebenso wie die australische AS/NZS 2063:2008. Auch hier ist die zulässige Einwirkdauer in Abhängigkeit von der Beschleunigungshöhe beschränkt.

\subsubsection{Festigkeit und Wirksamkeit der Trageeinrichtung}

Die Festigkeit der Trageeinrichtung wird mit einer annähernd einheitlichen Prüfapparatur ermittelt, die in Abbildung 2-3 skizziert ist. Dazu wird der Helm in aufrechter Position auf dem Prüfkopf aufgesetzt und der Kinnriemen geschlossen. Anschließend wird die Prüfeinrichtung frei hängend an der Trageeinrichtung des Helms, d.h. am Kinnriemen, befestigt. Diese besteht aus einer Stange, auf der ein Gewicht geführt aus einer definierten Höhe (typischerweise ca. $600 \mathrm{~mm}$ ) auf einen Stoppsockel herunterfallen kann. Zusätzlich ist auch eine Messapparatur angebracht, die sowohl die dynamische (elastische) Dehnung als auch in manchen Testverfahren die statische (plastische) Dehnung messen kann. 


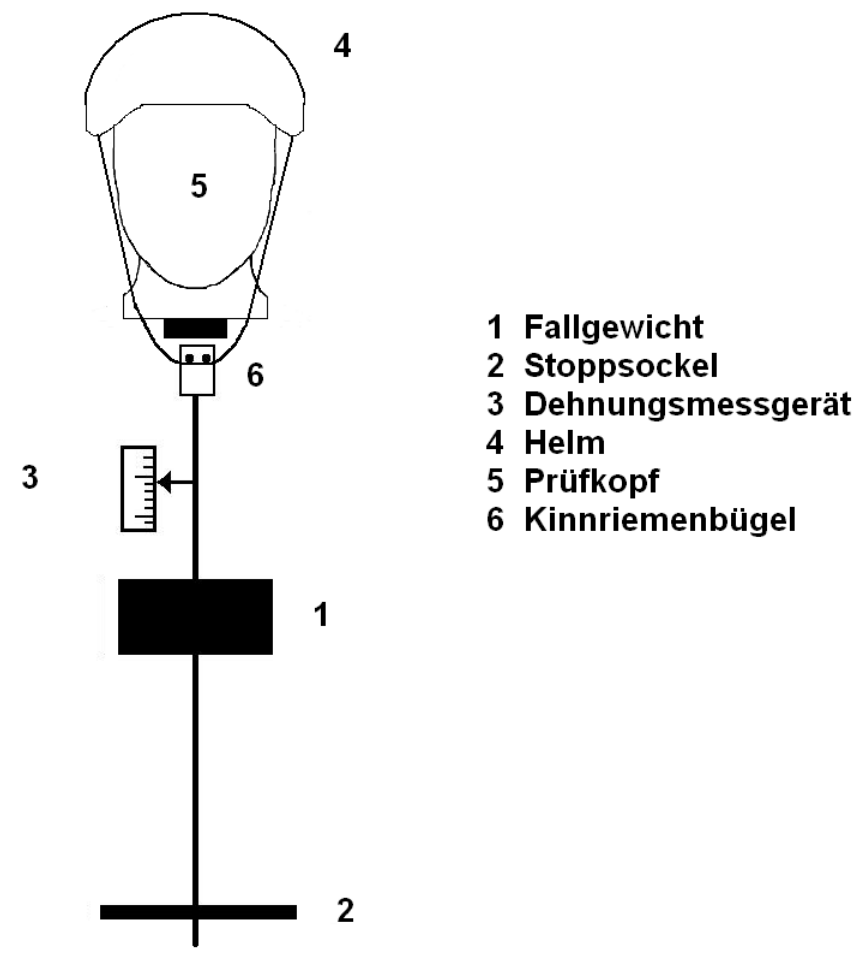

Abbildung 2-3: Prüfaufbau zum Test der Festigkeit der Trageeinrichtung

Der zulässige Maximalwert der dynamischen Dehnung beträgt je nach Vorschrift 30 $35 \mathrm{~mm}$, der Grenzwert der bleibenden Dehnung ist mit $25 \mathrm{~mm}$ angegeben. Das Schloss des Riemens muss ich nach dem Test immer noch leicht öffnen lassen. Beschädigungen der Trageeinrichtung sind zulässig, solange diese Grenzwerte eingehalten worden sind.

Zusätzlich zur Festigkeit der Trageeinrichtung wird deren Wirksamkeit geprüft. Dabei wird mit einer speziellen Vorrichtung (Abbildung 2-4) ein Abgleiten des Helms vom Prüfkopf provoziert. Dazu wird der Helm auf dem fixierten Prüfkopf befestigt. Auf der Rückseite des Helms wird ein Haken eingehängt, der über eine Umlenkrolle mit einem flexiblen Band an einer frei hängenden Führungseinrichtung befestigt ist. Dort wird ein Fallgewicht aus einer definierten Höhe fallen gelassen. 


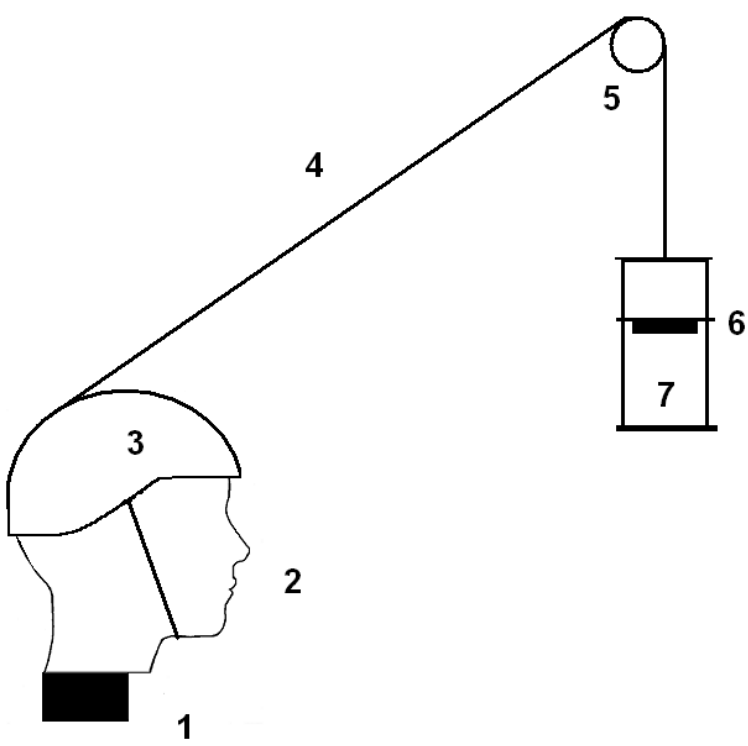
1 Sockel
2 Prüfkopf
3 Helm
4 Flexibles Band
5 Umlenkrolle
6 Geführtes Fallgewicht
7 Führungseinrichtung

Abbildung 2-4: Prüfaufbau zum Test der Wirksamkeit der Trageeinrichtung

Das Erfüllungskriterium für diesen Test ist das Verbleiben des Helms auf dem Prüfkopf, ohne dass dies in den Testvorschriften genauer definiert wird.

\subsubsection{Individuelle Besonderheiten}

Wie in den obigen Kapiteln beschrieben, ähneln sich die Vorschriften im Fahrradhelmbereich trotz einiger Unterschiede bei den Prüfverfahren und -bedingungen.

Neben diesen dargestellten "Standardprozeduren" ist jedoch in der australischen Norm ein weiteres Prüfverfahren hervorzuheben.

Es handelt sich hierbei um die "Determination of load distribution" (AS/NZS 2512.9:2006). Ziel dieses Tests ist es darzustellen, wie gut ein Helm die lokal auftretenden Kräfte eines Aufpralls verteilen kann.

Dazu wird ein Testsockel auf einen Helm fallengelassen, der auf einer speziellen Aufnahme aufgesetzt ist. Diese Aufnahme besteht aus einer halbkreisförmigen Kopfform, darunter befindet sich eine Kraftmesseinrichtung, die die beim Anprall einwirkende Kraft misst. Überschreitet diese einen Wert von 500 Newton, gilt der Test als nicht bestanden.

So wie dieses zusätzliche Prüfverfahren ausschließlich in der AS/NZS 2512.9:2006 zu finden ist, ist hervorzuheben, dass die Norm EN 1078 die einzige Vorschrift ist, welche eine Vorbehandlung des zu prüfenden Helms mit UV-Strahlung fordert, um damit einen bereits gealterten Helm zu simulieren. 


\subsection{2 Übersicht und Vergleich weiterer Helmnormen / Testverfahren}

Ergänzend zu den Fahrradhelmnormen/ -richtlinien werden im Folgenden die am häufigsten angewendeten Vorschriften in den Bereichen Motorrad, Ski/ Snowboard und Reiten betrachtet. Es soll ein effektiver Schutz vor Kopfverletzungen im Straßenverkehr, im Sport oder der Freizeit bei gleichzeitig höchstmöglichem Tragekomfort gewährleistet werden.

\subsubsection{Motorradhelm}

Im Vergleich zu den bisher betrachteten Fahrradhelmnormen liegen die Anforderungen an Motorradhelme generell etwas höher. Im Folgenden wird ein Querschnitt aus den gebräuchlichsten Normen und Richtlinien dargestellt. Diese sind die europäische Regelung (ECE R22, Version 05), die amerikanische Vorschrift (Federal Motor Vehicle Safety Standard No. 218 (49 CFR Sec. 571.218): Motorcycle Helmets), die Empfehlungen der Snell Memorial Foundation ${ }^{\circledR}$ M2010 und L98 sowie die für Australien und Neuseeland geltende Vorschrift AS/NZS 1698:2006.

Es sind auch hier jeweils mehrere Testverfahren vorgeschrieben. Es wird die Festigkeit des Trageriemens getestet, das Abstreifverhalten des Helms sowie in der europäischen Regelung die Oberflächenreibung des Helms. In der der FMVSS 218, der Empfehlung M2010 und der AS/NZS wird zudem ein Durchdringungstest gefordert. Der wichtigste Test in allen Vorschriften ist die Stoßdämpfungsprüfung auf dem Fallprüfstand.

In Tabelle 2-7 sind die Prüfgeschwindigkeiten der einzelnen Normen/ Richtlinien für die jeweiligen Sockel aufgelistet.

Tabelle 2-7: Prüfgeschwindigkeiten der Motorradhelmnormen/ -richtlinien der einzelnen Sockel

\begin{tabular}{|c|c|c|c|}
\hline & Flacher Sockel & Bordstein & Halbkugel \\
\hline & \multicolumn{2}{|c|}{ Prüfgeschwindigkeit [m/s] (Fallhöhe [m]) } \\
\hline ECE R 22 & $7,5(2,9)$ & $7,5(2,9)$ & - \\
\hline FMVSS 218 & $6(1,8)$ & - & $5,2(1,4)$ \\
\hline Snell M2010 & $7,75(3,1)$ & $7,75(3,1)$ & $7,75(3,1)$ \\
\hline AS/NZS 1698:2006 & $6(1,8)$ & - & $5,2(1,4)$ \\
\hline
\end{tabular}

Während die FMVSS 218 und die australische AS/NZS 1698:2006 mit Geschwindigkeiten testet, die denen im Fahrradhelmbereich ähneln, prüfen die ECE R22 und die M2010 mit deutlich höheren Geschwindigkeiten. Einzig die M2010 fordert einen Stoßdämpfungstest auf allen drei unterschiedlichen Sockelformen. Die ECE R22 schreibt die Lage der vier Prüfpunkte am Helm genau vor, während die anderen Vorschriften die genaue Positionierung des Kopfes mit Helm überwiegend dem Prüfer überlassen. Der Prüfbereich ist hingegen in allen Vorschriften festgelegt; der Helm muss den Kopf in der Prüfzone oberhalb einer virtuellen Linie von der Mitte der Stirn bis zum Hinterkopf schützen.

Die nachfolgende Tabelle 2-8 zeigt die zulässigen Grenzwerte für die Kopfbeschleunigung beim Test auf dem Fallprüfstand. 
Tabelle 2-8: Zulässige Grenzwerte der Kopfbeschleunigung der Motorradhelmnormen/ -richtlinien

\begin{tabular}{|c|c|c|}
\hline & \multicolumn{2}{|c|}{ Zulässige Grenzwerte Kopfbeschleunigung } \\
\hline ECE R22 & $275 \mathrm{~g}$ & HIC $<2400$ \\
\hline FMVSS 218 & $400 \mathrm{~g}$ & $<2 \mathrm{~ms}$, falls $>200 \mathrm{~g} ;<4 \mathrm{~ms}$, falls $>150 \mathrm{~g}$ \\
\hline Snell M2010 & $275 \mathrm{~g}$ & \\
\hline AS/NZS 1698:2006 & $300 \mathrm{~g}$ & max. 3ms falls $>200 \mathrm{~g} ;$ max. $6 \mathrm{~ms}$ falls $>150 \mathrm{~g}$ \\
\hline
\end{tabular}

Es wird deutlich, dass wiederum die ECE R22 wie auch die M2010 die höchsten Anforderungen stellen und einen verhältnismäßig niedrigen Maximalwert der Kopfbeschleunigung zulassen. Zudem fordert die ECE R22 auch die Einhaltung eines "Parameters der Kopfverletzung (HIC)" (ECE R22). Der HIC-Wert (Head Injury Criterion) wurde ursprünglich als dimensionslose Kenngröße zur Einschätzung des Risikos von Kopfverletzungen bei frontalen Automobilcrashtests entwickelt. Bei der Ermittlung des HIC wird neben der Höhe der einwirkenden Kopfbeschleunigung auch ihre Einwirkdauer (beim HIC 36 maximal 36 ms, beim HIC 15 maximal 15 ms) berücksichtigt. Je geringer die Einwirkdauer ist, umso höher darf die Beschleunigung ausfallen.

Auch die FMVSS 218 und die AS/NZS 1698:2006 begrenzen die maximal erlaubte Beschleunigung in Abhängigkeit von der Einwirkdauer.

Die Grenzwerte für die zulässige maximale Dehnung des Kinnriemens liegen bei allen Vorschriften im Bereich zwischen 25 und 35 mm, der Aufbau zur Ermittlung dieses Wertes ist annähernd identisch.

Die Prüfverfahren zum Abstreifverhalten des Helms unterscheiden sich zwar geringfügig, der Grenzwert für die Verschiebung des Helms gegenüber dem Kopf beträgt bei allen Vorschriften ungefähr $30^{\circ}$.

In den USA und Australien wird zudem ein Durchdringungstest gefordert. Dazu wird der Helm mit Prüfkopf auf einen spitzen Sockel fallen gelassen. Durchdringt die Spitze den Helm bis zum Kopf, gilt der Test als nicht bestanden.

\subsubsection{Ski- / Snowboardhelm}

Im Bereich der Vorschriften für Skihelme sind die Normen DIN EN 1077, ASTM F 2040-11 und die Richtlinie SNELL RS-98 zu nennen. Diese Helme zwar nicht zur Nutzung im öffentlichen Straßenverkehr vorgesehen, jedoch ist das Anforderungsprofil vergleichbar mit dem von Fahrradhelmen.

Dementsprechend ähneln sich auch die Prüfaufbauten und -abläufe. Die amerikanische Norm ASTM F2040-11 enthält zusätzlich einen Stoßdämpfungstest auf einem Sockel, der einen hochkant gestellten Ski abbilden soll.

In

Tabelle 2-9 sind die Prüfgeschwindigkeiten aufgeführt, die Bezeichnung "Eck-Sockel" stellt bei der ASTM 2040-11 den gerade beschriebenen Sockel dar. 
Tabelle 2-9: Prüfgeschwindigkeiten Skihelmnormen/-richtlinien

\begin{tabular}{|c|c|c|c|}
\hline & Flacher Sockel & Eck-Sockel & Halbkugel \\
\hline & \multicolumn{2}{|c|}{ Prüfgeschwindigkeit [m/s] (Fallhöhe [m]) } \\
\hline EN 1077 & $5,42(1,5)$ & - & - \\
\hline ASTM 2040-11 & $6,2(2)$ & $4,5(1)$ & $4,8(1,2)$ \\
\hline Snell RS 98 & $6,3(2)$ & $5,7(1,6)$ & $5,7(1,6)$ \\
\hline
\end{tabular}

Das Prüfgeschwindigkeitsniveau ist vergleichbar mit den Anforderungen an Fahrradhelme, die in Tabelle 2-10 aufgeführten Grenzwerte entsprechen denen im Fahrradhelmbereich.

Tabelle 2-10: Zulässige Grenzwerte Kopfbeschleunigung Skihelmnormen/-richtlinien

\begin{tabular}{|c|c|}
\hline & Grenzwerte Kopfbeschleunigung \\
\hline EN 1077 & $250 \mathrm{~g}$ \\
\hline ASTM 2040-11 & $300 \mathrm{~g}$ \\
\hline Snell RS 98 & $300 \mathrm{~g}$ \\
\hline
\end{tabular}

Zusätzlich zu den obligatorischen Tests bezüglich der Festigkeit und Wirksamkeit der Trageeinrichtung enthalten die DIN EN 1077 und die Snell RS 98 einen Durchdringungstest.

\subsubsection{Reithelm}

Von den Reithelmvorschriften sind die Norm DIN EN 1384, die ASTM 1163-04a sowie die Richtlinie SNELL E2001 dargestellt.

Die generelle Prüfprozedur stimmt in vielen Punkten mit denen der Fahrrad- und Skihelme überein. Auch hier definieren die ASTM 2040-11 und die Snell RS 98 einen speziellen Sockel, in der nachfolgenden Tabelle 2-11 als "Reithindernis" beschrieben. Dieser bildet die Spitze eines beschlagenen Pferdehufs ab.

Die Prüfgeschwindigkeiten beim Stoßdämpfungstest sind vergleichbar mit denen im Fahrradhelmbereich (siehe Tabelle 2-11).

Auch die zulässigen Grenzwerte der Kopfbeschleunigung, wie in Tabelle 2-12 abgebildet, sind annähernd identisch zu denen für Fahrrad und Skihelme. 
Tabelle 2-11: Prüfgeschwindigkeiten Reithelmnormen/-richtlinien

\begin{tabular}{|c|c|c|c|}
\hline & Flacher Sockel & Reithindernis & Halbkugel \\
\hline & \multicolumn{2}{|c|}{ Prüfgeschwindigkeit [m/s] (Fallhöhe [m]) } \\
\hline EN 1384 & $5,4(1,5)$ & - & - \\
\hline ASTM F1163-04a & $6(1,8)$ & $5(1,3)$ & - \\
\hline Snell E2001 & $6,3(2)$ & $5,3(1,4)$ & $5,7(1,6)$ \\
\hline
\end{tabular}

Tabelle 2-12: Zulässige Grenzwerte Kopfbeschleunigung Reithelmnormen/-richtlinien

\begin{tabular}{|c|c|c|}
\hline & \multicolumn{2}{|c|}{ Zulässige Grenzwerte Kopfbeschleunigung } \\
\hline EN 1384 & $250 \mathrm{~g}$ & $<5 \mathrm{~ms}$, falls $>150 \mathrm{~g}$ \\
\hline ASTM F1163-04a & $300 \mathrm{~g}$ & \\
\hline Snell E2001 & $300 \mathrm{~g}$ & \\
\hline
\end{tabular}

Lediglich die Europäische Norm setzt etwas strengere Grenzen, hier dürfen Werte über $150 \mathrm{~g}$ nur auftreten, wenn sie für weniger als $5 \mathrm{~ms}$ andauern. Mit $250 \mathrm{~g}$ legt die europäische Fahrradhelmnorm EN 1078 zwar denselben Grenzwert für die maximale Beschleunigung fest, eine zeitliche Einschränkung der zulässigen Beschleunigung enthält sie hingegen nicht.

Der Test der Trageeinrichtung ist vergleichbar zu den Verfahren und Grenzwerten der Vorschriften in den vorher genannten Bereichen.

Auch hier lässt sich abschließend als Besonderheit noch ein geforderter Durchdringungstest in der DIN EN 1384 und der Snell E2001 nennen.

\subsubsection{Einschätzung der verschiedenen Helmnormen / Testverfahren}

Nach dem Vergleich der Helmnormen/ Testverfahren lassen sich nach aktuellem Kenntnisstand folgende Einschätzungen treffen:

Unter allen betrachteten Vorschriften stellen diejenigen im Bereich Motorradhelm die höchsten Anforderungen, wenngleich sich die einzelnen Prüfverfahren in den wichtigsten Punkten kaum von den übrigen Normen/ Richtlinien unterscheiden.

Unter den Vorschriften im Fahrradhelmbereich stellt die Richtlinie Snell B95 die höchsten Anforderungen an die Prüfgeschwindigkeiten im Stoßdämpfungstest (bis zu 6,6 m/s). Die Norm EN 1078 sowie AS/NZS 2063:2008 sind am anspruchsvollsten hinsichtlich der maximal zulässigen Kopfbeschleunigung $(250 \mathrm{~g})$. 
Die einzelnen Vorschriften sind hinsichtlich der Prüfbedingungen und Grenzwerte unterschiedlich anspruchsvoll, es gibt jedoch keine Norm oder Richtlinie, die in allen Prüfpunkten den höchsten Maßstab aller Vorschriften ansetzt.

Die angewendeten Prüfverfahren der betrachteten Fahrradhelmnormen/ -richtlinien sind vergleichbar, lediglich die australisch-neuseeländische Vorschrift zeigt hier mit dem Test der Kraftverteilung eine individuelle Besonderheit.

Die Testverfahren im Gebiet der Ski-/ Snowboard- und Reithelme ähneln sehr den Vorschriften im Fahrradhelmbereich; teilweise wird zusätzlich ein Durchdringungstest gefordert. 


\section{$2.2 \quad$ Fahrradhelmmodellierung}

Um den Nutzen eines Fahrradhelms im Rahmen von detaillierten Simulationen abschätzen zu können, wurde von der Arbeitsgruppe Biomechanik des Instituts für Rechtsmedizin München ein Finite Elemente (FE)-Fahrradhelmmodell erstellt.

\subsubsection{Auswahl eines geeigneten Helms und geometrische Beschreibung}

Zunächst wurde ein für die Modellerstellung geeigneter Helm ausgewählt. Nach Analyse der auf dem Markt gängigen Produkte wurde ein Modell eines großen Helmherstellers (Kopfgröße 52-57cm, für Männer) als repräsentativ bestimmt. Der Helm war nach Auskunft des Herstellers das meistverkaufte Modell zum Zeitpunkt des Projektbeginns. Es handelt sich um einen typischen Mikroschalen-Helm, der Absorptionsschaumstoff ist außen von einer dünnen glatten Plastikschale umgeben. Der Helm kann mit einem frei einstellbaren Gurtsystem an die Kopfgröße angepasst werden. Zudem besitzt der Helm großflächige Belüftungsöffnungen.

Im nächsten Schritt wurde die Geometrie des Helms erfasst. Dieser Prozess wurde iterativ mit den nachfolgend beschriebenen Arbeitsschritten durchgeführt. Um eine möglichst hohe Qualität der später durchzuführenden numerischen Simulationen zu erlangen, wurde eine geometrische Modellierung auf Basis von CT-Scans durchgeführt. Dies erlaubt eine hohe Genauigkeit, ohne auf die Bereitstellung von Geometriedaten durch den Helmhersteller angewiesen zu sein.

Der gewählte Helm wurde in einem CT-Gerät der Firma Philips, Gerätbezeichnung Brilliance 64, gescannt. Es wurden insgesamt drei Datensätze in unterschiedlicher Fensterung angefertigt, um sicher zu gehen, dass auch der lufthaltige und somit kontrastarme Schaumstoff abgebildet wird. Der Begriff Fensterung beschreibt, in welchen Grauabstufungen (von weiß bis schwarz) Material unterschiedlicher Dichte im Bild dargestellt wird. Tabelle 2-13 gibt die technischen Daten der CT-Datensätze an. Abbildung 2-5 zeigt exemplarisch Datensatz 2 mit der Fensterung -888/350 nach Import in die Segmentierungssoftware und das zugehörige Histogramm der Grauwerte, welches auch das Material mit geringem Houndsfield-Wert $(\mathrm{HU})$ wiedergibt. Die Hounsfield Units $(\mathrm{HU})$ werden auf der Hounsfield Skala gemessen. Diese Skala beschreibt die Abschwächung von Röntgenstrahlung durch das bestrahlte Material. 
Tabelle 2-13: Technische Daten der angefertigten CT Datensätze

\begin{tabular}{|l|l|l|l|l|l|}
\hline $\begin{array}{l}\text { Datensatz } \\
\text { Nr. }\end{array}$ & $\begin{array}{l}\text { Fensterung } \\
\text { (window } \\
\text { center/window } \\
\text { width) in } \\
\text { Grauwerten }\end{array}$ & $\begin{array}{l}\text { Bild- } \\
\text { ausschnitts- } \\
\text { maße/mm }\end{array}$ & $\begin{array}{l}\text { Slice } \\
\text { Thickness/ } \\
\text { mm }\end{array}$ & Auflösung/mm ${ }^{2}$ & $\begin{array}{l}\text { Röhrenspannung/ } \\
\text { kVp }\end{array}$ \\
\hline 1 & $800 / 2000$ & $350 \times 350$ & 2 & $0,68 \times 0,68 \mathrm{~mm}^{2}$ & 120 \\
\hline 2 & $-888 / 350$ & $350 \times 350$ & 3 & $0,68 \times 0,68 \mathrm{~mm}^{2}$ & 120 \\
\hline 3 & $-600 / 1600$ & $350 \times 350$ & 3 & $0,68 \times 0,68 \mathrm{~mm}^{2}$ & 120 \\
\hline
\end{tabular}
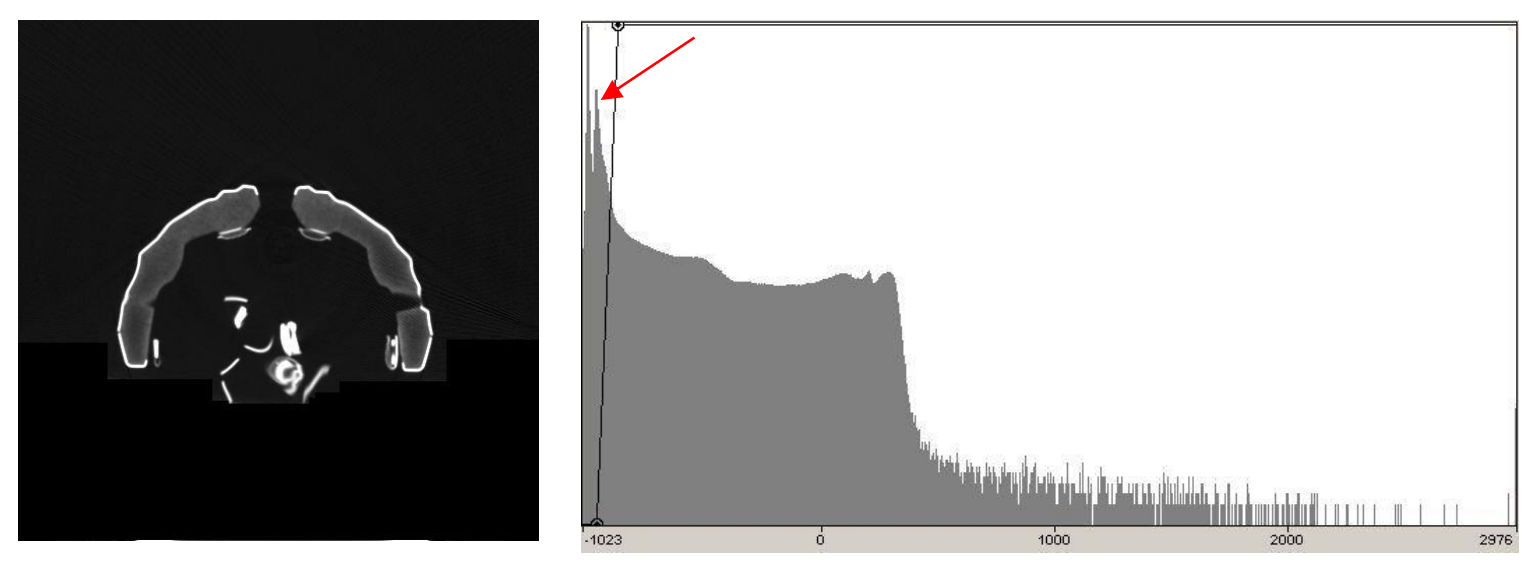

Abbildung 2-5: CT Aufnahme Datensatz 2 (Fensterung -888/350) mit Plot der Grauwerte

Abbildung 2-5 zeigt den Peak des luftdurchlässigen Materials auf der linken des Histogramms (markiert durch roten Pfeil) und das Abbild von Schaum und Schale.

Aus diesen Gründen eignete sich Datensatz 2 am besten zur Segmentierung.

\subsubsection{Segmentierung Helmgeometrien, Export / Netzerstellung FEM}

Um aus den angefertigten CT-Datensätzen ein dreidimensionales Modell zu generieren, wurde der ausgewählte Datensatz zunächst in eine gängige Segmentierungssoftware importiert. Dann wurden geeignete Schwellwerte zur Erstellung von Masken für den Schaumstoff und die Kunststoff-Außenhülle (Mikroschale) ausgewählt. Die Schwellwerte müssen dabei so gewählt werden, dass alle Grauwerte des Schaumstoffs sowie des Außenschalenmaterials in getrennten Masken erfasst werden. Abbildung 2-6 zeigt den für den Schaumstoff ausgewählten Schwellwert. 


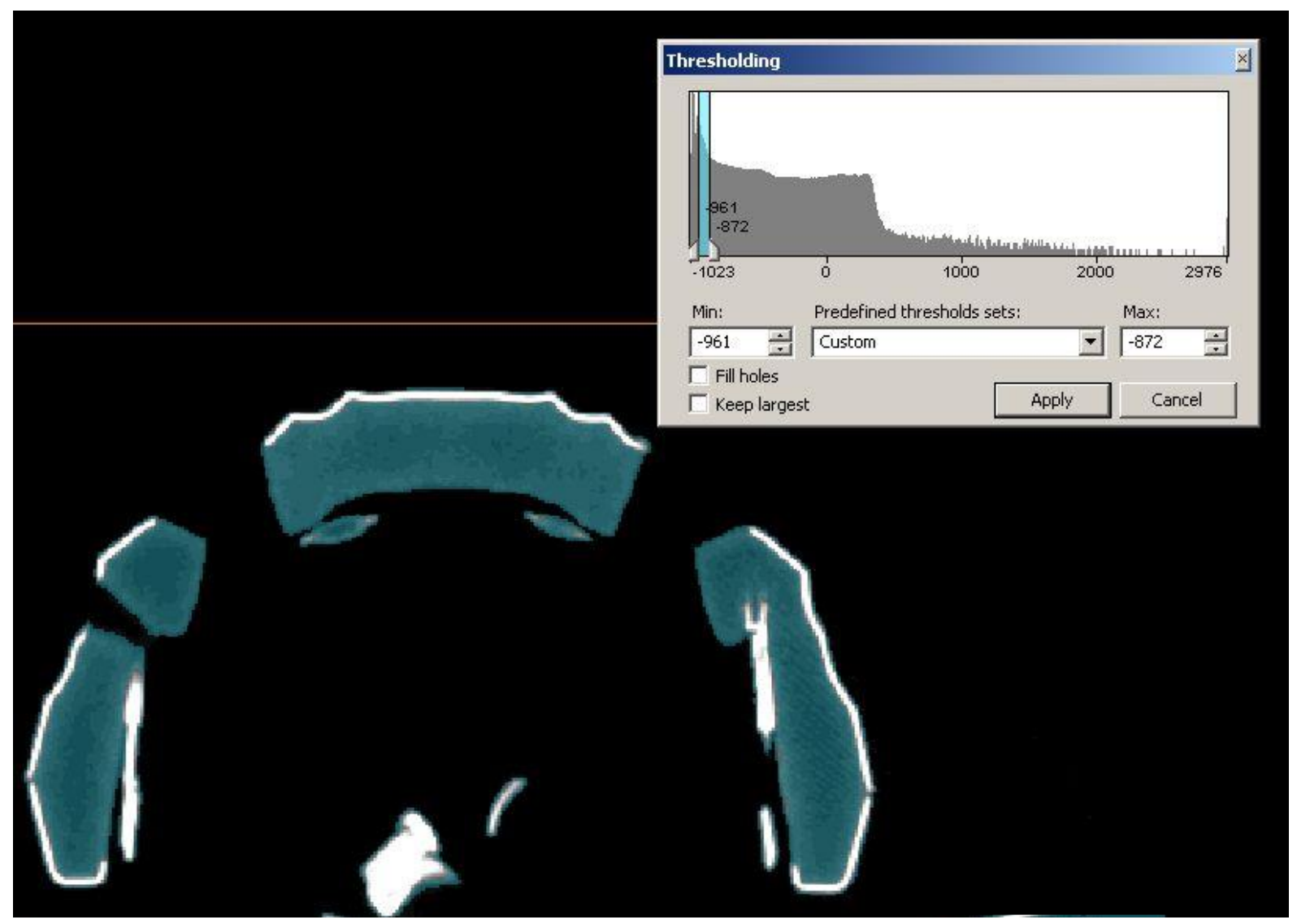

Abbildung 2-6: Schwellwert für das absorbierende Schaumstoffmateriald thermoplastischen Kunststoff an.

Tabelle 2-14 gibt die angewandten Schwellwerte für Schaumstoff und thermoplastischen Kunststoff an.

Tabelle 2-14: Schwellwerte bei der Segmentierung des absorbierenden Schaumstoffs und der Außenschale des Fahrradhelms in Hounsfield Einheiten

\begin{tabular}{|l|l|l|}
\hline & unterer Schwellwert / HU & oberer Schwellwert / HU \\
\hline Schaumstoff & -961 & -872 \\
\hline Außenschale & -819 & -491 \\
\hline
\end{tabular}

Durch weitere Algorithmen (Region-Growing-Funktion des Segmentierprogramms) verbleibt schließlich nur das gewünschte Objekt in der Maske. Abbildung 2-7 zeigt die resultierende Maske nach Anwendung der Region-Growing-Funktion. Anschließend wurden in manueller Arbeit die ebenfalls enthaltenen Halteriemen des Fahrradhelms aus der Maske entfernt. 


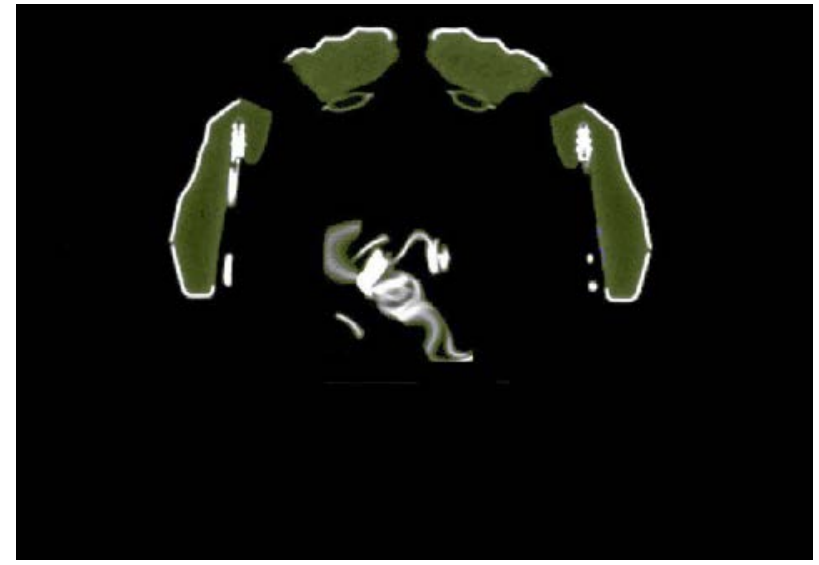

Abbildung 2-7: Maske des Schaumstoffs nach der Operation Region-Growing

Auf die gleiche Weise wie beim Schaumstoffmaterial wurde auch die Außenschale segmentiert.

Nach Festlegen der Maske wurden zwei 3D-Objekte von Schaumstoff und Außenschale aus den markierten Voxeln berechnet. Abbildung 2-8 zeigt beispielhaft noch einmal die drei Ansichten der Maske des Schaumstoffs sowie rechts unten das aus ihr berechnete 3DObjekt.
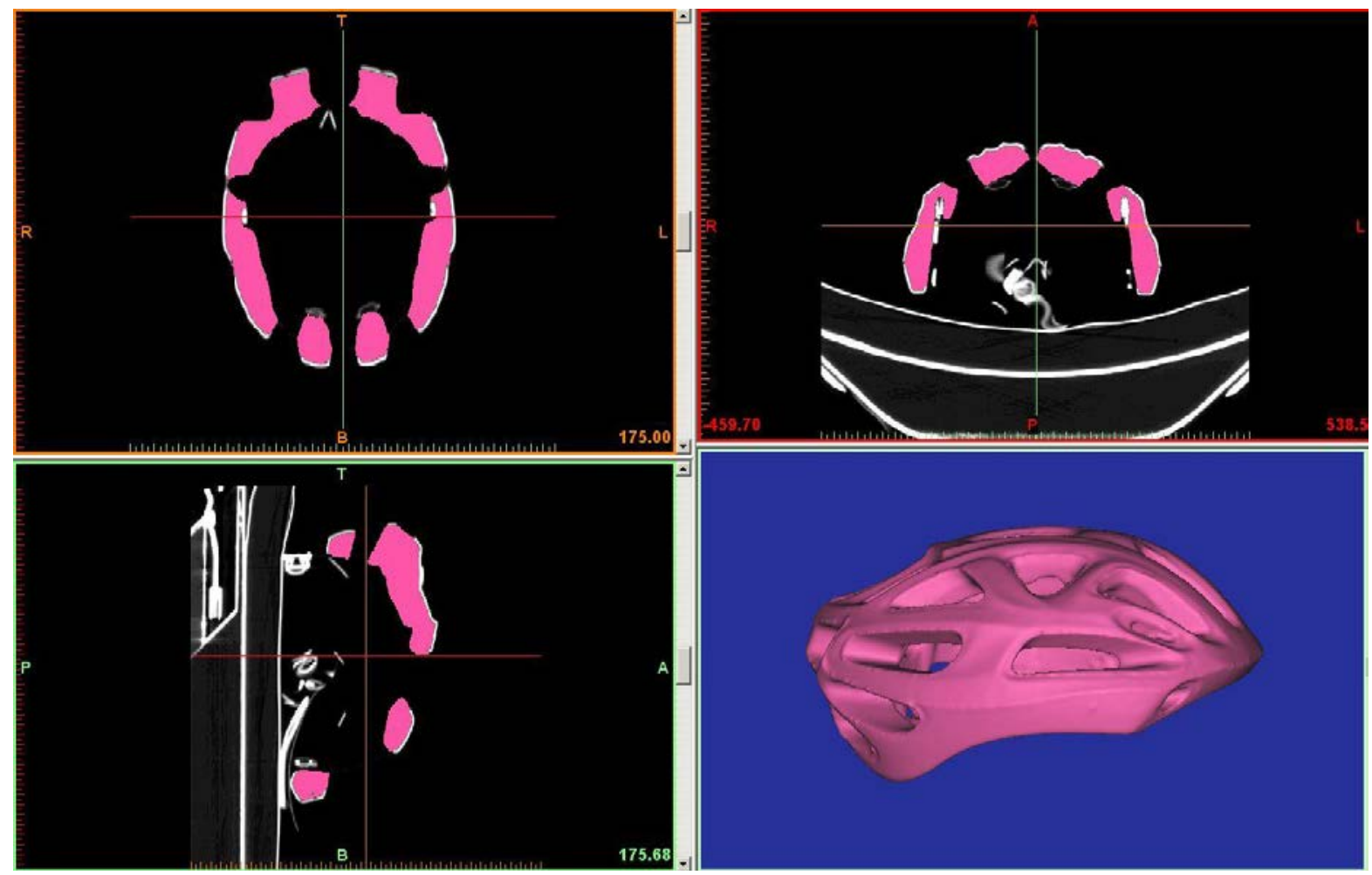

Abbildung 2-8: Maske des Schaumstoffs aus drei Raumansichten und daraus berechnetem 3D-Objekt

Nach Berechnung des 3D-Objektes wird mit Hilfe von Smooth- und Wrap- Algorithmen eine glatte Außenkontur ohne Löcher erzeugt.

Abbildung 2-9 zeigt die fertigen 3D-Objekte des Schaumstoffes und der Helmschale in Draufsicht und Seitenansicht. 

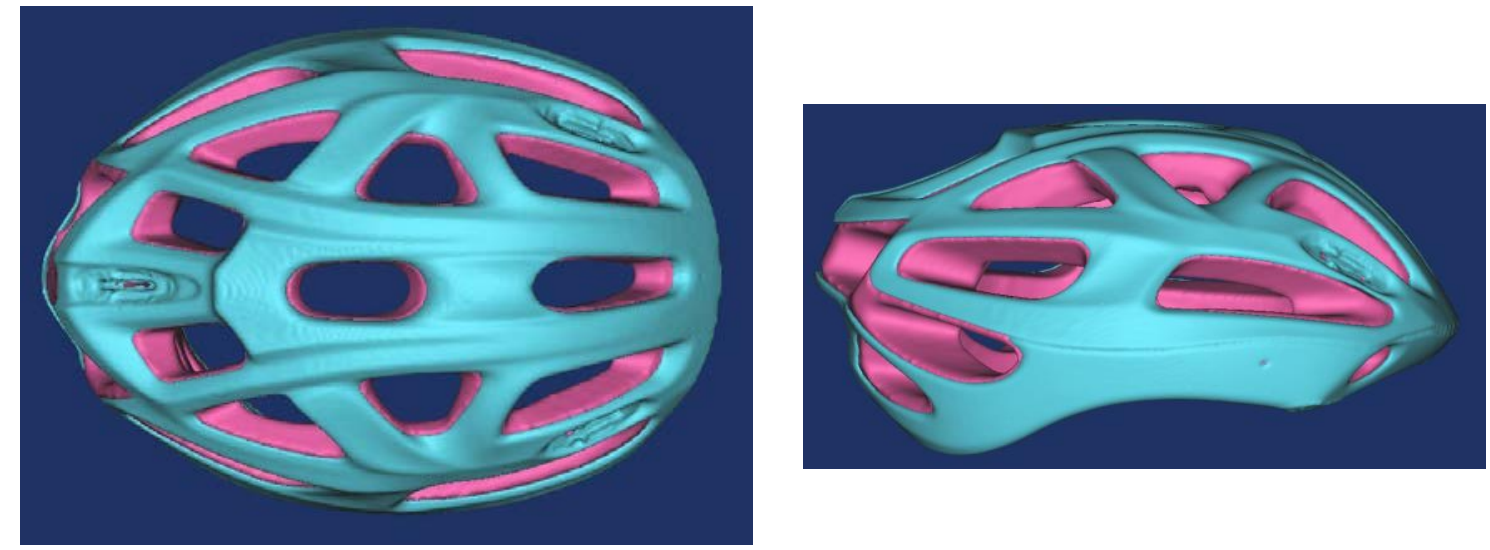

Abbildung 2-9: Fertige 3D-Modelle des Schaumstoffes und der Schale

Im Anschluss wurde das 3D-Modell im STL-Format gespeichert. Auf Basis dieser Daten wurde ein Finite-Elemente-Netz des Helms erstellt. Auf Grund der hohen Anforderungen an die Genauigkeit, Stabilität und Rechenzeit eines solchen Modells wurde eine Vernetzung mit Hexaeder-(dreidimensional) und Vierecks-Elementen (zweidimensional) angestrebt. Bei der Vernetzung von solch komplexen Geometrien wie der des vorliegenden Helms müssen dennoch Kompromisse zwischen Aufwand und Detailgenauigkeit eingegangen werden. Um eine durchgehende Vernetzung unter Verzicht auf Tetraeder-Elemente zu gewährleisten, mussten einige der kleineren geometrischen Formen approximiert werden. Abbildung 2-10 zeigt das fertig vernetzte Modell des Schaumstoffes. Die Schale wurde als zweidimensionales Objekt mit Verbindung zum Schaumstoff-Volumenmodell durch Knotenäquivalenz dargestellt. Die Helmschale ist dabei durch Viereckselemente und einige wenige Dreieckselemente modelliert.

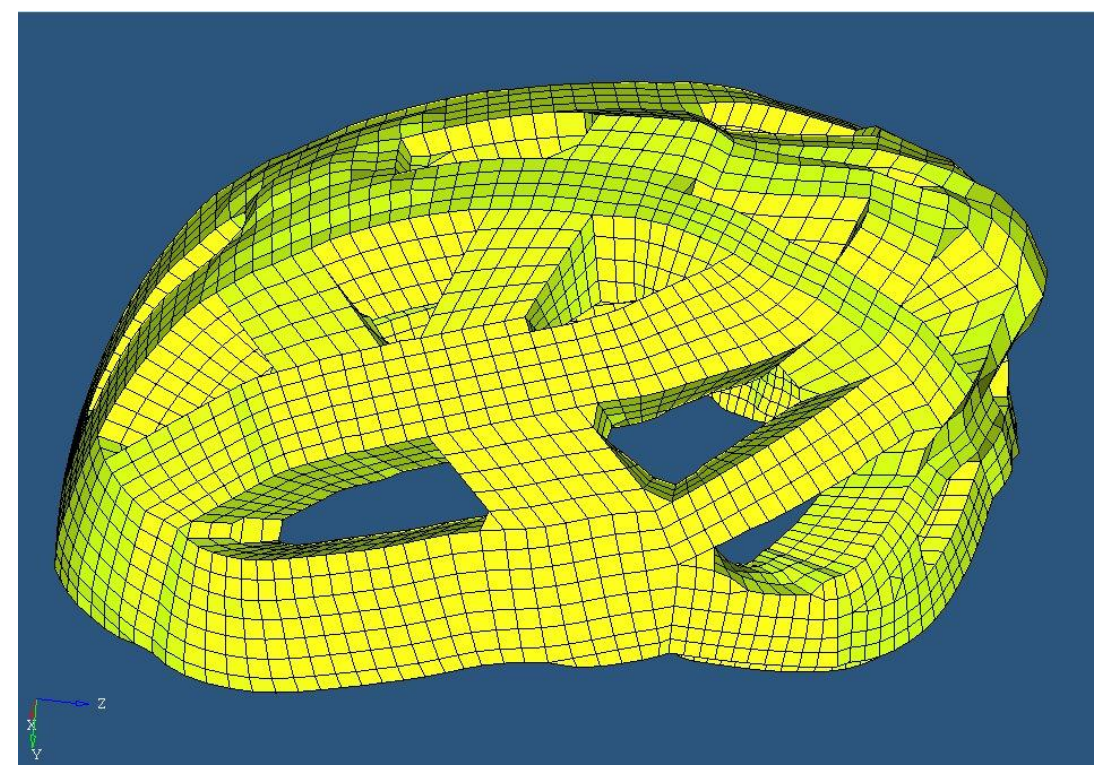

Abbildung 2-10: Netzgeometrie des Schaumstoffs des ausgewählten Fahrradhelms

Abbildung 2-11 enthält die Darstellung beider Helmkomponenten im Finite-Elemente-Netz im Vergleich zur geometrischen Darstellung. 

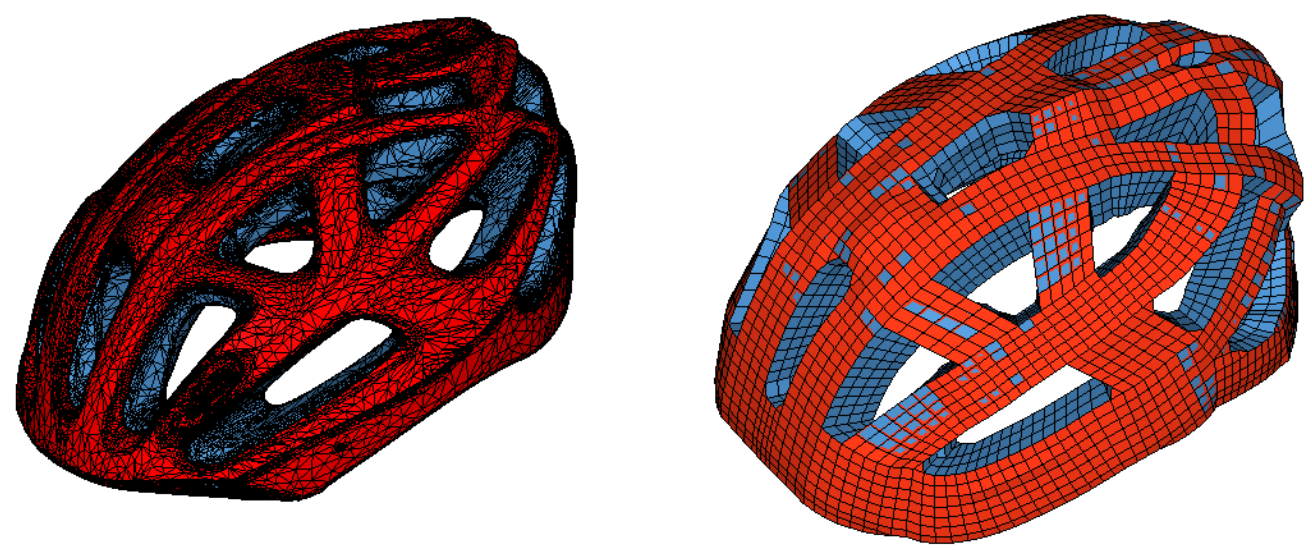

$\sum_{x}^{2}$

Abbildung 2-11: Geometrische Darstellung und FE-Modell

\subsubsection{Entwicklung und Validierung der Materialbeschreibung des Helmmodells}

Die Entwicklung der Materialbeschreibung des Helmmodells wurde zunächst mit dem Fokus auf der Charakterisierung des Absorptionsschaums durchgeführt, da diese Komponente die im biomechanischen Sinne mit Abstand wichtigste ist.

Neben dem Absorptionsschaum ist auch die Helmschale durch eine Materialbeschreibung charakterisiert worden. Hier wurde auf eine linear-elastische Materialmodellierung als Annäherung des Verhaltens eines thermoplastischen Kunststoffs zurückgegriffen. Der dabei entstehende Fehler dürfte sich in Grenzen halten, da nach Studien mit Beteiligung des Instituts für Rechtsmedizin selbst von faserverstärkten Kunststoffschalen im Motorradbereich weniger als ein Fünftel der absorbierten Energie beim Anprall aufgenommen wird. Bei den hier betrachteten Anprallvorgängen mit Fahrradhelmen dürfte dieser Anteilswert deutlich unterschritten werden, so dass der Verzicht auf aufwändige Materialprüfungen der Helmschale vertretbar ist. Im Übrigen wären diese kaum auf dem Wege des sogenannten „Reverse Engineering“, d.h. ohne Einbeziehung des Herstellers möglich.

\subsubsection{Entwicklung einer Materialbeschreibung für quasi-statische Belastung}

Grundlage für die Materialmodellierung von Schaumstoffen, die auf Kompression belastet werden, ist eine im Kompressionsversuch ermittelte Spannungs-Dehnungs-Kurve. Für deren Ermittlung wurden im vorliegenden Fall kleine quaderförmige Proben aus dem Schaumstoff geschnitten und diese quasi-statischen Kompressionsversuchen unterzogen. Abbildung 2-12 enthält die dabei gewonnenen Spannungs-Dehnungs-Kurven der ersten drei Proben. Diese zufällig ausgewählten Proben wurden aus demselben Helm, aber unterschiedlichen Bereichen geschnitten. Der Schaumstoff zeigt dabei im Bereich bis ca. 8\% Dehnung ein nahezu linear-elastisches Verhalten. Ab etwa 40\% Dehnung ist nach Abbildung 2-12 von einer zunehmenden Verdichtung bzw. Verblockung auszugehen. Dabei zeigte die Probe 3 ein deutlich anderes Verhalten als die Proben 1 und 2 mit ihren fast identischen Messergebnissen. 
Um dieses unterschiedliche Verhalten im quasi-statischen Kompressionstest besser zu verstehen, wurden neun weitere zufällig ausgewählte Proben getestet. Die daraus gewonnenen Spannungs-Dehnungskurven sind auf Abbildung 2-13 dargestellt. Auch diese Kurven zeigen kein identisches Verhalten, bewegen sich jedoch in einem relativ engen Korridor. Es wurde zusätzlich die Dichte der einzelnen Proben vor dem Kompressionstest gemessen. Die Werte waren annähernd gleich (ca. $0,078 \mathrm{~g} / \mathrm{cm}^{3}$ ). Somit ist anzunehmen, dass das Material annähernd homogen, aber nicht isotrop ist.

Die Materialmodellierung basiert auf den oben dargestellten Untersuchungsergebnissen.

Dazu wurden die Spannungs-Dehnungs-Kurven approximiert und in der Materialdefinition für LSDyna als Ausgangskurve verwendet.

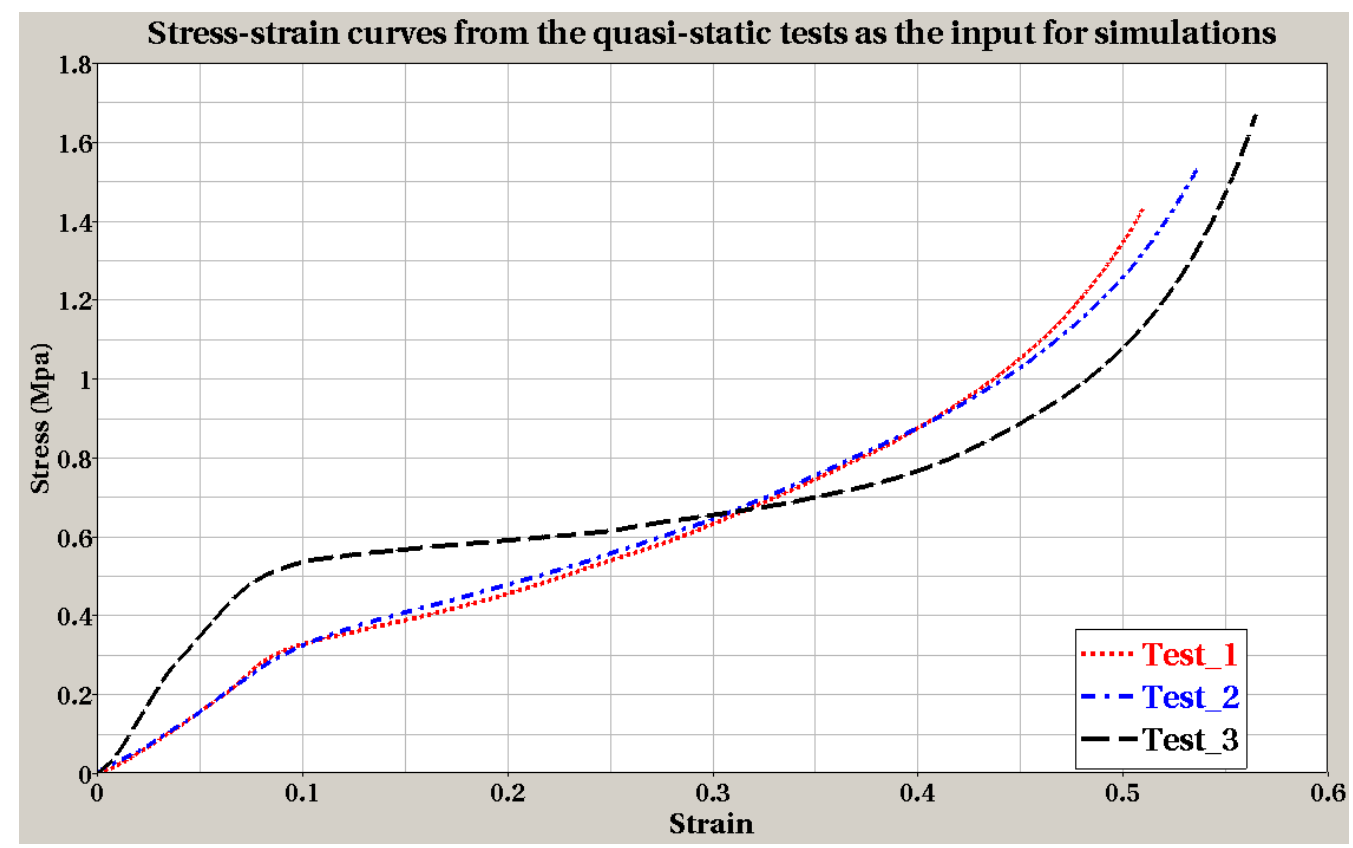

Abbildung 2-12: Spannungs-Dehnungskurven der ersten drei quasi-statischen Kompressionsversuche 


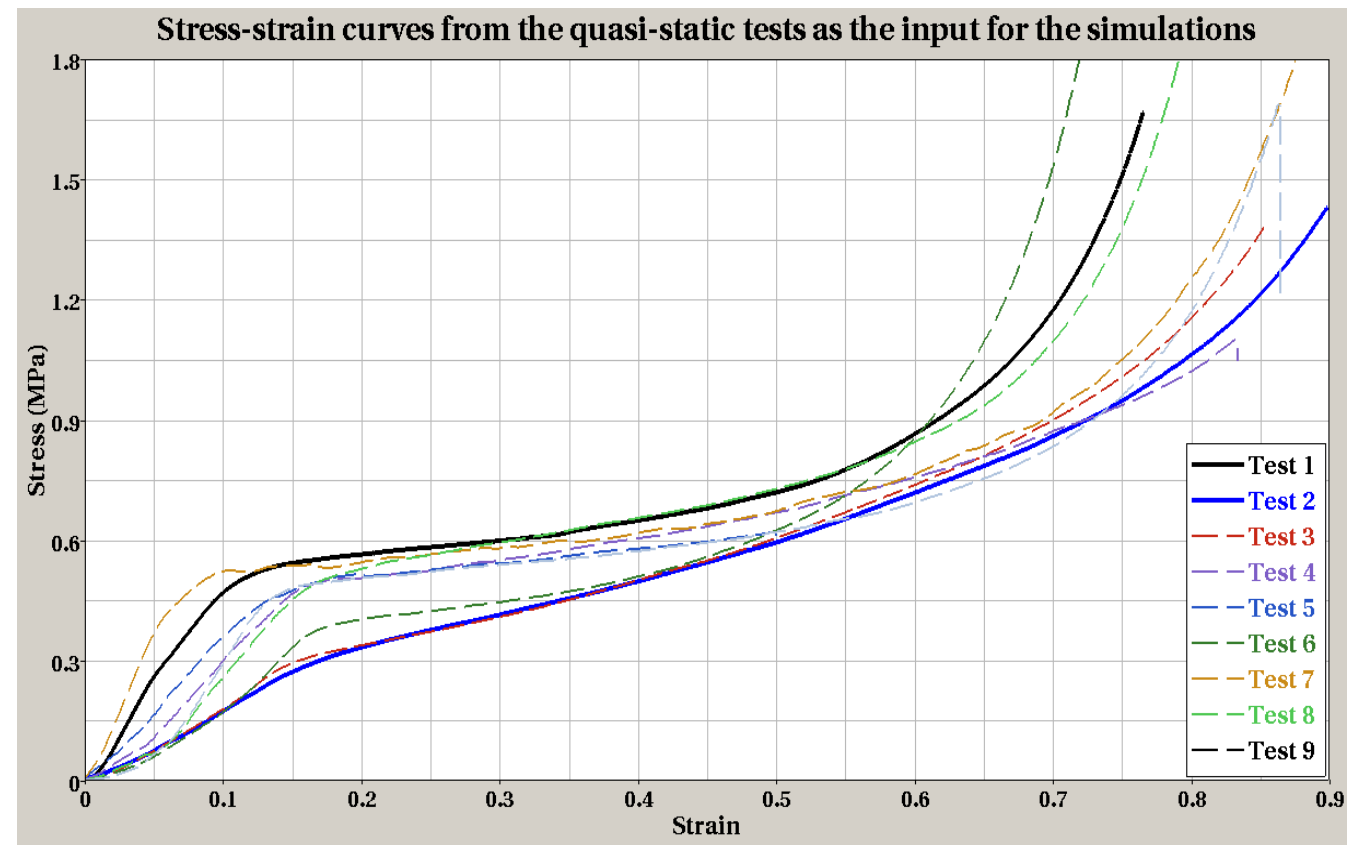

Abbildung 2-13: Spannungs-Dehnungskurven der weiteren neun quasi-statischen Kompressionsversuche

\subsubsection{Dynamische Materialprüfung und -modellierung}

Es ist davon auszugehen, dass der vorliegende Schaumstoff eine nicht zu vernachlässigende Dehnratenabhängigkeit im Materialverhalten unter Kompression aufweist. Um diese Charakteristik gezielt zu untersuchen, wurden dynamische Kompressionsversuche durchgeführt. Dazu wurden erneut Proben aus dem Helm geschnitten. Die Form und Lage dieser Proben sind in Abbildung 2-14 dargestellt. Diese Proben wurden mittels Fallversuch dynamisch auf Kompression mit einem sphärisch geformten Impaktor belastet. Abbildung 2-16 bis Abbildung 2-19 (S. 110 -111) geben die Sequenz eines solchen Versuches wieder, wie er in der Simulation nachgestellt wurde. 


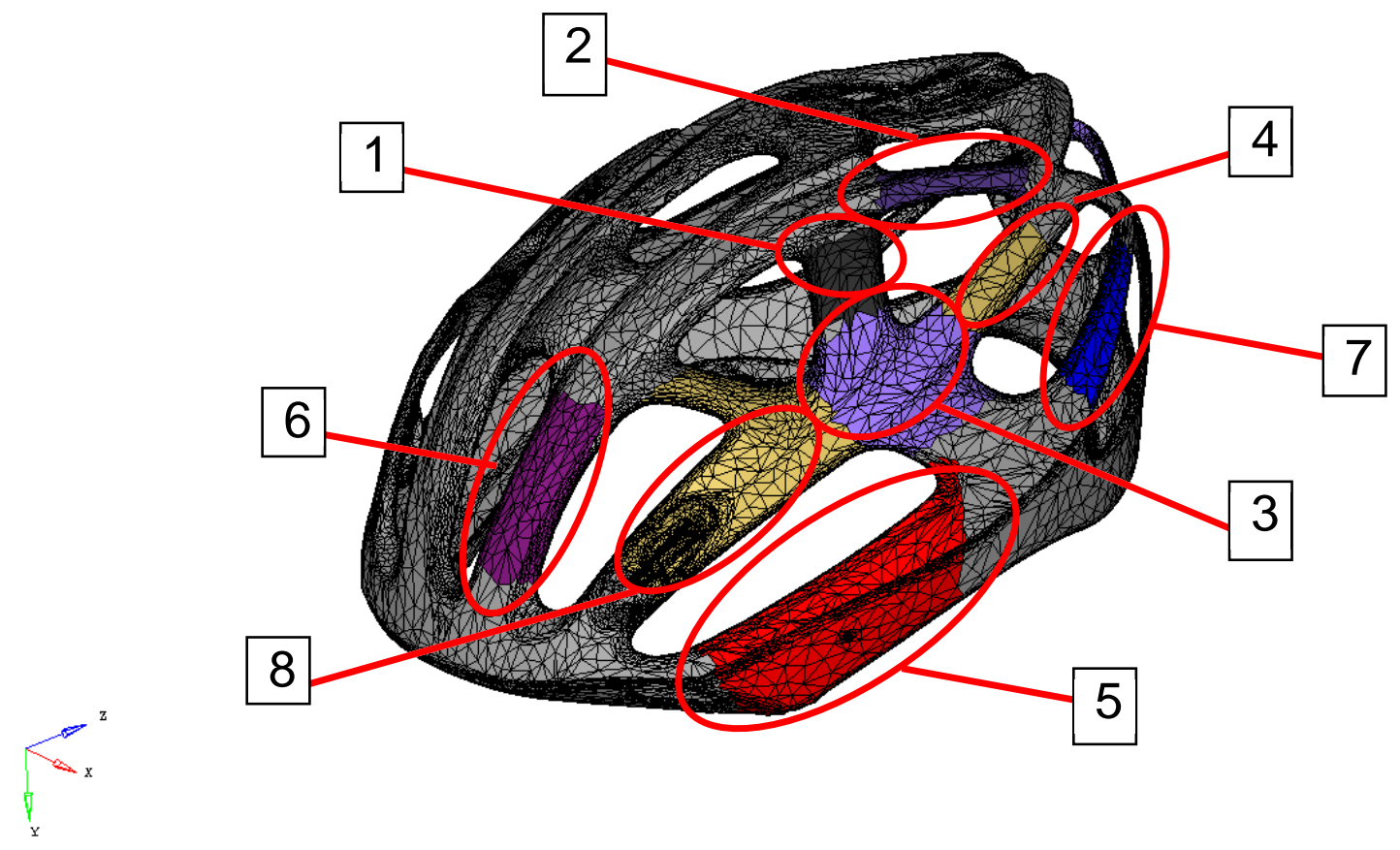

Abbildung 2-14: Form und Lage der Probenentnahme für die dynamischen Kompressionsversuche

Auf Abbildung 2-15 ist der Versuchsaufbau der dynamischen Materialprüfung abgebildet.

Der Impaktor wird aus einer definierten Höhe geführt auf die Materialprobe fallengelassen, die sich auf einem Sockel befindet und frei beweglich ist.

Es wurden zwei verschiedene Impaktoren verwendet, beide sind zylinderförmig mit einer aufgesetzten Halbkugel als Kopf. Der Radius der Halbkugel beträgt jeweils $50 \mathrm{~mm}$. Der leichtere Impaktor wiegt 960 Gramm und hat eine Länge des Zylinders von $150 \mathrm{~mm}$. Der schwerere Impaktor wiegt 2960 Gramm und hat eine Zylinderlänge von 200 mm.

Die einzelnen Proben wurden markiert und sorgfältig aus dem Helm entnommen. Die Proben wurden anschließend so auf dem Sockel positioniert, dass der Impaktor in das Zentrum traf. 


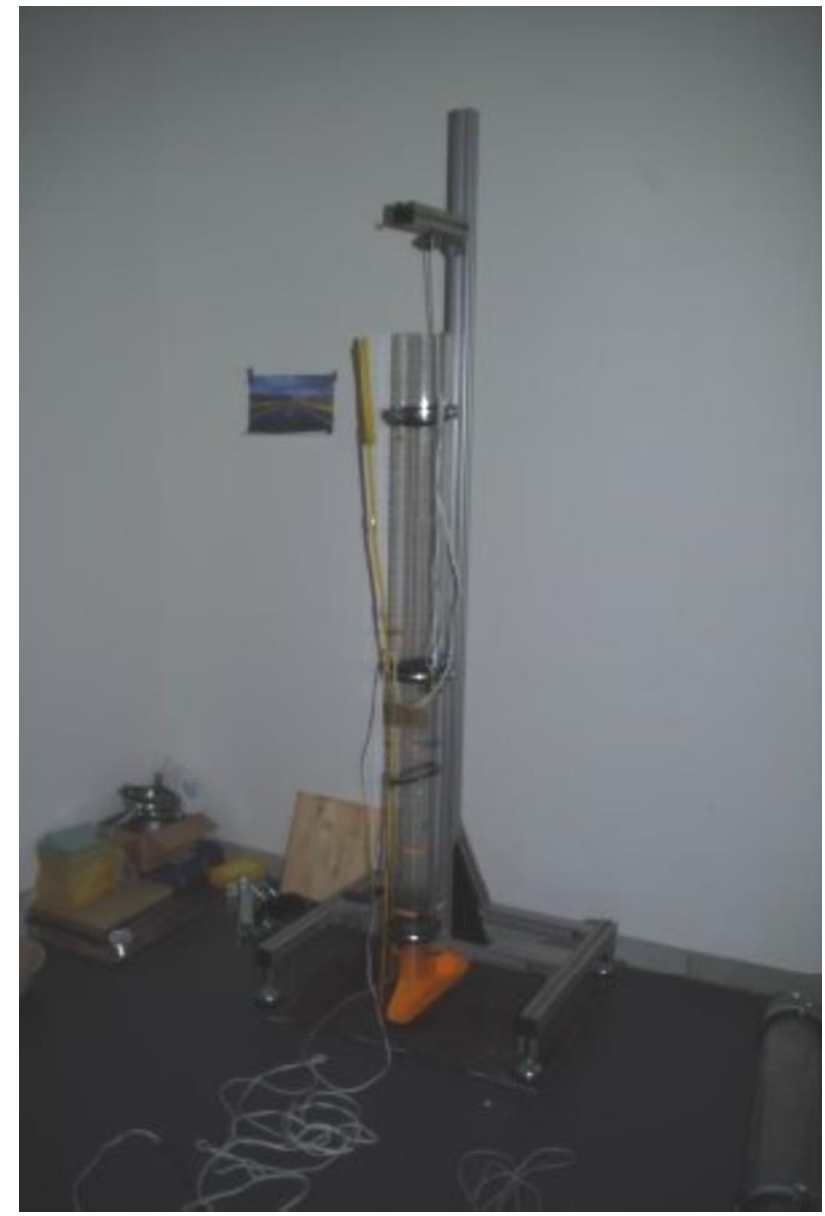

Abbildung 2-15: Versuchsaufbau der dynamischen Materialprüfung

Folgende Tabelle 2-15 zeigt den Versuchsablauf der getesteten Proben.

Tabelle 2-15: Versuchsablauf der dynamischen Materialprüfung

\begin{tabular}{|c|c|c|c|}
\hline Nummer & $\begin{array}{c}\text { Gewicht } \\
\text { Impaktor }\end{array}$ & Fallhöhe & Aufprallgeschwindigkeit \\
\hline 1 & $2960 \mathrm{~g}$ & $160 \mathrm{~cm}$ & $5,6 \mathrm{~m} / \mathrm{s}$ \\
\hline 2 & $2960 \mathrm{~g}$ & $125 \mathrm{~cm}$ & $4,95 \mathrm{~m} / \mathrm{s}$ \\
\hline 3 & $2960 \mathrm{~g}$ & $100 \mathrm{~cm}$ & $4,42 \mathrm{~m} / \mathrm{s}$ \\
\hline 4 & $2960 \mathrm{~g}$ & $80 \mathrm{~cm}$ & $3,96 \mathrm{~m} / \mathrm{s}$ \\
\hline 5 & $2960 \mathrm{~g}$ & $150 \mathrm{~cm}$ & $5,42 \mathrm{~m} / \mathrm{s}$ \\
\hline 6 & $960 \mathrm{~g}$ & $160 \mathrm{~cm}$ & $5,6 \mathrm{~m} / \mathrm{s}$ \\
\hline 7 & $960 \mathrm{~g}$ & $150 \mathrm{~cm}$ & $5,42 \mathrm{~m} / \mathrm{s}$ \\
\hline 8 & $960 \mathrm{~g}$ & $125 \mathrm{~cm}$ & $4,95 \mathrm{~m} / \mathrm{s}$ \\
\hline
\end{tabular}


110

Contour Plot

Stress(vonMises, Max)

Time $=0.000000$

Global System

Advanced Average

$-0.000 \mathrm{E}+00$

$-0.000 E+00$

$-0.000 \mathrm{E}+00$

$-0.000 E+00$

$-0.000 \mathrm{E}+00$

$-0.000 E+00$

$-0.000 E+00$

$-0.000 \mathrm{E}+00$

$\mathrm{Max}=0.000 \mathrm{E}+00$

Node 26463

Min $=0.000 \mathrm{E}+$
Node 26463
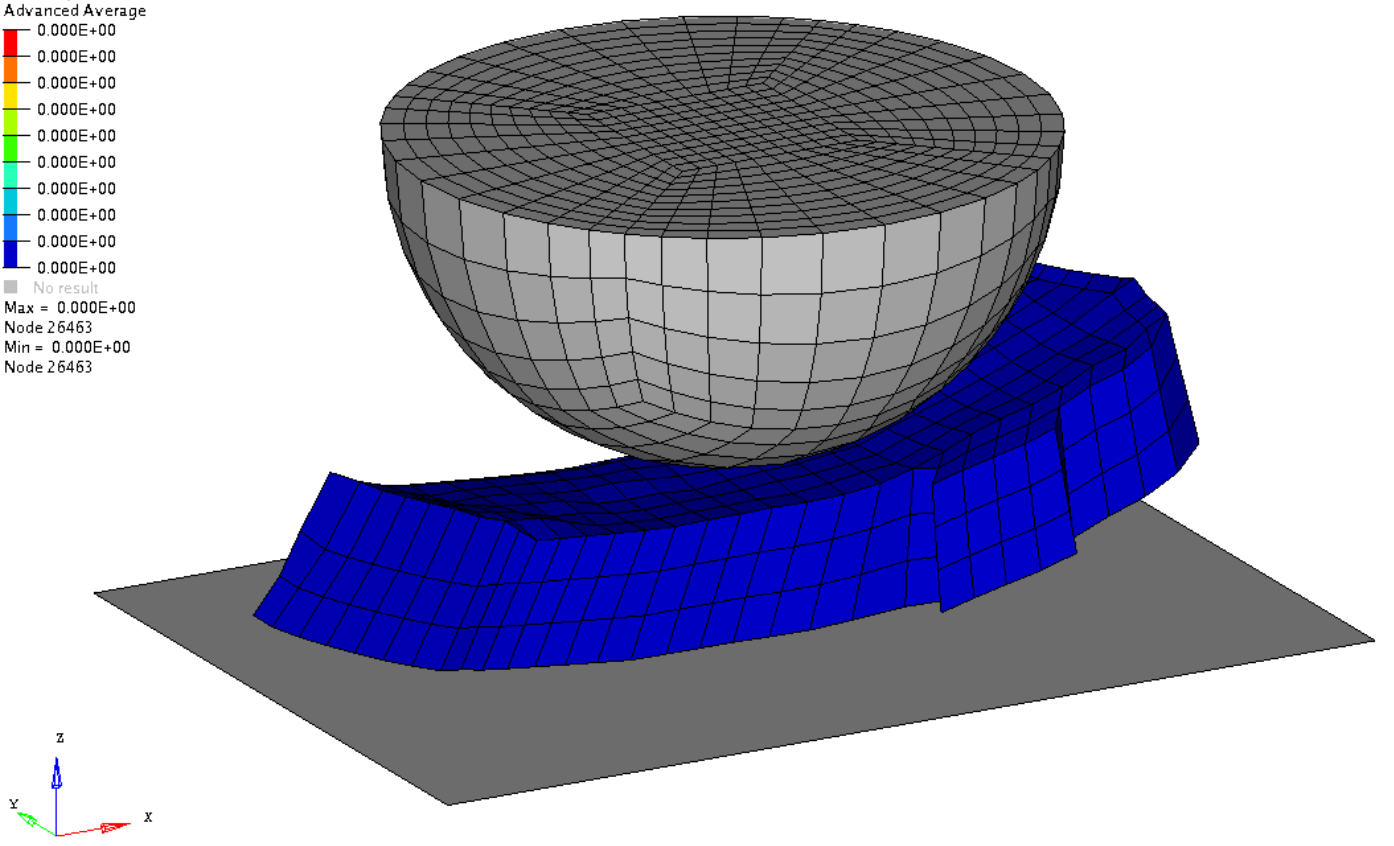

Abbildung 2-16: Dynamischer Kompressionsversuch Phase 1

Contour Plot

Stress(vonMises, Max)

Global System

Advanced Average

- $1.872 \mathrm{E} \cdot 03$

- 1.664E-03

$-1.456 \mathrm{E} \cdot 03$
$-1248 \mathrm{E} .03$

- $1.040 \mathrm{E} \cdot 03$

$1.040 \mathrm{E} \cdot 03$
$-8.323 \mathrm{E} \cdot 04$

- 6.244E.04

- 4.164E.04

$-2.084 \mathrm{E} \cdot 04$
$4.202 \mathrm{E} \cdot 07$

Max $=1.872 \mathrm{E} \cdot 03$

Node 27113

Min $=4.202 \mathrm{E} \cdot 07$
Node 37882

Time $=0.799991$ 


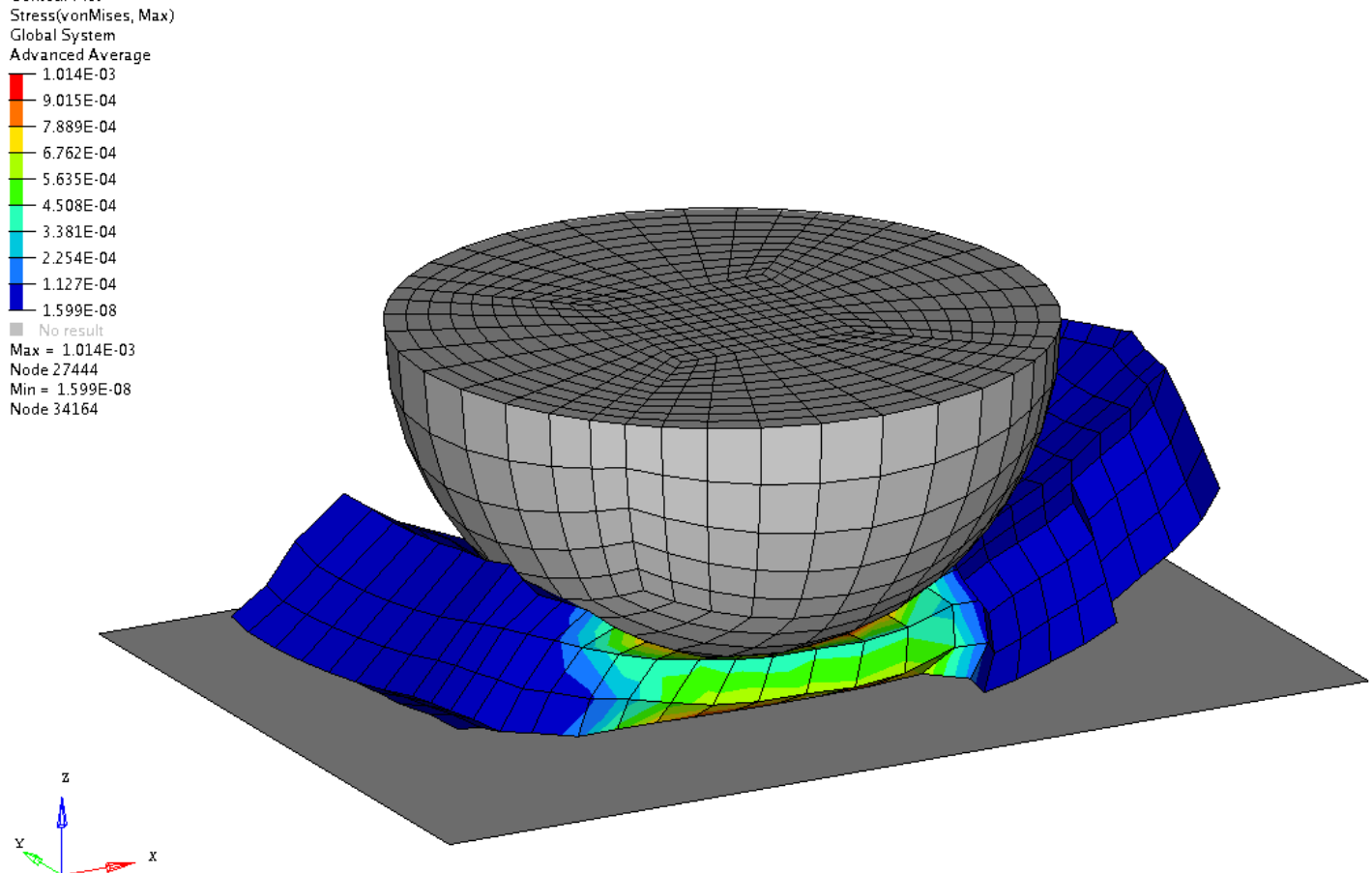

Abbildung 2-18: Dynamischer Kompressionsversuch Phase 3

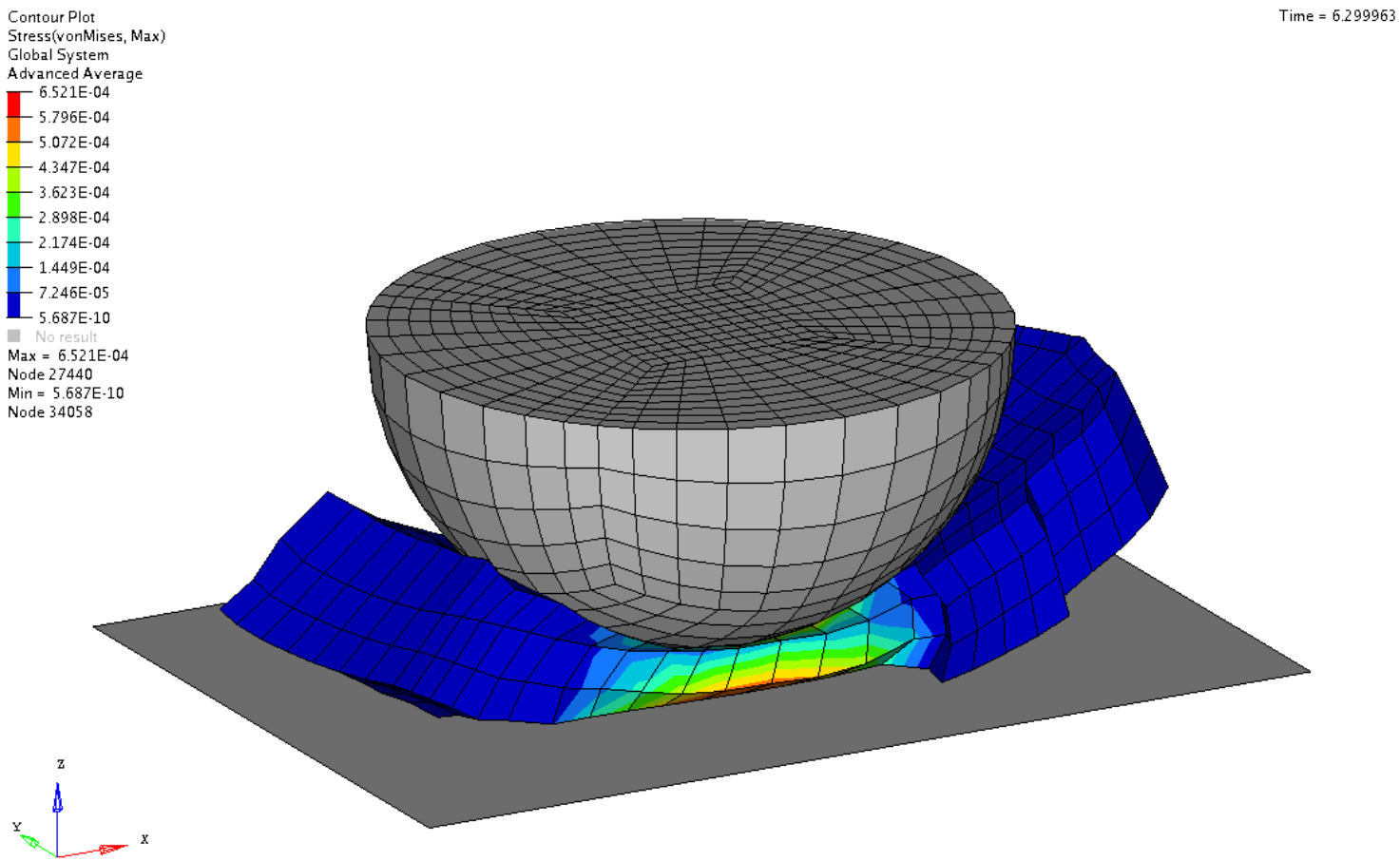

Abbildung 2-19: Dynamischer Kompressionsversuch Phase 4

Wie aus Tabelle 2-15 zu ersehen, wurden die dynamischen Versuche bei unterschiedlichen Fallhöhen und mit unterschiedlichen Fallgewichten durchgeführt. Dadurch ergeben sich gewisse Bandbreiten an anfänglichen Dehnraten und an Dehnratenverläufen. Basierend auf der Ausgangskurve wurde die Materialbeschreibung des Simulationsmodells um Skalierungen für verschiedene Dehnraten erweitert. Dieser iterative Prozess wurde so lange 
ausgeführt, bis eine ausreichende Übereinstimmung zwischen experimentell ermitteltem und berechnetem Kraft-Zeit-Verlauf für mehrere Versuche erlangt wurde. Abbildung 2-20 bis Abbildung 2-24 enthalten die Kraft-Zeit-Verläufe aus Simulation und Versuch für verschiedene Aufprallgeschwindigkeiten und Impaktorgewichte. In den Darstellungen sind die durchgezogenen Linien Kraft-Zeit-Kurven, die gestrichelten Linien sind Kraft-WegKurven.

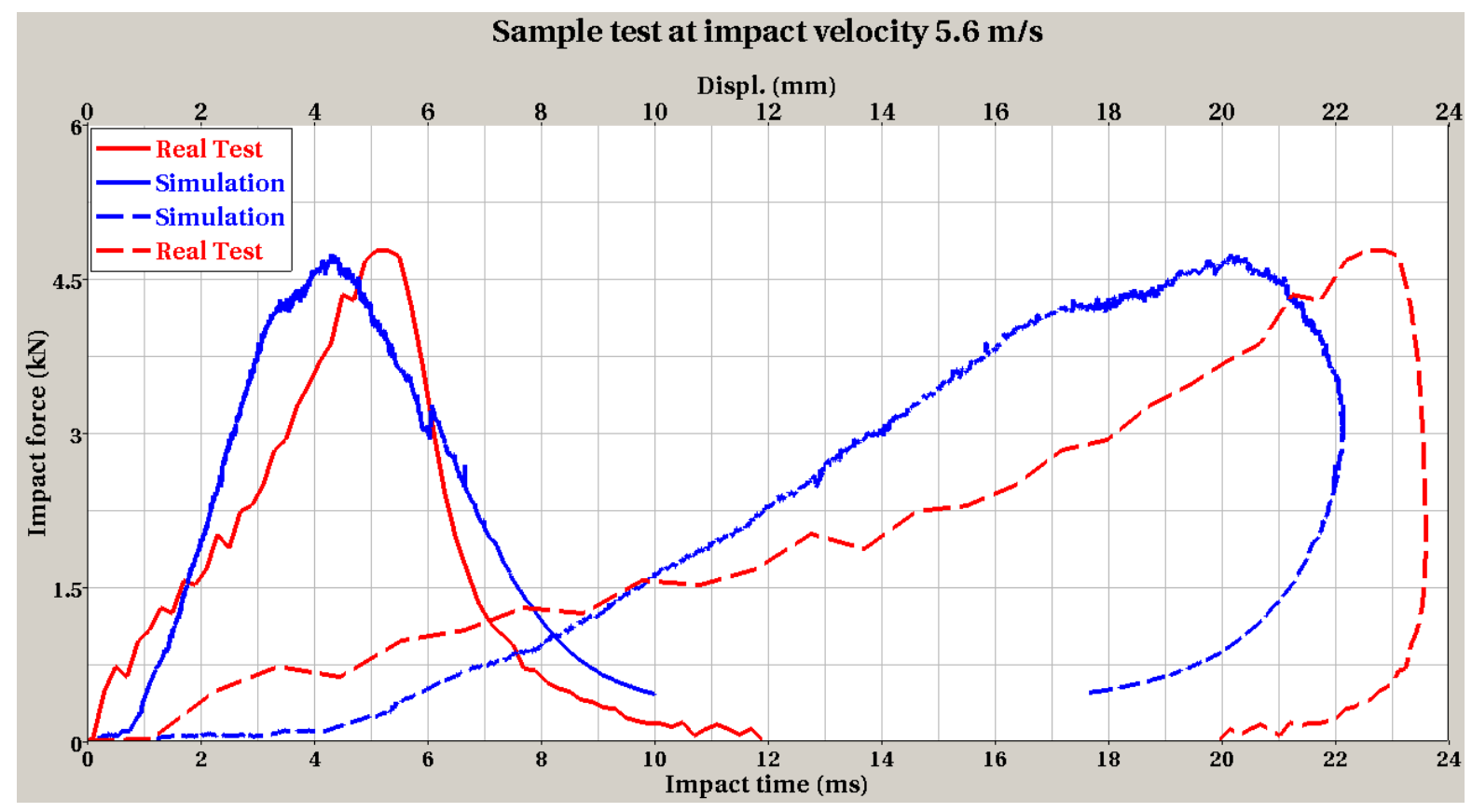

Abbildung 2-20: Vergleich der Kraft-Zeit-/Kraft-Weg-Verläufe aus Simulation und Versuch (Aufprallgeschwindigkeit 5,6 m/s, Impaktorgewicht $2960 \mathrm{~g}$ )

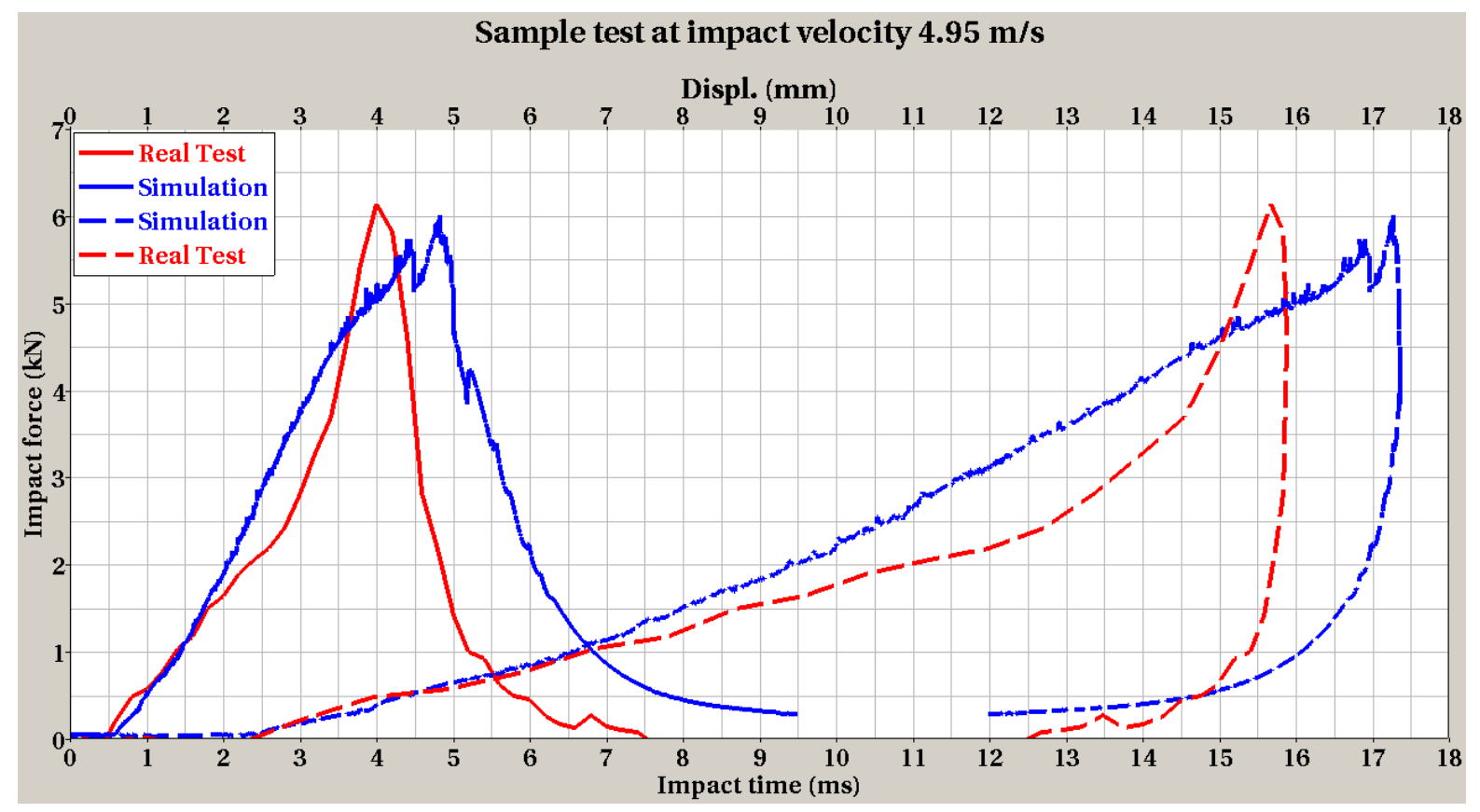

Abbildung 2-21: Vergleich der Kraft-Zeit-/Kraft-Weg-Verläufe aus Simulation und Versuch (Aufprallgeschwindigkeit 4,95 m/s, Impaktorgewicht $2960 \mathrm{~g}$ ) 


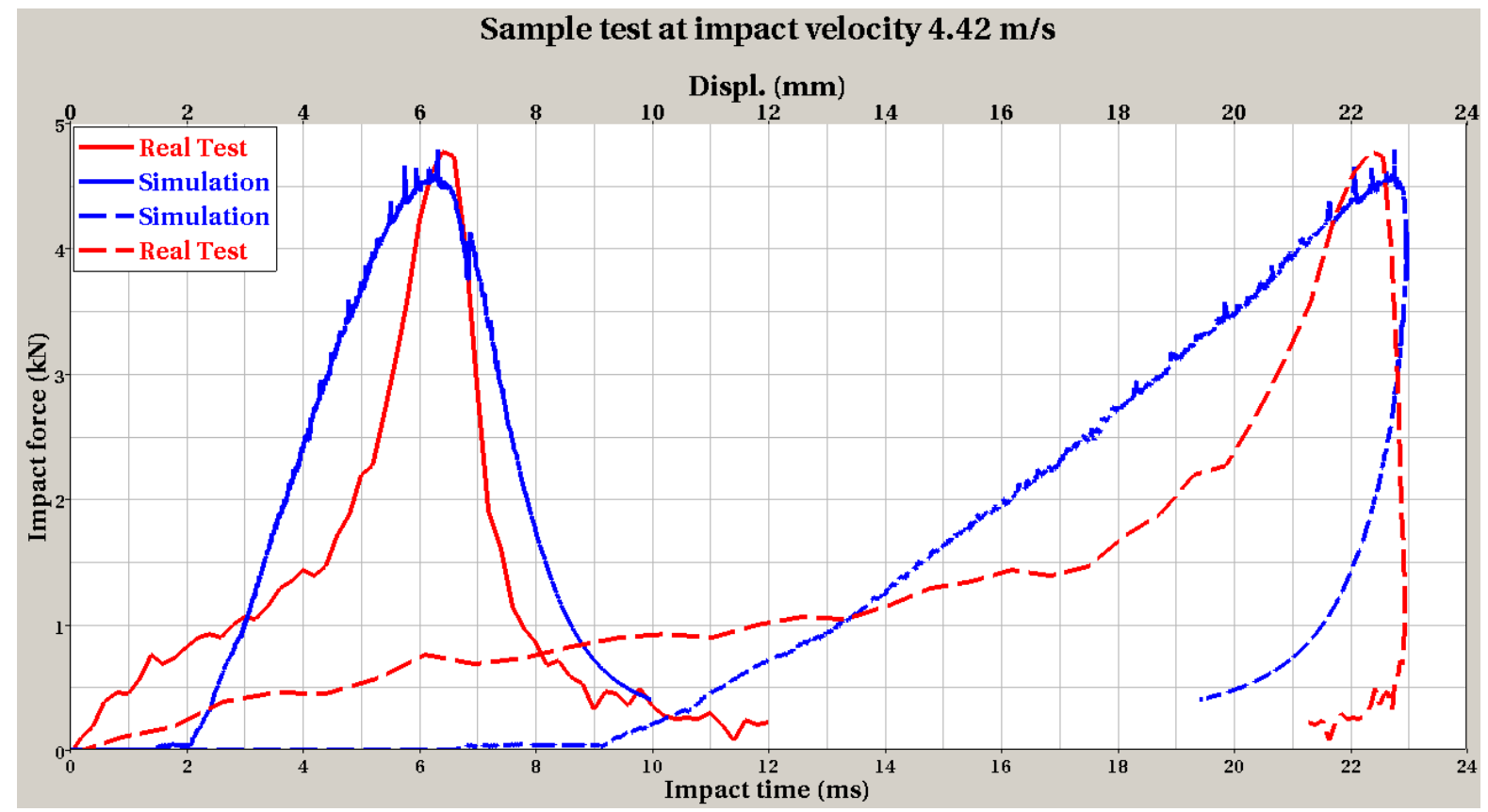

Abbildung 2-22: Vergleich der Kraft-Zeit-/Kraft-Weg-Verläufe aus Simulation und Versuch (Aufprallgeschwindigkeit 4,42 m/s, Impaktorgewicht $2960 \mathrm{~g}$ )

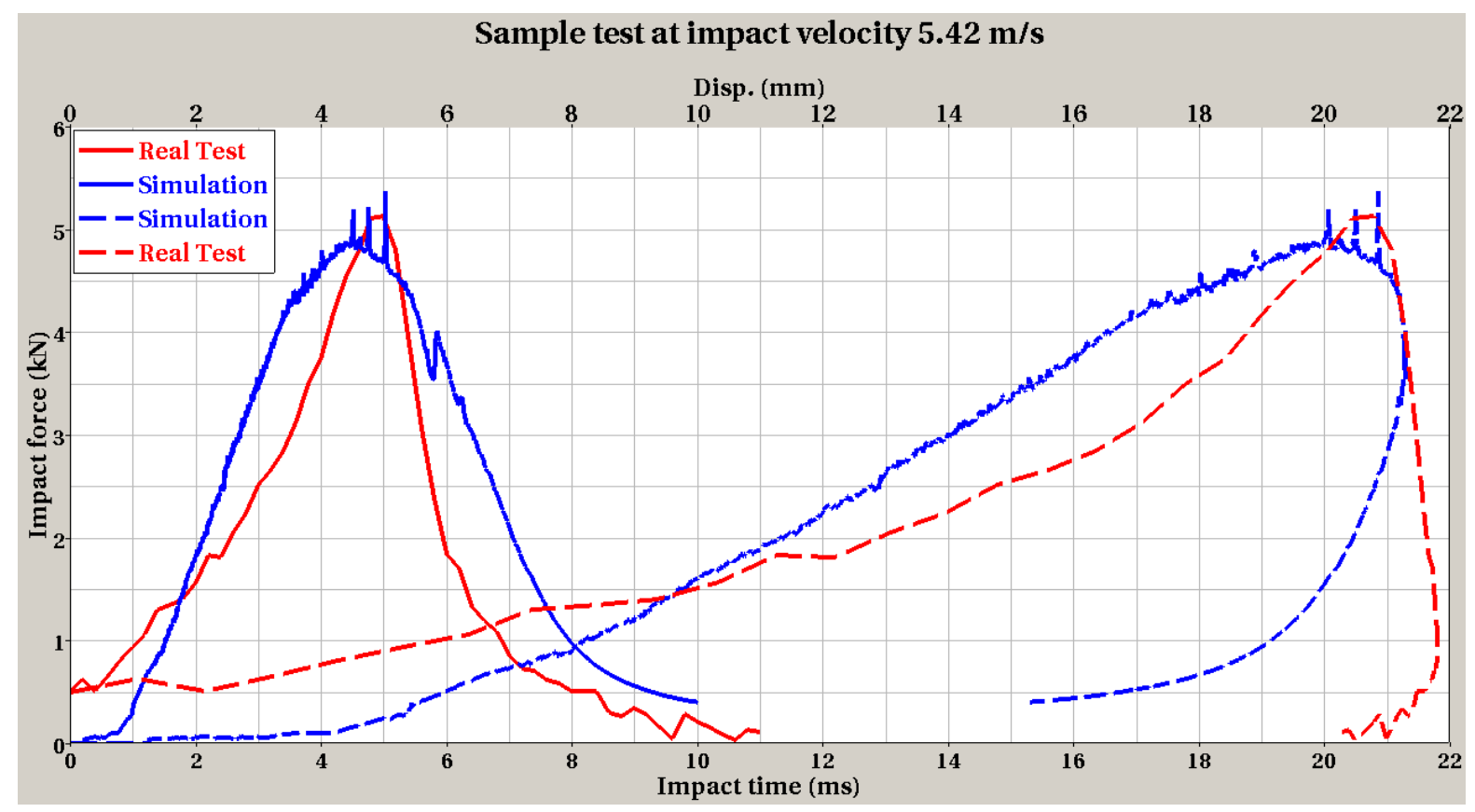

Abbildung 2-23: Vergleich der Kraft-Zeit-/Kraft-Weg-Verläufe aus Simulation und Versuch (Aufprallgeschwindigkeit 5,42 m/s, Impaktorgewicht $2960 \mathrm{~g}$ ) 


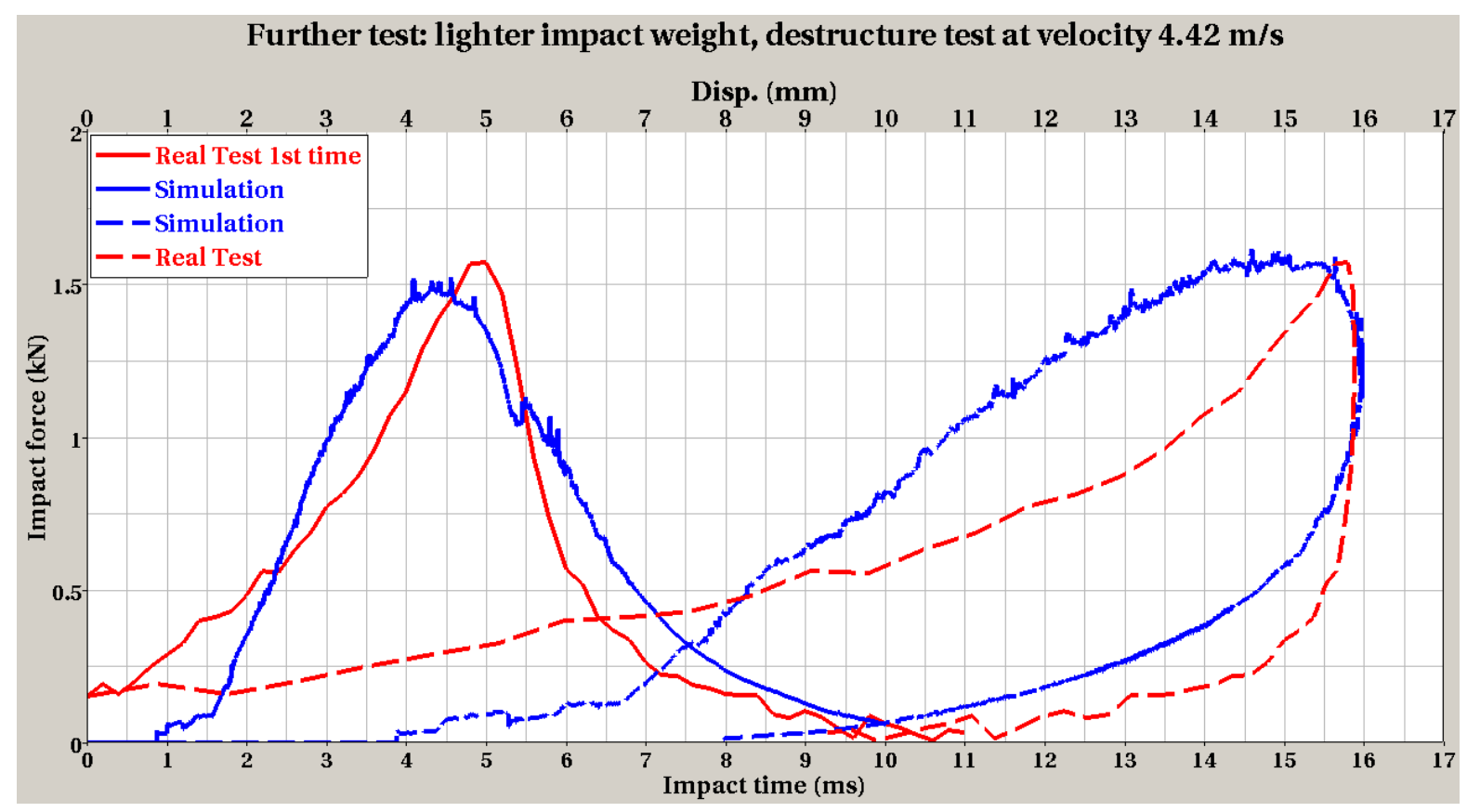

Abbildung 2-24: Vergleich der Kraft-Zeit-/Kraft-Weg-Verläufe aus Simulation und Versuch

(Aufprallgeschwindigkeit 4,42 m/s, Impaktorgewicht $960 \mathrm{~g}$ )

Es lässt sich in diesen Abbildungen eine Abhängigkeit der Kurvenverläufe von der Geschwindigkeit bei sonst gleichen Parametern erkennen. Diese Veränderung mit der Geschwindigkeit stellt sich in Simulation und Versuch in etwa gleich dar und wird von den Autoren als prioritär gegenüber absoluten Messwerten angesehen, insbesondere in dem hier dargestellten, für die Untersuchung von Standardlastfällen wichtigen

Geschwindigkeitsbereich. Die Maximalkräfte und damit auch die maximale Beschleunigung werden im Vergleich der Simulation zum Versuch minimal unterschätzt, während die zeitliche Ausdehnung des Anpralls in der Simulation überschätzt wird.

Zudem sind die ansteigenden Kurvenverläufe bis zum Maximalwert leicht abweichend, was durch Rotation, Reibung und andere nicht zu kontrollierende Bewegungen vor dem tatsächlichen Aufprall zu erklären ist.

Beides hat naturgemäß Einfluss auf die Qualität später durchzuführender Betrachtungen der Belastungen des menschlichen Kopfes, die beim Anprall mit einem solchen Helmmodell auftreten würden.

Die Modellierungsgüte des Absorptionsschaums hat wie im vorigen Abschnitt dargestellt ein angemessenes Niveau erreicht. Um weitere Unsicherheiten nach Möglichkeit noch weiter auszuschließen, wurden Fallversuche in Anlehnung an EN 1078 als zusätzliche Grundlage für die Simulation herangezogen. Dazu wurden in Kooperation mit der Université de Strasbourg (Prof. Willinger / IMFS) Versuche an einem Fallprüfstand mit dem hier betrachteten Helm durchgeführt. Naturgemäß kommen bei einer Darstellung eines solchen Versuches in der Simulation vielerlei Fehlerquellen zusätzlich zu möglichen Ungenauigkeiten in der Materialmodellierung zum Tragen. Nichtsdestotrotz wurden diese Versuche als zusätzliche Überprüfung für die Modellierungsqualität herangezogen.

Unter kontrollierten Bedingungen, die denen der EN 1078 entsprechen, wurden mehrere Versuche durchgeführt (Aufprall seitlich, frontal und auf die Rückseite).

Auf den Abbildung 2-25 bis Abbildung 2-27 sind die Aufprallkonstellation, die Beschleunigungs-Zeit-Kurven und die Kraft-Weg-Kurven des seitlichen Aufpralls dargestellt, auf den Abbildung 2-28 bis Abbildung 2-30 die analogen Ergebnisse für den frontalen 
Aufprall und auf Abbildung 2-31 bis Abbildung 2-33 die analogen Ergebnisse für den Aufprall auf die Rückseite des Helms.

Die Abbildungen enthalten zusätzlich zu den Ergebnissen der Fallversuchte (in rot) die Ergebnisse, die die Simulationen der jeweiligen Versuchskonstellationen lieferten (in blau). Die Kurvenverläufe, die maximale Beschleunigung und die maximale Kraft sind für den frontalen und insbesondere den seitlichen Anprall sehr ähnlich. Eine Ausnahme stellt der Aufprall auf die Rückseite des Helms dar, hier sind teilweise recht deutliche Abweichungen zu erkennen. Ursache hierfür ist die komplexe geometrische Form und damit die in der Simulation schwierig abzubildende Rückseite des Helms.

Die erzielten Ergebnisse der Simulation unter Norm-Testbedingungen mit dem kompletten Helmmodell und einer Kopfform sind somit näher an der Realität als die Ergebnisse der Komponententests.

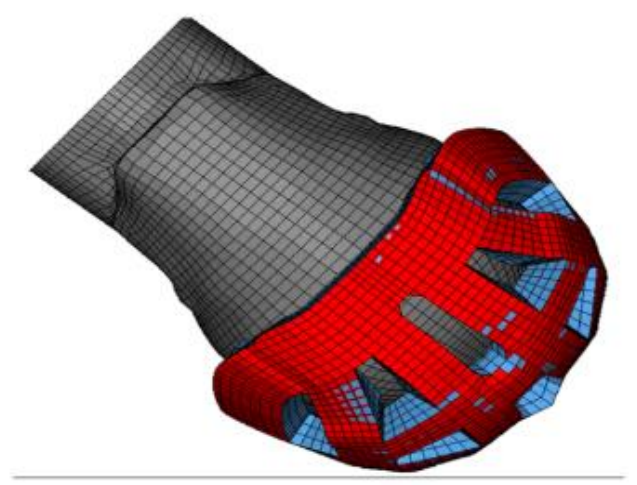

Abbildung 2-25: Aufprallkonstellation des seitlichen Aufpralls

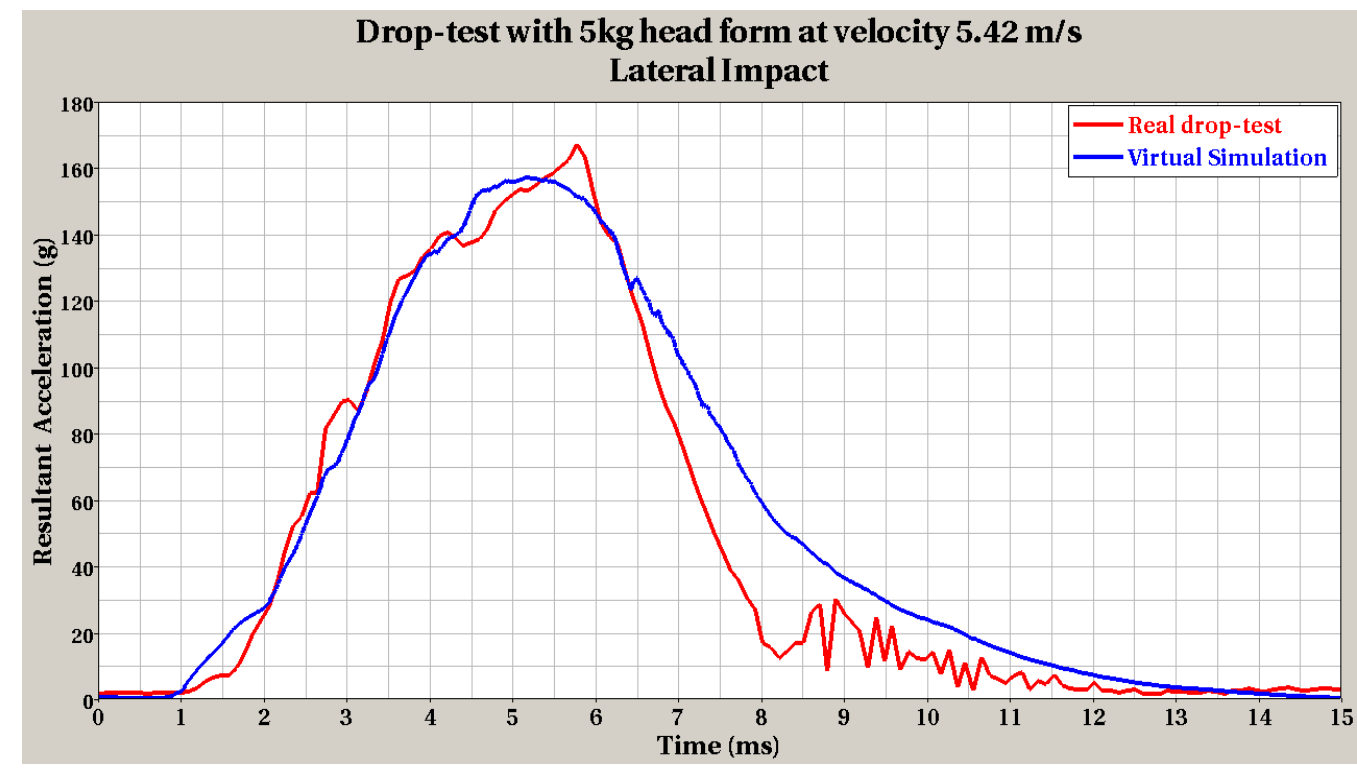

Abbildung 2-26: Vergleich der Beschleunigungs-Zeit-Kurven (seitlicher Aufprall, Gewicht der Kopfform 5 kg, Aufprallgeschwindigkeit 5,42 m/s) 


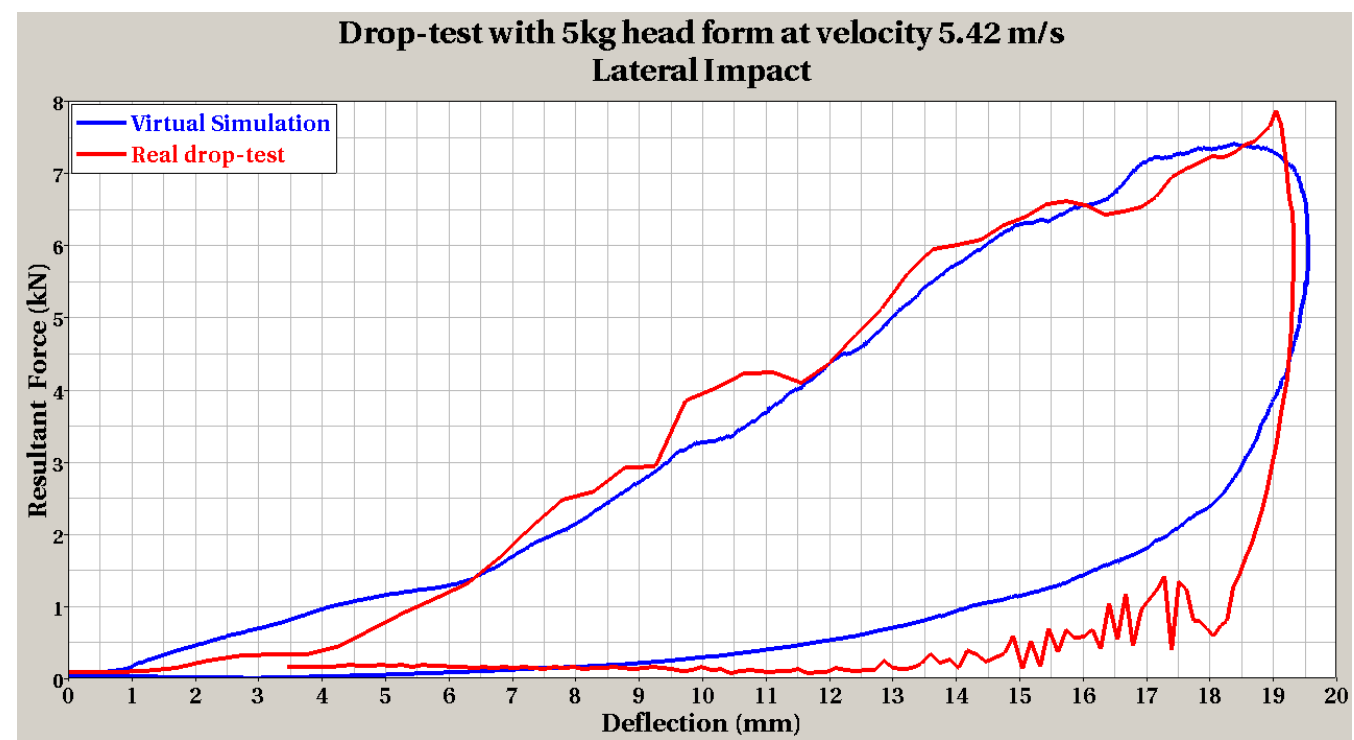

Abbildung 2-27: Vergleich der Kraft-Weg-Kurven (seitlicher Aufprall, Gewicht der Kopfform 5 kg, Aufprallgeschwindigkeit $5,42 \mathrm{~m} / \mathrm{s}$ )

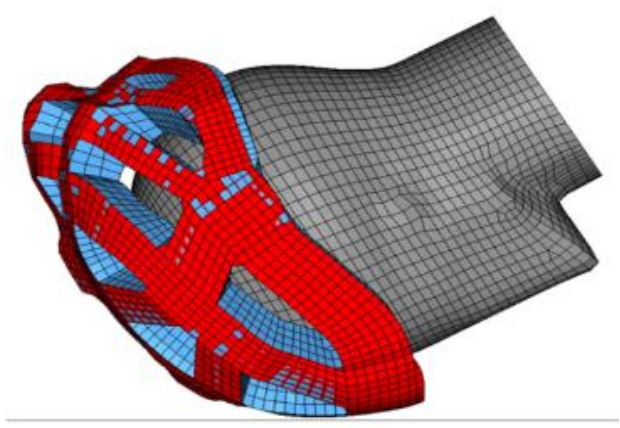

Abbildung 2-28: Aufprallkonstellation des frontalen Aufpralls

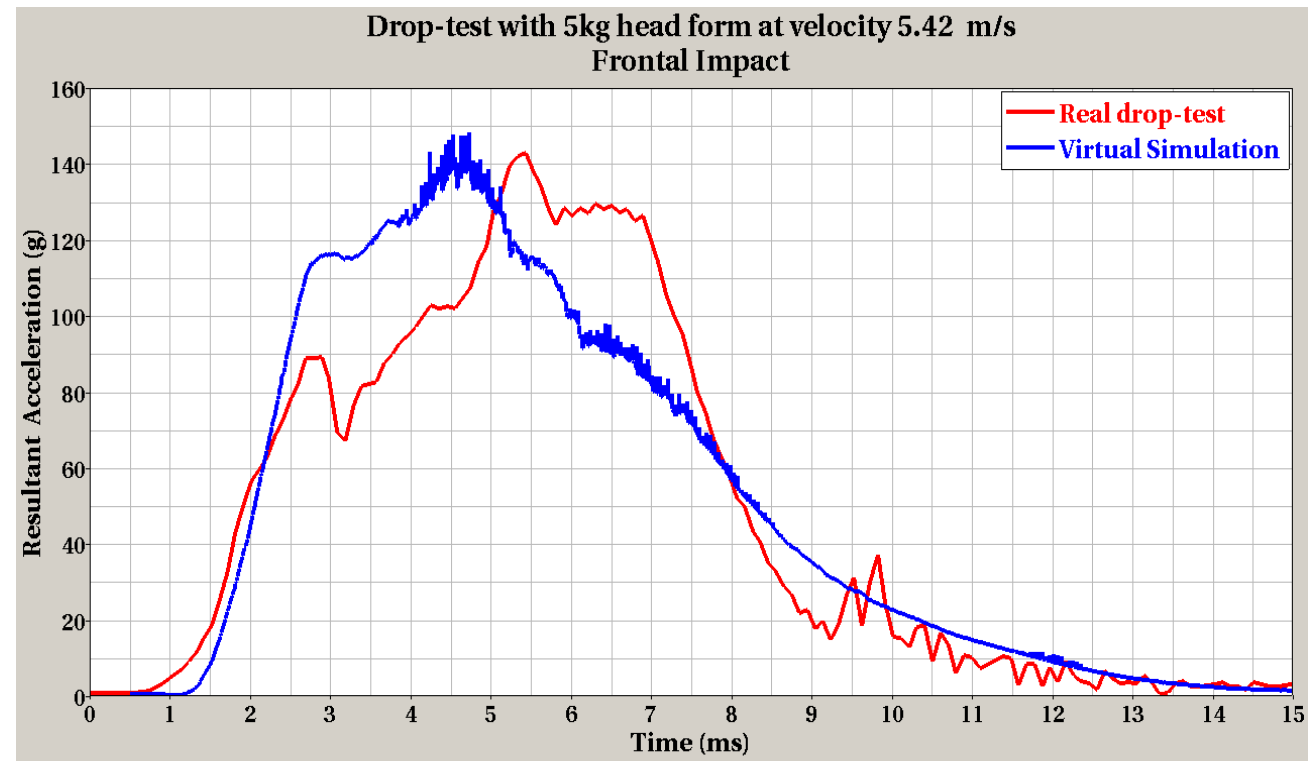

Abbildung 2-29: Vergleich der Beschleunigungs-Zeit-Kurven (frontaler Aufprall, Gewicht der Kopfform 5 kg, Aufprallgeschwindigkeit 5,42 m/s) 


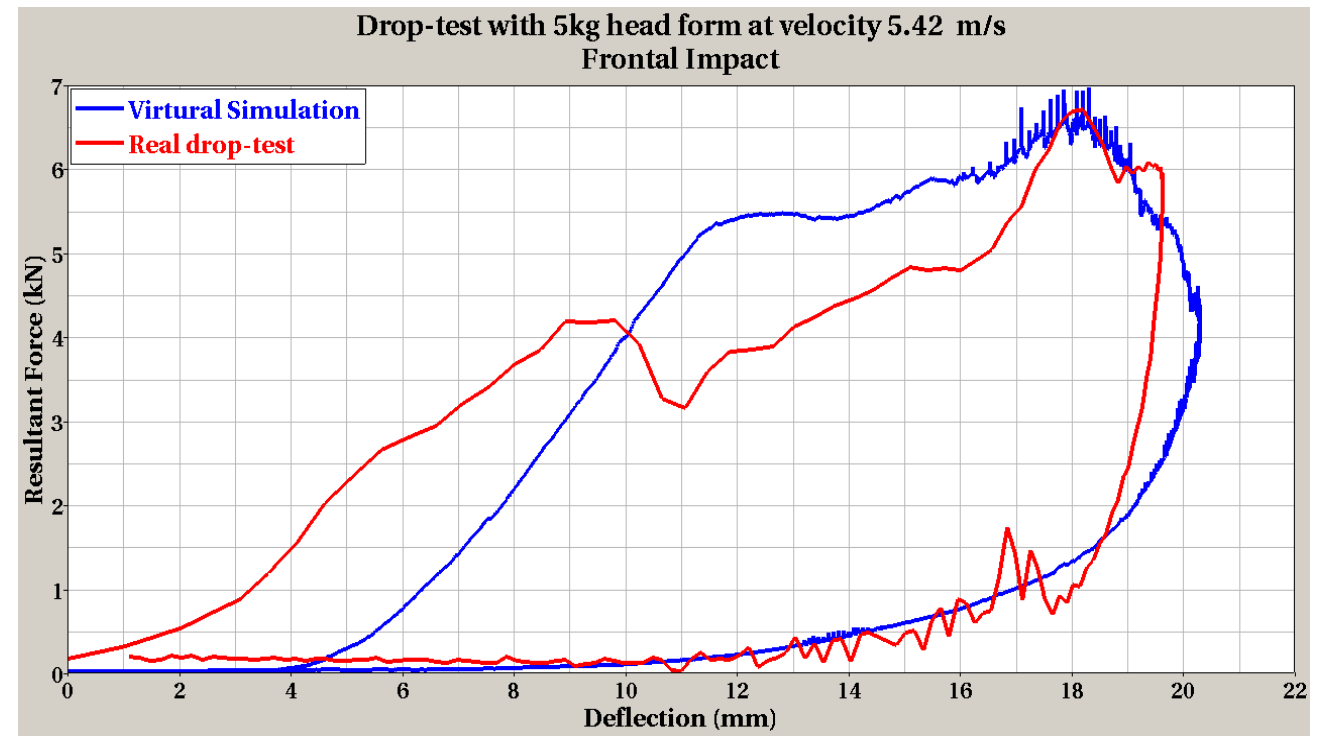

Abbildung 2-30: Vergleich der Kraft-Weg-Kurven (frontaler Aufprall, Gewicht der Kopfform 5 kg, Aufprallgeschwindigkeit 5,42 $\mathrm{m} / \mathrm{s}$ )

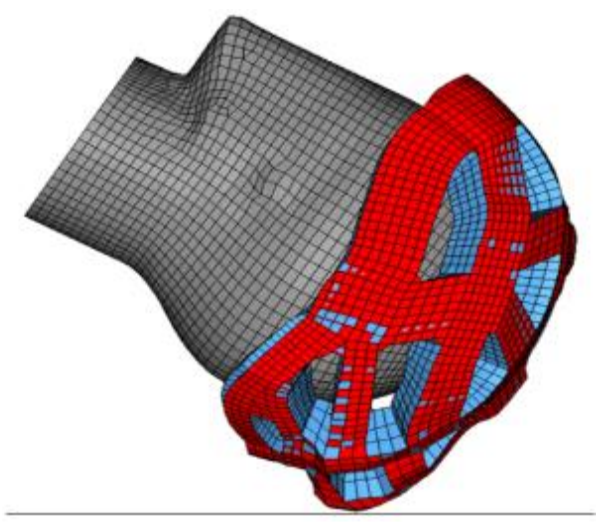

Abbildung 2-31: Aufprallkonstellation des Aufpralls auf die Rückseite

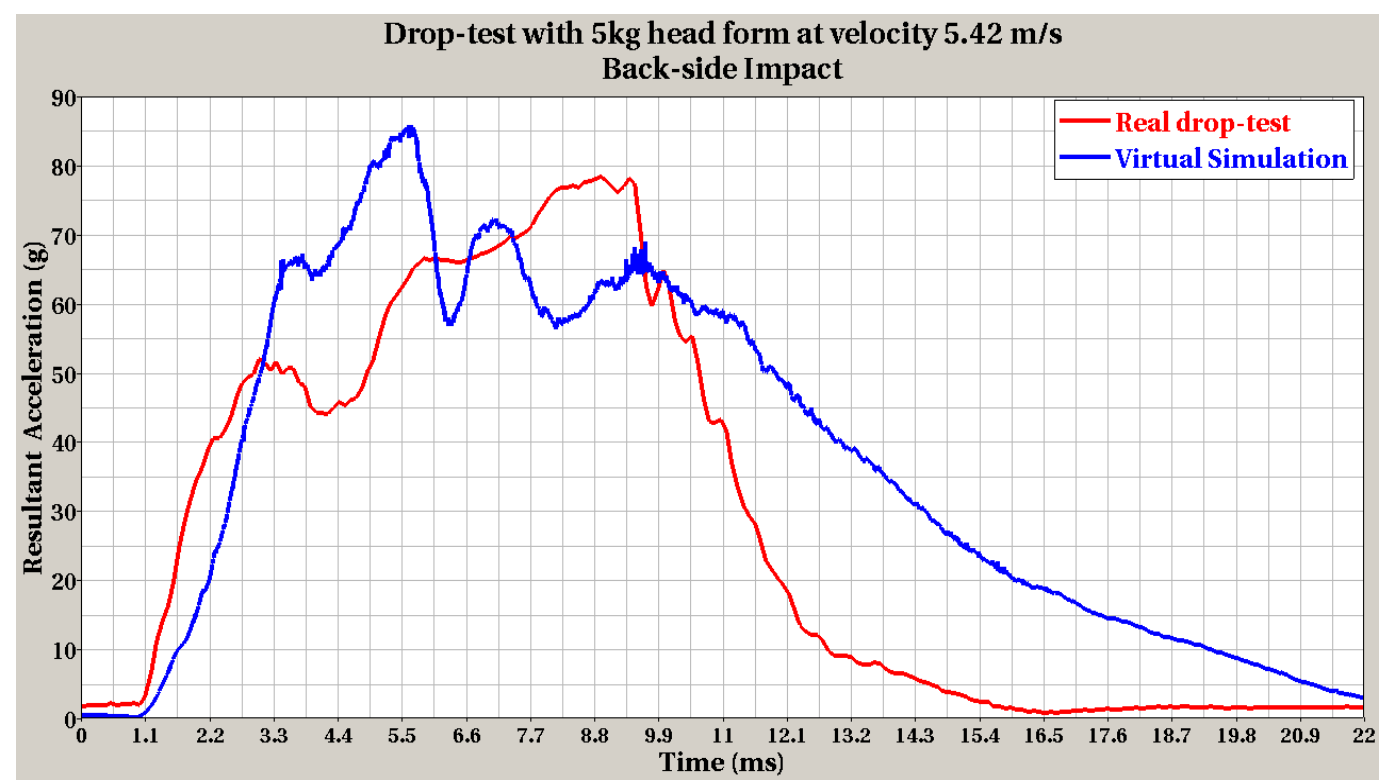

Abbildung 2-32: Vergleich der Beschleunigungs-Zeit-Kurven (Aufprall auf die Rückseite, Gewicht der Kopfform $5 \mathrm{~kg}$, Aufprallgeschwindigkeit 5,42 m/s) 


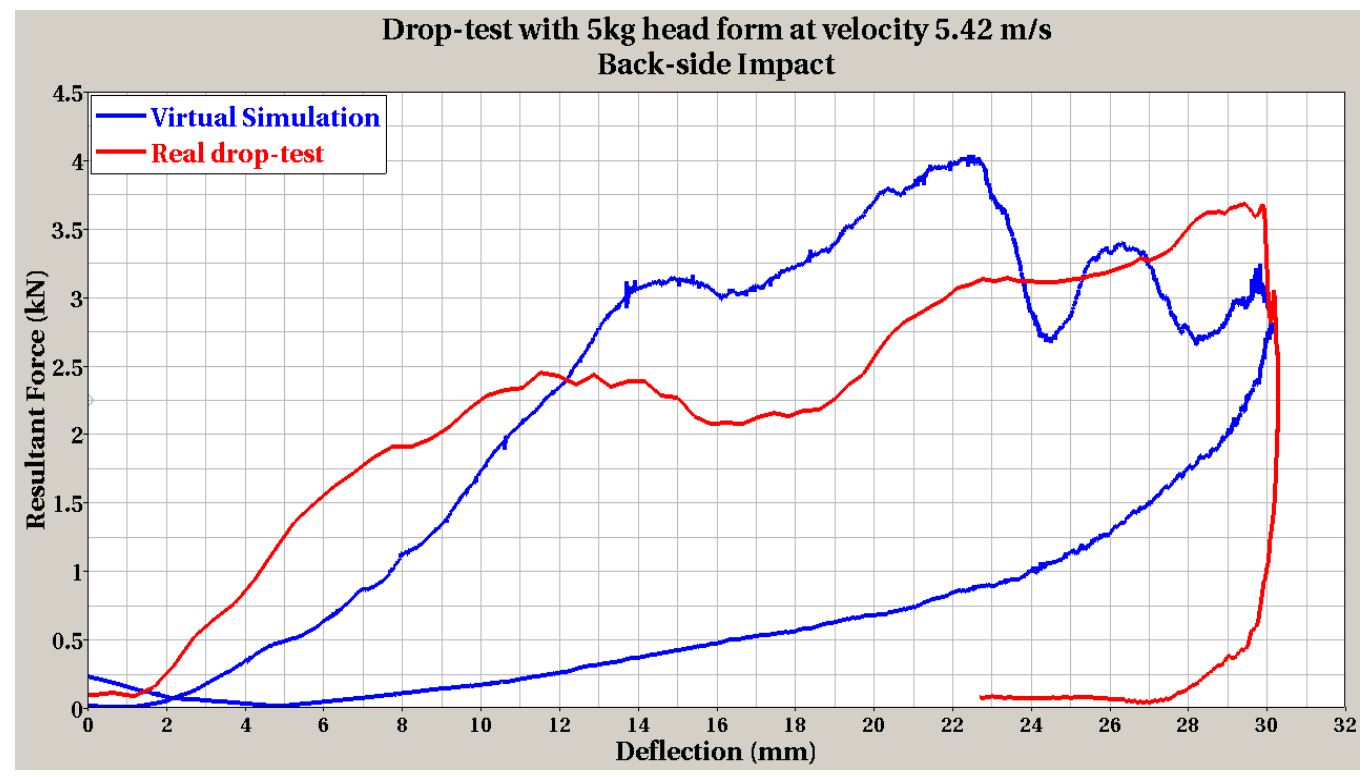

Abbildung 2-33: Vergleich der Kraft-Weg-Kurven (Aufprall auf die Rückseite, Gewicht der Kopfform 5 kg, Aufprallgeschwindigkeit 5,42 $\mathrm{m} / \mathrm{s}$ )

Zusätzlich zu den oben dargestellten Verläufen konnten die im realen Versuch aufgetretenen Risse im Helm in der Simulation annähernd genau dargestellt werden. Auf Abbildung 2-34 ist beispielhaft ein gerade beginnender Riss in der Simulation dargestellt. Die Lokalisation des Risses ist dabei identisch mit dem Riss aus dem Realversuch, wie auf Abbildung 2-35 dargestellt.

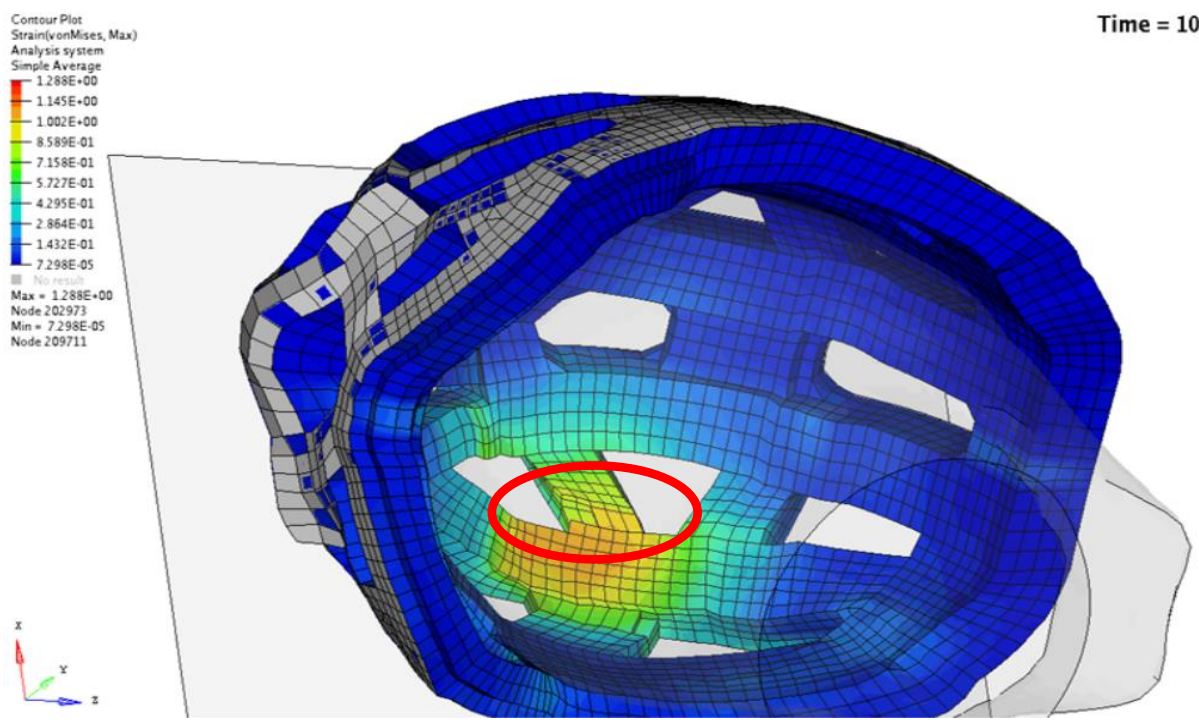

Abbildung 2-34: Darstellung eines beginnenden Risses im Helm in der Simulation 


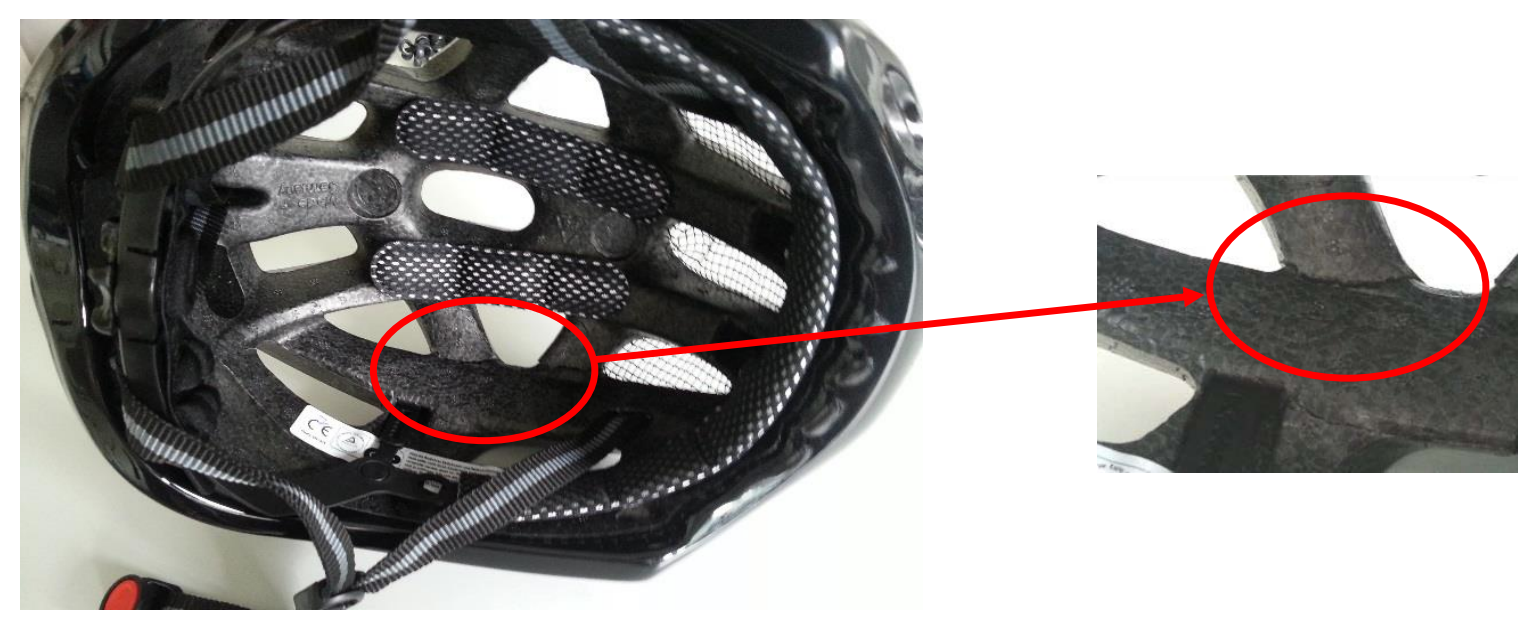

Abbildung 2-35: Riss des Helms im Realversuch

\subsubsection{Bewertung und Kopplung mit dem Kopfmodell}

Die Modellierungsgüte des Absorptionsschaums hat ein angemessenes Niveau erreicht. Zusätzlich zu den Komponententests wurden Versuche mit dem gesamten Helm durchgeführt. Die dabei festgestellten Abweichungen zwischen Simulation und Realversuch sind noch geringer als bei den Komponententests.

Im Rahmen des vorliegenden Forschungsprojekts ist die Kopplung des Helmmodells mit einem numerischen Schädel-Hirn-Modell vorgesehen, um das Kopfverletzungsrisiko unter variierenden Randbedingungen des Aufpralls bewerten zu können. Als „State-of-the-art“Simulationsmodell für den Kopf kommt das Modell an der Universität Straßburg entwickelte „Strasbourg University Finite Element Head Modell“ (SUFEHM) zur Anwendung.

Für das gekoppelte Simulationsverfahren bieten sich hier grundsätzlich zwei Wege an: Das SUFEHM kann, wie bereits mehrfach publiziert (u.a. Deck et al. 2008), als nachgeschaltetes Simulationsmodell zur Unfallrekonstruktion bzw. experimenteller oder virtueller Helmprüfung genutzt werden. Basis dieser Simulationsmethode sind Beschleunigungskurven, die über vorgeschaltete Arbeitsschritte ermittelt werden (z.B. experimentell oder durch Berechnungen). Das Kopfmodell wird mit diesen Beschleunigungskurven anschließend in der Simulationsumgebung beaufschlagt und die entstehenden Kopfbelastungswerte können ausgelesen werden. Mit dieser Methode können nur die Belastungen innerhalb des Schädels abgebildet werden. Die Belastung des Schädelknochens und damit verbunden das Risiko für eine Schädelfraktur kann nicht ermittelt werden. Es ist ebenfalls nicht möglich, das Kopfmodell ohne zusätzliche Arbeitsschritte für Anprallsimulationen, auch im Zusammenspiel mit anderen Modellen (z.B. eines Helms oder Pkws), zu verwenden.

Diese Einschränkungen kann man mit der zweiten Simulationsmethode umgehen. Hier erfolgt die Beaufschlagung des Kopfmodells nicht durch Beschleunigungskurven, sondern durch Geschwindigkeiten. So ist eine direkte Anprallsimulation (auch gekoppelt mit einem Helmmodell) möglich. Hat man ein geeignetes Modell eines Kollisionsgegners, z.B. eines Pkws, zur Verfügung, kann dieses ebenfalls in der gleichen Simulationsumgebung verwendet werden (s. 2.4.1.2). Die Anbindung des Helmmodells erfolgt über reibungsbehaftete Kontakte. Mit dieser Methodik kann auch das Risiko für eine Schädelfraktur bestimmt werden. 
Im Rahmen dieses Forschungsprojekts wurde die Entscheidung für die zweite Simulationsmethode (geschwindigkeitsbasiert) getroffen, da hier Anprallszenarien mit gekoppeltem Helmmodell und unter Verwendung eines Pkw-Modells in einer Simulationsumgebung erfolgen können. Dazu wird hier die LSDyna-Version des SUFEHM herangezogen.

Das Helmmodell wurde zur Kopplung mit dem Kopfmodell zuerst skaliert, d.h. die Größe des Helmmodells wurde dem Kopfmodell angepasst. Anschließend wurde das Helmmodell realitätsnah auf dem Kopfmodell positioniert, um eine möglichst große Auflagefläche zwischen Kopf und Helm zu erreichen. Dazu wurde die untere Kante im vorderen Bereich des Helms auf Höhe der Stirn platziert, so dass das Blickfeld des Kopfmodells nicht beeinträchtigt ist. Es wurde ebenfalls auf möglichst realitätsnahe und symmetrische Ausrichtung im seitlichen Kopfbereich und am Hinterkopf geachtet. Anschließend erfolgte das Anlegen der Bänderung zur besseren Fixierung bzw. zur Vermeidung von möglichen unnatürlichen Rotationsbewegungen des Helms auf dem Kopf. Diese liegt vereinfachend im Bereich der Schädelbasis auf, da hier eine größere Auflagefläche als am nur schematisch modellierten Kinn vorhanden ist. Trotzdem ist eine als realistisch zu betrachtende Fixierung des Helms zum Anprallzeitpunkt gegeben. Die Interaktion zwischen Kopfmodell und Helmmodell samt Bänderung wurde als reibungsbehafteter Kontakt abgebildet.

Auf Abbildung 2-36 ist das Helmmodell mit gekoppeltem Kopfmodell zu sehen.

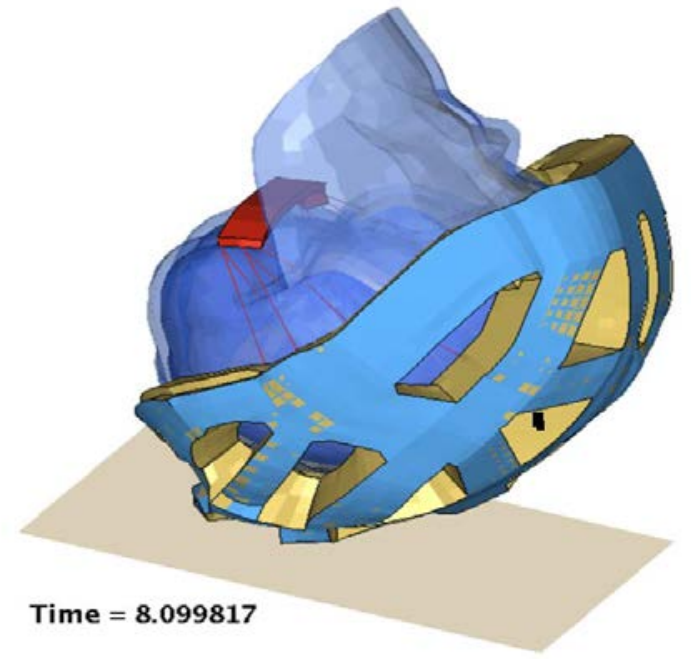

Abbildung 2-36: Kopfmodell mit gekoppeltem Helmmodell

Insgesamt wird die Modellierungsgüte des Helms als sehr gut eingeschätzt. Die Kopplung mit dem SUFEHM wurde im Rahmen der Möglichkeiten realitätsnah durchgeführt. 


\subsection{Datenbank rekonstruierter Unfälle}

Die unter 1.3 selektierten im Detail zu rekonstruierenden Unfälle aus der SUD (fünf Alleinunfälle, neun Unfälle mit Pkw-Beteiligung) wurden im weiteren Verlauf des Projekts durch vier prospektiv erfasste Unfälle ergänzt.

Diese Unfälle stimmen mit den ermittelten typischen Szenarien gut überein und eignen sich aufgrund einer sehr guten Dokumentationsgrundlage für detaillierte Betrachtungen.

Eine Übersicht dieser Unfälle ist Anhang 3 zu entnehmen.

Primäres Auswahlkriterium der Unfälle war zunächst die Übereinstimmung mit den in 0 bestimmten typischen Unfallszenarien, der Alleinunfall des Fahrradfahrers und die Kollision mit der Front eines Pkw in annähernd rechtem Winkel.

Der Unfallhergang wurde für jeden einzelnen der ausgewählten Unfälle rekonstruiert. Die teilweise schon vorhandenen Unfallgutachten wurden ebenfalls überprüft und gegebenenfalls korrigiert. Anschließend wurden die in der Tabelle (Anhang 3) aufgeführten Variablen und Werte bestimmt. Diese sind aufgeteilt in:

- Alter und Geschlecht des Fahrradfahrers

- Unfallrelevante Daten (Auto- und Fahrradtyp, Geschwindigkeiten, Kollisionsstellung etc.)

- Verletzungen des Fahrradfahrers

- Ergänzende Bemerkungen.

\subsection{Vergleichende Einschätzung der Testnormen in Relation zu 1.4 und 2.3}

\subsubsection{Simulierte Unfälle}

Aus den 18 gut dokumentierten und rekonstruierten Realunfällen wurden vier Unfälle ausgewählt, die detailliert simuliert wurden. Auswahlkriterien waren eine sehr gute Dokumentationsqualität sowie eine möglichst gute Übereinstimmung mit den unter 1.4 ermittelten typischen Unfallszenarien.

Um alle im Verlauf des Forschungsprojekts (insb. unter 1.4) ermittelten typischen Unfallszenarien detailliert simulieren zu können, wurden diese Realunfälle zusätzlich mit generischen Szenarien ergänzt.

Diese Szenarien wurden anschließend in zwei Schritten simuliert, um die Kinematik des Fahrradfahrers sowie die Kopfverletzungsrisiken beim Kopfanprall und die Wirkung eines Fahrradhelms darstellen zu können.

\subsubsection{Simulierte Unfälle in MADYMO}

Der erste Simulationsschritt wurde mit dem Mehrkörpersimulationsprogramm „MADYMO“, Version 7.1 (MADYMO Software 2014), durchgeführt. 
Basis dieses ersten Simulationsschritts war die Rekonstruktion des Unfallhergangs, insbesondere die ermittelten Kollisionsgeschwindigkeiten, die Kollisionsstellung und der Bewegungsablauf.

Mit Hilfe der Modelle wurde in der Simulation die Kinematik des Fahrradfahrers möglichst exakt abgebildet; besonderer Fokus lag auf der Ermittlung der translatorischen und rotatorischen Kopfanprallgeschwindigkeiten und dem Kopfanprallwinkel zum Zeitpunkt des Anpralls. Diese Daten dienten als Ausgangspunkt für den zweiten Simulationsschritt zur Ermittlung der Kopfverletzungsrisiken.

Die Tabelle aller in MADYMO simulierten Unfallszenarien mit den wichtigsten Unfallparametern ist in Anhang 4 ab Seite 187 aufgelistet.

\subsection{Verwendete Modelle}

Um die Unfälle möglichst genau darstellen zu können, wurden realitätsnahe Simulationsmodelle von Fahrradfahrer, Fahrrad und Pkw verwendet.

Als Fahrradfahrer wurden die in MADYMO integrierten Fußgänger-Mensch-Modelle verwendet (Abbildung 2-37).

Zur Simulation der Realunfälle und der generischen Szenarien wurde das 50-Perzentil männliche Mensch-Modell verwendet (Körpergröße $174 \mathrm{~cm}$, Gewicht 75,7 kg), lediglich bei einem Realunfall (ID 3) wurde das 5-Perzentil weibliche Mensch-Modell verwendet (Körpergröße 153 cm, Gewicht 49,8 kg).
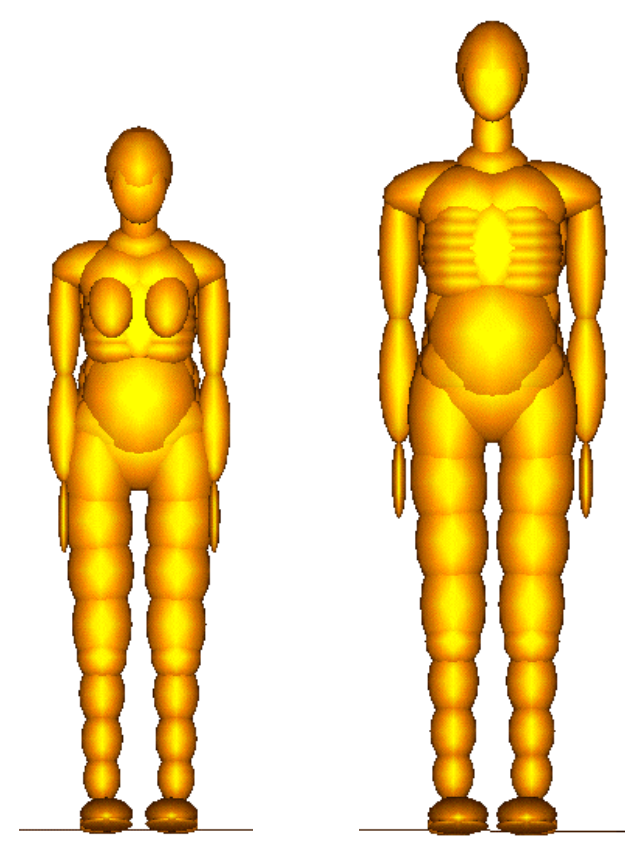

Abbildung 2-37: 5-Perzentil Frau und 50-Perzentil Mann Fußgänger-Mensch-Modelle MADYMO

Es wurden zwei unterschiedliche Fahrradmodelle erstellt, ein „Trekkingrad“ und ein „City“bzw. „Hollandrad“ (Abbildung 2-38).

Diese Modelle wurden in MADYMO als Mehrkörpersysteme aus starren Ellipsoiden entworfen, die über Gelenke miteinander verbunden wurden.

Die einzelnen Elemente des Rahmens wurden mittels starrer Gelenke verbunden. 
Die Fahrradgabel wurde durch ein ortsfest fixiertes, aber im Vorderrohr drehbares Gelenk mit dem Rahmen verbunden, so dass eine Drehung des Vorbaus (Lenker, Gabel und Vorderrad) relativ zum Rahmen ermöglicht wurde.

Das Vorder- und Hinterrad wurden mit der Gabel bzw. dem Hinterbau so verbunden, dass eine Rollbewegung der Räder ermöglicht wurde. Die Größe der Laufräder beträgt jeweils 26 Zoll.

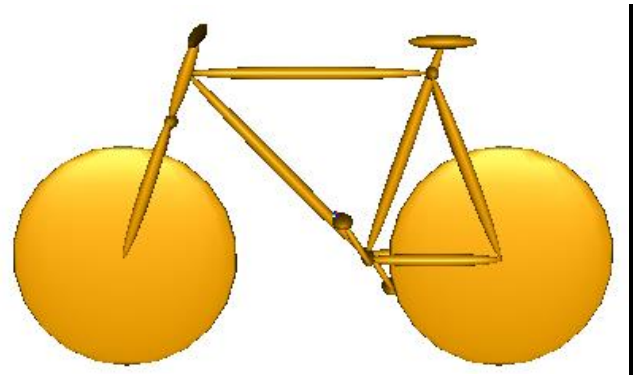

Trekkingrad

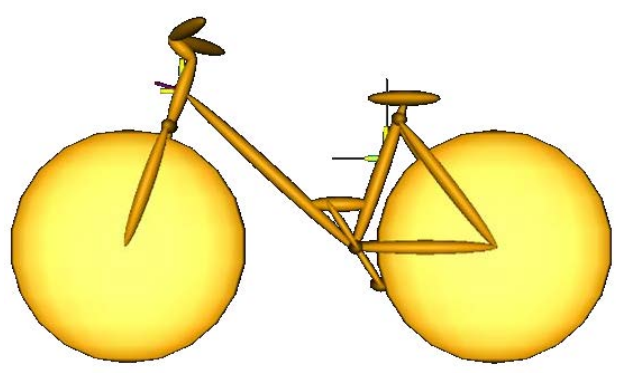

City-/Hollandrad

Abbildung 2-38: Fahrradmodelle in MADYMO

Zur Darstellung der Pkw-Front konnte auf ein der UDV zur Verfügung stehendes PkwModell in MADYMO (Kühn 2011; Hummel 2012) zurückgegriffen werden. Dieses Modell entspricht der Front eines aktuellen Fahrzeugmodells der oberen Mittelklasse. Es wurde sowohl die Geometrie als auch die Kontaktsteifigkeiten verwendet.

Auf Abbildung 2-39 ist das Modell der Fahrzeugfront in dreidimensionaler Ansicht zu sehen.

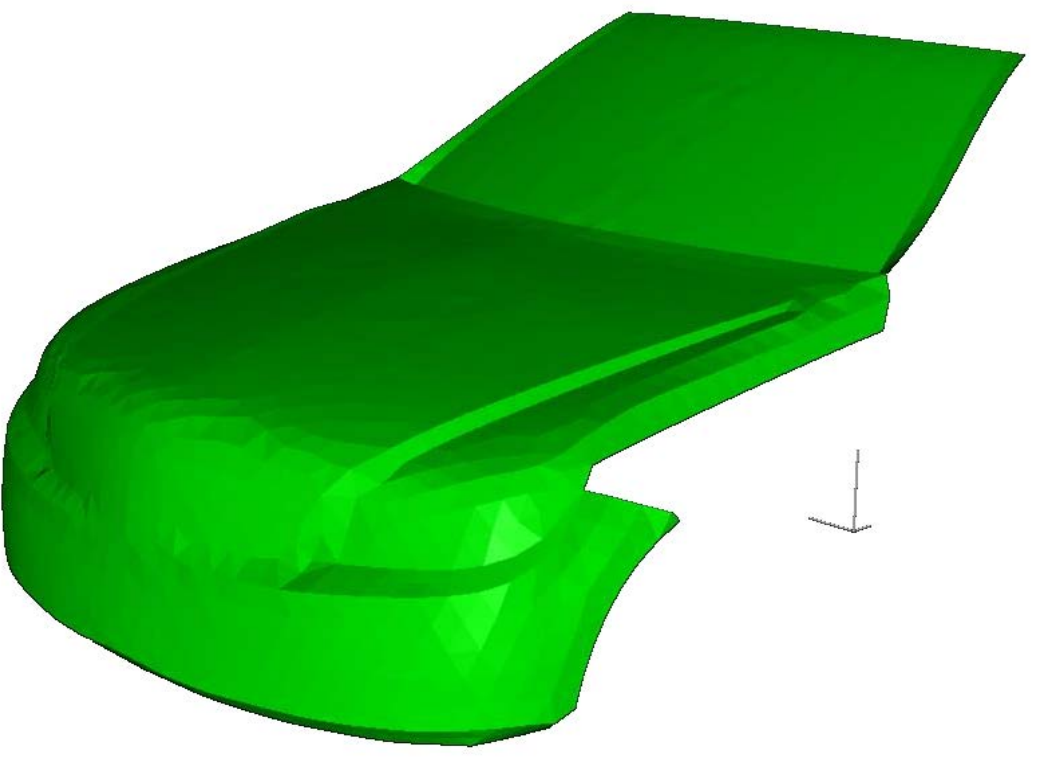

Abbildung 2-39: Pkw-Modell „Obere Mittelklasse“

Die Geometrie dieses Modells wurde zur Simulation eines Realunfalls (ID 3) an das dort beteiligte reale Fahrzeug (Kompaktvan) angepasst. Das entsprechende Modell ist auf Abbildung 2-40 abgebildet. Es wurde die Motorhaube verkürzt, das Fahrzeug insgesamt leicht erhöht und verbreitert und die Windschutzscheibe steiler gestellt. 


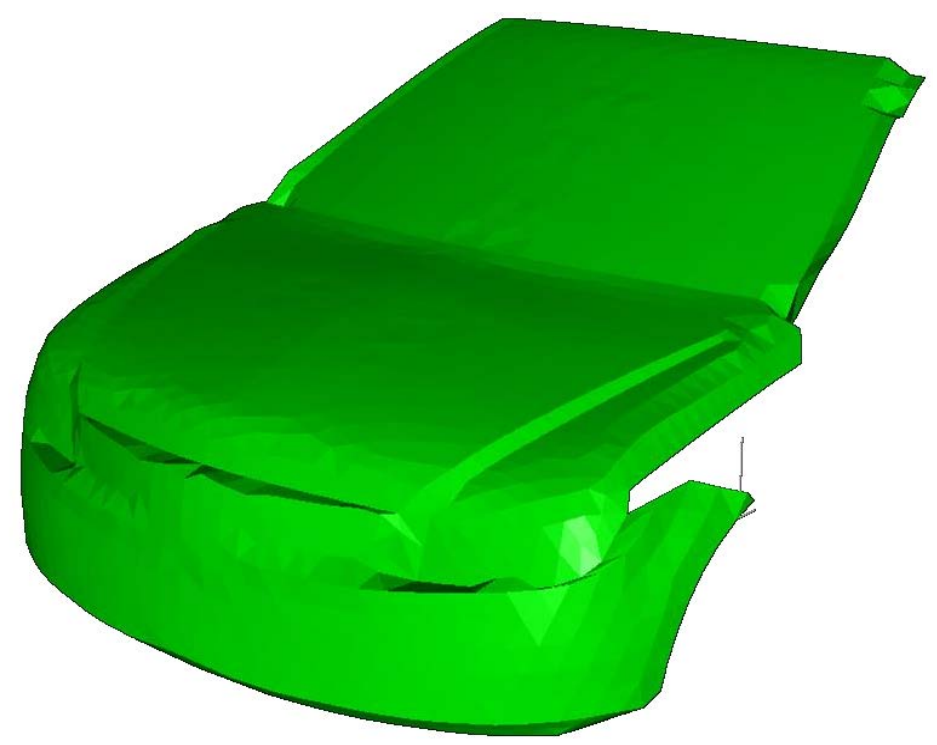

Abbildung 2-40: Pkw-Modell „Kompaktvan“

\subsection{Simulierte Realunfälle}

Folgende Realunfälle wurden aus der Datenbank der rekonstruierten Unfälle (2.3) zur detaillierten Simulation ausgewählt:

- SUD 1302; ab sofort ID 1 (Alleinunfall Sturz seitlich)

- SUD 1474; ab sofort ID 2 (Alleinunfall Sturz über Lenker)

- SUD 1376; ab sofort ID 3 (Kollision rechtwinklig mit Pkw-Front, 65 km/h)

- UKLMU 3; ab sofort ID 4 (Kollision rechtwinklig mit Pkw-Front, 29 km/h)

Die Unfälle ID 1 und ID 2 entsprechen dabei den unter 1.4 ermittelten typischen Alleinunfallszenarien 1a (Alleinunfall, Sturz seitlich) und 1b (Alleinunfall, Sturz über Lenker).

Die Kinematik des Fahrradfahrers bei den simulierten Realunfällen konnte aufgrund der vorliegenden detailliert beschrieben Verletzungen genau überprüft und validiert werden.

Auf den folgenden Abbildungen sind die in MADYMO simulierten Unfallszenarien zum Zeitpunkt des Kopfanpralls entweder auf die Straße oder am Pkw dargestellt. Der eingezeichnete Pfeil entspricht dabei dem Vektor der relativen Kopfanprallgeschwindigkeit und zeigt auf den Anprallpunkt am Kopf des Fahrradfahrers.

Auf Abbildung 2-41 ist der Kopfanprall des Fahrradfahrers beim Alleinunfall seitlich mit geringer Ausgangsgeschwindigkeit $(8 \mathrm{~km} / \mathrm{h})$ dargestellt. Verwendete Modelle sind das 50 Perzentil Mann Fußgänger-Mensch-Modell und das Trekkingrad.

Der Fahrradfahrer verliert das Gleichgewicht und stürzt seitlich auf die Straße, ohne sich dabei mit den Händen abzustützen. Wesentlichen Kontakt mit der Straße hat zuerst die linke Schulter des Fahrradfahrers, die den Sturz etwas abbremst und dadurch den Kopf in eine „Pendelbewegung“ zwingt. Der Kopfanprall erfolgt seitlich links im Bereich der Schläfenregion. 


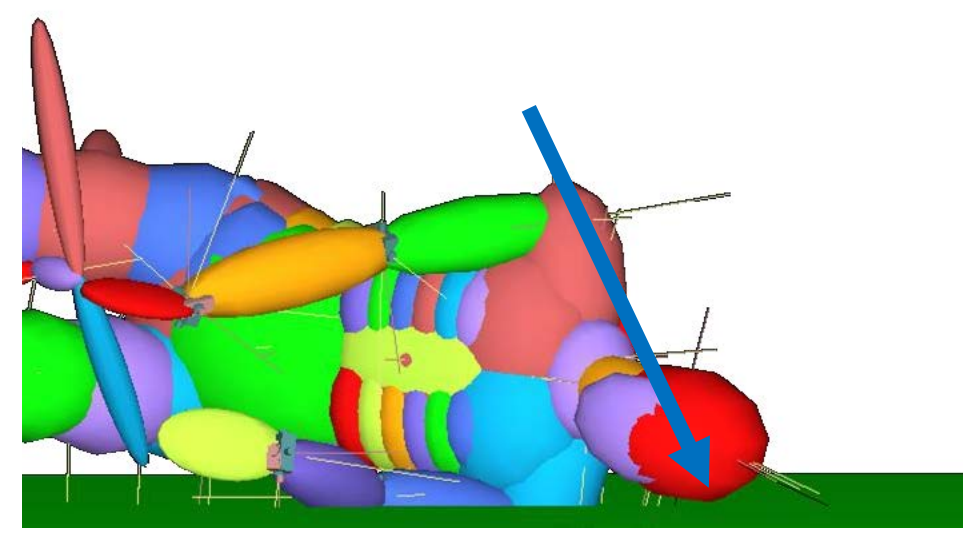

Abbildung 2-41: Kopfanprall Alleinunfall seitlich (ID 1)

Abbildung 2-42 zeigt den Kopfanprall beim Sturz des Fahrradfahrers über das blockierte Vorderrad. Verwendete Modelle sind das 50-Perzentil Mann Fußgänger-Mensch-Modell und das Trekkingrad.

Mit einer Ausgangsgeschwindigkeit von 15 km/h stürzt der Fahrradfahrer ohne eine ausgeprägte seitlichen Bewegung nach vorne über den Lenker, zeigt noch einen Abstützversuch mit den Händen, prallt dann aber mit der Stirn voraus auf die Straße.

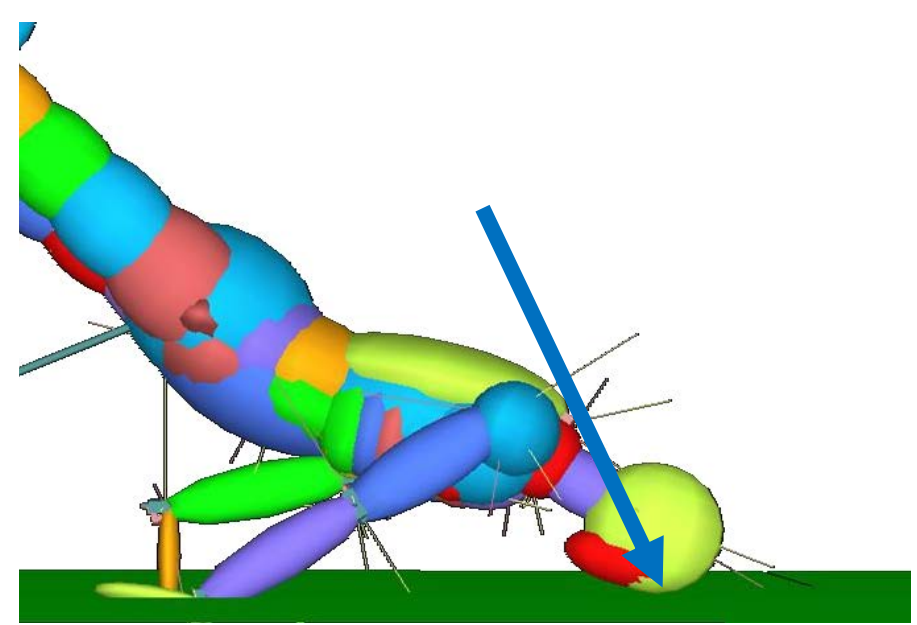

Abbildung 2-42: Kopfanprall Alleinunfall Sturz über Lenker (ID 2)

Auf Abbildung 2-43 ist das Szenario ID 3 zum Zeitpunkt des Kopfanpralls abgebildet. Die Fahrradfahrerin wird seitlich von einem Pkw erfasst, die Kollisionsgeschwindigkeit beträgt $65 \mathrm{~km} / \mathrm{h}$. Verwendete Modelle sind das 5-Perzentil Frau Fußgänger-Mensch-Modell, das Cityrad, sowie das Modell des Kompaktvans.

Die Fahrradfahrerin wird von der Front des Pkw erfasst und prallt mit dem Kopf zwischen Dachkante und A-Säule in die Ecke der Frontscheibe. Der Kopfanprall erfolgt seitlich links in der Schläfenregion. 


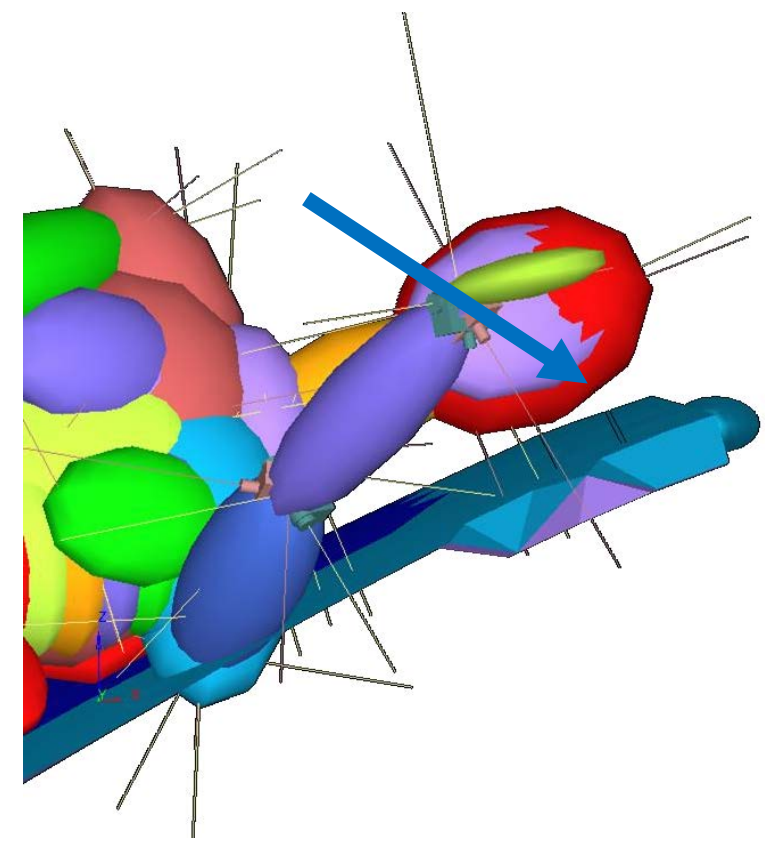

Abbildung 2-43: Kopfanprall Kollision Pkw (ID 3)

Auf Abbildung 2-44 ist der Realunfall ID 4 abgebildet. Verwendete Modelle sind das 50Perzentil Mann Fußgänger-Mensch-Modell, das Trekkingrad und das Modell der oberen Mittelklasse.

Der Fahrradfahrer wird von der Front des Pkws erfasst (Kollisionsgeschwindigkeit ca. $30 \mathrm{~km} / \mathrm{h}$ ), wird von diesem ohne weiteren Kontakt in die Luft geschleudert und prallt mit der Schläfenregion voran auf die Kante eines Bordsteins.

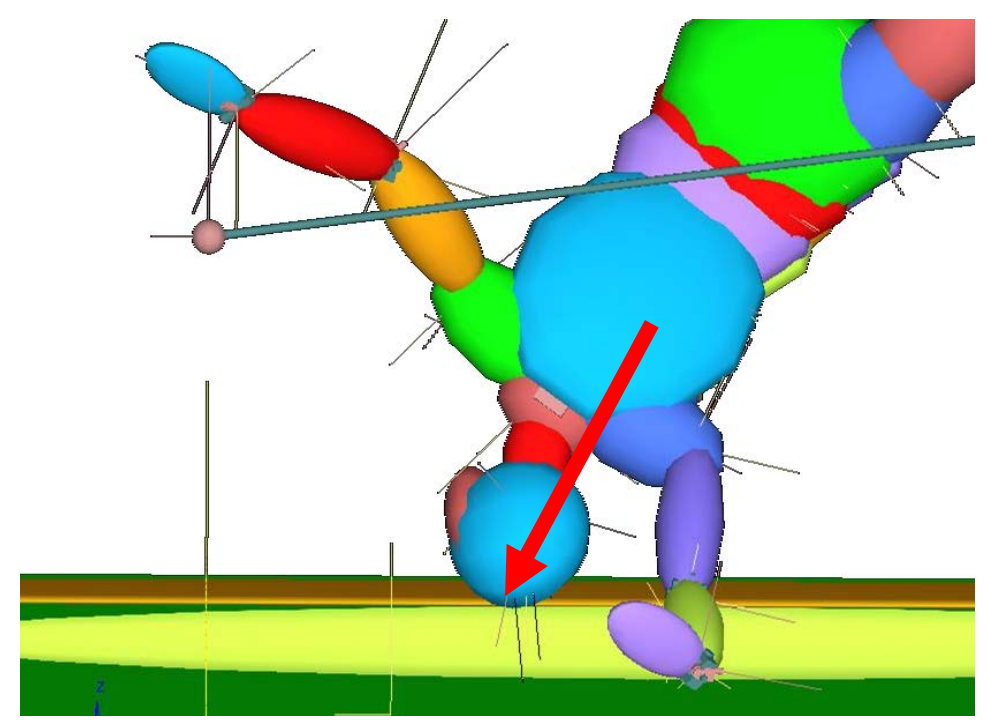

Abbildung 2-44: Kopfanprall Bordstein (ID 4) 


\subsection{Simulierte generische Szenarien}

Die Ergebnisse aus den oben dargestellten Simulationen decken sich sehr gut mit den Beschreibungen, Verletzungen und Beschädigungsbildern aus den realen Unfällen. Die verwendeten Modelle und die Simulationsmethode wurden als geeignet angesehen, um für generisch erstellte Szenarien mit Hilfe der Simulation realistische Ergebnisse zu erlangen.

In diesen weiteren Simulationen wurden die als unter 1.4 typisch bestimmten Unfallszenarien 2a (Kollision Pkw-Front gegen Fahrrad seitlich bei geringer Geschwindigkeit) und 2b (Kollision Pkw-Front gegen Fahrrad seitlich bei mittlerer Geschwindigkeit) abgebildet. Dabei sollte idealerweise der Kopfanprall auf die Motorhaube und die Windschutzscheibe abgebildet werden.

Die eigenen Studienerkenntnisse und Ergebnisse anderer Studien führten, wie in 1.4.2.7 und 1.4.3 diskutiert, zur Entscheidung, das Szenario „Pkw kollidiert frontal mit der Seite des Fahrrads in annähernd rechtem Winkel" mit Kollisionsgeschwindigkeiten des Pkw von 15 und $40 \mathrm{~km} / \mathrm{h}$ als typische Kollisionssituationen zu simulieren.

Bei der Geschwindigkeit von $15 \mathrm{~km} / \mathrm{h}$ kam es zum Kopfaufprall auf die Motorhaube, bei 40 km/h traf der Kopf den Pkw auf Höhe Mitte der Frontscheibe.

Auf Abbildung 2-45 ist die Kollision des Fahrradfahrers mit der Front des Pkw bei $15 \mathrm{~km} / \mathrm{h}$ Kollisionsgeschwindigkeit abgebildet (Kollisionswinkel $90^{\circ}$ ). Verwendete Modelle sind das 50-Perzentil Mann Fußgänger-Mensch-Modell, das Trekkingrad und das Modell der oberen Mittelklasse.

Der Fahrradfahrer prallt nach der primären Kollision mit dem Kopf auf den hinteren Bereich der Motorhaube. Anprallbereich am Kopf ist die Stirn und das Gesicht. Die Anprallgeschwindigkeit ist dabei verhältnismäßig gering; der Kopf erfährt außer der Schwerkraft keine nennenswerten kollisionsbedingten Krafteinwirkungen.

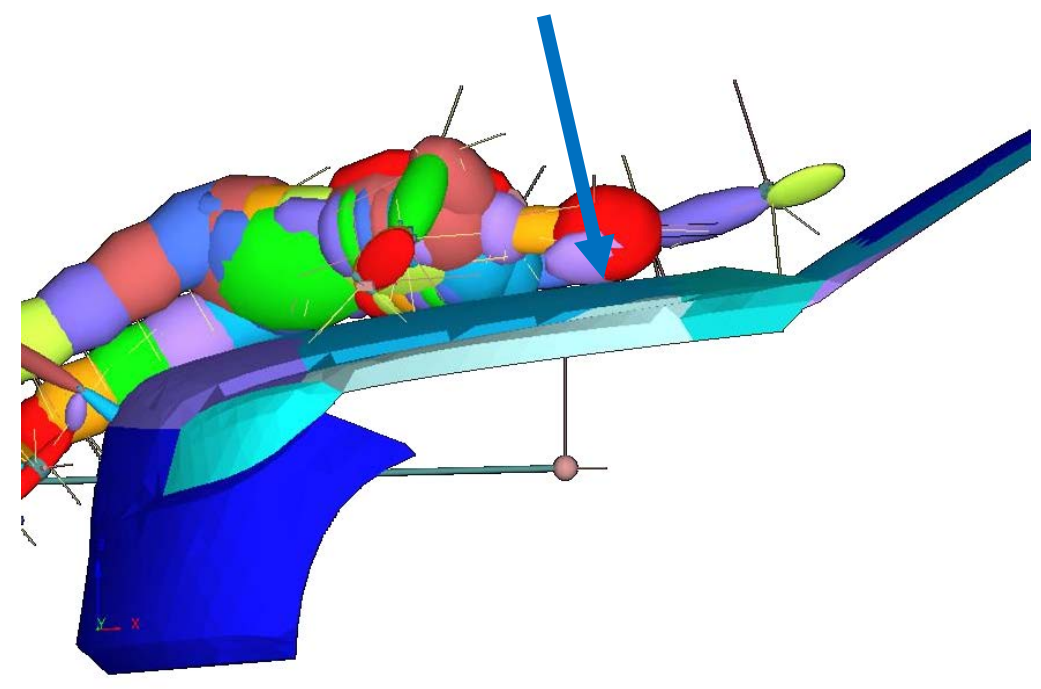

Abbildung 2-45: Kopfanprall auf Motorhaube (ID 5a)

Ausgehend von diesem Unfallszenario ID 5 a wurden unter Variation des Kollisionswinkels $\left(90^{\circ} \pm 35^{\circ}\right)$ und der Anprallstelle des Kopfs auf die Motorhaube mehrere Szenarien simuliert (ID 5b, 5c, 6a, 6b, 6c). 
Dabei stellte sich heraus, dass von diesen betrachteten Szenarien bei ID 5a sowohl die relative Kopfanprallgeschwindigkeit als auch die Kopfbelastung (für den Fall des

Kopfanpralls auf die Motorhaube) am höchsten war. In den Szenarien ID 6b und $6 c$ fand nicht einmal ein Kopfanprall auf die Motorhaube statt.

Da die Kopfverletzungsrisiken im Falle des Anpralls auf die Motorhaube ohnehin schon sehr gering sind, wurden für die Simulation der Kopfanprallszenarien auf die Motorhaube nur die Kopfanprallgeschwindigkeiten des „worst case“ ID 5a verwendet. Die Szenarien ID 5b bis 6c wurden nicht weiter betrachtet, da in diesen kein Kopfverletzungsrisiko nachgewiesen werden konnte.

Bedingt durch die Kinematik des Fahrradfahrers ist ein Kopfanprall auf die Motorhaube ohnehin nur bis zu einer Kollisionsgeschwindigkeit des simulierten Pkw bis knapp unter $20 \mathrm{~km} / \mathrm{h}$ zu erreichen. Die mögliche Kopfanprallgeschwindigkeit auf die Motorhaube ist somit stark begrenzt; höhere Kopfanprallgeschwindigkeiten treten nur im Bereich der Frontscheibe oder Dachkante auf.

Auf Abbildung 2-46 ist der Kopfanprall des Fahrradfahrers bei einer rechtwinkligen Kollision mit einem Pkw (Kollisionsgeschwindigkeit 40 km/h, ID 7) abgebildet. Verwendete Modelle sind das 50-Perzentil Mann Fußgänger-Mensch-Modell, das Trekkingrad und das Modell der oberen Mittelklasse.

Der Fahrradfahrer wird über die Front des Pkw abgewickelt und prallt mit dem Kopf zentral in die Frontscheibe. Anprallbereich am Kopf ist seitlich links im Bereich der temporalen Region.

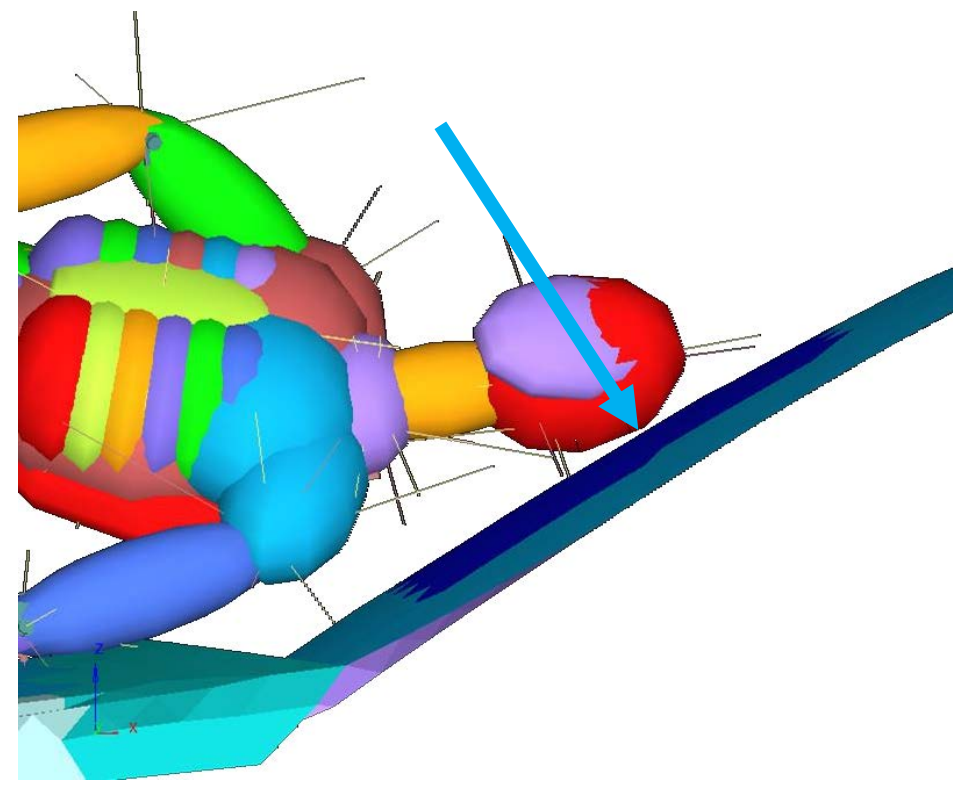

Abbildung 2-46: Kopfanprall an Frontscheibe zentral (ID 7)

Auf Abbildung 2-47 ist der Kopfanprall des Fahrradfahrers mit identischen Parametern zu ID 7 dargestellt. Der Fahrradfahrer wird vom Pkw derart erfasst, dass der Kopfanprall im Bereich der Frontscheibe direkt neben der linken A-Säule stattfindet.

Dieser Bereich ist im Vergleich zum Zentrum der Windschutzscheibe als wesentlich steifer und unnachgiebiger einzuschätzen. 


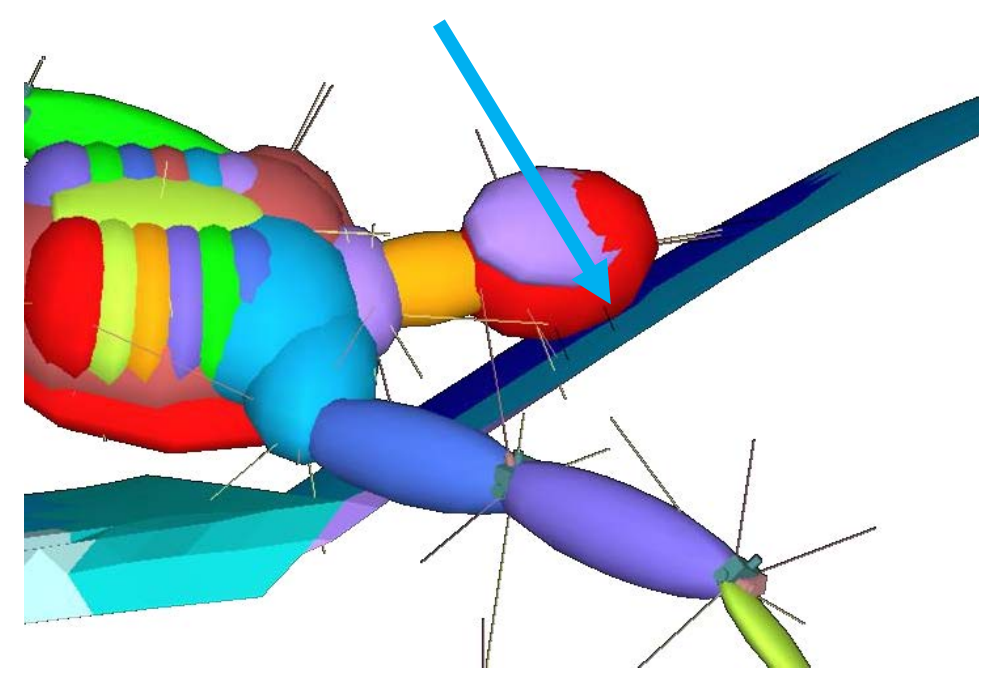

Abbildung 2-47: Kopfanprall an Frontscheibe außen (ID 8)

\subsubsection{Anprallsimulation in FE}

Der zweite Simulationsschritt erfolgt auf Basis von Finite-Elemente-Simulationen (FE) und wurde mit dem Solver LS-DYNA V971 R6.1.2 (LS-DYNA Software 2014) und dem Prä- und Postprozessor Altair HyperWorks v12.0 (HyperWorks Software 2014) durchgeführt.

Die wie oben dargestellt im ersten Simulationsschritt erhaltenen Daten (translatorische und rotatorische Kopfgeschwindigkeiten zum Anprallzeitpunkt, Anprallwinkel) wurden als Ausgangswerte für die detaillierte Simulation des Kopfanpralls in FE verwendet.

Die Tabelle aller in FE simulierten Kopfanprallszenarien mit den wichtigsten Parametern ist in Anhang 5 aufgelistet.

In der FE-Simulation wurden durch das SUFEHM-Modell die Kopfbelastungen (Von-MisesSpannung, innere Energie der Gehirnflüssigkeit CSF, innere Energie des Schädels) ermittelt. Diese Werte werden vom Modell anschließend anhand von Risikokurven in Kopfverletzungsrisiken umgerechnet.

Alle Kopfanprallszenarien wurden mit und ohne Helm simuliert. Anhand der Reduktion der Kopfverletzungsrisiken wurde der jeweilige Nutzen des Helms sichtbar.

\subsection{Verwendete Modelle}

Für eine detaillierte Simulation der Kopfbelastungen und der Kopfverletzungsrisiken konnte auf das von der Universität Straßburg entwickelte Kopfmodell (SUFEHM) (Bourdet et al. 2014) zurückgegriffen werden.

Die Entwicklung dieses Kopfmodells startete 1997 und wird weiterhin kontinuierlich vorangetrieben. Es sind alle grundlegenden anatomischen Komponenten und Eigenschaften umgesetzt (Abbildung 2-48). 

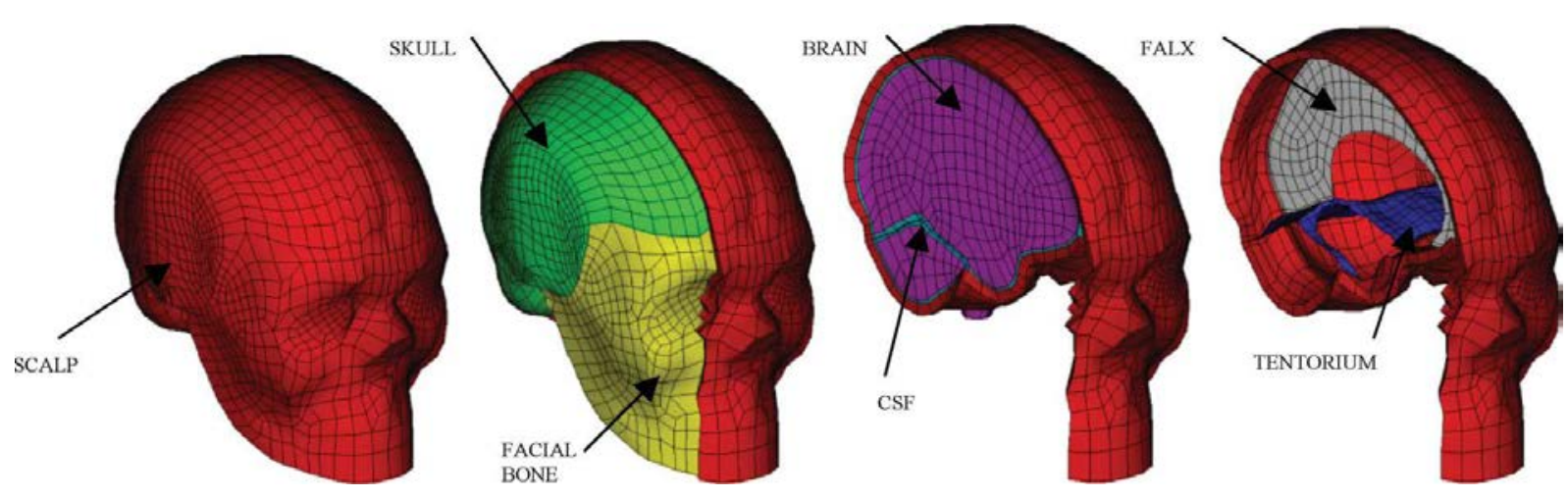

Abbildung 2-48: Kopfmodell der Universität Straßburg SUFEHM (Bourdet et al. 2014)

Dieses Modell erlaubt die Ermittlung der Risiken für diffusen Axonschäden (DAI, leicht/mittel und schwer), ein subdurales Hämatom (SDH) sowie einer Schädelfraktur. Dazu werden die in der Simulationsumgebung berechneten spezifischen inneren Belastungen in einem zusätzlichen Arbeitsschritt über Risikokurven in Kopfverletzungsrisiken umgerechnet. Anhand der Von-Mises-Spannung (VM) wird das Risiko für diffuse Axonschäden ermittelt; die innere Energie (IE) der Gehirnflüssigkeit (CSF) wird zur Ermittlung des Risikos für ein subdurales Hämatom herangezogen und die innere Energie des Schädels („Skull“) ermöglicht die Ermittlung des Risikos einer Schädelfraktur.

Der diffuse Axonschaden ist eine schwere traumatische Schädigung des Gehirns mit oft bleibenden Schäden. Der "leichte DAI“ wird mit einem AIS 4 kodiert; der "mittlere DAl“ und „schwere DAI“ mit einem AIS 5. Die Entwickler des Kopfmodells haben bezüglich der Terminologie von „leichtem“ und „mittlerem“ DAI und auch der dazugehörigen Risikokurve keine Unterscheidung getroffen und verwenden beide Begrifflichkeiten gleichwertig. Im Folgenden wird somit die Aufteilung "leichter/mittlerer" DAI mit einem AIS 4 bis AIS 5 und „schwerer“ DAI mit einem AIS 5 verwendet.

Das subdurale Hämatom bezeichnet einen Bluterguss unter der harten Hirnhaut und wird je nach betroffener Region und Ausdehnung mit einem AIS 2 bis AIS 5 kodiert (Großhirnbereich AIS 3 - AIS 5, Kleinhirnbereich AIS 2 - AIS 5).

Eine Schädelfraktur entsteht durch starke Gewalteinwirkung im Kopfbereich und wird mit einem AIS 2 bis AIS 4 kodiert (Schädelbasisfraktur AIS 3 - AIS 4).

Das verwendete FE Helmmodell wurde unter 2.2 bereits ausführlich vorgestellt.

Für die Simulation des Kopfanpralls an verschiedene Strukturen eines Pkws wurde das frei verfügbare FE-Modell eines Kleinwagens Toyota Yaris, Baujahr 2010 (National Crash Analysis Center 2011) verwendet. Dieses Fahrzeug wird in ähnlicher Form auch auf dem europäischen Markt angeboten.

Wie auf Abbildung 2-49 zu erkennen, sind bei diesem Modell alle relevanten Bauteile nachgebildet. Für die Simulation des Kopfanpralls wurden zur Reduktion der Rechenzeit nicht erforderliche Bauteile entfernt (z.B. im Heckbereich des Pkw). 


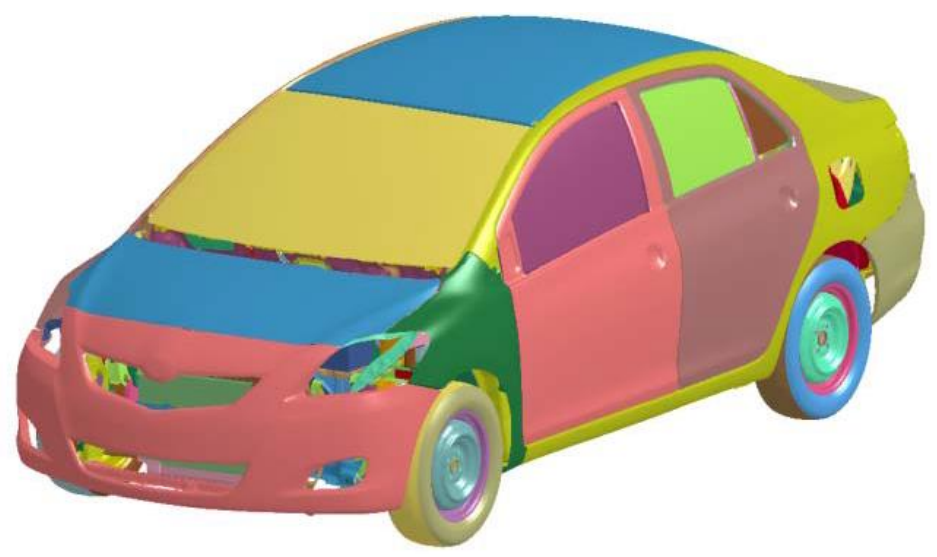

Abbildung 2-49: 2010 Toyota Yaris (National Crash Analysis Center 2011)

Ergänzend dazu wurde von der Arbeitsgruppe Biomechanik des Instituts für Rechtsmedizin München in Anlehnung an die Veröffentlichung von Peng et al. (2013) ein FE-Modell einer Frontscheibe erstellt, das für ergänzende Betrachtungen verwendet wurde.

\subsection{Simulierte Kopfanprallszenarien und Kopfverletzungsrisiken}

Wie bereits unter 2.4.1.1 dargestellt, wurden sowohl Kopfanprallszenarien resultierend aus Alleinunfällen und aus Kollisionen des Fahrradfahrers mit einem Pkw simuliert.

Beim Alleinunfall mit Sturz seitlich bei geringer Geschwindigkeit (Abbildung 2-50) wurde eine resultierende Kopfanprallgeschwindigkeit von 4,1 m/s ermittelt, der Winkel des Geschwindigkeitsvektors zur Fahrbahnoberfläche beträgt $67^{\circ}$. Für die Fahrbahn wurde eine unendlich steife Kennung angenommen.

Die Normalkomponente der Anprallgeschwindigkeit (der Anteil der Geschwindigkeit senkrecht zum Untergrund) beträgt $3,7 \mathrm{~m} / \mathrm{s}$.

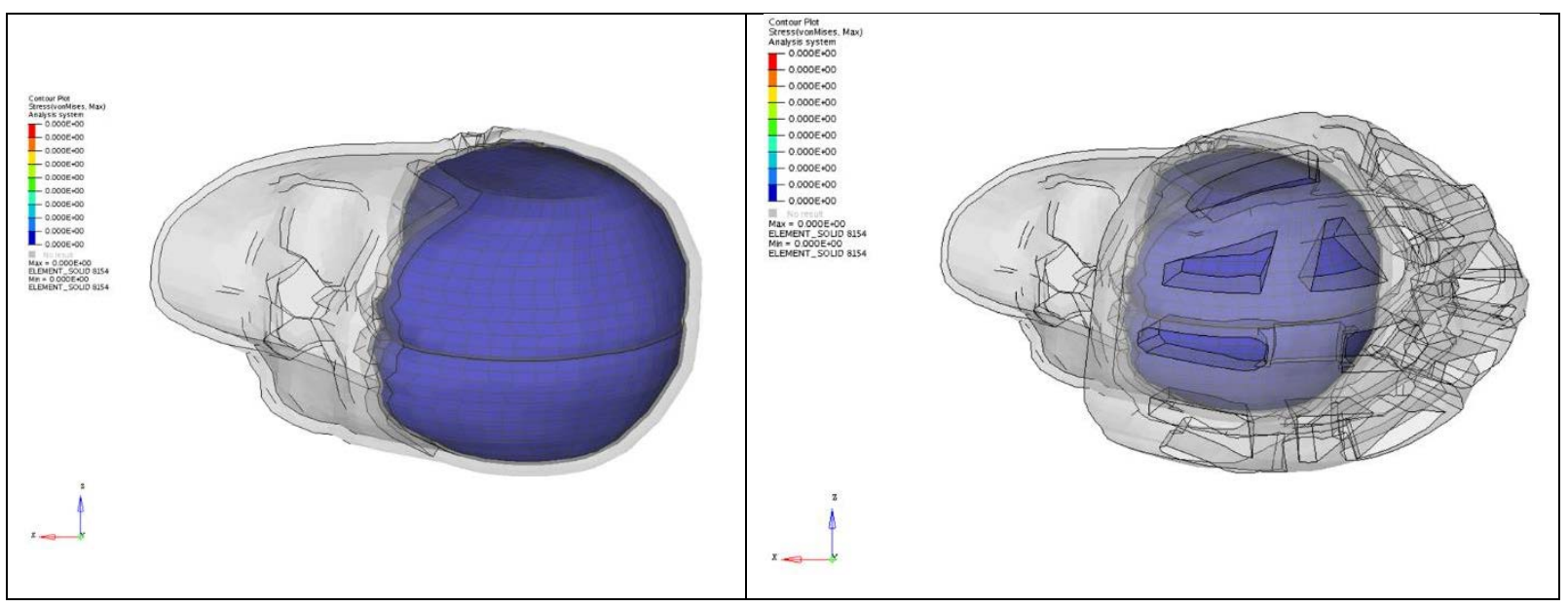

Abbildung 2-50: Kopfanprall Straße, Alleinunfall Sturz seitlich (ID 1a und 1b)

Das Risiko für ein leichtes/mittleres DAI beträgt ohne Helm $76 \%$, mit Helm reduziert sich das Risiko auf $47 \%$. Das Risiko für ein schweres DAI beträgt ohne Helm 12\%, mit Helm wird eine Reduktion auf $6 \%$ erreicht. Das Risiko für ein SDH beträgt ohne Helm 100\%, mit Helm wird das Risiko auf $40 \%$ reduziert. Das Risiko für eine Schädelfraktur beträgt mit und ohne Helm 100\% (VM-Spannung 34,2/27,6 kPa, IE CSF 15188/4509 mJ, IE Skull 4406/1376 mJ). 
Auf Abbildung 2-51 ist der Kopfanprall beim Alleinunfall mit Sturz über den Lenker bei blockiertem Vorderrad dargestellt. Die resultierende Kopfanprallgeschwindigkeit beträgt $5,2 \mathrm{~m} / \mathrm{s}$, der Winkel des Geschwindigkeitsvektors zum Untergrund beträgt $71^{\circ}$. Die Normalkomponente der Kopfanprallgeschwindigkeit beträgt 4,9 m/s.

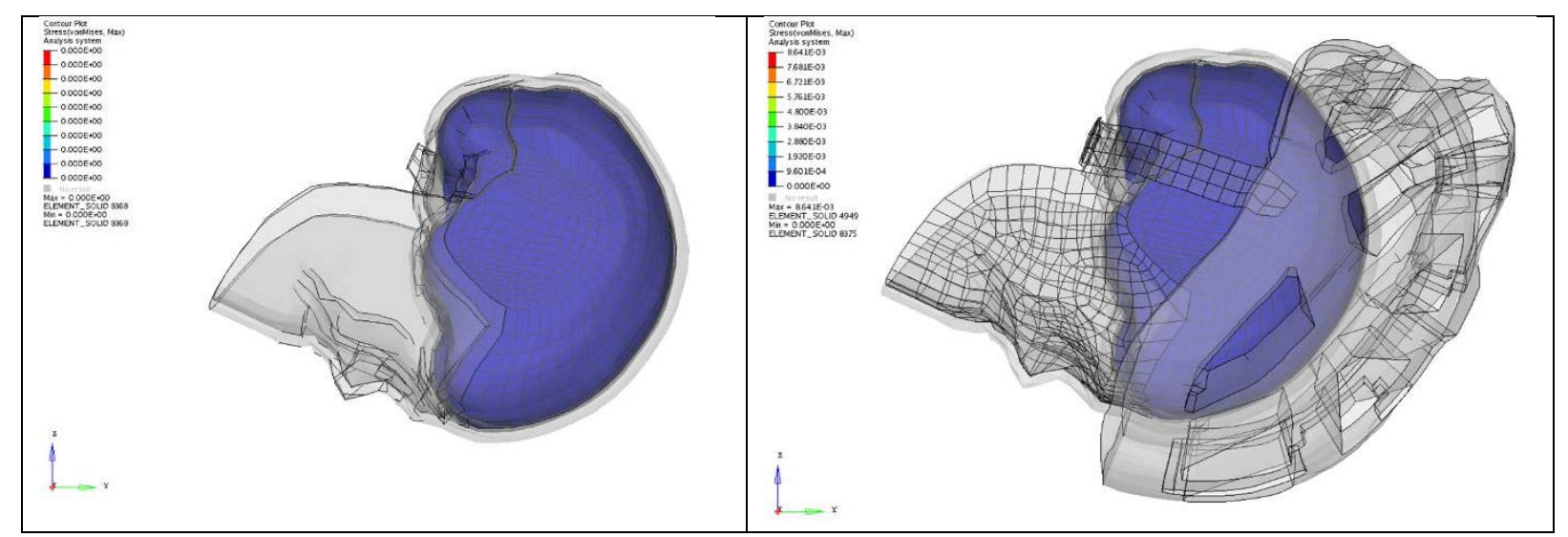

Abbildung 2-51: Kopfanprall Straße, Alleinunfall Sturz über Lenker (ID 2a und 2b)

Das Risiko für ein leichtes/mittleres DAI beträgt ohne Helm 52\%, mit Helm reduziert sich das Risiko auf 4\%. Das Risiko für ein schweres DAI beträgt ohne Helm 6\%, mit Helm wird eine Reduktion auf 0,5\% erreicht. Das Risiko für ein SDH beträgt ohne Helm 100\%, mit Helm wird das Risiko auf $8 \%$ reduziert. Das Risiko für eine Schädelfraktur beträgt ohne Helm 100\%, mit Helm wird eine Reduktion auf 74\% erreicht (VM-Spannung 28,0/8,7kPa, IE CSF 22198/2293 mJ, IE Skull 4210/619 mJ).

Auf Abbildung 2-52 ist der Kopfanprall gegen einen Bordstein dargestellt.

Die resultierende Kopfanprallgeschwindigkeit beträgt $8 \mathrm{~m} / \mathrm{s}$, der Winkel des

Geschwindigkeitsvektors zum Untergrund beträgt $101^{\circ}$. Die Normalkomponente der Kopfanprallgeschwindigkeit beträgt $5,9 \mathrm{~m} / \mathrm{s}$.

Der Anprall findet im Bereich der linken Schläfenregion statt. Bei der Simulation mit Helm wurde deutlich, dass der Anprall genau an der Kante des Helms stattfindet, der eine leichte Ausweichbewegung vollführt und für dieses Szenario sein Schutzpotential nicht entfalten kann.

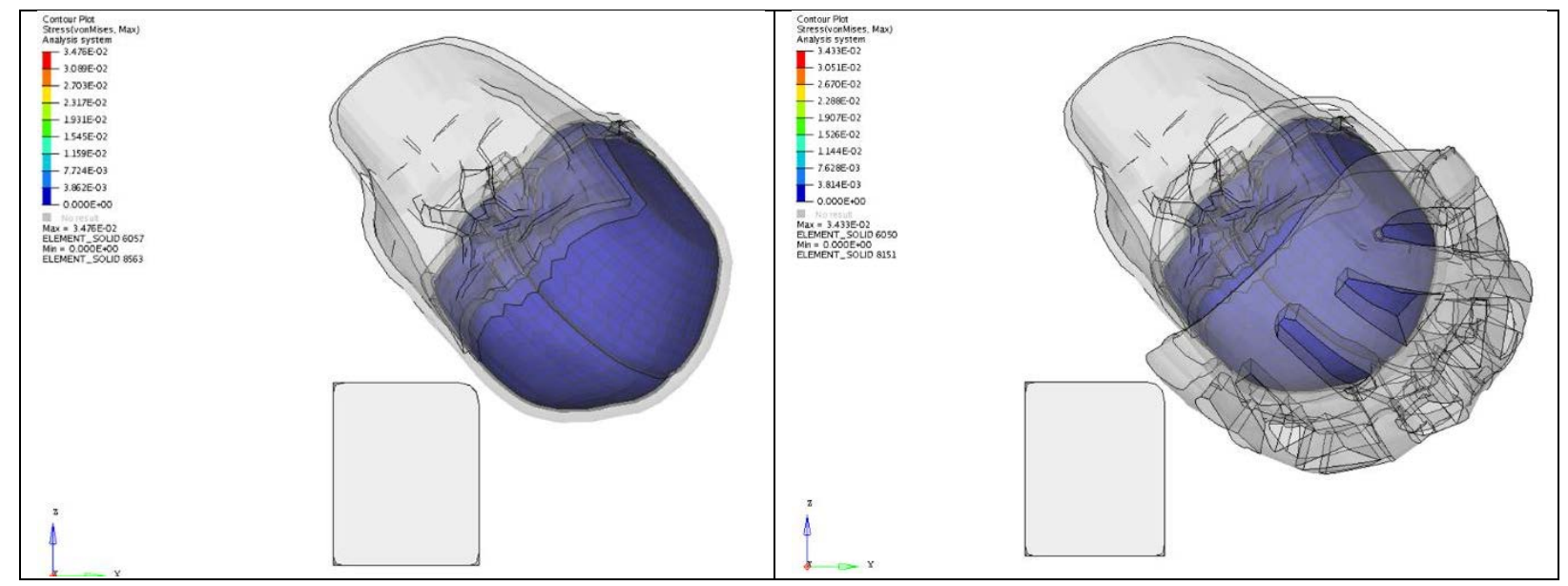

Abbildung 2-52: Kopfanprall gegen Bordstein (ID 4a und 4b) 
Das Risiko für ein leichtes/mittleres DAl beträgt ohne Helm 80\%, mit dem „Standard-Helm“ reduziert sich das Risiko nur unwesentlich auf 79\%. Das Risiko für ein schweres DAI beträgt ohne und mit Helm 13\%. Das Risiko für ein SDH beträgt ohne Helm 100\%, mit Helm wird das Risiko auf $99 \%$ reduziert. Das Risiko für eine Schädelfraktur beträgt ohne und mit Helm 100\% (VM-Spannung 34,8/34,4 kPa, IE CSF 17350/11554 mJ, IE Skull 23762/10309 mJ).

Auf Abbildung 2-53 ist das identische Kopfanprallszenario abgebildet, jedoch wurde ein "optimiertes“ Helmmodell verwendet. Der Schutzbereich dieses Helms wurde nach unten bis knapp unterhalb der Ohren erweitert, wobei die Materialeigenschaften auch auf den vergrößerten Schutzbereich angewendet wurden.
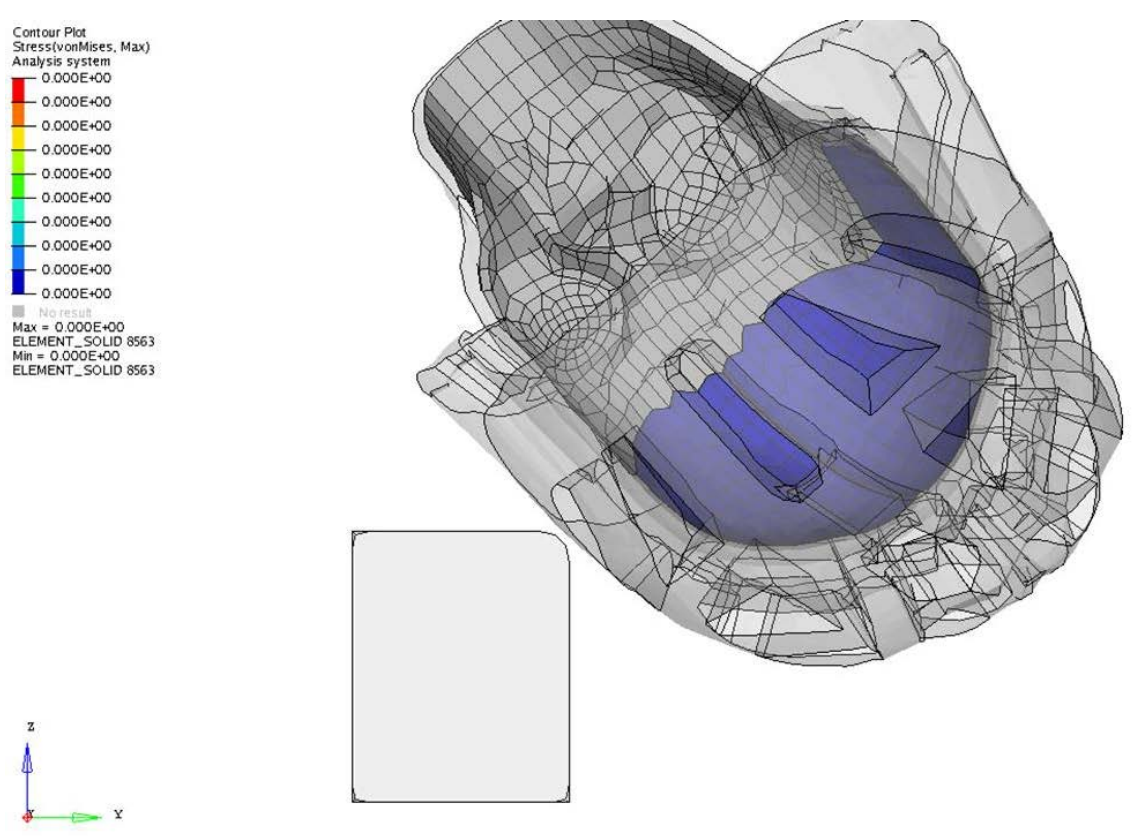

Abbildung 2-53: Kopfanprall Bordstein mit optimierten Helm (ID 4c)

Die Kopfverletzungsrisiken reduzieren sich mit diesem „optimierten“ Helmmodell deutlich, Das Risiko für ein leichtes/mittleres DAI beträgt nur noch 18\%, das Risiko für ein schweres DAI reduziert sich auf 3\%. Das Risiko für ein SDH wird auf 19\% reduziert. Das Risiko für eine Schädelfraktur bleibt bei 100\% (VM-Spannung 19,5 kPa, IE CSF 3526 mJ, IE Skull $1818 \mathrm{~mJ})$.

Auf Abbildung 2-54 das vorangegangene Kopfanprallszenario abgebildet, der Bordstein wurde jedoch aus dem Modell entfernt, so dass es zum Anprall auf die Straße kommt. Die Simulationsparameter (resultierende Kopfanprallgeschwindigkeit $8 \mathrm{~m} / \mathrm{s}$, identischer Winkel des Geschwindigkeitsvektors) sind dabei mit den Szenarien ID 4a bis 4c identisch. 


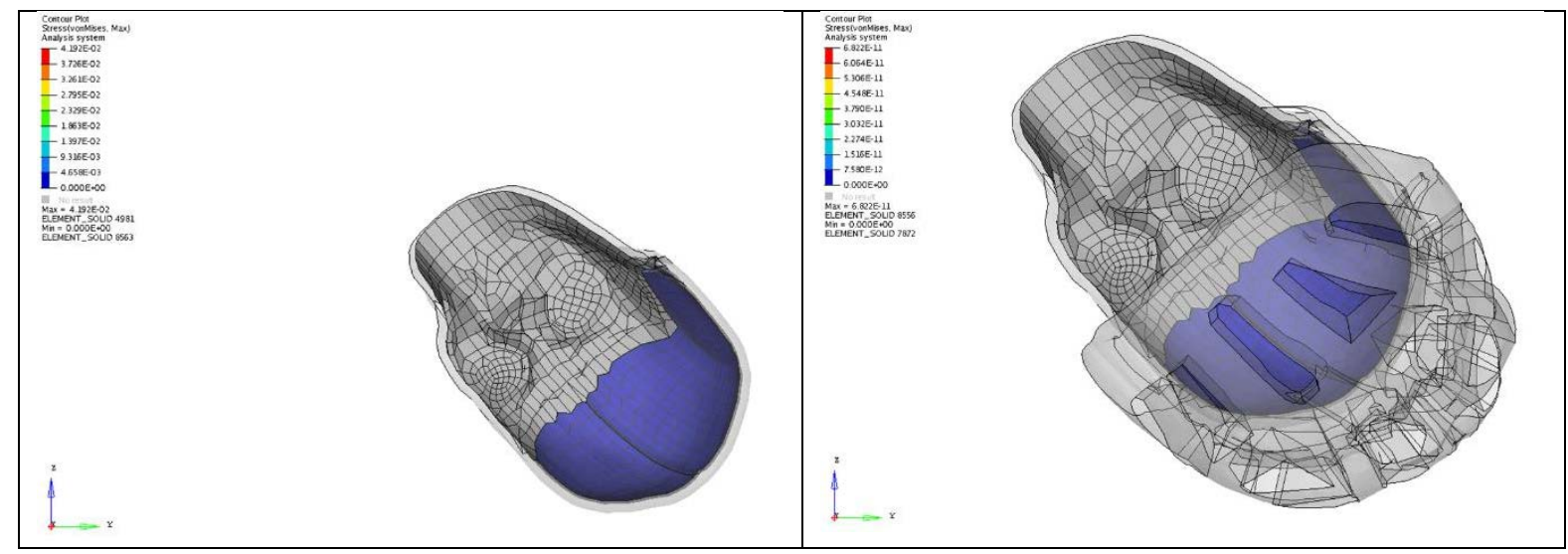

Abbildung 2-54: Kopfanprall Straße (ID P1 und P2)

Das Risiko für ein leichtes/mittleres DAI beträgt ohne Helm 93\%, mit Helm reduziert sich das Risiko auf 9\%. Das Risiko für ein schweres DAl beträgt ohne Helm 23\%, mit Helm wird eine Reduktion auf 2\% erreicht. Das Risiko für ein SDH beträgt ohne Helm 100\%, mit Helm wird das Risiko auf $1 \%$ reduziert. Das Risiko für eine Schädelfraktur beträgt ohne und mit Helm 100\% (VM-Spannung 41,9/16,6 kPa, IE CSF 18249/933 mJ, IE Skull 6753/3318 mJ).

Auf Abbildung 2-55 ist der Kopfanprall auf die Motorhaube dargestellt. Die Kollisionsgeschwindigkeit des Pkw beträgt $15 \mathrm{~km} / \mathrm{h}$. Der Kopf prallt auf einen Bereich, unter dem keine steifen Strukturen des Motors liegen.

Die resultierende Kopfanprallgeschwindigkeit beträgt 2,6 m/s, der Winkel des Geschwindigkeitsvektors zur Motorhaube $88^{\circ}$. Die Normalkomponente der Kopfanprallgeschwindigkeit beträgt $2,3 \mathrm{~m} / \mathrm{s}$.

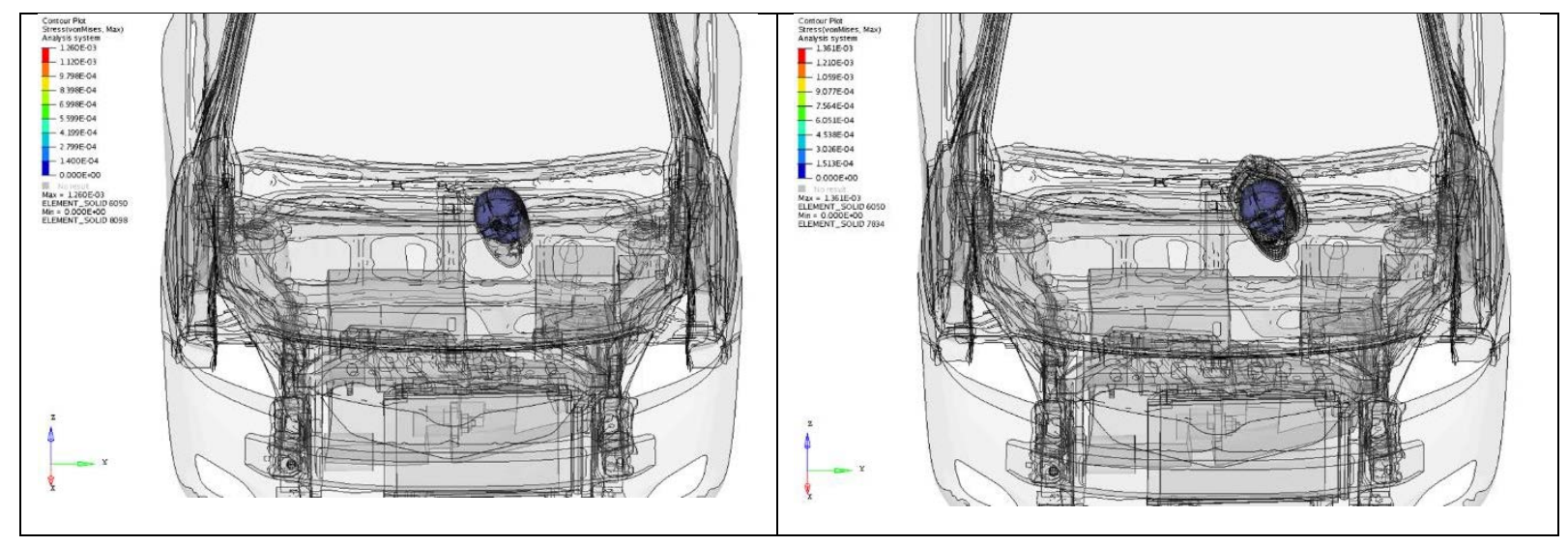

Abbildung 2-55: Kopfanprall Motorhaube (ID 5aa und 5ab)

Das Risiko für ein leichtes/mittleres und schweres DAI beträgt jeweils ohne und mit Helm $0,5 \%$.

Das Risiko für ein SDH beträgt ohne und mit Helm 0\%; das Risiko für eine Schädelfraktur beträgt ohne und mit Helm 2\% (VM-Spannung 1,26/1,26 kPa, IE CSF 10/9 mJ, IE Skull $265 / 228 \mathrm{~mJ})$.

Auf Abbildung 2-56 ist der Kopfanprall unter identischen Simulationsparametern dargestellt; allerdings liegt der Anprallpunkt des Kopfes auf der Motorhaube nun direkt über dem Motorblock. 


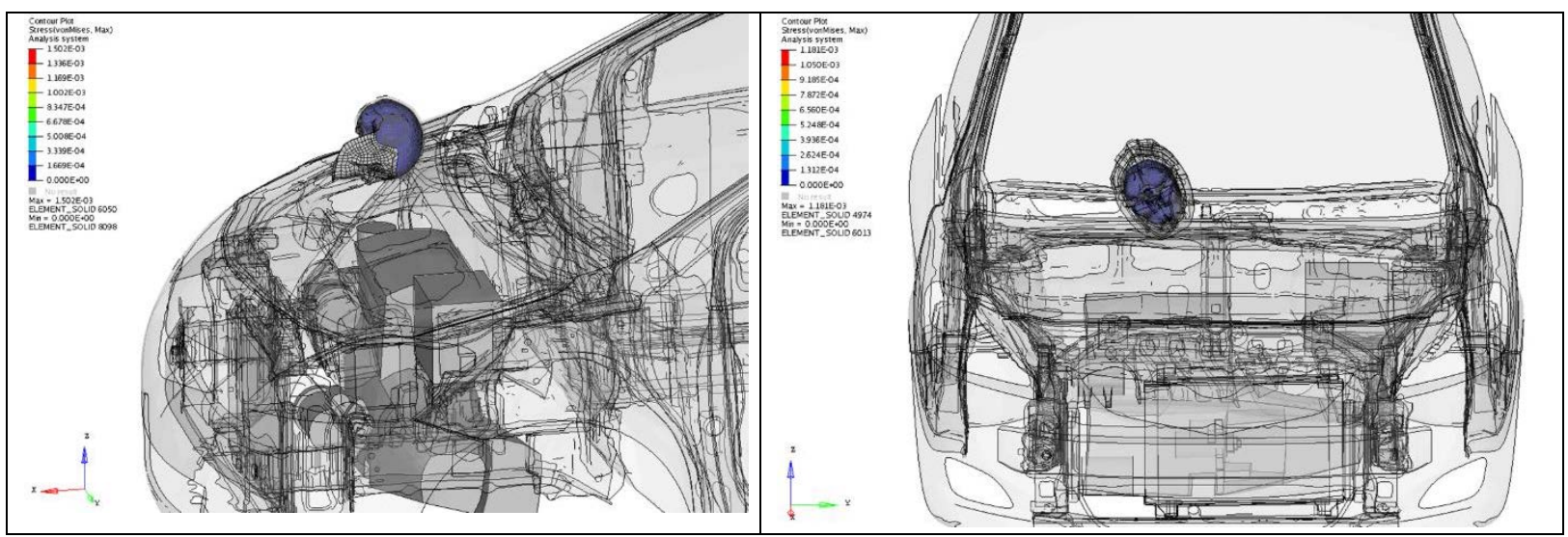

Abbildung 2-56: Kopfanprall Motorhaube „worst case“ (ID 5ac und 5ad)

Die Kopfverletzungsrisiken entsprechen dabei denen des vorherigen Szenarios und betragen nahezu Null (VM-Spannung 1,51/1,18 kPa, IE CSF 16/11 mJ, IE Skull 148/59 mJ).

Durch die Kinematik des Fahrradfahrers bedingt ist bei einem Kopfanprall auf die Motorhaube die mögliche Kopfanprallgeschwindigkeit limitiert. Durch diese geringe Geschwindigkeit wird (unter den Randbedingungen dieser Simulation) die Motorhaube nicht bis zum Kontakt mit darunter liegenden steifen Strukturen deformiert. Demnach prallt auch der Kopf nicht gegen solch steife Strukturen wie z.B. den Motorblock. Die Kopfverletzungsrisiken sind dementsprechend in diesem Szenario sehr gering.

Auf Abbildung 2-57 ist der Kopfanprall auf die Frontscheibe dargestellt. Die Kollisionsgeschwindigkeit des Pkw beträgt 40 km/h. Der Kopf prallt ins Zentrum der Frontscheibe.

Die resultierende Kopfanprallgeschwindigkeit beträgt $12 \mathrm{~m} / \mathrm{s}$, der Winkel des Geschwindigkeitsvektors zur Frontscheibe $83^{\circ}$. Die Normalkomponente der Kopfanprallgeschwindigkeit beträgt 11,9 m/s.

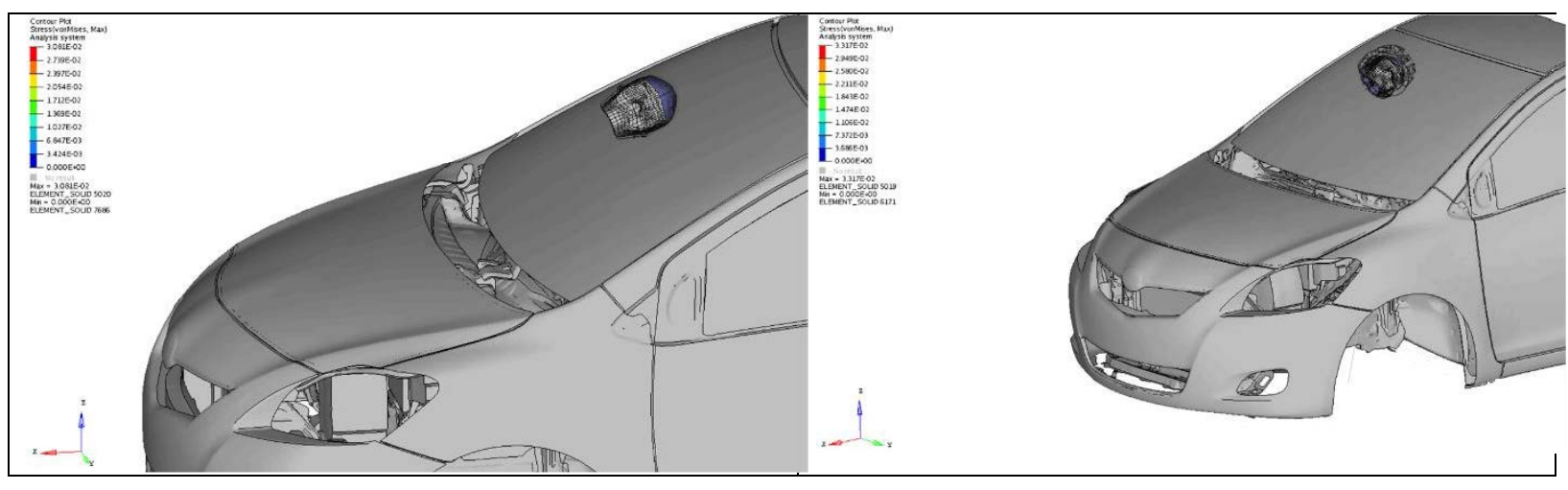

Abbildung 2-57: Kopfanprall Frontscheibe zentral (ID 7a und 7b)

Das Risiko für ein leichtes/mittleres DAI beträgt ohne Helm 68\%, mit Helm reduziert sich das Risiko auf 48\%. Das Risiko für ein schweres DAI beträgt ohne Helm 11\%, mit Helm wird eine Reduktion auf $6 \%$ erreicht. Das Risiko für ein SDH beträgt ohne Helm 53\%, mit Helm wird das Risiko auf 3\% reduziert. Das Risiko für eine Schädelfraktur beträgt ohne und mit Helm 100\% (VM-Spannung 32,7/27,9 kPa, IE CSF 5513/1040 mJ, IE Skull 14628/11521 mJ). 
Auf Abbildung 2-58 ist der Kopfanprall auf die Frontscheibe dargestellt. Die Kollisionsgeschwindigkeit des Pkw beträgt 40 km/h. Der Kopf prallt in den Randbereich der Frontscheibe nahe der A-Säule.

Die resultierende Kopfanprallgeschwindigkeit beträgt $12 \mathrm{~m} / \mathrm{s}$, der Winkel des Geschwindigkeitsvektors zur Frontscheibe $86^{\circ}$. Die Normalkomponente der Kopfanprallgeschwindigkeit beträgt $12 \mathrm{~m} / \mathrm{s}$.

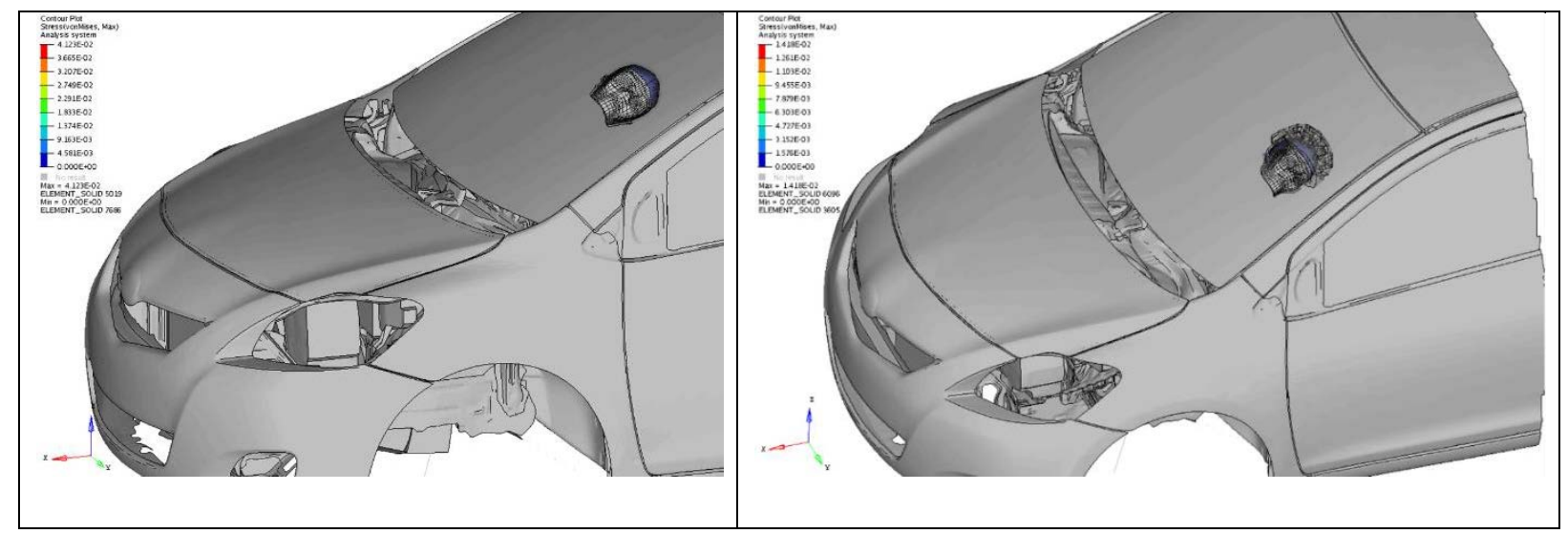

Abbildung 2-58: Kopfanprall Frontscheibe Randbereich (ID 8a und 8b)

Das Risiko für ein leichtes/mittleres DAI beträgt ohne Helm 92\%, mit Helm reduziert sich das Risiko auf 8\%. Das Risiko für ein schweres DAI beträgt ohne Helm 22\%, mit Helm wird eine Reduktion auf $2 \%$ erreicht. Das Risiko für ein SDH beträgt ohne Helm 4\%, mit Helm wird das Risiko auf 3\% reduziert. Das Risiko für eine Schädelfraktur beträgt ohne und mit Helm 100\% (VM-Spannung 41,2/14,2 kPa, IE CSF 1695/1219 mJ, IE Skull 13946/6334 mJ).

Auf Abbildung 2-59 ist ein Extremfall für den Kopfanprall auf die Frontscheibe dargestellt. Der Kopf prallt in den Randbereich nahe A-Säule und Dachkante. Die Kollisionsgeschwindigkeit des Pkw beträgt $65 \mathrm{~km} / \mathrm{h}$.

Die resultierende Kopfanprallgeschwindigkeit beträgt $15 \mathrm{~m} / \mathrm{s}$, der Winkel des Geschwindigkeitsvektors zur Frontscheibe $94^{\circ}$. Die Normalkomponente der Kopfanprallgeschwindigkeit beträgt 14,4 m/s.

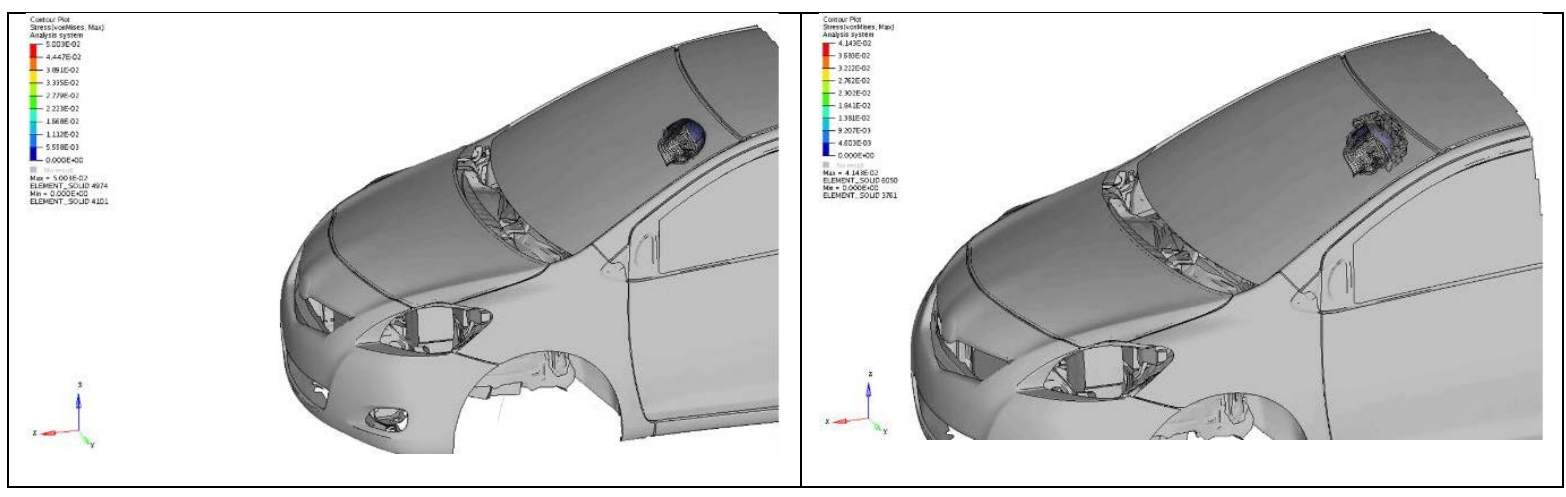

Abbildung 2-59: Extremszenario Kopfanprall Frontscheibe (ID 3a und 3b)

Das Risiko für ein leichtes/mittleres DAI beträgt ohne Helm 99\%, mit Helm reduziert sich das Risiko auf 92\%. Das Risiko für ein schweres DAI beträgt ohne Helm 42\%, mit Helm wird eine Reduktion auf 22\% erreicht. Das Risiko für ein SDH beträgt ohne Helm 31\%, mit Helm wird das Risiko auf 13\% reduziert. Das Risiko für eine Schädelfraktur beträgt ohne und mit Helm 100\% (VM-Spannung 50,0/41,4 kPa, IE CSF 4078/2976 mJ, IE Skull 22143/15032 mJ). 


\subsubsection{Bewertung der Simulation}

Die oben dargestellten Simulationen lieferten in Verbindung mit den verwendeten Modellen plausible Bewegungsabläufe und Kopfbelastungswerte. Gleichwohl sind jeder Form von Simulation Grenzen gesetzt, die man beachten muss. Jedes Modell stellt eine Vereinfachung der Realität dar und beeinträchtigt dadurch naturgemäß die Genauigkeit der Simulationsergebnisse. Die Verwendung von Modellen sollte immer in dafür den vorgesehenen Parameterbereichen erfolgen, um Ergebnisse zu erhalten, die sich auf die Realität übertragen lassen. Weitere Fehlerquellen können in der Ungenauigkeit von Ausgangsdaten liegen.

Die verwendeten Modelle in MADYMO sind zur Simulation der Kinematik eines Fahrradfahrers in den betrachteten Unfallabläufen gut geeignet. Die Mensch-Modelle wurden bereits erfolgreich in mehreren Studien zur kinematischen Betrachtung von Fahrradfahrern im Unfallablauf verwendet (u.a. Chen et al. 2008; Bourdet et al. 2012). Die Fahrradmodelle wurden realitätsnah erstellt und bieten naturgemäß eine geringe Fehlerquelle, zumal der Fokus der Betrachtungen ausschließlich auf der Kinematik der Radfahrer lag. Die verwendeten Modelle der Pkw-Front wurden zum Zweck von solchen Bewegungsanalysen erstellt und eignen sich somit sehr gut für oben dargestellte Simulationen.

Die Messung der Kopfanprallgeschwindigkeiten und Kopfanprallwinkel zum Zeitpunkt des Kopfanpralls erfolgte sehr genau im Postprozessor und bietet eine sehr geringe Fehlerquelle. Der Transfer dieser Daten in die FE-Umgebung als Ausgangswerte für die Simulation der Kopfbelastungen war problemlos und sehr genau möglich.

Die verschieden Schritte zur Erstellung und Validierung des verwendeten Helmmodells wurden bereits unter 2.2 genauer erläutert; die Modellierungsgüte ist als sehr gut zu bezeichnen. Das verwendete FE-Pkw-Modell wurde primär zur Simulation eines PkwAnpralls gegen starre oder deformierbare Barrieren entwickelt. Die Frontscheibe Modellierung der Frontscheibe erfolgte möglichst genau, gleichwohl ist die Modellierung eines so komplexen Materialverbunds (Verbundglas) in Zusammenhang mit der komplexen Form nicht trivial. Für die durchgeführten Simulationen erschien die Modellierungsgüte trotz möglicher Ungenauigkeiten als ausreichend.

Das Kopfmodell der Universität Straßburg wurde zur Simulation von Anprallvorgängen entwickelt eignet sich daher sehr gut für die oben durchgeführten Betrachtungen. Dieses Modell ist sehr komplex und wird seit vielen Jahren kontinuierlich verbessert.

Verbesserungspotential liegt im Kontext der in diesem Projekt realisierten Betrachtungen u.a. möglicherweise in der Risikokurve zur Ermittlung des Risikos für eine Schädelfraktur. In fast allen analysieren Kopfanprallszenarien betrug das Risiko für eine Schädelfraktur $100 \%$. Die dahinter liegenden Messwerte der inneren Energie des Schädels schwanken aber teils sehr deutlich. So beträgt dieser Wert beim Anprall des Kopfs gegen den seitlichen Randbereich der Frontscheibe (ID 8a und 8b) ohne Helm 13946 mJ und mit Helm 6334 mJ. Das entspricht einer Reduktion von 55\%. Das Kopfverletzungsrisiko anhand der Risikokurve beträgt jedoch bei einem Wert von ca. $1000 \mathrm{~mJ}$ bereits $100 \%$, so dass sich diese deutliche Reduktion der Kopfbelastung nicht in einer Reduktion des Verletzungsrisikos ausdrückt. 


\subsubsection{Vergleich der Kontaktparameter Unfallgeschehen/Norm}

Im Folgenden werden die durch Realunfallanalyse (siehe 1.4) sowie Simulation typischer Unfälle gewonnenen Erkenntnisse bezüglich des Kopfanpralls bei Fahrradunfällen mit den Anforderungen der in Deutschland gültigen Norm für Fahrradhelme (DIN EN 1078) verglichen.

\subsubsection{Kontaktparameter DIN EN 1078}

In der DIN EN 1078 wird zur Überprüfung des Stoßdämpfungsvermögens eines Fahrradhelms ein behelmter Prüfkopf aus mit einer definierten Geschwindigkeit auf einen starr befestigten Stahlsockel fallengelassen.

Der Anprallpunkt muss dabei in einer definierten Prüfzone auf der Helmoberfläche liegen (siehe 2.1).

\subsection{Prüfzone}

Bezüglich der Prüfzone wird in der DIN EN 1078 gefordert: "Der Helm muss die Stirn, den Hinterkopf, die Seiten, die Schläfen und den Oberkopf des Benutzers schützen" (DIN EN 1078). Dazu ist der Helm entsprechend eines vorgeschriebenen Schemas zu vermessen und die Prüfzone zu kennzeichnen.

Die Prüfzone deckt den Kopf oberhalb einer Umfangslinie um Stirn und Hinterkopf ab (siehe Abbildung 2-60). Bei Durchführung des Stoßdämpfungstests auf den flachen Sockel gilt die Zone über der Line von 1 nach 3 als Prüfzone. Wird auf den Bordsteinsockel getestet, gilt die Zone über der Linie zwischen den Punkten 1, 2 und 3' als Prüfzone. Im Vergleich zur Prüfzone des flachen Sockels ist von der Mitte des Kopfs nach hinten ein Teil des unteren Hinterkopfs ausgespart.

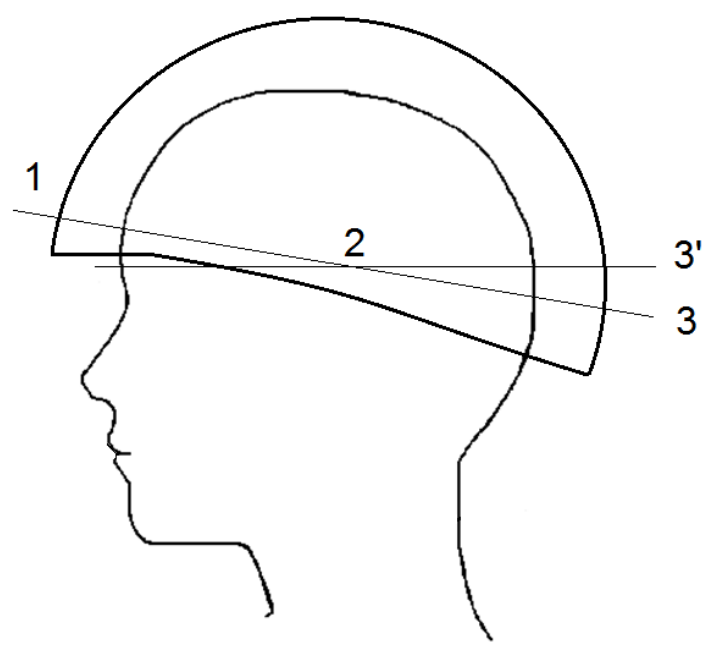

\section{Abbildung 2-60: Prüfzone}

Bei einem Test nach EN 1078 sind „Aufschlagspunkte auszuwählen, die für die Bedingungen "im schlimmsten Fall" stehen [...]. Bei jeder Prüfserie eines Prüfmusters werden die Aufschläge auf jede als schwach erkannte Zone [...] ausgeführt“. 


\subsection{Testsockel}

Es wird der Aufprall auf einen flachen und bordsteinähnlichen Sockel getestet. Der flache Sockel ist eine runde, ebene Stahlplatte und soll die Straßenoberfläche abbilden. Der Bordstein-Sockel ist ebenfalls aus Stahl gefertigt und bildet die Kante eines Bordsteins ab. Der Helm prallt im Test auf die nach oben gerichtete Kante.

\subsection{Prüfgeschwindigkeiten}

In der DIN EN 1078 wird der Aufschlag auf einen flachen Sockel bei 5,42 m/s und der Aufschlag auf einen bordsteinähnlichen Sockel mit $4,57 \mathrm{~m} / \mathrm{s}$ geprüft.

Das entspricht einer Geschwindigkeit von 19,5 bzw. 16,5 km/h und einer Fallhöhe von 1,5 bzw. 1,1 Metern.

\subsubsection{Kontaktparameter Unfallgeschehen}

\subsection{Anprallpunkte am Kopf / Helm}

Wie bereits unter 1.4 dargestellt, sind insbesondere bei schweren Unfällen die temporale Region, die Stirn und auch der Bereich des unteren Hinterkopfs häufig verletzt. Verletzungen im Bereich des Oberkopfs bzw. Scheitels sind im analysierten Fallmaterial fast nicht vorhanden.

Auch bei den untersuchten beschädigten Fahrradhelmen hat sich gezeigt, dass Schäden und damit Anprallpunkte im Bereich des Oberkopfs selten vorhanden sind.

Es sind häufig Schäden am Rand des Helmes dokumentiert. Somit liegt der Schluss nahe, dass auch Helmträger oft mit Stirn, Schläfe und Hinterkopf anprallen, möglicherweise auch außerhalb des Schutzbereichs.

Die aktuelle Testanforderung an den Schutzbereich von Fahrradhelmen bildet demnach das reale Unfallgeschehen nur unzureichend ab.

\subsection{Anpralloberflächen}

Im analysierten Unfallmaterial prallt der Kopf meist auf eine flache, harte Oberfläche. Bei Alleinunfällen findet in der überwiegenden Mehrheit der Fälle der Kopfanprall auf der Straße statt. In seltenen Ausnahmefällen ist auch ein Kopfanprall auf dem Bordstein dokumentiert, was aber meist schwerwiegende Verletzungen nach sich zieht.

Im Falle einer Kollision mit einem Pkw ist bei tödlichen Unfällen der SUD oft ein Kopfanprall auf die Frontscheibe, seltener auch in Bereiche bei der A-Säule oder Dachkante dokumentiert.

\subsection{Kopfanprallgeschwindigkeiten}

Die Kopfanprallgeschwindigkeit lässt sich je nach Kollisionstyp in verschiedene Bereiche einteilen.

Beim reinen Alleinunfall bewegt sich die Kopfanprallgeschwindigkeit (auch die Normalkomponente) typischerweise im Bereich von ca. 4 bis knapp über $5 \mathrm{~m} / \mathrm{s}$. 
Bei einer Kollision mit einem Pkw kann die Kopfanprallgeschwindigkeit deutlich variieren. Ist sie bei einem Kopfanprall auf die Motorhaube typischerweise sehr gering (unter $3 \mathrm{~m} / \mathrm{s}$ ), kann sie je nach Kollisionsgeschwindigkeit des Pkw deutlich ansteigen.

Bei oben dargestellter rechtwinkliger Kollisionsstellung und einer Kollisionsgeschwindigkeit des Pkw von $30 \mathrm{~km} / \mathrm{h}$ beträgt die Kopfanprallgeschwindigkeit gegen die Scheibe ca. $8 \mathrm{~m} / \mathrm{s}$, bei einer Kollisionsgeschwindigkeit von $40 \mathrm{~km} / \mathrm{h}$ ca. $12 \mathrm{~m} / \mathrm{s}$ und bei einer Kollisionsgeschwindigkeit von $65 \mathrm{~km} / \mathrm{h}$ ca. $15 \mathrm{~m} / \mathrm{s}$.

Der Kopfanprallwinkel relativ zur Windschutzscheibe ist dabei in den oben genannten Kollisionsszenarien typischerweise annähernd rechtwinklig und entspricht demnach vereinfachend dargestellt einem senkrecht nach unten gerichteten Fall ohne relevante Tangentialkomponente.

\subsubsection{Diskussion der Anforderungen durch Testnorm}

Die Analyse realer Unfälle hat aufgezeigt, dass die aktuelle Fahrradhelmnorm bereits ein guter Ansatz ist, um das Schutzvermögen von Fahrradhelmen zu bewerten. In einigen Punkten ist jedoch Verbesserungspotential vorhanden.

So bildet die aktuelle Prüfzone die Realität nur unzureichend ab.

Insbesondere bei schweren Unfällen sind die temporale Region, die Stirn und auch der Bereich des unteren Hinterkopfs häufig verletzt. Verletzungen im Bereich des Oberkopfs bzw. Scheitels sind fast nicht vorhanden.

Dabei ist unter anderem nach McIntosh et al. (1998) und Depreitere et al. (2007) gerade die temporoparietale Region des Schädels besonders anfällig für schwere Verletzungen.

Bei den untersuchten beschädigten Fahrradhelmen hat sich gezeigt, dass häufig Schäden am Rand des Helmes dokumentiert sind. Studien von Otte et al. 2013 sowie Bourdet et al. 2012 zeigen ebenfalls, dass oft ein Anprall im Randbereich des Helms oder unter der Helmkante stattfindet.

Es wird deshalb empfohlen, die Prüfzone in diesen kritischen Bereichen auszuweiten, insbesondere der temporale Bereich bedarf einer optimierten Abdeckung.

In der DIN EN 1078 sind keine spezifischen Prüfpunkte vorgeschrieben. Es sollen vom Prüfer die Punkte ausgewählt werden, die „für den schlimmsten Fall“ (DIN EN 1078) stehen. Es erscheint sinnvoll, die als kritisch erkannten Zonen im Bereich der Stirn und temporalen Region verpflichtend zur Prüfung festzulegen. Dabei soll die Gefahr der möglichen Optimierung von zukünftigen Fahrradhelmen nur „auf den Punkt“ vermieden werden; der Fahrradhelm muss im kompletten Schutzbereich sein volles Potential ausschöpfen.

Im realen Unfallgeschehen ist ein Kopfaufprall auf ein bordsteinähnliches Hindernis selten dokumentiert.

Bei Alleinunfällen prallt der Kopf meist auf die flache Straße; in wenigen Fällen ist ein Kopfanprall auf die Bordsteinkante oder eine ähnliche Oberfläche dokumentiert. Die Veröffentlichung von Deck et al. (2012) zeigt vergleichbare Ergebnisse.

Im Falle einer Kollision mit einem Pkw erfolgt der Kopfanprall typischerweise im Bereich der Motorhaube oder Windschutzscheibe. 
Ein Kopfanprall gegen eine steife Struktur eines Pkw (z.B. A-Säule, Dachkante) ist verhältnismäßig selten, jedoch meist mit schweren Verletzungen verbunden.

Es erscheint deshalb angemessen, weiterhin beide Testsockel für den Stoßdämpfungstest zu verwenden.

Die Kopfanprallgeschwindigkeit beträgt im Realunfallgeschehen in der Mehrheit der Unfälle ohne Fremdbeteiligung zwischen 4 und $5 \mathrm{~m} / \mathrm{s}$ und deckt sich annähernd mit Ergebnissen anderen Studien (z.B. Bourdet et al. 2012).

Bei Unfällen mit Pkw-Beteiligung findet bei niedriger Kollisionsgeschwindigkeit (bis ca. $20 \mathrm{~km} / \mathrm{h}$ ) der Kopfanprall auf die Motorhaube mit geringer Kopfanprallgeschwindigkeit (unter $3 \mathrm{~m} / \mathrm{s}$ ) statt. Für diese Fälle ist das Risiko für Kopfverletzungen sehr gering.

Je nach Kollisionsgeschwindigkeit des Pkw sind meist deutlich höhere

Kopfanprallgeschwindigkeiten zu beobachten. Die mittlere Kollisionsgeschwindigkeit bei Fahrradunfällen mit Pkw-Beteiligung liegt bei ca. 40 km/h (Fredriksson et al. 2012; Otte et al. 2013; UDV 2014). Dabei beträgt die Kopfanprallgeschwindigkeit dann ungefähr 12 m/s.

Die aktuelle Fahrradhelmnorm bildet demnach nur den typischen Geschwindigkeitsbereich von Alleinunfällen ab. Die bei Kollisionen des Fahrradfahrers mit einem Pkw teils deutlich höheren Kopfanprallgeschwindigkeiten werden nicht geprüft.

In der Diskussion um Verbesserungen bei Helmtestverfahren muss daher auch über eine Anhebung der Prüfgeschwindigkeit nachgedacht werden.

\section{$2.5 \quad$ Fallversuche}

\subsubsection{Versuchsaufbau und Testmatrix}

Zusätzlich zu den oben dargestellten Betrachtungen wurden in Anlehnung an die EN 1078 ergänzende Fallversuche durchgeführt. Dazu wurde das Modell eines großen Helmherstellers (Kopfgröße 52-57 cm, für Männer) ausgewählt, der nach Auskunft des Herstellers das meistverkaufte Modell zum Zeitpunkt des Projektbeginns war. Es handelt sich um einen typischen Mikroschalen-Helm.

Die durchgeführten Versuche sind in Tabelle 2-16 aufgelistet. Es wurden die Anprallpunkte und Aufprallgeschwindigkeiten variiert; ebenso wurden drei Versuche mit einer Hybrid IIIKopfform durchgeführt. Die Anprallkonstellation der Tests 1 bis 3 decken sich mit denen der Tests 8 bis 10, so dass eine direkte Vergleichbarkeit der ermittelten Belastungswerte zwischen den beiden verwendeten Kopfformen gegeben ist. Es wurden der EN-Prüfkopf der Kopfform J mit einer Masse von 4,85 kg und eine 50\% männliche Hybrid III-Kopfform mit einer Masse von 4,6 kg verwendet.

Für jeden Test wurden die linearen Beschleunigungen in allen drei Achsenrichtungen über die Zeit gemessen, aus denen im Anschluss die Kurven der resultierenden Beschleunigungen errechnet wurden.

Zudem wurde der HIC 15 berechnet. Dieser dimensionslose Wert wird durch die Integration der Kopfbeschleunigung über den Zeitraum von 15 ms ermittelt. Damit wird zusätzlich zum (Maximal-) Wert der Kopfbeschleunigung auch die zeitliche Ausdehnung der Belastung mit einbezogen. 
Tabelle 2-16: Testmatrix Fallversuche

\begin{tabular}{|c|c|c|c|}
\hline Versuch & Anprallort & Kopfform & $\begin{array}{c}\text { Geschwindigkeit } \\
{[\mathrm{m} / \mathbf{s}]}\end{array}$ \\
\hline 1 & seitlich & EN & 5,43 \\
\hline 2 & fronto-temporal & EN & 5,43 \\
\hline 3 & zentr. Scheitel & EN & 5,43 \\
\hline 4 & seitlich & EN & 7,7 \\
\hline 5 & seitlich & EN & 7,7 \\
\hline 6 & seitlich & EN & 6,5 \\
\hline 7 & fronto-temporal & EN & 6,5 \\
\hline 8 & seitlich & Hybrid III & 5,42 \\
\hline 9 & zentr., Scheitel & Hybrid III & 5,42 \\
\hline 10 & fronto-temporal & Hybrid III & 5,42 \\
\hline 11 & fronto-temporal & EN & 5,43 \\
\hline
\end{tabular}

\subsubsection{Ergebnisse}

Auf den folgenden Abbildung 2-61 bis Abbildung 2-71 sind die resultierenden Beschleunigungsverläufe für die durchgeführten Fallversuche abgebildet.

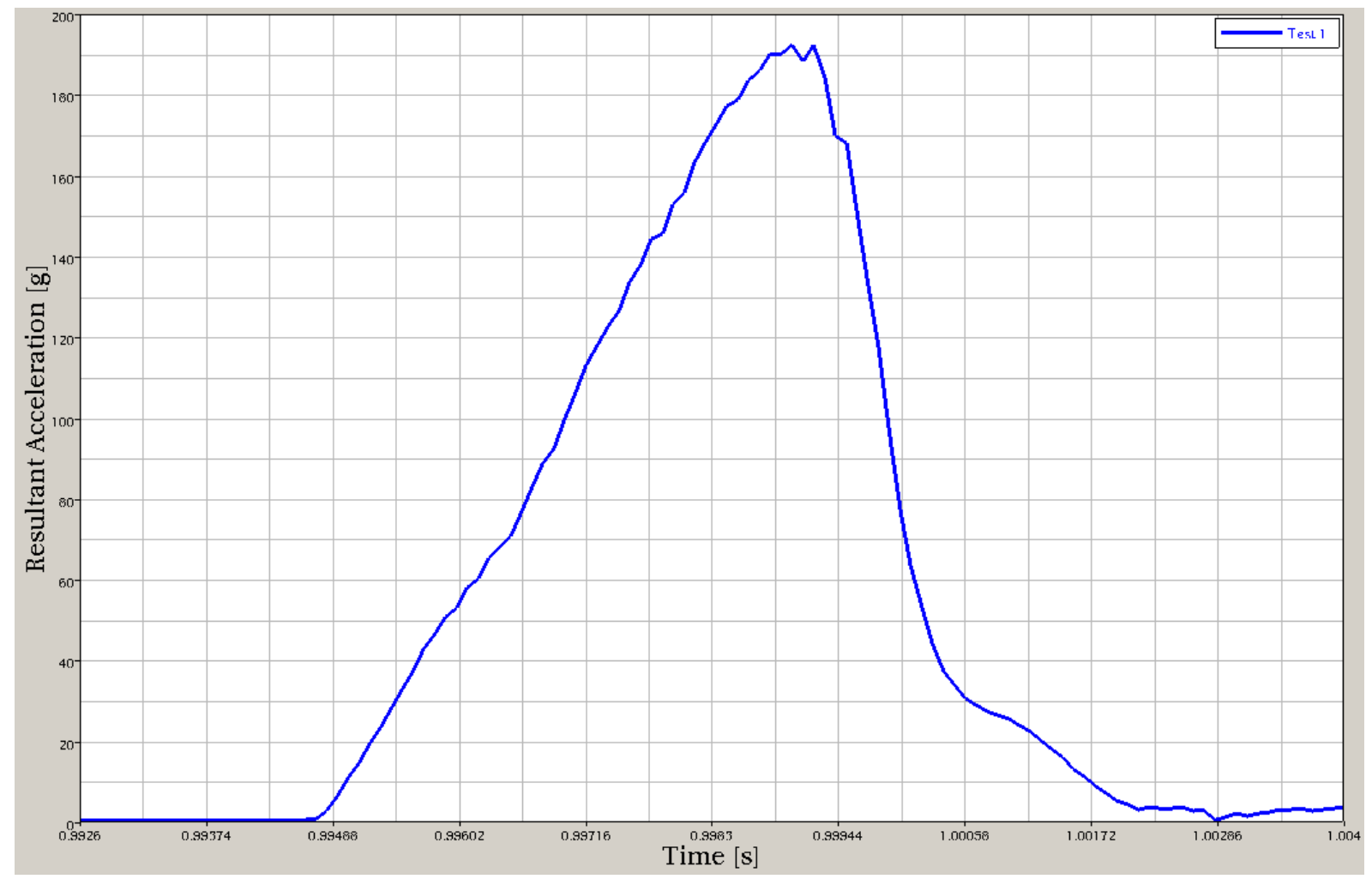

Abbildung 2-61: Fallversuch 1 (Anprall seitlich, Kopfform nach EN, Geschwindigkeit 5,43 m/s) 


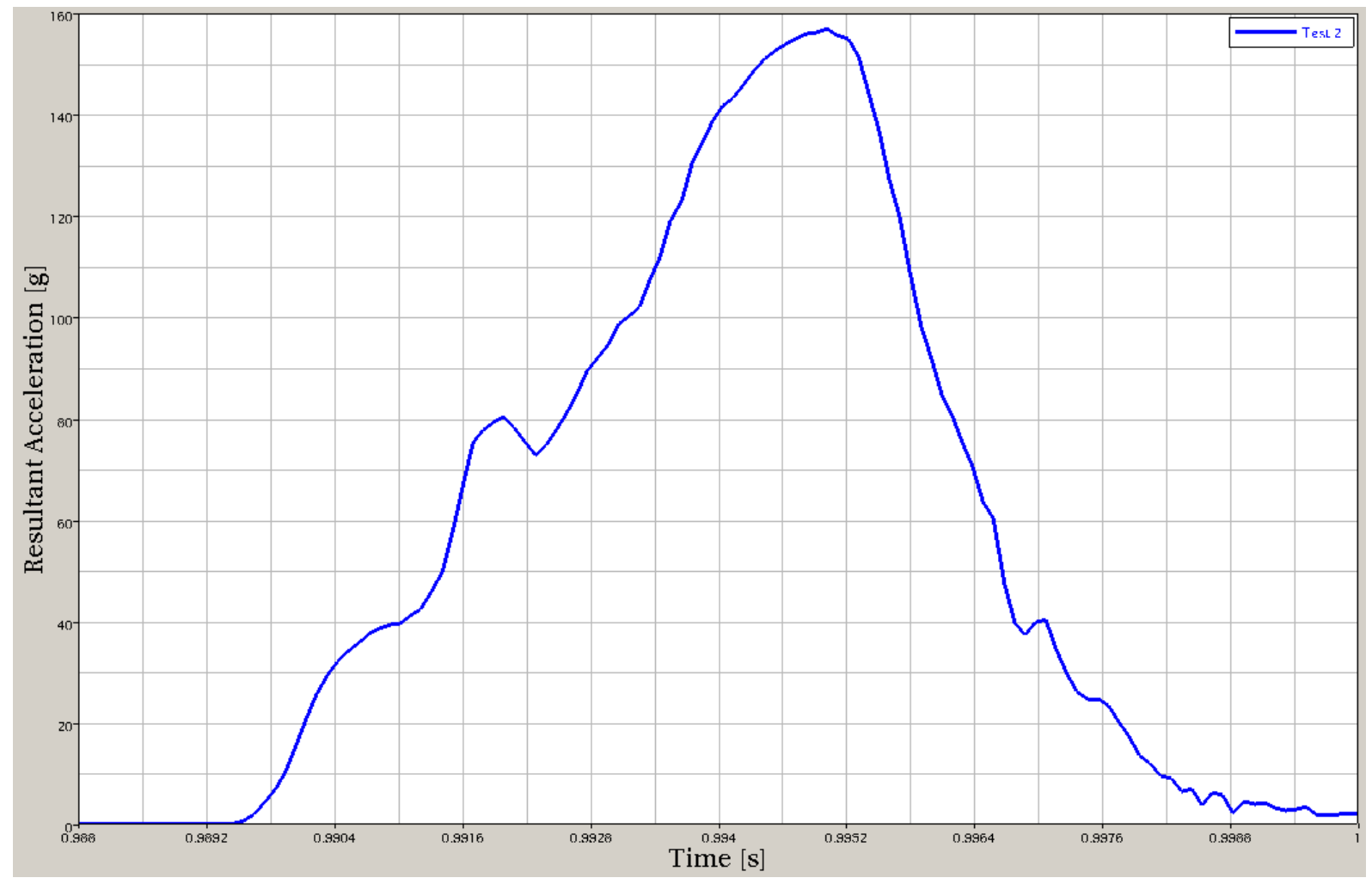

Abbildung 2-62: Fallversuch 2 (Anprall fronto-temporal, Kopfform nach EN, Geschwindigkeit 5,43 m/s)

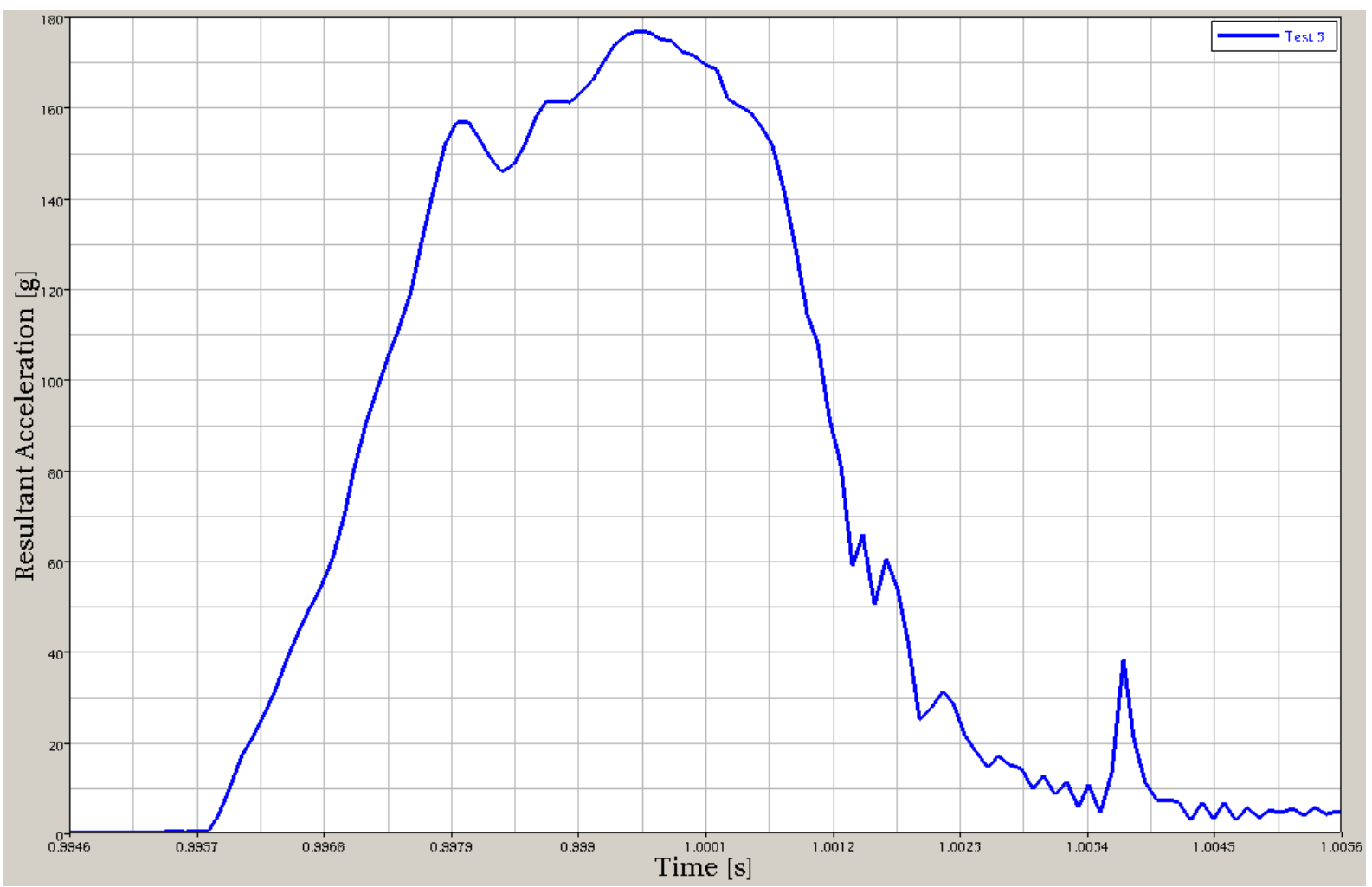

Abbildung 2-63: Fallversuch 3 (Anprall zentr. Scheitel, Kopfform nach EN, Geschwindigkeit 5,43 m/s) 


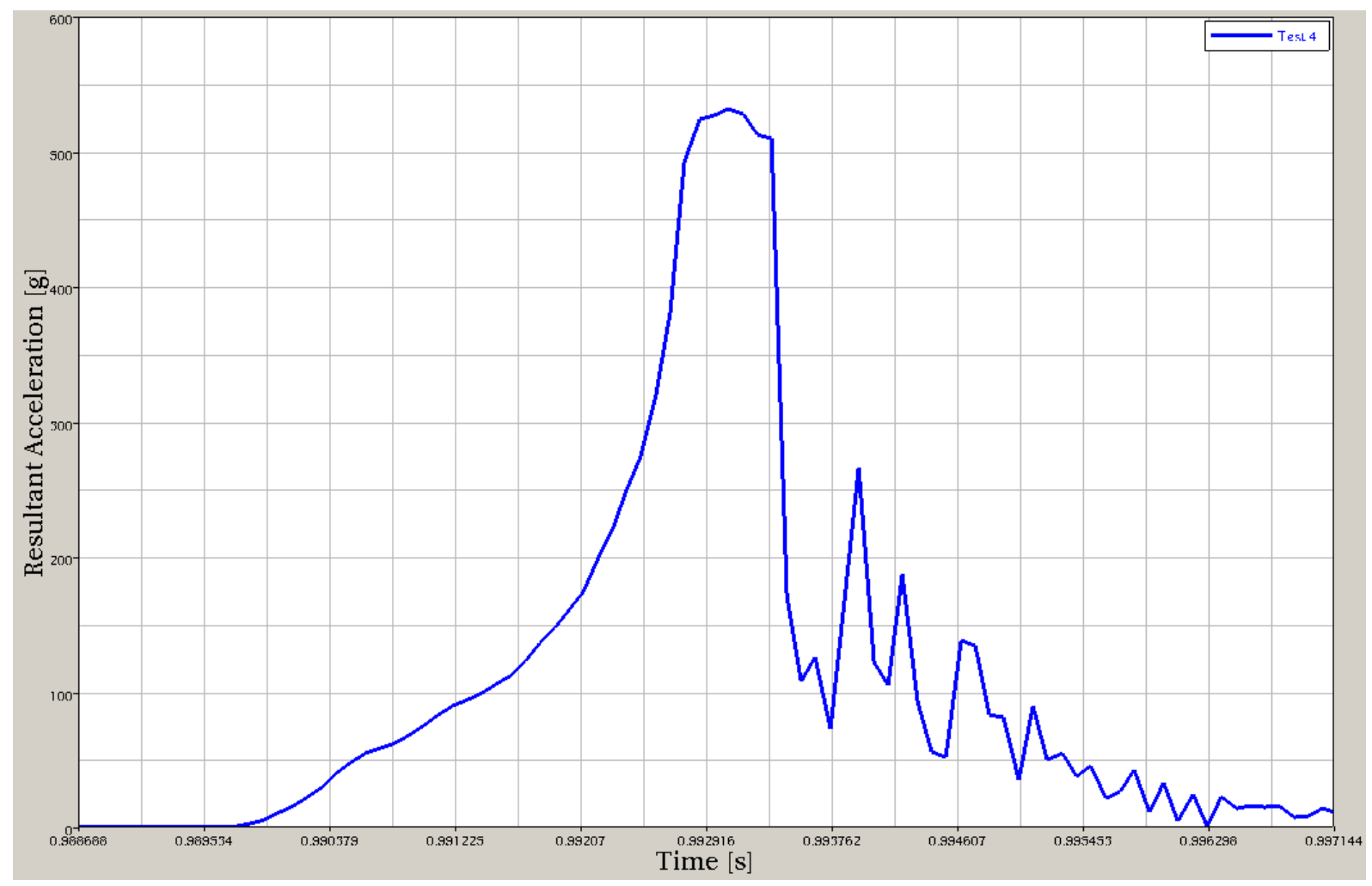

Abbildung 2-64: Fallversuch 4 (Anprall seitlich, Kopfform nach EN, Geschwindigkeit 7,7 m/s), Überschreitung des Beschleunigungsaufnehmer-Messbereichs

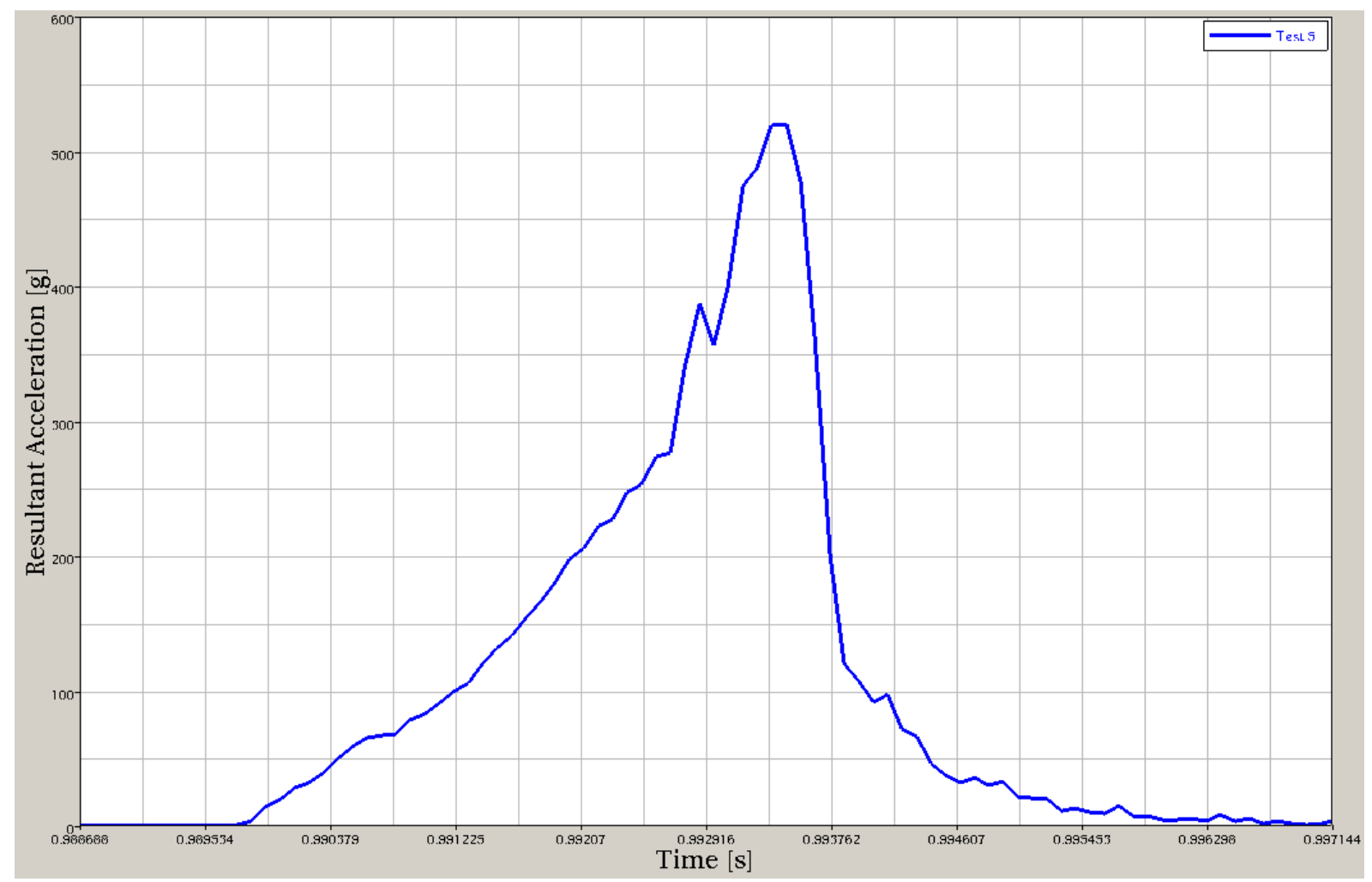

Abbildung 2-65: Fallversuch 5 (Anprall seitlich, Kopfform nach EN, Geschwindigkeit 7,7 m/s), Überschreitung des Beschleunigungsaufnehmer-Messbereichs 


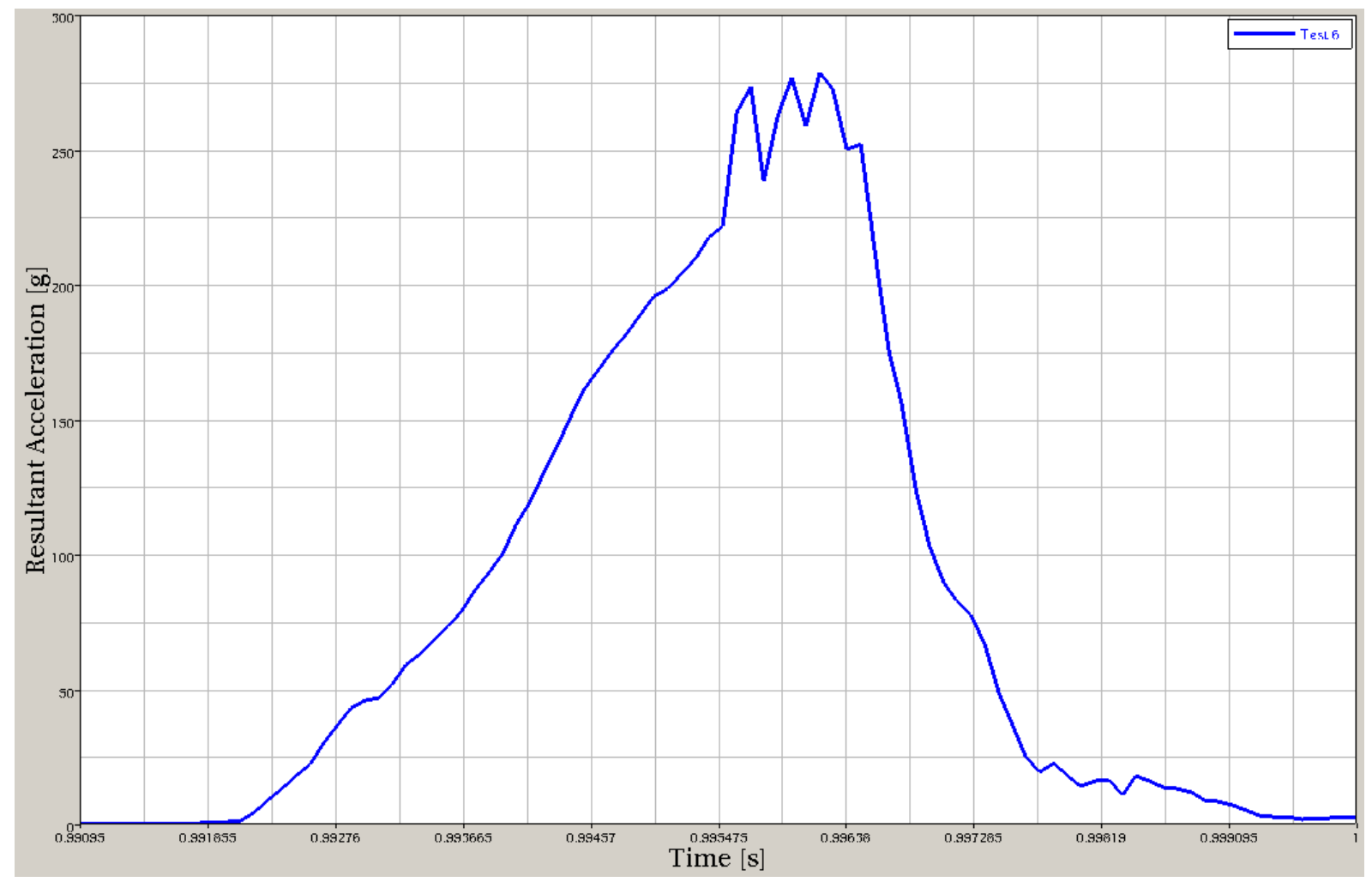

Abbildung 2-66: Fallversuch 6 (Anprall seitlich, Kopfform nach EN, Geschwindigkeit 6,5 m/s)

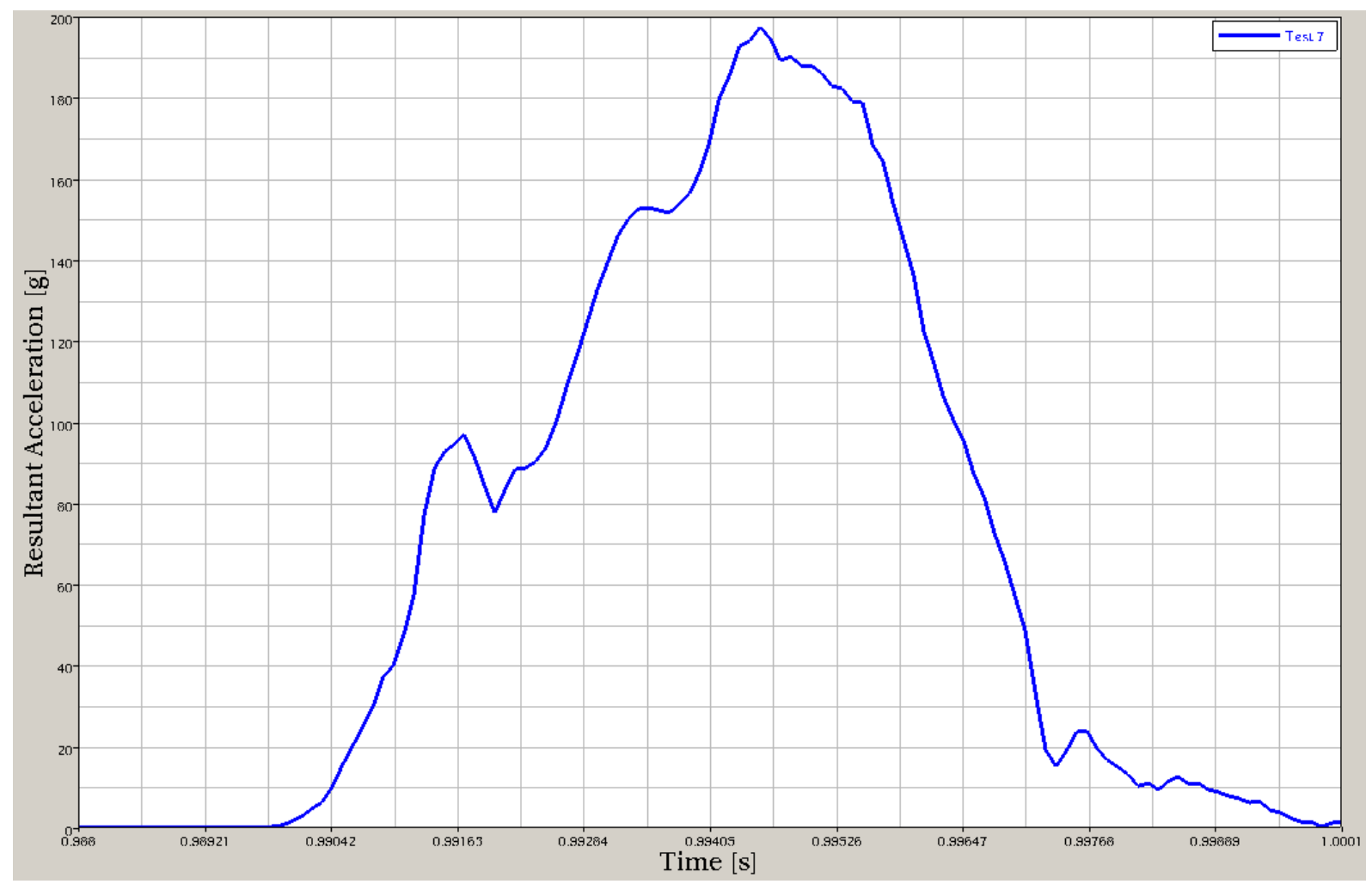

Abbildung 2-67: Fallversuch 7 (Anprall fronto-temporal, Kopfform nach EN, Geschwindigkeit 6,5 m/s) 


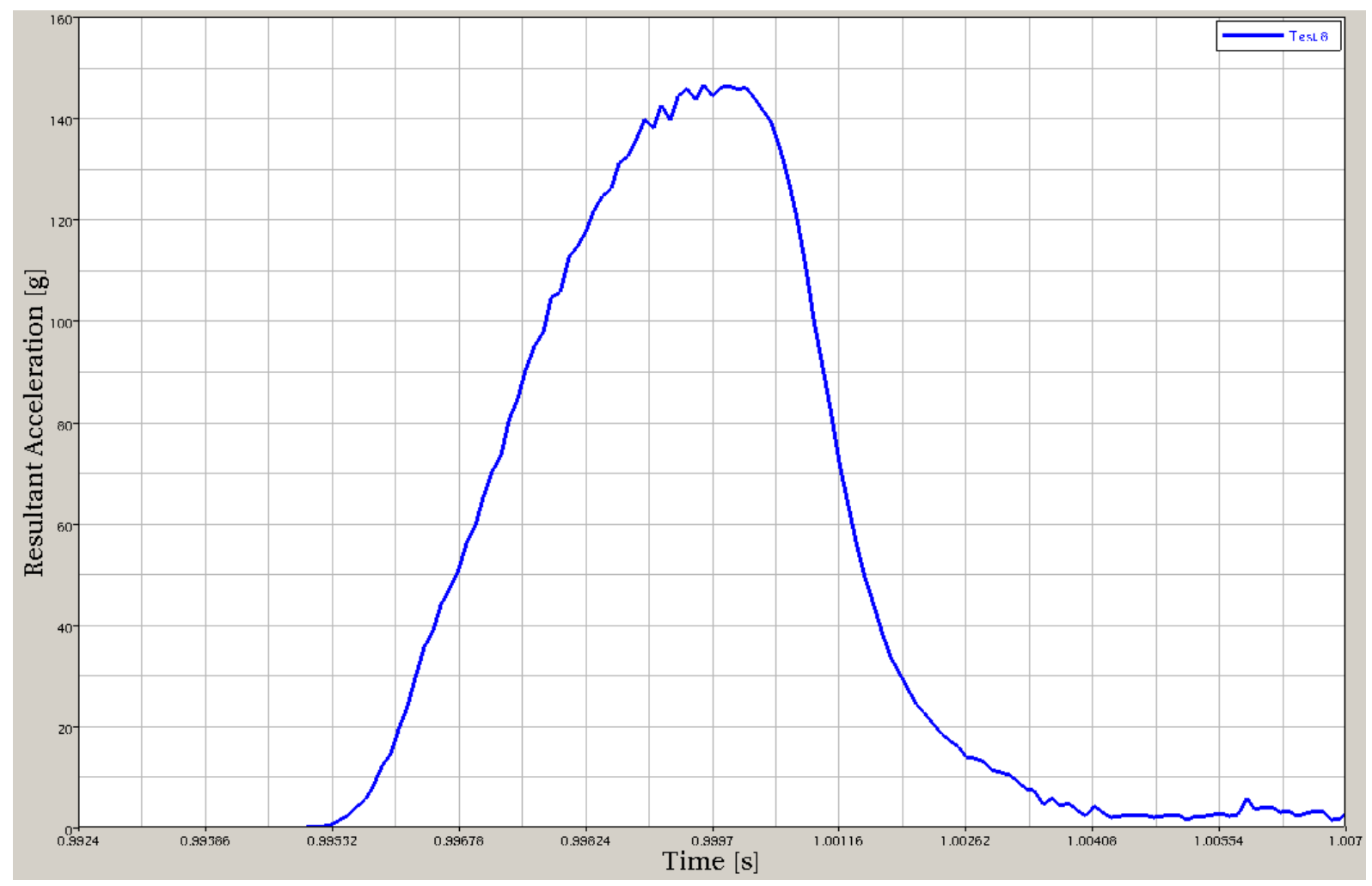

Abbildung 2-68: Fallversuch 8 (Anprall seitlich, Kopfform Hybrid III, Geschwindigkeit 5,42 m/s)

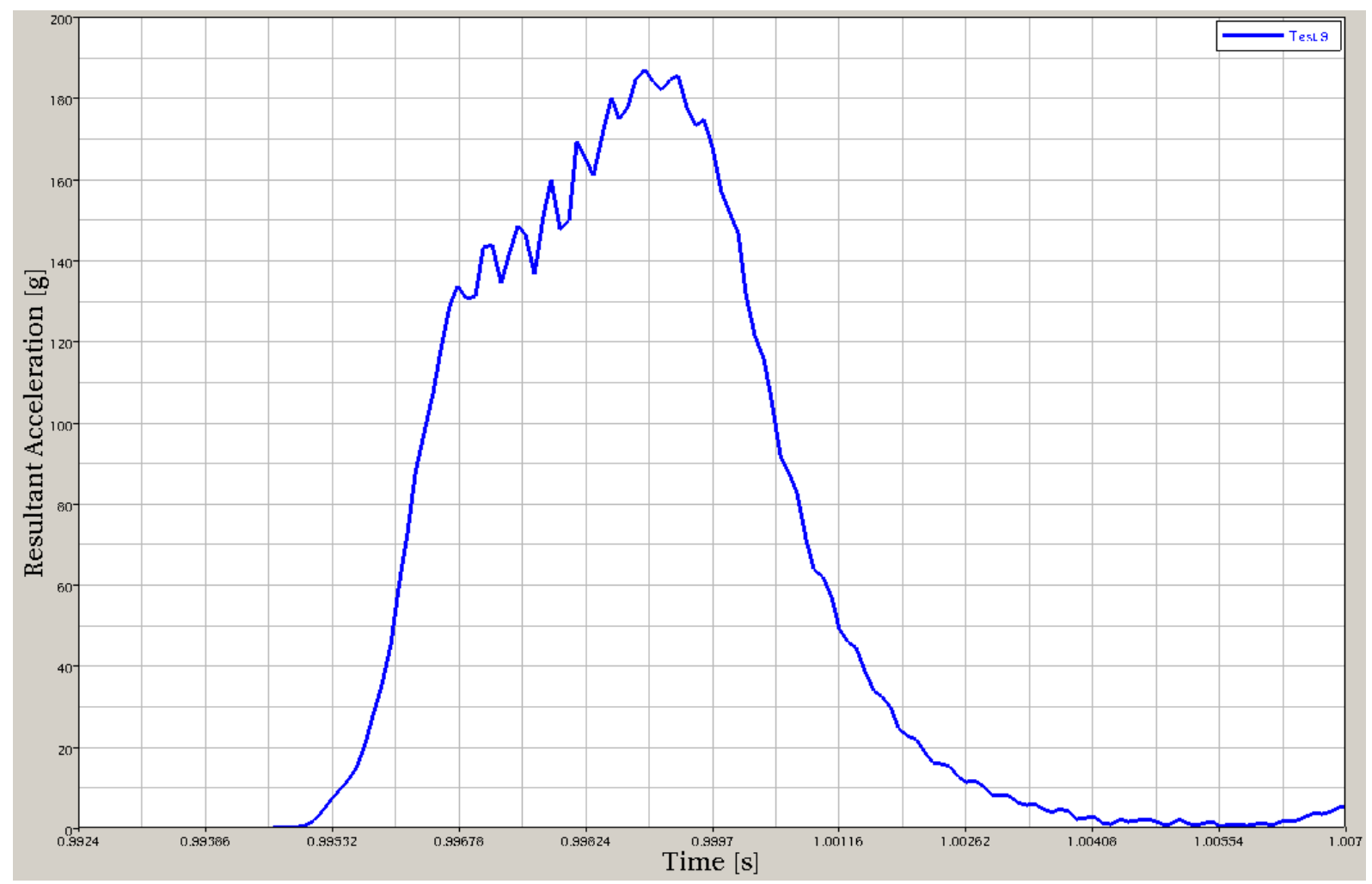

Abbildung 2-69: Fallversuch 9 (Anprall zentr. Scheitel, Kopfform Hybrid III, Geschwindigkeit 5,42 m/s) 


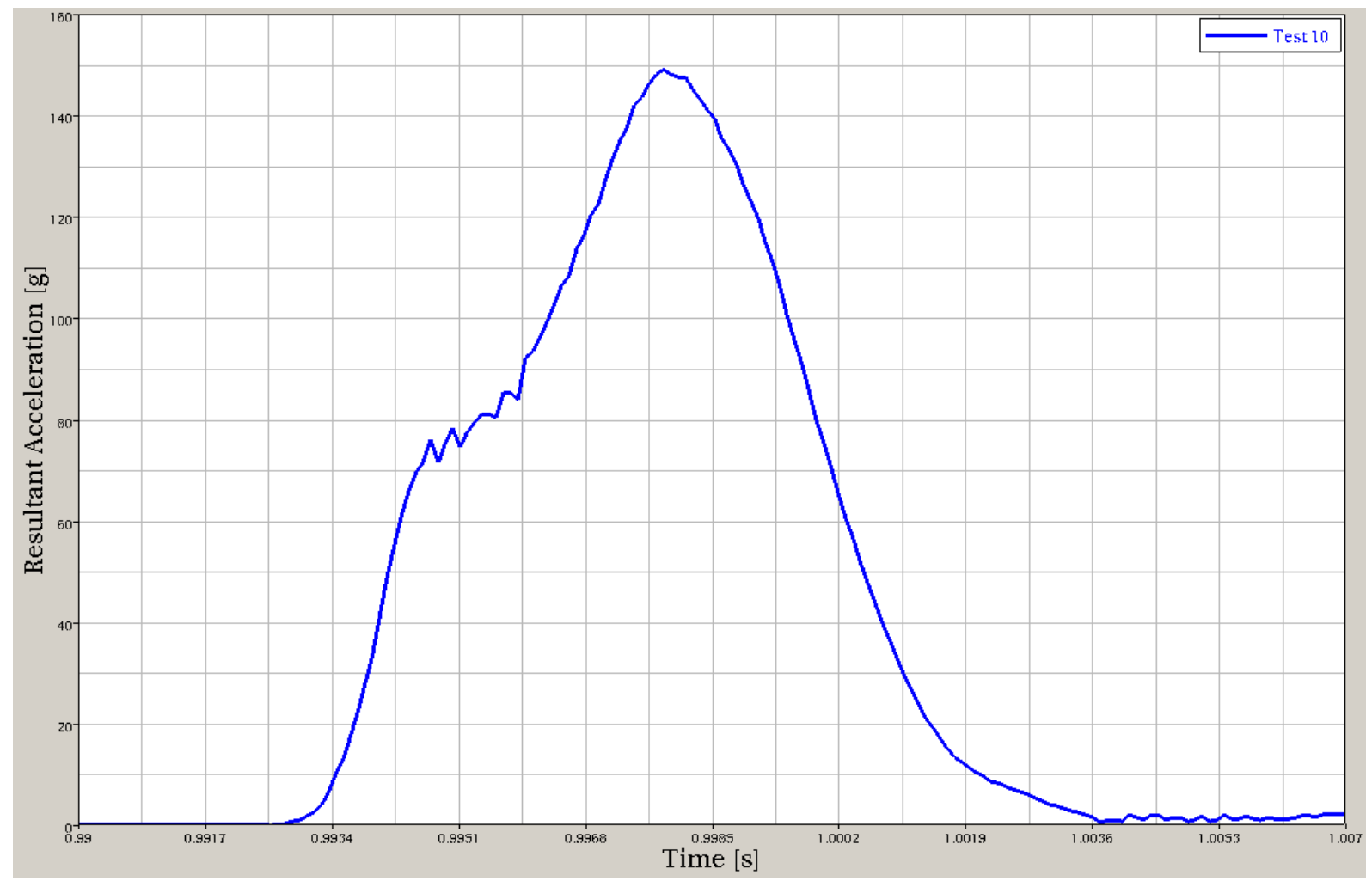

Abbildung 2-70: Fallversuch 10 (Anprall fronto-temporal, Kopfform Hybrid III, Geschwindigkeit 5,42 m/s)

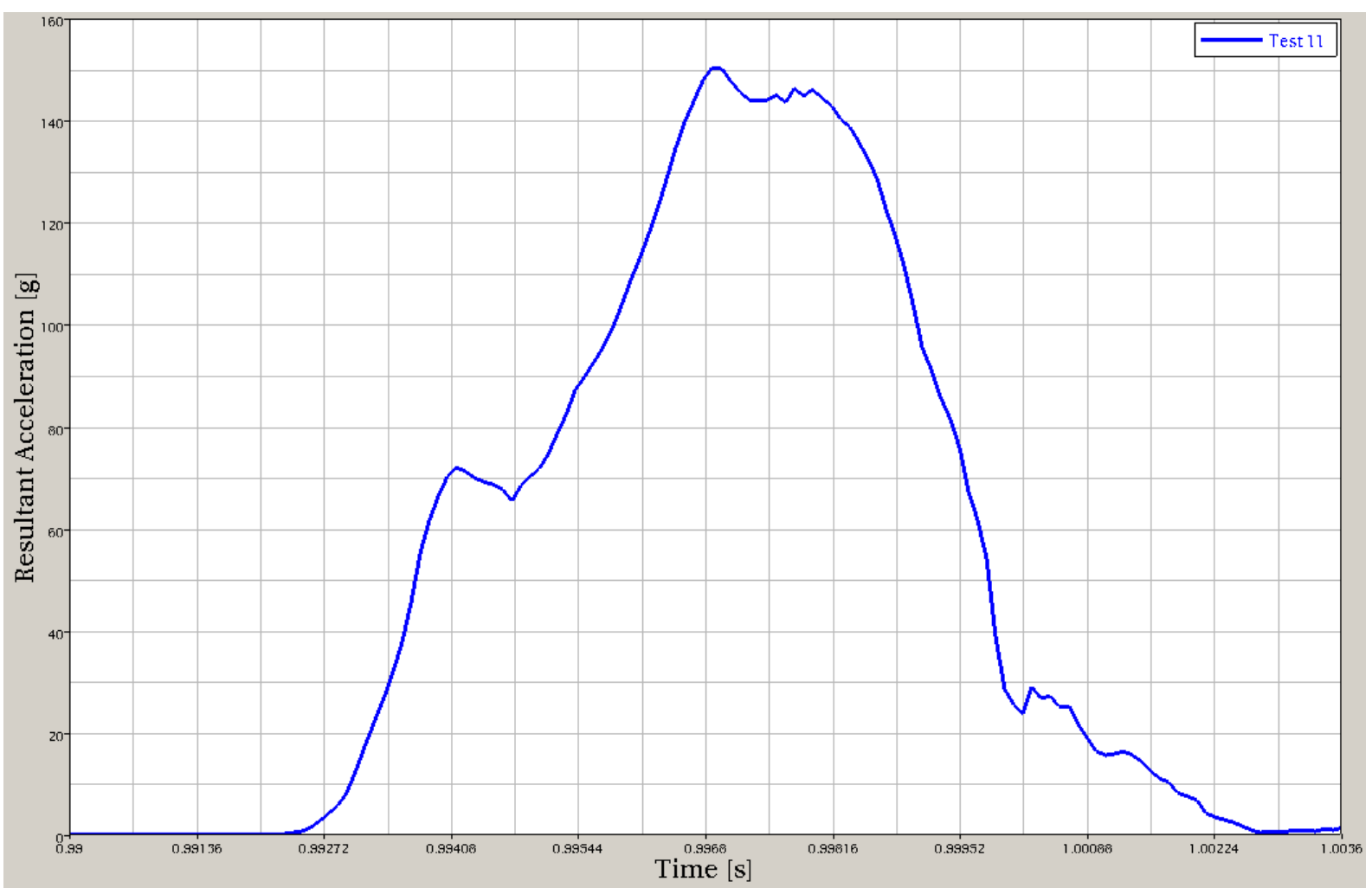

Abbildung 2-71: Fallversuch 11 (Anprall fronto-temporal, Kopfform nach EN, Geschwindigkeit 5,43 m/s) 
Ergänzend zu den jeweiligen Verläufen der resultierenden Beschleunigung sind in Tabelle 2-17 die Maximalwerte der resultierenden Beschleunigung sowie die HIC 15-Werte aufgeführt.

Tabelle 2-17: Ergebnisse Fallversuche

\begin{tabular}{|c|c|c|c|c|c|}
\hline Versuch & Anprallort & Kopfform & $\begin{array}{c}\text { Geschwindigkeit } \\
{[\mathbf{m} / \mathbf{s}]}\end{array}$ & $\begin{array}{c}\text { Max. } \\
\text { Beschleunigung [g] }\end{array}$ & HIC 15 \\
\hline 1 & seitlich & EN & 5,43 & 192 & 842 \\
\hline 2 & fronto-temporal & EN & 5,43 & 157 & 628 \\
\hline 3 & zentr. Scheitel & EN & 5,43 & 177 & 1102 \\
\hline 4 & seitlich & EN & 7,7 & $>500$ & 4256 \\
\hline 5 & seitlich & EN & 7,7 & $>500$ & 3917 \\
\hline 6 & seitlich & EN & 6,5 & 279 & 1696 \\
\hline 7 & fronto-temporal & EN & 6,5 & 197 & 1157 \\
\hline 8 & seitlich & Hybrid III & 5,42 & 147 & 617 \\
\hline 9 & zentr. Scheitel & Hybrid III & 5,42 & 187 & 1130 \\
\hline 10 & fronto-temporal & Hybrid III & 5,42 & 149 & 661 \\
\hline 11 & fronto-temporal & EN & 5,43 & 150 & 680 \\
\hline
\end{tabular}

Die Erkenntnisse aus den Fallversuchen lassen sich folgendermaßen zusammenfassen:

Einfluss der Anprallgeschwindigkeit: Die Versuche 1, 4, 5 und 6 wurden jeweils unter identischer Anprallkonfiguration (Kopfform nach EN, gleicher Anprallpunkt und gleiche Ausrichtung des Kopfes) durchgeführt, es wurde lediglich die Anprallgeschwindigkeit variiert. Beträgt beim Test in Anlehnung an die EN 1078 mit 5,4 m/s die maximale Kopfbeschleunigung $192 \mathrm{~g}$ und bewegt sich damit deutlich unter dem Grenzwert von $250 \mathrm{~g}$, wird eben dieser Grenzwert bei Versuch mit einer Anprallgeschwindigkeit von $6,5 \mathrm{~m} / \mathrm{s}$ schon überschritten (279 g). Erhöht man die Anprallgeschwindigkeit weiter auf 7,7 m/s, was auf dem Niveau von Falltests für Motorradhelme ist, überschreitet man an die Grenzen des Messbereichs des Beschleunigungsaufnehmers, der nur Werte bis $500 \mathrm{~g}$ aufzeichnen kann.

Einfluss der Kopfform: Die Versuche 1, 2 und 3 wurden jeweils unter identischen Randbedingungen zu den Versuchen 8, 10 und 9 durchgeführt, hier wurde nur die Kopfform variiert. Für den seitlichen Anprall wurde mit dem Hybrid III-Kopf $(147 \mathrm{~g})$ im Vergleich zur Kopfform nach EN (192 g) eine deutliche geringere maximale Beschleunigung erreicht. Beim Anprall auf den zentralen Scheitel wurde mit dem Hybrid III-Kopf (187 g) im Vergleich zur Kopfform nach EN (177 g) eine minimal höhere maximale Beschleunigung gemessen. Beim fronto-temporalen Anprall sind die ermittelten Werte der Kopfform nach EN (157 g) unwesentlich höher wie unter Verwendung des Hybrid III-Kopfes (149 g).

Einfluss des Anprallortes: Unter Verwendung der Kopfform nach EN wurde unter sonst identischen Randbedingungen beim seitlichen Anprall mit $197 \mathrm{~g}$ die höchste maximale Beschleunigung gemessen. Beim Anprall auf den zentralen Scheitel wurden $177 \mathrm{~g}$ gemessen, der geringste Wert mit $157 \mathrm{~g}$ wurde beim fronto-temporalen Anprall gemessen. Beim den Versuchen unter Verwendung der Hybrid III-Kopfform wurde beim Anprall auf den zentralen Scheitel mit $187 \mathrm{~g}$ die höchste maximale Beschleunigung gemessen. Der seitliche und der fronto-temporale Anprall liefern mit $147 \mathrm{~g}$ bzw. $149 \mathrm{~g}$ annähernd identische Werte. 
Im Rahmen des Fahrradhelmprojekts der UDV wurde basierend auf einer detaillierten Unfallanalyse und weiterführenden biomechanischen Betrachtungen eine Empfehlung für verbesserte Fahrradhelmtests erarbeitet.

Durch Analyse von realen Fahrradunfällen mit leicht, schwer und tödlich verletzten Fahrradfahrern wurden typische Unfallsituationen und Verletzungen erarbeitet.

Basierend auf diesen Ergebnissen wurden typische Unfallabläufe rekonstruiert und in mehreren Schritten simuliert. Schwerpunkt dieser Betrachtungen war die Kinematik des Fahrradfahrers während der Kollision sowie die Ermittlung von Kopfverletzungsrisiken. Die folgenden Empfehlungen für verbesserte Anforderungen im Bereich der stoßdämpfenden Eigenschaften von Fahrradhelmen stehen somit in engem Bezug zum realen Unfallgeschehen.

\subsection{Beschreibung der Fahrradhelmnorm EN 1078}

Fahrradhelme, die in Deutschland und der restlichen Europäischen Union auf den Markt kommen, müssen die Anforderungen der EN 1078 erfüllen. Es sind Anforderungen und entsprechende Prüfverfahren bezüglich folgender Punkte beschrieben (s. 2.1.1):

- Konstruktion, einschließlich Sichtfeld

- Stoßdämpfende Eigenschaften

- Eigenschaften der Trageeinrichtung, einschließlich Kinnriemen und

Befestigungsvorrichtungen

- Kennzeichnung und Information.

Im Rahmen des Fahrradhelmprojekts der UDV wurden mögliche Verbesserungsvorschläge im Bereich des primären und wichtigsten Schutzkriteriums eines Fahrradhelms, den stoßdämpfenden Eigenschaften, erarbeitet.

\subsection{Empfehlung für verbesserte Fahrradhelmtests}

Im Folgenden sollen nun, basierend auf den Methoden der aktuell gültigen Norm für Fahrradhelme (DIN EN 1078), verbesserte Anforderungen im Bereich der Stoßdämpfungsprüfung beschrieben werden.

\subsubsection{Prüfzone und Prüfpunkte}

Die Analyse realer Unfälle hat aufgezeigt, dass die Prüfzone eines Fahrradhelms insbesondere im Bereich der Stirn, der temporalen Region und auch dem Hinterkopf Verbesserungspotential aufweist.

Es wird deshalb empfohlen, die Prüfzone in diesen kritischen Bereichen auszuweiten. Auf Abbildung 3-1 ist eine optimierte Prüfzone dargestellt. Diese stellt nicht zwangsläufig den durch einen Helm komplett abzudeckenden Bereich dar. Beispielsweise könnten im Bereich der Ohren Aussparungen vorgesehen werden, ohne die Schutzwirkung beim seitlichen Anprall gegen ein flächiges oder linienhaftes Objekt maßgeblich zu reduzieren. 


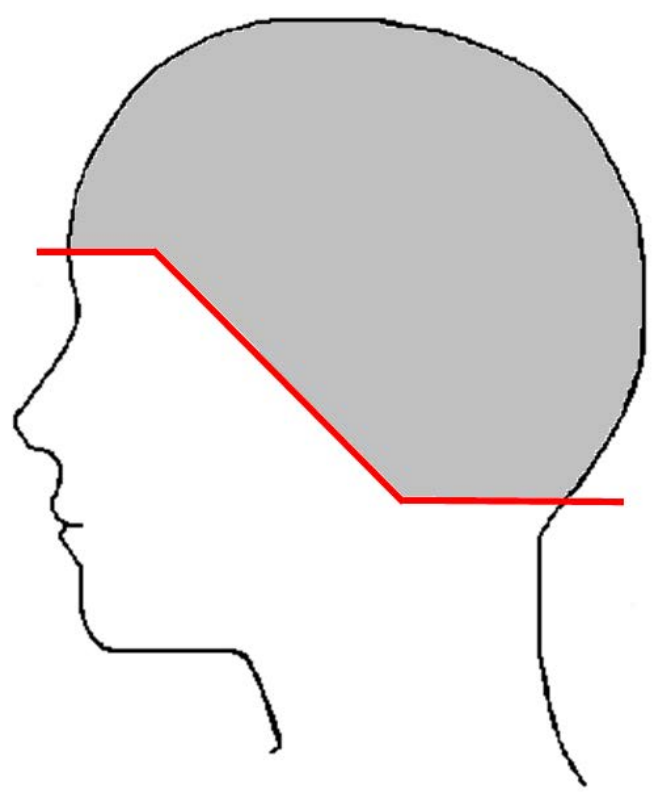

\section{Abbildung 3-1: Optimierte Prüfzone}

Zudem erscheint es sinnvoll, die als kritisch erkannten Zonen im Bereich der Stirn und temporalen Region verpflichtend zur Prüfung festzulegen. Dabei soll die Gefahr der möglichen Optimierung von zukünftigen Fahrradhelmen nur „auf den Punkt“ vermieden werden; der Fahrradhelm muss im kompletten Schutzbereich sein volles Potential ausschöpfen.

\subsubsection{Prüfgeschwindigkeiten und Testsockel}

Im realen Unfallgeschehen ist ein Kopfaufprall auf ein bordsteinähnliches Hindernis selten dokumentiert. Der Kopf prallt meist gegen ein flaches und hartes Hindernis. Allerdings ist ein Kopfanprall gegen ein bordsteinähnliches Hindernis häufig mit besonders schweren Verletzungen verbunden.

Es erscheint deshalb weiterhin angemessen, beide Testsockel für den Stoßdämpfungstest zu verwenden.

Der Bereich der Kopfanprallgeschwindigkeit um $5 \mathrm{~m} / \mathrm{s}$ bildet im Realunfallgeschehen die Mehrheit der Unfälle ohne Fremdbeteiligung, in erster Linie also der Fahrradsturz, ab. Bei Unfällen mit Pkw-Beteiligung sind häufig deutlich höhere Kopfanprallgeschwindigkeiten zu beobachten.

Es sollte überlegt werden, eine zusätzliche Prüfgeschwindigkeit des Stoßdämpfungstests mit etwa $10-12 \mathrm{~m} / \mathrm{s}$ einzuführen, für die gegebenenfalls reduzierte Anforderungen hinsichtlich der zu erfüllenden Grenzwerte zu diskutieren wären. Auf diese Weise könnte die Helmschutzwirkung bei den zahlenmäßig häufigen Alleinunfällen weiterhin bestehen bleiben, aber ein völliges Versagen der Schutzwirkung bei höheren Anprallgeschwindigkeiten, wie sie für schwere Kollisionen mit Kraftfahrzeugen typisch sind, vermieden werden. 


\subsubsection{Kopfbelastung}

Die Bewertung der Kopfbelastung und des Schutzpotentials eines Helms mit Hilfe des Maximalwerts der linearen Kopfbeschleunigung erscheint nicht mehr zeitgemäß.

Es wird empfohlen, zur Bewertung der Schutzfunktion eines Helms eine geeignetere Methode zur Beschreibung der Kopfbelastung zu verwenden.

Die Kopfbelastung kann auch durch den dimensionslosen HIC (Head Injury Criterion) angegeben werden, wie es in der Kraftfahrzeugsicherheit schon lange praktiziert wird. Damit wird zusätzlich zum (Maximal-) Wert der Kopfbeschleunigung auch die zeitliche Ausdehnung der Belastung miteinbezogen.

In der europäischen Vorschrift für Motorradhelme, der ECE R22, wird zusätzlich zum Maximalwert der Kopfbeschleunigung die Einhaltung eines HIC von 2400 gefordert; über die Einhaltung eines angepassten HIC-Wertes kann auch im Bereich der Vorschriften für Fahrradhelme nachgedacht werden. 


\section{Zusammenfassung}

Im Forschungsprojekt „Fahrradhelm“ der Unfallforschung der Versicherer (UDV) wurden durch detaillierte Realunfallanalyse typische Fahrradunfallszenarien erarbeitet und durch darauf aufbauenden biomechanischen Betrachtungen mögliches Verbesserungspotential für Fahrradhelme dargestellt.

Die aktuellen Prüfmethoden für Fahrradhelme entsprechen nicht immer dem realen Schutzwirkungsbedarf. Da das Sturzverhalten von Fahrradfahrern unzulänglich erforscht ist, wurden die Parameter des Kontaktes zwischen Helm und Hindernis kritisch hinterfragt und Empfehlungen für verbesserte Testverfahren für Fahrradhelme erarbeitet.

Laut Gesundheitsberichtserstattung des Bundes starben im Jahr 2010 über 50\% aller getöteten Fahrradfahrer in Deutschland an Verletzungen des Kopfes.

Studien von Fife et al. (1983), Oström et al. (1993), Wood et al. (1988) führen auf, dass in $69 \%$ bis $86 \%$ aller tödlichen Fahrradunfälle eine Kopfverletzung die direkte Todesursache war. Es ist ein essentieller Zusammenhang zwischen Kopfverletzungen, tödlichen Unfällen und dem möglichen Schutzpotential von Fahrradhelmen gegeben.

Thompson et al. $(1989,1996)$ beschrieben in ihren häufig zitierten, aber auch kritisierten Fall-Kontroll-Studien, dass sich bei Fahrradfahrern mit Helm das Risiko für Kopfverletzungen um bis zu $85 \%$ und das Risiko für Hirnverletzungen um bis zu $88 \%$ reduziert.

Attewell et al. (2001) zeigen in ihrem Review auf, dass Fahrradfahrer mit Helm ein geringeres Risiko tragen, eine Kopf- oder Hirnverletzung zu erleiden. Die Werte für eine mögliche Reduktion der Kopf- und Gehirnverletzungen schwanken dabei von 33\% bis $88 \%$, sind aber nach Elvik (2011) aufgrund methodischer Mängel der betrachteten Studien zu hoch angesetzt.

Die Studien von Amoros et al. (2011), Otte et al. (2008), Pang et al. (2009), Richter et al. (2007) sprechen von einer deutlichen Reduktion des Risikos für alle Kopfverletzungen. Nach Otte et al. (2008), Amoros et al. (2011), Berg et al. (2007) kann ein aktueller Fahrradhelm auch schwerste (AIS 3+) Kopfverletzungen verhindern. Nach Amoros et al. (2011) ist die Risikoreduzierung für schwere Kopfverletzungen (-69\%) größer als für allgemeine Kopfverletzungen ohne Berücksichtigung des Schweregrads (-31\%). Liers (2011) zeigt auf, dass die Helmbenutzung sowohl zu einem Rückgang von schweren Verletzungen als auch einem Anstieg der Zahl im Kopfbereich unverletzter Fahrradfahrer führen kann. Berg et al. (2007) stellen aufgrund ihrer Fall-Kontroll-Studie fest, dass der Helm einen protektiven Effekt haben muss, ohne dabei konkrete Zahlen zur Risikoreduktion zu nennen.

McIntosh et al. (1998) stellen in ihrer Fallstudie dar, dass in der temporoparietalen Region des Kopfs ein hoher Anteil an Aufprallpunkten lokalisiert ist. Depreitere et al (2007) kommen in ihrer Experimentalstudie zu dem Ergebnis, dass aktuelle Fahrradhelme die temporoparietale Region des Kopfs nur unzureichend schützen. Mills et al. $(2006,2008)$ bemängeln ebenfalls den Schutz gängiger Helmmodelle in der Schläfenregion.

Depreitere et al. (2004), Eilert-Pertersson et al. (1997) und Fredriksson et al. (2012) beschreiben typische Unfallszenarien für Fahrradfahrer. Während Eilert-Petersson et al. (1997) den Alleinunfall mit über $80 \%$ als typischen Fahrradunfall einschätzen, ist es bei Depreitere et al. (2004) und Fredriksson et al. (2012) die Kollision mit einem anderen Fahrzeug. Kollisionen mit einem Fahrzeug ziehen im Allgemeinen schwerere Verletzungen nach sich. Nach Kim et al. (2007) steigt die Verletzungsschwere dabei mit der Fahrzeuggeschwindigkeit. 
Oström et al. (1993) betrachteten tödliche Fahrradunfälle und stellten fest, dass $88 \%$ der Fahrradfahrer bei einer Kollision mit einem andern Fahrzeug zu Tode kamen. In 69\% war dabei eine Kopfverletzung die Todesursache. Tschernitschek et al. (2010) stellten fest, dass im betrachteten Fallkollektiv kein verunfallter Fahrradfahrer mit Helm eine Verletzung AIS $\geq 4$ davontrug.

Deck et al. (2012) liefern konkrete Vorschläge, wie man aktuelle Testverfahren optimieren kann. Milne et al. (2012) haben ein numerisches Fahrradhelmmodell entwickelt und damit die aktuellen Testbedingungen in Simulationen überprüft. Bourdet et al. (2012) haben in ihrer Parameterstudie die Anprallbedingungen eines Kopfanpralls simuliert. Dabei stellten sie fest, dass die Aufprallpunkte oft an oder unter der Helmkante lokalisiert sind.

Es bleibt an dieser Stelle festzuhalten, dass ein beträchtlicher Anteil an verunfallten Fahrradfahrern schwere oder tödliche Verletzungen im Bereich des Kopfs erleiden. Dabei ist die Verletzungsentstehung und -mechanik noch nicht im Detail geklärt. Einzelne Studien versuchen bisher nur, Lösungsvorschläge für einzelne Teile dieser komplexen Problematik anzubieten.

Der im vorliegenden Forschungsprojekt realisierte Ansatz mit der Verknüpfung von Unfallforschung und Biomechanik schafft die Möglichkeit für komplexere und weiterführende Betrachtungen. Diese können in Zukunft nicht nur als Grundlage für verbesserte Testverfahren für Fahrradhelme dienen, sondern auch als Diskussionsgrundlage für darauf aufbauende Problemstellungen zur Verfügung stehen.

Im vorliegenden Forschungsprojekt wurden Fahrradunfälle aus drei unterschiedlichen Fallkollektiven näher betrachtet und ausgewertet. Als Datengrundlage dienten 71 prospektiv erfasste Unfälle mit leicht- bis schwerverletzten Fahrradfahrern aus dem Universitätsklinikum München (UKM) und 500 prospektiv erfasste Unfälle mit leicht- bis schwerverletzten Fahrradfahrern aus dem Universitätsklinikum Münster (UKM). Nach erster Analyse wurden für die Auswertungen 34 Radfahrer ausgeschlossen, die entweder unverletzt blieben oder ihr Fahrrad als Fußgänger schoben. Somit standen insgesamt 543 Unfälle mit leicht- bis schwerverletzten Radfahrern zu Verfügung. Diese wurden ergänzt durch 117 retrospektiv erfasste gut dokumentierte tödliche Fahrradunfälle aus der Sicherheits-Unfall-Datenbank (SUD) des Instituts für Rechtsmedizin München.

Bei den Fahrradunfällen des UKLMU und des UKM war die Mehrheit der Fahrradfahrer zwischen 15 und 64 Jahren alt. Bei den getöteten Fahrradfahrern war ein deutlicher Anstieg der erfassten Fälle mit dem Alter zu erkennen.

$13 \%$ der verunfallten Fahrradfahrer in Münster (UKM) trugen einen Fahrradhelm. In München (UKLMU) trugen $21 \%$ der verunglückten Fahrradfahrer einen Helm. In der SUD trugen nur 6 der 117 getöteten Fahrradfahrer (5\%) einen Helm. Die Fahrer eines Sportrads, Rennrads, Mountain- oder Trekkingbikes trugen sowohl in München als auch in Münster überdurchschnittlich häufig einen Fahrradhelm.

Es konnten vier typische Unfallszenarien bestimmt werden:

\section{1) Alleinunfall des Fahrradfahrers}

1a) Sturz seitlich

1b) Sturz über Lenker bei blockierendem Vorderrad 
2) Kollision "Pkw-Front gegen Fahrrad seitlich"

2a) mit geringer Geschwindigkeit (ca. $15 \mathrm{~km} / \mathrm{h}$ )

2b) mit mittlerer Geschwindigkeit (ca. $40 \mathrm{~km} / \mathrm{h}$ )

In München (UKLMU) waren 45\% der Fahrradfahrer am Kopf verletzt, in Münster (UKM) waren knapp über 20\% am Kopf verletzt. Schwerste Kopfverletzung ist in den Daten des UKLMU AIS 4, in den Daten des UKM AIS 3. In 42\% der Fälle des UKLMU war der Kopf als schwerstverletzte Körperregion beteiligt bzw. stellte allein die schwerstverletzte Körperregion dar. In den Daten des UKM war in 19\% der Kopf bei der schwerstverletzten Körperregion beteiligt bzw. alleinig die schwerstverletzte Körperregion. Über $80 \%$ der getöteten Fahrradfahrer der SUD hatten schwerste Verletzungen AIS 3+ im Bereich des Kopfs. Bei diesen getöteten Fahrradfahrern war der Kopf in 69\% der Unfälle als schwerstverletzte Körperregion beteiligt.

Fahrradfahrer, die einen Helm trugen, erlitten im Fallmaterial des UKLMU und UKM maximal eine Kopfverletzung AIS 1 oder AIS 2. Kopfverletzungen AIS 3+ traten bei Helmträgern nicht auf.

183 der 543 verunfallten Fahrradfahrer aus dem UKLMU und dem UKM hatten äußerliche Kopfverletzungen. 25 Fahrradfahrer mit oberflächlichen Kopfverletzungen trugen einen Helm; 126 Personen mit Kopfverletzungen trugen keinen Helm.

Verletzungen im Bereich des Scheitels (Bereich von Stirn bis Hinterkopf) waren bei den unbehelmten Fahrradfahrer aus dem UKLMU und dem UKM sehr selten. Die untersuchten Fahrradhelme hatten in diesem Bereich ebenfalls selten Beschädigungen.

Lediglich im Bereich des Ober-/Hinterkopfs, des temporalen Bereichs und der Stirn wurden oberflächliche Kopfverletzungen dokumentiert, die im unmittelbaren Schutzbereich eines Fahrradhelms liegen. Die Häufigkeit von Gesichtsverletzungen (z.B. Orbit, Kinn) war bei den Helmträgern reduziert, obwohl diese nicht im unmittelbaren Schutzbereich des Fahrradhelms liegen. Es dominierten bei den Unfällen mit verletzten Fahrradfahrern der vordere Gesichtsbereich, insbesondere der Bereich um die Augen, die Nase und das Kinn.

Von den 117 getöteten Fahrradfahrern der SUD erlitt jeder mindestens eine oberflächliche Kopfverletzung, meist sogar mehrere. In der SUD waren die temporale Region des Kopfs sowie die Stirn am häufigsten verletzt.

Die aktuelle Prüfzone bildet das reale Unfallgeschehen nur unzureichend ab. Insbesondere bei schweren Unfällen sind die temporale Region, die Stirn und auch der Bereich des unteren Hinterkopfs häufig verletzt. Verletzungen im Bereich des Oberkopfs bzw. Scheitels sind fast nicht vorhanden. Es wird deshalb empfohlen, die Prüfzone in diesen kritischen Bereichen auszuweiten, insbesondere der temporale Bereich bedarf einer optimierten Abdeckung.

Um einen Eindruck von Anforderungen in Testverfahren/Normen für Helme aus unterschiedlichen Gebieten (Fahrrad, Motorrad, Ski/Snowboard, Reiten) zu erlangen, wurden verschiedene Normen und Testverfahren für Helme näher betrachtet.

Im Bereich der Fahrradhelme wurden die Vorschriften DIN EN 1078 (Europa), CPSC 16 CFR Part 1203, ASTM F1447-06 (USA), AS/NZS 2063:2008 (Australien/ Neuseeland), JIS T 
8134:2007 (Japan) sowie die Richtlinie Snell B95 (USA) und die Prüfverfahren der deutschen Stiftung Warentest und des ADAC betrachtet.

In den Bereichen Motorrad-, Ski/Snowboard- und Reithelme wurden die aktuellen Vorschriften aus Europa, den USA und Australien dargestellt.

In allen Vorschriften sind ähnliche Prüfverfahren/-abläufe vorgeschrieben. Primär wird das Stoßdämpfungsvermögen sowie die Festigkeit und Wirksamkeit der Trageeinrichtung getestet. Einige Verfahren schreiben weitere individuelle Tests vor. Dabei gilt es jeweils vorgeschriebene Grenzwerte einzuhalten; der wichtigste Wert ist die maximal zulässige Kopfbeschleunigung im Stoßdämpfungstest.

Unter den Vorschriften im Fahrradhelmbereich stellt die Richtlinie Snell B95 die höchsten Anforderungen an die Prüfgeschwindigkeiten im Stoßdämpfungstest (bis zu 6,6 m/s). Die Norm EN 1078 sowie AS/NZS 2063:2008 sind am anspruchsvollsten hinsichtlich der maximal zulässigen Kopfbeschleunigung $(250 \mathrm{~g})$.

Die einzelnen Vorschriften sind hinsichtlich der Prüfbedingungen und Grenzwerte unterschiedlich anspruchsvoll, es gibt jedoch keine Norm oder Richtlinie, die in allen Prüfpunkten den höchsten Maßstab aller Vorschriften ansetzt.

Die Vorschriften im Motorradhelmbereich stellen die höchsten Anforderungen an die getesteten Helme, das Niveau der Prüfgeschwindigkeiten im Stoßdämpfungstest ist verhältnismäßig hoch (bis zu 7,7 m/s). Es werden jedoch auch verhältnismäßig hohe Kopfbeschleunigungen toleriert $(275-400 \mathrm{~g})$.

Die Testverfahren im Gebiet der Ski-/Snowboard- und Reithelme ähneln sehr den Vorschriften im Fahrradhelmbereich; teilweise wird zusätzlich ein Durchdringungstest gefordert.

Um durch Rekonstruktion und Simulation ausgewählter Fälle aus den vorliegenden Datenkollektiven das Potential eines aktuellen Fahrradhelms bestimmen zu können, wurde ein detailliertes FE-Modell eines repräsentativen Fahrradhelms erstellt.

Die Wahl fiel auf den zu Projektbeginn meistverkauften Helm eines großen Helmherstellers.

Die Konstruktion des virtuellen Helmmodells erfolgte auf der Basis von CT- Aufnahmen. Das 3D Modell wurde mit Hilfe moderner Image-Processing Software segmentiert und exportiert. Auf der Basis dieses Modells wurde anschließend ein Netz für die Finite-ElementeSimulation erstellt.

Das Materialverhalten des Absorptionsschaums wurde mit Komponententests und „fullscale" Fallversuchen ermittelt und validiert. Die Modellierungsgüte des Absorptionsschaums hat ein angemessenes Niveau erreicht. Die festgestellten Abweichungen zwischen Simulation und Realversuch sind bei Fallversuchen nach EN 1078 noch geringer als bei den Komponententests. Neben dem Absorptionsschaum ist auch die Helmschale durch eine Materialbeschreibung charakterisiert worden. Hier wurde auf eine linear-elastische Materialmodellierung als Annäherung des Verhaltens eines thermoplastischen Kunststoffs zurückgegriffen.

Im Rahmen des vorliegenden Forschungsprojekts wurde das Helmmodell in Kopplung mit einem Schädel-Hirn-Modell für die Bewertung des Kopfverletzungsrisikos mit Hilfe eines "State-of-the-art"-Simulationsmodells verwendet. Die Entscheidung ist hier für das Modell „Strasbourg University Finite Element Head Modell“ (SUFEHM) gefallen. Zur besseren 
Fixierung bzw. zur Vermeidung von möglichen unnatürlichen Rotationsbewegungen des Helms auf dem Kopf wurde ebenfalls die Bänderung des Helms nachgebildet.

Anschließend wurden aus den vorliegenden Datenkollektiven ausgewählte Fälle rekonstruiert und in zwei Stufen simuliert. Auswahlkriterium der Unfälle war die Übereinstimmung mit den in den vorherigen Arbeitsschritten bestimmten typischen Unfallszenarien sowie eine sehr gute Dokumentationsqualität.

Diese Szenarien sind zum einen der Alleinunfall des Fahrradfahrers, zum zweiten die frontale Kollision mit einem Pkw in annähernd rechtem Winkel.

Aus 18 gut dokumentierten und rekonstruierten Fällen wurden vier Fälle bestimmt, die anschließend möglichst realitätsnah simuliert wurden. Diese Realunfälle wurden im weiteren Verlauf durch generische Szenarien ergänzt. Durch die Simulation in zwei Stufen wurden die Kinematik des Fahrradfahrers sowie die Kopfverletzungsrisiken beim Kopfanprall und die Wirkung eines Fahrradhelms dargestellt.

Dieser erste Simulationsschritt wurde mit Hilfe eines Mehrkörpersimulationsprogramms durchgeführt. Ziel dabei war es, die Kinematik des Fahrradfahrers während des Unfallablaufs möglichst realitätsnah abzubilden, insbesondere den Kopfanprall auf die Straße oder den beteiligten Pkw. Es wurden vier Realunfälle und elf generische Szenarien in MADYMO simuliert.

Zur Simulation dieser Szenarien wurde das in MADYMO integrierte Fußgänger-MenschModell verwendet, zusätzlich wurden zwei Fahrradmodelle erstellt. Ergänzend dazu konnte auf ein der UDV vorliegendes Modell der Front eines Pkws der oberen Mittelklasse zurückgegriffen werden.

Ausgehend von den Kopfanprallgeschwindigkeiten (translatorisch und rotatorisch) zum Anprallzeitpunkt wurden die Kopfbelastungen und Kopfverletzungsrisiken in Finite-ElementeSimulation (FE) berechnet.

Es wurden 19 verschiedene Szenarien in FE simuliert. Dabei wurde auf das Kopfmodell der Straßburger Universität (SUHEFM), das selbst entwickelte Fahrradhelmmodell und das frei verfügbare Modell eines Toyota Yaris (National Crash Analysis Center 2011) zurückgegriffen.

Es konnte anhand der FE-Simulation aufgezeigt werden, dass ein aktueller Fahrradhelm das Risiko für Kopfverletzungen teils deutlich senken kann, jedoch Verbesserungspotential insbesondere bei Kollisionen des Fahrradfahrers mit einem Pkw bietet.

So ist die Kopfanprallgeschwindigkeit bei solchen Kollisionen oft deutlich höher, als in aktuellen Testverfahren für Helme gefordert.

Ergänzend zu den oben dargestellten Betrachtungen wurden elf Fallversuche in Anlehnung an die EN 1078 durchgeführt, um den Einfluss der Prüfgeschwindigkeit, des Anprallpunkts und der verwendeten Kopfform aufzuzeigen. Mit steigender Prüfgeschwindigkeit erhöht sich auch die maximale resultierende Kopfbeschleunigung. Unter Verwendung der Kopfform nach EN 1078 ist die resultierende Beschleunigung beim seitlichen Anprall am höchsten.

Die Analyse realer Unfälle hat aufgezeigt, dass die Prüfzone eines Fahrradhelms insbesondere im Bereich der Stirn, der temporalen Region und auch dem Hinterkopf Verbesserungspotential aufweist. Dabei soll die Gefahr der möglichen Optimierung von zukünftigen Fahrradhelmen nur „auf den Punkt“ vermieden werden; der Fahrradhelm muss im kompletten Schutzbereich sein volles Potential ausschöpfen. 
Im realen Unfallgeschehen ist ein Kopfaufprall auf ein bordsteinähnliches Hindernis selten dokumentiert. Der Kopf prallt meist gegen ein flaches und hartes Hindernis. Allerdings ist ein Kopfanprall gegen ein bordsteinähnliches Hindernis häufig mit besonders schweren Verletzungen verbunden. Es erscheint deshalb weiterhin angemessen, beide Testsockel für den Stoßdämpfungstest zu verwenden.

Der Bereich der Kopfanprallgeschwindigkeit um $5 \mathrm{~m} / \mathrm{s}$ bildet im Realunfallgeschehen die Mehrheit der Unfälle ohne Fremdbeteiligung, in erster Linie also den Fahrradsturz, ab. Bei Unfällen mit Pkw-Beteiligung sind häufig deutlich höhere Kopfanprallgeschwindigkeiten zu beobachten. Es sollte überlegt werden, eine zusätzliche Prüfgeschwindigkeit des Stoßdämpfungstests mit etwa $10-12 \mathrm{~m} / \mathrm{s}$ einzuführen, für die gegebenenfalls reduzierte Anforderungen hinsichtlich der zu erfüllenden Grenzwerte zu diskutieren wären. Auf diese Weise könnte die Helmschutzwirkung bei den zahlenmäßig häufigen Alleinunfällen weiterhin bestehen bleiben, aber ein völliges Versagen der Schutzwirkung bei höheren Anprallgeschwindigkeiten, wie sie für schwere Kollisionen mit Kraftfahrzeugen typisch sind, vermieden werden.

Die Bewertung der Kopfbelastung und des Schutzpotentials eines Helms allein auf Grundlage des Maximalwerts der linearen Kopfbeschleunigung erscheint nicht mehr zeitgemäß. Es wird empfohlen, zur Bewertung der Schutzfunktion eines Helms gegebenenfalls ergänzend - eine geeignetere Methode zur Beschreibung der Kopfbelastung zu verwenden. 


\section{Literaturverzeichnis}

AS/NZS 1698:2006, 2006. Protective helmets for vehicle users.

AS/NZS 2063:2008, 2008. Bicycle helmets.

AS/NZS 2512.3.1:2007, 2007. Methods of testing protective helmets, Part 1: Definitions and headforms.

AS/NZS 2512.9:2006, 2006. Methods of testing protective helmets, Method 9: Determination of load distribution.

ASTM F 1163-04a, 2004. Standard Specification for Protective Headgear Used in Horse Sports and Horseback Riding.

ASTM F 1446-11a, 2011. Standard Test Methods for Equipment and Procedures Used in Evaluating the Performance Characteristics of Protective Headgear.

ASTM F 1447-06, 2006. Standard Specification for Helmets used in Recreational Bicycling or Roller Skating.

ASTM F 2040-11, 2011. Standard Specification for Helmets used for Recreational Snow Sports.

CPSC 16 CFR Part 1203, 1996. Safety Standard for Bicycle Helmets; Final Rule.

DIN EN 960, 2006. Prüfköpfe zur Prüfung von Schutzhelmen; Deutsche Fassung EN 960:2006.

DIN EN 1077, 2007. Helme für alpine Skiläufer und für Snowboarder; Deutsche Fassung EN 1077:2007.

DIN EN 1078, 2006. Helme für Radfahrer und Benutzer von Skateboards und Rollschuhen;

Deutsche Fassung EN 1078:1997 + A1:2005.

DIN EN 1078, 2014. Helme für Radfahrer und für Benutzer von Skateboards und Rollschuhen; Deutsche Fassung EN 1078:2012+A1:2012.

DIN EN 1384, 1996. Schutzhelme für reiterliche Aktivitäten; Deutsche Fassung EN 1384:1996.

ECE R22, Version 05, 2002. Einheitliche Bedingungen für die Genehmigung der Schutzhelme und Visiere für Fahrer und Mitfahrer von Krafträdern und Mopeds.

Federal Motor Vehicle Safety Standard No. 218 (49 CFR Sec. 571.218): Motorcycle Helmets, 1988.

JIS T 8134:2007, 2007. Protective helmets for bicycle users.

SNELL B95, 1998. 1995 Standard for Protective Headgear (1998 Revision) for Use in Bicycling.

SNELL E2001, 2001. 2001 Standard for Protective Headgear for Use in Horseback Riding.

SNELL L98, 1998. 1998 Standard for Protective Headgear for Use with Low Powered Vehicles, Mopeds, and Motorized Bicycles.

SNELL M2010, 2007. 2010 Standard for Protective Headgear for Use with Motorcycles and Other motorized Vehicles.

SNELL RS-98, 1998. 1998 Standard for Protective Headgear for Use in Recreational Skiing and Snowboarding. 
HyperWorks Software (2014). Version 12.0: Altair Engineering Inc. Online verfügbar unter http://www.altairhyperworks.com, zuletzt geprüft am 21.11.2014.

MADYMO Software (2014). Version 7.1: Tass International. Online verfügbar unter https://www.tassinternational.com/madymo, zuletzt geprüft am 21.11.2014.

LS-DYNA Software (2014). Version V971 R6.1.2: Livermore Software Technology Corporation. Online verfügbar unter http://www.Istc.com/products/ls-dyna, zuletzt geprüft am 21.11.2014.

American Association for Automotive Medicine (AAAM) (2008). The Abbreviated Injury Scale 2005. Update 2008.

Amoros, E.; Chiron, M.; Martin, J.-L.; Thélot, B.; Laumon, B. (2012). Bicycle helmet wearing and the risk of head, face, and neck injury: a French case--control study based on a road trauma registry. Injury Prevention 18 (1), S. 27-32. DOI: 10.1136/ip.2011.031815.

Attewell, R. G.; Glase, K.; McFadden, M. (2001). Bicycle helmet efficacy: a meta-analysis. Accident Analysis \& Prevention 33 (3), S. 345-352. DOI: 10.1016/S0001-4575(00)00048-8.

Bachmann, K. (2011). Retrospektive Analyse tödlicher Fahrradunfälle und deren Präventionsmöglichkeiten. Diplomarbeit. München.

Berg, P.; Westerling, R. (2007). A decrease in both mild and severe bicycle-related head injuries in helmet wearing ages--trend analyses in Sweden. Health Promotion International 22 (3), S. 191-197. DOI: 10.1093/heapro/dam020.

Bourdet, N.; Deck, C.; Carreira, R. P.; Willinger, R. (2012). Head impact conditions in the case of cyclist falls. Proceedings of the Institution of Mechanical Engineers, Part P: Journal of Sports Engineering and Technology 226 (3-4), S. 282-289. DOI:

10.1177/1754337112442326.

Bourdet, N.; Deck, C.; Serre, T.; Perrin, C.; Llari, M.; Willinger, R. (2014). In-depth real-world bicycle accident reconstructions. International Journal of Crashworthiness 19 (3), S. 222232. DOI: $10.1080 / 13588265.2013 .805293$.

Bundesanstalt für Straßenwesen (2013). Gurte, Kindersitze, Helme und Schutzkleidung 2012. Forschung kompakt 06/13.

Chen, Y.; Yang, J.; Otte, D. (2008). Load and Impact Conditions for Head Injuries in Car-toPedestrian and Car-to-Cyclist Accidents - A Comparison of Real Accidents and Simulations. 4th International Conference on ESAR "Expert Symposium on Accident Research".

COST 327. Final Report. Online verfügbar unter

http://ec.europa.eu/transport/roadsafety_library/publications/cost327_final_report.pdf, zuletzt geprüft am 18.07.2014.

Curnow, W. J. (2003). The efficacy of bicycle helmets against brain injury. Accident Analysis \& Prevention 35 (2), S. 287-292.

Curnow, W. J. (2005). The Cochrane Collaboration and bicycle helmets Accident Analysis \& Prevention 37 (3), S. 569-573. DOI: 10.1016/j.aap.2005.01.009.

Deck, C.; Bourdet, N.; Calleguo, A.; Carreira, P. R.; Willinger, R. (2012). Proposal of an improved bicycle helmet standards. ICRASH 2012. Mailand, Italien.

Deck, C.; Willinger, R. (2008). Improved head injury criteria based on head FE model. International Journal of Crashworthiness 13 (6), S. 667-678. DOI:

$10.1080 / 13588260802411523$. 
Depreitere, B.; van Lierde, C.; Maene, S.; Plets, C.; Vander Sloten, J.; van Audekercke, R. et al. (2004). Bicycle-related head injury: a study of 86 cases. Accident Analysis \& Prevention 36 (4), S. 561-567. DOI: 10.1016/S0001-4575(03)00062-9.

Depreitere, B.; van Lierde, C.; Vander Sloten, J.; Van der Perre, Georges; van Audekercke, R.; Plets, C.; Goffin, J. (2007). Lateral head impacts and protection of the temporal area by bicycle safety helmets. The Journal of Trauma 62 (6), S. 1440-1445. DOI:

10.1097/01.ta.0000221472.68873.fb.

Eilert-Petersson, E.; Schelp, L. (1997). An epidemiological study of bicycle-related injuries. Accident Analysis \& Prevention 29 (3), S. 363-372.

Elvik, R. (2011). Publication bias and time-trend bias in meta-analysis of bicycle helmet efficacy: a re-analysis of Attewell, Glase and McFadden, 2001 Accident Analysis \& Prevention 43 (3), S. 1245-1251. DOI: 10.1016/j.aap.2011.01.007.

Fahlstedt, M.; Baeck, K.; Halldin, P.; Vander Sloten, J.; Goffin, J.; Depreitere, B.; Kleiven, S. (2012). Influence of Impact Velocity and Angle in a Detailed Reconstruction of a Bicycle Accident. IRCOBI 2012 (84).

Fife, D.; Davis, J.; Tate, L.; Wells, J. K.; Mohan, D.; Williams, A. (1983). Fatal injuries to bicyclists: the experience of Dade County, Florida. The Journal of Trauma 23 (8), S. 745755.

Fredriksson, R.; Rosén, E. (2012). Priorities for Bicyclist Protection in Car Impacts - a Real life Study of Severe Injuries and Car Sources. IRCOBI 2012 (83).

Gesamtverband der Deutschen Versicherungswirtschaft e.V. (2003). Sicherung des Verkehrs auf Straßen - SVS. Anhang 8 Unfalltypen-Katalog, S. 78-93: Brandenburgische Universitätsdruckerei und Verlagsgesellschaft mbH, Potsdam, Berlin 2003, ISSN 0724-3685.

Gesundheitsberichtserstattung des Bundes: Sterbefälle nach äußeren Ursachen und ihren Folgen (ab 1998). Gliederungsmerkmale: Jahre, Region, Alter, Geschlecht, Nationalität, ICD10 (V-Y), ICD-10 (S-T). Online verfügbar unter http://www.gbe-bund.de, zuletzt geprüft am 08.12.2014

Ghajari, M.; Galvanetto, U.; lannucci, L.; Willinger, R. (2011). Intracranial Response in Helmet Oblique Impacts. IRCOBI 2011 (34, S. 90 - 93).

Hansen, K. S.; Engesaeter, L. B.; Viste, A. (2003). Protective effect of different types of bicycle helmets. Traffic Injury Prevention 4 (4), S. 285-290. DOI: 10.1080/714040486.

Heesch, K. C.; Garrard, J.; Sahlqvist, S. (2011). Incidence, severity and correlates of bicycling injuries in a sample of cyclists in Queensland, Australia. Accident Analysis \& Prevention 43 (6), S. 2085-2092. DOI: 10.1016/j.aap.2011.05.031.

Heng, K W J; Lee, A H P; Zhu, S.; Tham, K. Y.; Seow, E. (2006). Helmet use and bicyclerelated trauma in patients presenting to an acute hospital in Singapore. Singapore Medical Journal 47 (5), S. 367-372.

Hummel, T. (2012). Unfallforschung kompakt - Bewertung von Fußgängerschutzmaßnahmen. Unfallforschung der Versicherer, Gesamtverband der Deutschen Versicherungswirtschaft e.V., Berlin.

Kim, J.-K.; Kim, S.; Ulfarsson, G. F.; Porrello, L. A. (2007). Bicyclist injury severities in bicycle-motor vehicle accidents. Accident Analysis \& Prevention 39 (2), S. 238-251. DOI: 10.1016/j.aap.2006.07.002. 
Kühn, M. (2011). Unfallforschung kompakt - Intelligente Fahrzeugbewertung zum Fußgängerschutz. Unfallforschung der Versicherer, Gesamtverband der Deutschen Versicherungswirtschaft e.V., Berlin.

Linn, S.; Smith, D.; Sheps, S. (1998). Epidemiology of bicycle injury, head injury, and helmet use among children in British Columbia: a five year descriptive study. Canadian Hospitals Injury, Reporting and Prevention Program (CHIRPP). Injury Prevention 4 (2), S. 122-125.

Liu, D.-S.; Chang, C.-Y.; Fan, C.-M.; Hsu, S.-L. (2003). Influence of environmental factors on energy absorption degradation of polystyrene foam in protective helmets. Engineering Failure Analysis 10 (5), S. 581-591. DOI: 10.1016/S1350-6307(03)00040-2.

Malczyk, A.; Bauer, K.; Juhra, C.; Schick, S. (2014). Head Injuries in Bicyclists and Associated Crash Characteristics. IRCOBI 2014.

McIntosh, A.; Dowdell, B.; Svensson, N. (1998). Pedal cycle helmet effectiveness: a field study of pedal cycle accidents. Accident Analysis \& Prevention 30 (2), S. 161-168.

Mills, N. J.; Gilchrist, A. (2006). Bicycle helmet design. Proceedings of the Institution of Mechanical Engineers, Part L: Journal of Materials: Design and Applications 220 (4), S. 167180. DOI: 10.1243/14644207JMDA100.

Mills, N. J.; Gilchrist, A. (2008a). Finite-element analysis of bicycle helmet oblique impacts. International Journal of Impact Engineering 35 (9), S. 1087-1101. DOI:

10.1016/j.jijimpeng.2007.05.006.

Mills, N. J.; Gilchrist, A. (2008b). Oblique impact testing of bicycle helmets. International Journal of Impact Engineering 35 (35), S. 1075-1086.

Milne, G.; Deck, C.; Bourdet, N.; Carreira, R. P.; Allinne, Q.; Willinger, R. (2012).

Development and validation of a bicycle helmet: Assessment of head injury risk under standard impact conditions. IRCOBI 2012 (86).

Moore, D. N.; Schneider, W. H.; Savolainen, P. T.; Farzaneh, M. (2011). Mixed logit analysis of bicyclist injury severity resulting from motor vehicle crashes at intersection and nonintersection locations. Accident Analysis \& Prevention 43 (3), S. 621-630. DOI: 10.1016/j.aap.2010.09.015.

National Crash Analysis Center (2011). 2010 Toyota Yaris. Online verfügbar unter http://www.ncac.gwu.edu/vml/models.html. Zuletzt geprüft am 21.11.2014

Oström, M.; Björnstig, U.; Näslund, K.; Eriksson, A. (1993). Pedal cycling fatalities in northern Sweden. International Journal of Epidemiology 22 (3), S. 483-488.

Otte, D.; Facius, T.; Wiese, B. (2013). Einflüsse auf das Verletzungsrisiko des Kopfes von Radfahrern und Nutzen von Radhelmen zur Vermeidung und Minderung von Verletzungen. Verkehrsunfall und Fahrzeugtechnik 2013 (09), S. 298-309.

Otte, D.; Haasper, C.; Wiese, B. (2008). Wirksamkeit von Fahrradhelmen bei Verkehrsunfällen von Radfahrern auf Kopfverletzungshäufigkeit und Verletzungsschwere. Verkehrsunfall und Fahrzeugtechnik (11), S. 2-12.

Pang, T. Y.; Sakeran, H.; Short, A.; McIntosh, A. S.; Rechnitzer, G.; Thai, K. (2008). Numerical analysis of real-world cyclist crashes: impact speed, collision mechanism and movement trajectories. 2008 Australasian Road Safety Research, Policing and Education Conference 7, 10. - 12. November 2008. Adelaide, Südaustralien.

Pang, T. Y.; Thai, K. T.; Rankin, T.; Curtis, K.; Schilter, E.; Mclntosh, A. S. (2009). Risk of Head, Facial and Neck Injury in Bicycle and Motorcycle Crashes in relation to Helmet Use. 2009 Australasian Road Safety Research, Policing and Education Conference. 
Peng, Y.; Yang, J.; Deck, C.; Willinger, R. (2013). Finite element modeling of crash test behavior for windshield laminated glass. International Journal of Impact Engineering 57, S. 27-35. DOI: 10.1016/j.jimpeng.2013.01.010.

Pschyrembel (2012). Klinisches Wörterbuch. 264. neu bearb. Aufl. Berlin [u.a.]. De Gruyter.

Richter, M.; Otte, D.; Haasper, C.; Knobloch, K.; Probst, C.; Westhoff, J. et al. (2007). The current injury situation of bicyclists--a medical and technical crash analysis. The Journal of Trauma 62 (5), S. 1118-1122. DOI: 10.1097/01.ta.0000221060.78894.cb.

Rivara, F. P.; Thompson, D. C.; Thompson, R. S. (1997). Epidemiology of bicycle injuries and risk factors for serious injury. Injury Prevention 1997 (3), S. 110-114.

Scheiman, S.; Moghaddas, H. S.; Björnstig, U.; Bylund, P.-O.; Saveman, B.-I. (2010). Bicycle injury events among older adults in Northern Sweden: a 10-year population based study. Accident Analysis \& Prevention 42 (2), S. 758-763. DOI: 10.1016/j.aap.2009.11.005.

Siman-Tov, M.; Jaffe, D. H.; Peleg, K. (2012). Bicycle injuries: a matter of mechanism and age. Accident Analysis \& Prevention 44 (1), S. 135-139. DOI: 10.1016/j.aap.2010.10.006.

Thai, K. T.; Pang, T. Y.; Mclnstosh, A. S. (2009). Comparison of Helmet Stability on Headforms and Human Subjects. 2009 Australasian Road Safety Research, Policing and Education Conference. Sydney, N.S.W.

Thompson, D. C.; Rivara, F.; Thompson, R. (2009). Helmets for preventing head and facial injuries in bicyclists. In: The Cochrane Library 2009, Issue 1. Online verfügbar unter http://www.thecochranelibrary.com/userfiles/ccoch/file/Safety_on_the_road/CD001855.pdf, zuletzt geprüft am 18.07.2014.

Thompson, D. C.; Rivara, F. P.; Thompson, R. S. (1996). Effectiveness of bicycle safety helmets in preventing head injuries. A case-control study. The Journal of the American Medical Association 276 (JAMA) (24), S. 1968-1973.

Thompson, R. S.; Rivara, F. P.; Thompson, D. C. (1989). A case-control study of the effectiveness of bicycle safety helmets. New England Journal of Medicine 320 (21), S. 13611367. DOI: 10.1056/NEJM198905253202101.

Tschernitschek, J.; Hannawald, L.; Koch, R.; Zwipp, H. (2010). Evaluation of the protective effect of bicycle helmets (A GIDAS-analysis 2000-2007). 4th International Conference on ESAR "Expert Symposium on Accident Research".

van Schijndel, M.; de Hair, S.; Rodarius, C.; Fredriksson, R. (2012). Cyclist kinematics in car impacts reconstructed in simulations and full scale testing with Polar dummy. IRCOBI 2012 (85).

Watson, J.; Hardy, R.; Kayvantash, K. (2009). Understanding the Nature of Cyclists Head Impacts. IRCOBI 2009, S. 301-313.

Williams, M. (1991). The protective performance of bicyclists' helmets in accidents. Accident Analysis \& Prevention 23 (2-3), S. 119-131.

Wood, T.; Milne, P. (1988). Head injuries to pedal cyclists and the promotion of helmet use in Victoria, Australia. Accident Analysis \& Prevention 20 (3), S. 177-185. 


\section{Abbildungsverzeichnis}

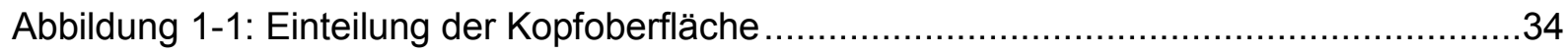

Abbildung 1-2: Unfallursachen der Fahrradfahrer nach Polizeibericht ...............................36

Abbildung 1-3: Unfallursachen der Unfallgegner nach Polizeibericht .................................37

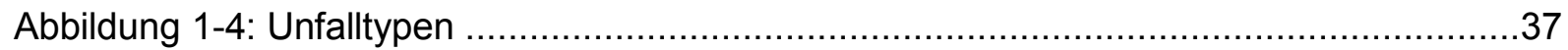

Abbildung 1-5: Tödlich verunglückte Fahrradfahrer nach Kollisionsgegner..........................38

Abbildung 1-6: Kollisionsstellungen Unfallbeteiligte Fahrzeuge (Fahrrad - PKW), $n=61$......39

Abbildung 1-7: Kollisionsgeschwindigkeiten aller Kollisionsgegner ...................................40

Abbildung 1-8: Kollisionsgeschwindigkeiten Pkw bei Unfällen mit Pkw-Beteiligung .............41

Abbildung 1-9: Kollisionsgeschwindigkeiten der Pkw bei den Kollisionsstellungen 4-8 ........41

Abbildung 1-10: Anprallpunkte am gegnerischen Pkw ...............................................42

Abbildung 1-11: Gefahrene Geschwindigkeiten der Fahrradfahrer bei Alleinunfällen ............43

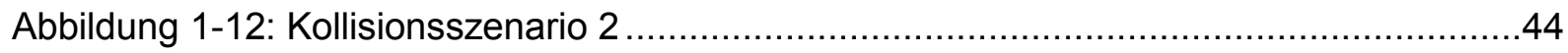

Abbildung 1-13: Verunfallte Fahrradfahrer nach Altersgruppen (UKLMU und UKM, $n=543$ ) 47

Abbildung 1-14: Tödlich verunglückte Fahrradfahrer nach Altersgruppen (SUD, $n=117) \ldots \ldots .47$

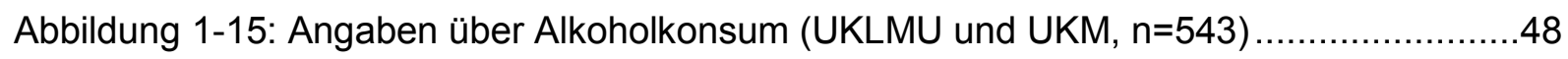

Abbildung 1-16: Erfasste BAK-Werte in Promille (UKM, n=24) .....................................49

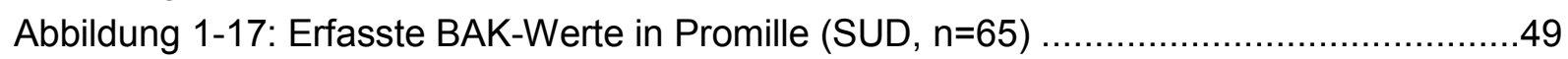

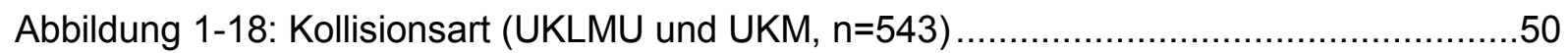

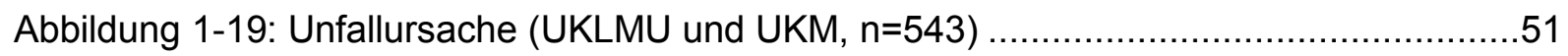

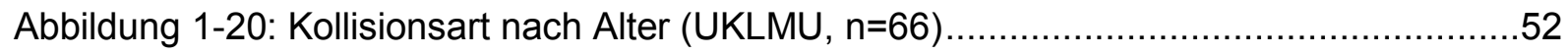

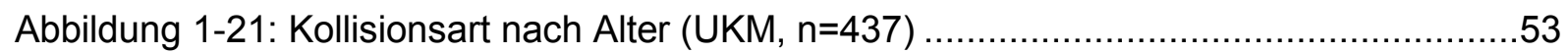

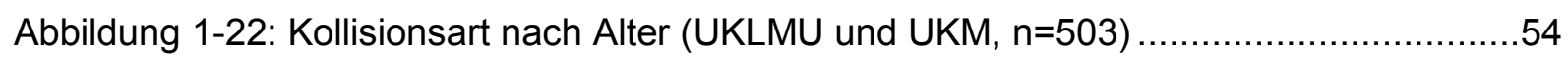

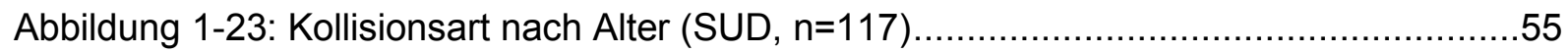

Abbildung 1-24: Kollisionsart nach Datenquelle (UKLMU, UKM, SUD) ...............................55

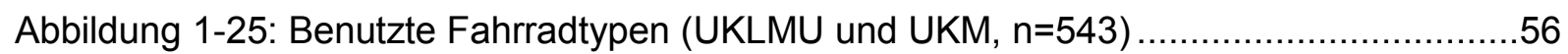

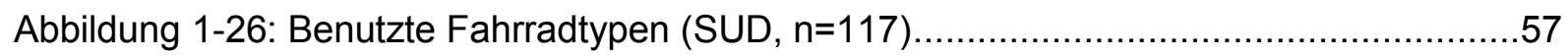

Abbildung 1-27: Eigene Geschwindigkeit bei Alleinunfall (UKLMU und UKM, $n=242) \ldots \ldots \ldots . .58$

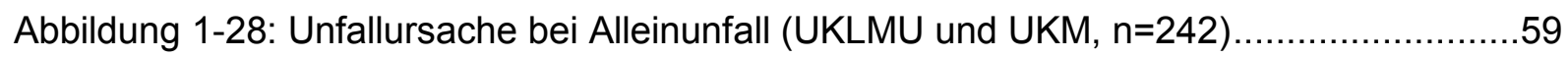

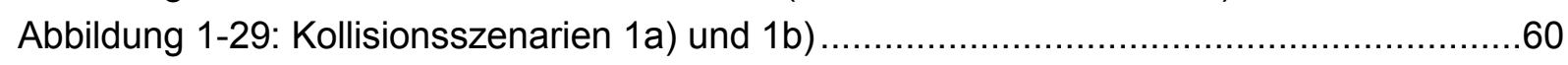

Abbildung 1-30: Anprallbereich am eigenen Fahrrad bei Unfällen mit Pkw-Beteiligung

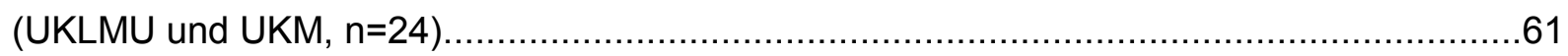

Abbildung 1-31: Anprallbereich am gegnerischen Pkw (UKLMU und UKM, $n=18) \ldots \ldots \ldots \ldots . . .62$

Abbildung 1-32: Kollisionsgeschwindigkeiten des gegnerischen Pkw (UKLMU und UKM,

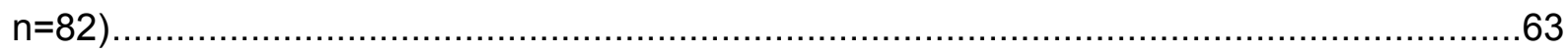

Abbildung 1-33: Fahrgeschwindigkeit des Fahrradfahrers bei Unfällen mit Pkw-Beteiligung

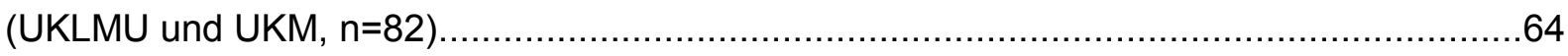

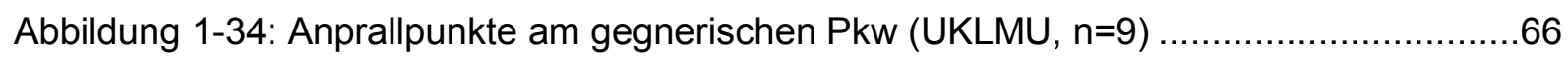

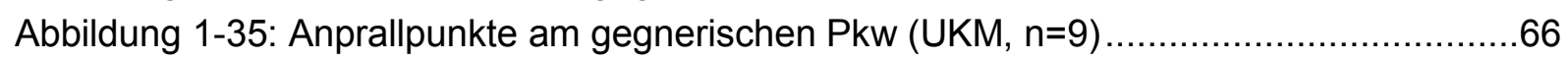

Abbildung 1-36: Form der Fahrzeugfront (UKLMU und UKM, $n=22) \ldots \ldots \ldots \ldots \ldots \ldots \ldots \ldots \ldots \ldots . . . \ldots 7$

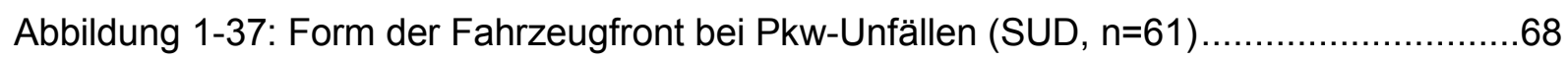

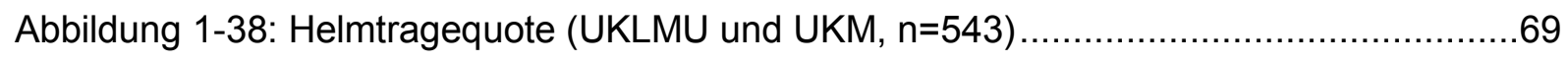

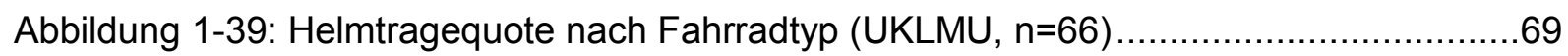


Abbildung 1-40: Helmtragequote nach Fahrradtyp (UKM, $n=477)$..................................

Abbildung 1-41: Lokalisation von Beschädigungen am Helm (UKLMU, $n=17$ ) .....................71

Abbildung 1-42: Lokalisation von Beschädigungen am Helm (UKM, $n=9)$.........................71

Abbildung 1-43: MAIS der verletzten Fahrradfahrer (UKLMU und UKM, $n=543$ ) .................72

Abbildung 1-44: Körperregion des MAIS der verletzten Fahrradfahrer (UKLMU, $n=66$ ) .......73

Abbildung 1-45: Körperregion des MAIS der verletzten Fahrradfahrer (UKM, n=477) ..........74

Abbildung 1-46: ISS der verletzten Fahrradfahrer (UKLMU und UKM, $n=543$ ) ...................75

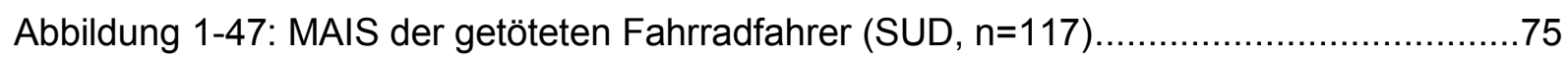

Abbildung 1-48: Körperregionen des MAIS der getöteten Fahrradfahrer (SUD, $n=117) \ldots \ldots . .76$

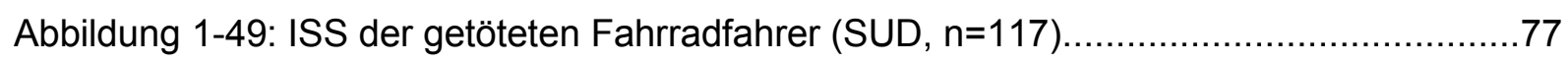

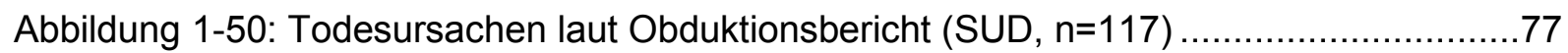

Abbildung 1-51: Größter AIS am Kopf der verletzten Fahrradfahrer (UKLMU und UKM,

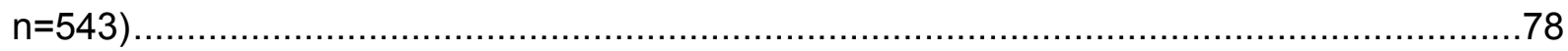

Abbildung 1-52: Größter AIS am Kopf der getöteten Fahrradfahrer (SUD, $n=117$ ) ..............79

Abbildung 1-53: Größter AIS am Kopf in Abhängigkeit von Helmnutzung (UKLMU und UKM, $\mathrm{n}=543)$...... .80

Abbildung 1-54: Häufigkeit der oberflächlichen Kopfverletzungen der Fahrradfahrer ohne Helm (UKLMU und UKM, $\mathrm{n}=126$ )

Abbildung 1-55: Häufigkeit der Oberflächlichen Kopfverletzungen der verletzten

Fahrradfahrer mit Helm (UKLMU und UKM, $n=25)$

Abbildung 1-56: Häufigkeit der oberflächlichen Kopfverletzungen der getöteten Fahrradfahrer

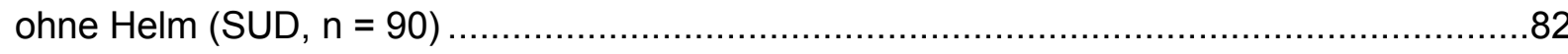

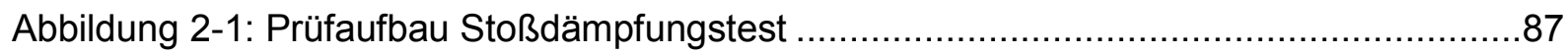

Abbildung 2-2: Prüfzone beim Stoßdämpfungstest in Anlehnung an die EN 1078 ...............91

Abbildung 2-3: Prüfaufbau zum Test der Festigkeit der Trageeinrichtung .........................93

Abbildung 2-4: Prüfaufbau zum Test der Wirksamkeit der Trageeinrichtung .......................94

Abbildung 2-5: CT Aufnahme Datensatz 2 (Fensterung -888/350) mit Plot der Grauwerte 101

Abbildung 2-6: Schwellwert für das absorbierende Schaumstoffmaterial ............................102

Abbildung 2-7: Maske des Schaumstoffs nach der Operation Region-Growing ..................103

Abbildung 2-8: Maske des Schaumstoffs aus drei Raumansichten und daraus berechnetem

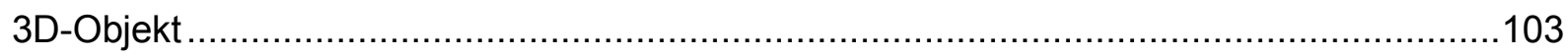

Abbildung 2-9: Fertige 3D-Modelle des Schaumstoffes und der Schale .............................104

Abbildung 2-10: Netzgeometrie des Schaumstoffs des ausgewählten Fahrradhelms .........104

Abbildung 2-11: Geometrische Darstellung und FE-Modell ..........................................105

Abbildung 2-12: Spannungs-Dehnungskurven der ersten drei quasi-statischen

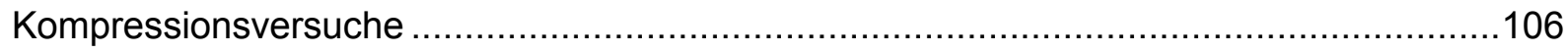

Abbildung 2-13: Spannungs-Dehnungskurven der weiteren neun quasi-statischen

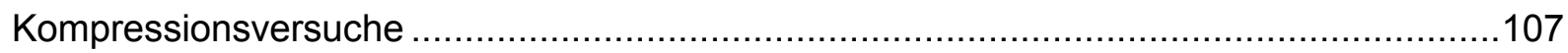

Abbildung 2-14: Form und Lage der Probenentnahme für die dynamischen

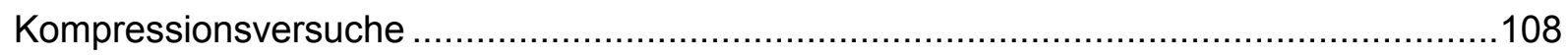

Abbildung 2-15: Versuchsaufbau der dynamischen Materialprüfung ................................109

Abbildung 2-16: Dynamischer Kompressionsversuch Phase 1 .......................................110

Abbildung 2-17: Dynamischer Kompressionsversuch Phase 2 .....................................110

Abbildung 2-18: Dynamischer Kompressionsversuch Phase 3 ......................................111

Abbildung 2-19: Dynamischer Kompressionsversuch Phase 4 .....................................111 
Abbildung 2-20: Vergleich der Kraft-Zeit-/Kraft-Weg-Verläufe aus Simulation und Versuch (Aufprallgeschwindigkeit 5,6 m/s, Impaktorgewicht $2960 \mathrm{~g}$ ).

Abbildung 2-21: Vergleich der Kraft-Zeit-/Kraft-Weg-Verläufe aus Simulation und Versuch (Aufprallgeschwindigkeit 4,95 m/s, Impaktorgewicht $2960 \mathrm{~g}$ ).

Abbildung 2-22: Vergleich der Kraft-Zeit-/Kraft-Weg-Verläufe aus Simulation und Versuch

(Aufprallgeschwindigkeit 4,42 m/s, Impaktorgewicht $2960 \mathrm{~g}$ )

Abbildung 2-23: Vergleich der Kraft-Zeit-/Kraft-Weg-Verläufe aus Simulation und Versuch

(Aufprallgeschwindigkeit 5,42 m/s, Impaktorgewicht $2960 \mathrm{~g}$ ).

Abbildung 2-24: Vergleich der Kraft-Zeit-/Kraft-Weg-Verläufe aus Simulation und Versuch

(Aufprallgeschwindigkeit 4,42 m/s, Impaktorgewicht $960 \mathrm{~g}$ ).... 114

Abbildung 2-25: Aufprallkonstellation des seitlichen Aufpralls. .115

Abbildung 2-26: Vergleich der Beschleunigungs-Zeit-Kurven (seitlicher Aufprall, Gewicht der Kopfform $5 \mathrm{~kg}$, Aufprallgeschwindigkeit $5,42 \mathrm{~m} / \mathrm{s}$ )

Abbildung 2-27: Vergleich der Kraft-Weg-Kurven (seitlicher Aufprall, Gewicht der Kopfform 5

$\mathrm{kg}$, Aufprallgeschwindigkeit 5,42 m/s) .............................................................116

Abbildung 2-28: Aufprallkonstellation des frontalen Aufpralls.......................................116

Abbildung 2-29: Vergleich der Beschleunigungs-Zeit-Kurven (frontaler Aufprall, Gewicht der Kopfform $5 \mathrm{~kg}$, Aufprallgeschwindigkeit $5,42 \mathrm{~m} / \mathrm{s}$ )

Abbildung 2-30: Vergleich der Kraft-Weg-Kurven (frontaler Aufprall, Gewicht der Kopfform 5

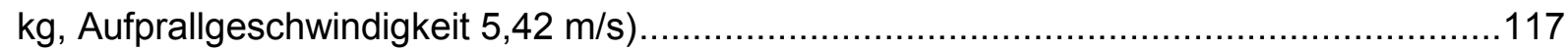

Abbildung 2-31: Aufprallkonstellation des Aufpralls auf die Rückseite .............................117

Abbildung 2-32: Vergleich der Beschleunigungs-Zeit-Kurven (Aufprall auf die Rückseite,

Gewicht der Kopfform 5 kg, Aufprallgeschwindigkeit 5,42 m/s) ...............................117

Abbildung 2-33: Vergleich der Kraft-Weg-Kurven (Aufprall auf die Rückseite, Gewicht der

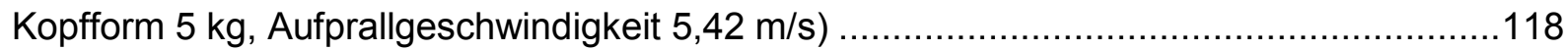

Abbildung 2-34: Darstellung eines beginnenden Risses im Helm in der Simulation............118

Abbildung 2-35: Riss des Helms im Realversuch .......................................................119

Abbildung 2-36: Kopfmodell mit gekoppeltem Helmmodell .............................................120

Abbildung 2-37: 5-Perzentil Frau und 50-Perzentil Mann Fußgänger-Mensch-Modelle

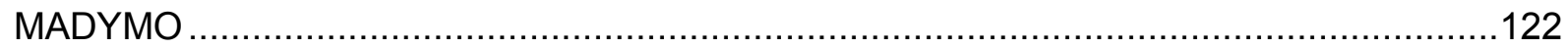

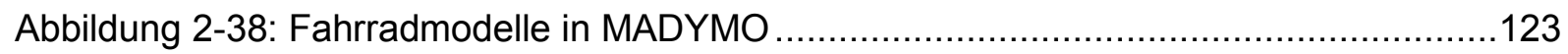

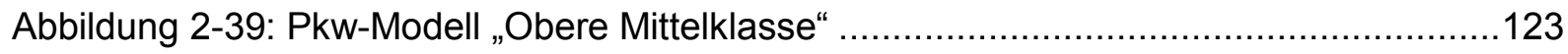

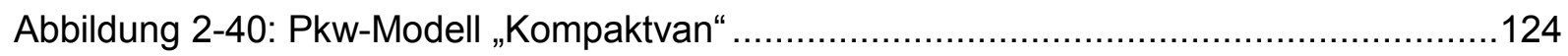

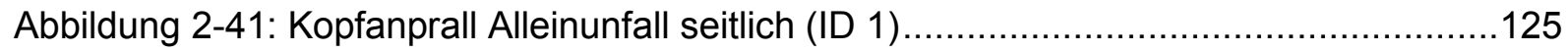

Abbildung 2-42: Kopfanprall Alleinunfall Sturz über Lenker (ID 2) .................................125

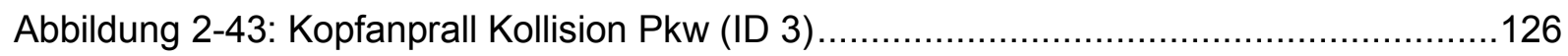

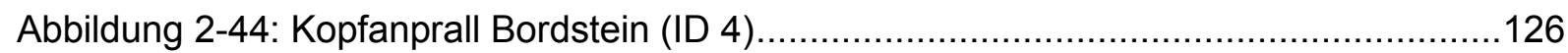

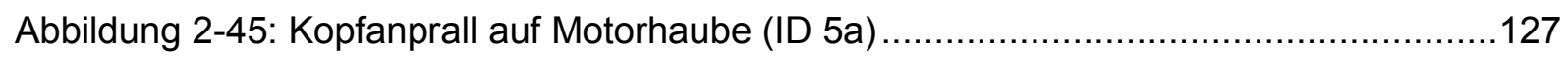

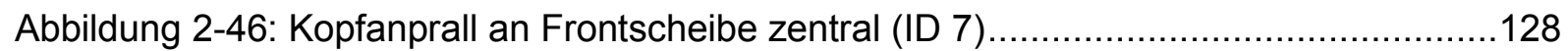

Abbildung 2-47: Kopfanprall an Frontscheibe außen (ID 8) .........................................129

Abbildung 2-48: Kopfmodell der Universität Straßburg SUFEHM (Bourdet et al. 2014) .....130

Abbildung 2-49: 2010 Toyota Yaris (National Crash Analysis Center 2011) ......................131

Abbildung 2-50: Kopfanprall Straße, Alleinunfall Sturz seitlich (ID 1a und 1b) ...................131

Abbildung 2-51: Kopfanprall Straße, Alleinunfall Sturz über Lenker (ID 2a und 2b) ...........132

Abbildung 2-52: Kopfanprall gegen Bordstein (ID 4a und 4b) .....................................132 
Abbildung 2-53: Kopfanprall Bordstein mit optimierten Helm (ID 4c) ................................133

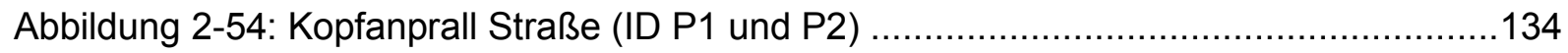

Abbildung 2-55: Kopfanprall Motorhaube (ID 5aa und 5ab) ........................................134

Abbildung 2-56: Kopfanprall Motorhaube „worst case“ (ID 5ac und 5ad) ...........................135

Abbildung 2-57: Kopfanprall Frontscheibe zentral (ID 7a und 7b) ..................................135

Abbildung 2-58: Kopfanprall Frontscheibe Randbereich (ID 8a und 8b) ............................136

Abbildung 2-59: Extremszenario Kopfanprall Frontscheibe (ID 3a und 3b) ......................136

Abbildung 2-60: Prüfzone ................................................................................. 138

Abbildung 2-61: Fallversuch 1 (Anprall seitlich, Kopfform nach EN, Geschwindigkeit 5,43

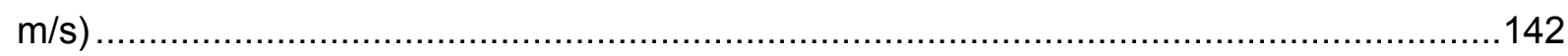

Abbildung 2-62: Fallversuch 2 (Anprall fronto-temporal, Kopfform nach EN, Geschwindigkeit

$5,43 \mathrm{~m} / \mathrm{s}$ )

Abbildung 2-63: Fallversuch 3 (Anprall zentr. Scheitel, Kopfform nach EN, Geschwindigkeit

$5,43 \mathrm{~m} / \mathrm{s})$

Abbildung 2-64: Fallversuch 4 (Anprall seitlich, Kopfform nach EN, Geschwindigkeit 7,7 m/s), Überschreitung des Beschleunigungsaufnehmer-Messbereichs. 144

Abbildung 2-65: Fallversuch 5 (Anprall seitlich, Kopfform nach EN, Geschwindigkeit 7,7 m/s), Überschreitung des Beschleunigungsaufnehmer-Messbereichs. .144 Abbildung 2-66: Fallversuch 6 (Anprall seitlich, Kopfform nach EN, Geschwindigkeit 6,5 m/s)

Abbildung 2-67: Fallversuch 7 (Anprall fronto-temporal, Kopfform nach EN, Geschwindigkeit

$6,5 \mathrm{~m} / \mathrm{s})$ 145

Abbildung 2-68: Fallversuch 8 (Anprall seitlich, Kopfform Hybrid III, Geschwindigkeit 5,42 $\mathrm{m} / \mathrm{s}$ )

Abbildung 2-69: Fallversuch 9 (Anprall zentr. Scheitel, Kopfform Hybrid III, Geschwindigkeit $5,42 \mathrm{~m} / \mathrm{s})$ 146

Abbildung 2-70: Fallversuch 10 (Anprall fronto-temporal, Kopfform Hybrid III, Geschwindigkeit 5,42 m/s)

Abbildung 2-71: Fallversuch 11 (Anprall fronto-temporal, Kopfform nach EN, Geschwindigkeit $5,43 \mathrm{~m} / \mathrm{s})$

Abbildung 3-1: Optimierte Prüfzone 150 


\section{Tabellenverzeichnis}

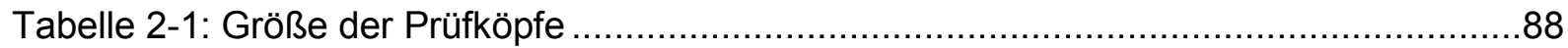

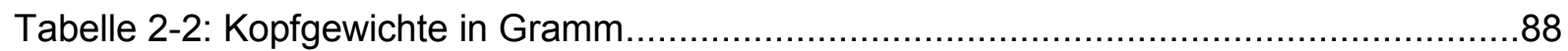

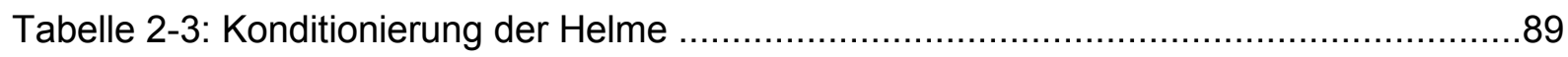

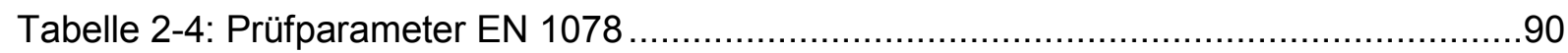

Tabelle 2-5: Prüfgeschwindigkeiten der Fahrradhelmnormen/ -richtlinien in Abhängigkeit der

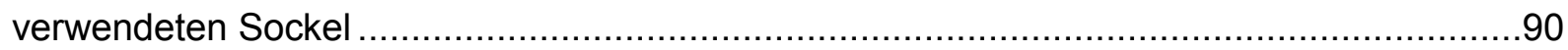

Tabelle 2-6: Zulässige Grenzwerte der Kopfbeschleunigung der Fahrradhelmnormen/ richtlinien

Tabelle 2-7: Prüfgeschwindigkeiten der Motorradhelmnormen/ -richtlinien der einzelnen

Sockel

Tabelle 2-8: Zulässige Grenzwerte der Kopfbeschleunigung der Motorradhelmnormen/ -

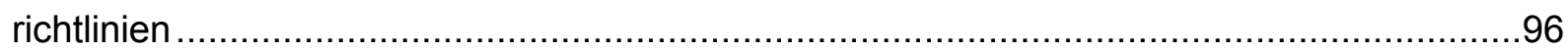

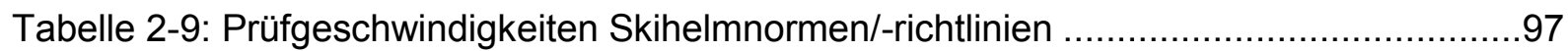

Tabelle 2-10: Zulässige Grenzwerte Kopfbeschleunigung Skihelmnormen/-richtlinien..........97

Tabelle 2-11: Prüfgeschwindigkeiten Reithelmnormen/-richtlinien ................................98

Tabelle 2-12: Zulässige Grenzwerte Kopfbeschleunigung Reithelmnormen/-richtlinien ........98

Tabelle 2-13: Technische Daten der angefertigten CT Datensätze..................................101

Tabelle 2-14: Schwellwerte bei der Segmentierung des absorbierenden Schaumstoffs und der Außenschale des Fahrradhelms in Hounsfield Einheiten...........................................102

Tabelle 2-15: Versuchsablauf der dynamischen Materialprüfung......................................109

Tabelle 2-16: Testmatrix Fallversuche .................................................................... 142

Tabelle 2-17: Ergebnisse Fallversuche ............................................................... 148 


\section{Anhang}

\section{Anhang 1: Fallauswahl der zu rekonstruierenden Unfälle}

Fälle, die in der Liste weiter oben stehen, sind besser dokumentiert

Alleinunfall, Sturz seitlich:

\begin{tabular}{|l|l|l|}
\hline Straße / Asphalt & $\begin{array}{l}\text { RF (w, 47) fährt mit ihrer Familie innerorts eine breite } \\
\text { Straße entlang, sieht über die Schulter nach links, verliert } \\
\text { dabei das Gleichgewicht und stürzt zu Boden. RF } \\
\text { verstirbt einen Tag später im Krankenhaus. } \\
\text { Kopf AIS 3, Hals AIS 0, Thorax AIS 0, Extremitäten AIS 1 }\end{array}$ \\
\hline Straße / Asphalt & $\begin{array}{l}\text { RF (m, 89) fährt aus einer Tankstelle auf den Radweg } \\
\text { und kommt alleinbeteiligt zu Sturz. RF stirbt 4 Tage } \\
\text { später im Krankenhaus. } \\
\text { Kopf AIS 3, Hals AIS 0, Thorax AIS 0, Extremitäten AIS 2 }\end{array}$ \\
\hline Straße / Asphalt & $\begin{array}{l}\text { RF (m, 73) stürzt aus ungeklärter Ursache seitlich zu } \\
\text { Boden. RF stirbt einen Tag später im Krankenhaus. } \\
\text { Fahrradhelm wurde getragen. } \\
\text { Kopf AIS 6, Hals AIS 0, Thorax AIS 3, Extremitäten AIS 2 }\end{array}$ \\
\hline
\end{tabular}

\section{Alleinunfall, Sturz über Lenker:}

\begin{tabular}{|l|l|l|}
\hline Schranke & $\begin{array}{l}\text { RF (m, 77) fährt talabwärts (ca.20 km/h), erkennt eine } \\
\text { vor ihm schließende Schranke zu spät, überbremst das } \\
\text { Vorderrad und stürzt. RF stirbt sechs Tage später im } \\
\text { Krankenhaus. } \\
\text { Kopf AIS 1, Hals AIS 3, Thorax AIS 0, Extremitäten AIS 1 }\end{array}$ \\
\hline Straße / Asphalt & $\begin{array}{l}\text { RF (m, 83) stürzt auf gerader Strecke (Einkaufstaschen } \\
\text { verhaken sich im Vorderrad) und schlägt mit dem Gesicht } \\
\text { auf den Asphalt auf. RF stirbt wenige Stunden später im } \\
\text { Krankenhaus. } \\
\text { Kopf AIS 5, Hals AIS 3, Thorax AIS 4, Extremitäten AIS 1 }\end{array}$ \\
\hline
\end{tabular}


Pkw erfasst Fahrradfahrer seitlich (geringe Geschwindigkeit):

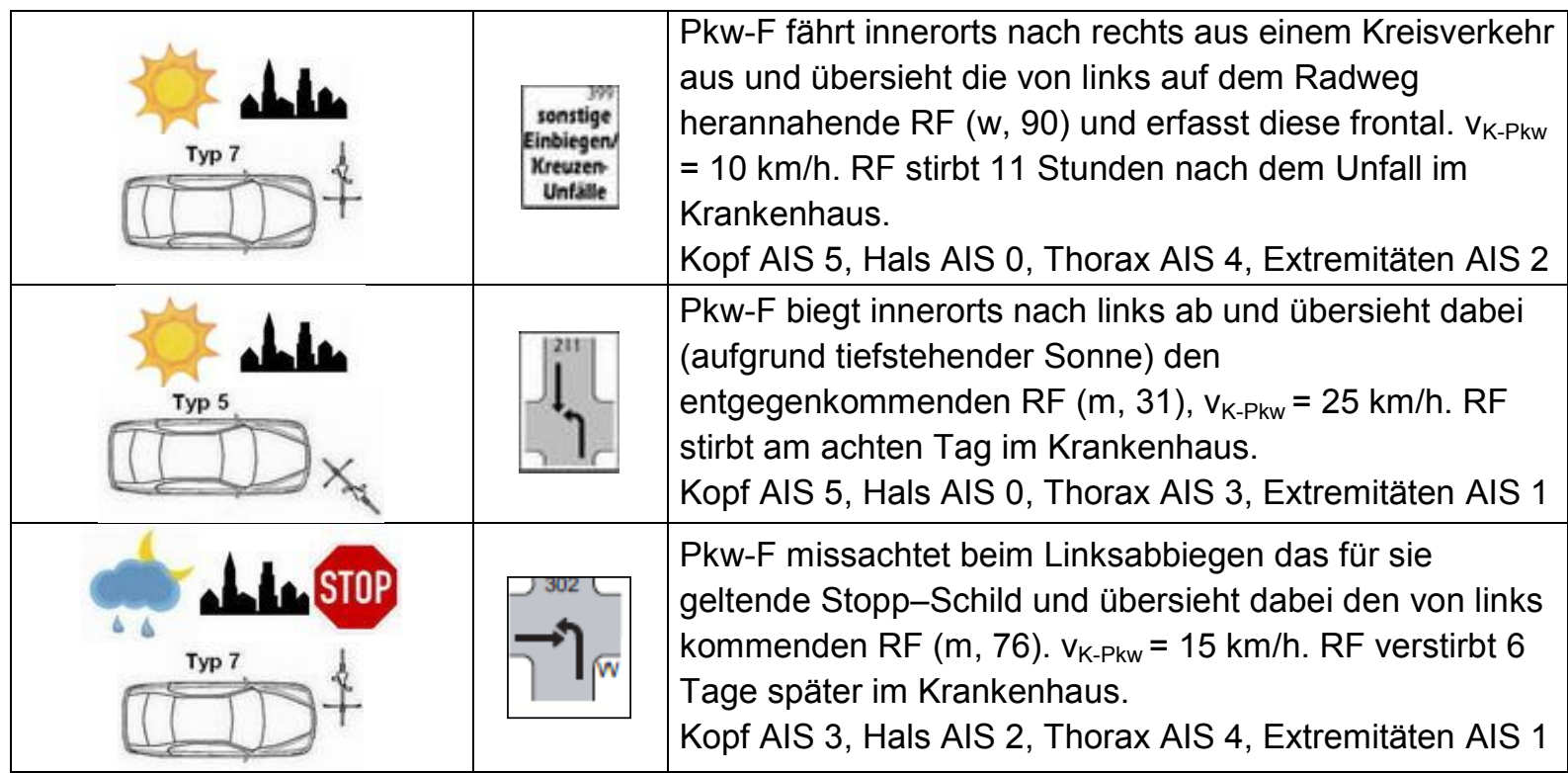

Pkw erfasst Fahrradfahrer seitlich (hohe Geschwindigkeit):

\begin{tabular}{|l|l|}
\hline Kopf AIS 4, Hals AIS 0, Thorax AIS 5, Extremitäten AIS 2 \\
\hline $\begin{array}{l}\text { KF (m, 66) übersieht beim Linksabbiegen außerorts } \\
\text { Kinen entgegenkommenden Pkw-F. }\end{array}$
\end{tabular}

\begin{tabular}{|l|l|}
\hline RF (w, 17) überquert außerorts an einer Kreuzung eine \\
bevorrechtigte Straße und übersieht den von links \\
kommenden Pkw-F. VK-Pkw $=75 \mathrm{~km} / \mathrm{h}$. RF stirbt am \\
zweiten Tag im Krankenhaus. \\
Kopf AIS 4, Hals AIS 3, Thorax AIS 3, Extremitäten AIS 5
\end{tabular}




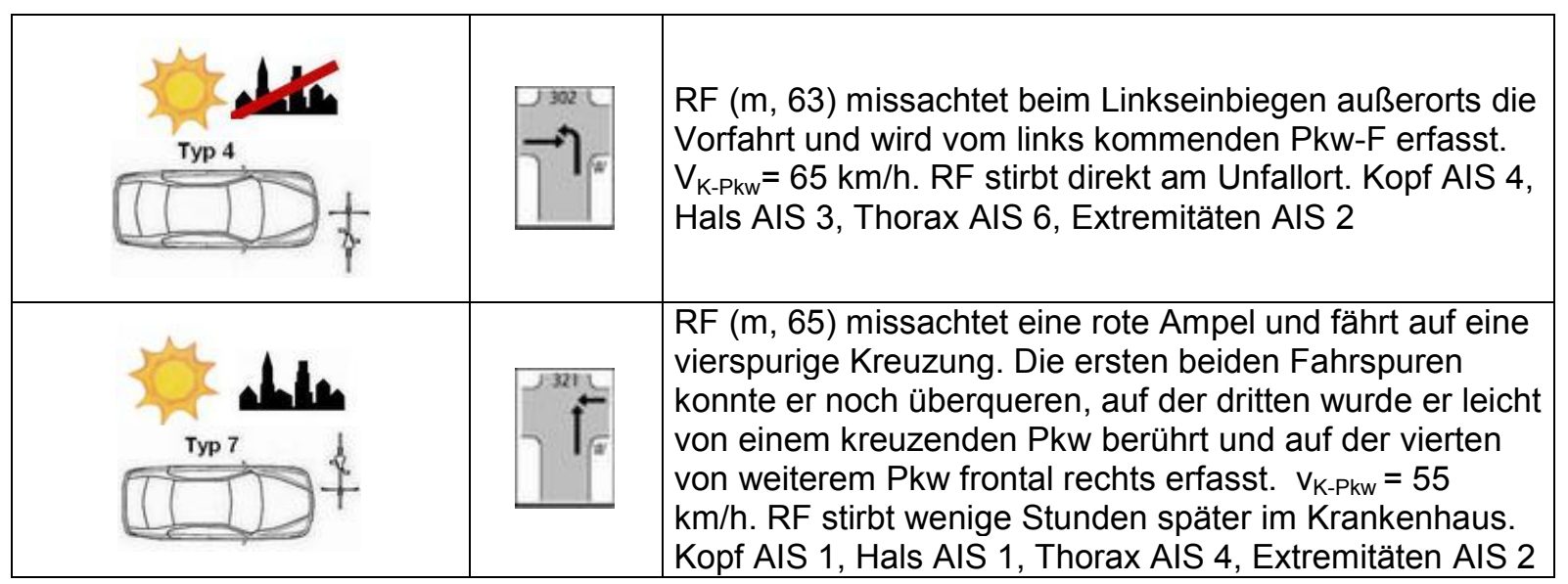




\section{Anhang 2: Patientenfragebogen UKLMU und UKM}

\section{Fragebogen Patient}

\section{Sehr geehrte Patientin / Sehr geehrter Patient,}

Leider mussten Sie sich heute aufgrund eines Radfahrunfalls in ärztliche Behandlung begeben. Wir möchten Sie bitten, uns zu helfen, die Zahl der Radfahrunfälle in Zukunft zu senken. Zu diesem Zweck sammeln wir ein Jahr lang (selbstverständlich freiwillig und anonym) die Daten aller Radfahrunfälle in München und Münster. Weitere Informationen finden Sie im beiliegenden Flyer.

Teil A: Allgemeine Angaben
1. Geschlecht:
$\square \mathbf{m} / \square \mathbf{w}$
2. Alter: Jahre

3. Größe: cm 4. Gewicht: kg

5. Wie häufig fahren Sie mit dem Fahrrad:

$\square$ Sehr selten (1x/Monat)

$\square$ Gelegentlich (1x/Woche)

Häufig (>1x/Woche, aber nicht täglich)

Sehr häufig (täglich)

\section{Warum fahren Sie Fahrrad:}

Freitext: 


\section{Teil B: Angaben zum Unfall}

1. Wurde der Unfall polizeilich erfasst? $\square$ ja $\square$ nein

2. Datum und Zeitpunkt des Unfalls:

\section{Unfallort:}

Straße und Hausnummer: $\mathrm{Nr}$.

Oder Kreuzung/Einmündung mit Straße:

\section{Ursache des Unfalls:}

Entweder a.) Bei Alleinunfall/Sturz:

$\square$ Schlechter Untergrund (z.B. Glatteis, Straßenbahnschienen,

Kopfsteinpflaster, Schlagloch)

$\square$ Hindernis übersehen

$\square$ Gleichgewicht verloren

$\square$ Abgelenkt gewesen

$\square$ Vom Pedal abgerutscht

$\square$ Technischer Defekt am Fahrrad

Anderer Grund:

Oder b.) Kollision mit anderem Verkehrsteilnehmer/Objekt:

$\square$ Kollision mit fahrendem Auto
$\square$ Kollision mit fahrendem Lkw/ Bus
$\square$ Kollision mit Fußgänger
$\square$ Kollision mit Motorrad
$\square$ Kollision mit anderem Fahrradfahrer
$\square$ Kollision mit Gegenstand (auch stehendes Auto)
$\square$ Kollision mit offener FZ-Tür
$\square$ Unbekannt $\square$ Sonstiges:

\section{Fahrradtyp:}

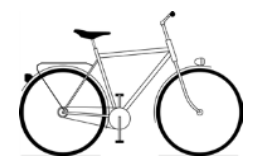

Sportrad, Rennrad

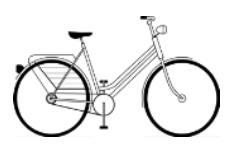

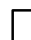

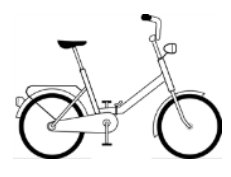

Klapprad

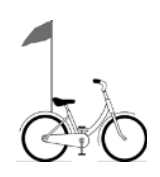

Kinderrad

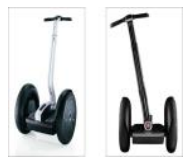

Segway

Mountain-/ Trekkingbike

E-Bike/ Pedelec

sonstiges:

\section{Straßenverhältnisse beim Unfall}

$\square$ trocken

$\square$ nass

$\square$ verschmutzt

$\square$ Schnee(-matsch) vereist 
7. Lichtverhältnisse: $\square$ Tag $\square$ Nacht $\square$ Dämmerung künstliche Beleuchtung

8. Straßenbelag: $\square$ Asphalt $\square$ Schotter $\square$ sonstiges:

9. Helm getragen:

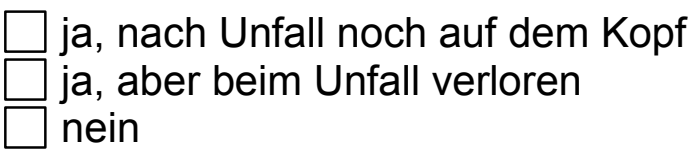

a) falls ja, hatten Sie mit Ihrem Helm Kontakt mit einem Objekt/Straße?

$\square$ ja $\square$ nein

b) falls ja, Anprallstelle des Helms (Mehrfachnennung möglich)

Ansicht von rechts hinten

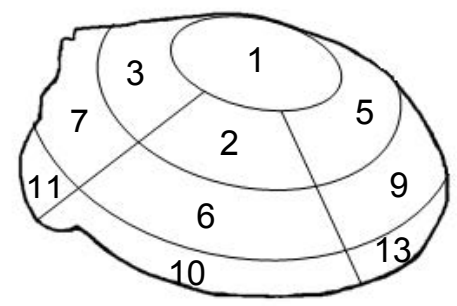

Ansicht von oben

vorne hinten

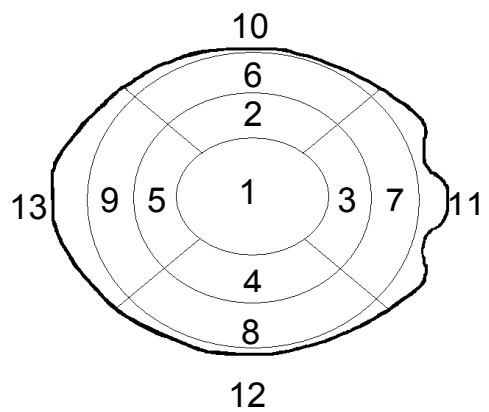

Ansicht von links

vorne

hinten

Anprallstelle:

$\begin{array}{lll}\square & \text { Kratzer } \square \text { Delle } \square \text { Bruch } \square \text { sonstiges } \\ \square & \text { Kratzer } \square \text { Delle } \square \text { Bruch } \square \text { sonstiges } \\ \square & \text { Kratzer } \square \text { Delle } \square \text { Bruch } \square \text { sonstiges } \\ & \text { Kratzer } \square \text { Delle } \square \text { Bruch } \square \text { sonstiges }\end{array}$

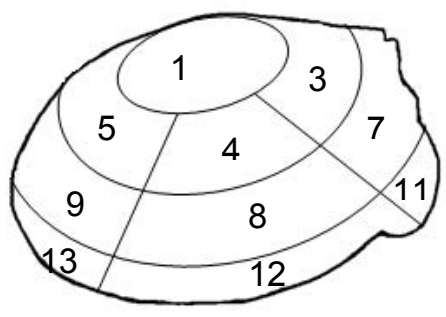

Genauere Beschreibung/Ausmaß in cm etc. (Freitext):

c) falls ja, würden Sie Ihren Helm für weitere Untersuchungen der Forschung zur Verfügung stellen?

10. Wo fuhren Sie, als sich der Unfall ereignete:

$\square$ Auf einer normalen Strasse (kein Fahrradweg)/Fahrradstraße 
$\square$ Auf einem baulich getrennten Fahrradweg

Auf einem nicht baulich getrennten Fahrradweg auf der Strasse

Auf einem nicht baulich getrennten Fahrradweg auf dem Bürgersteig

Auf dem Bürgersteig (kein Fahrradweg)

Auf dem dafür vorgesehenen Weg zum Überqueren eine Fahrstrasse (z.B.

Ampel)

$\square$ Auf einem Feldweg / Waldweg etc.

$\square$ Sonstiges:

11. Kurzbeschreibung des Unfalls:

12. Geschätzte Geschwindigkeit des Kollisionsgegners:

$\square<10 \mathrm{~km} / \mathrm{h} \square 10-30 \mathrm{~km} / \mathrm{h} \square 30-50 \mathrm{~km} / \mathrm{h} \square>50 \mathrm{~km} / \mathrm{h}$

13. Geschätzte eigene Geschwindigkeit bevor der Unfall geschah:

\begin{tabular}{|c|c|c|}
\hline nd & $\square$ anfahrend & $\begin{array}{c}\square \text { langsam fahrend } \\
(<20 \mathrm{~km} / \mathrm{h})\end{array}$ \\
\hline
\end{tabular}

14.

A) Bitte tragen Sie im Bild unten ein, aus welcher Richtung der Unfallgegner für Sie kam, als der Anprall erfolgte (Fahrtrichtung Unfallgegner mit Pfeil einzeichnen)

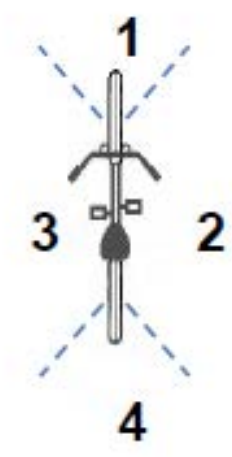

B)

Bitte tragen Sie im Bild unten ein, an welchem Bereich des Kraftfahrzeugs Sie bzw. Ihr Fahrrad zuerst anprallten

(Bereich ankreuzen) 


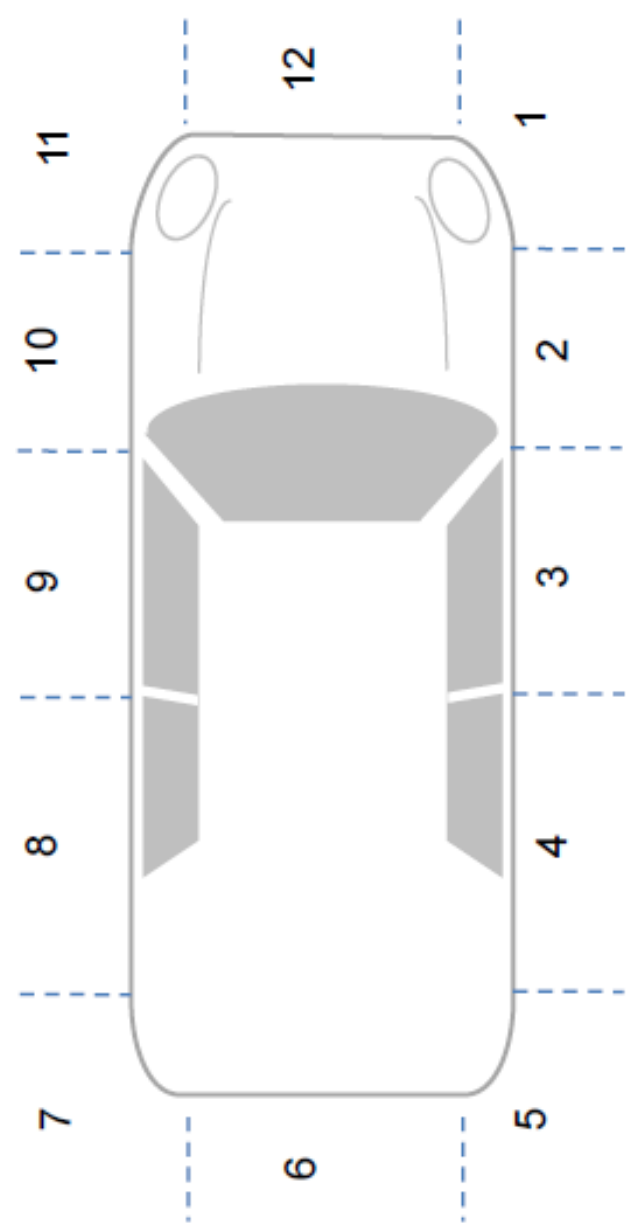

15. Anprallbereich Fahrradfahrer am Kfz (falls möglich):
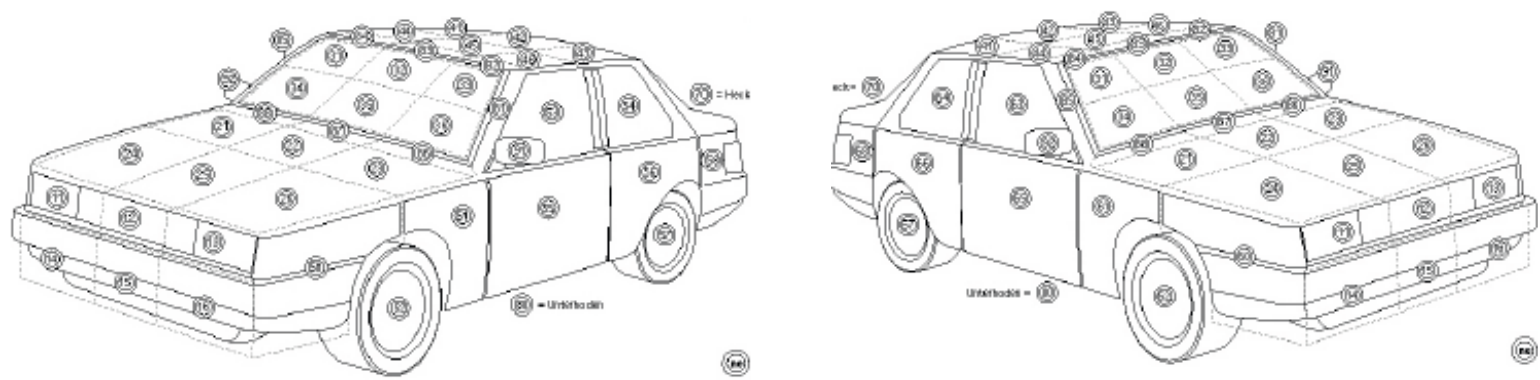

16. Fahrzeugtyp (Fokus auf der Fahrzeugfront)

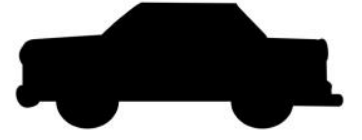

Limousine/ Kombi

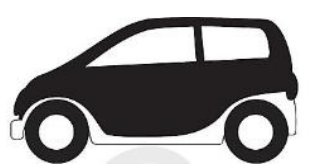

Kleinstwagen (Smart o.ä.)

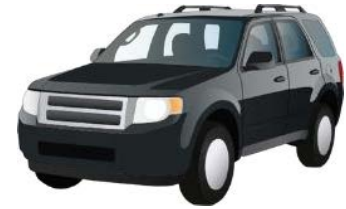

SUV I

Geländewagen 


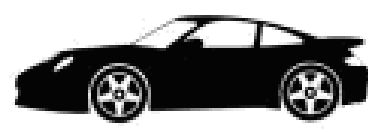

$\square$ Sportwagen

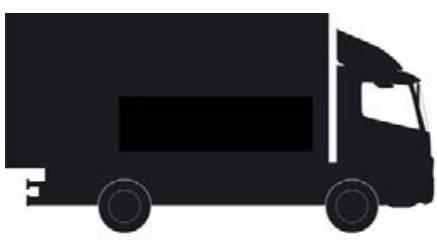

$\square$ Transporter/ Lkw

$\square$ sonstiges:

17. Hatten Sie vor dem Unfall Alkohol getrunken?

$\square$ ja $\square$ nein 
Teil C: Angaben zum klinischen Verlauf

1. Datum und Zeitpunkt der Aufnahme:

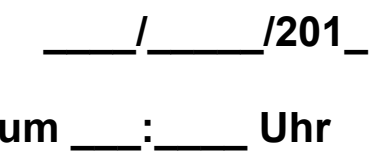

2. Primäre Behandlung erfolgte:

$\square$ ambulant

stationär (auch, wenn zuerst ambulant!)

$\square$ stationär, intensiv

3. Einlieferung durch:

\begin{tabular}{ll}
$\square$ & Privaten PKW / ÖPNV / zu Fuss \\
\hline$\square$ & RTW (Unfallort) \\
\hline$\square$ & NEF / NAW (Unfallort) \\
\hline$\square$ & RTH (Unfallort) \\
\hline$\square$ Verlegung von anderer Klinik \\
$\square$ Direkte Zuweisung vom Hausarzt
\end{tabular}

4. Klinische Parameter bei Aufnahme:

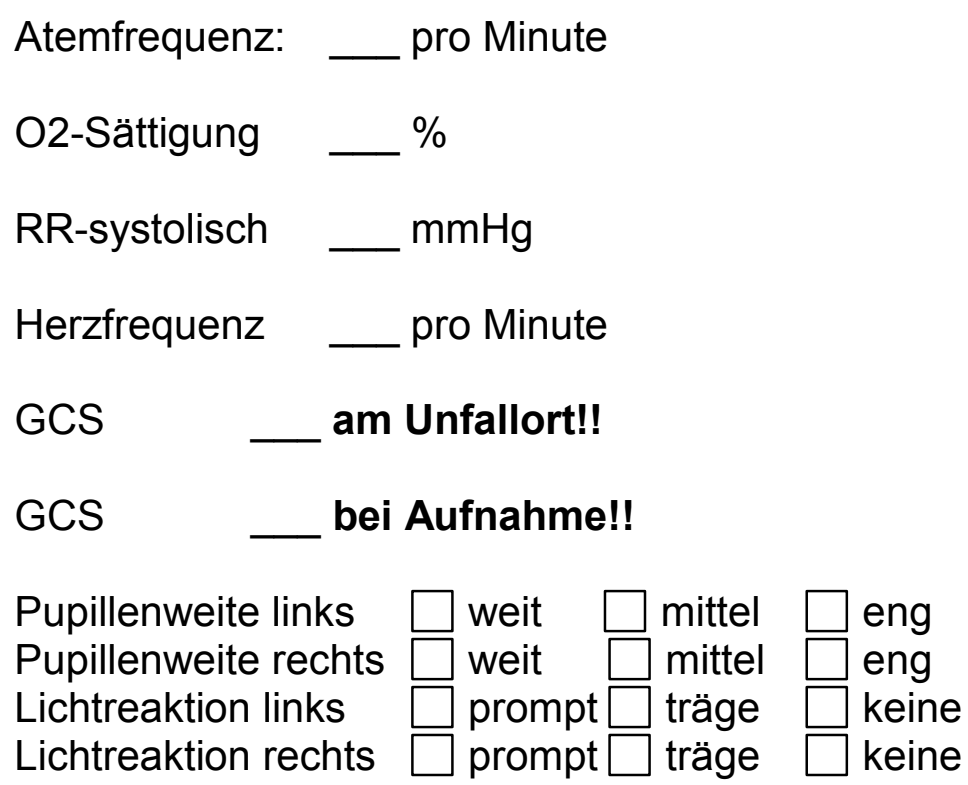


5. Diagnosen/Befunde (Freitext):

\section{Dauer der Bewusstlosigkeit:}

$\square<1$ Std. $\square$ 1-6 Std. $\square 6-24$ Std. $\square>24$ Std. 
7. Oberflächliche Kopfverletzungen (Mehrfachnennung möglich):
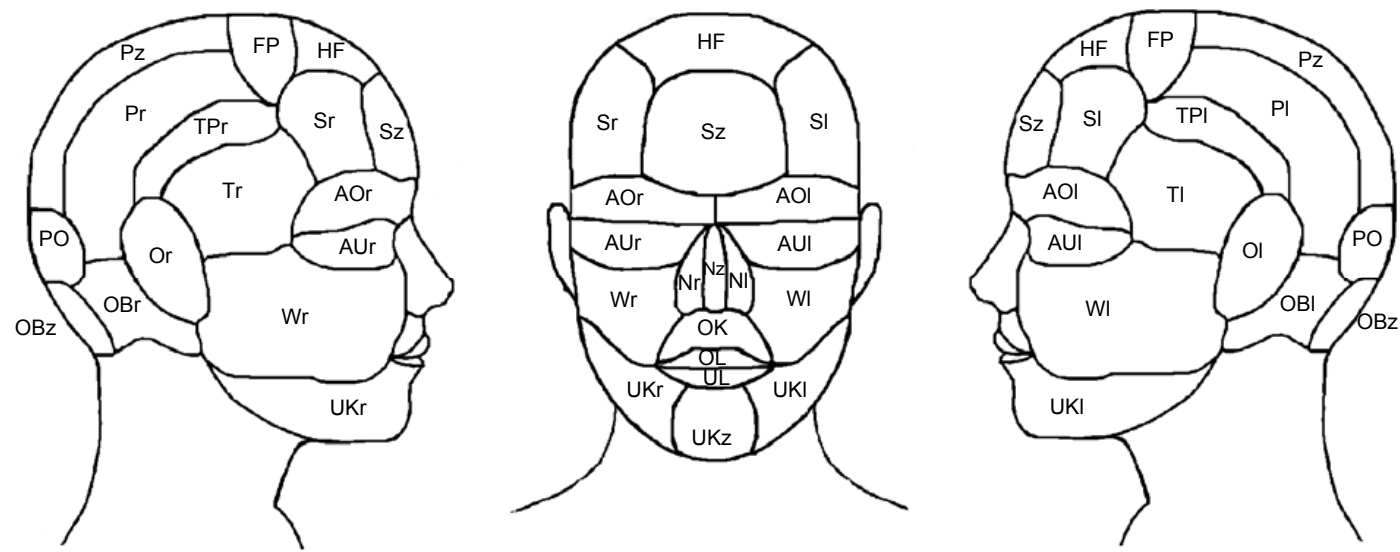

(zu kodieren mit 1. Ort gemäß obigen Abbildung und 2. Art der Verletzung nach folgender Einteilung, z.B. HF 1)

1 Hämatom 2 Schürfung

3 Riss-/ Quetschwunde 4 Schnittwunde

5 Kopfschwarteneinblutung 6 Skalpierungsverletzung

7 Crush

Ort Art Ort Art Ort Art

Ort Art Ort Art Ort Art

Genauere Beschreibung/Ausmaß in cm etc. (Freitext):

8. CT des Schädels erfolgt?

$\square$ ja $\square$ nein 


\section{Teil D: Angaben zum Outcome}

1. Verweildauer Intensiv: _ _ Tage

2. Beatmungsstunden insgesamt: __ Stunden

3. Gesamtverweildauer: Tage

4. DRG:

5. Entlassung:

$\square$ Nach Hause

$\square$ In Rehabilitationsklinik

$\square$ In andere Klinik verlegt

$\square$ Verstorben

6. Voraussichtliche Dauer der Arbeitsunfähigkeit : Tage 


\section{Anhang 3: Datenbank rekonstruierter Unfälle}

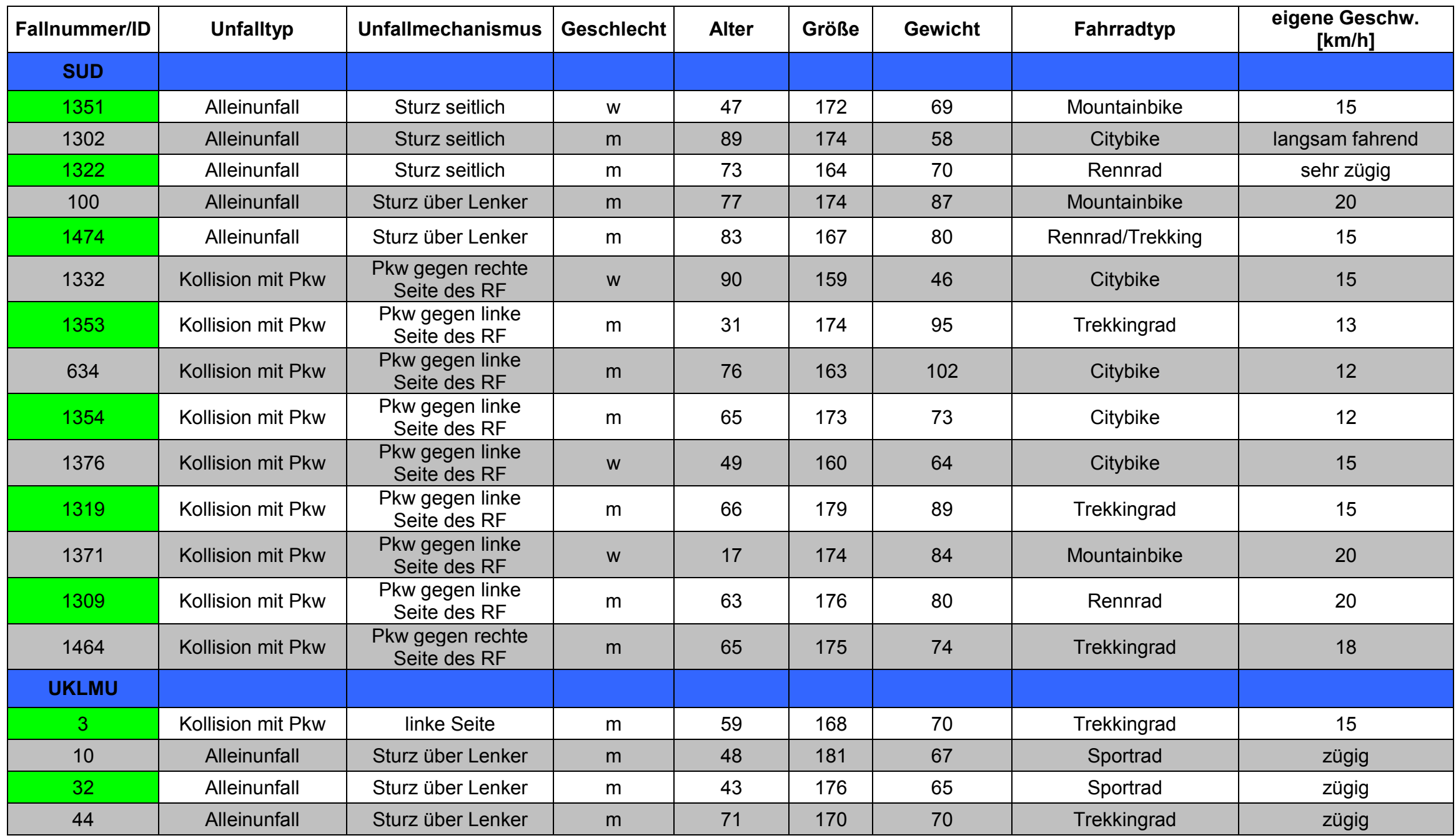

\begin{tabular}{|l|l|l|l|l|l|l|l|}
\hline Fallnummer/ID & Typ Pkw-Front & Koll.-Geschw. & Gebremst & Kollisionswinkel & Anprallstelle Erstkontakt & Längswurfweite RF & Querwurfweite RF \\
\hline
\end{tabular}




\begin{tabular}{|c|c|c|c|c|c|c|c|}
\hline & & Pkw [km/h] & & & & [m] & {$[\mathrm{m}]$} \\
\hline \multicolumn{8}{|l|}{ SUD } \\
\hline 1351 & - & - & - & - & - & - & - \\
\hline 1302 & - & - & - & - & - & - & - \\
\hline 1322 & - & - & - & - & - & - & - \\
\hline 100 & - & - & - & - & - & - & - \\
\hline 1474 & - & - & - & - & - & - & - \\
\hline 1332 & Limousine & 7 & ja & $50^{\circ}$ & 16 & 1 & 0 \\
\hline 1353 & Citroen C3 & 25 & nein & $135^{\circ}$ & 15 & 6 & 0 \\
\hline 634 & Limousine & 15 & nein & $90^{\circ}$ & 14 & - & - \\
\hline 1354 & Limousine & 52 & ja & $60^{\circ}$ & 14 & ca. 20 & n.b. \\
\hline 1376 & Corolla Verso & 65 & nein & $90^{\circ}$ & 15 & 20 & 2 \\
\hline 1319 & Limousine & 70 & ja & $100^{\circ}$ & 15 & 25 & n.b. \\
\hline 1371 & Kleinstfz/Lim. & 75 & ja & $90^{\circ}$ & 15 & n.b. & n.b. \\
\hline 1309 & suv & 60 & ja & $80^{\circ}$ & 14 & 8,5 & n.b. \\
\hline 1464 & MB A-Klasse & 55 & ja & $90^{\circ}$ & 16 & 21 & 1,3 \\
\hline \multicolumn{8}{|c|}{ UKLMU } \\
\hline 3 & Limousine & 30 & ja & $90^{\circ}$ & 16 & 1 & 1 \\
\hline 10 & - & - & - & - & - & - & - \\
\hline 32 & - & - & - & - & - & - & - \\
\hline 44 & - & - & - & - & - & - & - \\
\hline
\end{tabular}




\begin{tabular}{|c|c|c|c|c|c|c|}
\hline Fallnummer/ID & Längswurfweite Fahrrad [m] & $\begin{array}{c}\text { Querwurfweite } \\
\text { Fahrrad [m] }\end{array}$ & $\begin{array}{c}\text { Beulenversatz } \\
{[\mathrm{cm}]}\end{array}$ & Anprallstelle Hüfte & $\begin{array}{l}\text { Anprallstelle } \\
\text { Oberkörper }\end{array}$ & Anprallstelle Kopf \\
\hline \multicolumn{7}{|l|}{ SUD } \\
\hline 1351 & - & - & - & flache Straße & flache Straße & flache Straße \\
\hline 1302 & - & - & - & flache Straße & flache Straße & flache Straße \\
\hline 1322 & - & - & - & flache Straße & flache Straße & flache Straße \\
\hline 100 & - & - & - & flache Straße & flache Straße & flache Straße \\
\hline 1474 & - & - & - & flache Straße & flache Straße & flache Straße \\
\hline 1332 & 1 & 0 & - & flache Straße & flache Straße & flache Straße \\
\hline 1353 & n.b. & n.b & 0 & 25 & 22 & 35 \\
\hline 634 & - & - & - & flache Straße & flache Straße & flache Straße \\
\hline 1354 & ca. 20 & n.b. & 10 & 26 & 36 & 82 \\
\hline 1376 & 25 & 4 & 20 & 26 & 36 & 82 \\
\hline 1319 & 43 & n.b. & 10 & 26 & 36 & 82 \\
\hline 1371 & n.b. & n.b. & 20 & 25 & 87 & 33 \\
\hline 1309 & 6 & n.b. & 10 & 24 & 21 & 34 \\
\hline 1464 & 18 & 2,6 & 20 & 26 & 23 & 36 \\
\hline \multicolumn{7}{|l|}{ UKLMU } \\
\hline 3 & n.b. & n.b. & - & flache Straße & flache Straße & Bordsteinkante \\
\hline 10 & - & - & - & flache Straße & flache Straße & flache Straße \\
\hline 32 & - & - & - & flache Straße & flache Straße & flache Straße \\
\hline 44 & - & - & - & flache Straße & flache Straße & flache Straße \\
\hline
\end{tabular}




\begin{tabular}{|c|c|}
\hline Fallnummer/ID & schwerste Verletzungen \\
\hline \multicolumn{2}{|l|}{ SUD } \\
\hline 1351 & 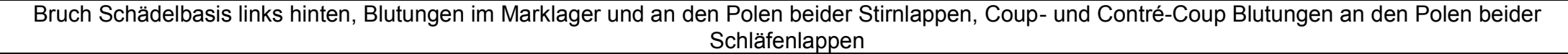 \\
\hline 1302 & Prellungen an Stirn- und Schläfenlappen, Epiduralblutung oberer Keilbeinflügel links, Schädelbruchlinie im Bereich der linken Schläfenschuppe \\
\hline 1322 & $\begin{array}{c}\text { Bruchsystem im Bereich der linken Schläfenregion, Brücke im Zentrum mit einer breiten Hirngewebsdefektzone, ausgedehnte Blutungen im Bereich der } \\
\text { Brücke und rechten Hirnhälfte }\end{array}$ \\
\hline 100 & Quetschung des Halsmarks mit Luxation des 1. Halswirbelkörpers nach hinten, Bruch des 3. HWK, kräftige Einblutungen im Bereich der rechten Hirnhälfte \\
\hline 1474 & $\begin{array}{l}\text { Quetschung des verlängerten Markes mit Blutungen, Multiple Einblutungen Hirn, Bruch der Schädelbasis am Augenhöhlendach links, Multiple Verletzungen } \\
\text { im zentralen Gesichtsbereich }\end{array}$ \\
\hline 1332 & $\begin{array}{c}\text { subdurales Hämatom rechts als Folge eines Sturzes auf den Hinterkopf, Teileinriß des Aortenbogens, die übrigen Verletzungen lassen auf einem Sturz auf } \\
\text { das Hinterhaupt und die rechte Körperhälfte schließen }\end{array}$ \\
\hline 1353 & $\begin{array}{c}\text { großflächige Hirnrindenprellungsblutungen beider Stirnpole sowie Schläfenlappen, Flächenhafte Blutungen auf und unter der harten Hirnhaut, Fraktur linkes } \\
\text { Augenhöhlendach, Unterblutungen Lungenfell }\end{array}$ \\
\hline 634 & $\begin{array}{l}\text { mäßige subdurale Blutungen, beidseits in den hinteren Schädelgruben, Bruch der Schädelbasis und des Schädeldachs, Multiple Rippenfrakturen und Blut } \\
\text { und der Brusthöhle }\end{array}$ \\
\hline 1354 & $\begin{array}{c}\text { Druckblutungen im Zwischenhirnbereich, Die Schädelbasis mit einem Diagonalbruch vom linken Felsenbein durch den Türkensattel bis zum rechten } \\
\text { Augenhöhlendach reichend }\end{array}$ \\
\hline 1376 & $\begin{array}{c}\text { Überdehnung der Großhirnschenkel am Übergang in die Brücke, offene Hirngewebszertrümmerungen an der Außenseite des linken Großhirns, } \\
\text { Teilskalpierungsverletzung an der linken vorderen Schädelseite }\end{array}$ \\
\hline 1319 & $\begin{array}{c}\text { Bruchlinie in der hinteren Schläfenregion rechtsseitig nach oben zur Scheitelregion hin verlaufend, Einblutungen unter der harten Hirnhaut, Bruch sämtlicher } \\
\text { Rippen rechts }\end{array}$ \\
\hline 1371 & $\begin{array}{c}\text { Ausgedehnte Hirnrindenprellungsbezirke/Einblutungen im Bereich der Hirnrinde entlang der gesamten Stirnbasis rechts, Bruch von der hinteren seitlichen } \\
\text { Schädelregion und der hinteren Schläfenregion rechts }\end{array}$ \\
\hline 1309 & $\begin{array}{c}\text { Schädelbasistrümmerbrüche in der vorderen Schädelgrube linksseitig stärker, Schädeldach linksseitig Impressionsfraktur, Nahezu Brüche aller Rippen } \\
\text { beidseits, teils mit Durchspießungen insbes. linksseitig }\end{array}$ \\
\hline 1464 & Einriss Aortenbogen, Multiple Rippenfrakturen mit Fokus auf rechter Seite, starke Einblutung beider Lungenstiele, \\
\hline \multicolumn{2}{|l|}{ UKLMU } \\
\hline 3 & Rippenfraktur 1+2 links, offene Schädelfraktur parieto-occipital links, Intracerebrale und subarachnoidale Blutungen parieto-occipital links \\
\hline 10 & multiple Gesichtsverletzungen, Nasenbeinfraktur, kleine intraparenchymatöse Gehirnblutung \\
\hline 32 & Prellmarke Schädel links occipital, Claviculafraktur links \\
\hline 44 & Mittelgesichtsfrakturen, Fraktur BWK 6 Vorderkante \\
\hline
\end{tabular}




\begin{tabular}{|c|c|c|c|c|}
\hline & & & Kopfverl. 3 & \\
\hline \multicolumn{5}{|l|}{ SUD } \\
\hline 1351 & POI, Schürfung & OBI, RQW & $\mathrm{PI}, \mathrm{RQW}$ & Tr, Hämatom \\
\hline 1302 & TI, Einblutung & TPI, Einblutung & SI, Einblutung & AOI, Hämatom \\
\hline 1322 & TI, Einblutung & TPI, Einblutung & UKz, Schürfung & AUI, Schürfung \\
\hline 100 & Sr, Einblutung & Pr, Einblutung & TPr, Einblutung & Tr, Einblutung \\
\hline 1474 & AOI, Hämatom & AOr, Hämatom & $\mathrm{Nz}, \mathrm{RQW}$ & Sz, Schürfung \\
\hline 1332 & POz, Einblutung & Tr, Einblutung & $\mathrm{OBr}$, Hämatom & POz, Hämatom \\
\hline 1353 & Stirn komplett Einblutung & FPI+FPr, Einblutung & $\begin{array}{c}\mathrm{Tr}+\mathrm{Tl} \\
\text { Einblutung }\end{array}$ & Stirn Schürfungen \\
\hline 634 & OBr, Einblutung & Tr, Einblutung & $\mathrm{OBr}, \mathrm{RQW}$ & OL, Hämatom \\
\hline 1354 & \multicolumn{4}{|c|}{ Einblutungen linksseitig über gesamten Schädel (Stirn bis OBI) } \\
\hline 1376 & \multicolumn{4}{|c|}{ Crush gesamter Schädel } \\
\hline 1319 & Tr, Einblutung & TPr, Einblutung & - & - \\
\hline 1371 & $\begin{array}{c}\text { Ausgedehnte Einblutungen linksseitig, } \mathrm{POz}+\mathrm{POI}+\mathrm{POr} \\
\text { Einblutungen }\end{array}$ & & & Gesicht rechts Schürf. \\
\hline 1309 & Schürfungen im gesamten Gesicht & & OBI Einblutung & AOI, RQW \\
\hline 1464 & Pz, Hämatom & FPr, Hämatom & $A U I+A U r, R Q W$ & $\begin{array}{l}\text { Stirn, Schürfungen + } \\
\text { RQW }\end{array}$ \\
\hline \multicolumn{5}{|c|}{ UKLMU } \\
\hline 3 & PI, RQW & Tpl, RQW & - & - \\
\hline 10 & SI, RQW & WI, RQW & - & - \\
\hline 32 & TPI, Schürfung & - & - & - \\
\hline 44 & SI, RQW & WI, Schürfung & - & - \\
\hline
\end{tabular}




\begin{tabular}{|c|c|c|c|c|c|c|}
\hline Fallnummer/ID & Kopf MAIS & Hals MAIS & Thorax MAIS & Extremitäten MAIS & Körperregion MAIS & ISS \\
\hline \multicolumn{7}{|l|}{ SUD } \\
\hline 1351 & 3 & 0 & 0 & 1 & Kopf & 11 \\
\hline 1302 & 3 & 0 & 0 & 2 & Kopf & 14 \\
\hline 1322 & 6 & 0 & 3 & 2 & Kopf & 75 \\
\hline 100 & 1 & 3 & 0 & 1 & Hals & 11 \\
\hline 1474 & 5 & 3 & 4 & 1 & Kopf & 45 \\
\hline 1332 & 5 & 0 & 4 & 2 & Kopf & 45 \\
\hline 1353 & 5 & 0 & 3 & 1 & Kopf & 35 \\
\hline 634 & 3 & 2 & 4 & 1 & Thorax & 29 \\
\hline 1354 & 5 & 0 & 0 & 1 & Kopf & 26 \\
\hline 1376 & 6 & 3 & 3 & 3 & Kopf & 75 \\
\hline 1319 & 4 & 0 & 5 & 2 & Thorax & 45 \\
\hline 1371 & 4 & 3 & 3 & 5 & Beine & 57 \\
\hline 1309 & 4 & 3 & 6 & 2 & Thorax & 75 \\
\hline 1464 & 1 & 1 & 4 & 2 & Thorax & 24 \\
\hline \multicolumn{7}{|l|}{ UKLMU } \\
\hline 3 & 4 & 0 & 2 & 0 & Kopf & 20 \\
\hline 10 & 3 & 1 & 0 & 0 & Kopf & 10 \\
\hline 32 & 1 & 0 & 0 & 2 & Obere Extremitäten & 5 \\
\hline 44 & 3 & 0 & 0 & 0 & Kopf & 17 \\
\hline
\end{tabular}


Anhang 4: Simulierte Unfälle in MADYMO

\begin{tabular}{|c|c|c|c|c|c|c|c|c|}
\hline & ID & Kurzbeschreibung & $\begin{array}{c}\text { Kollisionswinkel } \\
\text { RF/Pkw }\end{array}$ & $\begin{array}{c}\text { Geschw. RF } \\
{[\mathrm{km} / \mathrm{h}]}\end{array}$ & $\begin{array}{l}\text { Geschw. } \\
\text { Pkw }[\mathrm{km} / \mathrm{h}]\end{array}$ & Pkw Modell & Kopfanprall an & $\begin{array}{c}\text { Anprallstelle } \\
\text { am Kopf }\end{array}$ \\
\hline \multirow[t]{4}{*}{ Realunfälle: } & 1 & Alleinunfall, Sturz seitlich & - & 8 & - & - & Straße & seitlich links \\
\hline & 2 & Alleinunfall, Sturz über Lenker & - & 15 & - & - & Straße & Stirn zentral \\
\hline & 3 & Koll. Pkw, Extremfall & $90^{\circ}$ & 7,2 & 65 & Toyota Corolla Verso & Windschutzscheibe/Dachkante & seitlich links \\
\hline & 4 & $\begin{array}{l}\text { Koll. Pkw, Kopf gegen } \\
\text { Bordsteinkante }\end{array}$ & $100^{\circ}$ & 18 & 29 & Mittelklasse & Bordsteinkante & seitlich links \\
\hline \multirow[t]{11}{*}{ Generisch: } & $5 a$ & Koll. Pkw, Kopf gegen Motorhaube & $125^{\circ}$ & 0 & 15 & Mittelklasse & Motorhaube & $\begin{array}{l}\text { frontal } \\
\text { (Gesicht) }\end{array}$ \\
\hline & $5 b$ & Koll. Pkw, Kopf gegen Motorhaube & $90^{\circ}$ & 0 & 15 & Mittelklasse & Motorhaube & Hinterkopf \\
\hline & 5c & Koll. Pkw, Kopf gegen Motorhaube & $55^{\circ}$ & 0 & 15 & Mittelklasse & Motorhaube & Hinterkopf \\
\hline & $6 a$ & Koll. Pkw, Kopf gegen Motorhaube & $125^{\circ}$ & 0 & 10 & Mittelklasse & Motorhaube & $\begin{array}{c}\text { frontal } \\
\text { (Gesicht) }\end{array}$ \\
\hline & $6 b$ & Koll. Pkw, Kopf gegen Motorhaube & $90^{\circ}$ & 0 & 10 & Mittelklasse & - & - \\
\hline & $6 c$ & Koll. Pkw, Kopf gegen Motorhaube & $55^{\circ}$ & 0 & 10 & Mittelklasse & - & - \\
\hline & 7 & $\begin{array}{l}\text { Koll. Pkw, Kopf gegen } \\
\text { Frontscheibe }\end{array}$ & $90^{\circ}$ & 7,2 & 40 & Mittelklasse & Frontscheibe, zentral & seitlich links \\
\hline & 8 & $\begin{array}{l}\text { Koll. Pkw, Kopf gegen } \\
\text { Frontscheibe }\end{array}$ & $90^{\circ}$ & 7,2 & 40 & Mittelklasse & Frontscheibe, Rand & seitlich links \\
\hline & 9 & $\begin{array}{l}\text { Koll. Pkw, Kopf gegen } \\
\text { Frontscheibe }\end{array}$ & $90^{\circ}$ & 7,2 & 30 & Mittelklasse & Frontscheibe, zentral & seitlich links \\
\hline & 10 & $\begin{array}{l}\text { Koll. Pkw, Kopf gegen } \\
\text { Frontscheibe }\end{array}$ & $90^{\circ}$ & 7,2 & 50 & Mittelklasse & Frontscheibe, zentral & seitlich links \\
\hline & 11 & $\begin{array}{l}\text { Koll. Pkw, Kopf gegen } \\
\text { Frontscheibe }\end{array}$ & $90^{\circ}$ & 7,2 & 20 & Mittelklasse & Frontscheibe, zentral & Oberkopf \\
\hline
\end{tabular}




\section{Anhang 5: Simulierte Kopfanprallszenarien FE}

\begin{tabular}{|c|c|c|c|c|c|c|c|c|c|}
\hline & & & & & Kop & schw. & & & \\
\hline ID & Kurzbeschreibung & Helm & Anprallobjekt & $\begin{array}{c}\text { Kopfanprallwinkel } \\
{\left[{ }^{\circ}\right]}\end{array}$ & $\begin{array}{l}\text { linear } \\
{[\mathrm{m} / \mathrm{s}]}\end{array}$ & $\begin{array}{l}\text { angular } \\
\text { [rad/s] }\end{array}$ & $\begin{array}{l}\text { Geschwindigkeit } \\
\text { Pkw }[\mathrm{m} / \mathrm{s}]\end{array}$ & $\begin{array}{c}\text { rel. } \\
\text { Kopfanprallgeschw. } \\
{[\mathrm{m} / \mathrm{s}]}\end{array}$ & $\begin{array}{l}\text { Normalkomponente } \\
\text { Kopfanprallgeschw. } \\
{[\mathrm{m} / \mathrm{s}]}\end{array}$ \\
\hline $1 a$ & Alleinunfall, seitlich & nein & Straße & 67 & 4,1 & 17,0 & - & 4,1 & 3,7 \\
\hline $1 \mathrm{~b}$ & Alleinunfall, seitlich & ja & Straße & 67 & 4,1 & 17,0 & - & $"$ & $"$ \\
\hline $2 a$ & Alleinunfall, über Lenker & nein & Straße & 71 & 5,2 & 1,0 & - & 5,2 & 4,9 \\
\hline $2 b$ & Alleinunfall, über Lenker & ja & Straße & 71 & 5,2 & 1,0 & - & $"$ & $"$ \\
\hline $3 a$ & Kollision Pkw, Extremfall & nein & Frontscheibe /Dachkante & 94 & 9,6 & 56,9 & 15,8 & 15,0 & 14,4 \\
\hline $3 b$ & Kollision Pkw, Extremfall & ja & Frontscheibe/Dachkante & 94 & 9,6 & 56,9 & 15,8 & $"$ & " \\
\hline $4 a$ & Kopf gegen Bordsteinkante & nein & Bordsteinkante & 101 & 8,0 & 6,7 & - & 8,0 & 5,9 \\
\hline $4 b$ & Kopf gegen Bordsteinkante & ja & Bordsteinkante & 101 & 8,0 & 6,7 & - & " & " \\
\hline $4 \mathrm{c}$ & Kopf gegen Bordsteinkante & Opt. & Bordsteinkante & 101 & 8,0 & 6,7 & - & $"$ & $"$ \\
\hline 5 aа & $\begin{array}{c}\text { Kollision Pkw, Kopf gegen } \\
\text { Motorhaube }\end{array}$ & nein & Motorhaube & 88 & 4,5 & 13,8 & 4,2 & 2,6 & 2,6 \\
\hline $5 a b$ & $\begin{array}{c}\text { Kollision Pkw, Kopf gegen } \\
\text { Motorhaube }\end{array}$ & ja & Motorhaube & 88 & 4,5 & 13,8 & 4,2 & " & " \\
\hline $5 \mathrm{ac}$ & $\begin{array}{c}\text { Kollision Pkw, Kopf gegen } \\
\text { Motorhaube }\end{array}$ & nein & Motorhaube worst case & 88 & 4,5 & 13,8 & 4,2 & $"$ & $"$ \\
\hline $5 a d$ & $\begin{array}{c}\text { Kollision Pkw, Kopf gegen } \\
\text { Motorhaube }\end{array}$ & ja & Motorhaube worst case & 88 & 4,5 & 13,8 & 4,2 & $"$ & $"$ \\
\hline $7 a$ & $\begin{array}{c}\text { Kollision Pkw, Kopf gegen } \\
\text { Frontscheibe }\end{array}$ & nein & Frontscheibe zentral & 83 & 10,3 & 31,3 & 11,0 & 12,0 & 11,9 \\
\hline $7 \mathrm{~b}$ & $\begin{array}{c}\text { Kollision Pkw, Kopf gegen } \\
\text { Frontscheibe }\end{array}$ & ja & Frontscheibe zentral & 83 & 10,3 & 31,3 & 11,0 & $"$ & $"$ \\
\hline $8 a$ & $\begin{array}{c}\text { Kollision Pkw, Kopf gegen } \\
\text { Frontscheibe }\end{array}$ & nein & Frontscheibe worst case & 86 & 10,8 & 31,0 & 11,0 & 12,0 & 12,0 \\
\hline $8 b$ & $\begin{array}{c}\begin{array}{c}\text { Kollision Pkw, Kopf gegen } \\
\text { Windschutzscheibe }\end{array} \\
\end{array}$ & ja & Frontscheibe worst case & 86 & 10,8 & 31,0 & 11,0 & $"$ & $"$ \\
\hline P1 & $\begin{array}{c}\text { Szenario 4a, ohne } \\
\text { Bordsteinkante, flache Straße } \\
\end{array}$ & nein & flache Straße & 56 & 8,0 & 6,7 & - & 8,0 & 4,5 \\
\hline $\mathrm{P} 2$ & $\begin{array}{c}\text { Szenario 4b, ohne } \\
\text { Bordsteinkante, flache Straße }\end{array}$ & ja & flache Straße & 56 & 8,0 & 6,7 & - & $"$ & $"$ \\
\hline
\end{tabular}




\section{GDV}

DIE DEUTSCHEN VERSICHERER

\section{Gesamtverband der Deutschen Versicherungswirtschaft e. V.}

Wilhelmstraße 43 / 43 G, 10117 Berlin

Postfach 0802 64, 1002 Berlin

Tel. $030 / 2020$-50 00, Fax 030 / 2020 - 6000

www.gdv.de, www.udv.de 